Division

Chemical Technology

Division

Chemical Technology

Division

Chemical Technology

Division

Chemical Technology

Division

Chemical Technology

Division

Chemical Technology

Division

chemical Technology

Division

Chemical Technology

Duvision

Chemical Technology

Division

Chemical Technology

Division

Chemical Technology

Division

Chemical Technology

Division

Chemical Technology

Division

Chemical Technology

Division

Chemical Technology

Division

Chemical Technology

Division

\title{
Radiation Effects in Moist-Air Systems and the Influence of Radiolytic Product Formation on Nuclear Waste Glass Corrosion
}

by D. J. Wronkiewicz, J. K. Bates, E. C. Buck, J. C. Hoh, J. W. Emery, and L. M. Wang

RECEIVED

NOY 121997

OSTI

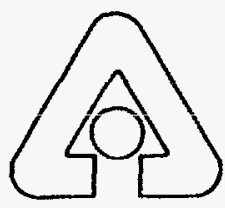

Argonne National Laboratory, Argonne, Illinois 60439

operated by The University of Chicago

for the United States Department of Energy under Contract W-31-109-Eng-38

Chemical Technology

Division

Chemical Technology

Division

Chemical Technology

Division

Chemical Technology

Duision 
Argonne National Laboratory, with facilities in the states of Illinois and Idaho, is owned by the United States government, and operated by The University of Chicago under the provisions of a contract with the Department of Energy.

\section{DISCLAIMER}

This report was prepared as an account of work sponsored by an agency of the United States Government. Neither the United States Government nor any agency thereof, nor any of their employees, makes any warranty, express or implied, or assumes any legal liability or responsibility for the accuracy, completeness, or usefulness of any information, apparatus, product, or process disclosed, or represents that its use would not infringe privately owned rights. Reference herein to any specific commercial product, process, or service by trade name, trademark, manufacturer, or otherwise, does not necessarily constitute or imply its endorsement, recommendation, or favoring by the United States Government or any agency thereof. The views and opinions of authors expressed herein do not necessarily state or reflect those of the United States Government or any agency thereof.

Reproduced from the best available copy.

Available to DOE and DOE contractors from the Office of Scientific and Technical Information

P.O. Box 62

Oak Ridge, TN 37831

P.ices available from (423) 576-8401

Available to the public from the National Technical Information Service

U.S. Department of Commerce 5285 Port Royal Road Springfield, VA 22161 


\section{DISCLAMMER}

Portions of this document may be illegible in electronic image products. Images are produced from the best available original document. 
Distribution Category:

General

(UC-2000)

ANL-97/15

\author{
ARGONNE NATIONAL LABORATORY \\ 9700 South Cass Avenue \\ Argonne, Illinois 60439
}

\title{
RADIATION EFFECTS IN MOIST-AIR SYSTEMS AND THE INFLUENCE OF RADIOLYTIC PRODUCT FORMATION ON NUCLEAR WASTE GLASS CORROSION
}

by

D. J. Wronkiewicz, J. K. Bates, E. C. Buck, J. C. Hoh, J. W. Emery, and L. M. Wang*

Chemical Technology Division

Argonne National Laboratory

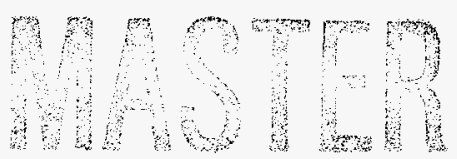

July 1997

*Department of Geology, The University of New Mexico. 


\section{TABLE OF CONTENTS}

Page

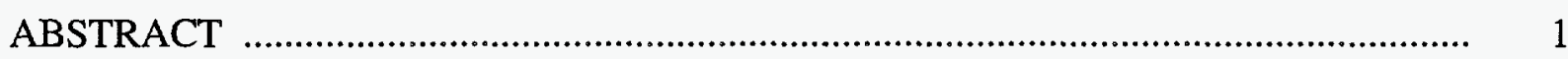

EXECUTIVE SUMMARY ……………...................................................................

I INTRODUCTION ................................................................................ 5

II. EXPERIMENTAL _...................................................................................... 7

A. Sample Matrix ......................................................................................... 7

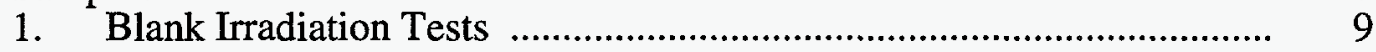

2. Irradiation Tests with Glass ............................................................... 11

B. Glass Sample Preparation ................................................................. 14

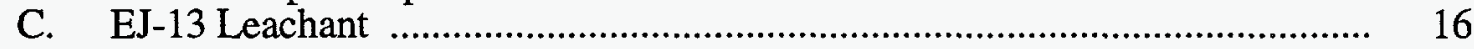

D. Analytical Procedure ……............................................................................ 18

1. Test Termination ....................................................................... 18

2. Leachate Analytical Techniques …………………………………... 19

3. Solid-Phase Analytical Techniques ..................................................... 19

E. Oven Dosimetry ...................................................................................... 21

III. SOLUTION RESULTS ……….................................................................... 23

A. Blank Tests with Gamma Irradiation ........................................................ 23

1. Anion Concentrations .......................................................................... 23

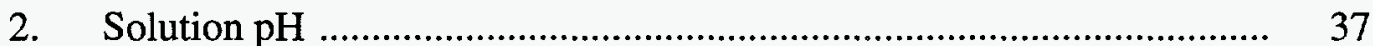

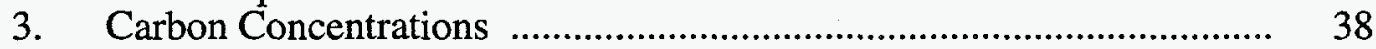

B. Blank Tests with Alpha Irradiation ................................................................. 40

1. Anion Concentrations .......................................................................... 40

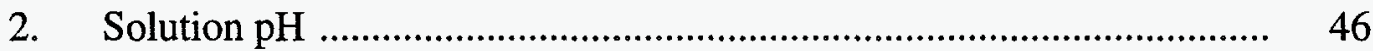

3. Carbon Concentrations ........................................................................ 46

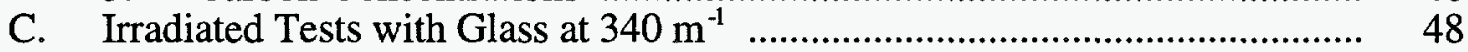

1. Anion Concentrations ........................................................................ 48

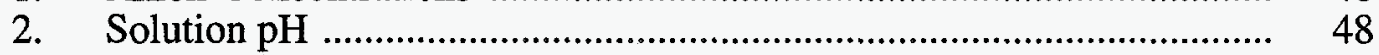

3. Carbon Concentrations .................................................................... 56

4. Cation Concentrations (Nonradionuclides) …………………………..... 57

5. Normalized Elemental Mass Loss (Nonradionuclides) ........................ $\quad 62$

6. Transuranic Element and Technetium Concentrations ......................... 68

IV. SURFACE ANALYTICAL RESULTS _......................................................... 71

A. Batch Leach Tests at $340 \mathrm{~m}^{-1}$............................................................... 71

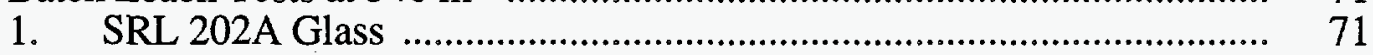

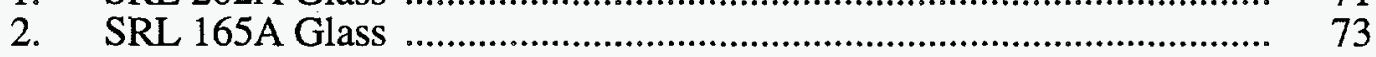

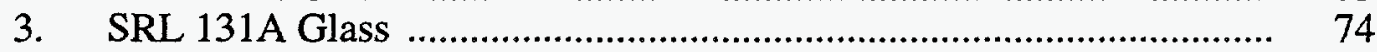

B. Vapor Hydration Tests ............................................................................ 76

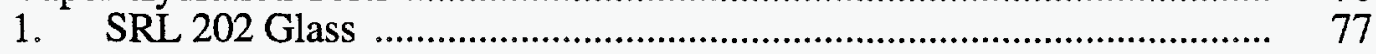

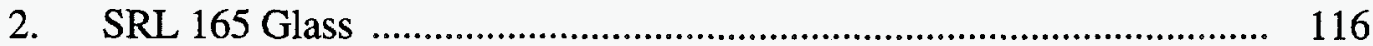

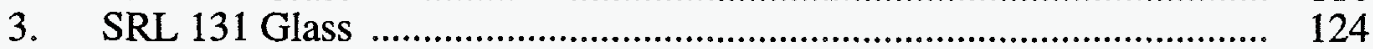


A. Blank Irradiation Tests

B. Batch Tests at $340 \mathrm{~m}^{-1}$

C. Vapor Hydration Tests

1. Reaction Layer Development on SRL 202 Glass

2. Surface Precipitate Formation on SRL 202 Glass

3. Reaction Layer Development on SRL 165 Glass

4. Surface Precipitate Formation on SRL 165 Glass

5. Reaction Layer Development on SRL 131 Glass

6. Surface Precipitate Formation on SRL 131 Glass

D. Comparison with Natural Analogues

VI. CONCLUSIONS

APPENDIX A. Raw Analytical Data _..................................................................... 208

APPENDIX B. Alpha Counting Results ............................................................... 211

APPENDIX C. Gamma Counting Results 


\section{LIST OF TABLES}

1. Sampling Matrix for Alpha and Gamma Blank Tests ................................ 8

2. Sampling Matrix for Saturated Batch Leach Tests ............................................. 8

3. Sampling Matrix for Vapor Hydration Tests .................................................. 9

4a. Compositions of Simulated Reference Nuclear Waste Glasses ........................... 15

4b. Technetium and Transuranic Dopant Levels for SRL 131A, SRL 202A, and SRL 165A Glasses

5. Alpha and Beta Activity Recorded for Selected Monoliths of SRL 202A and SRL 165 Glass

6. Equilibrated J-13 (EJ-13) Groundwater Leachant Composition (ppm) ................ . 17

7. Sampling Matrix for Blank Gamma Tests ...................................................... 25

8a. Anion Results for Blank Gamma Tests ..................................................... 26

8b. Raw Anion Results for Blank Gamma Tests ................................................. 28

9a. Carbon Species $(\mu \mathrm{mol})$ and pH Results for Gamma Blank Tests ....................... 30

9b. Carbon Species (ppm) and pH Results for Gamma Blank Tests......................... 32

10. Results from G-Value Calculations for Alpha and Gamma Blank Tests .............. 34

11. Sampling Matrix for Blank Alpha Tests .......................................................... 41

12a. Anion Results for Alpha Blank Tests (values in total $\mu$ mol produced).................. 42

12b. Anion Results for Alpha Blank Tests (values in ppm) ................................. 43

13a. Carbon Species $(\mu \mathrm{mol})$ and $\mathrm{pH}$ Results for Alpha Blank Tests ........................... 44

13b. Carbon Species (ppm) and pH Results for Alpha Blank Tests ........................... 45

14. Sampling Matrix for Batch Leach Tests ...................................................... 49

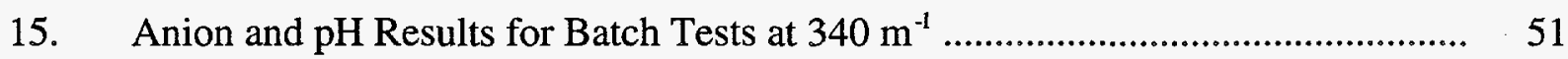

16. $\mathrm{NO}_{3}{ }^{-} / \mathrm{NO}_{2}{ }^{-}$Ratios from Irradiation Tests ............................................... 53

17. Dilution-Corrected Cation Results for $340 \mathrm{~m}^{-1}$ Batch Leach Tests ....................... 58

18. Normalized Release $\left(\mathrm{g} / \mathrm{m}^{2}\right)$ of Elements for $340 \mathrm{~m}^{-1}$ Batch Leach Tests ............... 63

19. Averaged Alteration Layer Thickness from Vapor Hydration Tests.................... 80 


\section{LIST OF TABLES (contd.)}

20. X-Ray Diffraction Indexing Data for Selected Mineral Phases Obtained from Sample Surfaces in Vapor Hydration Tests with 202U Glass

21. SEM/EDS Compositional Analysis of Clay Layer and Unaltered Glass from SRL 202U Sample Reacted in Vapor Hydration Test for 14 Days at $200^{\circ} \mathrm{C}$

22. Selected Area Diffraction Data Obtained from Inner Clay Layer of Three-Day 131U Samples and JCPDS Data for Montmorillonite

23. AEM/EDS Compositional Analysis of Clay Layers Formed on SRL 131U Glass Samples Reacted in Vapor Hydration Tests

24. Calculated Clay Mineral Fractions (in percent) in Reacted Clay Layers Based on Analyzed Layer Composition of 131U Samples 


\section{LIST OF FIGURES}

1. Experimental Flowchart for Radiation Testing ………......................................... 7

2. Experimental Apparatus for Alpha Radiolysis Tests.............................................. 10

3. Experimental Apparatus for $340 \mathrm{~m}^{-1}$ Batch Tests with Glass Monoliths. .............. 11

4. Experimental Apparatus for Vapor Hydration Tests with Glass Monoliths............ 12

5. Total $\mu$ moles of Nitrate + Nitrite Recovered in Blank Gamma Tests as a Function of Cumulative Exposure Dose at $25^{\circ} \mathrm{C}$.

6. Measured Nitrate + Nitrite Formation in $\sim 35 \mathrm{~Gy} / \mathrm{h}$ Blank Gamma Tests as a Function of Cumulative Exposure Dose.

7. Solution $\mathrm{pH}$ Values for the Gamma Blank Tests Plotted as a Function of Cumulative Exposure Dose at $25^{\circ} \mathrm{C}$

8. Measured Organic Carbon Formation in Blank Gamma Tests as a Function of Cumulative Exposure Dose for the $\sim 35$ and $\sim 500 \mathrm{~Gy} / \mathrm{h}$ Tests at $25^{\circ} \mathrm{C}$.

9. Measured Organic Carbon Formation in Gamma Blank Tests as a Function of Cumulative Exposure Dose for the $\sim 35$ and $\sim 500 \mathrm{~Gy} / \mathrm{h}$ Tests.

10. Total $\mu$ moles of Nitrate + Nitrite Recovered in Blank Alpha Tests as a Function of Cumulative Dose at $25^{\circ} \mathrm{C}$. The $\mathrm{G}\left(\mathrm{NO}_{\mathrm{x}}\right)=2.4$ line represents the average $\mathrm{G}\left(\mathrm{NO}_{3}{ }^{-}+\mathrm{NO}_{2}{ }^{-}\right)$yield for all alpha tests.

11. Solution $\mathrm{pH}$ Values for the Blank Alpha Tests Plotted as a Function of Cumulative Exposure Dose.

12. Measured Organic Carbon Formation in Blank Alpha Tests (Stainless

Steel Support Rods) as a Function of Cumulative Exposure Dose.

13. Concentration of Nitrate Plus Nitrite as a Function of Cumulative Exposure

Time in Solutions from Gamma-Irradiated Batch Tests with SRL 202A, SRL 165A, and SRL 131A Glasses $\left(\mathrm{S} / \mathrm{V}=340 \mathrm{~m}^{-1}, \mathrm{~T}=90^{\circ} \mathrm{C}\right.$, Dose

Rate $\sim 35 \mathrm{~Gy} / \mathrm{h}$ )

14. Nitrate/Nitrite Ratios in Blank Irradiation Tests as a Function of Cumulative Exposure Time

15. Solution $\mathrm{pH}$ Trends from $340 \mathrm{~m}^{-1}$ Batch Tests Exposed to an External ${ }^{60} \mathrm{Co}$ Gamma Source of $\sim 35 \mathrm{~Gy} / \mathrm{h}$.

16. Concentration Trends from $340 \mathrm{~m}^{-1}$ Batch Tests Exposed to External ${ }^{60} \mathrm{Co}$ Gamma Source of $\sim 35 \mathrm{~Gy} / \mathrm{h}$.

17. Normalized Element Release Results from $340 \mathrm{~m}^{-1}$ Batch Tests Exposed to an External ${ }^{60} \mathrm{Co}$ Gamma Radiation Source of $\sim 35 \mathrm{~Gy} / \mathrm{h}$. 


\section{LIST OF FIGURES (contd.)}

18. Transuranic Element Release from $340 \mathrm{~m}^{-1}$ Batch Tests Exposed to External

${ }^{60} \mathrm{Co}$ Gamma Radiation Source of $\sim 35 \mathrm{~Gy} / \mathrm{h}$.

19. AEM Photomicrograph of Alteration Layer Cross Sections from $340 \mathrm{~m}^{-1}$

Batch Test with SRL 202A Glass

20. AEM Photomicrograph of Colloidal Material from $340 \mathrm{~m}^{-1}$ Batch Test with

SRL 202A Glass at 180 Days

21. AEM Photomicrograph of Colloidal Clay Particles Filtered from $340 \mathrm{~m}^{-1}$ Test with SRL 165A Glass at 91 Days

22. AEM Photomicrograph of Colloidal Material from $340 \mathrm{~m}^{-1}$ Test with SRL $131 \mathrm{~A}$ Glass at 180 Days

23. Optical Photographs of the Reacted Surfaces of SRL 202U Glass Samples from Vapor Hydration Tests at $200^{\circ} \mathrm{C}$

24. SEM/EDS Spectra of SRL 202U Sample from $200^{\circ} \mathrm{C}$ Vapor Hydration

Test at 7 Days

25. SEM Images of Secondary Alteration Phases Formed on SRL 202U Glass

Reacted 7 Days in Vapor Hydration Test

26. Secondary Electron Image of Secondary Alteration Phase Formed on

SRL 202U Glass Reacted 14 Days in Vapor Hydration Test.

27. AEM/EDS Spectra from SRL 202U Glass Samples Used in Vapor

Hydration Testing

28. AEM Images of Slightly Porous Smectite Layer from SRL 202U

Glass Reacted for 14 Days in Saturated Vapor Environment at $200^{\circ} \mathrm{C}$

29. AEM Images of Na-Rich Chabazite (var. Herschelite) Phase from SRL 202U

Glass Reacted for 14 Days in Saturated Vapor Environment at $200^{\circ} \mathrm{C}$

30. Photomicrographs of Unidentified Ca-Si Phase from SRL 202U Glass Reacted for 14 Days in Saturated Vapor Environment at $200^{\circ} \mathrm{C}$

31. SEM Images of Secondary Alteration Phases Formed on SRL 202U Glass

Reacted for 21 Days in Saturated Vapor Environment at $200^{\circ} \mathrm{C}$

32. SEM/EDS Spectra of Phases from SRL 202U Samples Reacted For 21

Days in Saturated Vapor Environment at $200^{\circ} \mathrm{C}$

33. SEM Images of Secondary Alteration Phases Formed on SRL 202U

Glass Reacted for 35 Days in Saturated Vapor Environment at $200^{\circ} \mathrm{C}$

34. SEM/EDS Spectra of SRL 202U Glass from 35-Day Vapor Hydration Test 


\section{LIST OF FIGURES (contd.)}

35. SEM Images of Secondary Alteration Phases Formed on SRL 202U Glass from Vapor Hydration Tests

36. SEM/EDS Spectrum of SRL 202U Sample from Vapor Hydration Test at 56 Days: $\mathrm{K}-\mathrm{U}-\mathrm{Al}-\mathrm{Si}$ Layer Material....

37. SEM Images of Secondary Alteration Phases Formed on SRL 202U Glass

from Vapor Hydration Test at 56 Days

38. SEM/EDS Spectra of SRL 202U Samples from Vapor Hydration

Testing at 56 Days ....

39. SEM Images of Secondary Alteration Phases Formed on SRL 202U Glass from Vapor Hydration Tests at 56 Days

40. Optical Photographs of the Reacted Surfaces of SRL 202A Glass Samples from the $200^{\circ} \mathrm{C}$ Vapor Hydration Tests Exposed to a Dose Rate of $\sim 35 \mathrm{~Gy} / \mathrm{h}$.......

41. SEM Images of Secondary Alteration Phases Forming on SRL 202A Glass Samples from the $200^{\circ} \mathrm{C}$ Vapor Hydration Tests Exposed to a Dose Rate of $\sim 35 \mathrm{~Gy} / \mathrm{h}$

42. SEM/EDS Spectra from Various Alteration Phases Formed on the 7-Day SRL 202A Samples from the $200^{\circ} \mathrm{C}$ Vapor Hydration Tests Exposed at Dose Rate of $\sim 35 \mathrm{~Gy} / \mathrm{h}$

43. SEM Images of Secondary Alteration Phases Forming on SRL 202A

Glass Samples from the $200^{\circ} \mathrm{C}$ Vapor Hydration Tests Exposed for 14 Days at Dose Rate of $\sim 35 \mathrm{~Gy} / \mathrm{h}$

44. SEM Image of Blocky Adularia Crystals and Bright Copper Oxide Crystals Forming on 21-Day SRL 202A Sample from the $200^{\circ} \mathrm{C}$ Vapor Hydration Test Exposed to Dose Rate of $\sim 35 \mathrm{~Gy} / \mathrm{h}$.

45. SEM Images of Secondary Alteration Phases Forming on 35-Day SRL 202A Samples from the $200^{\circ} \mathrm{C}$ Vapor Hydration Tests Exposed to Dose Rate of $\sim 35 \mathrm{~Gy} / \mathrm{h}$.

46. SEM/EDS Spectra from Various Alteration Phases Formed on 35-Day

SRL 202A Samples from the $200^{\circ} \mathrm{C}$ Vapor Hydration Tests Exposed to a Dose

Rate of $\sim 35 \mathrm{~Gy} / \mathrm{h}$.

47. SEM Images of Secondary Alteration Phases Forming on 35-Day

SRL 202A Samples from the $200^{\circ} \mathrm{C}$ Vapor Hydration Tests Exposed to

Dose Rate of $\sim 35 \mathrm{~Gy} / \mathrm{h}$

48. SEM/EDS Spectra from Various Alteration Phases Formed on 35-Day SRL 202A Samples from the $200^{\circ} \mathrm{C}$ Vapor Hydration Tests Exposed to Dose Rate of $\sim 35 \mathrm{~Gy} / \mathrm{h}$ 


\section{LIST OF FIGURES (contd.)}

49. SEM Images of Secondary Alteration Phases Forming on 56-Day

SRL 202A Samples from the $200^{\circ} \mathrm{C}$ Vapor Hydration Tests Exposed to

Dose Rate of $\sim 35 \mathrm{~Gy} / \mathrm{h}$

50. Optical Photographs of the Reacted Surfaces of SRL 165A Samples

from the $200^{\circ} \mathrm{C}$ Vapor Hydration Tests Exposed to Dose Rate of $\sim 35 \mathrm{~Gy} / \mathrm{h}$

51. SEM/EDS Spectra from Various Alteration Phases Formed on 14-Day SRL 165A Samples from the $200^{\circ} \mathrm{C}$ Vapor Hydration Tests Exposed to Dose Rate of $\sim 35 \mathrm{~Gy} / \mathrm{h}$.

52. SEM Images of Secondary Alteration Phases Forming on SRL

165A Samples from the $200^{\circ} \mathrm{C}$ Vapor Hydration Tests Exposed to

Dose Rate of $\sim 35 \mathrm{~Gy} / \mathrm{h}$

53. SEM Images of Secondary Alteration Phases Forming on 35-Day SRL

165A Samples from the $200^{\circ} \mathrm{C}$ Vapor Hydration Tests Exposed to Dose

Rate of $\sim 35 \mathrm{~Gy} / \mathrm{h}$.

54. SEM/EDS Spectra from Various Alteration Phases Formed on 35-day

SRL 165A Samples from the $200^{\circ} \mathrm{C}$ Vapor Hydration Tests Exposed to

Dose Rate of $\sim 35 \mathrm{~Gy} / \mathrm{h}$

55. SEM Images of Secondary Alteration Phases Forming on 56-Day

SRL 165A Samples from the $200^{\circ} \mathrm{C}$ Vapor Hydration Tests Exposed

to Dose of $\sim 35 \mathrm{~Gy} / \mathrm{h}$

56. AEM Photomicrographs of Alteration Layer Cross Section from

SRL 131U Glass Samples Reacted in Vapor Hydration Test at $150^{\circ} \mathrm{C}$

for Three Days

57. Low-Magnification AEM Photomicrograph of Alteration Layer Cross Section from SRL 131U Glass Samples Reacted in Vapor Hydration Test at $150^{\circ} \mathrm{C}$ for Five Days Showing Oriented Outer Clay Layer and Random-Oriented Inner Clay Layer

58. SEM Image of Fine-Grained Smectite Growing over Glass Surface on 3-Day Sample of SRL 131U Glass

59. Optical Photographs of Reacted Surfaces on SRL 131U Samples from the $150^{\circ} \mathrm{C}$ Vapor Hydration Tests. All photographs at $9 \mathrm{X}$ with disk diameter $\sim 10 \mathrm{~nm}$

60. Secondary Electron Image of Na-Rich Chabazite (Herschelite) Disk Growing on Smectite Surface for 14-Day Sample of SRL 131U Glass

61. AEM Photomicrograph of Alteration Layer Cross Section from SRL 131U Glass Samples Reacted in Vapor Hydration Test at $150^{\circ} \mathrm{C}$ for 56 Days. 


\section{LIST OF FIGURES (contd.)}

62. AEM Photomicrograph of Alteration Layer Cross Section from SRL 131U Glass Samples Reacted in Vapor Hydration Test at $150^{\circ} \mathrm{C}$ for 91 Days

63. SEM Image of Ca-Si Nodules Forming on 91-Day Sample of SRL 131U Glass

64. SEM/EDS Spectrum of Si-Al-P-La Phase Formed on the SRL 131U

Samples from Vapor Hydration Tests at 91 Days. Spectrum taken at $20 \mathrm{keV}$ accelerating potential

65. AEM Photomicrograph of Random-Oriented Inner Clay Layer from

SRL 131U Glass Samples Reacted in Vapor Hydration Tests at $150^{\circ} \mathrm{C}$ for 180 Days

66. SEM Images of Secondary Alteration Phases Forming on 182-Day Sample of SRL 131U Glass.

67. SEM/EDS Spectrum of Haiweeite Formed on 182-Day SRL 131U Sample from Vapor Hydration Tests.

68. SEM/EDS Spectra of Alteration Phases Formed on 365-Day SRL 131U Samples from Vapor Hydration Test.

69. SEM Images of Secondary Alteration Phases Forming on 365-Day Sample of SRL $131 \mathrm{U}$ Glass

70. SEM/EDS Spectra of Alteration Phases Formed on the SRL 131U Samples in Vapor Hydration Tests at 365 Days

71. SEM Secondary Electron Image of Tobermorite Needles on Clay Surface of 540-Day Sample of SRL 131 U Glass.

72. SEM/EDS Spectrum of Si-P Buttons Formed on the SRL 131U Samples from Vapor Hydration Test after 540 Days.

73. SEM/EDS Spectra of (a) Tobermorite and (b) Soddyite Formed on the SRL 131U Sample in Vapor Hydration Tests after 720 Days

74. SEM Secondary Electron Image of Bladed Soddyite Crystals Displaying Delamina-tion Features and Fine-Grained Radiating Ca-Si Phase on 720-Day Sample of SRL 131U Glass.

75. Optical Photographs of Reacted Surfaces of SRL 131A Glass Samples from the $150^{\circ} \mathrm{C}$ Vapor Hydration Tests Exposed to a Dose Rate of $\sim 35 \mathrm{~Gy} / \mathrm{h}$

76. SEM Image of Large Agglomeration of Radiating Weeksite Needles, $\mathrm{Ca}-\mathrm{Si}$ Fibers (upper-center), and Ca-Si Flower-Shaped Phase on 7-Day Sample of SRL 131A Glass from the $150^{\circ} \mathrm{C}$ V apor Hydration Tests Exposed to Dose Rate of $\sim 35 \mathrm{~Gy} / \mathrm{h}$. 


\section{LIST OF FIGURES (contd.)}

77. SEM/EDS Spectrum of Ca-Si Needles Formed on SRL 131A Samples from $150^{\circ} \mathrm{C}$ Vapor Hydration Tests Exposed to Dose Rate of $\sim 35 \mathrm{~Gy} / \mathrm{h}$ at 7 Days

78. SEM Images of Secondary Alteration Phases Forming on 14-Day Sample of SRL 131A Glass from the $150^{\circ} \mathrm{C}$ Vapor Hydration Tests Exposed to Dose Rate of $\sim 35 \mathrm{~Gy} / \mathrm{h}$

79. SEM/EDS Spectrum of (a) Weeksite and (b) Ca-Si-P Disks Formed on the SRL 131A Samples from the $150^{\circ} \mathrm{C}$ Vapor Hydration Tests Exposed to Dose Rate of $\sim 35 \mathrm{~Gy} / \mathrm{h}$.

80. AEM Photomicrograph of Alteration Layer Cross Section from SRL 131A Glass Samples Reacted in Vapor Hydration Test at $150^{\circ} \mathrm{C}$ for 14 Days

81. AEM/EDS Spectra from 14-Day SRL 131A Glass Sample from Fig. 80a

82. SEM/EDS Spectrum of (a) Surface Clays, (b) Raised Mounds, and (c) Flat-Lying Altered Glass Surface Formed on SRL 131A Samples from the $150^{\circ} \mathrm{C}$ Vapor Hydration Tests Exposed to Dose Rate of $\sim 35 \mathrm{~Gy} / \mathrm{h}$ for 21 Days

83. SEM Images of Secondary Alteration Phases Forming on 21-Day Sample of SRL 131A Glass from the $150^{\circ} \mathrm{C}$ Vapor Hydration Tests Exposed to Dose Rate of $\sim 35 \mathrm{~Gy} / \mathrm{h}$.

84. SEM/EDS Spectrum of Low Atomic Weight Salt Deposited on Pt-Rh Wire from Vapor Hydration Test Sample with SRL $131 \mathrm{~A}$ Glass at $150^{\circ} \mathrm{C}$

85. SEM Images of Secondary Alteration Phases Forming on 35-Day Sample of SRL 131A Glass from the $150^{\circ} \mathrm{C}$ Vapor Hydration Tests Exposed to Dose Rate of $\sim 35 \mathrm{~Gy} / \mathrm{h}$

86. SEM/EDS Spectrum of Apatite Formed on 35-Day SRL 131A Samples from $150^{\circ} \mathrm{C}$ Vapor Hydration Tests Exposed to Dose Rate of $\sim 35 \mathrm{~Gy} / \mathrm{h}$.

87. SEM/EDS Spectrum of Smectite Clay Layer Formed on 56-Day SRL 131A Samples from the $150^{\circ} \mathrm{C}$ Vapor Hydration Tests Exposed to Dose Rate of $\sim 35 \mathrm{~Gy} / \mathrm{h}$

88. Calcium-Silicon Phase (possibly tobermorite) Found within Altered Clay Layer in SRL 131A Glass Sample Reacted in Vapor Hydration Test at $150^{\circ} \mathrm{C}$ for 56 Days

89. SEM Backscattered Electron Image of Phase Forming on 56-Day Sample of SRL 131A Glass from $150^{\circ} \mathrm{C}$ Vapor Hydration Tests Exposed to Dose Rate of $\sim 35 \mathrm{~Gy} / \mathrm{h}$.

90. Nitrate/Nitrite Ratio for Blank Gamma Tests 


\section{LIST OF FIGURES (contd.)}

91. Average Alteration Layer Thickness Measured from Cross-Sectioned

SRL 202A and SRL 202U Glass Samples Reacted in Vapor Hydration

Tests at $200^{\circ} \mathrm{C}$

92. Alteration Mineral Paragenetic Sequence Identified for SRL 202 Glasses

Reacted in Vapor Hydration Tests at $200^{\circ} \mathrm{C}$

93. SEM Photomicrographs of SRL 202 Glasses Reacted in the Vapor Hydration

Tests for 35 Days at $200^{\circ} \mathrm{C}$.

94. Average Alteration Layer Thickness Measured from Cross-Sectioned

SRL 165A and SRL 165U Glass Samples Reacted in Vapor Hydration

Tests at $200^{\circ} \mathrm{C}$

95. Alteration Mineral Paragenetic Sequence Identified for SRL 165 Glasses

Reacted in Vapor Hydration Tests at $200^{\circ} \mathrm{C}$ While Exposed to a Gamma

Dose Rate of $\sim 35 \mathrm{~Gy} / \mathrm{h}$.

96. Average Alteration Layer Thickness Measured from Cross-Sectioned

SRL 131A and SRL 131U Glass Samples Reacted in Vapor Hydration

Tests at $150^{\circ} \mathrm{C}$

97. Average Alteration Layer Thickness Measured from Cross-Sectioned

SRL 131 U Glass Samples Reacted in Vapor Hydration Tests as a Function

of Reaction Time and Temperature

98. Element Concentrations in the Outer Oriented and Inner Random Clay

Alteration Layers in SRL 131U Glass Normalized to the Concentration of the Respective Elements in the Unaltered Glass

99. Alteration Mineral Paragenetic Sequence Identified for SRL 131 Glasses

Reacted in Vapor Hydration Tests at $150^{\circ} \mathrm{C}$

100. SEM Photomicrographs of SRL 131 Glasses Reacted in Vapor Hydration Tests for 35 Days at $150^{\circ} \mathrm{C}$

101. (a) Mineral Zonation Patterns and Paragenesis Developed during Volcanic Glass Alteration in Saline-Alkaline Lakes as Function of Increasing Water Salinity and Alkalinity from Basin Margin to Basin Center. (b) Mineral Zonation Patterns and Paragenesis Developed during Simulated SRL 202U Borosilicate Waste Glass Alteration in Vapor Saturated-Air Environment at $200^{\circ} \mathrm{C}$.

102. Mineral Paragenesis Developed During Silicic Volcanogenic Sediment Alteration, and Mineral Zonation Patterns Developed During Simulated SRL 202U Borosilicate Waste Glass Alteration in a Vapor Saturated-Air Environment at $200^{\circ} \mathrm{C}$ 


\title{
RADIATION EFFECTS IN MOIST-AIR SYSTEMS AND THE INFLUENCE OF RADIOLYTIC PRODUCT FORMATION ON NUCLEAR WASTE GLASS CORROSION
}

\author{
D. J. Wronkiewicz, J. K. Bates, E. C. Buck, J. C. Hoh, J. W. Emery, \\ and L. M. Wang*
}

Chemical Technology Division

Argonne National Laboratory

*Department of Geology

The University of New Mexico

\begin{abstract}
Ionizing radiation may affect the performance of glass in an unsaturated repository site by interacting with air, water vapor, or liquid water to produce a variety of radiolytic products. Tests were conducted to examine the effects of radiolysis under high gas/liquid ratios. Results indicate that nitrate is the predominant radiolytic product produced following both gamma and alpha radiation exposure, with lesser amounts of nitrite and carboxylic acids. The formation of nitrogen acids during exposure to long-lived, alpha-particle-emitting transuranic elements indicates that these acids may play a role in influencing nuclear waste form reactions in a long-term unsaturated disposal scenario.

Experiments were also conducted with samples that simulate the composition of Savannah River Plant nuclear waste glasses. Radiolytic product formation in batch tests $\left(340 \mathrm{~m}^{-1}, 90^{\circ} \mathrm{C}\right)$ resulted in a small increase in the release rates of many glass components, such as alkali and alkaline earth elements, although silicon and uranium release rates were slightly reduced indicating an overall beneficial effect of radiation on waste form stability. The silicon decrease probably results from the lowering of the activity of silicic acid with decreasing $\mathrm{pH}$, while the uranium decrease may be correlated to the increased stability of the host silicate glass matrix, as well as a decrease in the stability of uranyl carbonate complexes at lower $\mathrm{pH}$ values. By contrast, radiation exposure in the vapor hydration tests $\left(150-200^{\circ} \mathrm{C}\right.$; predominantly external gamma exposure) has resulted in a four- to ten-fold increase of alteration layer thickness relative to samples reacted without radiation exposure. These increases for the irradiated experiments appear to result from the condensation of radiolytic acids into the thin film of water contacting the glass surface. The radiolytic acids increased the rate of ion exchange between the glass and the thin film of condensate, resulting in accelerated corrosion rates for the glass.
\end{abstract}


The paragenetic sequence of alteration phases formed on both the irradiated and nonirradiated glass samples reacted in the vapor hydration tests matches closely with those developed during volcanic glass alteration in naturally occurring saline-alkaline lake systems. This similarity arises because the thin film of water present on the simulated waste glass samples is transformed into a salinealkaline fluid by the release of glass components. This correspondence suggests that the high temperatures used in these tests have not changed the underlying glass reaction mechanism relative to that which controls glass reactions under ambient surficial conditions. 


\section{EXECUTIVE SUMMARY}

Ionizing radiation may affect the long-term performance of glass in an unsaturated repository site by interacting with air, water vapor, or liquid water to produce a variety of radiolytic products. The present study examines (1) the effects of alpha and gamma irradiation in producing radiolytic products in an unsaturated environment dominated by water vapor; (2) the influence of radiolytic products on the rate of glass alteration; and (3) the influence of radiolytic products on the stability of alteration minerals.

A series of "blank tests" was conducted without glass samples present to examine the effects of radiolysis on gas/liquid (G/L) ratio $(10$ and 100$)$, temperature $\left(25-200^{\circ} \mathrm{C}\right)$, and dose rate $(30$ and $500 \mathrm{~Gy} / \mathrm{h})$. Results indicate that nitrate is the predominant radiolytic product formed under both gamma and alpha radiation exposure, with high $\mathrm{NO}_{3}{ }^{-} \mathrm{NO}_{2}{ }^{-}$ratios indicating that oxidizing conditions persist during irradiation. Lesser amounts of carboxylic acid species are also produced. For a given cumulative dose, nitrogen acid species concentrations were noted to vary inversely with temperature, but there was little change as a function of dose rate or gas/liquid ratios. The decrease with temperature is important from the standpoint of waste form and container stability in that the elevated temperatures expected early in the life of the repository may mitigate against large decreases in $\mathrm{pH}$ resulting from nitrogen acid production. The formation of nitrogen acid species due to irradiation from long-lived, alpha-particle-emitting transuranic elements indicates that nitrogen acids may play a role in influencing nuclear waste form reactions in a long-term disposal scenario.

Tests were also conducted with samples of SRL 131, SRL 165, and SRL 202 glasses irradiated at $30 \mathrm{~Gy} / \mathrm{h}$ in both vapor hydration $\left(150\right.$ and $\left.200^{\circ} \mathrm{C}\right)$ and batch $\left(90^{\circ} \mathrm{C}, 340 \mathrm{~m}^{-1}\right)$ test conditions in an air-dominated, air + water system. A comparison of glass reactions between irradiated and nonirradiated test conditions is used to evaluate what influence the irradiation environment has on glass alteration rates and alteration phase stability. Radiolytic product formation in the batch tests resulted in little change in the release rate of many components from the glass. A small increase in $\mathrm{B}, \mathrm{Na}$, and $\mathrm{K}$ release from the irradiated tests is noted, relative to the release of these components from the nonirradiated SRL 202 glass, for experiments conducted over the one-to-two-year testing interval. By contrast, $\mathrm{Si}$ and $\mathrm{U}$ release rates were slightly reduced in the irradiated tests relative to nonirradiated tests, indicating a beneficial effect of irradiation exposure on waste glass durability. This decrease is attributed to a decrease in the solution $\mathrm{pH}$ during radiation exposure and a resultant decrease in the activity of silicic acid and uranyl bicarbonate complexes.

Exposure to the irradiated environment in the vapor hydration experiments has led to the rate of alteration layer development being increased by a factor of ten in tests with SRL 202A glass at $200^{\circ} \mathrm{C}$ and by a factor of only four to five in tests with SRL 131 and SRL 165 glasses. These increases are attributed to the condensation of radiolytic acids in the thin film of water present on the glass surface. The radiolytic acids increased the rate of ion exchange between the glass and the thin film of water contacting the sample, thereby leading to accelerated corrosion rates for the glass. Some enhanced growth of alteration layers was also noted along fractures in the irradiated glasses, indicating penetration of fluids along these fracture surfaces. The irradiation environment also accelerated the formation sequence of secondary phases on the altered glass surfaces of the vapor hydration test samples. In some cases, however, the irradiation exposure produced phases that were not present on the nonirradiated tests, although the phases may have eventually formed if the nonirradiated tests were allowed to continue for longer periods. It appears that the release of glass components via an ion-exchange process in the irradiated tests neutralized the radiolytic acids that condensed on the samples to a sufficient extent where the alteration mineralogy was not dramatically changed. The accelerated alteration paragenesis in the irradiated tests is, therefore, attributed to the increased release rate of glass 
components, which, in turn, results in an accelerated saturation of the leachate with respect to the various secondary phases that have formed. The increased release rate of glass components would also result in the increased rate of alteration layer development.

- The paragenetic sequence of phases identified on the simulated nuclear waste glasses altered in the vapor hydration tests also matches closely with those that developed as a result of the alteration of volcanic glasses deposited in saline-alkaline lake systems. This similarity occurs because the rapid concentration increases in the thin films of water present on the altered glass samples quickly transform this film into a saline-alkaline fluid. This observation also suggests that the high temperatures used in these tests have not changed the reaction mechanism relative to that which will control glass reactions under ambient surficial conditions. 


\section{INTRODUCTION}

The emplacement of nuclear waste in a geologic repository will subject the immediate environment of the waste container to radiation effects. In the event of container failure, the radioactive waste will also be exposed to radiolytic products formed by the interaction of radiation with the air and liquid present in the repository environment. The influence of these radiolytic products is an important consideration for radionuclide immobilization because of their ability to corrode the waste containers and radioactive waste forms, thereby increasing the potential for the release of radioactive materials to the accessible environment. The purpose of this present study is to examine the potential effects of radionuclide decay on a moist-air environment and the effects that radiolysis products may have on waste glass corrosion in an unsaturated repository setting.

The intensities of the various types of radioactivity emanating from glass and spent nuclear fuel waste forms will vary as the nuclear waste ages. During the first several hundred years of repository operation, the radioactive energy is expected to be dominated by beta and gamma emissions from fission products with relatively short half-lives, such as ${ }^{137} \mathrm{Cs}$ and ${ }^{90} \mathrm{Sr}$ [1]. Transuranic elements that decay to emit alpha particles generally have longer half-lives (from hundreds to tens of thousands of years) and will become the dominant radiation source at longer times. Because of their low penetrability in solids, alpha and beta particles can interact with the environment surrounding the waste container only after it has been breached and the air plus water environment of the repository has contacted the waste. Gamma radiation can penetrate the waste container walls and thus may interact with the environment surrounding the waste package immediately after emplacement. The relatively short half-lives of gammaemitting sources, however, lessen their importance as the waste ages [1].

Many studies have been conducted to investigate the effects of radiation on glass alteration in air-water systems. Ionizing radiation will electronically excite and ionize water molecules and dissolved gases to form reactive radicals and new molecules [2]. The predominant water radiolysis species include hydrated electrons $\left(\mathrm{e}_{\mathrm{aq}}^{-}\right)$, hydrogen ions $\left(\mathrm{H}^{+}\right)$, hydroxyl $(\bullet \mathrm{OH})$, hydroperoxyl $\left(\mathrm{HO}_{2}{ }^{-}\right)$, hydrogen atoms $(\mathrm{H} \bullet)$, molecular oxygen anions $\left(\mathrm{O}_{2}{ }^{-}\right)$, the molecular species hydrogen $\left(\mathrm{H}_{2}\right)$, and hydrogen peroxide $\left(\mathrm{H}_{2} \mathrm{O}_{2}\right)$. Molecular nitrogen and carbon dioxide dissolved in the water may also undergo radiolytic decomposition with a several-step recombination of the dissociation products with oxygen, water, and other associated radiolytic products to form nitrogen and carboxylic acids [3-7]. Irradiation of moist air has been shown to produce nitric acid $\left(\mathrm{HNO}_{3}\right)$, nitrous oxide $\left(\mathrm{N}_{2} \mathrm{O}\right)$, and ozone $\left(\mathrm{O}_{3}\right)$ [8]. These radiolytic products may influence glass stability by altering the solution $\mathrm{pH}$ and $\mathrm{Eh}$, or by complexing with waste radionuclides and glass-forming components.

The efficiency of radiation in producing radicals or molecules is expressed as a $G$ value, which is the average number of radiolytic species created (positive $G$ ) or destroyed (negative $G$ ) per $100 \mathrm{eV}$ absorbed radiation energy. Because the solubility of nitrogen in water is low, $\mathrm{G}$ values for nitric acid production in air-saturated water are also small. The $\mathrm{G}\left(\mathrm{NO}_{3}\right)$ values reported for oxidized water systems irradiated by a variety of sources range from 0 to less than 0.2 [9-14], while values for radiolytic production of nitric acid from moist air or two-phase air/liquid water systems are $\sim 2$ [11,14-18]. Both nitric acid and water dissociation products may accelerate nuclear waste and metal container reaction rates [5,19-25].

The formation of nitric acid in air-dominated systems and the subsequent dissolution of these acids in the limited amount of water present will decrease the $\mathrm{pH}$ of the water. The concentration of these radiolytic acids under high gas/liquid (G/L) ratios should rapidly lead to the formation of thin films of acidified water contacting the waste package components [23]. Radiolytic acidification of aqueous fluids will be most pronounced in a geologically unsaturated 
environment, where air and only small amounts of liquid water are expected to be present. The potential site at Yucca Mountain, Nevada, is to be located in such an unsaturated setting; therefore, the effects of radionuclide decay on the waste repository environment and waste package components need to be considered for licensing of the repository.

In a comprehensive review of the available literature on the effects of radionuclide decay on waste glass corrosion, Wronkiewicz [26] identified several potential needs for additional radiation study. These identified topics include (1) studies of the release of intermediate-decayseries daughter radionuclides from alpha tracks in the glass; (2) annealing processes and rates in glass, especially at temperatures that are relevant to nuclear waste disposal $\left(<300^{\circ} \mathrm{C}\right)$; (3) the effects of ionization-induced phase segregations and bubble formation on glass corrosion; (4) the identification of water radiolysis radical species responsible for accelerated glass corrosion; (5) the influence of air and water radiolysis products on glass corrosion in an unsaturated [high glass surface area/leachant volume (S/V) ratio] environment; (6) the influence of air and radiolysis products on the stability of secondary alteration products, especially those that may retard the migration of radionuclide components from the corroding glass waste form; (7) the effects of alpha radiolysis in producing corrosive species in an unsaturated environment; and (8) studies comparing the corrosion behavior of simulated versus fully radioactive waste forms.

The present study specifically addresses research needs 5,6 , and 7 , which are also discussed by Wronkiewicz et al. [26,27]. The initial ("blank") tests were conducted without the presence of glass and were designed to examine the distributions and types of radiolytic products formed under a variety of test conditions. These tests concentrated on examining the formation and distribution of radiolysis products under the high $\mathrm{G} / \mathrm{L}$ ratios that may be expected in an unsaturated repository setting. The temperatures and dose rates used in these tests were chosen to represent potential bounding conditions for the repository environment after waste emplacement. For example, tests at high temperature $\left(150-200^{\circ} \mathrm{C}\right)$ and high dose rate $(500 \mathrm{~Gy} / \mathrm{h})$ represent environmental conditions shortly after waste emplacement into the repository, while tests at low temperature $\left(90^{\circ} \mathrm{C}\right)$ and low dose rate $(30 \mathrm{~Gy} / \mathrm{h})$ represent conditions expected several hundred years after waste emplacement. The gamma tests simulate an early repository scenario, dominated by beta- and gamma-radiation sources from short-lived fission products, while the alpha-radiation tests simulate a long-term scenario dominated by alpha emissions from actinide sources. The $G / L$ ratios used in these tests $(G / L=10$ to 100$)$ include those chosen to overlap with those of previously published results [11] and those that simulate an unsaturated repository setting with limited amounts of water present $(G / L=100)$.

The second phase of this study examines the effects of radiation on glass reactions. Of concern is the potential for increases in the rates of glass reaction resulting from the rapid acidification of small amounts of aqueous fluids contacting the glass. In such a scenario, the bicarbonate $\mathrm{pH}$-buffering capacity of the thin film of water contacting the sample may be rapidly overwhelmed by the condensation of radiolytically produced acids. The resultant $\mathrm{pH}$ decrease may accelerate glass alteration rates [19-21,28,29], or alternatively decrease glass alteration rates [30-33] depending on the exposure conditions. Results from the radiation tests without glass will form a basis for understanding the types of radiolysis products present and the locations where they condense in the glass radiation tests at high $\mathrm{G} / \mathrm{L}$ ratio. The effect of these radiolysis products on the formation and stability of secondary alteration minerals will also be examined by comparing the alteration trends developed on glasses reacted both with and without the presence of radiation. 


\section{EXPERIMENTAL}

\section{A. $\quad$ Sample Matrix}

Irradiation experiments have been performed using several configurations, with each test in duplicate. All test were conducted in a heterogeneous air-water system. Tests are divided into (1) those conducted without the presence of glass (blank tests) and (2) those where simulated nuclear waste glass was irradiated in the presence of an air-water environment. An experimental flowchart, linking the similarities between the various tests, appears in Fig. 1. The glass and leachant compositions are discussed in Sec. II.B. and II.C.

Dose Rate Test
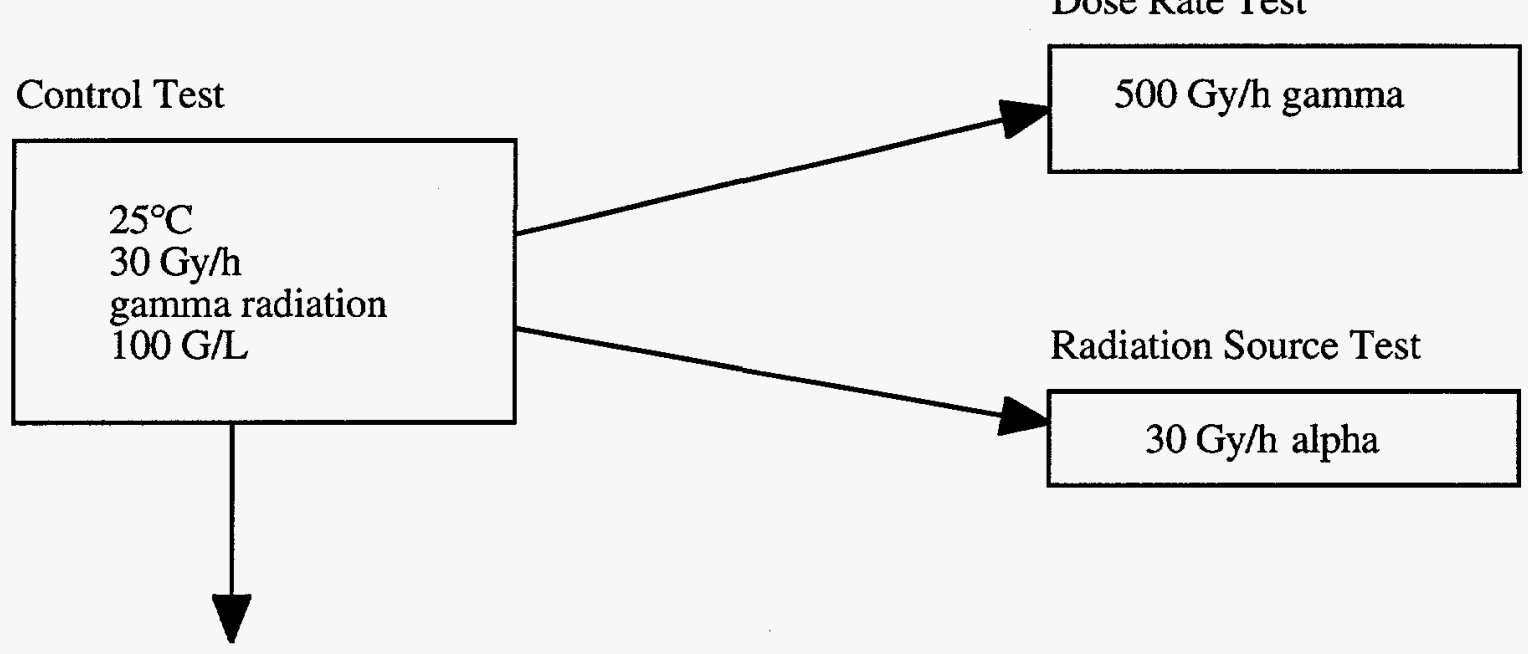

Temperature Test

Gas/Liquid Ratio Test

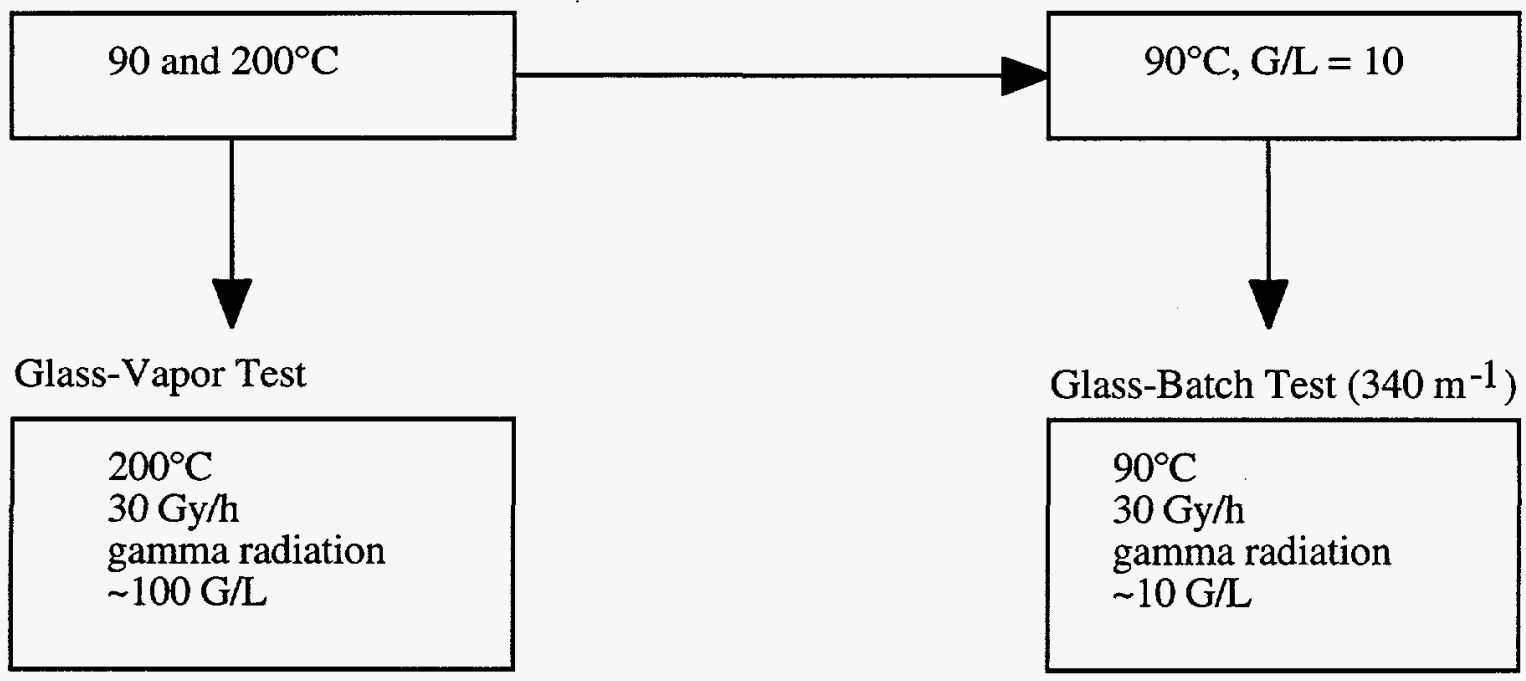

Fig. 1. Experimental Flowchart for Radiation Testing 
With the blank tests, separate experiments were carried out under both gamma and alpha irradiation (Table 1). Forty-eight gamma and 23 alpha radiolysis tests were performed over time intervals ranging from 14 to 180 days. Eight additional tests were conducted without radiation exposure to check on element background concentrations. In addition to the separate examination of alpha and gamma radiation effects, we also examined the effects of temperature, dose rate, and $\mathrm{G} / \mathrm{L}$ ratio on the production of radiolytic species.

To examine the effects of the irradiated environment on waste glass corrosion and alteration phase development, experiments were conducted in two configurations. The first test configuration includes batch tests conducted with monolithic glass samples completely immersed in water at a $S / V$ ratio of $340 \mathrm{~m}^{-1}$ (Table 2). For this study, tests were conducted only in the presence of gamma radiation. Results from tests run without irradiation but otherwise under identical reaction conditions [34] are compared with results from the present task to provide an evaluation of the radiation effects on glass durability.

The second test configuration employs monolithic glass samples suspended by a thread in a vapor environment (Table 3 ). These tests were conducted under both gamma irradiated and nonirradiated conditions.

Table 1. Sampling: Matrix for Alpha and Gamma Blank Tests

\begin{tabular}{llrrrr}
\hline $\begin{array}{l}\text { Expt. } \\
\text { Number }\end{array}$ & $\begin{array}{c}\text { Radiation Type } \\
\text { Alpha/Gamma }\end{array}$ & $\begin{array}{c}\text { Temp. } \\
\left({ }^{\circ} \mathrm{C}\right)\end{array}$ & $\begin{array}{c}\text { Test Length } \\
\text { (days) }\end{array}$ & $\begin{array}{c}\text { Dose Rate } \\
(\mathrm{Gy} / \mathrm{h})\end{array}$ & $\begin{array}{c}\text { Gas/Liquid } \\
\text { Ratio (L/L) }\end{array}$ \\
\hline & & & & & \\
IV9000 & Gamma & 25 & $14,28,56,120$ & 500 & 100 \\
IV9000 & Gamma & 25 & $14,28,56,120$ & 30 & 100 \\
IV1000 & Gamma & 90 & $56,120,180$ & 30 & 100 \\
IV2000 & Gamma & 200 & $56,120,180$ & 30 & 100 \\
IV9000 & Gamma & 25 & 56,120 & 500 & 10 \\
IV9000 & Alpha-Lucite Support & 25 & $14,28,56$ & 30 & 100 \\
IV9000 & Alpha-SS Support & 25 & $14,28,45,56$, & 30 & 100 \\
& & & $85,110,129$ & & \\
\hline
\end{tabular}

Table 2. Sampling Matrix for Saturated Batch Leach Tests

\begin{tabular}{|c|c|c|c|c|c|c|}
\hline $\begin{array}{l}\text { Expt. } \\
\text { Number }\end{array}$ & $\begin{array}{l}\text { Glass } \\
\text { Type }\end{array}$ & $\begin{array}{c}\text { Temp. } \\
\left({ }^{\circ} \mathrm{C}\right)\end{array}$ & $\begin{array}{c}\text { Test Length } \\
\text { (days) }\end{array}$ & $\begin{array}{l}\text { Water } \\
\text { Type }\end{array}$ & $\begin{array}{l}\mathrm{S} / \mathrm{V}^{\mathrm{a}} \\
\left(\mathrm{m}^{-1}\right)\end{array}$ & $\begin{array}{c}\text { Dose Rate } \\
(\mathrm{Gy} / \mathrm{h})\end{array}$ \\
\hline IV9202A & SRL 202A & 90 & $\begin{array}{l}14,28,56, \\
91,180,360 \text {, } \\
540,720\end{array}$ & $\mathrm{EJ}-13$ & 340 & 30 \\
\hline IV9165A & SRL 165A & 90 & $\begin{array}{l}91,180 \\
360,720\end{array}$ & EJ-13 & 340 & 30 \\
\hline IV9131A & SRL 131A & 90 & $\begin{array}{l}14,28,56, \\
180,360, \\
720\end{array}$ & EJ-13 & 340 & 30 \\
\hline
\end{tabular}

${ }^{\mathrm{a}} \mathrm{S} / \mathrm{V}=$ sample surface area/leachant volume 
Table 3. Sampling Matrix for Vapor Hydration Tests

\begin{tabular}{|c|c|c|c|c|c|}
\hline $\begin{array}{l}\text { Expt. } \\
\text { Number }\end{array}$ & $\begin{array}{l}\text { Glass } \\
\text { Type }^{\mathrm{a}}\end{array}$ & $\begin{array}{l}\text { Temp. } \\
\left({ }^{\circ} \mathrm{C}\right)\end{array}$ & $\begin{array}{l}\text { Water } \\
\text { Type }^{b}\end{array}$ & Test Length (days) & $\begin{array}{c}\text { Dose Rate } \\
(\mathrm{Gy} / \mathrm{h})\end{array}$ \\
\hline IVE202A & SRL 202A & 200 & DIW & $7,14,21,35,56$ & 30 \\
\hline IVE202U & SRL 202U & 200 & DIW & $7,14,21,35,56$ & 0 \\
\hline IVE165A & SRL 165A & 200 & DIW & $7,14,21,35,56$ & 30 \\
\hline IVE131A & SRL 131A & 150 & DIW & $7,14,21,35,56$ & 30 \\
\hline IVE131U & SRL 131U & 150 & DIW & $3,5,7,14,28,56,91,180$ & 0 \\
\hline
\end{tabular}

" $\mathrm{A}$ " suffix designates $\mathrm{U}, \mathrm{Pu}, \mathrm{Am}, \mathrm{Np}$, and Tc doped glass; "B" suffix designates glass doped with uranium. Composition of all glasses is presented in Section IIB.

${ }^{b}$ DIW = deionized water.

\section{Blank Irradiation Tests}

\section{a. Gamma-Irradiation Tests}

All dosimetry and gamma irradiation tests were performed in Parr reaction vessels fabricated with type 304L stainless steel and manufactured by Parr Instrument Company, Moline, IL. Internal volumes of the Parr vessels were measured at $21.4 \mathrm{~cm}^{3}$.

Dosimetry measurements for the vessel locations were made by adding a ferrous sulfate solution in a sealed glass tube, which was then inserted into an open Parr vessel for irradiation. The dosimetry tests were conducted at ambient hot cell temperatures. The absorbed dose is determined by measuring the amount of ferrous sulfate converted to ferric salt during irradiation [35]. Desired radiation dose rates were obtained by altering the distance of the test vessels from the ${ }^{60} \mathrm{Co}$ source. The measured dose rates were then adjusted over time to account for decay of the ${ }^{60} \mathrm{Co}$ source by applying the isochron equation [36] and a half-life of 5.272 yr to derive the ${ }^{60} \mathrm{Co}$ decay constant. The decay constant and median time of the irradiation tests following the dosimetry tests were then used to calculate a median dose rate for the samples. Further discussion of this dosimetry technique is given in Sec. II.E, and the results of the dosimetry tests are presented in Table A.1. The calculated dose rates and cumulative doses absorbed by the solutions in the individual gamma irradiation tests are also described further in Sec. III.

Prior to testing, a premeasured amount of high-purity deionized water was added to the bottom of each vessel, which was then fitted with an annealed copper gasket and hermetically sealed with a compression fitting to 160 foot-pound $(2170 \mathrm{~N} \cdot \mathrm{m})$ torque. The assembled vessels were then placed in an oven (model Blue M, General Signal Co., Blue Island, IL) that is controlled at the desired temperature to within $\pm 1^{\circ} \mathrm{C}$. The oven was monitored with a thermocouple and attached data logger that recorded the temperature of the oven every $12 \mathrm{~h}$. Heating due to irradiation was assumed to be insignificant for the elevated temperature tests.

"Baseline" gamma-irradiation tests were initiated by adding $0.22 \mathrm{~mL}$ of deionized water into each $21.4 \mathrm{~cm}^{3}$ Parr vessel to achieve a $\mathrm{G} / \mathrm{L}$ ratio of $\sim 100$ (Table 1 ). These samples were exposed to a ${ }^{60} \mathrm{Co}$ gamma radiation source at a dose rate of $\sim 30 \mathrm{~Gy} / \mathrm{h}(\sim 3000 \mathrm{rad} / \mathrm{h})$ at an ambient hot cell temperature of $\sim 25^{\circ} \mathrm{C}$, and form a basis against which tests using other parametric values could be compared. For example, additional tests were run at higher temperatures $\left(90\right.$ and $\left.200^{\circ} \mathrm{C}\right)$ and dose rates of $500 \mathrm{~Gy} / \mathrm{h}(50,000 \mathrm{rad} / \mathrm{h})$ to evaluate their effects on radiolytic product yields. Other tests were run to examine the effects of variable $\mathrm{G} / \mathrm{L}$ ratios 
by adding $2.0 \mathrm{~mL}$ of deionized water to achieve a G/L ratio of $\sim 10$ in the vessels. Test intervals for the various gamma radiation experiments ranged from 14 to 180 days.

\section{b. $\quad$ Alpha-Irradiation Tests}

The alpha-irradiation tests utilized a ${ }^{241} \mathrm{Am}$-foil source $(1500 \mu \mathrm{Ci})$ coated with a thin, gold film as a radiation source. Alpha decay energies for the ${ }^{241} \mathrm{Am}$ isotope are $\sim 4.6 \mathrm{MeV} /$ alpha decay. In initial tests, the foil was cemented to a Lucite support rod, while in later experiments, it was attached to a type $304 \mathrm{~L}$ stainless steel rod to eliminate potential sources of organic carbon contamination. This foil-rod assembly was attached to the underside of the vessel lid and inserted and sealed into a glass reaction vessel (Fig. 2). The measured internal volume of the alpha test vessel with the rod assembly inserted was determined to be $1764 \mathrm{~cm}^{3}$. The assembled test vessel configuration ensured that all of the alpha particle energy was absorbed by the air inside the vessel rather than the container walls.

Dosimetry measurements for the alpha-foil tests at ambient room temperatures $\left(-25^{\circ} \mathrm{C}\right)$ were performed with a $\mathrm{N}_{2} \mathrm{O}$ gas dosimeter. This dosimeter determines the fraction of energy transferred to the gas phase by measuring the rate of $\mathrm{N}_{2}$ gas production and comparing relative yields to an expected $\mathrm{G}\left(\mathrm{N}_{2}\right)$ value of 10.0 . Nitrogen production was found to be linear for periods of $0,2,7,17$, and 24 days, with $~ 37 \%$ of the energy produced by the foils being deposited in the gas phase. The remainder of the alpha radiation energy was absorbed by the foil material itself or the protective outer gold film. The calculated dose rate absorbed by the air surrounding the foil apparatus is $\sim 26 \mathrm{~Gy} / \mathrm{h}(\sim 2600 \mathrm{rad} / \mathrm{h})$, with maximum alpha-particle travel distances in air of 3-4 cm.

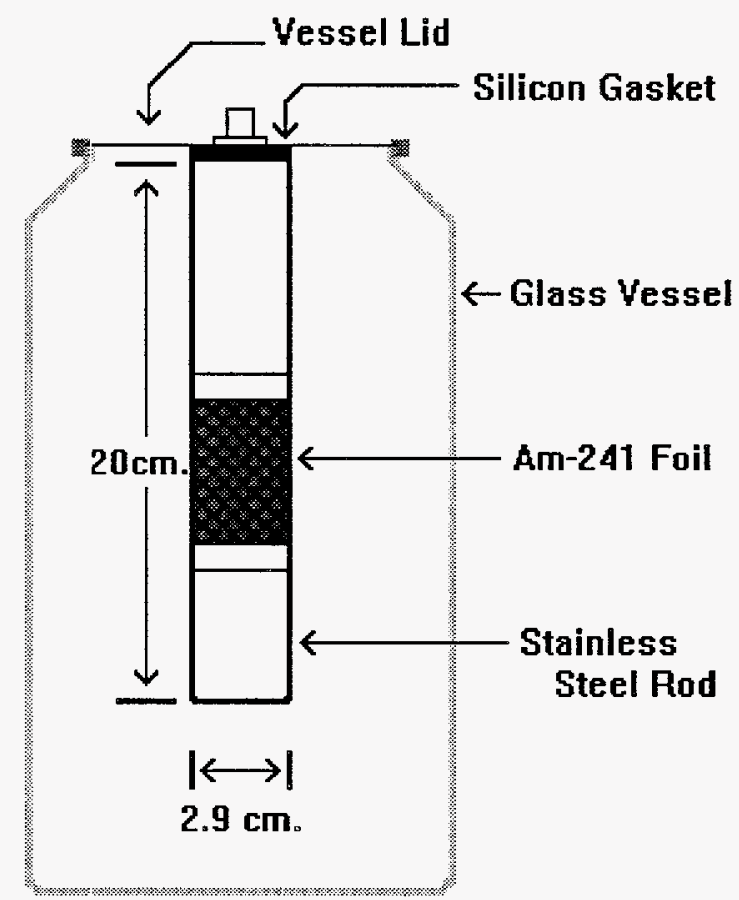

Fig. 2. Experimental Apparatus for Alpha Radiolysis Tests. Vessels were exposed to an internal ${ }^{241} \mathrm{Am}$-foil irradiation source at a dose rate of $\sim 26 \mathrm{~Gy} / \mathrm{h}$. 
Alpha-irradiation tests were initiated by adding $\sim 17.6 \mathrm{~mL}$ of deionized water into each $1764 \mathrm{~cm}^{3}$ vessel to achieve a $\mathrm{G} / \mathrm{L}$ ratio of $\sim 100$. These tests were exposed to alpha radiation at ambient room temperatures and time periods of 14 to 129 days (Table 1). The similarity of experimental conditions between these experiments and the baseline gammairradiation experiments allows direct comparison of the effects of these two radiation sources at high $\mathrm{G} / \mathrm{L}$ ratios.

\section{Irradiation Tests with Glass}

\section{a. $\quad$ Batch Tests at $340 \mathrm{~m}^{-1}$}

Batch test experiments were initiated by placing four glass disks of a given composition into a Parr reaction vessel identical to that used in the blank gamma-irradiation tests. The glass disks rested on top of a perforated 304L stainless steel support plate, which was previously laid on the bottom of the vessel (Fig. 3). A volume of $2.0 \mathrm{~mL}$ of equilibrated J-13 (EJ-13) well water was added to each vessel to attain a $\mathrm{S} / \mathrm{V}$ ratio of $340 \mathrm{~m}^{-1}$. This amount of leachant was determined to be the minimum amount of fluid that could be added to the vessels

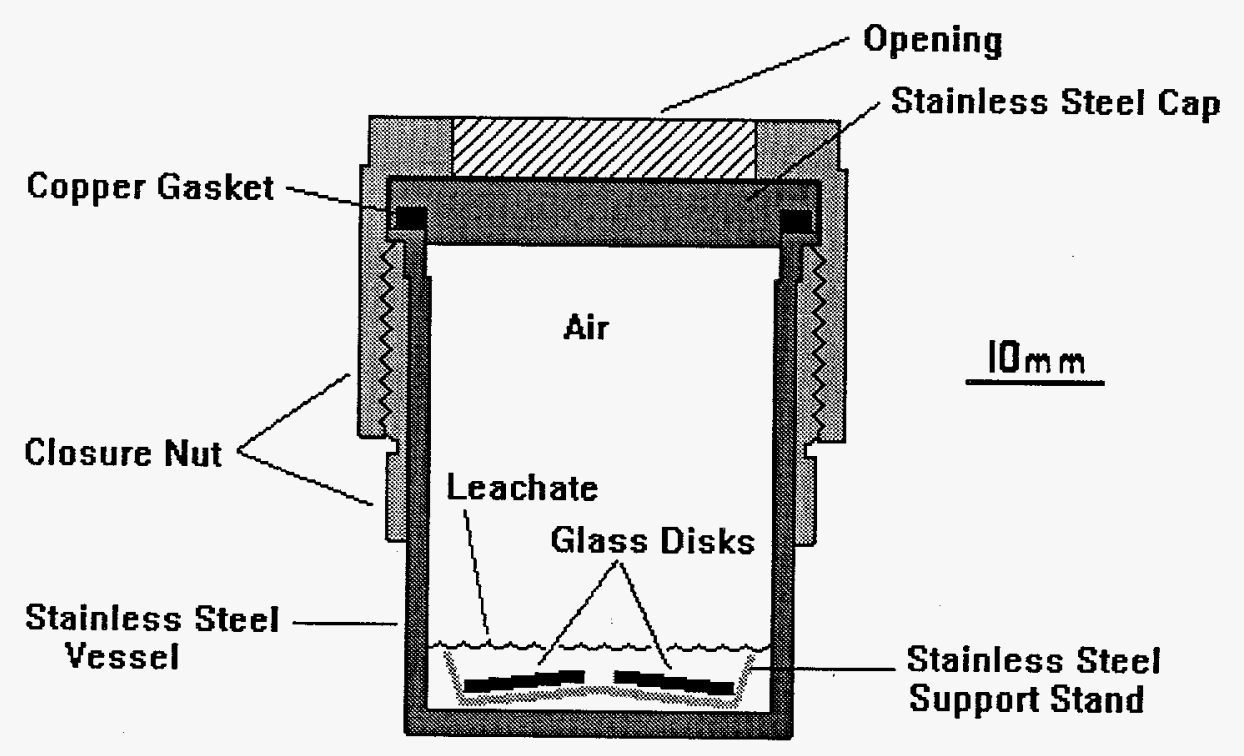

Fig. 3. Experimental Apparatus for $340 \mathrm{~m}^{-1}$ Batch Tests with Glass Monoliths. Samples were exposed to an external ${ }^{60} \mathrm{Co}$ irradiation source at a dose rate of $\sim 35 \mathrm{~Gy} / \mathrm{h}$. 
and yet ensure that the glass disks were completely immersed in the bath of water. The preparation and compositional analysis of the EJ-13 solution are described in Sec. II.C. Testing procedures, including vessel closure, oven temperature maintenance, dosimetry measurements, and dose rate calculations were identical to those used in the blank gamma-irradiation tests. All tests were conducted at temperatures of $90 \pm 1^{\circ} \mathrm{C}$ and intervals ranging from 14 to 720 days. These tests were also subjected to a gamma-radiation dose of $\sim 35 \mathrm{~Gy} / \mathrm{h}$. These dose rates were chosen as an upper bound for radiation exposure in a repository and match those used in the baseline blank irradiation tests.

\section{b. Vapor Hydration Tests}

Vapor hydration tests were initiated by suspending two glass disks of a given composition inside the test vessels. Irradiated and nonirradiated glass samples were suspended via $\mathrm{Pt}-\mathrm{Rh}$ and Teflon wires, respectively, inside stainless steel Parr reaction vessels identical to those used in the blank gamma-irradiation tests (Fig. 4). High-purity deionized water was added to each vessel in an amount necessary to saturate the air inside the heated vessel, but limited so as to not cause solution runoff from the suspended samples. This latter aspect of test solution volume was necessary to prevent the dilution of corrosion products in the film of water

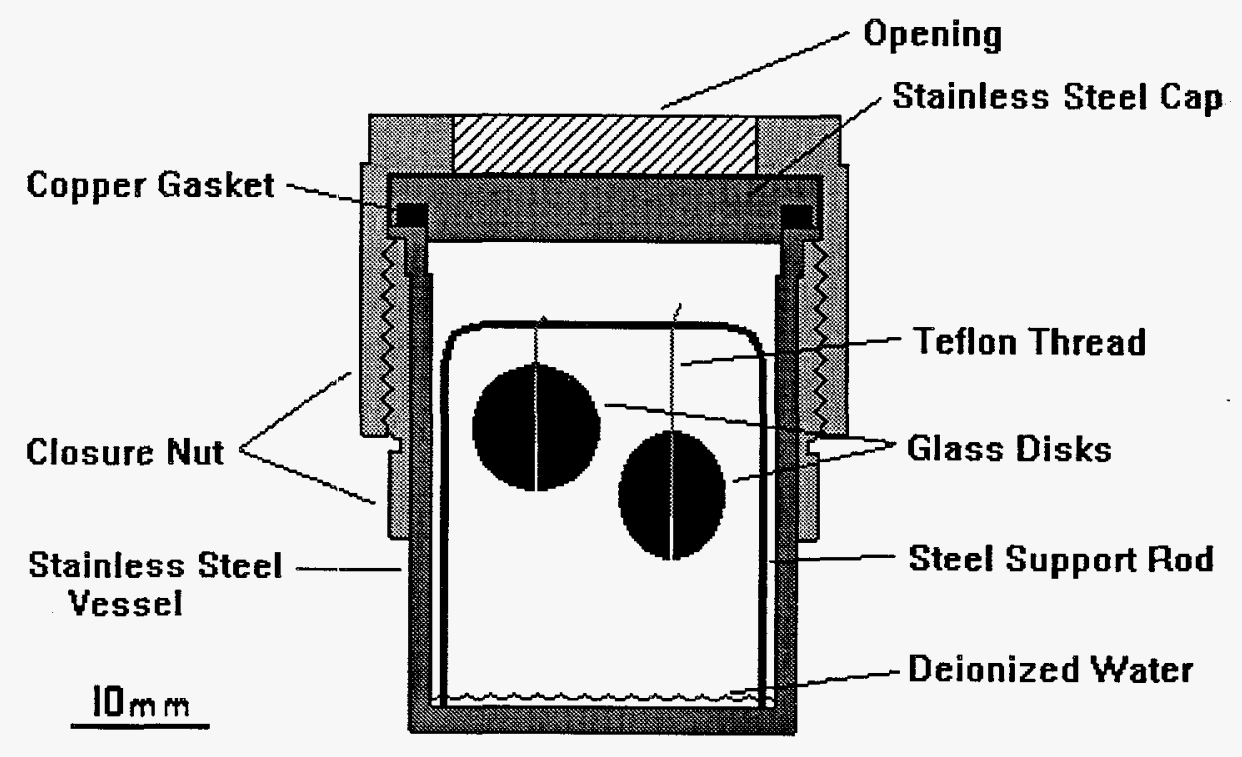

Fig. 4. Experimental Apparatus for Vapor Hydration Tests with Glass Monoliths. Samples were exposed to an external ${ }^{60} \mathrm{Co}$ irradiation source at a dose rate of $\sim 35 \mathrm{~Gy} / \mathrm{h}$. 
contacting the glass through the condensation of water and subsequent dripping of leachate from the samples. The expression "vapor hydration test" reflects the predominant water vapor or steam environment inside the test vessels. Note, however, that the reaction on the glass surface occurs between the glass and a thin film of water that has condensed on the glass surface. The condensation occurs due to the confining pressure of the test vessel walls on the vapor pressure of the water added at the beginning of the test.

For the irradiation tests, experimental vessels housing actinide-plus${ }^{99} \mathrm{Tc}$-doped glass samples were exposed to an external ${ }^{60} \mathrm{Co}$ gamma source at a dose rate of $\sim 35 \mathrm{~Gy} / \mathrm{h}$. The actinides decay primarily through alpha decay, and ${ }^{99} \mathrm{Tc}$ primarily through beta decay. Thus, the internal doping of the samples exposed the vessel atmosphere to both alpha and beta sources during testing. This exposure was in addition to the gamma radiation supplied by the external ${ }^{60} \mathrm{Co}$ source. Total radiation exposure was dominated by the gamma source, with an approximate exposure ratio for the atmosphere and fluid immediately surrounding the glass monoliths at a level of $\sim 40$ gamma: 1 alpha + beta. Test intervals ranged from 7 to 56 days, with cumulative gamma doses for the 56-day tests being $\sim 5 \times 10^{4} \mathrm{~Gy}$. Dosimetry measurements for these tests followed the same procedure as outlined in Sec. II.A.1.

For the nonirradiated vapor hydration tests, uranium-doped samples were reacted without being exposed to any external gamma source. Test intervals for these experiments ranged from 7 to 56 days for the SRL $202 \mathrm{U}$ glasses, while the test matrix for the SRL $131 \mathrm{U}$ glasses was expanded to cover intervals from 3 to 728 days.

The gamma dose exposures used in the irradiated tests are $\sim 50 \%$ of the level projected for "as cast" glass from the Savannah River Plant. This level of exposure will probably exceed that experienced by actual waste glass following geologic disposal in a nuclear waste repository. In a realistic waste disposal scenario, the waste container is expected to isolate the waste form from the repository environment for several hundred years following emplacement. This initial period will allow radionuclide levels to decay to sufficiently low enough levels where the radiation exposure to the surrounding environment will be significantly lower than the levels used in these tests. The gamma dose rates used in these tests were chosen only as an upper bounding condition and are used to evaluate whether there is a potential for radiation to affect glass reactions.

The elevated temperatures of these tests $\left(150\right.$ and $\left.200^{\circ} \mathrm{C}\right)$ were used to accelerate glass reactions into reasonably short experimental durations. These elevated temperatures thus allow examination of long-term corrosion behavior of the glass waste. Previous studies have indicated that the glass reaction mechanism remains unchanged within the range of $70-240^{\circ} \mathrm{C}[24,37]$. Additional comparisons will also be investigated to examine the consistency of the high-temperature experimental reactions with those of natural analogue reactions with volcanic glass at low temperature. As with the elevated dose rates, the temperatures used for these experiments approximate an upper bound for waste disposal. Although actual borosilicate waste glasses may initially be exposed to temperatures and gamma dose rates as high as those used in these tests, these initial conditions will only be realized at a time when the waste container integrity is maintained, thus preventing contact of the glass by the repository environment. The waste containers are not expected to be breached until several hundred years or more after the waste has been placed in the repository. At these later times, the radioactivity level of the waste will be significantly reduced, resulting in a corresponding decrease in the temperature of the waste package to less than $100^{\circ} \mathrm{C}$. 
Elevated test pressures also result from the confining pressure of the test vessel walls on the vapor pressure of water. These confining pressures will be $\sim 5$ atm for the $150^{\circ} \mathrm{C}$ tests and $\sim 16 \mathrm{~atm}$ for the $200^{\circ} \mathrm{C}$ tests, while actual waste materials at Yucca Mountain will be exposed only to local atmospheric pressures. Previous studies, conducted under saturated test conditions, have indicated that pressures as high as 100 atm will not affect the rate of glass reactions [38].

\section{B. Glass Sample Preparation}

Experiments were performed using three synthetic waste glass formulations: SRL 131, SRL 165, and SRL 202 glass frits, provided by the vitrification facility of Savannah River Plant. These sample frits were blended and homogenized with desired levels of radionuclide components to produce the glass samples used in the tests. For the irradiated tests, the glass samples were doped with ${ }^{99} \mathrm{Tc}$ and actinides $\left({ }^{237} \mathrm{~Np},{ }^{238} \mathrm{U},{ }^{239} \mathrm{Pu},{ }^{241} \mathrm{Am}\right)$ at levels approximating $23 \mu \mathrm{Ci} / \mathrm{g}$ total activity (designated SRL 131A, SRL 165A, and SRL 202A glasses). These levels represent a nuclear waste glass material that has "aged" for a period of $\sim 1000$ years prior to its exposure to moisture in a nuclear waste repository. Stable isotopes of $\mathrm{Sr}$ and $\mathrm{Cs}$ also have been added at concentration levels approximating those expected for radioactive $\mathrm{Sr}$ and $\mathrm{Cs}$ in a 1000 year glass. Glass used in the nonirradiated tests was doped with only ${ }^{238} \mathrm{U}$ (designated SRL 131U, SRL 165U, and SRL 202U glasses). The compositions of these glasses are given in Table 4.

The glasses were prepared by blending the actinide dopants with the glass frit, homogenizing the glass-dopant mixture, and vitrifying for six hours at $1150^{\circ} \mathrm{C}$. The resultant melt was quenched in water, reground, homogenized with the technetium dopant (for the " $A$ " suffix glasses only), and then remelted at $1150^{\circ} \mathrm{C}$ for one hour. The glass melt was then poured 
Table 4a. Compositions of Simulated Reference Nuclear Waste Glasses (Oxide Weight \%)

SRL 131U/A SRL 202U/A SRL 165U/A

\begin{tabular}{lccc}
\hline $\mathrm{SiO}_{2}$ & 44.3 & 50.2 & 52.4 \\
$\mathrm{Fe}_{2} \mathrm{O}_{3}$ & 12.8 & 11.9 & 12.2 \\
$\mathrm{Na}_{2} \mathrm{O}$ & 12.2 & 7.8 & 11.4 \\
$\mathrm{~B}_{2} \mathrm{O}_{3}$ & 9.8 & 8.0 & 6.7 \\
$\mathrm{Li}_{2} \mathrm{O}^{2}$ & 3.0 & 4.4 & 4.5 \\
$\mathrm{Al}_{2} \mathrm{O}_{3}$ & 3.3 & 3.9 & 4.5 \\
$\mathrm{~K}_{2} \mathrm{O}$ & 3.9 & 3.7 & 0.20 \\
$\mathrm{MnO}$ & 2.5 & 2.2 & 2.8 \\
$\mathrm{CaO}$ & 0.94 & 1.3 & 1.6 \\
$\mathrm{MgO}$ & 1.3 & 1.4 & 0.73 \\
$\mathrm{UO}$ & & 2.0 & 1.1 \\
$\mathrm{TiO}_{2}$ & 3.0 & & \\
$\mathrm{ZrO}_{2}$ & & 0.93 & 0.15 \\
$\mathrm{Cs}_{2} \mathrm{O}$ & 0.66 & 0.06 & 0.70 \\
$\mathrm{SrO}^{\mathrm{NiO}}$ & 0.22 & $\mathrm{NA}$ & 0.072 \\
$\mathrm{CuO}$ & $\mathrm{NA}$ & 0.03 & 0.11 \\
$\mathrm{ZnO}$ & 0.01 & 0.85 & 0.85 \\
$\mathrm{BaO}$ & 1.2 & 0.41 & $\mathrm{NA}$ \\
$\mathrm{ThO}_{2}$ & 0.02 & 0.29 & 0.02 \\
$\mathrm{MoO}$ & 0.02 & 0.21 & 0.03 \\
$\mathrm{Cr}_{2} \mathrm{O}_{3}$ & 0.02 & 0.28 & $\mathrm{NA}$ \\
$\mathrm{P}_{2} \mathrm{O}_{5}$ & 0.01 & 0.05 & 0.01 \\
${ }^{2} \mathrm{NA}=$ not analyzed & & 0.12 & 0.02 \\
& 0.01 & $\mathrm{NA}$ & 0.023 \\
\hline
\end{tabular}

Table 4b.Technetium and Transuranic Dopant Levels for SRL 131A, SRL 202A, and SRL 165A Glasses. Values in Oxide Weight Percent and Element Weight Percent in Parenthesis.

\begin{tabular}{llll} 
& \multicolumn{1}{c}{ SRL 131A } & \multicolumn{1}{c}{ SRL 202A } & \multicolumn{1}{c}{ SRL 165A } \\
\hline${ }^{99} \mathrm{Tc}$ & $0.0035(0.0026)$ & $0.0030(0.0023)$ & $0.0015(0.0011)$ \\
${ }^{237} \mathrm{~Np}$ & $0.013(0.011)$ & $0.014(0.012)$ & $0.014(0.012)$ \\
${ }^{239} \mathrm{Pu}$ & $0.012(0.011)$ & $0.013(0.011)$ & $0.0087(0.0077)$ \\
${ }^{241} \mathrm{Am}$ & $0.00045(0.00040)$ & $0.00044(0.00039)$ & $0.00018(0.00016)$ \\
\hline
\end{tabular}


into a $95 \%$ platinum $+5 \%$ gold mold preheated to $700^{\circ} \mathrm{C}$. This mold was then immediately transferred to a $500^{\circ} \mathrm{C}$ annealing oven for $15 \mathrm{~min}$; cooled at a rate of $\sim 100^{\circ} \mathrm{C} / \mathrm{h}$ down to $350^{\circ} \mathrm{C}$; then removed from the annealing oven and allowed to cool to room temperature.

Monolithic glass disks used in the tests were prepared by coring 10 -mm-dia logs from the cast glass and then cutting the core into $\sim 1-\mathrm{mm}$-thick wafers. The flat surfaces of the glass disks were then polished in water to a 500 -grit finish for subsequent vapor hydration tests and a 320 -grit finish for the saturated-water $340 \mathrm{~m}^{-1}$ tests. The rounded edges of the disks were not polished and represent the as-cored glass surface. The glass disks designated for use in the vapor hydration tests were then notched in two locations on opposite edges of the samples to allow for later attachment with hanging threads. All disks were then rinsed in water and ultrasonically cleaned three times in ethanol.

Glass disks were examined for sample homogeneity by transmitted optical microscopy and scanning electron microscopy/energy dispersive X-ray spectrometry (SEM/EDS). Color variations were noted on most disks during optical examinations, indicating that some heterogeneity exists in the samples. These variations appeared as irregular, dark bands within a lighter, yellow-brown glass. The irregular wavy texture of the bands suggests that they were formed as solidified flow textures within the melt. No compositional variations in major oxides were noted during SEM/EDS examinations of the glass, although trace amounts of minute spinel crystals and small gas bubbles have been noted in the glass disks [39]. Random disks of SRL 165A and SRL 202A glass were also chosen for beta- and alpha-decay counts to determine if technetium and the actinide elements were distributed homogeneously between the glass samples. Individual glass samples were counted for one minute each (Table 5), with a one-sigma variation in alpha and beta counts between different glass disks being $<5 \%$ of the mean number of counts for all disks counted. Differences in mean values between SRL 165A and SRL 202A glass (but with identical dopant levels) were $<10 \%$.

\section{EJ-13 Leachant}

The leachant used in the $340 \mathrm{~m}^{-1}$ batch tests consists of groundwater from the $\mathrm{J}-13$ well at Yucca Mountain that has been equilibrated at $90^{\circ} \mathrm{C}$ for 14 days, with samples of caliche-free Topopah Springs volcanic tuff obtained from the Yucca Mountain site. The composition of this leachant, referred to as EJ-13, is given in Table 6. A detailed preparation procedure for the EJ-13 leachant solution is given by Bates [30].

The EJ-13 leachant used in these tests is believed to be the best available representation of the groundwater expected to exist at the unsaturated horizon at Yucca Mountain where the potential nuclear waste repository will be located. Differences may exist, however, between the composition of this test leachant and that of the fluid actually present in the geologically unsaturated Yucca Mountain repository site. These differences may arise because the J-13 well water was drawn from a water-saturated horizon of the Topopah Springs tuff, while the proposed Yucca Mountain repository is located within an unsaturated zone in the same geologic horizon. Differences may arise in water compositions from these two horizons because of the influence that variable water/rock ratios may have in controlling solution compositions, and the preTopopah Springs flow history may have influenced the water composition from the $\mathrm{J}-13$ well. Also, thermal heating of the repository due to waste package emplacement will dehydrate the pore space surrounding the waste packages and subsequently deposit salts, which are derived from the residue of groundwater components [40]. The initial reincursion of liquids into the repository horizon after cooling of the waste packages may be characterized by the presence of high-ionic strength brines that have formed as evaporative minerals previously deposited during evaporative episodes are redissolved in the encroaching groundwater front. 
Table 5. Alpha and Beta Activity Recorded for Selected Monoliths of SRL 202A and SRL 165 Glass

\begin{tabular}{lcc}
\hline & & \\
Sample Number & Alpha Counts/min & Beta Counts/min \\
\hline IV9202A-180-2B & 50,078 & 100,682 \\
IV9202A-360-1A & 49,547 & 98,897 \\
IV9202A-360-1C & 49,081 & 95,473 \\
IV9202A-360-2D & 48,713 & 93,173 \\
IV9202A-540-1C & 49,189 & 95,245 \\
IV9202A-540-1D & 49,292 & 98,708 \\
IV9202A-540-2D & 49,428 & 99,683 \\
IV9202A-720-1A & 53,368 & 108,755 \\
IV9202A-720-1D & 48,593 & 96,949 \\
IV9202A-720-2D & 48,611 & 96,459 \\
\hline Average (s.d.) & $49,593( \pm 1405)$ & $98,462( \pm 4219)$ \\
\hline & & \\
IV9165A-180-1B & 47,166 & 87,899 \\
IV9165A-180-2A & 45,989 & 86,893 \\
IV9165A-360-1A & 45,544 & 90,533 \\
IV9165A-360-1D & 46,398 & 93,206 \\
IV9165A-360-2A & 46,060 & 91,120 \\
IV9165A-360-2C & 46,446 & 90,157 \\
IV9165A-720-1A & 44,982 & 89,612 \\
IV9165A-720-1B & 45,980 & 89,810 \\
IV9165A-720-2C & 47,368 & 92,859 \\
IV9165A-720-2D & 44,617 & 87,763 \\
\hline Average (s.d.) & $46,055( \pm 864)$ & $89,985( \pm 2089)$ \\
\hline
\end{tabular}

Table 6. Equilibrated J-13 (EJ-13) Groundwater

\begin{tabular}{lc}
\multicolumn{2}{c}{ Leachant Composition $(\mathrm{ppm})^{\mathrm{a}}$} \\
\hline $\mathrm{Si}$ & 34.4 \\
$\mathrm{Na}$ & 46.5 \\
$\mathrm{~K}$ & 8.08 \\
$\mathrm{Mg}$ & 0.96 \\
$\mathrm{Ca}$ & 9.08 \\
$\mathrm{U}$ & 0.0024 \\
$\mathrm{NO}^{-}$ & $<0.5$ \\
$\mathrm{NO}_{3}^{-}$ & 8.2 \\
$\mathrm{~F}^{-}$ & 2.8 \\
$\mathrm{Cl}^{-}$ & 8.6 \\
$\mathrm{HCO}_{3}^{-}$ & 93.5 \\
$\mathrm{SO}_{4}{ }^{-}$ & 21 \\
\hline${ }^{2} \mathrm{pH}=8.2$ &
\end{tabular}


D. Analytical Procedure

1. Test Termination

Gamma-irradiated samples were removed from their respective radiation sources upon completion of the prescribed test intervals. The vessels for both the elevated-temperature ( 90 to $200^{\circ} \mathrm{C}$ ) blank gamma-irradiation tests and vapor hydration tests with glass were cooled by immersing the vessel bottom in an ice-water bath. This procedure induced the condensation of moisture on the bottom of the vessel rather than on the sides of the vessel wall or on the glass test components if present. Solution aliquots from the $340 \mathrm{~m}^{-1}$ batch tests were collected while the solution was maintained at the experimental temperature of $90^{\circ} \mathrm{C}$. This sampling at elevated temperature prevented the precipitation of dissolved components before the solution aliquots were collected.

The weights of the test vessels plus contents were measured both before, during, and after testing to assess the extent of fluid loss, if any, from the vessels. Weight losses from the vessels in the gamma-irradiation tests at 25 and $90^{\circ} \mathrm{C}$ were insignificant $(<0.02 \mathrm{~g})$, while average losses for the gamma tests at $200^{\circ} \mathrm{C}$ and the alpha tests at $25^{\circ} \mathrm{C}$ were $0.03 \pm 0.03$ and $3.21 \pm 3.47 \mathrm{~g}$, respectively. For the latter two test types, the average weight loss represents $\sim 15 \%$ of the total water mass added to each test vessel. With the alpha tests, an increase in water loss as a function of increasing reaction time was also observed; however, a separate liquid-water phase remained in the bottom of all vessels during testing. In the tests with glass, the maximum water loss from the 150 and $200^{\circ} \mathrm{C}$ gamma tests was always less than that required to maintain vapor-phase saturation inside the vessels. Vessel leakage from the $340 \mathrm{~m}^{-1}$ batch tests was also insignificant relative to the initial $2.0 \mathrm{~mL}$ added to the test vessels.

The previously calculated weight loss from the samples may have two contributing sources: (1) loss of water from the interior of the vessels and (2) evaporation of the silica-organic lubricant applied to the closure nut assembly used with tests conducted in 304L stainless steel containers (gamma blank and vapor hydration tests with glass). The calculated water weight losses are thus maximum estimates due to the potential contributing weight loss from the lubricant material. Any leakage of water from the interior of the vessels would be expected to lower the concentration of "dry" atmospheric gases as evaporation of water vapor continuously replaced gas components such as $\mathrm{N}_{2}, \mathrm{O}_{2}$, and $\mathrm{CO}_{2}$. The test results from vessels with large amounts of water loss indicated that vessel leakage did not influence radiolytic product yields to any measurable degree.

\section{a. Leachate Collection from Blank Irradiation Tests}

After weighing, the blank test vessels were opened, and "prerinse" aliquots were collected from the solution that collected in the bottom of the vessel. For the gamma irradiation tests, prerinse solutions were analyzed only for anions $\left(\mathrm{NO}_{3}{ }^{-}, \mathrm{NO}_{2}{ }^{-}, \mathrm{Cl}^{-}, \mathrm{SO}_{4}{ }^{2}\right.$, $\mathrm{F}^{-}, \mathrm{COOH}^{-}$, and $\mathrm{C}_{2} \mathrm{O}_{4}{ }^{2-}$, while anions, $\mathrm{pH}$, and carbon were analyzed from the prerinse alphairradiation solutions. The surfaces of the test vessels and components were next sprayed with a fine mist of high-purity deionized water to flush any radiolytic products that had condensed on the solid surfaces to the bottom of the vessel. Aliquots of this rinse solution were analyzed for anions, $\mathrm{pH}$, and carbon for both the gamma- and alpha-irradiation tests. Corrections were applied to rinse solution analyses to account for the mass of elements that were removed with the prerinse aliquot. The rinse solution values reported in Sec. III thus represent the total production (in micro-moles) of elemental species in these tests. Actual concentrations of radiolytic products in the fluid condensed on the test vessel walls could not be calculated because it was impossible to determine the actual weight distribution of water between the prerinse solution and the condensed fluid. 


\section{b. Leachate Collection from Batch Tests at $340 \mathrm{~m}^{-1}$}

After being weighed, the batch test vessels were opened, and aliquots were drawn from the leachate solution for determination of anions, cations, $\mathrm{pH}$, and carbon, as well as alpha spectroscopy analysis. These aliquots were taken immediately after the vessels were opened to avoid cooling of the test solution and subsequent precipitation of elements from solution. The aliquot drawn for cation analysis was also acidified with ultrapure nitric acid to keep dissolved components in solution. A small amount of solution was also drawn from a selected number of tests for filtering and microscopic examination of colloidal material.

After $\mathrm{pH}$ measurements were taken, the remaining solution was passed through a 5-nm centrifuge filter. This filtrate was also submitted for alpha spectroscopy analysis. Both the $\mathrm{pH}$ analysis and all filtration steps were conducted at room temperature.

The reacted glass samples were next removed from the test vessels and retained for future solid-phase analyses. The test vessel was then refilled with deionized water plus six drops of nitric acid and returned to a $90^{\circ} \mathrm{C}$ test oven. After soaking overnight, a third alpha spectroscopy sample was collected from this acid strip solution. This final aliquot is representative of the fraction of alpha-emitting actinide elements that plated out on the test vessel walls and support stand during the testing period.

\section{c. Leachate Analysis from Vapor Hydration Tests}

The minute amount of fluid present in these tests precluded the collection of solution aliquots for compositional analysis. However, it was desirable to analyze the $\mathrm{pH}$ of the solution collected in the vessel bottom to determine whether condensed fluid had dripped from the glass samples. The presence of these drops of fluid would be readily apparent in the elevated $\mathrm{pH}$ values of the solution in the bottom of the vessel. These $\mathrm{pH}$ determinations were performed with litmus paper.

\section{Leachate Analytical Techniques}

Anion determinations were made by ion chromatography, carbon with a Dohrman total carbon analyzer, and $\mathrm{pH}$ with a combination electrode. All analytical measurements, including $\mathrm{pH}$, were made at room temperature. Accuracy of anion results, reported as a percentage deviation from known values of a standard, ranges from 2 to $7 \%$. Anion precision, determined by triplicate analyses of a single sample and reported as the coefficient of variation (SD/Mean $\times 100 \%$ ), ranges from 2 to $5 \%$. Cation accuracy and precision are both $<10 \%$. The accuracy determined for carbon values is within $3 \%$ error, while analytical drift over the time period required for $\mathrm{pH}$ analyses is typically less than $0.02 \mathrm{pH}$ units. For the alpha spectroscopy measurements, accuracy was determined to be $\sim 20 \%$ for americium and plutonium, while neptunium accuracy was $\sim 50 \%$.

\section{Solid-Phase Analytical Techniques}

Solid-phase analytical investigations focused on examining the thickness of the alteration layer, identifying the sequence of secondary precipitates that developed on the glass surface, and identifying colloids formed in solution. Solid-phase analyses provide complementary information to solution results for the $340 \mathrm{~m}^{-1}$ tests, while they are the primary information source for the vapor hydration tests. These analyses are thus important in determining the true extent of glass reaction. Analysis of the alteration phases will provide information on elements that are released to solution during the glass corrosion processes, including phases that are depleted from altered glass regions and those that are subsequently reprecipitated as alteration minerals on the reacted glass surface. Knowledge of the conditions 
that favor the precipitation of these phases may also provide complementary information on the solution environments contacting the glass. This latter aspect will provide critical information on fluid composition for the vapor hydration tests, when the limited amounts of fluid present preclude direct solution analyses.

Two general types of alteration phases develop on the sample surfaces as the glass begins to corrode. As the glass hydrates, the composition of the outer surface will change due to a combination of hydration and selective ion exchange (leaching) processes. The net effect of this process is the formation of an in situ alteration layer that is depleted in more soluble glass components and enriched in hydration components relative to the original glass. This layer also displays a residual enrichment in less-soluble components that remain behind after glass leaching. This alteration layer typically originates as an amorphous gel material but will, in time, restructure to form a layer composed predominantly of clay minerals. Leached components from the glass may reprecipitate on the glass surface as the solution in contact with the glass surface becomes saturated with respect to various mineral phases. These ex situ phases are typically crystalline and may precipitate on the altered glass surface as discrete mineral phases and/or blanketing layers covering the in situ layer.

The altered sample surfaces were examined using a variety of analytical techniques including optical microscopy, SEM/EDS, X-ray diffraction (XRD), and analytical electron microscopy (AEM). This combination of techniques was diagnostic in identifying many of the mineral phases present on the nonirradiated test samples. The altered materials from the samples doped with actinides plus ${ }^{99} \mathrm{Tc}$ were only examined by optical and SEM/EDS analyses because of the difficulties in handling very fine-grained radioactive materials. Phase identification on these latter samples was also based partially on knowledge gained during previous examinations of nonirradiated samples.

The general approach used in these sample examinations is to first complete an optical survey of each sample. This survey is used to identify the degree of sample heterogeneity and regions of interest for more detailed SEM analysis. Optical surveys were conducted with a stereomicroscope with magnification capabilities up to $150 \mathrm{X}$. Color and black-and-white photographs were taken to provide a record of the sample surfaces.

Representative samples of the various crystalline phases of interest were carefully removed from the surface for analysis using XRD and/or AEM analysis. Crystallographic data using XRD analysis were obtained with a Phillips XRD powder diffraction camera of $114.6-\mathrm{mm}$ dia using filtered $\mathrm{CuK}_{\text {alpha }}$ or $\mathrm{FeK}_{\mathrm{alpha}}$ radiation. Intensities of the refraction lines were visually estimated and normalized to the strongest line intensity. Crystallographic data using the AEM instrumentation were obtained by selected area electron diffraction (SAED) analysis . Intensities of the refraction lines were visually estimated and normalized to the strongest line intensity.

Crystalline material deposited on the sample surfaces was typically examined in situ by SEM/EDS after the altered glass was mounted directly on the aluminum mounting stub and the samples were carbon coated. Mineral paragenetic trends were identified by examining spatial relationships developed between the various mineral phases on a single sample and by correlating the genesis of phases from a temporal sequence of samples. In some cases, after extracted crystalline phases had been analyzed by XRD analysis, the phases were mounted on an aluminum stub, carbon coated, and analyzed by SEM/EDS. 
The SEM investigations provided a detailed examination of at least one of the four glass monoliths that were reacted each sampling interval. Additional sample examinations may be warranted based on sample heterogeneities. The SEM analyses were performed using both secondary electron and backscattered electron (BSE) detectors. The latter technique rapidly identifies regions of contrasting elemental density. The BSE analysis was found to be invaluable in the rapid detection of mineral phases bearing uranium and high-density transition metal.

Spatial resolutions using SEM analysis are typically in the sub-micron range. The EDS resolution is inversely proportional to the sample density and accelerating voltage used in the analysis. The EDS resolution is typically poorer than both the SEM resolution and the electron beam diameter. A typical EDS spatial resolution on the order of $8 \mu \mathrm{m}$ is obtainable for a material with a density similar to glass $\left(2.7 \mathrm{~g} / \mathrm{cm}^{3}\right)$ and an accelerating voltage of $20 \mathrm{kV}$, while a spatial resolution of $\sim 3 \mu \mathrm{m}$ would be expected for the same material at an accelerating voltage of $10 \mathrm{kV}$. The EDS resolution can be improved by using a lower accelerating voltage or by examining higher density materials.

Layer thickness measurements were made by SEM analysis of altered glass specimens that had been mounted in epoxy, sectioned normal to the original glass surface, and polished to a one-micron finish using a water lubricant. The epoxy mounting resin provided a strong binding medium, which prevented the loosely attached alteration minerals from being dislodged and the more soluble phases from being completely dissolved during the cutting and polishing processes. Because of the fragmentary nature of some of the more highly altered samples in the irradiated vapor hydration tests, there was some concern that the pouring of epoxy resin onto the samples would disturb the delicate nature of the altered layers by a flotation process. A procedure was devised for these samples, where the altered glass was first impregnated with a low-viscosity Medcast medium. This initial casting process appears to have solidified the samples without separating the layers from the glass. The mounts for the Medcast samples were then set into an epoxy resin, cut with a Buehler saw, and polished for cross-section analysis as done with previous samples.

Approximately 20 layer measurements were taken from each sectioned glass sample, with measurement locations being made at regularly spaced intervals along the sample perimeter. A single sample was analyzed from each of the testing intervals for each respective glass composition. These layer thickness measurements include the aggregate thicknesses of all clay-rich layers that occur on the samples, regardless of whether they formed as residual in situ layers or ex situ precipitate layers. These measurements do not, however, include the formation of discrete crystalline precipitates such as zeolites and uranyl silicates.

The AEM analysis was used to determine the thickness and structure of very thin clay layers and the identity of colloidal materials filtered from solution. This technique uses a combination of morphological information generated by transmission electron microscopy (TEM), mineral structural data generated by SAED analysis, and quantitative EDS compositional analysis. Spatial resolutions using TEM analysis are typically in the sub-nanometer to angstrom range and are thus particularly useful in imaging the 0.7 to $1.5 \mathrm{~nm}$ lattice planes that are characteristic of clay crystallites.

\section{E. Oven Dosimetry}

A ferrous sulfate (Fricke) dosimeter was employed to determine radiation dose rates for these tests. With the ferrous sulfate dosimetry technique, the oxidation level of the test solution is measured as a function of the level of conversion of ferrous sulfate to a ferric salt after the solution has been irradiated in the presence of oxygen for a specific period. This conversion occurs as a function of the amount of energy absorbed by an aqueous system and thus can be used as a dosimeter [35]. The ferrous sulfate solution was prepared by dissolving $0.14 \mathrm{~g}$ 
$\mathrm{FeSO}_{4} \cdot 7 \mathrm{H}_{2} \mathrm{O}$ and $11 \mathrm{~mL}$ concentrated $\mathrm{H}_{2} \mathrm{SO}_{4}$ into enough high-purity deionized water to produce $500 \mathrm{~mL}$ of test solution.

Several vessel configurations for the dosimetry tests were considered for these analyses. The design mode chosen for the test components maximized the amount of ferrous sulfate solution added to each container, yet maintained the level of the solution below the upper lip of the Parr vessel. Eleven dram glass tubes were placed in uncapped $22 \mathrm{~mL}$ Parr stainless steel vessels with assembled closure nuts. This experimental design simulates the exposure conditions expected for the actual test vessels. Thirteen milliliters of fresh ferrous sulfate solution was then added to the each glass tube. This quantity of solution allows for triplicate spectral analysis and one rinse of the UV spectra absorption cells. The glass tubes were next capped with aluminum foil, and the assembled components were transported to the irradiation facility for testing.

After irradiation, the samples were removed from the irradiation cell and transported to a separate laboratory for UV spectroscopy analysis. The ferric ions produced during radiolysis show a maximum absorption at about $304 \mathrm{~nm}$ [35]. The difference between peak heights for irradiated and nonirradiated Fricke solutions gives the difference in absorbance or optical density (OD) for the solution. The mean absorbed dose $\left(D_{D}\right)$, in $G y / h$, can be calculated by the equation

$$
\mathrm{D}_{\mathrm{D}}=\frac{\mathrm{OD} \times \mathrm{k}}{\text { irradiation time }(\mathrm{h})}
$$

where $\mathrm{k}$ is a constant with a value of $2.7556 \times 10^{2}$.

Results of the dosimetry tests are presented Table A.1. Tests were performed in two ${ }^{60} \mathrm{Co}$ irradiation facilities at Argonne National Laboratory (ANL): one in the Building $200 \mathrm{M}$-wing facility , and the other in Building 223. The former was used for ambient temperature hot-cell tests and all blank gamma tests at $90^{\circ} \mathrm{C}$ and all batch tests. The dosimetry tests measured (1) the radiation dose received $0.67 \mathrm{~m}$ from the ${ }^{60} \mathrm{Co}$ source, (2) the radiation dose received $3.11 \mathrm{~m}$ from the source, and (3) the radiation dose received $3.11 \mathrm{~m}$ from the source with the samples behind $2.5 \mathrm{~cm}$ of lead shielding. These last tests utilized the same positions as the previous unshielded $3.11-\mathrm{m}$ samples. The first set of tests was designed to identify a suitable location for the blank gamma tests at high dose rate and $25^{\circ} \mathrm{C}$. The completed analyses indicate a dose yield of $\sim 500 \mathrm{~Gy} / \mathrm{h}$. The second set of tests determined the optimum location for placement of the batch tests at $340 \mathrm{~m}^{-1}$. Calculated dose yields obtained from the ferrous sulfate tests for these $3.11 \mathrm{~m}$ locations are between 31 and $37 \mathrm{~Gy} / \mathrm{h}$. The third set of tests, conducted at $3.11 \mathrm{~m}$ but with the samples behind $2.5-\mathrm{cm}$ layer of lead shielding, indicates that the shielding attenuated gamma yields by a factor of $\sim 67 \%$.

The Building $223{ }^{60} \mathrm{Co}$ irradiation facility was used for the blank gamma tests at $200^{\circ} \mathrm{C}$ and all vapor hydration tests. Results from the dosimetry tests at this location are also presented in Table A.1. Distances measured from the ${ }^{60} \mathrm{Co}$ source to the samples ranged from 0.20 to $0.43 \mathrm{~m}$. 


\section{SOLUTION RESULTS}

Solution analyses were completed for the blank tests with gamma and alpha irradiation and for the $340 \mathrm{~m}^{-1}$ batch leach tests.

\section{A. Blank Tests with Gamma Irradiation}

\section{Anion Concentrations}

A detailed matrix listing all blank gamma-irradiation tests and experimental parameters is given in Table 7. Solution volumes used to calculate anion species $\mu$ mol values are given in Table B.1.1. Dilution and aliquot removed corrected $\mu \mathrm{mol}$ values are given in Tables $8 \mathrm{a}$ and $9 \mathrm{a}$, while as-received solution aliquot concentrations appear in Tables $8 \mathrm{~b}$ and $9 \mathrm{~b}$. Significant amounts of nitrate $\left(\mathrm{NO}_{3}{ }^{-}\right)$and minor amounts of nitrite $\left(\mathrm{NO}_{2}{ }^{-}\right)$were produced during these tests (Table 8). Between 40 and $80 \%$ of the nitrate and nitrite recovered from the vessels was concentrated in the prerinse solution, with the remaining fraction being located in the condensate that had collected on the vessel walls. The vessel bottom represents only $10 \%$ of the geometric surface area, indicating that nitrogen acids are preferentially concentrated in the prerinse solution at the vessel bottom. This concentration mechanism probably involves gravitational settling of aerosols and/or dripping of condensate from the walls into the bottom of the vessel. The concentration of $\mathrm{F}^{-}$in irradiated solutions remained near background levels for most rinse and prerinse solutions. Concentrations of $\mathrm{SO}_{4}{ }^{2-}$ were enriched in solutions for many of the respective gamma irradiation test scenarios, relative to the concentrations detected in the blank tests. Sulfur is a minor component in the stainless steel test vessels, thus the enrichment patterns observed may reflect some enhanced corrosion of the vessels under the influence of an irradiated moist air environment. Chlorine is also enriched in many of the irradiated solutions as well. Levels of enrichment for both elements tended to be higher in the lower temperature relative to the higher temperature tests, and higher concentrations of these elements were noted in the prerinse relative to the rinse solutions (Table $8 \mathrm{~b}$ ).

Data for G-value yields in Table 10 are presented for nitrate, nitrite, and nitrate + nitrite summed together. The latter summation accounts for the potential influence of redox reactions on nitrogen acid yields. For most tests, the contribution of nitrite toward the total yields is negligible. Exceptions to this trend are found in the high-dose gamma tests at 56 and 120 days, where nitrite levels represent $\sim 10 \%$ of the total production, and in the gamma tests with $\mathrm{G} / \mathrm{L}=10$, where nitrite yields represent $\sim 65 \%$ of the total yield.

The $G$ values were calculated from the following equation [11]:

$$
\mathrm{G}=\left(\frac{\mathrm{n} \mathrm{N}_{\mathrm{A}}}{\mathrm{DkgN_{2 }}}\right) \times 100
$$


where $\mathrm{n}=$ the number of moles of $\mathrm{NO}_{2}{ }^{-}+\mathrm{NO}_{3}{ }^{-}$produced,

$\mathrm{N}_{\mathrm{A}}=$ Avogadro's constant $\left(6.022 \times 10^{23}\right.$ molecules $\left.\mathrm{mol}^{-1}\right)$,

$\mathrm{D}=$ the cumulative dose in rad,

$\mathrm{k}=\quad \mathrm{a}$ constant, $6.24 \times 10^{13} \mathrm{eV} \cdot \mathrm{g}^{-1} \cdot \operatorname{rad}^{-1}$, and

$\mathrm{gN}_{2}=$ the number of grams of $\mathrm{N}_{2}$ gas contained in each $21.4 \mathrm{~cm}^{3}$ stainless steel ( 0.0213 for the $G / L=100$ tests and 0.0195 for the $G / L=10$ tests) gamma tests or $1764 \mathrm{~cm}^{3}$ glass vessel for the alpha tests $(1.72 \mathrm{~g})$.

The value of 100 in the numerator reflects the G-value units of molecules produced per $100 \mathrm{eV}$. 
Table 7. Sampling Matrix for Blank Gamma Tests

\begin{tabular}{|c|c|c|c|c|c|c|}
\hline $\begin{array}{l}\text { Test } \\
\text { Number }\end{array}$ & $\begin{array}{l}\text { Glass } \\
\text { Type }\end{array}$ & $\begin{array}{l}\text { Temp. } \\
\left({ }^{\circ} \mathrm{C}\right)\end{array}$ & $\begin{array}{l}\text { Test } \\
\text { Length } \\
\text { (days) }\end{array}$ & $\begin{array}{l}\text { Dose } \\
\text { Rate } \\
(\mathrm{Gy} / \mathrm{h})\end{array}$ & $\begin{array}{c}\text { Gas/Liquid } \\
\text { Ratio }\end{array}$ & $\begin{array}{c}\text { Cumulative } \\
\text { Dose } \\
\text { (Mrad) }\end{array}$ \\
\hline IV9000-7-G1B-100 & None & 25 & 7 & 515 & 100 & 86.5 \\
\hline IV9000-7-G2B-100 & None & 25 & 7 & 515 & 100 & 86.5 \\
\hline IV9000-14-G1B-100 & None & 25 & 14 & 561 & 100 & 188.5 \\
\hline IV9000-14-G2B-100 & None & 25 & 14 & 561 & 100 & 188.5 \\
\hline IV9000-28-G1B-100 & None & 25 & 28 & 548 & 100 & 368.3 \\
\hline IV9000-28-G2B-100 & None & 25 & 28 & 532 & 100 & 357.5 \\
\hline IV9000-56-G1B-100 & None & 25 & 56 & 515 & 100 & 692.2 \\
\hline IV9000-56-G2B-100 & None & 25 & 56 & 515 & 100 & 692.2 \\
\hline IV9000-120-G1B-100 & None & 25 & 120 & 514 & 100 & 1480.3 \\
\hline IV9000-120-G2B-100 & None & 25 & 120 & 514 & 100 & 1480.3 \\
\hline IV9000-28-G1-100 & None & 25 & 34 & 36 & 100 & 29.4 \\
\hline IV9000-28-G2-100 & None & 25 & 34 & 36 & 100 & 29.4 \\
\hline IV9000-56-G1-100 & None & 25 & 56 & 36 & 100 & 48.4 \\
\hline IV9000-56-G2-100 & None & 25 & 56 & 36 & 100 & 48.4 \\
\hline IV9000-120-G1-100 & None & 25 & 126 & 36 & 100 & 108.9 \\
\hline IV9000-120-G2-100 & None & 25 & 126 & 36 & 100 & 108.9 \\
\hline IV9000-14-G1-100 & None & 25 & 126 & 36 & 100 & 108.9 \\
\hline IV9000-14-G2-100 & None & 25 & 126 & 36 & 100 & 108.9 \\
\hline IV1000-56-G3-100 & None & 90 & 49 & 22 & 100 & 25.9 \\
\hline IV1000-56-G4-100 & None & 90 & 49 & 22 & 100 & 25.9 \\
\hline IV1000-85-G1-100 & None & 90 & 85 & 34 & 100 & 69.4 \\
\hline IV1000-85-G2-100 & None & 90 & 85 & 34 & 100 & 69.4 \\
\hline IV1000-120-G1-100 & None & 90 & 122 & 22 & 100 & 64.4 \\
\hline IV1000-120-G2-100 & None & 90 & 122 & 22 & 100 & 64.4 \\
\hline IV1000-150-G1-100 & None & 90 & 150 & 32 & 100 & 115.2 \\
\hline IV1000-150-G2-100 & None & 90 & 150 & 32 & 100 & 115.2 \\
\hline IV1000-56-G1-100 & None & 90 & 171 & 22 & 100 & 90.3 \\
\hline IV1000-56-G2-100 & None & 90 & 171 & 22 & 100 & 90.3 \\
\hline IV2000-56-G3-100 & None & 200 & $\overline{49}$ & $\overline{42}$ & 100 & 49.4 \\
\hline IV2000-56-G4-100 & None & 200 & 49 & 42 & 100 & 49.4 \\
\hline IV2000-85-G1-100 & None & 200 & 85 & 24 & 100 & 49.0 \\
\hline IV2000-85-G2-100 & None & 200 & 85 & 24 & 100 & 49.0 \\
\hline IV2000-120-G1-100 & None & 200 & 122 & 42 & 100 & 123.0 \\
\hline IV2000-120-G2-100 & None & 200 & 122 & 42 & 100 & 123.0 \\
\hline IV2000-150-G1-100 & None & 200 & 150 & 25 & 100 & 90.0 \\
\hline IV2000-150-G2-100 & None & 200 & 150 & 22 & 100 & 79.2 \\
\hline IV2000-56-G1-100 & None & 200 & 171 & 42 & 100 & 172.4 \\
\hline IV2000-56-G2-100 & None & 200 & 171 & 42 & 100 & 172.4 \\
\hline IV1000-56-G1-10 & None & 90 & 56 & $\overline{32}$ & 10 & 43.0 \\
\hline IV1000-56-G2-10 & None & 90 & 56 & 32 & 10 & 43.0 \\
\hline IV1000-120-G1-10 & None & 90 & 120 & 37 & 10 & 106.6 \\
\hline IV1000-120-G2-10 & None & 90 & 120 & 37 & 10 & 106.6 \\
\hline IV9000-28-BLANK-100 & None & 25 & 28 & None & 100 & None \\
\hline IV9000-120-BLANK-100 & None & 25 & 120 & None & 100 & None \\
\hline IV1000-56-BLANK-100 & None & 90 & 56 & None & 100 & None \\
\hline IV $1000-120-B L A N K-100$ & None & 90 & 120 & None & 100 & None \\
\hline IV2000-56-BLANK-100 & None & 200 & 56 & None & 100 & None \\
\hline IV2000-120-BLANK-100 & None & 200 & 120 & None & 100 & None \\
\hline
\end{tabular}


Table 8a. Anion Results for Blank Gamma Tests (values in total $\mu$ mol produced)

\begin{tabular}{|c|c|c|c|c|c|c|c|c|c|c|c|c|c|c|c|}
\hline \multirow[b]{2}{*}{ Test Number } & \multirow[b]{2}{*}{$\begin{array}{c}\text { Temp. } \\
\left({ }^{\circ} \mathrm{C}\right)\end{array}$} & \multirow{2}{*}{$\begin{array}{c}\text { Test } \\
\text { Length } \\
\text { (days) }\end{array}$} & \multirow{2}{*}{$\begin{array}{l}\text { Dose } \\
\text { Rate } \\
(\mathrm{Gy} / \mathrm{h})\end{array}$} & \multirow{2}{*}{$\begin{array}{l}\text { Gas: } \\
\text { Liq. } \\
\text { Ratio }\end{array}$} & \multirow{2}{*}{$\begin{array}{l}\text { Expos } \\
\text { Time } \\
\text { (h) }\end{array}$} & \multicolumn{2}{|c|}{$\mathrm{Cl}$} & \multicolumn{2}{|c|}{$\mathrm{SO}_{4}$} & \multicolumn{2}{|c|}{$\mathrm{NO}_{3}$} & \multicolumn{2}{|c|}{$\mathrm{NO}_{2}$} & \multicolumn{2}{|l|}{$\mathrm{F}$} \\
\hline & & & & & & Prerinse & Rinse & Prerinse & Rinse & Prerinse & Rinse & Prerinse & Rinse & Prerinse & Rinse \\
\hline IV $9000-7-G 1 B-100$ & 25 & 7 & 515 & 100 & 165 & 0.02 & 0.03 & 0.00 & 0.02 & 0.62 & 0.48 & $<0.00$ & 0.02 & 0.03 & 0.05 \\
\hline IV $9000-7-G 2 B-100$ & 25 & 7 & 515 & 100 & 165 & 0.01 & 0.02 & 0.00 & 0.01 & 0.56 & 0.47 & $<0.00$ & 0.02 & 0.02 & 0.04 \\
\hline IV $9000-14-G 1 B-100$ & 25 & 13 & 561 & 100 & 304 & 0.03 & 0.08 & 0.01 & 0.03 & 1.59 & 1.23 & $<0.00$ & 0.04 & 0.01 & $<0.02$ \\
\hline IV $9000-14-G 2 B-100$ & 25 & 13 & 561 & 100 & 304 & 0.03 & 0.05 & 0.01 & 0.02 & 1.44 & 1.21 & $<0.00$ & 0.04 & 0.02 & $<0.02$ \\
\hline IV $9000-28-G 1 B-100$ & 25 & 28 & 548 & 100 & 657 & 0.04 & 0.05 & 0.00 & 0.02 & 2.35 & 1.86 & $<0.00$ & 0.03 & 0.02 & 0.07 \\
\hline IV $9000-28-G 2 B-100$ & 25 & 28 & 532 & 100 & 657 & 0.01 & 0.03 & $<0.00$ & $<0.01$ & 2.16 & 2.05 & $<0.00$ & 0.04 & 0.01 & $<0.01$ \\
\hline IV $9000-56-G 1 B-100$ & 25 & 56 & 515 & 100 & 1324 & 0.03 & 0.05 & 0.04 & 0.02 & 4.94 & 4.22 & 0.54 & 0.26 & 0.02 & 0.05 \\
\hline IV $9000-56-G 2 B-100$ & 25 & 56 & 515 & 100 & 1324 & 0.02 & 0.05 & 0.13 & 0.04 & 6.71 & 4.89 & 0.51 & 0.27 & 0.03 & 0.04 \\
\hline IV $9000-120-G 1 B-100$ & 25 & 120 & 514 & 100 & 2805 & 0.02 & 0.03 & 0.00 & $<0.01$ & 6.34 & 5.06 & 0.48 & 0.24 & 0.01 & 0.06 \\
\hline IV $9000-120-G 2 B-100$ & 25 & 120 & 514 & 100 & 2805 & 0.06 & 0.07 & 0.06 & 0.02 & 10.55 & 7.33 & 1.25 & $<0.57$ & 0.02 & 0.06 \\
\hline IV $9000-28-G 1-100$ & 25 & 34 & 36 & 100 & 816 & 0.30 & 0.52 & 0.00 & 0.06 & 0.06 & 0.22 & 0.00 & 0.02 & 0.02 & 0.05 \\
\hline IV $9000-28-G 2-100$ & 25 & 34 & 36 & 100 & 816 & 0.75 & 1.98 & 0.07 & 0.21 & 0.10 & 0.27 & 0.01 & 0.05 & 0.00 & 0.07 \\
\hline IV $9000-56-G 1-100$ & 25 & 56 & 36 & 100 & 1342 & 0.12 & 0.30 & 0.04 & 0.08 & 0.04 & 0.33 & 0.00 & 0.01 & 0.02 & 0.07 \\
\hline IV $9000-56-G 2-100$ & 25 & 56 & 36 & 100 & 1342 & 0.10 & 0.13 & 0.00 & 0.03 & 0.25 & 0.46 & 0.00 & 0.01 & 0.02 & 0.03 \\
\hline IV $9000-120-G 1-100$ & 25 & 126 & 36 & 100 & 3017 & 0.64 & 1.18 & 0.00 & 0.14 & 0.37 & 0.71 & 0.00 & 0.02 & 0.00 & 0.00 \\
\hline IV $9000-120-G 2-100$ & 25 & 126 & 36 & 100 & 3017 & 0.68 & 1.44 & 0.06 & 0.19 & 0.38 & 0.82 & 0.00 & 0.02 & 0.00 & 0.00 \\
\hline IV $9000-14-G 1-100$ & 25 & 126 & 36 & 100 & 3017 & 1.11 & 4.23 & 0.11 & 0.26 & 0.22 & 0.56 & 0.00 & 0.02 & 0.00 & 0.00 \\
\hline IV $9000-14-G 2-100$ & 25 & 126 & 36 & 100 & 3017 & 0.83 & 2.76 & 0.06 & 0.17 & 0.09 & 0.51 & 0.00 & 0.02 & 0.00 & 0.00 \\
\hline IV $1000-56-G 3-100$ & 90 & 49 & 22 & 100 & 1179 & 0.01 & 0.11 & 0.06 & 0.11 & 0.12 & 0.14 & 0.00 & 0.05 & 0.00 & 0.00 \\
\hline IV1000-56-G4-100 & 90 & 49 & 22 & 100 & 1179 & 0.02 & 0.04 & 0.39 & 0.26 & 0.04 & 0.05 & 0.02 & 0.03 & 0.00 & 0.00 \\
\hline IV $1000-85-G 1-100$ & 90 & 85 & 34 & 100 & 2014 & 0.08 & 0.10 & 0.05 & 0.06 & 0.01 & 0.03 & $<0.00$ & 0.06 & 0.07 & 0.10 \\
\hline IV $1000-85-G 2-100$ & 90 & 85 & 34 & 100 & 2014 & 0.04 & 0.07 & 0.19 & 0.13 & 0.01 & 0.03 & 0.01 & 0.05 & 0.04 & 0.08 \\
\hline IV $1000-120-G 1-100$ & 90 & 122 & 22 & 100 & 2922 & 0.04 & 0.10 & 0.03 & 0.06 & 0.15 & 0.18 & 0.05 & 0.07 & 0.05 & 0.02 \\
\hline IV $1000-120-G 2-100$ & 90 & 122 & 22 & 100 & 2922 & 0.02 & 0.17 & 0.01 & 0.11 & 0.05 & 0.08 & 0.06 & 0.08 & 0.00 & 0.00 \\
\hline IV $1000-150-G 1-100$ & 90 & 150 & 32 & 100 & 3470 & 0.07 & 0.08 & 0.30 & 0.12 & 0.04 & 0.04 & $<0.00$ & $<0.01$ & 0.01 & 0.05 \\
\hline IV $1000-150-G 2 \cdot 100$ & 90 & 150 & 32 & 100 & 3470 & 0.21 & 0.19 & 0.05 & 0.06 & 0.05 & 0.05 & $<0.00$ & $<0.01$ & 0.02 & 0.03 \\
\hline IV $1000-56-G 1-100$ & 90 & 173 & 22 & 100 & 4101 & 0.03 & 0.27 & 0.14 & 0.35 & 0.33 & 0.32 & 0.00 & 0.04 & 0.11 & 0.11 \\
\hline IV $1000-56-G 2-100$ & 90 & 173 & 22 & 100 & 4101 & 0.07 & 0.16 & 0.01 & 0.11 & 0.032 & 0.28 & 0.03 & 0.05 & 0.05 & 0.06 \\
\hline
\end{tabular}


Table 8a - contd.

\begin{tabular}{|c|c|c|c|c|c|c|c|c|c|c|c|c|c|c|c|}
\hline \multirow[b]{2}{*}{ Test Number } & \multirow{2}{*}{$\begin{array}{c}\text { Temp. } \\
\left({ }^{\circ} \mathrm{C}\right)\end{array}$} & \multirow{2}{*}{$\begin{array}{c}\text { Test } \\
\text { Length } \\
\text { (days) }\end{array}$} & \multirow{2}{*}{$\begin{array}{c}\text { Dose } \\
\text { Rate } \\
(\mathrm{Gy} / \mathrm{h}) \\
\end{array}$} & \multirow{2}{*}{$\begin{array}{l}\text { Gas: } \\
\text { Liq. } \\
\text { Ratio } \\
\end{array}$} & \multirow{2}{*}{$\begin{array}{c}\text { Expos. } \\
\text { Time } \\
\text { (h) }\end{array}$} & \multicolumn{2}{|c|}{$\mathrm{Cl}$} & \multicolumn{2}{|c|}{$\mathrm{SO}_{4}$} & \multicolumn{2}{|c|}{$\mathrm{NO}_{3}$} & \multicolumn{2}{|c|}{$\mathrm{NO}_{2}$} & \multicolumn{2}{|c|}{$\bar{F}$} \\
\hline & & & & & & Prerinse & Rinse & Prerinse & Rinse & Prerinse & Rinse & Prerinse & Rinse & Prerinse & Rinse \\
\hline IV2000-56-G3-100 & 200 & 49 & 42 & 100 & 1179 & 0.00 & 0.03 & 0.03 & 0.05 & 0.00 & 0.05 & 0.00 & 0.01 & 0.00 & 0.00 \\
\hline IV2000-56-G4-100 & 200 & 49 & 42 & 100 & 1179 & 0.00 & 0.02 & 0.05 & 0.04 & 0.07 & 0.06 & 0.00 & 0.04 & 0.00 & 0.00 \\
\hline IV $2000-85-G 1-100$ & 200 & 85 & 24 & 100 & 3737 & 0.00 & 0.02 & 0.01 & 0.01 & 0.02 & 0.02 & 0.00 & $<0.01$ & 0.01 & 0.03 \\
\hline IV2000-85-G2-100 & 200 & 85 & 24 & 100 & 4318 & 0.00 & 0.01 & 0.00 & 0.01 & 0.00 & 0.00 & 0.00 & 0.02 & 0.02 & 0.05 \\
\hline IV $2000-120-G 1-100$ & 200 & 122 & 42 & 100 & 2922 & 0.01 & 0.06 & 0.05 & 0.08 & 0.05 & 0.12 & 0.00 & 0.02 & 0.00 & 0.00 \\
\hline IV2000-120-G2-100 & 200 & 122 & 42 & 100 & 2922 & 0.00 & 0.18 & 0.02 & 0.13 & 0.08 & 0.16 & 0.00 & 0.01 & 0.00 & 0.00 \\
\hline IV2000-150-G1-100 & 200 & 150 & 25 & 100 & 4318 & 0.00 & 0.01 & 0.00 & 0.01 & 0.00 & 0.01 & 0.00 & 0.03 & 0.05 & 0.08 \\
\hline IV2000-150-G2-100 & 200 & 150 & 22 & 100 & 3737 & 0.00 & 0.02 & 0.03 & 0.02 & 0.00 & 0.00 & 0.00 & 0.03 & 0.11 & 0.12 \\
\hline IV2000-56-G1-100 & 200 & 173 & 42 & 100 & 4101 & 0.00 & 0.02 & 0.07 & 0.07 & 0.02 & 0.12 & 0.00 & 0.01 & 0.00 & 0.00 \\
\hline IV2000-56-G2-100 & 200 & 173 & 42 & 100 & 4101 & 0.01 & 0.05 & 0.05 & 0.03 & 0.00 & 0.02 & 0.00 & 0.04 & 0.00 & 0.00 \\
\hline IV1000-56-G1-10 & 90 & 56 & 32 & 10 & 1324 & 0.07 & 0.08 & 0.23 & 0.15 & 0.04 & 0.04 & 0.06 & 0.09 & 0.07 & 0.08 \\
\hline IV1000-56-G2-10 & 90 & 56 & 32 & 10 & 1324 & 0.08 & 0.11 & 0.37 & 0.24 & 0.06 & 0.08 & 0.07 & 0.12 & 0.08 & 0.10 \\
\hline IV1000-120-G1-10 & 90 & 120 & 37 & 10 & 2805 & 0.11 & 0.14 & 0.16 & 0.13 & 0.04 & 0.05 & 0.16 & 0.19 & 0.11 & 0.12 \\
\hline IV1000-120-G2-10 & 90 & 120 & 37 & 10 & 2805 & 0.11 & 0.12 & 0.25 & 0.18 & 0.01 & 0.03 & 0.07 & 0.13 & 0.08 & 0.17 \\
\hline IV9000-28-BLANK-100 & 25 & 56 & None & 100 & 0 & 0.00 & 0.07 & 0.00 & 0.01 & 0.00 & 0.03 & 0.00 & 0.09 & 0.03 & 0.05 \\
\hline IV $9000-120-B L A N K-100$ & 25 & 120 & None & 100 & 0 & 0.00 & 0.02 & 0.01 & 0.01 & 0.00 & 0.01 & 0.01 & 0.02 & 0.03 & 0.03 \\
\hline IV1000-56-BLANK-100 & 90 & 56 & None & 100 & 0 & 0.00 & 0.01 & 0.00 & 0.01 & 0.01 & 0.02 & 0.01 & 0.02 & 0.02 & 0.01 \\
\hline IV1000-120-BLANK-100 & 90 & 120 & None & 100 & 0 & 0.04 & 0.06 & 0.00 & 0.02 & 0.03 & 0.04 & 0.00 & 0.04 & 0.05 & NA \\
\hline IV2000-56-BLANK-100 & 200 & 56 & None & 100 & 0 & 0.01 & 0.07 & 0.00 & 0.04 & 0.00 & 0.05 & 0.00 & 0.08 & 0.00 & 0.01 \\
\hline IV2000-120-BLANK-100 & 200 & 120 & None & 100 & 0 & 0.00 & 0.06 & 0.00 & 0.01 & 0.00 & 0.01 & 0.00 & 0.03 & NA & NA \\
\hline
\end{tabular}

Rinse solution values include a correction factor for both the proportion of aliquots removed and the dilution of the test solution by rinse fluid. Prerinse total $\mu$ moles $=($ Prerinse concentration in $\mu \mathrm{g} / \mathrm{ml} * \mathrm{ml}$ leachate $) /$ molecular weight. Rinse total $\mu \mathrm{mole}=($ Rinse concentration in $\mu \mathrm{g} / \mathrm{ml}$ (ml leachant $-\mathrm{ml}$ aliquots removed $+\mathrm{ml}$ rinse solution added) + prerinse concentration in $\mu \mathrm{g} / \mathrm{ml} * \mathrm{ml}$ aliquots removed)/molecular weight. 
Table 8b. Raw Anion Results for Blank Gamma Tests (concentrations in ppm)

\begin{tabular}{|c|c|c|c|c|c|c|c|c|c|c|c|c|c|c|c|}
\hline \multirow[b]{2}{*}{ Test Number } & \multirow{2}{*}{$\begin{array}{c}\text { Temp. } \\
\left({ }^{\circ} \mathrm{C}\right)\end{array}$} & \multirow{2}{*}{$\begin{array}{c}\text { Test } \\
\text { Length } \\
\text { (days) }\end{array}$} & \multirow{2}{*}{$\begin{array}{c}\text { Dose } \\
\text { Rate } \\
(\mathrm{Gy} / \mathrm{h}) \\
\end{array}$} & \multirow{2}{*}{$\begin{array}{l}\text { Gas: } \\
\text { Liq. } \\
\text { Ratio }\end{array}$} & \multirow{2}{*}{$\begin{array}{c}\text { Expos. } \\
\text { Time } \\
\text { (h) }\end{array}$} & \multicolumn{2}{|c|}{$\mathrm{Cl}$} & \multicolumn{2}{|c|}{$\mathrm{SO}_{4}$} & \multicolumn{2}{|c|}{$\mathrm{NO}_{3}$} & \multicolumn{2}{|c|}{$\mathrm{NO}_{2}$} & \multicolumn{2}{|l|}{$\mathrm{F}$} \\
\hline & & & & & & Prerinse & Rinse & Prerinse & Rinse & Prerinse & Rinse & Prerinse & Rinse & Prerinse & Rinse \\
\hline IV9000-7-G1B-100 & 25 & 7 & 515 & 100 & 165 & 2.6 & 0.68 & 0.69 & 1.2 & 176 & 11 & $<0.20$ & 0.84 & 2.8 & 0.58 \\
\hline IV $9000-7-G 2 B-100$ & 25 & 7 & 515 & 100 & 165 & 1.4 & 0.43 & 0.24 & 0.61 & 159 & 8.5 & $<0.20$ & 0.59 & 1.3 & 0.37 \\
\hline IV $9000-14-G 1 B-100$ & 25 & 13 & 561 & 100 & 304 & 4.3 & 1.0 & 3.1 & 1.2 & 448 & 14 & $<0.2$ & 0.78 & 1.2 & $<0.1$ \\
\hline IV $9000-14-G 2 B-100$ & 25 & 13 & 561 & 100 & 304 & 4.7 & 0.62 & 2.9 & 0.92 & 407 & 18 & $<0.2$ & 0.93 & 1.6 & $<0.1$ \\
\hline IV9000-28-G1B-100 & 25 & 28 & 548 & 100 & 657 & 6.6 & 0.67 & 1.1 & 0.73 & 694 & 25 & $<0.2$ & 0.84 & 2.2 & 0.56 \\
\hline IV9000-28-G2B-100 & 25 & 28 & 532 & 100 & 657 & 2.4 & 0.54 & $<0.5$ & $<0.5$ & 637 & 37 & $<0.2$ & 1.1 & 0.9 & $<0.1$ \\
\hline IV $9000-56-G 1 B-100$ & 25 & 56 & 515 & 100 & 1324 & 5.3 & 1.3 & 16 & 1.5 & 1392 & 132 & 112 & 0.85 & 1.6 & 0.83 \\
\hline IV9000-56-G2B-100 & 25 & 56 & 515 & 100 & 1324 & 3.0 & 1.1 & 58 & 2.6 & 1890 & 86 & 106 & 1.2 & 2.3 & 0.39 \\
\hline IV $9000-120-G 1 B-100$ & 25 & 120 & 514 & 100 & 2805 & 3.2 & 0.5 & 1.0 & $<0.2$ & 1872 & 95 & 105 & 0.57 & 0.70 & 0.86 \\
\hline IV $9000-120-G 2 B-100$ & 25 & 120 & 514 & 100 & 2805 & 10.0 & 1.4 & 28 & 0.89 & 2974 & 139.0 & 262 & $<0.20$ & 2.0 & 0.86 \\
\hline IV9000-28-G1-100 & 25 & 34 & 36 & 100 & 816 & 48 & 10 & $<0.5$ & 1.0 & 17 & 9 & $<1.0$ & 0.76 & 1.3 & 0.62 \\
\hline IV9000-28-G2-100 & 25 & 34 & 36 & 100 & 816 & 127 & 38 & 33 & 5.0 & 31 & 9 & $<1.2$ & 1.4 & 0.0 & 0.89 \\
\hline IV9000-56-G1-100 & 25 & 56 & 36 & 100 & 1342 & 20 & 5.4 & 19.0 & 3.6 & 12 & 12 & $<0.2$ & 0.3 & 1.9 & 0.7 \\
\hline IV $9000-56-G 2-100$ & 25 & 56 & 36 & 100 & 1342 & 16 & 2.3 & 1.4 & 1.1 & 71 & 17 & $<0.2$ & 0.4 & 1.3 & 0.4 \\
\hline IV9000-120-G1-100 & 25 & 126 & 36 & 100 & 3017 & 120 & 14 & $<0.5$ & $<0.5$ & 120 & 15.0 & $<0.5$ & $<0.5$ & 0.00 & 0.00 \\
\hline IV $9000-120-G 2-100$ & 25 & 126 & 36 & 100 & 3017 & 120 & 18 & 29 & 2.9 & 118 & 18.0 & $<0.5$ & $<0.5$ & 0.00 & 0.00 \\
\hline IV9000-14-G1-100 & 25 & 126 & 36 & 100 & 3017 & 231 & 76 & 60.0 & 1.0 & 82 & 16.0 & 0.0 & $<0.5$ & 0.00 & 0.00 \\
\hline IV9000-14-G2-100 & 25 & 126 & 36 & 100 & 3017 & 147 & 52 & 29 & 1.1 & 28 & 18.0 & 0.0 & $<0.5$ & 0.00 & 0.00 \\
\hline IV1000-56-G3-100 & 90 & 49 & 22 & 100 & 1179 & 2.3 & 1.1 & 25 & 3.0 & 34 & 1.6 & $<0.2$ & 0.75 & 0.00 & 0.00 \\
\hline IV1000-56-G4-100 & 90 & 49 & 22 & 100 & 1179 & 2.9 & 0.61 & 170 & 12 & 12 & 0.95 & 4.4 & 0.50 & 0.00 & 0.00 \\
\hline IV $1000-85-G 1-100$ & 90 & 85 & 34 & 100 & 2014 & 14 & 1.6 & 26 & 3.2 & 1.6 & 1.4 & $<0.2$ & 1.8 & 6.2 & 0.83 \\
\hline IV $1000-85-G 2-100$ & 90 & 85 & 34 & 100 & 2014 & 6.2 & 1.4 & 86 & 9.3 & 1.8 & 1.1 & 2.8 & 1.8 & 3.5 & 1.0 \\
\hline IV $1000-120-G 1-100$ & 90 & 122 & 22 & 100 & 2922 & 6.2 & 0.95 & 12 & 1.7 & 42 & 2.1 & 11 & 0.67 & 4.1 & 0.00 \\
\hline IV $1000-120-G 2-100$ & 90 & 122 & 22 & 100 & 2922 & 2.9 & 1.4 & 5.2 & 2.6 & 14 & 0.81 & 12.0 & 0.61 & 0.00 & 0.00 \\
\hline IV $1000-150-G 1-100$ & 90 & 150 & 32 & 100 & 3470 & 11 & 0.86 & 130 & 5.5 & 10 & 0.69 & $<0.2$ & $<0.2$ & 0.73 & 0.46 \\
\hline IV $1000-150-G 2-100$ & 90 & 150 & 32 & 100 & 3470 & 36 & 2.2 & 22 & 1.7 & 14 & 1.1 & $<0.2$ & $<0.2$ & 1.8 & 0.23 \\
\hline IV $1000-56-G 1-100$ & 90 & 173 & 22 & 100 & 4101 & 5.2 & 3.9 & 59 & 14 & 94 & 4.4 & 0.6 & 0.69 & 9.1 & 0.49 \\
\hline IV $1000-56-G 2-100$ & 90 & 173 & 22 & 100 & 4101 & 12 & 2.0 & 3.3 & 4.3 & 90 & 3.8 & 7.1 & 0.67 & 4.5 & 0.32 \\
\hline
\end{tabular}


Table $8 \mathrm{~b}$ - contd.

\begin{tabular}{|c|c|c|c|c|c|c|c|c|c|c|c|c|c|c|c|}
\hline \multirow[b]{2}{*}{ Test Number } & \multirow[b]{2}{*}{$\begin{array}{c}\text { Temp. } \\
\left({ }^{\circ} \mathrm{C}\right)\end{array}$} & \multirow{2}{*}{$\begin{array}{c}\text { Test } \\
\text { Length } \\
\text { (days) }\end{array}$} & \multirow{2}{*}{$\begin{array}{c}\text { Dose } \\
\text { Rate } \\
\text { (Gy/h) }\end{array}$} & \multirow{2}{*}{$\begin{array}{l}\text { Gas: } \\
\text { Liq. } \\
\text { Ratio }\end{array}$} & \multirow{2}{*}{$\begin{array}{l}\text { Expos. } \\
\text { Time } \\
\text { (h) }\end{array}$} & \multicolumn{2}{|c|}{$\mathrm{Cl}$} & \multicolumn{2}{|c|}{$\mathrm{SO}_{4}$} & \multicolumn{2}{|c|}{$\mathrm{NO}_{3}$} & \multicolumn{2}{|c|}{$\mathrm{NO}_{2}$} & \multicolumn{2}{|l|}{ F } \\
\hline & & & & & & Prerinse & Rinse & Prerinse & Rinse & Prerinse & Rinse & Prerinse & Rinse & Prerinse & Rinse \\
\hline IV2000-56-G3-100 & 200 & 49 & 42 & 100 & 1179 & 0.59 & 0.44 & 13 & 2.0 & $<0.5$ & 1.4 & $<0.2$ & $<0.2$ & 0.0 & 0.0 \\
\hline IV $2000-56-G 4-100$ & 200 & 49 & 42 & 100 & 1179 & 0.13 & 0.31 & 24 & 1.6 & 21 & $<0.5$ & 0.60 & 0.59 & 0.0 & 0.0 \\
\hline IV $2000-85-G 1-100$ & 200 & 85 & 24 & 100 & 3737 & 0.56 & 0.52 & 3.8 & 0.74 & 5.4 & 0.77 & 0.95 & $<0.2$ & 0.80 & 0.39 \\
\hline IV $2000-85-G 2-100$ & 200 & 85 & 24 & 100 & 4318 & $<0.3$ & $<0.3$ & 1.2 & 0.58 & 0.55 & 0.24 & 1.20 & 0.66 & 1.9 & 0.79 \\
\hline IV $2000-120-G 1-100$ & 200 & 122 & 42 & 100 & 2922 & 1.2 & 0.53 & 26 & 1.9 & 17 & 1.6 & $<0.2$ & $<0.2$ & 0.0 & 0.0 \\
\hline IV $2000-120-G 2-100$ & 200 & 122 & 42 & 100 & 2922 & 0.40 & 2.4 & 11 & 4.8 & 32 & 2.5 & $<0.2$ & $<0.2$ & 0.0 & 0.0 \\
\hline IV $2000-150-G 1-100$ & 200 & 150 & 25 & 100 & 4318 & $<0.3$ & $<0.3$ & 1.3 & 1.1 & 0.51 & 0.67 & 0.96 & 1.4 & 4.3 & 1.4 \\
\hline IV $2000-150-G 2-100$ & 200 & 150 & 22 & 100 & 3737 & $<0.2$ & 0.91 & 16 & 2.8 & 0.56 & $<0.2$ & $<0.2$ & 1.6 & 13 & 1.2 \\
\hline IV $2000-56-\mathrm{G} 1-100$ & 200 & 173 & 42 & 100 & 4101 & 0.31 & 0.30 & 31 & 2.2 & 5.1 & 2.5 & 1.0 & $<0.2$ & 0.37 & 0.0 \\
\hline IV $2000-56-G 2-100$ & 200 & 173 & 42 & 100 & 4101 & 3.2 & 0.63 & 33 & 1.2 & $<0.5$ & $<0.5$ & $<0.2$ & 0.76 & 0.33 & 0.0 \\
\hline IV1000-56-G1-10 & 90 & 56 & 32 & 10 & 1324 & 1.3 & 0.77 & 11 & 5.6 & 1.1 & 0.70 & 1.5 & 1.1 & 0.68 & 0.43 \\
\hline IV $1000-56-G 2-10$ & 90 & 56 & 32 & 10 & 1324 & 1.4 & 0.88 & 18 & 6.8 & 1.8 & 1.0 & 1.6 & 1.3 & 0.78 & 0.39 \\
\hline IV $1000-120-G 1-10$ & 90 & 120 & 37 & 10 & 2805 & 1.9 & 1.6 & 7.6 & 5.2 & 1.1 & 1.0 & 3.6 & 2.8 & 1.0 & 0.72 \\
\hline IV1000-120-G2-10 & 90 & 120 & 37 & 10 & 2805 & 1.9 & 1.3 & 12 & 6.9 & 0.35 & 0.58 & 1.7 & 2.1 & 0.8 & 1.2 \\
\hline IV $9000-28-B L A N K-100$ & 25 & 56 & None & 100 & 0 & 0.62 & 1.0 & 0.50 & 0.50 & 1.3 & 0.78 & 0.88 & 1.6 & 3.0 & 0.30 \\
\hline IV $9000-120-B L A N K-100$ & 25 & 120 & None & 100 & 0 & 0.73 & 0.36 & 2.6 & 0.68 & 0.99 & 0.30 & 1.30 & 0.45 & 2.4 & 0.22 \\
\hline IV1000-56-BLANK- 100 & 90 & 56 & None & 100 & 0 & 0.79 & 0.55 & 1.1 & 1.3 & 1.6 & 1.0 & 1.3 & 0.79 & 1.9 & 0.11 \\
\hline IV $1000-120-B L A N K-100$ & 90 & 120 & None & 100 & 0 & 6.8 & 0.86 & 1.2 & 0.49 & 8.0 & 0.79 & $<0.20$ & 0.91 & 4.7 & N.R. \\
\hline IV2000-56-BLANK-100 & 200 & 56 & None & 100 & 0 & 1.8 & 0.91 & 0.76 & 1.7 & 0.34 & 1.3 & 0.68 & 1.4 & $<0.1$ & $<0.1$ \\
\hline IV $2000-120$-BLANK-100 & 200 & 120 & None & 100 & 0 & 0.46 & 1.4 & 1.1 & 0.44 & 0.36 & 0.23 & 0.23 & 0.85 & N.A. & N.A. \\
\hline
\end{tabular}

Prerinse values represent undiluted anion concentrations collected from fluid in bottom of test vessel. Rinse values represent diluted concentrations after the vessel walls were rinsed with deionized water. 
Table 9a. Carbon Species $(\mu \mathrm{mol})$ and pH Results for Gamma Blank Tests ${ }^{\mathrm{a}}$

\begin{tabular}{|c|c|c|c|c|c|c|c|c|c|c|c|c|}
\hline \multirow[b]{2}{*}{ Test Number } & \multicolumn{2}{|c|}{$\mathrm{pH}^{\mathrm{b}}$} & \multicolumn{3}{|c|}{ Prerinse } & \multicolumn{3}{|c|}{ Rinse } & \multicolumn{2}{|c|}{ Oxalate } & \multicolumn{2}{|c|}{ Formate } \\
\hline & Prerinse & Rinse & $\begin{array}{c}\text { Total } \\
\text { Carbon }\end{array}$ & $\begin{array}{c}\text { Organic } \\
\text { Carbon } \\
\end{array}$ & $\begin{array}{c}\text { Inorganic } \\
\text { Carbon }\end{array}$ & $\begin{array}{c}\text { Total } \\
\text { Carbon } \\
\end{array}$ & $\begin{array}{l}\text { Organic } \\
\text { Carbon }\end{array}$ & $\begin{array}{c}\text { Inorganic } \\
\text { Carbon }\end{array}$ & Prerinse & Rinse & Prerinse & Rinse \\
\hline IV9000-7-G1B-100 & & & & & & 0.35 & 0.24 & 0.11 & $<0.001$ & $<0.007$ & $\mathrm{NR}$ & NR \\
\hline IV $9000-7-G 2 B-100$ & & & & & & 0.49 & 0.32 & 0.17 & $<0.001$ & $<0.009$ & NR & NR \\
\hline IV $9000-14-G 1 B-100$ & & & & & & 0.65 & 0.40 & 0.25 & 0.010 & 0.026 & $<0.001$ & 0.131 \\
\hline IV9000-14-G2B-100 & & & & & & 0.45 & 0.28 & 0.17 & 0.025 & 0.027 & $<0.001$ & 0.107 \\
\hline IV $9000-28-G 1 B-100$ & & & & & & 0.32 & 0.13 & 0.19 & 0.003 & $<0.016$ & $<0.001$ & $<0.009$ \\
\hline IV $9000-28-G 2 B-100$ & & & & & & 0.22 & 0.00 & 0.22 & $<0.002$ & $<0.014$ & $<0.001$ & 0.084 \\
\hline IV9000-56-G1B-100 & & & & & & 0.11 & 0.03 & 0.08 & 0.002 & 0.006 & $<0.001$ & $<0.005$ \\
\hline IV $9000-56-G 2 B-100$ & & & & & & 0.21 & 0.09 & 0.13 & $<0.001$ & $<0.008$ & $<0.001$ & $<0.006$ \\
\hline IV9000-120-G1B-100 & & & & & & 0.29 & 0.19 & 0.10 & $<0.001$ & $<0.008$ & NR & NR \\
\hline IV $9000-120-G 2 B-100$ & & & & & & 0.31 & 0.23 & 0.08 & $<0.001$ & $<0.007$ & NR & NR \\
\hline IV9000-28-G!-100 & & & & & & 1.29 & 0.88 & 0.41 & 0.001 & 0.008 & 0.029 & 0.105 \\
\hline IV $9000-28-G 2-100$ & & & & & & 2.28 & 1.50 & 0.78 & 0.029 & $0 . \overline{0} \overline{2}$ & 0.261 & 0.413 \\
\hline IV $9000-56-G 1-100$ & & & & & & 1.10 & 0.70 & 0.41 & 0.001 & 0.040 & 0.002 & 0.075 \\
\hline IV $9000-56-G 2-100$ & & & & & & 0.53 & 0.22 & 0.32 & 0.001 & 0.029 & 0.008 & 0.065 \\
\hline IV9000-120-G1-100 & & & & & & 0.80 & 0.00 & 0.80 & 0.005 & 0.128 & 0.068 & 0.200 \\
\hline IV $9000-120-G 2-100$ & & & & & & 1.91 & 1.06 & 0.84 & 0.001 & 0.013 & 0.076 & 0.264 \\
\hline IV9000-14-G1-100 & & & & & & 6.79 & 4.98 & 1.82 & 0.058 & 0.262 & 0.000 & 0.594 \\
\hline IV $9000-14-G 2-100$ & & & & & & 2.05 & 1.22 & 0.83 & 0.064 & 0.059 & 0.102 & 0.197 \\
\hline IV1000-56-G3-100 & & & & & & 1.37 & 0.80 & 0.57 & 0.002 & 0.039 & 0.059 & 0.096 \\
\hline IV1000-56-G4-100 & & & & & & 0.48 & 0.25 & 0.23 & 0.035 & 0.039 & 0.068 & 0.056 \\
\hline IV1000-85-G1-100 & & & & & & 0.26 & 0.09 & 0.17 & 0.022 & 0.024 & $<0.001$ & $<0.007$ \\
\hline IV $1000-85-G 2-100$ & & & & & & 0.14 & 0.00 & 0.14 & 0.036 & 0.040 & $<0.001$ & $<0.006$ \\
\hline IV1000-120-G1-100 & & & & & & 0.63 & 0.33 & 0.30 & 0.008 & 0.022 & 0.002 & 0.478 \\
\hline IV1000-120-G2-100 & & & & & & 2.20 & 1.43 & 0.77 & 0.001 & 0.024 & 0.068 & 0.628 \\
\hline IV1000-150-G1-100 & & & & & & 0.49 & 0.27 & 0.22 & 0.007 & $<0.010$ & NR & NR \\
\hline IV $1000-150-G 2-100$ & & & & & & 0.54 & 0.34 & 0.20 & 0.003 & $<0.006$ & NR & NR \\
\hline IV1000-56-G1-100 & & & & & & 3.78 & 1.49 & 2.29 & 0.010 & 0.031 & 0 & 0.000 \\
\hline IV $1000-56-G 2-100$ & & & & & & 1.47 & 0.64 & 0.84 & 0.002 & 0.351 & 0.000 & 0.000 \\
\hline
\end{tabular}


Table 9a-contd.

\begin{tabular}{|c|c|c|c|c|c|c|c|c|c|c|c|c|}
\hline \multirow[b]{2}{*}{ Test Number } & \multicolumn{2}{|c|}{$\mathrm{pH}$} & \multicolumn{3}{|c|}{ Prerinse } & \multicolumn{3}{|c|}{ Rinse } & \multicolumn{2}{|c|}{ Oxalate } & \multicolumn{2}{|c|}{ Formate } \\
\hline & Prerinse & Rinse & $\begin{array}{c}\text { Total } \\
\text { Carbon }\end{array}$ & $\begin{array}{l}\text { Organic } \\
\text { Carbon }\end{array}$ & $\begin{array}{c}\text { Inorganic } \\
\text { Carbon }\end{array}$ & $\begin{array}{c}\text { Total } \\
\text { Carbon }\end{array}$ & $\begin{array}{c}\text { Organic } \\
\text { Carbon } \\
\end{array}$ & $\begin{array}{c}\text { Inorganic } \\
\text { Carbon }\end{array}$ & Prerinse & Rinse & Prerinse & Rinse \\
\hline IV2000-56-G3-100 & & & & & & 0.33 & 0.08 & 0.26 & 0.002 & 0.026 & 0.005 & 0.050 \\
\hline IV2000-56-G4-100 & & & & & & 0.38 & 0.04 & 0.34 & 0.002 & 0.031 & 0.004 & 0.072 \\
\hline IV2000-85-G1-100 & & & & & & 0.10 & 0.05 & 0.05 & $<0.002$ & $<0.015$ & NR & NR \\
\hline IV2000-85-G2-100 & & & & & & 0.11 & 0.07 & 0.04 & 0.001 & 0.0067 & NR & NR \\
\hline IV2000-120-G1-100 & & & & & & 0.70 & 0.19 & 0.50 & 0.001 & 0.022 & 0.023 & 0.527 \\
\hline IV2000-120-G2-100 & & & & & & 0.98 & 0.24 & 0.74 & 0.001 & 0.016 & 0.021 & 0.393 \\
\hline IV2000-150-G1-100 & & & & & & 0.09 & 0.06 & 0.03 & 0.001 & 0.005 & NR & NR \\
\hline IV2000-150-G2-100 & & & & & & 0.06 & 0.06 & $<0.05$ & $<0.002$ & $<0.010$ & NR & $\mathrm{NR}$ \\
\hline IV2000-56-G1-100 & & & & & & 0.51 & 0.09 & 0.42 & 0.002 & 0.034 & $0.000^{\circ}$ & 0.042 \\
\hline IV $2000-56-G 2-100$ & & & & & & 0.73 & 0.06 & 0.66 & 0.002 & 0.029 & 0.000 & 0.051 \\
\hline IV9000-56-G1-10 & 5.66 & 5.60 & 0.14 & 0.26 & -0.11 & 0.21 & 0.27 & $<0.05$ & 0.012 & $<0.019$ & $<0.009$ & $<0.014$ \\
\hline IV9000-56-G2-10 & 5.49 & 5.68 & 0.29 & 0.17 & 0.12 & 0.32 & 0.27 & 0.06 & $<0.011$ & $<0.023$ & $<0.009$ & $<0.018$ \\
\hline IV9000-120-G1-10 & 6.01 & 6.16 & 0.21 & 0.05 & 0.17 & 0.21 & 0.27 & $<0.05$ & 0.014 & $<0.018$ & NR & NR \\
\hline IV9000-120-G2-10 & 6.34 & 6.60 & 0.17 & 0.00 & 0.17 & 0.32 & 0.27 & 0.06 & $<0.011$ & $<0.017$ & NR & NR \\
\hline IV9000-28-BLANK-100 & & & & & & 0.29 & 0.10 & 0.20 & $<0.002$ & $<0.021$ & $<0.001$ & $<0.011$ \\
\hline IV9000-120-BLANK-100 & & & & & & 0.42 & 0.50 & $<0.05$ & $<0.001$ & $<0.010$ & NR & NR \\
\hline IV1000-56-BLANK-100 & & & & & & 0.13 & 0.06 & 0.07 & $<0.001$ & $<0.005$ & $<0.001$ & $<0.004$ \\
\hline IV 1000-120-BLANK-100 & & & & & & 0.40 & 0.44 & $<0.05$ & $<0.001$ & $<0.011$ & NR & NR \\
\hline IV2000-56-BLANK-100 & & & & & & 0.49 & 0.26 & 0.23 & $<0.001$ & $<0.014$ & 0.002 & 0.120 \\
\hline IV2000-120-BLANK-100 & & & & & & 0.46 & NA & NA & $<0.001$ & $<0.009$ & 0.007 & 0.100 \\
\hline
\end{tabular}

${ }^{a}$ All values other than $\mathrm{pH}$ given in $\mu$ moles; carbon $\mu$ moles calculated with assumed molecular weight $=12$; NA $=$ not analyzed; $\mathrm{NR}=$ not able to resolve from adjacent $\mathrm{Cl}^{-}$peak.

Rinse solution values include a correction factor for both the proportion of aliquots removed and the dilution of the test solution by the rinse fluid.

${ }^{b}$ The $\mathrm{pH}$ values were only measured for $\mathrm{G} / \mathrm{L}=10$ tests. Measurements were not made for the $\mathrm{G} / \mathrm{L}=100$ tests due to the small amount of solution present in the test vessels. 
Table 9b. Carbon Species (ppm) and pH Results for Gamma Blank Tests ${ }^{\mathrm{a}}$

\begin{tabular}{|c|c|c|c|c|c|c|c|c|c|c|c|c|}
\hline \multirow[b]{2}{*}{ Test Number } & \multicolumn{2}{|c|}{$\mathrm{pH}^{\mathrm{b}}$} & \multicolumn{3}{|c|}{ Prerinse } & \multicolumn{3}{|c|}{ Rinse } & \multicolumn{2}{|c|}{ Oxalate } & \multicolumn{2}{|c|}{ Formate } \\
\hline & Prerinse & Rinse & $\begin{array}{c}\text { Total } \\
\text { Carbon }\end{array}$ & $\begin{array}{l}\text { Organic } \\
\text { Carbon }\end{array}$ & $\begin{array}{c}\text { Inorganic } \\
\text { Carbon }\end{array}$ & $\begin{array}{c}\text { Total } \\
\text { Carbon }\end{array}$ & $\begin{array}{l}\text { Organic } \\
\text { Carbon }\end{array}$ & $\begin{array}{c}\text { Inorganic } \\
\text { Carbon }\end{array}$ & Prerinse & Rinse & Prerinse & Rinse \\
\hline IV9000-7-G1B-100 & & & & & & 3.87 & 2.66 & 1.20 & $<0.50$ & $<0.50$ & N.R. & N.R. \\
\hline IV9000-7-G2B-100 & & & & & & 3.78 & 2.48 & 1.30 & $<0.50$ & $<0.50$ & N.R. & N.R. \\
\hline IV9000-14-G1B-100 & & & & & & 3.48 & 2.15 & 1.34 & 4.0 & 0.85 & $<0.2$ & 2.6 \\
\hline IV $9000-14-G 2 B-100$ & & & & & & 2.83 & 1.74 & 1.09 & 10 & 0.74 & $<0.2$ & 2.5 \\
\hline IV9000-28-G1B-100 & & & & & & 2.07 & 0.82 & 1.25 & 1.1 & $<0.7$ & $<0.2$ & $<0.2$ \\
\hline IV $9000-28-G 2 B-100$ & & & & & & 1.54 & 0.00 & 1.54 & $<0.7$ & $<0.7$ & $<0.2$ & 2.2 \\
\hline IV9000-56-G1B-100 & & & & & & 1.37 & 0.39 & 0.98 & 0.85 & 0.5 & $<0.2$ & $<0.2$ \\
\hline IV $9000-56-G 2 B-100$ & & & & & & 1.94 & 0.78 & 1.16 & $<0.5$ & $<0.5$ & $<0.2$ & $<0.2$ \\
\hline IV9000-120-G1B-100 & & & & & & 2.63 & 1.74 & 0.89 & $<0.5$ & $<0.5$ & N.R & N.R. \\
\hline IV $9000-120-G 2 B-100$ & & & & & & 3.24 & 2.40 & 0.84 & $<0.5$ & $<0.5$ & N.R. & N.R. \\
\hline IV $9000-28-G 1-100$ & & & & & & 11.21 & 7.68 & 3.53 & $<0.5$ & $<0.5$ & 6.0 & 3.0 \\
\hline IV $9000-28-G 2-100$ & & & & & & 18.17 & 11.96 & 6.21 & $<12$ & $<0.5$ & 56 & 8.6 \\
\hline IV $9000-56-G 1-100$ & & & & & & 8.40 & 5.30 & 3.10 & $<0.5$ & 2.2 & $<0.5^{\circ}$ & 2.1 \\
\hline IV9000-56-G2-100 & & & & & & 5.07 & 2.06 & 3.01 & $<0.5$ & 2.0 & 1.7 & 2.2 \\
\hline IV $9000-120-G 1-100$ & & & & & & 4.55 & 0.00 & 4.55 & 2.5 & 5.2 & 16 & 3.5 \\
\hline IV $9000-120-G 2-100$ & & & & & & 10.55 & 5.88 & 4.67 & $<0.5$ & $<0.5$ & 17 & 4.7 \\
\hline IV9000-14-G1-100 & & & & & & 48.86 & 35.78 & 13.08 & 30 & 12.0 & 0.0 & 16 \\
\hline IV $9000-14-G 2-100$ & & & & & & 15.42 & 9.16 & 6.26 & 28 & 1.5 & 23 & 4.1 \\
\hline IV1000-56-G3-100 & & & & & & 4.92 & 2.89 & 2.03 & $<1.0$ & $<1.0$ & 12.0 & 0.93 \\
\hline IV $1000-56-G 4-100$ & & & & & & 2.80 & 1.46 & 1.34 & 14 & $<1.0$ & 14.0 & 0.55 \\
\hline IV1000-85-G1-100 & & & & & & 2.20 & 0.80 & 1.40 & 9.7 & 0.78 & $<0.2$ & $<0.2$ \\
\hline IV $1000-85-G 2-100$ & & & & & & 1.39 & 0.00 & 1.39 & 15 & 1.6 & $<0.2$ & $<0.2$ \\
\hline IV $1000-120-G 1-100$ & & & & & & 2.34 & 1.22 & 1.12 & 3.4 & $<0.5$ & $<0.5$ & 6.6 \\
\hline IV $1000-120-G 2-100$ & & & & & & 6.48 & 4.21 & 2.27 & $<0.5$ & $<0.5$ & 14 & 6.6 \\
\hline IV1000-150-G1-100 & & & & & & 3.03 & 1.67 & 1.36 & 2.9 & $<0.3$ & N.R. & N.R. \\
\hline IV $1000-150-G 2-100$ & & & & & & 4.37 & 2.73 & 1.64 & 1.1 & $<0.3$ & N.R. & N.R. \\
\hline IV1000-56-G1-100 & & & & & & 19.26 & 7.59 & 11.67 & 3.9 & $<1.0$ & 0.0 & 0.0 \\
\hline IV1000-56-G2-100 & & & & & & 8.05 & 3.48 & 4.57 & $<1.0$ & 14 & 0.0 & 0.0 \\
\hline
\end{tabular}


Table $9 b$ - contd

\begin{tabular}{|c|c|c|c|c|c|c|c|c|c|c|c|c|}
\hline \multirow[b]{2}{*}{ Test Number } & \multicolumn{2}{|c|}{$\mathrm{pH}$} & \multicolumn{3}{|c|}{ Prerinse } & \multicolumn{3}{|c|}{ Rinse } & \multicolumn{2}{|c|}{ Oxalate } & \multicolumn{2}{|c|}{ Formate } \\
\hline & Prerinse & Rinse & $\begin{array}{c}\text { Total } \\
\text { Carbon }\end{array}$ & $\begin{array}{l}\text { Organic } \\
\text { Carbon }\end{array}$ & $\begin{array}{c}\text { Inorganic } \\
\text { Carbon }\end{array}$ & $\begin{array}{c}\text { Total } \\
\text { Carbon }\end{array}$ & $\begin{array}{c}\text { Organic } \\
\text { Carbon } \\
\end{array}$ & $\begin{array}{c}\text { Inorganic } \\
\text { Carbon }\end{array}$ & Prerinse & Rinse & Prerinse & Rinse \\
\hline IV2000-56-G3-100 & & & & & & 1.85 & 0.43 & 1.42 & $<1$ & $<1$ & 1.1 & 0.99 \\
\hline IV2000-56-G4-100 & & & & & & 1.73 & 0.18 & 1.56 & $<1$ & $<1$ & 0.77 & 1.2 \\
\hline IV2000-85-G1-100 & & & & & & 1.04 & 0.51 & 0.53 & $<1$ & $<1$ & N.R. & N.R. \\
\hline IV2000-85-G2-100 & & & & & & 1.49 & 0.95 & 0.55 & $<0.5$ & $<0.5$ & N.R. & N.R. \\
\hline IV2000-120-G1-100 & & & & & & 2.24 & 0.62 & 1.63 & $<0.5$ & $<0.5$ & 6.1 & 6.2 \\
\hline IV2000-120-G2-100 & & & & & & 4.42 & 1.09 & 3.33 & $<0.5$ & $<0.5$ & 6.0 & 6.4 \\
\hline IV2000-150-G1-100 & & & & & & 1.30 & 0.86 & 0.44 & $<0.5$ & $<0.5$ & N.R. & N.R. \\
\hline IV2000-150-G2-100 & & & & & & 0.93 & 0.86 & 0.07 & $<1$ & $<1$ & N.R. & N.R. \\
\hline IV2000-56-G1-100 & & & & & & 2.14 & 0.38 & 1.76 & $<1$ & $<1$ & 0.0 & 0.67 \\
\hline IV2000-56-G2-100 & & & & & & 3.57 & 0.32 & 3.25 & $<1$ & $<1$ & 0.0 & 0.95 \\
\hline IV9000-56-G1-10 & 5.66 & 5.60 & 0.87 & 1.56 & -0.69 & 0.73 & 0.804 & -0.07 & 0.54 & $<0.5$ & $<0.2$ & $<0.2$ \\
\hline IV9000-56-G2-10 & 5.49 & 5.68 & 1.75 & 1.04 & 0.71 & 0.75 & 0.729 & 0.02 & $<0.5$ & $<0.5$ & $<0.2$ & $<0.2$ \\
\hline IV9000-120-G1-10 & 6.01 & 6.16 & 1.28 & 0.28 & 1.01 & 2.22 & 0.82 & 1.40 & 0.60 & $<0.5$ & N.R. & N.R. \\
\hline IV $9000-120-G 2-10$ & 6.34 & 6.60 & 1.02 & 0.00 & 1.02 & 2.22 & 0.62 & 1.60 & $<0.5$ & $<0.5$ & N.R. & N.R. \\
\hline IV $9000-28-B L A N K-100$ & & & & & & 1.42 & 0.48 & 0.95 & $<0.7$ & $<0.7$ & $<0.2$ & $<0.2$ \\
\hline IV9000-120-BLANK-100 & & & & & & 2.97 & 3.53 & -0.56 & $<0.5$ & $<0.5$ & N.R. & N.R. \\
\hline IV1000-56-BLANK-100 & & & & & & 1.90 & 0.877 & 1.02 & $<0.5$ & $<0.5$ & $<0.2$ & $<0.2$ \\
\hline IV $1000-120-B L A N K-100$ & & & & & & 2.61 & 2.87 & -0.26 & $<0.5$ & $<0.5$ & N.R. & 2.5 \\
\hline IV2000-56-BLANK-100 & & & & & & 2.43 & 1.306 & 1.13 & $<0.5$ & $<0.5$ & 0.48 & 2.2 \\
\hline IV $2000-120-B L A N K-100$ & & & & & & 3.58 & N.A. & N.A. & $<0.5$ & $<0.5$ & 1.5 & 2.8 \\
\hline
\end{tabular}

${ }^{a}$ All values other than $\mathrm{pH}$ given in $\mathrm{ppm}$. $\mathrm{NA}=$ not analyzed; $\mathrm{NR}=$ not able to resolve from adjacent $\mathrm{Cl}^{-}$peak.

Prerinse values represent undiluted anion concentrations collected from fluid in bottom of test vessel. Rinse values represent diluted concentrations after the vessel walls were rinsed with deionized water.

${ }^{b}$ The $\mathrm{pH}$ values were only measured for $\mathrm{G} / \mathrm{L}=10$ tests. Measurements were not made for the $\mathrm{G} / \mathrm{L}=100$ tests due to the small amount of soltion present in the test vessels. 
Table 10. Results from G-Value Calculations for Alpha and Gamma Blank Tests

\begin{tabular}{|c|c|c|c|c|c|c|c|c|c|}
\hline Sample & $\begin{array}{c}\text { Actual } \\
\text { Days }\end{array}$ & $\begin{array}{l}\text { Total } \mathrm{NO}_{3}{ }^{\circ} \\
\text { in } \mu \text { moles }\end{array}$ & $\begin{array}{l}\text { Total } \mathrm{NO}_{2}^{-} \\
\text {in umoles } \\
\end{array}$ & $\begin{array}{l}\text { Total } \mathrm{NO}_{3}^{-} \\
\text {and } \mathrm{NO}_{2}^{-}\end{array}$ & $\begin{array}{c}\text { Dose } \\
\text { Rate } \\
(\mathrm{Gy} / \mathrm{h}) \\
\end{array}$ & $\begin{array}{c}\text { Expos. } \\
\text { Time } \\
\text { (h) }\end{array}$ & $\begin{array}{r}\text { Total } \\
\text { Expos. } \\
\text { (kGy) } \\
\end{array}$ & $\mathrm{G}\left(\mathrm{NO}_{3}\right)^{-}$ & $\mathrm{G}\left(\mathrm{NO}_{3}^{-}+\mathrm{NO}_{2}^{-}\right)$ \\
\hline \multicolumn{10}{|c|}{ Alpha Sample, $25^{\circ} \mathrm{C}$, Lucite Support Rod } \\
\hline IV9000-14-A1-100 & 22 & 1.21 & 0.58 & 1.79 & 26 & 528 & 13.89 & 0.49 & 0.72 \\
\hline IV9000-14-A2-100 & 22 & 4.65 & 0.47 & 5.12 & 26 & 528 & 13.89 & 1.88 & 2.07 \\
\hline IV $9000-28-A 1-100$ & 31 & 5.88 & 1.83 & 7.71 & 26 & 744 & 19.57 & 1.68 & 2.21 \\
\hline IV $9000-28-A 2-100$ & 31 & 7.88 & $<0.21$ & 8.09 & 26 & 744 & 19.57 & 2.26 & 2.32 \\
\hline IV $9000-56-A 1-100$ & 65 & 15.68 & $<0.79$ & 16.47 & 26 & 1560 & 41.03 & 2.14 & 2.25 \\
\hline \multirow[t]{2}{*}{ IV9000-56-A2-100 } & 65 & 16.83 & $<0.75$ & 17.58 & 26 & 1560 & 41.03 & 2.30 & 2.40 \\
\hline & & & & & & & $\begin{array}{r}\text { Avg. } \\
\text { SD }\end{array}$ & $\begin{array}{l}1.79 \\
0.68\end{array}$ & $\begin{array}{l}2.00 \\
0.63\end{array}$ \\
\hline \multicolumn{10}{|c|}{ Alpha Sample, $25^{\circ} \mathrm{C}$, Stainless Steel Support Rod } \\
\hline IV $9000-14-A 1 S-100$ & 14 & 3.60 & $<0.19$ & 3.79 & 26 & 336 & 8.84 & 2.28 & 2.40 \\
\hline IV $9000-14-A 2 S-100$ & 14 & 0.93 & 1.80 & 2.73 & 26 & 336 & 8.84 & 0.59 & 1.73 \\
\hline IV9000-28-A1S-100 & 33 & 7.37 & $<0.67$ & 8.04 & 26 & 792 & $20 . \overline{8} 3$ & 1.98 & 2.16 \\
\hline IV $9000-28-A 2 S-100$ & 33 & 7.90 & $<0.60$ & 8.50 & 26 & 792 & 20.83 & 2.13 & 2.29 \\
\hline IV $9000-45-A 1 S-100$ & 46 & 24.00 & 0.27 & 24.27 & 26 & 1104 & 29.04 & 4.63 & 4.69 \\
\hline IV $9000-45-A 2 S-100$ & 46 & 26.39 & 0.54 & 26.93 & 26 & 1104 & 29.04 & 5.10 & 5.20 \\
\hline IV9000-56-A1S-100 & 60 & 15.29 & $<0.58$ & 15.87 & 26 & 1440 & 37.87 & 2.26 & 2.35 \\
\hline IV $9000-56-A 2 S-100$ & 60 & 15.81 & $<0.59$ & 16.40 & 26 & 1440 & 37.87 & 2.34 & 2.43 \\
\hline IV9000-85-A1S-100 & 84 & 2.06 & $<0.14$ & 2.20 & 26 & 2016 & 53.02 & 0.22 & 0.23 \\
\hline IV $9000-85-A 2 S-100$ & 84 & 13.13 & $<0.13$ & 13.26 & 26 & 2016 & 53.02 & 1.39 & 1.40 \\
\hline IV $9000-110-A 1 S-100$ & 110 & 33.44 & $<0.10$ & 33.54 & 26 & 2640 & 69.43 & 2.70 & 2.71 \\
\hline IV9000-110-A2S-100 & 110 & 37.31 & $<0.12$ & 37.43 & 26 & 2640 & 69.43 & 3.01 & 3.02 \\
\hline IV $9000-120-A 1 S-100$ & 129 & 41.56 & $<1.03$ & 42.59 & 26 & 3096 & 81.42 & 2.86 & 2.93 \\
\hline \multirow[t]{3}{*}{ IV $9000-120-A 2 S-100$} & 129 & 39.46 & $<0.00$ & 39.46 & 26 & 3096 & 81.42 & 2.72 & 2.72 \\
\hline & & & & & & & Avg. & 2.44 & 2.59 \\
\hline & & & & & & & SD & 1.31 & 1.23 \\
\hline \multicolumn{10}{|c|}{ Gamma Sample, $25^{\circ} \mathrm{C}, 500 \mathrm{~Gy} / \mathrm{h}$} \\
\hline IV $9000-7-G 1 B-100$ & 7 & 0.48 & 0.02 & 0.50 & 448 & 164.8 & 73.83 & 2.95 & 3.07 \\
\hline IV $9000-7-G 2 B-100$ & 7 & 0.47 & 0.02 & 0.49 & 448 & 164.8 & 73.83 & 2.89 & 3.01 \\
\hline IV $9000-14-G 1 B-100$ & 13 & 1.23 & 0.04 & 1.27 & 508 & 304.1 & 154.48 & 3.61 & 3.73 \\
\hline IV9000-14-G2B-100 & 13 & 1.21 & 0.04 & 1.25 & 508 & 304.1 & 154.48 & 3.55 & 3.67 \\
\hline IV9000-28-G1B-100 & 28 & 1.86 & 0.03 & 1.89 & 496 & 657 & 325.87 & 2.59 & 2.63 \\
\hline IV9000-28-G2B-100 & 28 & 2.05 & 0.04 & 2.09 & 482 & 657 & 316.67 & 2.94 & 3.00 \\
\hline IV9000-56-G1B-100 & 56 & 4.22 & 0.26 & 4.48 & 464 & 1323.6 & 614.15 & 3.12 & 3.31 \\
\hline IV $9000-56-G 2 B-100$ & 56 & 4.89 & 0.27 & 5.16 & 474 & 1323.6 & 614.15 & 3.61 & 3.81 \\
\hline IV $9000-120-G 1 B-100$ & 120 & 5.06 & 0.25 & 5.31 & 458 & 2408.9 & 1284.64 & 1.79 & 1.88 \\
\hline \multirow[t]{3}{*}{ IV9000-120-G2B-100 } & 120 & 7.33 & 0.57 & 7.90 & 458 & 2804.9 & 1284.64 & 2.59 & 2.79 \\
\hline & & & & & & & Avg. & 2.96 & 3.09 \\
\hline & & & & & & & SD & 0.57 & 0.59 \\
\hline
\end{tabular}


Table 10 - contd.

\begin{tabular}{|c|c|c|c|c|c|c|c|c|c|}
\hline Sample & $\begin{array}{c}\text { Actual } \\
\text { Days }\end{array}$ & $\begin{array}{l}\text { Total } \mathrm{NO}_{3} \\
\text { in umoles }\end{array}$ & $\begin{array}{l}\text { Total } \mathrm{NO}_{2} \\
\text { in umoles }\end{array}$ & $\begin{array}{l}\text { Total } \mathrm{NO}_{3}^{-} \\
\text {and } \mathrm{NO}_{2}^{-}\end{array}$ & $\begin{array}{c}\text { Dose } \\
\text { Rate } \\
(\mathrm{Gy} / \mathrm{h})\end{array}$ & $\begin{array}{l}\text { Expos. } \\
\text { Time } \\
\text { (h) }\end{array}$ & $\begin{array}{l}\text { Total } \\
\text { Expos. } \\
(\mathrm{kGy})\end{array}$ & $\mathrm{G}\left(\mathrm{NO}_{3}^{-}\right)$ & $\mathrm{G}\left(\mathrm{NO}_{3}+\mathrm{NO}_{2}\right)^{-}$ \\
\hline \multicolumn{10}{|c|}{ Gamma Sample, $25^{\circ} \mathrm{C}, 36 \mathrm{~Gy} / \mathrm{h}, \mathrm{G} / \mathrm{L}=100$} \\
\hline IV9000-28-G1-100 & 34 & 0.220 & 0.02 & 0.24 & 37 & 815 & 30.17 & 3.31 & 3.61 \\
\hline IV9000-28-G2-100 & 34 & 0.270 & 0.05 & 0.32 & 37 & 815 & 30.17 & 4.06 & 4.81 \\
\hline IV9000-56-G1-100 & 56 & 0.330 & 0.01 & 0.34 & 37 & 1342 & 49.66 & 3.02 & 3.11 \\
\hline IV9000-56-G2-100 & 56 & 0.460 & 0.01 & 0.47 & 37 & 1342 & 49.66 & 4.20 & 4.30 \\
\hline IV9000-120-G1-100 & 126 & 0.710 & 0.02 & 0.73 & 36 & 3017 & 108.60 & 2.07 & 3.05 \\
\hline IV $9000-120-G 2-100$ & 126 & 0.820 & 0.02 & 0.84 & 36 & 3017 & 108.60 & 3.43 & 3.51 \\
\hline IV9000-14-G1-100 & 126 & 0.560 & 0.02 & 0.58 & 36 & 3017 & 108.60 & 2.34 & 2.42 \\
\hline \multirow[t]{3}{*}{ IV9000-14-G2-100 } & 126 & 0.510 & 0.02 & 0.53 & 36 & 3017 & 108.60 & 2.13 & 2.21 \\
\hline & & & & & & & Avg. & 3.18 & 3.38 \\
\hline & & & & & & & SD & 0.73 & 0.88 \\
\hline \multicolumn{10}{|c|}{ Gamma Sample, $90^{\circ} \mathrm{C}, 36 \mathrm{~Gy} / \mathrm{h}, \mathrm{G} / \mathrm{L}=10$} \\
\hline IV9000-56-G1-10 & 56 & 0.04 & 0.09 & 0.13 & 29 & 1323.6 & 38.38 & 0.52 & 1.67 \\
\hline IV9000-56-G2-10 & 56 & 0.08 & 0.12 & 0.20 & 29 & 1323.6 & 38.38 & 1.03 & 2.58 \\
\hline IV $9000-120-G 1-10$ & 120 & 0.05 & 0.19 & 0.24 & 33 & 2804.9 & 92.56 & 0.27 & 1.28 \\
\hline \multirow[t]{3}{*}{ IV9000-120-G2-10 } & 120 & 0.03 & 0.13 & 0.16 & 33 & 2804.9 & 92.56 & 0.16 & 0.85 \\
\hline & & & & & & & Avg. & 0.49 & 1.60 \\
\hline & & & & & & & $\mathrm{SD}$ & 0.39 & 0.73 \\
\hline \multicolumn{10}{|c|}{ Gamma Sample, $90^{\circ} \mathrm{C}, 30 \mathrm{~Gy} / \mathrm{h}, \mathrm{G} / \mathrm{L}=100$} \\
\hline IV1000-56-G3-100 & 49 & 0.14 & 0.05 & 0.19 & 21 & 1179 & 24.75 & 2.57 & 3.48 \\
\hline IV1000-56-G4-100 & 49 & 0.05 & 0.03 & 0.08 & 21 & 1179 & 24.75 & 0.92 & 1.47 \\
\hline IV $1000-85-G 1-100$ & 85 & 0.04 & 0.06 & 0.10 & 31 & 2014 & 62.42 & 0.29 & 0.73 \\
\hline IV1000-85-G2-100 & 85 & 0.03 & 0.06 & 0.09 & 31 & 2014 & 62.42 & 0.22 & 0.65 \\
\hline IV1000-120-G1-100 & 122 & 0.18 & 0.07 & 0.25 & 21 & 2922 & 61.36 & 1.33 & 1.85 \\
\hline IV1000-120-G2-100 & 122 & 0.08 & 0.08 & 0.16 & 21 & 2922 & 61.36 & 0.59 & 1.18 \\
\hline IV $1000-150-G 1-100$ & 150 & 0.04 & 0.00 & 0.04 & 29 & 3470 & 100.62 & 0.18 & 0.18 \\
\hline IV1000-150-G2-100 & 150 & 0.05 & 0.00 & 0.05 & 29 & 3470 & 100.62 & 0.23 & 0.23 \\
\hline IV1000-56-G1-100 & 171 & 0.32 & 0.04 & 0.36 & 21 & 4101 & 86.11 & 1.69 & 1.90 \\
\hline \multirow[t]{2}{*}{ IV1000-56-G2-100 } & 171 & 0.28 & 0.05 & 0.33 & 21 & 4101 & 86.11 & 1.48 & 1.74 \\
\hline & & & & & & & Avg. & 0.95 & 1.34 \\
\hline
\end{tabular}

Method of G-value calculation described in Section III.A.1, equation (2). 
The gamma tests run at $\sim 500 \mathrm{~Gy} / \mathrm{h}(\sim 50,000 \mathrm{rad} / \mathrm{h})$ and $\sim 36 \mathrm{~Gy} / \mathrm{h}(\sim 3600 \mathrm{rad} / \mathrm{h})$ do not show any consistent variation in nitrate + nitrite levels or G-value yields with dose, indicating that there is no measurable effect on nitrogen acid formation as a function of dose rate (Fig. 5 and Table 10). The final two tests at cumulative doses of $1.48 \times 10^{6} \mathrm{~Gy}(148 \mathrm{Mrad})$ do, however, display a decrease in $\mathrm{G}$ values relative to the earlier tests. The significance of these latter two data points is discussed further in Section V.A. Averaged G values were $3.2 \pm 0.4$ for the high-dose tests (excluding the two $1.48 \times 10^{6}$ Gy tests) and $3.4 \pm 0.9$ for the low-dose tests at $25^{\circ} \mathrm{C}$. A comparison of the $90^{\circ} \mathrm{C}$ gamma tests run at $\mathrm{G} / \mathrm{L}$ ratios of 10 versus 100 also indicates no variation in average yields, although these results showed considerably more scatter. As shown in Table 10 , tests run at $G / L=: 100$ had $G$ values of $1.3 \pm 1.0$, while tests run at $G / L=10$ had a $\mathrm{G}$ value $=1.6 \pm 0.7$ for the air-filled portion of the vessel

The gamma tests run at 25,90 , and $200^{\circ} \mathrm{C}$ measure the formation of radiolytic products as a function of temperature. Results indicate that nitrate + nitrite production varies inversely with temperature, with the lowest quantities being detected for the higher-temperature experiments (Fig. 6. and Table 10). Average $\mathrm{G}\left(\mathrm{NO}_{3}{ }^{\circ}+\mathrm{NO}_{2}{ }^{\circ}\right)$ values for the 25,90 , and $200^{\circ} \mathrm{C}$ rinse solutions are $3.2 \pm 0.7,1.3 \pm 1.0$, and $0.4 \pm 0.3$, respectively, while results from the $25^{\circ} \mathrm{C}$ tests differ from the $\mathrm{G}\left(\mathrm{NO}_{3}{ }^{\circ}\right)=1.2$ value obtained during $1.0-\mathrm{MeV}$ electron irradiation tests at ambient temperatures [8]. These results indicate that yields for the $90^{\circ} \mathrm{C}$ experiments are $\sim 40 \%$ of those that characterize the $25^{\circ} \mathrm{C}$ results, while the $200^{\circ} \mathrm{C}$ yields are $\sim 10 \%$. The $90^{\circ} \mathrm{C}$ yields are also comparable to the $\mathrm{G}\left(\mathrm{NO}_{3}{ }^{-}\right)=1.9$ values obtained in neutron irradiation tests conducted at $80^{\circ} \mathrm{C}$ [11]. The proportionally larger degree of data scatter for the higher temperatures tests reflects analyzed concentrations that are approaching limits of determination $(\sim 0.2 \mathrm{ppm})$.

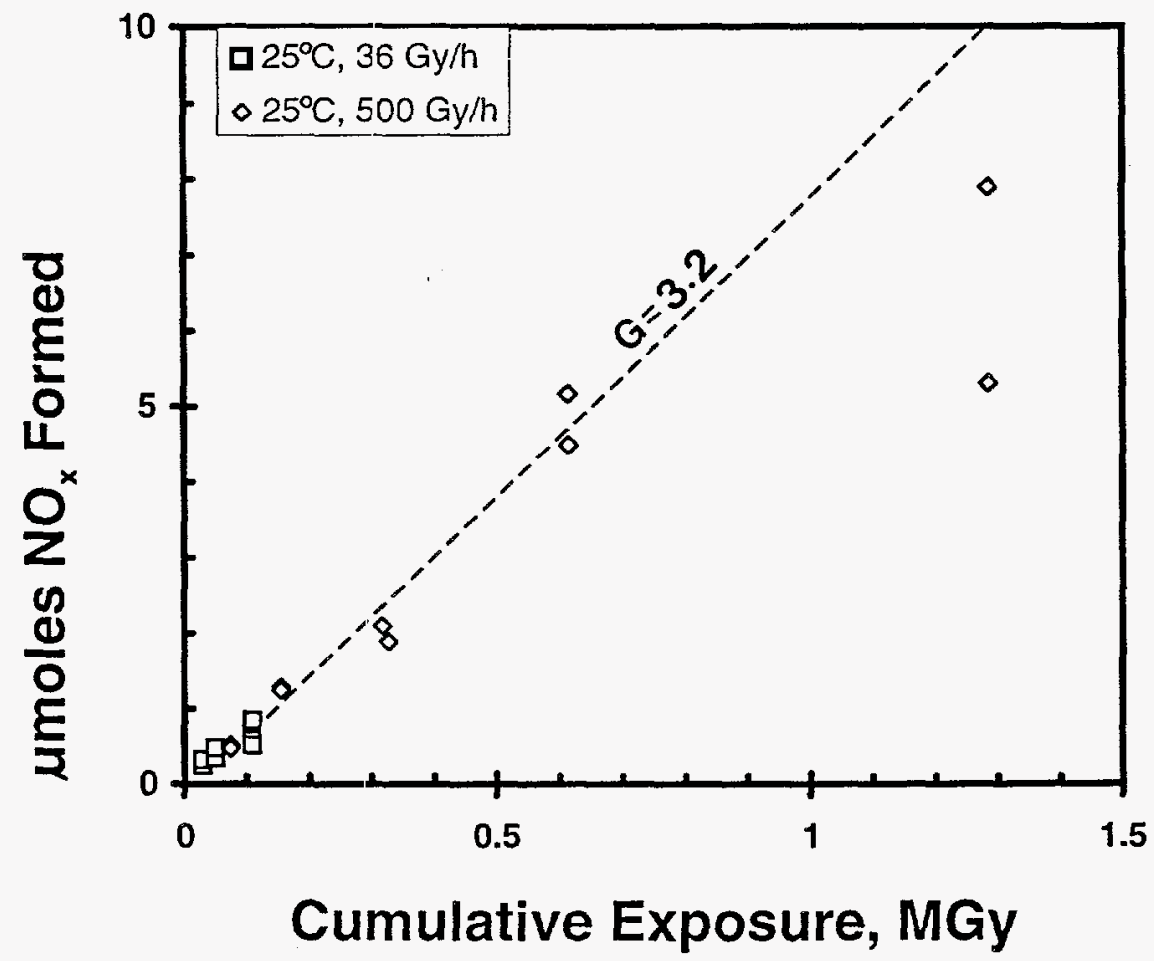

Fig. 5. Total $\mu$ moles of Nitrate + Nitrite Recovered in Blank Gamma Tests as a Function of Cumulative Exposure Dose at $25^{\circ} \mathrm{C}$. Dose rates are $\sim 36$ and $\sim 500 \mathrm{~Gy} / \mathrm{h}$. The $\mathrm{G}=3.2$ line represents the average $\mathrm{G}\left(\mathrm{NO}_{3}{ }^{-}+\mathrm{NO}_{2}{ }^{\circ}\right)$ yield for all $25^{\circ} \mathrm{C}$ gamma tests. 


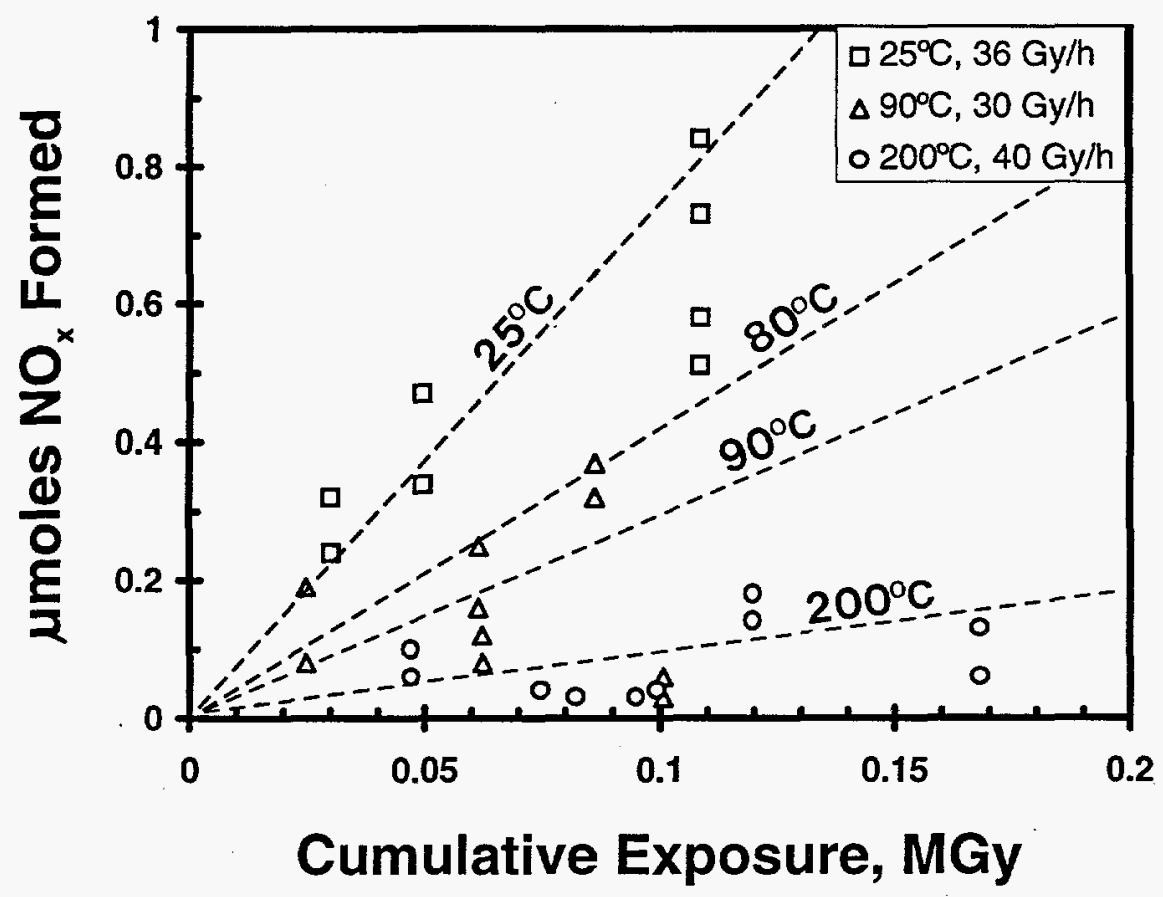

Fig. 6. Measured Nitrate + Nitrite Formation in $\sim 35 \mathrm{~Gy} / \mathrm{h}$ Blank Gamma Tests as a Function of Cumulative Exposure Dose. Dotted lines represent average yields for tests at temperatures of 25,90 , and $200^{\circ} \mathrm{C}$. Line for the $80^{\circ} \mathrm{C}$ trend derived from neutron irradiation study of Linacre and Marsh [11].

\section{Solution $\mathrm{pH}$}

Solution $\mathrm{pH}$ measurements for the four $\mathrm{G} / \mathrm{L}$ ratio $=10$ tests indicate a $\mathrm{pH}$ decrease from the starting deionized water solution to $5.9 \pm 0.4$ (Table 9). This value is indistinguishable from that expected for water in equilibrium with atmospheric $\mathrm{CO}_{2}$ at ambient temperatures $(\mathrm{pH}=5.7)$, indicating that radiation exposure has had no discernible effect on the $\mathrm{pH}$ of these relatively dilute solutions. A pH of 4.0 to 4.2 would be expected based on the measured $\mathrm{NO}_{3}{ }^{-}+$ $\mathrm{NO}_{2}{ }^{-}$concentrations determined for the test solutions. The difference between the measured $\mathrm{pH}$ of 5.9 and the calculated values reflects the ability of the solution in the tests to buffer against $\mathrm{pH}$ changes.

The limited amounts of solution present in the $\mathrm{G} / \mathrm{L}$ ratio $=100$ tests precluded any direct measurement of solution $\mathrm{pH}$. Solution $\mathrm{pH}$ values have, however, been estimated based on the $\mathrm{NO}_{3}{ }^{-}+\mathrm{NO}_{2}{ }^{-}$contents detected in the rinse. These calculations suggest that the limited quantities of solution will attain a $\mathrm{pH}$ of $\sim 3$ after absorption of $50,000 \mathrm{~Gy}(5 \mathrm{Mrad})$ and would continue to decrease to a $\mathrm{pH}$ of $\sim 2$ after $7 \times 10^{5} \mathrm{~Gy}$ ( $70 \mathrm{Mrad}$ ) exposure (Fig. 7). These calculations assume that nitric acid is the predominant acid present, complete dissociation of nitric acid occurs, and external pH buffering systems (e.g., bicarbonate) do not greatly influence the solution $\mathrm{pH}$. 


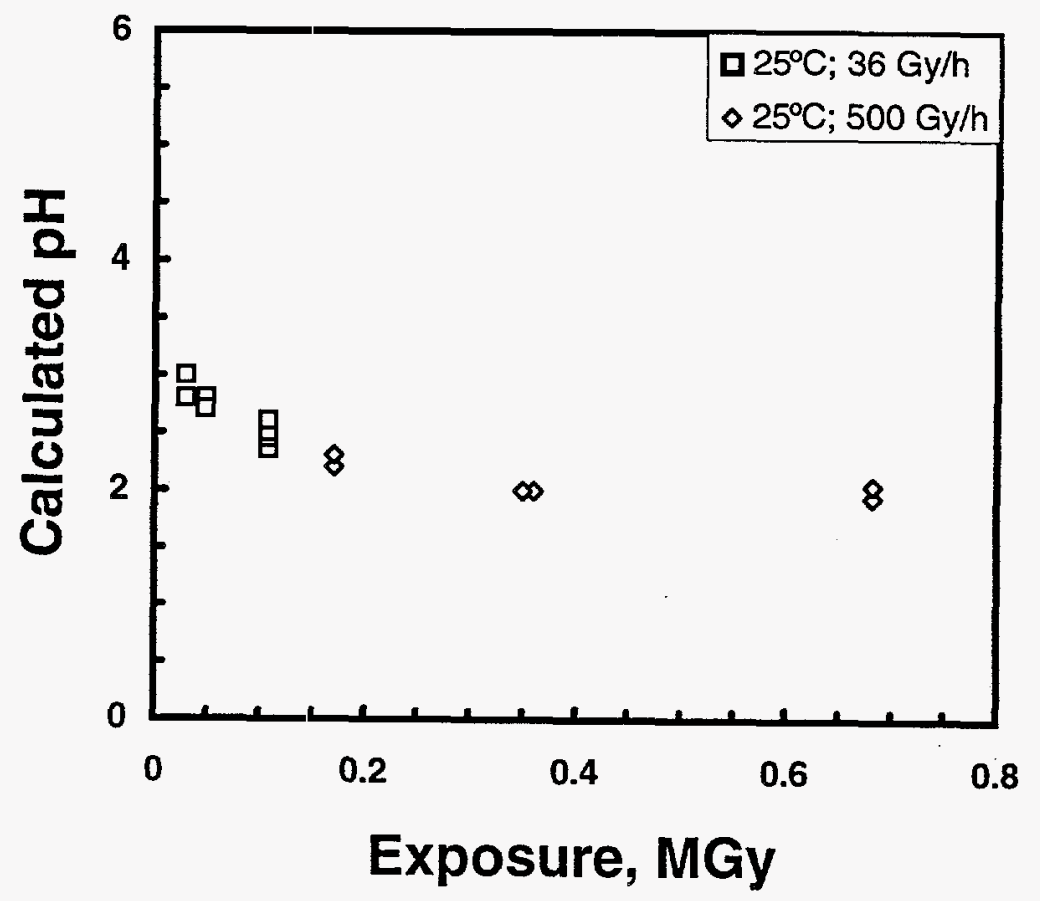

Fig. 7. Solution $\mathrm{pH}$ Values for the Gamma Blank Tests Plotted as a Function of Cumulative Exposure Dose at $25^{\circ} \mathrm{C}$. The $\mathrm{pH}$ values were calculated based on the $\mathrm{NO}_{3}{ }^{-}+\mathrm{NO}_{2}{ }^{-}$ solution concentrations, assuming complete dissociation of the acid species. Dose rates at $\sim 36$ and $\sim 500 \mathrm{~Gy} / \mathrm{h}$.

The $\mathrm{pH}$ value calculations are representative of the averaged $\mathrm{pH}$ for the entire $0.22 \mathrm{~mL}$ of solution initially present in the test vessels. This solution, however, is actually divided into two fractions: a thin film of condensate located on the inside vessel walls and cap, and the solution remaining in the bottom of the vessel. The thin film of condensate may have a higher $\mathrm{NO}_{3}{ }^{-}$concentration than the solution at the bottom of the test vessel due to the relatively larger $\mathrm{S} / \mathrm{V}$ expected for the former. Thus, the calculated $\mathrm{pH}$ values may be considered minimums when being applied to model the characteristics of solutions that would potentially condense on the surfaces of radioactive waste and waste containers.

\section{Carbon Concentrations}

Only the rinse solutions could be analyzed for carbon concentrations in the gamma-irradiation tests, due to the small amount of fluid in each reaction vessel. The gamma tests at low dose rates are enriched in total carbon (TC), organic carbon (OC), and inorganic carbon (IC) relative to the background concentrations determined from blank test runs without radiation (Table 9). In contrast, carbon contents from the high-dose tests were at or below levels that characterized the background tests. Samples exposed to $>2 \times 10^{5}$ Gy $(>20 \mathrm{Mrad})$ cumulative exposure display the greatest depletion of carbon (Fig. 8). 


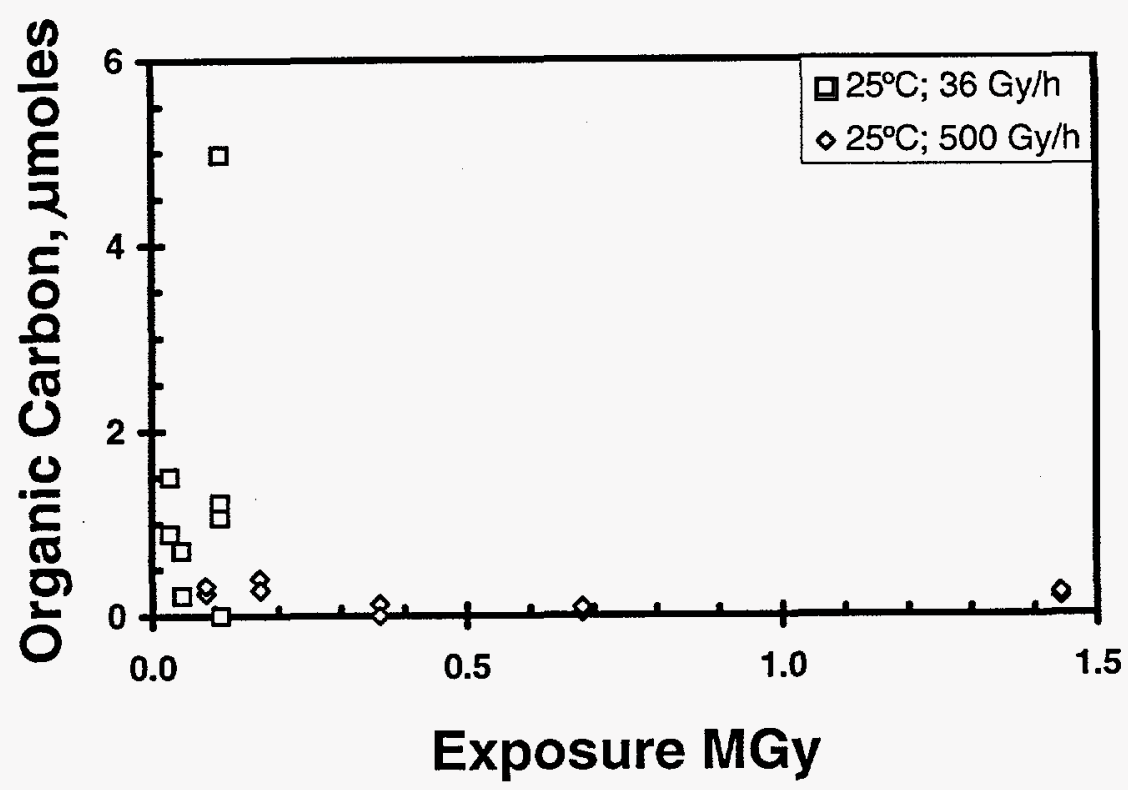

Fig. 8. Measured Organic Carbon Formation in Blank Gamma Tests as a Function of Cumulative Exposure Dose for the $\sim 35$ and $\sim 500 \mathrm{~Gy} / \mathrm{h}$ Tests at $25^{\circ} \mathrm{C}$

The carboxylic acids (formate and oxalate) were analyzed from both rinse and prerinse solutions. Concentrations of these species occur at levels that parallel the $\mathrm{OC}$ data, suggesting that they are at least partially responsible for the observed increase in $\mathrm{OC}$ in these tests. The low-dose rate tests show production levels of formate and oxalate that are significantly enriched relative to blank tests without radiation (Table 9). Although there is a considerable amount of data scatter, the carboxylic acid contents appear to show a progressive increase with the absorbed dose in these tests. This increase is more strongly developed in the rinse solutions relative to the prerinse samples.

The various carbon fractions were also examined as a function of temperature. Overall results indicate that the $\mathrm{OC}$ fraction was lower in the $200^{\circ} \mathrm{C}$ tests compared to most of the 25 and $90^{\circ} \mathrm{C}$ tests; however, some overlap is noted in results from all three temperature populations (Fig. 9 and Table 9). No variation is noted in the IC concentrations as a function of temperature. Both the formate and oxalate contents show a progressive decrease with increasing temperature, especially in the prerinse fractions. 


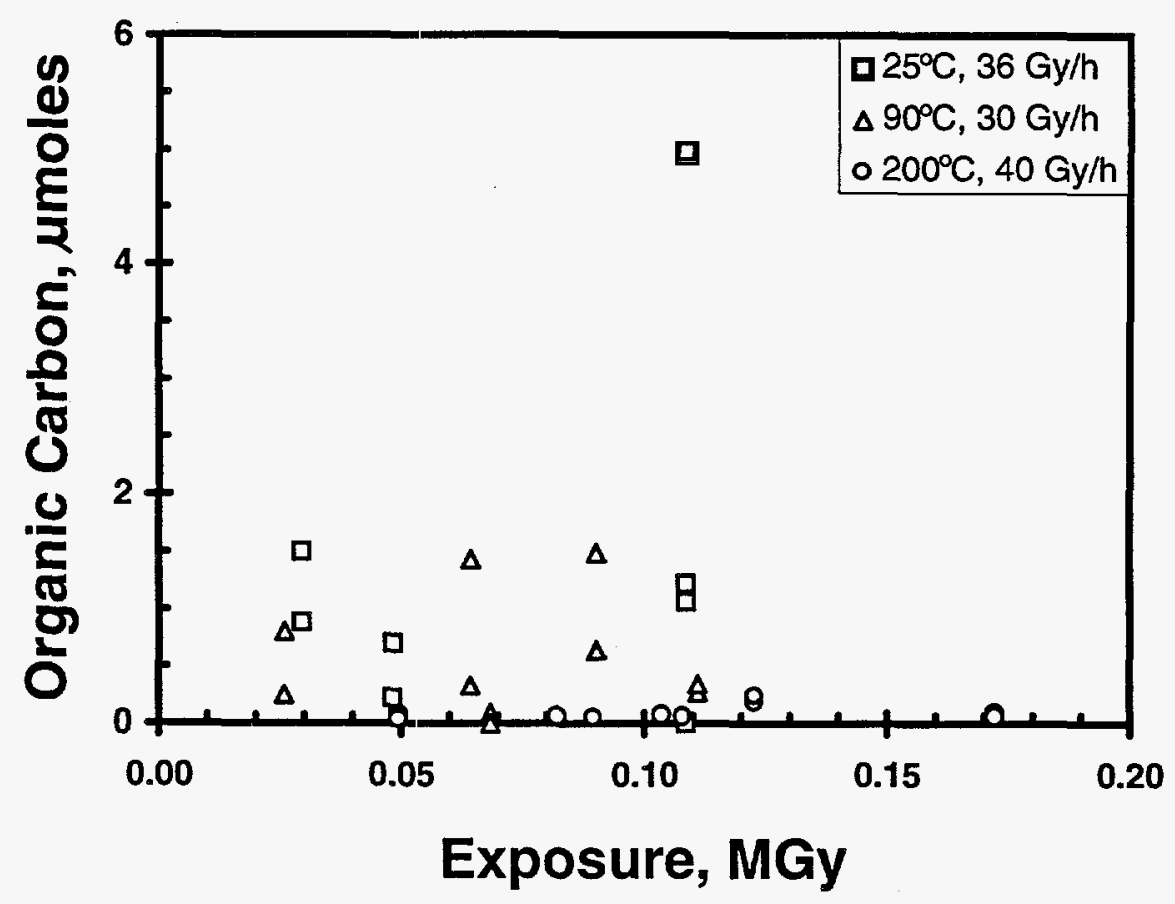

Fig. 9. Measured Organic Carbon Formation in Gamma Blank Tests as a Function of Cumulative Exposure Dose for the $\sim 35$ and $\sim 500 \mathrm{~Gy} / \mathrm{h}$ Tests. Data plotted for temperatures of 25,90 , and $200^{\circ} \mathrm{C}$.

\section{B. Blank Tests with Alpha Irradiation}

\section{Anion Concentrations}

A detailed test matrix listing all alpha blank tests and experimental parameters is given in Table 11. Solution volumes used to calculate anion species $\mu \mathrm{m}$ values are given in Table B.1.2. Dilution and aliquot removed corrected $\mu \mathrm{mol}$ values are given in Tables $12 \mathrm{a}$ and 13a, while as-received solution aliquot concentrations appear in Tables $12 \mathrm{~b}$ and 13b. Thirty percent of the total nitrate and nitrite present in the tests at the end of the sampling period was recovered from the prerinse solution that collected in the bottom of the test vessel. This surface represents only $\sim 5 \%$ of the geometric surface area of the vessels (including the surface area of the ${ }^{241} \mathrm{Am}$ foil assembly). As with the gamma tests, the concentration of the acid in the vessel bottom probably results from gravitational settling of aerosols and/or dripping of condensed solution from the vessel walls and foil assembly. Nitrate is the principal anion recovered in these tests, with minor amounts of nitrite also being recovered (Table 12). Tests run at $25^{\circ} \mathrm{C}$ with an alpha-radiation source indicate a $\mathrm{G}\left(\mathrm{NO}_{3}{ }^{-}+\mathrm{NO}_{2}{ }^{-}\right)$value of $2.4 \pm 1.1$ for the air-filled portion of the vessel (Fig. 10). These values are slightly lower than the $3.4 \pm 0.9$ obtained in the gamma blank tests at low dose rates. 
Table 11. Sampling Matrix for Blank Alpha Tests

\begin{tabular}{|c|c|c|c|c|c|c|}
\hline Test Number & $\begin{array}{l}\text { Glass } \\
\text { Type } \\
\end{array}$ & $\begin{array}{c}\text { Temp. } \\
\left({ }^{\circ} \mathrm{C}\right)\end{array}$ & $\begin{array}{c}\text { Test } \\
\text { Length } \\
\text { (days) }\end{array}$ & $\begin{array}{l}\text { Dose } \\
\text { Rate } \\
(\mathrm{Gy} / \mathrm{h}) \\
\end{array}$ & $\begin{array}{c}\text { Gas:Liq. } \\
\text { Ratio }\end{array}$ & $\begin{array}{l}\text { Cumulative } \\
\text { Dose (kGy) }\end{array}$ \\
\hline IV9000-14-A1-100 & None & 25 & 22 & 26 & 100 & 13.7 \\
\hline IV9000-14-A2-100 & None & 25 & 22 & 26 & 100 & 13.7 \\
\hline IV9000-28-A1-100 & None & 25 & 31 & 26 & 100 & 19.3 \\
\hline IV $9000-28-A 2-100$ & None & 25 & 31 & 26 & 100 & 19.3 \\
\hline IV9000-56-A1-100 & None & 25 & 65 & 26 & 100 & 40.6 \\
\hline IV9000-56-A2-100 & None & 25 & 65 & 26 & 100 & 40.6 \\
\hline IV9000-14-A1S-100 & None & 25 & 14 & 26 & 100 & 8.7 \\
\hline IV9000-14-A2S-100 & None & 25 & 14 & 26 & 100 & 8.7 \\
\hline IV9000-28-A1S-100 & None & 25 & 33 & 26 & 100 & 20.6 \\
\hline IV9000-28-A2S-100 & None & 25 & 33 & 26 & 100 & 20.6 \\
\hline IV9000-45-A1S-100 & None & 25 & 45 & 26 & 100 & 28.1 \\
\hline IV9000-45-A2S-100 & None & 25 & 45 & 26 & 100 & 28.1 \\
\hline IV $9000-56-A 1 S-100$ & None & 25 & 60 & 26 & 100 & 37.4 \\
\hline IV $9000-56-A 2 S-100$ & None & 25 & 60 & 26 & 100 & 37.4 \\
\hline IV $9000-85-A 1 S-100$ & None & 25 & 63 & 26 & 100 & 39.3 \\
\hline IV $9000-85-A 2 S-100$ & None & 25 & 63 & 26 & 100 & 39.3 \\
\hline IV $9000-110-A 1 S-100$ & None & 25 & 110 & 26 & 100 & 68.6 \\
\hline IV $9000-110-A 2 S-100$ & None & 25 & 110 & 26 & 100 & 68.6 \\
\hline IV9000-120-A1S-100 & None & 25 & 129 & 26 & 100 & 80.5 \\
\hline IV $9000-120-A 2 S-100$ & None & 25 & 129 & 26 & 100 & 80.5 \\
\hline IV $9000-45-B L A N K-100$ & None & 25 & 45 & None & 100 & None \\
\hline IV $9000-56-B L A N K-100$ & None & 25 & 56 & None & 100 & None \\
\hline IV9000-110-BLANK-100 & None & 25 & 110 & None & 100 & None \\
\hline
\end{tabular}


Table 12a. Anion Results for Alpha Blank Tests (values in total $\mu \mathrm{mol}$ produced)

\begin{tabular}{|c|c|c|c|c|c|c|c|c|c|c|c|c|c|c|}
\hline \multirow[b]{2}{*}{ Test Number } & \multirow[b]{2}{*}{$\begin{array}{l}\text { Temp. } \\
\left({ }^{\circ} \mathrm{C}\right)\end{array}$} & \multirow{2}{*}{$\begin{array}{c}\text { Test } \\
\text { Length } \\
\text { (days) }\end{array}$} & \multirow{2}{*}{$\begin{array}{c}\text { Dose } \\
\text { Rate } \\
(\mathrm{Gy} / \mathrm{h})\end{array}$} & \multirow[b]{2}{*}{$\begin{array}{c}\text { Gas:Liq. } \\
\text { Ratio }\end{array}$} & \multicolumn{2}{|c|}{$\mathrm{Cl}$} & \multicolumn{2}{|c|}{$\mathrm{SO}_{4}$} & \multicolumn{2}{|c|}{$\mathrm{NO}_{3}$} & \multicolumn{2}{|c|}{$\mathrm{NO}_{2}$} & \multicolumn{2}{|c|}{$\mathrm{F}$} \\
\hline & & & & & Prerinse & Rinse & Prerinse & Rinse & Prerinse & Rinse & Prerinse & Rinse & Prerinse & Rinse \\
\hline IV9000-14-Al-100 & 25 & 22 & 26 & 100 & $<0.15$ & $<0.26$ & $<0.19$ & $<0.32$ & 0.66 & 1.21 & $<0.15$ & 0.58 & $<0.28$ & 0.49 \\
\hline IV9000-14-A2-100 & 25 & 22 & 26 & 100 & 0.25 & 1.53 & $<0.19$ & 0.64 & 2.85 & 4.65 & $<0.16$ & 0.47 & 0.56 & 0.83 \\
\hline IV9000-28-Al-100 & 25 & 31 & 26 & 100 & $<0.30$ & 0.97 & 0.24 & 0.83 & 1.08 & 5.88 & 1.40 & 1.83 & $<0.56$ & $<1.13$ \\
\hline IV $9000-28-A 2-100$ & 25 & 31 & 26 & 100 & $<0.30$ & 1.02 & $<0.11$ & 0.81 & 2.36 & 7.88 & 0.17 & $<0.21$ & $<0.56$ & $<1.14$ \\
\hline IV9000-56-A1-100 & 25 & 65 & 26 & 100 & $<0.49$ & $<1.03$ & $<0.91$ & $<1.89$ & 3.66 & 15.68 & $<0.38$ & $<0.79$ & 2.11 & 2.95 \\
\hline IV $9000-56-\mathrm{A} 2-100$ & 25 & 65 & 26 & 100 & $<0.49$ & $<0.97$ & $<0.91$ & $<1.79$ & 3.96 & 16.83 & $<0.38$ & $<0.75$ & 3.97 & 4.68 \\
\hline IV9000-14-A1S-100 & 25 & 14 & 26 & 100 & $<0.29$ & 0.80 & $<0.18$ & 0.94 & 0.63 & 3.60 & 0.17 & $<0.19$ & $<0.54$ & $<0.97$ \\
\hline IV $9000-14-A 2 S-100$ & 25 & 14 & 26 & 100 & $<0.28$ & 2.20 & $<0.17$ & 3.09 & 1.12 & 0.93 & 0.65 & 1.80 & $<0.52$ & 2.06 \\
\hline IV $9000-28-A 1 S-100$ & 25 & 33 & 26 & 100 & $<0.18$ & 0.74 & $<0.84$ & $<1.06$ & 1.93 & 7.37 & $<0.35$ & $<0.67$ & $<0.34$ & 0.68 \\
\hline IV $9000-28-A 2 S-100$ & 25 & 33 & 26 & 100 & $<0.16$ & 1.17 & $<0.74$ & 1.60 & 1.47 & 7.90 & $<0.31$ & $<0.60$ & 0.39 & 1.18 \\
\hline IV $9000-45-A 1 S-100$ & 25 & 46 & 26 & 100 & 0.14 & 0.70 & 0.06 & 0.77 & 3.44 & $24.0 \hat{0}$ & 0.10 & 0.27 & $<0.08$ & $<0.22$ \\
\hline IV $9000-45-A 2 S-100$ & 25 & 46 & 26 & 100 & 0.36 & 1.68 & 0.06 & 0.63 & 2.84 & 26.39 & 0.16 & 0.54 & 1.31 & 1.30 \\
\hline IV $9000-56-A 1 S-100$ & 25 & 60 & 26 & 100 & $<0.36$ & 0.93 & $<0.66$ & 1.76 & 4.11 & 15.29 & $<0.28$ & $<0.58$ & 0.80 & 1.52 \\
\hline IV $9000-56-A 2 S-100$ & 25 & 60 & 26 & 100 & $<0.41$ & 0.96 & $<0.75$ & 1.59 & 3.72 & 15.81 & $<0.31$ & $<0.59$ & $<0.76$ & 1.52 \\
\hline IV $9000-85-A 1 S-100$ & 25 & 63 & 26 & 100 & 0.33 & 0.67 & 0.11 & 0.44 & 1.29 & 2.06 & $<0.07$ & $<0.14$ & $<0.08$ & $<0.17$ \\
\hline IV $9000-85-A 2 S-100$ & 25 & 63 & 26 & 100 & 0.30 & 0.56 & 0.08 & 0.36 & 1.21 & 13.13 & $<0.06$ & $<0.13$ & $<0.08$ & $<0.16$ \\
\hline IV $9000-110-A 1 S-100$ & 25 & 110 & 26 & 100 & 0.25 & 0.49 & 0.08 & 0.99 & 3.77 & 33.44 & $<0.03$ & $<0.10$ & $<0.04$ & 0.88 \\
\hline IV $9000-110-A 2 S-100$ & 25 & 110 & 26 & 100 & 0.16 & 0.70 & 0.05 & 1.01 & 3.37 & 37.31 & $<0.02$ & $<0.12$ & $<0.03$ & 0.91 \\
\hline IV $9000-120-A 1 S-100$ & 25 & 129 & 26 & 100 & 0.69 & 1.87 & 0.46 & 2.43 & 22.43 & 41.56 & $<0.27$ & $<1.03$ & 0.12 & 0.49 \\
\hline IV $9000-120-A 2 S-100$ & 25 & 129 & 26 & 100 & N.A. & N.A. & N.A. & N.A. & 6.31 & 39.46 & N.A. & N.A. & 0.31 & 0.91 \\
\hline IV $9000-45-B L A N K-100$ & 25 & 46 & None & 100 & 0.11 & 0.61 & $<0.07$ & 1.18 & $<0.07$ & 1.24 & $<0.09$ & 0.29 & 0.44 & 1.64 \\
\hline IV9000-110-BLANK-100 & 25 & 110 & None & 100 & 0.24 & 0.84 & 0.06 & 0.57 & 0.11 & 0.74 & 0.23 & $<0.33$ & 0.20 & $<0.33$ \\
\hline
\end{tabular}

Rinse solution values include a correction factor for both the proportion of aliquots removed and the dilution of the test solution by rinse fluid. Prerinse total $\mu$ moles $=($ Prerinse concentration in $\mu \mathrm{g} / \mathrm{ml} * \mathrm{ml}$ leachate $) /$ molecular weight. Rinse total $\mu \mathrm{mole}=($ Rinse concentration in $\mu \mathrm{g} / \mathrm{ml}$ ( $\mathrm{ml}$ leachant $-\mathrm{ml}$ aliquots removed $+\mathrm{ml}$ rinse solution added) + prerinse concentration in $\mu \mathrm{g} / \mathrm{ml} * \mathrm{ml}$ aliquots removed)/molecular weight. 
Table 12b. Anion Results for Alpha Blank Tests (values in ppm)

\begin{tabular}{|c|c|c|c|c|c|c|c|c|c|c|c|c|c|c|}
\hline \multirow[b]{2}{*}{ Test Number } & \multirow[b]{2}{*}{$\begin{array}{l}\text { Temp. } \\
\left({ }^{\circ} \mathrm{C}\right)\end{array}$} & \multirow{2}{*}{$\begin{array}{c}\text { Test } \\
\text { Lengt } \\
\text { (days) }\end{array}$} & \multirow{2}{*}{$\begin{array}{l}\text { Dose } \\
\text { Rate } \\
(\mathrm{Gy} / \mathrm{h})\end{array}$} & \multirow{2}{*}{$\begin{array}{c}\text { Gas:Liq. } \\
\text { Ratio }\end{array}$} & \multicolumn{2}{|c|}{$\mathrm{Cl}$} & \multicolumn{2}{|c|}{$\mathrm{SO}_{4}$} & \multicolumn{2}{|c|}{$\mathrm{NO}_{3}$} & \multicolumn{2}{|c|}{$\mathrm{NO}_{2}$} & \multicolumn{2}{|c|}{$\mathrm{F}$} \\
\hline & & & & & Prerinse & Rinse & Prerinse & Rinse & Prerinse & Rinse & Prerinse & Rinse & Prerinse & Rinse \\
\hline IV9000-14-A1-100 & 25 & 22 & 26 & 100 & $<0.3$ & $<0.3$ & $<1.0$ & $<1.0$ & 2.3 & 2.5 & $<0.4$ & 1.1 & $<0.3$ & 0.30 \\
\hline IV $9000-14-A 2-100$ & 25 & 22 & 26 & 100 & 0.50 & 2.2 & $<1.0$ & 2.3 & 9.8 & 8.2 & $<0.4$ & 0.77 & 0.59 & 0.42 \\
\hline IV9000-28-A1-100 & 25 & 31 & 26 & 100 & $<0.6$ & 1.12 & 1.29 & 2.65 & 3.79 & 13.1 & 3.66 & 1.78 & $<0.6$ & $<0.6$ \\
\hline IV $9000-28-A 2-100$ & 25 & 31 & 26 & 100 & $<0.6$ & 1.17 & $<0.6$ & 2.84 & 8.26 & 15.8 & 0.44 & $<0.2$ & $<0.6$ & $<0.6$ \\
\hline IV $9000-56-A 1-100$ & 25 & 65 & 26 & 100 & $<1.0$ & $<1.0$ & $<5.0$ & $<5.0$ & 13 & 33 & $<1.0$ & $<1.0$ & 2.3 & 1.2 \\
\hline IV9000-56-A2-100 & 25 & 65 & 26 & 100 & $<1.0$ & $<1.0$ & $<5.0$ & $<5.0$ & 14 & 38 & $<1.0$ & $<1.0$ & 4.3 & 1.8 \\
\hline IV $9000-14-A 1 S-100$ & 25 & 14 & 26 & 100 & $<0.6$ & 1.1 & $<1.0$ & 4.0 & 2.3 & 10 & 0.45 & $<0.20$ & $<0.60$ & $<0.60$ \\
\hline IV $9000-14-A 2 S-100$ & 25 & 14 & 26 & 100 & $<0.6$ & 3.5 & $<1.0$ & 14 & 4.2 & 0.60 & 1.8 & 3.1 & $<0.60$ & 1.6 \\
\hline IV $9000-28-A 1 S-100$ & 25 & 33 & 26 & 100 & $<0.4$ & 1.1 & $<5.0$ & $<5.0$ & 7.4 & 19 & $<1.0$ & $<1.0$ & $<0.40$ & 0.43 \\
\hline IV $9000-28-A 2 S-100$ & 25 & 33 & 26 & 100 & $<0.4$ & 2.2 & $<5.0$ & 5.9 & 6.4 & 25 & $<1.0$ & $<1.0$ & 0.52 & 1.0 \\
\hline IV $9000-45-A 1 S-100$ & 25 & 46 & 26 & 100 & 0.32 & 0.70 & 0.38 & 2.3 & 14 & 44 & 0.30 & 0.30 & $<0.10$ & $<0.10$ \\
\hline IV $9000-45-A 2 S-100$ & 25 & 46 & 26 & 100 & 0.86 & 1.7 & 0.40 & 1.9 & 12 & 51 & 0.51 & 0.65 & 1.7 & 0.20 \\
\hline IV $9000-56-A 1 S-100$ & 25 & 60 & 26 & 100 & $<1.0$ & 1.4 & $<5.0$ & 7.2 & 20 & 46 & $<1.0$ & $<1.0$ & 1.2 & 1.0 \\
\hline IV $9000-56-A 2 S-100$ & 25 & 60 & 26 & 100 & $<1.0$ & 1.4 & $<5.0$ & 6.0 & 16 & 49 & $<1.0$ & $<1.0$ & $<1.0$ & 1.1 \\
\hline IV $9000-85-A 1 S-100$ & 25 & 63 & 26 & 100 & 0.78 & 0.75 & 0.70 & 1.7 & 5.3 & 3.4 & $<0.2$ & $<0.2$ & $<0.10$ & $<0.10$ \\
\hline IV $9000-85-A 2 S-100$ & 25 & 63 & 26 & 100 & 0.73 & 0.63 & 0.54 & 1.5 & 5.2 & 40 & $<0.2$ & $<0.2$ & $<0.10$ & $<0.10$ \\
\hline IV $9000-110-A 1 S-100$ & 25 & 110 & 26 & 100 & 1.2 & 0.55 & 1.0 & 5.5 & 32 & 116 & $<0.2$ & $<0.2$ & $<0.10$ & 1.0 \\
\hline IV $9000-110-A 2 S-100$ & 25 & 110 & 26 & 100 & 1.0 & 0.85 & 0.93 & 4.0 & 38 & 92 & $<0.2$ & $<0.2$ & $<0.10$ & 0.73 \\
\hline IV $9000-120-A 1 S-100$ & 25 & 129 & 26 & 100 & 2.0 & 1.2 & 3.6 & 5.3 & 114 & 35.5 & $<1.0$ & $<1.0$ & 0.18 & 0.20 \\
\hline IV $9000-120-A 2 S-100$ & 25 & 129 & 26 & 100 & N.A. & 0.76 & N.A. & 3.0 & 38.0 & 31.5 & N.A. & $<0.1$ & 0.57 & 0.18 \\
\hline IV9000-45-BLANK-100 & 25 & 46 & None & 100 & 0.28 & 0.70 & $<0.5$ & 4.1 & $<0.30$ & 2.8 & $<0.3$ & 0.39 & 0.61 & 0.93 \\
\hline IV9000-110-BLANK-100 & 25 & 110 & None & 100 & 0.91 & 0.83 & 0.62 & 1.9 & 0.73 & 1.5 & 1.1 & $<0.2$ & 0.40 & $<0.10$ \\
\hline
\end{tabular}

Prerinse values represent undiluted anion concentrations collected from fluid in bottom of test vessel. Rinse values represent diluted concentrations after the vessel walls were rinsed with deionized water. 
Table 13a. Carbon Species $(\mu \mathrm{mol})$ and $\mathrm{pH}$ Results for Alpha Blank Tests ${ }^{\mathrm{a}}$

\begin{tabular}{|c|c|c|c|c|c|c|c|c|c|c|c|c|}
\hline \multirow[b]{2}{*}{ Test Number } & \multicolumn{2}{|c|}{$\mathrm{pH}$} & \multicolumn{3}{|c|}{ Prerinse } & \multicolumn{3}{|c|}{ Rinse } & \multicolumn{2}{|c|}{ Oxalate } & \multicolumn{2}{|c|}{ Formate } \\
\hline & Prerinse & Rinse & $\begin{array}{c}\text { Total } \\
\text { Carbon }\end{array}$ & $\begin{array}{l}\text { Organic } \\
\text { Carbon }\end{array}$ & $\begin{array}{c}\text { Inorganic } \\
\text { Carbon }\end{array}$ & $\begin{array}{c}\text { Total } \\
\text { Carbon }\end{array}$ & $\begin{array}{l}\text { Organic } \\
\text { Carbon }\end{array}$ & $\begin{array}{c}\text { Inorganic } \\
\text { Carbon }\end{array}$ & Prerinse & Rinse & Prerinse & Rinse \\
\hline IV9000-14-A1-100 & 7.05 & 7.25 & 17.25 & 11.29 & 5.96 & 44.64 & 29.55 & 15.09 & $<0.20$ & $<0.35$ & 1.11 & 2.11 \\
\hline IV $9000-14-A 2-100^{b}$ & 6.90 & 6.65 & 27.84 & 20.73 & 7.11 & 63.00 & 46.95 & 16.05 & $<0.20$ & $<0.38$ & $<0.24$ & $<0.44$ \\
\hline IV $9000-28-A 1-100^{b}$ & 4.94 & 5.54 & 76.35 & 63.72 & 12.63 & 156.86 & 131.34 & 25.52 & 0.26 & 0.60 & 1.29 & 3.36 \\
\hline IV $9000-28-A 2-100^{b}$ & 3.60 & 4.13 & 75.94 & 65.59 & 10.34 & 156.40 & 134.00 & 22.40 & 0.15 & 0.35 & 3.18 & 4.12 \\
\hline IV $9000-56-A 1-100^{b}$ & 3.83 & 4.19 & 107.20 & 91.09 & 16.11 & 143.52 & 116.08 & 27.44 & $<0.99$ & $<2.06$ & $<0.78$ & $<1.61$ \\
\hline IV9000-56-A2-100 & 4.18 & 4.60 & 102.11 & 80.71 & 21.40 & 134.90 & 103.92 & 30.97 & $<1.00$ & $<1.95$ & 5.45 & 9.11 \\
\hline IV9000-14-A1S-100 & 6.94 & 6.70 & 10.83 & 5.30 & 5.53 & 24.69 & 14.96 & 9.73 & $<0.19$ & $<0.35$ & 0.49 & 1.50 \\
\hline IV $9000-14-A 2 S-100$ & 7.20 & 6.65 & 15.28 & 8.11 & 7.16 & 52.26 & 34.77 & 17.48 & $<0.19$ & $<1.54$ & 0.59 & 2.38 \\
\hline IV $9000-28-A 1 S-100$ & 6.98 & 6.65 & 7.70 & 3.44 & 4.26 & 22.02 & 12.93 & 9.09 & $<1.29$ & $<2.44$ & 0.43 & 1.87 \\
\hline IV $9000-28-A 2 S-100$ & 6.82 & 6.53 & 10.52 & 6.43 & 4.09 & 35.68 & 23.84 & 11.84 & $<1.13$ & $<2.20$ & 0.41 & 2.89 \\
\hline IV $9000-45-A 1 S-100$ & 4.60 & 4.84 & 8.18 & 7.78 & 0.40 & 30.36 & 26.85 & 3.50 & $<0.12$ & $<0.33$ & 1.76 & $3 . \overline{3}$ \\
\hline IV $9000-45-A 2 S-100$ & 5.61 & 6.27 & 5.18 & 4.49 & 0.70 & 16.21 & 13.98 & 2.23 & $<0.12$ & 0.72 & $<0.07$ & $<0.18$ \\
\hline IV9000-56-A1S-100 & 6.55 & 6.30 & 15.68 & 9.23 & 6.45 & 35.98 & 24.28 & 11.71 & $<1.01$ & $<2.13$ & $<0.57$ & $<1.19$ \\
\hline IV9000-56-A2S-100 & 6.80 & 6.65 & 6.75 & 3.19 & 3.55 & 24.90 & 15.81 & 9.10 & $<1.15$ & $<2.17$ & $<0.64$ & $<1.21$ \\
\hline IV9000-85-AIS-100 & 4.10 & 4.50 & 8.10 & 8.44 & 0.00 & 14.00 & 13.30 & 0.70 & $<0.12$ & 0.37 & 1.41 & 2.47 \\
\hline IV $9000-85-A 2 S-100$ & 4.74 & 4.83 & 5.68 & 5.21 & 0.47 & 8.74 & 7.74 & 1.00 & $<0.11$ & 0.32 & 1.38 & 2.19 \\
\hline IV $9000-110-A 1 S-100$ & 4.07 & 4.59 & 6.24 & 4.87 & 1.36 & 17.39 & 14.87 & 2.52 & $<0.06$ & 0.80 & 1.28 & 2.62 \\
\hline IV $9000-110-A 2 S-100$ & 5.10 & 4.92 & 6.60 & 6.26 & 0.34 & 19.19 & 21.13 & 0.00 & $<0.04$ & 0.75 & 2.32 & 4.17 \\
\hline IV $9000-120-A 1 S-100$ & 7.41 & 6.64 & 37.29 & 14.23 & 23.06 & 62.43 & 33.69 & 28.74 & $<0.97$ & $<3.79$ & N.R. & N.R. \\
\hline IV $9000-120-A 2 S-100$ & 5.63 & 6.23 & 9.44 & 7.49 & 1.95 & 37.97 & 30.97 & 7.00 & N.A. & N.A. & N.A. & N.A. \\
\hline IV9000-45-BLANK-100 & 6.69 & 6.54 & 3.18 & 1.87 & 1.32 & 23.27 & 19.48 & 3.79 & $<0.11$ & $<0.30$ & $<0.06$ & $<0.17$ \\
\hline IV $9000-110$-BLANK-100 & 6.52 & 6.67 & 3.75 & 2.18 & 1.57 & 13.07 & 8.24 & 4.83 & $<0.08$ & $<0.28$ & 0.46 & 1.66 \\
\hline
\end{tabular}

${ }^{a}$ All values other than $\mathrm{pH}$ in $\mu$ moles; carbon $\mu$ moles calculated with assumed molecular weight $=12 ;$ N.A. $=$ not analyzed; N.R. $=$ not able to resolve from adjacent $\mathrm{Cl}^{-}$peak.

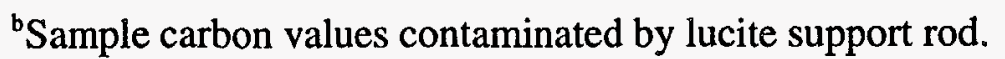

Rinse solution values include a correction factor for both the proportion of aliquots removed and the dilution of the test solution by the rinse fluid. 
Table 13b. Carbon Species (ppm) and pH Results for Alpha Blank Tests ${ }^{a}$

\begin{tabular}{|c|c|c|c|c|c|c|c|c|c|c|c|c|}
\hline \multirow[b]{2}{*}{ Test Number } & \multicolumn{2}{|c|}{$\mathrm{pH}$} & \multicolumn{3}{|c|}{ Prerinse } & \multicolumn{3}{|c|}{ Rinse } & \multicolumn{2}{|c|}{ Oxalate } & \multicolumn{2}{|c|}{ Formate } \\
\hline & Prerinse & Rinse & $\begin{array}{l}\text { Total } \\
\text { Carbon }\end{array}$ & $\begin{array}{l}\text { Organic } \\
\text { Carbon }\end{array}$ & $\begin{array}{c}\text { Inorganic } \\
\text { Carbon }\end{array}$ & $\begin{array}{c}\text { Total } \\
\text { Carbon }\end{array}$ & $\begin{array}{l}\text { Organic } \\
\text { Carbon }\end{array}$ & $\begin{array}{c}\text { Inorganic } \\
\text { Carbon }\end{array}$ & Prerinse & Rinse & Prerinse & Rinse \\
\hline IV9000-14-A1-100 & 7.05 & 7.25 & 11.63 & 7.61 & 4.02 & 20.32 & 13.50 & 6.82 & $<1.0$ & $<1.0$ & 2.8 & 3.2 \\
\hline IV9000-14-A2-100 & 6.90 & 6.65 & 18.53 & 13.80 & 4.73 & 25.02 & 18.65 & 6.37 & $<1.0$ & $<1.0$ & $<0.6$ & $<0.6$ \\
\hline IV $9000-28-A 1-100$ & 4.94 & 5.54 & 51.88 & 43.30 & 8.58 & 53.19 & 44.60 & 8.59 & 1.28 & 1.56 & 3.28 & 4.67 \\
\hline IV $9000-28-A 2-100$ & 3.60 & 4.13 & 51.54 & 44.52 & 7.02 & 52.02 & 44.41 & 7.61 & 0.77 & 0.88 & 8.10 & 3.82 \\
\hline IV9000-56-A1-100 & 3.83 & 4.19 & 73.72 & 62.64 & 11.08 & 35.42 & 27.28 & 8.14 & $<5.0$ & $<5.0$ & $<2.0$ & $<2.0$ \\
\hline IV9000-56-A2-100 & 4.18 & 4.60 & 69.94 & 55.48 & 14.66 & 36.64 & 27.58 & 9.06 & 5.0 & $<5.0$ & 14 & 11 \\
\hline IV $9000-14-A 1 S-100$ & 6.94 & 6.70 & 7.60 & 3.72 & 3.88 & 10.77 & 7.01 & 3.76 & $<1.0$ & $<1.0$ & 1.3 & 2.7 \\
\hline IV $9000-14-A 2 S-100$ & 7.20 & 6.65 & 11.07 & 5.88 & 5.19 & 24.84 & 17.32 & 7.52 & $<1.0$ & $<6.1$ & 1.6 & 4.4 \\
\hline IV9000-28-A1S-100 & 6.98 & 6.65 & 5.71 & 2.55 & 3.16 & 10.20 & 6.43 & 3.77 & $<7.0$ & $<7.0$ & 1.2 & 3.4 \\
\hline IV9000-28-A2S-100 & 6.82 & 6.53 & 8.85 & 5.41 & 3.44 & 19.76 & 13.53 & 6.23 & $<7.0$ & $<7.0$ & 1.3 & 6.9 \\
\hline IV $9000-45-A 1 S-100$ & 4.60 & 4.84 & 6.44 & 6.12 & 0.32 & 9.68 & 8.41 & 1.27 & $<0.7$ & $<0.7$ & 5.2 & 3.8 \\
\hline IV $9000-45-A 2 S-100$ & 5.61 & 6.27 & 4.24 & 3.67 & 0.57 & 5.01 & 4.32 & 0.69 & $<0.7$ & 1.9 & $<0.2$ & $<0.2$ \\
\hline IV9000-56-A1S-100 & 6.55 & 6.30 & 14.77 & 8.69 & 6.08 & 17.09 & 12.40 & 4.69 & $<7.0$ & $<7.0$ & $<2.0$ & $<2.0$ \\
\hline IV $9000-56-A 2 S-100$ & 6.80 & 6.65 & 5.62 & 2.66 & 2.96 & 14.45 & 9.77 & 4.68 & $<7.0$ & $<7.0$ & $<2.0$ & $<2.0$ \\
\hline IV9000-85-A1S-100 & 4.10 & 4.50 & 6.42 & 6.69 & 0.00 & 4.78 & 4.22 & 0.56 & $<0.7$ & 1.2 & 4.2 & 3.2 \\
\hline IV $9000-85-A 2 S-100$ & 4.74 & 4.83 & 4.73 & 4.34 & 0.39 & 2.79 & 2.39 & 0.41 & $<0.7$ & 1.1 & 4.3 & 2.7 \\
\hline IV $9000-110-A 1 S-100$ & 4.07 & 4.59 & 10.24 & 8.00 & 2.24 & 8.56 & 7.65 & 0.91 & $<0.7$ & 4.1 & 7.9 & 3.9 \\
\hline IV $9000-110-A 2 S-100$ & 5.10 & 4.92 & 14.39 & 13.65 & 0.74 & 6.65 & 7.84 & 0.00 & $<0.7$ & 2.7 & 19 & 3.7 \\
\hline IV $9000-120-A 1 S-100$ & 7.41 & 6.64 & 36.68 & 14.00 & 22.68 & 9.22 & 6.78 & 2.44 & $<7.0$ & $<7.0$ & N.R. & N.R. \\
\hline IV $9000-120-A 2 S-100$ & 5.63 & 6.23 & 11.00 & 8.73 & 2.27 & 5.33 & 4.38 & 0.95 & N.A. & $<7.0$ & N.R. & N.R. \\
\hline IV9000-45-BLANK-100 & 6.69 & 6.54 & 3.18 & 1.87 & 1.32 & 9.30 & 8.12 & 1.18 & $<0.7$ & $<0.7$ & $<0.2$ & $<0.2$ \\
\hline IV $9000-110-B L A N K-100$ & 6.52 & 6.67 & 3.75 & 2.18 & 1.57 & 4.71 & 3.04 & 1.67 & $<0.7$ & $<0.7$ & 2.2 & 2.1 \\
\hline
\end{tabular}

${ }^{a}$ All values other than $\mathrm{pH}$ in ppm; N.A. $=$ not analyzed; N.R. $=$ not able to resolve from adjacent $\mathrm{Cl}^{-}$peak.

Prerinse values represent undiluted anion concentrations collected from fluid in bottom of test vessel. Rinse values represent diluted concentrations after the vessel walls were rinsed with deionized water. 


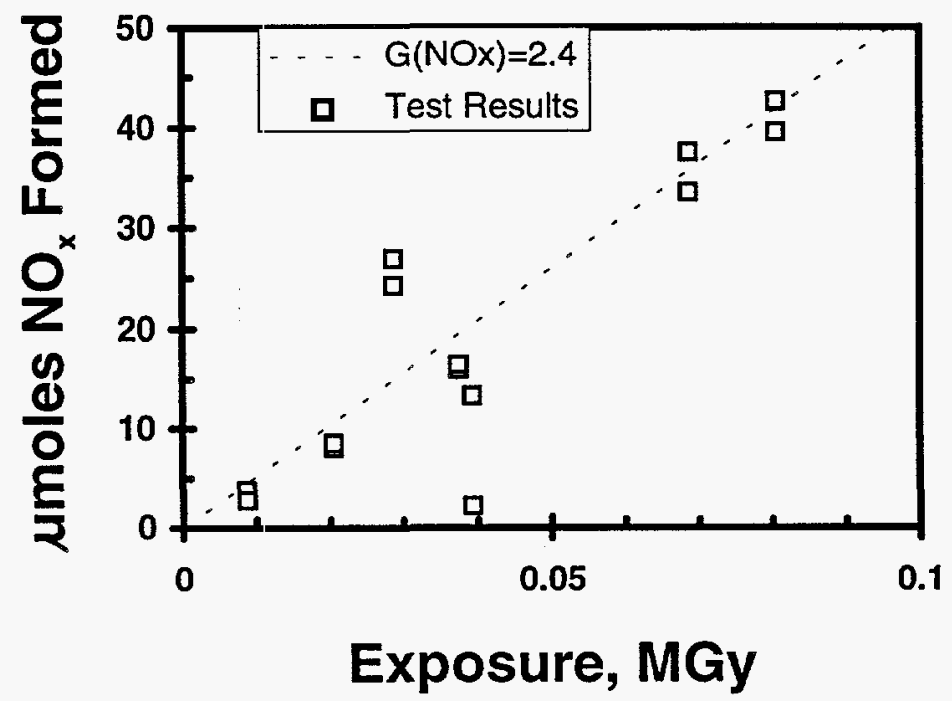

Fig. 10. Total $\mu$ moles of Nitrate + Nitrite Recovered in Blank Alpha Tests as a Function of Cumulative Dose at $225^{\circ} \mathrm{C}$. The $\mathrm{G}\left(\mathrm{NO}_{\mathrm{x}}\right)=2.4$ line represents the average $\mathrm{G}\left(\mathrm{NO}_{3}^{-}+\mathrm{NO}_{2}^{-}\right)$yield for all alpha tests. Samples exposed to ${ }^{24} \mathrm{Am}$ foil at a dose rate of $\sim 26 \mathrm{~Gy} / \mathrm{h}$.

\section{Solution $\mathrm{pH}$}

As with the gamma tests, the measured $\mathrm{NO}_{3}{ }^{-}$contents were employed to predict the solution $\mathrm{pH}$. These calculations indicate a $\mathrm{pH}$ decrease to $\sim 4$ after $10,000 \mathrm{~Gy}$ ( $1 \mathrm{Mrad}$ ) of radiation exposure, and to $\sim 2.5$ after 70,000 Gy (7 Mrad) exposure (Fig. 11). The relatively large quantities of solution present in the alpha tests also allowed for a direct measurement of solution $\mathrm{pH}$. Analytically measured $\mathrm{pH}$ values display a large degree of scatter and are notably higher than the calculated values (Fig. 11 and Table 13). This comparison suggests that the measured $\mathrm{pH}$ may have been buffered through reactions with the glass test vessels and/or by bicarbonate derived from the dissolution of $\mathrm{CO}_{2}$ present in the vessel atmosphere.

\section{Carbon Concentrations}

Results from alpha tests with Lucite-supported ${ }^{241} \mathrm{Am}$ foils indicate that the Lucite contributed to the OC production; therefore, no further consideration of these carbon values was given. For the tests with the stainless steel support rods, the average TC and OC values were increased relative to background tests without radiation, although the data display a large amount of scatter (Fig. 12 and Table 13). These trends indicate that OC species are produced during 


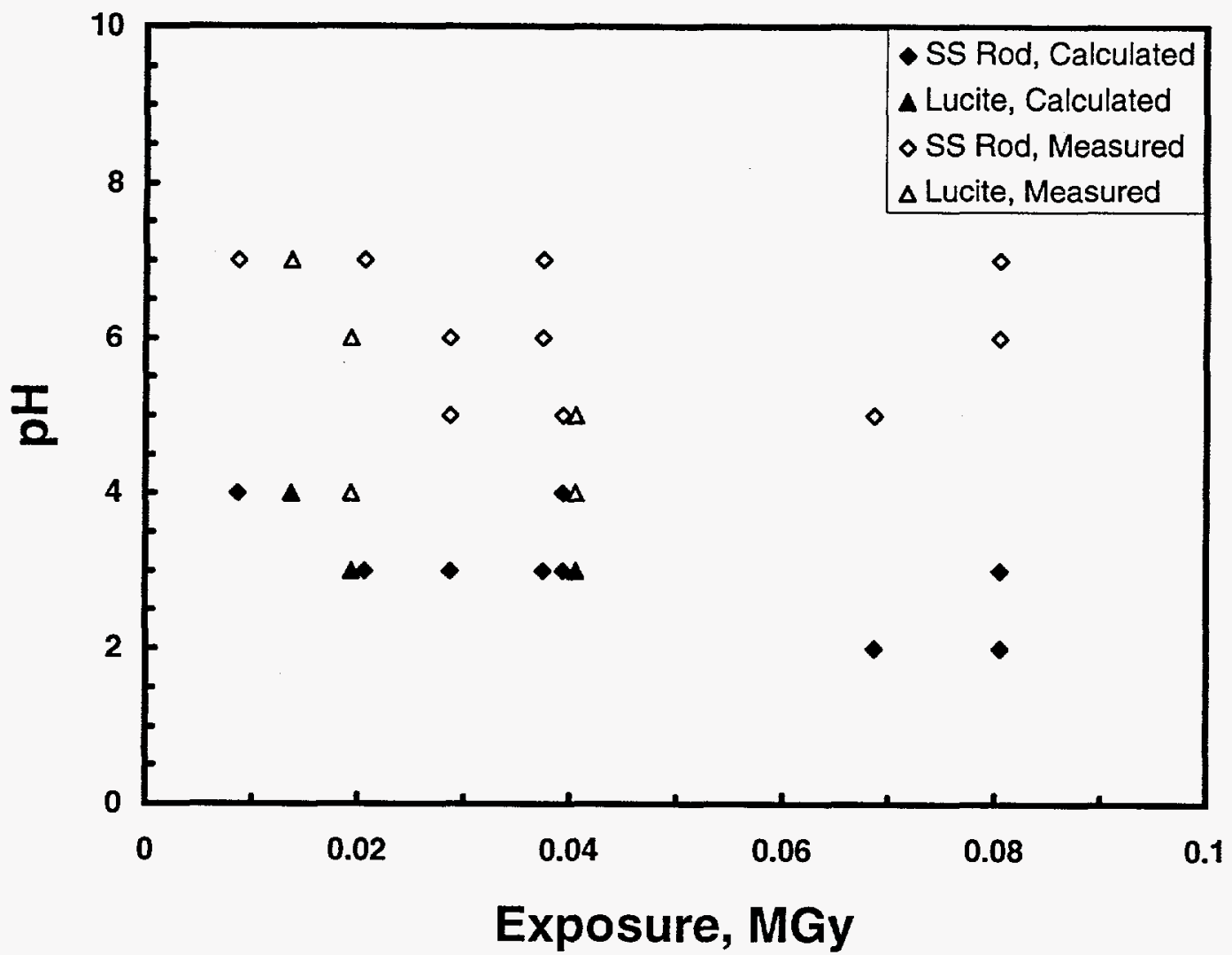

Fig. 11. Solution pH Values for the Blank Alpha Tests Plotted as a Function of Cumulative Exposure Dose. The $\mathrm{pH}$ values are plotted for both the "as measured" solution concentrations and calculated $\mathrm{pH}$ values based on the $\mathrm{NO}_{3}{ }^{-}+\mathrm{NO}_{2}{ }^{-}$concentrations, assuming complete dissociation of the acid species. Samples exposed to an ${ }^{241} \mathrm{Am}$ foil at a dose rate of $\sim 26 \mathrm{~Gy} / \mathrm{h}$ and a temperature of $25^{\circ} \mathrm{C}$.

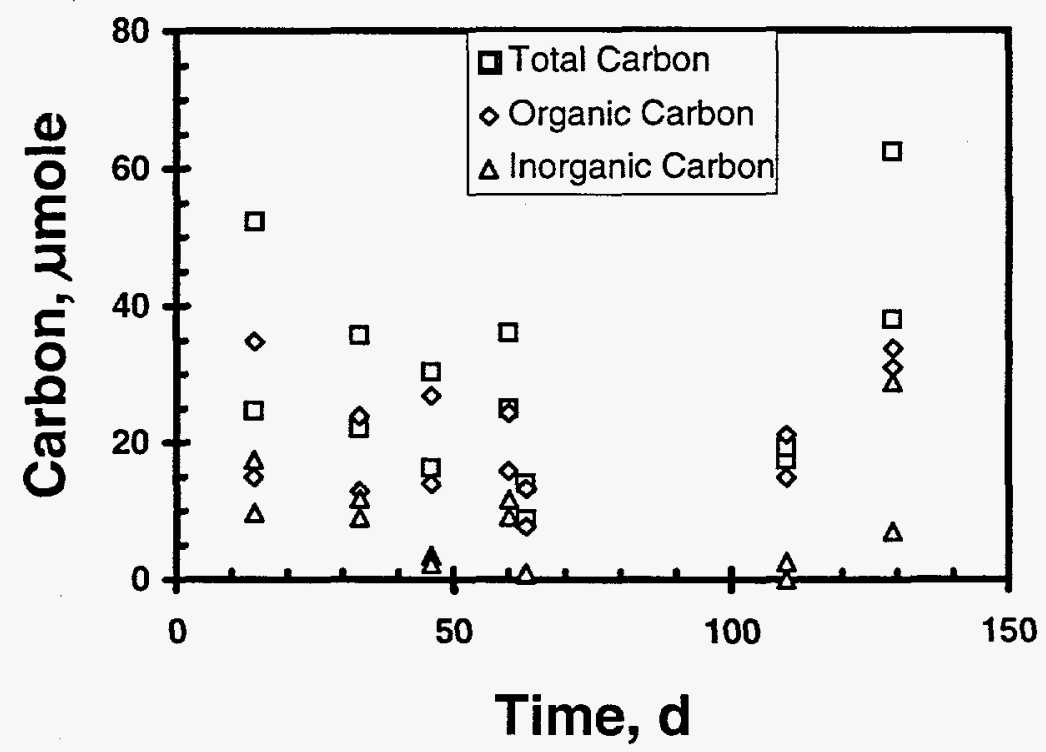

Fig. 12. Measured Organic Carbon Formation in Blank Alpha Tests (Stainless Steel Support Rods) as a Function of Cumulative Exposure Dose. Samples exposed to ${ }^{241} \mathrm{Am}$ foil at a dose rate of $\sim 26 \mathrm{~Gy} / \mathrm{h}$ and a temperature of $25^{\circ} \mathrm{C}$. 
alpha exposure. Both the rinse and prerinse solutions show a decrease in the IC with increasing exposure up to $\sim 70,000 \mathrm{~Gy}(\sim 7 \mathrm{Mrad})$, and thereafter an increase occurred. Contents of oxalate and formate in rinse solutions, as well as formate contents in prerinse solutions, increase after 50,000 Gy ( 5 Mrad) of cumulative alpha exposure.

\section{Irradiation Tests with Glass at $340 \mathrm{~m}^{-1}$}

\section{Anion Concentrations}

A detailed test matrix listing experimental parameters for all $340 \mathrm{~m}^{-1}$ irradiation tests is given in Table 14. Results from analyses of solutions in contact with SRL 202A, SRL 165A, and SRL 131A glasses during irradiation (Table 15) show little variation with absorbed dose for $\mathrm{NO}_{3}^{-}, \mathrm{COOH}^{-}$, and $\mathrm{C}_{2} \mathrm{O}_{4}^{2}$, while concentrations of $\mathrm{F}^{-}, \mathrm{Cl}^{-}$, and $\mathrm{SO}_{4}$ are only slightly enriched relative to the starting EJ-13 composition. These patterns suggest that the presence of reacting glass in the tests does not have a major influence on the anion chemistry of the leachate; however, some complexation of released glass reaction products may still occur. An exception in this trend is found in the concentration of nitrite. Levels of nitrite increase with exposure time for each glass, although there appears to be an initial induction period in which the nitrite levels remain relatively unchanged. Nitrite levels increase in solutions in contact with SRL 202A and SRL 165A glasses starting with the 360-day samples ( $0.3 \mathrm{MGy}$ cumulative exposure). Solutions in contact with the SRL 131A glasses also displayed an increase in nitrite levels between 180 and 720 days (0.13 to 0.60 MGy cumulative exposure), however, 360 day tests were not conducted with these samples.

The total concentration of nitrogen acid species increases over the course of the tests, from a starting value of $\sim 8 \mathrm{ppm}$ for the EJ-13 leachant, to $\sim 15 \mathrm{ppm}$ for 28 -day irradiation tests, and 35 to $75 \mathrm{ppm}$ for the 720 -day tests (Fig. 13 and Table 15). The $\mathrm{NO}_{3}^{-} \mathrm{NO}_{2}^{-}$ratio is significantly decreased relative to the level that characterizes both the starting EJ-13 leachant and the blank irradiation tests (Fig. 14 and Table 16). Total nitrate concentrations are also significantly depleted in irradiation tests with glass, relative to EJ-13 and the gamma blank tests (Table 15). There does not appear to be any significant effect of the specific glass composition on the nitrogen species concentrations. Further discussion of the effects of glass samples on the solution anion contents are presented in Section V.B.

\section{2. $\quad$ Solution $\mathrm{pH}$}

Solution results for batch leach tests with SRL 202A glass indicate a peak $\mathrm{pH}$ of $\sim 9.5$ after 180 days, followed by a slight acidification of the leachate down to a $\mathrm{pH} \sim 9.0$ between 180 and 720 days of testing (Table 15). The pH trends displayed in Fig. 15 have been plotted against averaged $\mathrm{pH}$ values of nonirradiated and non-transuranic doped samples from the data of Ebert [34] (dotted line in the figure). These latter tests have been performed under identical conditions as the present samples, except no external gamma radiation exposure was used. A comparison of the two $\mathrm{pH}$ trends indicates that the irradiated SRL 202A tests display pH values that are typically reduced $\sim 0.5$ to $1.0 \mathrm{pH}$ units relative to the nonirradiated SRL 202U samples. The 720 -day tests show the $\mathrm{pH}$ values converging from the irradiated and nonirradiated tests.

Analyses also suggest an increase in $\mathrm{pH}$ values for the SRL 165A glass solution during the entire 720 days of testing. These values increase from the starting value of 8.1 for the EJ-13 leachant, to about 8.8 after 91 days of testing (Fig. 15 and Table 15). The 180-day results show some data scatter with $\mathrm{pH}$ values of 8.4 and 9.6. Thereafter, the $\mathrm{pH}$ values for these tests display a continuous gradual increase to $\sim 9.1$ at 360 days and 9.7 at 720 days. Comparative analyses of nonirradiated SRL $165 \mathrm{U}$ glasses are not available. 
Table 14. Sampling Matrix for Batch Leach Tests $\left(340 \mathrm{~m}^{-1}\right)$

\begin{tabular}{|c|c|c|c|c|c|c|}
\hline Test Number & Glass Type & $\begin{array}{c}\text { Temp. } \\
\left({ }^{\circ} \mathrm{C}\right)\end{array}$ & $\begin{array}{c}\text { Test } \\
\text { Length } \\
\text { (days) }\end{array}$ & $\begin{array}{c}\text { Dose } \\
\text { Rate } \\
\text { (Gy/h) }\end{array}$ & $\begin{array}{l}\text { Expos. } \\
\text { Time } \\
\text { (h) }\end{array}$ & $\begin{array}{l}\text { Cumulative } \\
\text { Exposure } \\
\text { (MGy) }\end{array}$ \\
\hline IV9202A-14-1 & SRL 202A & 90 & 14 & 36 & 339 & 0.0122 \\
\hline IV9202A-28-1 & SRL 202A & 90 & 28 & 36 & 670 & 0.0241 \\
\hline & SRL 202A & 90 & 28 & 36 & 670 & 0.0241 \\
\hline IV9202A-56-1 & SRL 202A & 90 & 56 & 36 & 1,342 & 0.0483 \\
\hline IV9202A-56-2 & SRL 202A & 90 & 56 & 36 & 1,342 & 0.0483 \\
\hline IV9202A-56-3 & SRL 202A & 90 & 56 & 32 & 1,385 & 0.0443 \\
\hline IV9202A-56-4 & SRL 202A & 90 & 56 & 32 & 1,385 & 0.0443 \\
\hline IV9202A-180-3 & SRL 202A & 90 & 180 & 28 & 4,218 & 0.1181 \\
\hline IV9202A-180-4 & SRL 202A & 90 & 180 & 30 & 4,218 & 0.1265 \\
\hline IV9202A-360-1 & SRL 202A & 90 & 360 & 34 & 8,638 & 0.2937 \\
\hline IV9202A-360-2 & SRL 202A & 90 & 360 & 34 & 8,638 & 0.2937 \\
\hline IV9202A-360-3 & SRL 202A & 90 & 360 & 34 & 8,638 & 0.2937 \\
\hline IV9202A-540-1 & SRL 202A & 90 & 540 & 32 & 12,960 & 0.4147 \\
\hline IV9202A-540-2 & SRL 202A & 90 & 540 & 32 & 12,960 & 0.4147 \\
\hline IV9202A-720-1 & SRL 202A & 90 & 720 & 31 & 17,280 & 0.5357 \\
\hline IV9202A-720-2 & SRL 202A & 90 & 720 & 31 & 17,280 & 0.5357 \\
\hline IV9165A-91-3 & SRL 165A & 90 & 91 & 32 & 2,155 & 0.0689 \\
\hline IV9165A-180-3 & SRL 165A & 90 & 180 & 30 & 4,218 & 0.1265 \\
\hline IV9165A-180-4 & SRL 165A & 90 & 180 & 30 & 8,638 & 0.2591 \\
\hline IV9165A-360-1 & SRL 165A & 90 & 360 & 37 & 8,638 & 0.3196 \\
\hline IV9165A-360-2 & SRL 165A & 90 & 360 & 37 & 8,638 & 0.3196 \\
\hline IV9165A-720-1 & SRL 165A & 90 & 720 & 30 & 17,280 & 0.5184 \\
\hline IV9165A-720-2 & SRL 165A & 90 & 720 & 30 & 17,280 & 0.5184 \\
\hline IV9131A-14-1 & SRL 131A & $\overline{90}$ & 14 & $\overline{36}$ & 339 & 0.0122 \\
\hline IV9131A-14-2 & SRL 131A & 90 & 14 & 36 & 339 & 0.0122 \\
\hline IV9131A-28-1 & SRL 131A & 90 & 28 & 36 & 670 & 0.0241 \\
\hline IV9131A-28-2 & SRL 131A & 90 & 28 & 36 & 670 & 0.0241 \\
\hline IV9131A-56-1 & SRL 131A & 90 & 56 & 36 & 1,342 & 0.0483 \\
\hline IV9131A-56-2 & SRL 131A & 90 & 56 & 36 & 1,342 & 0.0483 \\
\hline IV9131A-56-3 & SRL 131A & 90 & 56 & 30 & 1,385 & 0.0416 \\
\hline IV9131A-56-4 & SRL 131A & 90 & 56 & 31 & 1,385 & 0.0429 \\
\hline IV9131A-180-3 & SRL 131A & 90 & 180 & 31 & 4,218 & 0.1307 \\
\hline IV9131A-720-1 & SRL 131A & 90 & 720 & 35 & 17,280 & 0.6048 \\
\hline IV9131A-7202 & SRL 131A & 90 & 720 & 35 & 17,280 & 0.6048 \\
\hline
\end{tabular}




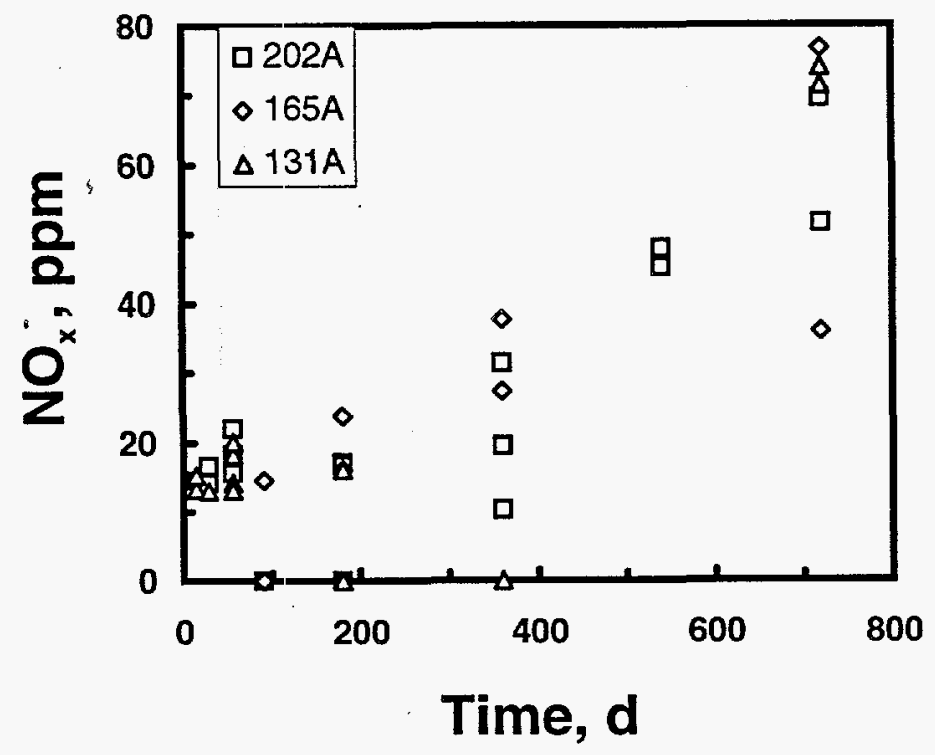

Fig. 13. Concentration of Nitrate Plus Nitrite as a Function of Cumulative Exposure Time in Solutions from Gamma-Irradiated Batch Tests with SRL 202A, SRL 165A, and SRL 131A Glasses $\left(\mathrm{S} / \mathrm{V}=340 \mathrm{~m}^{-1}, \mathrm{~T}=90^{\circ} \mathrm{C}\right.$, Dose Rate $\sim 35 \mathrm{~Gy} / \mathrm{h}$ ) 
Table 15. Anion and $\mathrm{pH}$ Results for Batch Tests at $340 \mathrm{~m}^{-1}$

\begin{tabular}{|c|c|c|c|c|c|c|c|c|c|c|c|c|c|}
\hline Test Number & $\begin{array}{c}\text { Test } \\
\text { Length } \\
\text { (days) }\end{array}$ & $\mathrm{pH}$ & $\begin{array}{c}\text { Total } \\
\text { Carbon } \\
\text { (ppm) }\end{array}$ & $\begin{array}{l}\text { Organic } \\
\text { Carbon } \\
(\mathrm{ppm})\end{array}$ & $\begin{array}{c}\text { Inorganic } \\
\text { Carbon } \\
\text { (ppm) }\end{array}$ & $\begin{array}{c}F^{*} \\
(\mathrm{ppm})\end{array}$ & $\begin{array}{c}\mathrm{Cl}^{-} \\
\text {(ppm) }\end{array}$ & $\begin{array}{c}\mathrm{NO}_{2} \\
(\mathrm{ppm})\end{array}$ & $\begin{array}{c}\mathrm{NO}_{3} \\
(\mathrm{ppm})\end{array}$ & $\begin{array}{c}\mathrm{HPO}_{4}{ }^{2} \\
\text { (ppm) }\end{array}$ & $\begin{array}{c}\mathrm{SO}_{4}^{2-} \\
\text { (ppm) }\end{array}$ & $\begin{array}{c}\text { Formate } \\
\mathrm{CHO}_{2}^{\circ} \\
(\mathrm{ppm})\end{array}$ & $\begin{array}{l}\text { Oxalate } \\
\mathrm{C}_{2} \mathrm{O}_{4}^{2-} \\
\text { (ppm) }\end{array}$ \\
\hline IV9202A-14-1 & 14 & 8.91 & 44.38 & 3.35 & 41.03 & 3.5 & 11 & 8.7 & 5.8 & $<0.5$ & 28 & $<0.5$ & $<0.5$ \\
\hline IV9202A-28-1 & 28 & 8.70 & 50.68 & 9.22 & 41.46 & 3.4 & 10 & 11 & 3.1 & $<0.5$ & 26 & $<0.5$ & 1.9 \\
\hline IV9202A-28-2 & 28 & 8.55 & 45.14 & 4.74 & 40.40 & 4.6 & 11 & 12 & 4.6 & $<0.5$ & 31 & $<0.5$ & 2.7 \\
\hline IV9202A-56-1 & 56 & 8.95 & 49.74 & 0.83 & 48.91 & 3.4 & 12 & 10 & 12 & $<0.5$ & 45 & $<0.5$ & 1.7 \\
\hline IV9202A-56-2 & 56 & 9.01 & 54.08 & 0.87 & 53.21 & 4.7 & 11 & 7.3 & 11 & $<0.5$ & 33 & $<0.5$ & 1.4 \\
\hline IV9202A-56-3 & 56 & 9.26 & 60.83 & 0.00 & 60.83 & 5.1 & 12 & 13 & 2.6 & $<2$ & 45 & $<0.3$ & 6.4 \\
\hline IV9202A-56-4 & 56 & 8.83 & 49.66 & 0.00 & 49.66 & 4.3 & 10 & 13 & 2.6 & $<$ & 31 & $<0.3$ & 2.5 \\
\hline IV9202A-180-3 & 180 & 9.59 & 50.05 & 6.86 & 43.20 & 4.3 & 13 & 15 & 2.1 & 1.8 & 36 & $<0.3$ & 8.6 \\
\hline IV9202A-180-4 & 180 & 9.35 & 43.59 & 5.26 & 38.33 & 2.9 & 9.5 & 15 & 1.7 & $<1$ & 28 & $<0.3$ & 2 \\
\hline IV9202A-360-1 & 360 & 9.31 & 82.50 & 0.71 & 81.79 & 3.7 & 11 & 17 & 2.4 & $<3$ & 35 & $<0.3$ & $<1.0$ \\
\hline IV $9202 A-360-2$ & 360 & 8.92 & 78.05 & 2.66 & 75.39 & 2.8 & 10 & 26 & 5.3 & $<$ & 22 & $<0.3$ & 1.4 \\
\hline IV9202A-360-3 & 360 & 10.03 & 41.02 & 9.19 & 31.83 & 3.1 & 10 & $<0.2$ & 10 & 3.3 & 9.6 & N.R. & $<1.0$ \\
\hline IV $9202 A-540-1$ & 540 & 9.01 & 65.35 & 59.55 & 5.81 & 4.1 & 11 & 40 & 7.8 & $<1$ & 47 & $<0.2$ & 2.3 \\
\hline IV9202A-540-2 & 540 & 8.68 & 69.40 & 0.00 & 69.40 & 4.9 & 9.4 & 34 & 11 & $<1$ & 28 & $<0.2$ & 2.0 \\
\hline IV9202A-720-1 & 720 & 9.13 & 82.55 & 4.23 & 78.33 & 4.6 & 13 & 47 & 4.4 & $<0.5$ & 32 & N.R. & 2.5 \\
\hline IV9202A-720-2 & 720 & 8.91 & 80.50 & 2.83 & 77.67 & 5.4 & 17 & 61 & 8.4 & 1.0 & 30 & N.R. & 2.9 \\
\hline IV9165A-91-3 & 91 & 8.80 & 44.56 & 6.94 & 37.62 & 6.2 & 9.1 & 11 & 3.5 & $<1$ & 24 & $<0.2$ & 3.2 \\
\hline IV9165A-180-3 & 180 & 9.55 & 40.03 & 33.99 & 6.04 & 3.6 & 9.4 & 14 & 2.1 & $<1$ & 28 & $<0.3$ & 3.6 \\
\hline IV9165A-180-4 & 180 & 8.35 & 50.15 & 3.80 & 46.36 & 3.8 & 9.8 & 18 & 5.8 & $<1$ & 30 & $<0.3$ & 3.2 \\
\hline IV9165A-360-1 & 360 & 9.18 & 61.65 & 0.98 & 60.67 & 3.2 & 10 & 24 & 3.4 & $<3$ & 28 & $<0.3$ & 3.2 \\
\hline IV9165A-360-2 & 360 & 9.00 & 64.38 & 0.00 & 64.38 & 3.7 & 9.7 & 33 & 4.7 & $<3$ & 35 & $<0.3$ & 1.4 \\
\hline IV9165A-720-1 & 720 & 9.66 & 74.55 & 12.76 & 61.80 & 5.6 & 29 & 72 & 4.6 & 3.4 & 28 & $<0.2$ & 5.7 \\
\hline IV9165A-720-2 & 720 & 9.75 & 62.75 & 8.49 & 52.47 & 2.1 & 8.6 & 33 & 2.9 & 5.1 & 13 & 2.8 & 2.2 \\
\hline IV9131A-14-1 & 14 & 8.58 & 68.92 & 10.39 & 58.54 & 3.7 & 11 & 10 & 3.2 & $<0.5$ & 34 & $<0.5$ & 1.8 \\
\hline IV9131A-14-2 & 14 & $9.0-9$ & 52.66 & 2.93 & 49.73 & 4.1 & 11 & 12 & 3.2 & $<0.5$ & 39 & $<0.5$ & 1.9 \\
\hline IV9131A-28-1 & 28 & 9.33 & 51.92 & 2.21 & 49.71 & 3.7 & 9.6 & 10 & 3.0 & $<0.5$ & 27 & $<0.5$ & $<0.5$ \\
\hline IV $9131 \mathrm{~A}-28-2$ & 28 & 9.32 & 69.20 & 2.51 & 66.69 & \multicolumn{8}{|c|}{ Not Enough Solution for Analysis } \\
\hline IV9131A-56-1 & 56 & 9.27 & 46.48 & 1.95 & 44.53 & 3.8 & 11 & 11 & 7.3 & $<0.5$ & 39 & $<0.5$ & 1.9 \\
\hline IV9131A-56-2 & 56 & 9.28 & 62.82 & 1.32 & 61.50 & 3.6 & 12 & 12 & 8.1 & $<0.5$ & 44 & $<0.5$ & $<0.5$ \\
\hline IV9131A-56-3 & 56 & 9.80 & 79.60 & 11.48 & 68.12 & 4.6 & 15 & 13 & 1.2 & $<2$ & 51 & $<0.3$ & 6.1 \\
\hline IV9131A-56-4 & 56 & 9.40 & 67.05 & 5.40 & 61.65 & 3.3 & 12 & 11 & 2.1 & $<$ & 38 & $<0.3$ & 5.1 \\
\hline IV9131A-180-3 & 180 & 9.37 & 52.50 & 2.92 & 49.59 & 2.8 & 9.4 & 14 & 2.1 & $<1$ & 28 & $<0.3$ & 3.6 \\
\hline IV9131A-720-1 & 720 & 8.88 & 98.75 & 11.09 & 87.67 & 5.6 & 10 & 64 & 10 & $<1$ & 31 & $<0.2$ & 4.7 \\
\hline IV9131A-720-2 & 720 & 9.16 & 96.70 & 5.33 & 91.37 & 3.0 & 11 & 64 & 7.4 & 1.7 & 42 & 2.8 & 3.7 \\
\hline $\mathrm{EJ}-13$ & - & 8.1 & 22.44 & 0.75 & 21.69 & 2.8 & 8.6 & $<0.05$ & 8.2 & - & 21 & - & - \\
\hline
\end{tabular}

N.R. $=$ not able to resolve from adjacent $\mathrm{Cl}^{-}$peak . 

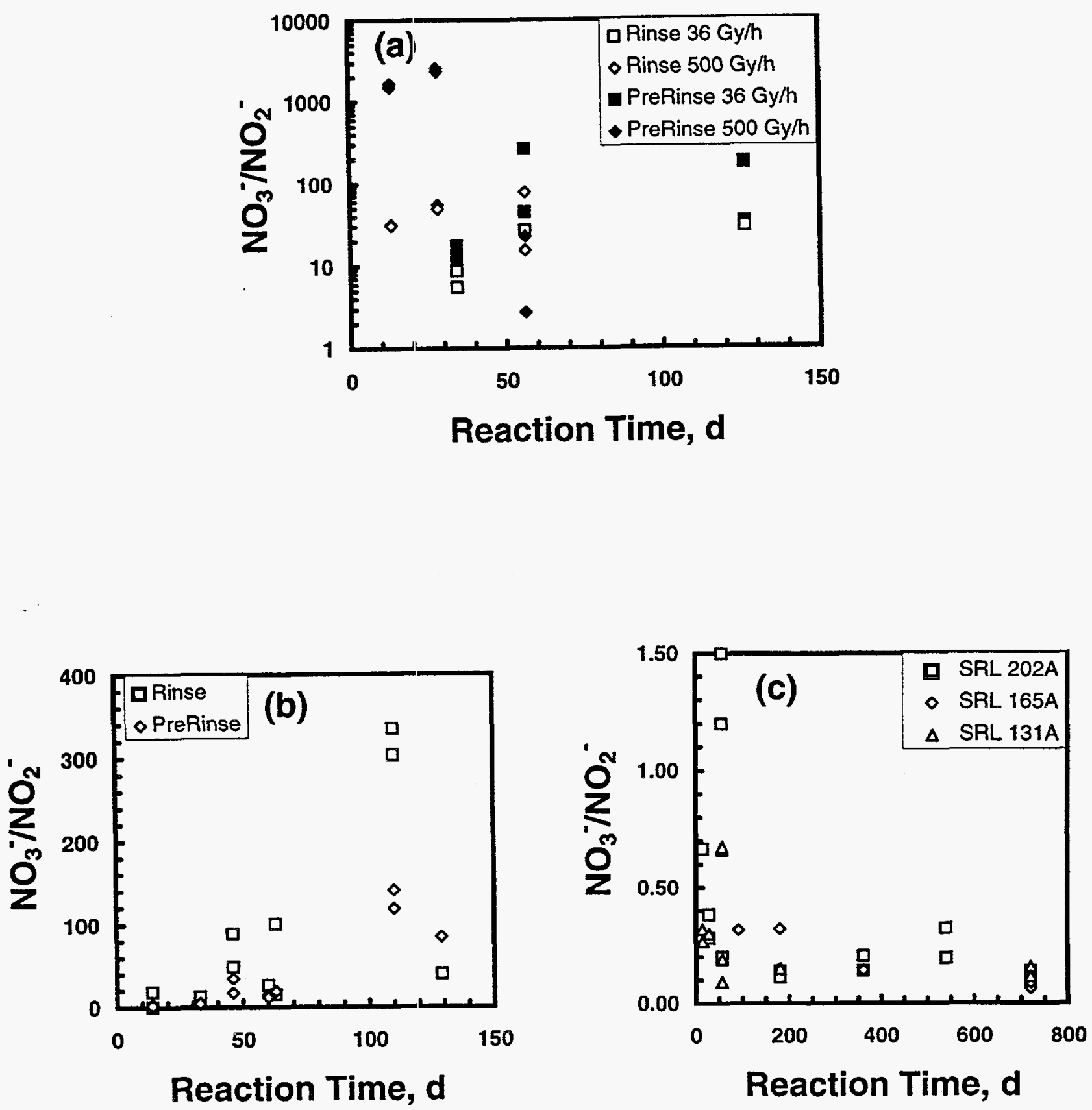

Fig. 14. Nitrate/Nitrite Ratios in Blank Irradiation Tests as a Function of Cumulative Exposure Time. (a) Blank gamma tests at dose rates of $\sim 35$ and $\sim 500 \mathrm{~Gy} / \mathrm{h}$ and $25^{\circ} \mathrm{C}$. (b) Blank alpha tests for both the rinse and prerinse solution fractions. Samples exposed to an ${ }^{241} \mathrm{Am}$-foil irradiation source at a dose rate of $\sim 26 \mathrm{~Gy} / \mathrm{h}$ and a temperature of $25^{\circ} \mathrm{C}$. (c) Batch leach tests at $340 \mathrm{~m}^{-1}$. Samples exposed to an external ${ }^{60} \mathrm{Co}$ irradiation source at a dose rate of $\sim 35 \mathrm{~Gy} / \mathrm{h}$ and $90^{\circ} \mathrm{C}$. 
Table 16. $\mathrm{NO}_{2}^{-}-\mathrm{NO}_{2}{ }^{-}$Ratios from Irradiation Tests

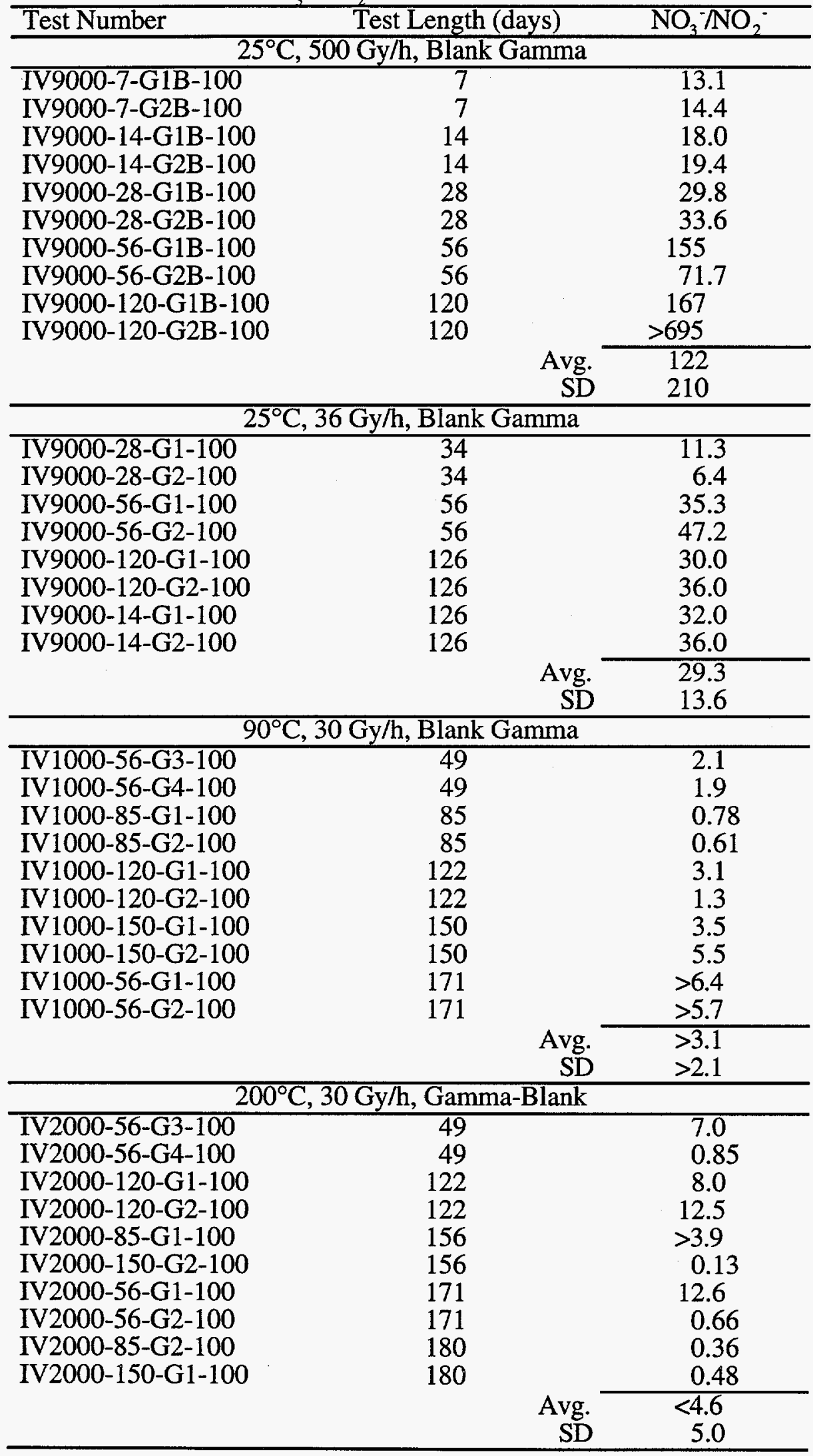


Table 16 - contd.

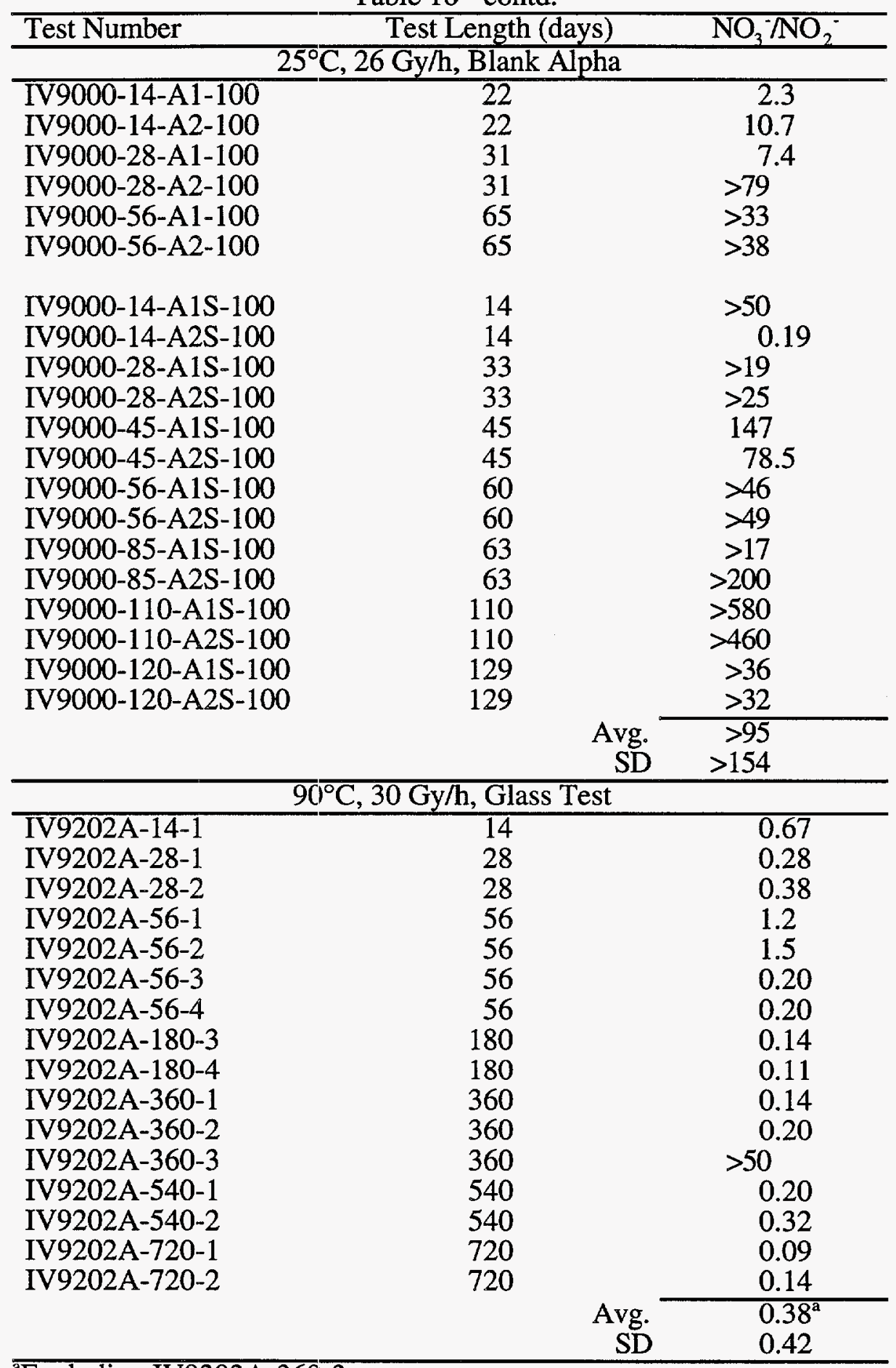

$\bar{a}$ Excluding IV9202A-360 -3. 
Table 16 - contd.

\begin{tabular}{|c|c|c|}
\hline Test Number & Test Length (days) & $\mathrm{NO}_{3}{ }^{-} \mathrm{NO}_{2}^{-}$ \\
\hline \multicolumn{3}{|c|}{$90^{\circ} \mathrm{C}, 30 \mathrm{~Gy} / \mathrm{h}$, Glass Test } \\
\hline IV9165A-91-3 & 91 & $\overline{0.32}$ \\
\hline IV9165A-180-3 & 180 & 0.15 \\
\hline IV9165A-180-4 & 180 & 0.32 \\
\hline IV9165A-360-1 & 360 & 0.14 \\
\hline IV9165A-360-2 & 360 & 0.14 \\
\hline IV9165A-720-1 & 720 & 0.06 \\
\hline IV9165A-720-2 & 720 & 0.09 \\
\hline & 0.17 \\
\hline & SD & 0.10 \\
\hline \multicolumn{3}{|c|}{$90^{\circ} \mathrm{C}, 30 \mathrm{~Gy} / \mathrm{h}$, Glass Test } \\
\hline IV9131A-14-1 & 14 & 0.32 \\
\hline IV9131A-14-2 & 14 & 0.27 \\
\hline IV9131A-28-1 & 28 & 0.30 \\
\hline IV9131A-28-2 & 28 & N.A. $b$ \\
\hline IV9131A-56-1 & 56 & 0.66 \\
\hline IV9131A-56-2 & 56 & 0.68 \\
\hline IV9131A-56-3 & 56 & 0.09 \\
\hline IV9131A-56-4 & 56 & 0.19 \\
\hline IV9131A-180-3 & 180 & 0.15 \\
\hline IV9131A-720-1 & 720 & 0.16 \\
\hline IV $9131 \mathrm{~A}-720-2$ & 720 & 0.12 \\
\hline \multicolumn{2}{|r|}{ Avg. } & $\begin{array}{l}0.29 \\
021\end{array}$ \\
\hline
\end{tabular}

${ }^{5}$ N.A. = not analyzed. 


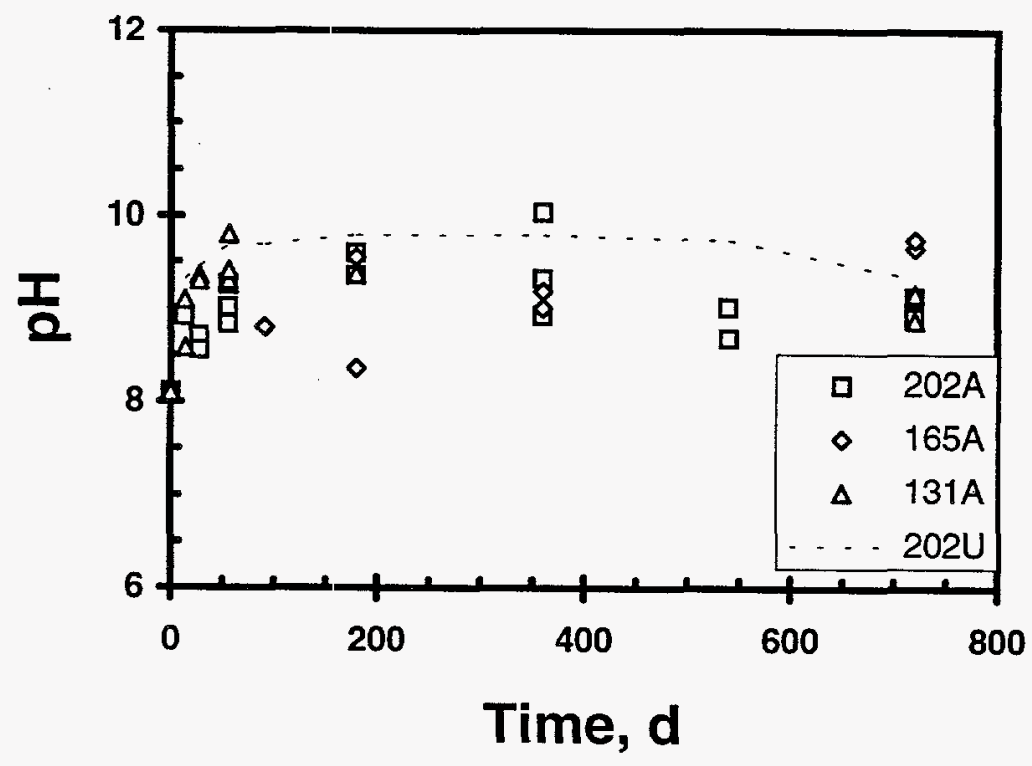

Fig. 15. Solution $\mathrm{pH}$ Trends from $340 \mathrm{~m}^{-1}$ Batch Tests Exposed to an External ${ }^{60} \mathrm{Co}$ Gamma Source of $\sim 35 \mathrm{~Gy} / \mathrm{h}$. Data are presented for individual solution analyses of tests conducted with SRL 202A, SRL 165A, and SRL 131A glasses. Averaged results from nonirradiated tests with SRL 202U glass [34] are included for reference (dotted lines). The $\mathrm{pH}$ of the initial EJ-13 leachant is 8.2

The SRL 131A samples display a similar $\mathrm{pH}$ trend as that of the SRL 202A samples, with an early increase in $\mathrm{pH}$ followed by a decline with the long-term samples. With these tests, the $\mathrm{pH}$ values rapidly increase from a starting value of 8.1 to a value of $\sim 9.4$ within the first 28 to 56 days of testing (Fig. 15 and Table 15). An isolated sample shows a pH rise to 9.8 after 56 days of testing. A single analysis at 180 days indicates a $\mathrm{pH}$ of 9.4 , while the 720-day results display a $\mathrm{pH}$ decrease to a value of 9.0 . As with the SRL 165A tests, no comparative analyses are available for the nonirradiated SRL 165U glasses.

\section{Carbon Concentrations}

Results of carbon analyses for the irradiated leachate solutions in contact with the glass samples are presented in Table 15. Although there is a considerable amount of data scatter in the tests, the concentrations of TC and IC to appear to rise with increasing reaction time. Most TC and IC values are also enriched relative to the starting EJ-13 solution, whereas OC concentrations are typically depleted relative to the leachant. Formate and oxalate do not display any consistent trends as a function of radiation exposure. 


\section{Cation Concentrations (Nonradionuclides)}

As-received analytical results, total $\mu \mathrm{g}$ element release, solution volumes, and sample aliquot data are presented for the nonradioactive cations in Table B.2, while glass monolith surface areas and monolith weights are presented in Table B.3. Dilution-corrected cation release data are presented in Table 17. These concentrations have been determined by including correction factors for aliquot removal. Thus, these results represent the fluid concentration resulting from the original cation concentrations present in the EJ-13 leachant, plus the contribution of various cations released into solution from the glass. The cation concentrations are also displayed in Fig. 16. (The composition of the starting EJ-13 solution is given in Tables 6 and 17.) Results are also presented for averaged normalized release trends of nonirradiated and nontransuranic-doped SRL 202U glass samples from the study of Ebert [34] (dotted lines in Fig. 16). These latter tests have been conducted under identical conditions as the present samples, except that no external gamma radiation was used.

Boron is often considered a good indicator of borosilicate glass corrosion because of its high solubility in aqueous solutions and relatively low concentration in most natural groundwaters. Boron released from the glass during corrosion processes will therefore generally not be incorporated into secondary alteration products that form on the altered glass surface. Leachate concentrations of boron increase progressively for the SRL 202A glass test throughout the entire 720 days of sampling (Fig. 16a and Table 17). Initial increases are relatively rapid, reaching approximately $18 \mathrm{ppm}$ by 180 days, and a single analysis of $44 \mathrm{ppm}$ at 360 days. The 540- and 720-day results display a gradual concentration increase over the 180-day tests, but are depleted relative to the 360 -day results. Boron concentrations from the nonirradiated SRL 202U glass tests display the same overall trend as the irradiated SRL 202A samples, with solution concentrations being slightly lower in the former tests. Tests with SRL 131U glasses display the same rapid increase in boron concentrations during the first 180 days, and a subsequent decrease up to 720 days, that characterized the SRL 202A samples. Results from the tests with SRL 165A glass display the lowest concentrations of boron for the first 360 days, but then display a large increase in boron concentrations between 360 and 720 days. This trend differs from that of the SRL 202A and SRL 131A tests, which displayed only minor concentration increases after 180 days. The final boron concentrations from the 720-day tests with the SRL 165A glass are slightly higher than those of the SRL 202A glass, despite the lower overall boron content of the SRL 165 glass (Table 4).

In tests with SRL 202A glass, the concentrations of alkali metals ( $\mathrm{Li}, \mathrm{Na}$, and $\mathrm{K}$ ) rapidly increased during the first 180 days, then gradually increased up to the 720 -day values (Fig. 16b-d and Table 17). Element concentrations from tests with SRL 202A glasses are also similar to those of the SRL $202 U$ glasses, except that the concentrations from the former are higher with $\mathrm{Li}$ and $\mathrm{Na}$ for 720-day samples, and higher with potassium for tests between 360 and 720 days. The SRL 131A samples also display initially rapid concentration increases of alkali metals, especially during the first 56 days. Thereafter, the rate of increase declines, although the concentrations still increase more rapidly than they did for the SRL 202A glass. With the SRL 165A samples, $\mathrm{Li}$ and $\mathrm{Na}$ concentrations initially increase relatively rapidly, level off to a slow increase between 180 and 360 days, and then increase rapidly up to the 720 days. This pattern mirrors the concentration levels of boron release. The concentration levels of potassium for SRL 165A, however, are markedly different from those of the other alkali metals. The potassium concentration stays at or below the EJ-13 leachant level throughout the tests. Potassium concentrations also show an overall decrease during the course of the tests, although this decrease is not uniform over the testing period. 
Table 17. Dilution-Corrected Cation Results for $340 \mathrm{~m}^{-1}$ Batch Leach Tests. All concentrations in ppm. ${ }^{\mathrm{a}}$

\begin{tabular}{|c|c|c|c|c|c|c|c|c|c|c|c|}
\hline Test Number & $\begin{array}{l}\begin{array}{l}\text { Length } \\
\text { (days) }\end{array}\end{array}$ & $\mathrm{Al}$ & B & $\mathrm{Ba}$ & $\mathrm{Ca}$ & $\mathrm{Cr}$ & $\mathrm{Cu}$ & $\mathrm{Fe}$ & $\mathrm{K}$ & $\mathrm{Li}$ & $\mathrm{Mg}$ \\
\hline IV9202A-14-1 & 14 & $\overline{1.33}$ & 6.05 & $<0.24$ & 1.09 & $<0.24$ & $\overline{0.36}$ & 2.30 & 13.4 & 6.9 & 0.31 \\
\hline IV9202A-28-1 & 28 & 1.51 & 7.03 & $<0.25$ & 1.13 & $<0.25$ & 0.50 & 3.01 & 13.8 & 7.9 & 0.40 \\
\hline IV $9202 \mathrm{~A}-28-2$ & 28 & 1.27 & 6.86 & $<0.25$ & 1.52 & $<0.25$ & 0.25 & 2.92 & 15.4 & 8.1 & 0.36 \\
\hline IV9202A-56-1 & 56 & $<1.34$ & 9.26 & $<0.27$ & 0.94 & $<0.27$ & 0.67 & 3.76 & 16.8 & 10.1 & 0.48 \\
\hline IV9202A-56-2 & 56 & $<1.28$ & 12.3 & $<0.26$ & 0.78 & $<0.26$ & 1.04 & 5.39 & 17.7 & 10.9 & 0.64 \\
\hline IV9202A-56-3 & 56 & 2.66 & 4.0 & 0.24 & 3.15 & 5.16 & 74.4 & 34.1 & 7.0 & 4.2 & 1.45 \\
\hline IV9202A-56-4 & 56 & $<1.16$ & 6.4 & $<0.23$ & 1.63 & $<0.23$ & 0.23 & 1.86 & 13.6 & 7.9 & 0.35 \\
\hline IV9202A-180-3 & 180 & $<1.60$ & 20.5 & $<0.32$ & 1.28 & $<0.32$ & 0.80 & 7.51 & 24.6 & 17.7 & 0.80 \\
\hline IV9202A-180-4 & 180 & 2.96 & 14.5 & $<0.27$ & 1.88 & $<0.27$ & 1.61 & 14.5 & 21.6 & 13.3 & 1.61 \\
\hline IV9202A-360-1 & 360 & 4.56 & 17.1 & $<0.27$ & 2.69 & 0.81 & 2.95 & 24.6 & 26.0 & 15.8 & 2.69 \\
\hline IV9202A-360-2 & 360 & 2.88 & 19.3 & $<0.23$ & 1.38 & 1.15 & 0.46 & 4.61 & 24.6 & 15.1 & 0.58 \\
\hline IV9202A-360-3 & 360 & 2.24 & 44.1 & $<0.28$ & 3.22 & $<0.42$ & $<0.28$ & 4.06 & 30.2 & 19.5 & 0.70 \\
\hline IV $9202 A-540-1$ & 540 & $<1.33$ & 21.3 & $<0.27$ & 0.67 & 3.46 & 0.53 & 6.13 & 25.6 & 18.9 & $<0.13$ \\
\hline IV9202A-540-2 & 540 & $<1.37$ & 18.3 & $<0.27$ & 10.5 & 0.68 & $<0.27$ & 2.32 & 26.1 & 17.8 & 0.27 \\
\hline IV9202A-720-1 & 720 & 4.95 & 24.8 & $<0.27$ & 2.3 & 1.07 & 2.41 & 20.0 & 32.8 & 21.8 & 2.14 \\
\hline IV9202A-720-2 & 720 & 1.47 & 21.8 & $<0.27$ & 1.5 & 1.07 & 0.27 & 3.21 & 28.9 & 19.5 & 0.40 \\
\hline IV9165A-91-3 & $\overline{91}$ & $<1.21$ & 4.97 & $<0.24$ & 1.33 & $<0.24$ & 0.24 & 2.31 & 7.64 & 7.64 & 0.24 \\
\hline IV9165A-180-3 & 180 & $<1.35$ & 10.6 & $<0.27$ & 1.61 & 0.27 & $<0.27$ & 1.08 & 4.7 & 13.6 & $<0.13$ \\
\hline IV9165A-180-4 & 180 & $<1.18$ & 6.2 & $<0.24$ & 2.23 & $<0.24$ & $<0.24$ & 0.71 & 5.8 & 11.2 & $<0.12$ \\
\hline IV9165A-360-1 & 360 & 1.34 & 7.7 & $<0.27$ & 1.34 & 0.27 & $<0.27$ & 0.27 & 7.4 & 13.6 & $<0.13$ \\
\hline IV $9165 A-360-2$ & 360 & 1.15 & 9.6 & $<0.23$ & 1.84 & 0.58 & 0.81 & 4.96 & 7.0 & 14.5 & 0.35 \\
\hline IV9165A-720-1 & 720 & 2.64 & 27.3 & $<0.09$ & 2.90 & 2.37 & 0.26 & 4.66 & 1.2 & 30.0 & 0.44 \\
\hline IV9165A-720-2 & 720 & 3.52 & 31.7 & $<0.09$ & 2.73 & 0.35 & 0.18 & 4.84 & 1.6 & 24.4 & 0.44 \\
\hline IV9131A-14-1 & 14 & 1.35 & 17.6 & $<0.25$ & $<0.12$ & $<0.25$ & $<0.25$ & 3.19 & 21.4 & 9.0 & $\overline{0.63}$ \\
\hline IV $9131 \mathrm{~A}-14-2$ & 14 & 1.70 & 18.2 & $<0.24$ & 0.12 & $<0.24$ & 0.61 & 4.00 & 21.5 & 9.2 & 0.74 \\
\hline IV9131A-28-1 & 28 & 1.52 & 7.1 & $<0.25$ & 1.14 & $<0.25$ & 0.51 & 3.05 & 14.0 & 8.0 & 0.41 \\
\hline IV $9131 \mathrm{~A}-28-2$ & 28 & 1.90 & 25.4 & $<0.25$ & 0.38 & $<0.25$ & 0.51 & 6.60 & 26.5 & 11.8 & 0.94 \\
\hline IV9131 A-56-1 & 56 & $<1.45$ & 27.5 & $<0.29$ & $<0.29$ & $<0.29$ & 0.39 & 6.98 & 27.8 & 12.9 & 0.96 \\
\hline IV $9131 \mathrm{~A}-56-2$ & 56 & 1.71 & 27.2 & $<0.26$ & $<0.26$ & $<0.26$ & 0.47 & 8.31 & 25.8 & 12.0 & 1.16 \\
\hline IV9131A-56-3 & 56 & $<1.50$ & 33.0 & $<0.30$ & 0.45 & $<0.30$ & $<0.30$ & 2.85 & 32.7 & 15.5 & 0.60 \\
\hline IV $9131 \mathrm{~A}-56-4$ & 56 & 2.04 & 26.9 & $<0.27$ & 0.54 & 0.41 & $<0.27$ & 7.47 & 27.2 & 12.4 & 1.36 \\
\hline IV9131A-180-3 & 180 & 2.18 & 38.9 & 0.27 & 0.55 & 0.55 & 0.55 & 12.1 & 33.4 & 16.8 & 1.50 \\
\hline IV9131A-720-1 & 720 & 3.45 & 54 & $<0.14$ & 3.17 & 2.90 & 0.3 & 5.24 & 44 & 25 & 0.97 \\
\hline IV9131A-720 & 720 & 7.59 & 58 & 0.14 & 4.69 & 3.45 & 0.4 & 31.6 & 49 & 26 & 4.83 \\
\hline EJ-13(4/18/90) & - & 1.07 & 0.16 & $\overline{-}$ & 5.08 & - & $\overline{-}$ & $<0.01$ & $\overline{7.37}$ & 0.05 & 0.39 \\
\hline
\end{tabular}


Table 17 - contd.

\begin{tabular}{|c|c|c|c|c|c|c|c|c|c|c|}
\hline Test Number & $\overline{\mathrm{Mn}}$ & $\overline{\mathrm{Na}}$ & $\mathrm{Ni}$ & $\overline{\mathrm{Si}}$ & $\overline{\mathrm{Sr}}$ & Th & $\overline{\mathrm{U}}$ & $\overline{\mathrm{Ti}}$ & $\overline{\mathrm{Zn}}$ & $\overline{\mathrm{Zr}}$ \\
\hline IV9202A-14-1 & 0.35 & 68.9 & $<0 . \overline{36}$ & 63.9 & $<0.12$ & $<1.21$ & 1.83 & $<0.12$ & $<0.12$ & $<0.24$ \\
\hline IV9202A-28-1 & 0.35 & 72.7 & $<0.38$ & 76.6 & $<0.13$ & $<1.26$ & 1.32 & $<0.13$ & $<0.13$ & $<0.25$ \\
\hline IV $9202 \mathrm{~A}-28-2$ & 0.42 & 73.6 & $<0.38$ & 68.9 & $<0.13$ & $<1.27$ & 4.14 & $<0.13$ & $<0.13$ & $<0.25$ \\
\hline IV9202A-56-1 & 0.55 & 84.6 & $<0.40$ & 82.3 & $<0.13$ & $<1.34$ & 0.97 & $<0.13$. & 0.31 & $<0.27$ \\
\hline IV9202A-56-2 & 0.83 & 82.9 & 0.51 & 84.7 & $<0.13$ & $<1.28$ & 1.01 & $<0.13$ & 0.26 & $<0.26$ \\
\hline IV9202A-56-3 & 5.33 & 35 & 2.82 & 47.1 & $<0.08$ & $<0.40$ & 0.86 & 0.32 & 5.81 & $<0.40$ \\
\hline IV9202A-56-4 & 0.23 & 74 & $<0.35$ & 64.9 & $<0.12$ & $<0.58$ & 1.24 & $<0.23$ & $<0.23$ & $<0.58$ \\
\hline IV9202A-180-3 & 1.28 & 117 & $<0.48$ & 113 & $<0.08$ & $<0.08$ & 1.85 & $<0.32$ & $<0.32$ & $<0.80$ \\
\hline IV9202A-180-4 & 2.15 & 94 & 0.67 & 101 & $<0.07$ & $<0.67$ & 1.20 & 0.40 & $<0.27$ & $<0.67$ \\
\hline IV9202A-360-1 & 3.49 & $<0.7$ & 1.07 & 116 & $<0.07$ & $<0.67$ & 1.52 & 0.67 & $<0.27$ & $<0.67$ \\
\hline IV9202A-360-2 & 0.58 & 95 & 0.69 & 71.9 & $<0.06$ & $<0.58$ & 1.27 & 0.23 & $<0.23$ & $<0.58$ \\
\hline IV9202A-360-3 & 0.98 & 127 & $<0.42$ & 91.0 & $<0.14$ & $<0.70$ & 1.92 & $<0.28$ & 0.28 & $<0.70$ \\
\hline IV9202A-540-1 & 0.27 & 111 & 0.67 & 80 & $<0.07$ & $<0.67$ & 1.95 & $<0.27$ & $<0.27$ & $<0.67$ \\
\hline IV9202A-540-2 & 0.27 & 110 & $<0.41$ & 75 & $<0.07$ & $<0.68$ & 1.44 & $<0.27$ & 1.09 & $<0.68$ \\
\hline IV9202A-720-1 & 2.95 & 127 & 1.21 & 123 & 0.04 & $<0.67$ & 1.65 & 0.67 & $<0.27$ & $<0.67$ \\
\hline IV9202A-720-2 & 0.40 & 121 & 0.53 & 80 & 0.03 & $<0.67$ & 1.71 & $<0.27$ & $<0.27$ & $<0.67$ \\
\hline IV9165A-91-3 & 0.24 & 70 & $<0.36$ & 55 & $<0.06$ & $<0.61$ & 1.59 & $<0.24$ & $<0.24$ & $<0.61$ \\
\hline IV9165A-180-3 & 0.27 & 92.1 & $<0.40$ & 73.6 & $<0.07$ & $<0.67$ & 2.56 & $<0.27$ & $<0.27$ & $<0.67$ \\
\hline IV $9165 \mathrm{~A}-180-4$ & $<0.12$ & 85.4 & $<0.35$ & 63.9 & $<0.06$ & $<0.59$ & 1.70 & $<0.24$ & $<0.24$ & $<0.59$ \\
\hline IV9165A-360-1 & $<0.13$ & 88.1 & $<0.40$ & 67.9 & $<0.07$ & $<0.67$ & 1.97 & $<0.27$ & $<0.27$ & $<0.67$ \\
\hline IV9165A-360-2 & 0.92 & 94.6 & $<0.35$ & 85.3 & $<0.06$ & $<0.58$ & 2.64 & $<0.23$ & $<0.23$ & $<0.58$ \\
\hline IV9165A-720-1 & 1.85 & 142 & 0.53 & 107 & N.A. & $<0.09$ & 0.46 & 0.09 & 0.18 & 0.26 \\
\hline IV9165A-720-2 & 2.20 & 131 & 0.62 & 112 & N.A. & $<0.09$ & 0.49 & 0.09 & 0.18 & 0.26 \\
\hline IV9131A-14-1 & 0.47 & 101 & $<0.37$ & 80 & $<0.12$ & $<1.23$ & 4.88 & $<0.12$ & $<0.12$ & $<0.25$ \\
\hline IV9131A-14-2 & 0.78 & 103 & $<0.36$ & 83 & $<0.12$ & $<1.21$ & 5.00 & $<0.12$ & $<0.12$ & $<0.24$ \\
\hline IV9131A-28-1 & 0.36 & 74 & $<0.38$ & 77 & $<0.13$ & $<1.27$ & 2.93 & $<0.13$ & $<0.13$ & $<0.25$ \\
\hline IV9131A-28-2 & 1.07 & 121 & $<0.38$ & 96 & $<0.13$ & $<1.27$ & 4.14 & $<0.13$ & $<0.13$ & $<0.25$ \\
\hline IV9131A-56-1 & 1.12 & 101 & $<0.44$ & 103 & $<0.15$ & $<1.45$ & 3.50 & $<0.15$ & $<0.15$ & $<0.29$ \\
\hline IV9131A-56-2 & 1.38 & 124 & 0.53 & 104 & $<0.13$ & $<1.32$ & 3.05 & $<0.13$ & 0.16 & $<0.26$ \\
\hline IV9131A-56-3 & 0.45 & 162 & $<0.45$ & 119 & $<0.08$ & $<0.75$ & 3.29 & $<0.30$ & $<0.30$ & $<0.75$ \\
\hline IV $9131 \mathrm{~A}-56-4$ & 1.22 & 127 & 0.54 & 95 & $<0.07$ & $<0.68$ & 4.27 & $<0.27$ & $<0.27$ & $<0.68$ \\
\hline IV9131A-180-3 & 1.91 & 160 & 0.55 & 109 & $<0.07$ & $<0.68$ & 3.58 & $<0.27$ & $<0.27$ & $<0.68$ \\
\hline IV9131A-720-1 & 1.52 & 235 & 0.97 & 122 & N.A. & $<0.14$ & 4.54 & 0.28 & 0.28 & 0.14 \\
\hline IV9131A-720 & 6.62 & 239 & 4.14 & 192 & N.A. & $<0.14$ & 4.32 & 0.69 & 0.55 & 0.28 \\
\hline EJ-13 (4/18/90) & $<0.01$ & 53.9 & $<0 . \overline{03}$ & 45.8 & 0.02 & $\overline{-}$ & 0.00 & $\overline{-}$ & $\overline{-}$ & - \\
\hline
\end{tabular}

${ }^{\mathrm{a}}$ All concentrations corrected for experimental dilutions. N.A. $=$ Not analyzed. Concentration $=$ Leachate concentration $\mu \mathrm{g} / \mathrm{ml}((\mathrm{ml}$ leachant $+\mathrm{ml}$ rinse solution added)/ml leachant). 

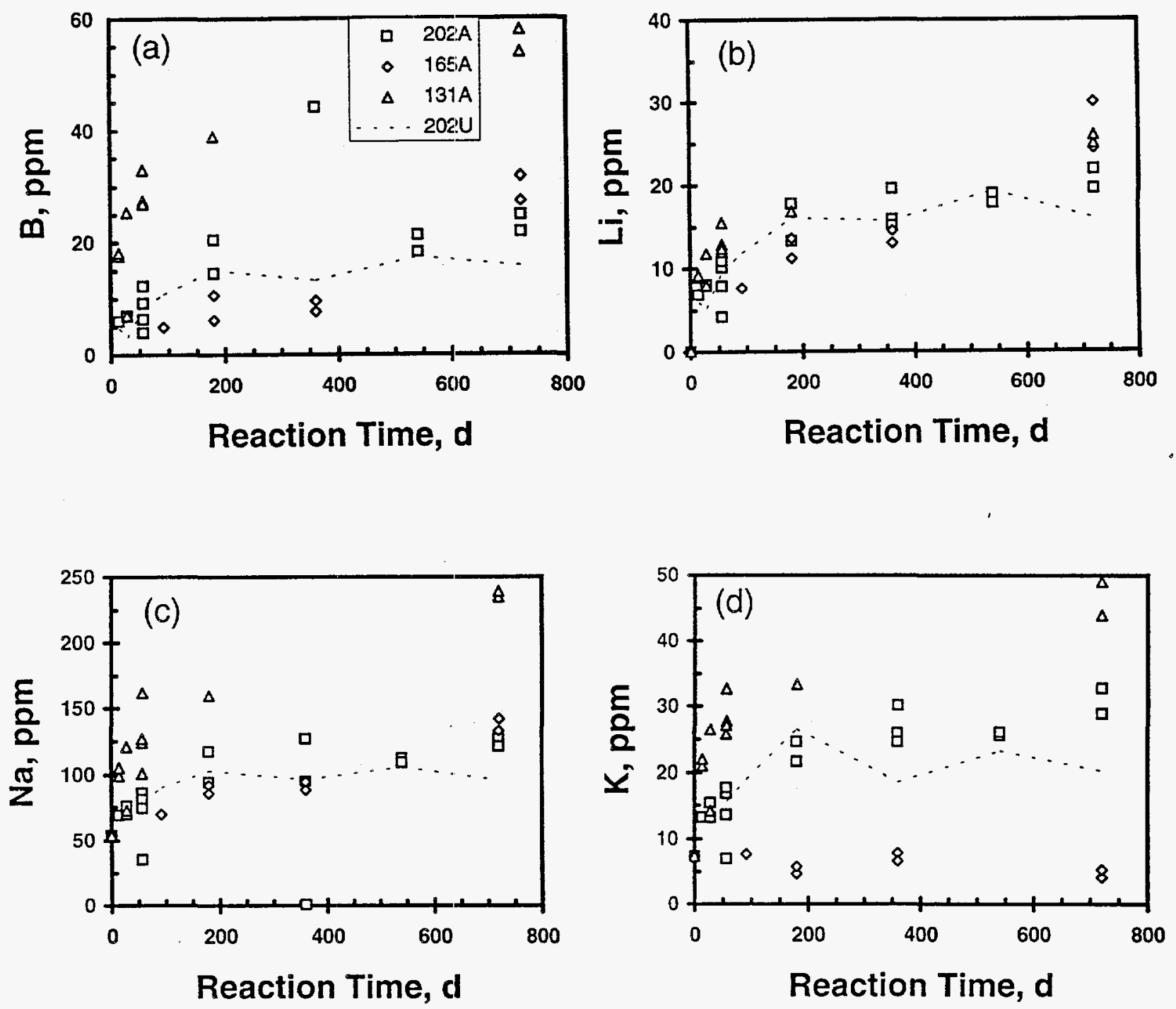

Fig. 16. Concentration Trends from $340 \mathrm{~m}^{-1}$ Batch Tests Exposed to External ${ }^{60} \mathrm{Co}$ Gamma Source of $\sim 35 \mathrm{~Gy} / \mathrm{h}$. Data are presented for individual solution analyses of tests conducted with SRL 202A, SRL 165A, and SRL 131A glasses. Averaged results from nonirradiated tests with SRL 202U glass [34] are included for reference (dotted lines). The concentration of elements in the EJ-13 leachant is indicated with the symbol intersection on the $\mathrm{Y}$-axis of each respective plot. All values are in ppm. (a) boron, (b) lithium, (c) sodium, (d) potassium, (e) magnesium, (f) calcium, (g) silicon, and (h) uranium. 

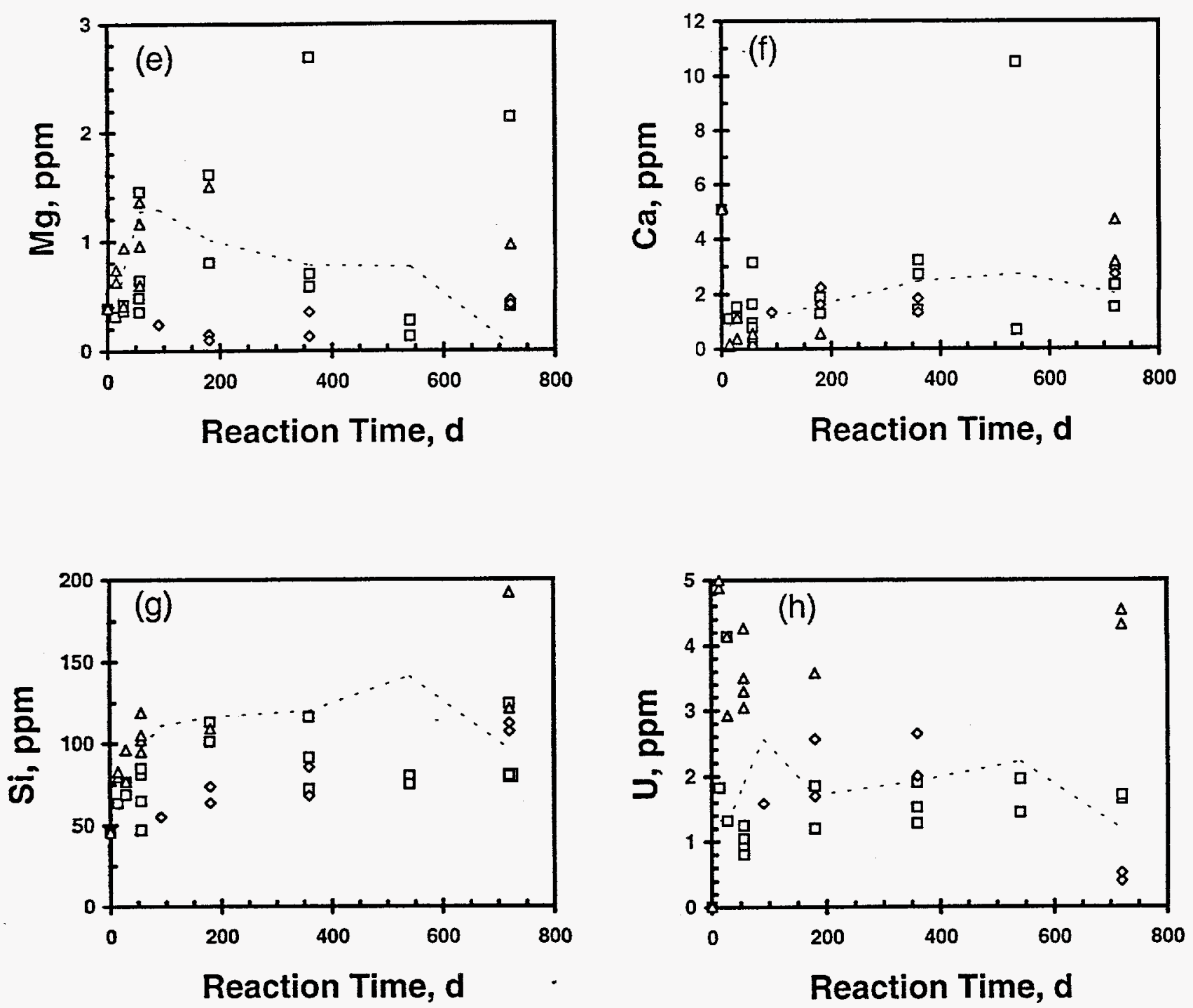
Alkaline earth element (Mg and $\mathrm{Ca}$ ) trends for tests with SRL 202A and SRL 202U glasses show initial concentration increases followed by decreasing concentrations (Figs. 16e-f and Table 17). With magnesium, some of the longer term tests display considerable data scatter. Overall trends suggest a peak in average concentrations occurring at 180 days, with a decline in concentrations thereafter; however, anomalously high magnesium concentrations are displayed for single samples terminated at 360 and 720 days. The magnesium concentrations of the SRL 202U sample are generally not distinguishable from those of its irradiated counterpart, except during the later testing intervals. For example, the 540-day results display lower concentrations for the SRL 202A samples, while the 720-day results indicate that the SRL 202U concentrations are lower. Magnesium levels also peak after 180 days in tests with SRL 131A glasses and then decline thereafter, while tests with SRL 165A glasses display relatively low concentrations throughout the testing period, with only a slight concentration increase displayed between 180 and 720 days.

Calcium concentrations from tests with SRL 202A and SRL 165A glasses show relatively little increase throughout the tests; however, a single SRL 202A sample at 540 days displays an anomalously high calcium concentration level. Calcium concentrations are also indistinguishable between tests of irradiated SRL 202A and nonirradiated SRL 202U glasses. Calcium concentrations from tests with the SRL 131A glass increase continuously over the course of the tests.

Silicon concentrations: also increase during the first 180 days of testing with the SRL 202A glasses and then remain relatively constant thereafter (Fig. 16g and Table 17). Silicon concentrations from the unirradiated tests with SRL 202U glasses are generally higher than those of the irradiated SRL 202A for all periods except 720 days, where the results from the two sets of tests are similar. Silicon concentrations rapidly increase during the first 56 days of testing with SRL 131A glasses and then gradually increase thereafter. Concentrations of silicon are also generally highest in tests with the SRL 131A glass when compared to the other two glass types, despite the fact that the SRL 131 glass has the lowest overall $\mathrm{SiO}_{2}$ content of the three glass types (Table 4). The SRL 165A glasses display a gradual increase in concentration throughout the entire 720 days of testing. Concentrations levels are also generally lower for the SRL 165A glasses relative to the other two glass compositions until 720 days, when the SRL 202A and SRL 165A compositions are similar.

Uranium results from the SRL 202A and SRL 131A glass tests fluctuated during the early stages of the tests, with decreasing concentrations between 14 and 56 days, and then a gradual increase throughout the remainder of the testing interval (Fig. 16h and Table 17). Uranium concentra-tions in irradiated tests with SRL 202A glass are generally lower than those in nonirradiated tests with SRL 202U glass. The 720-day tests, however, display a reversal in the trend, with the nonirradiated samples displaying the lowest concentrations. The relative differences in uranium concentrations noted between the irradiated and nonirradiated tests parallel the previously described trends for silicon. The SRL 165A leachate samples display a consistent increase in concentration up to 360 days, and then a strong decrease in the 720 -day results.

\section{Normalized Elemental Mass Loss (Nonradionuclides)}

The normalized elemental mass loss of species $i, N L(i)$, is the mass of element $i$ as measured in solution normalized to the geometric surface area of the glass samples and the weight fraction of element $i$ in the glass. The NL(i) values are also corrected to account for the mass of species i present in the original $2.0 \mathrm{~mL}$ of EJ-13 leachant. This normalized value thus allows for a direct comparison of the release of elements for different glasses or a comparison of release patterns from tests conducted at slightly different $\mathrm{S} / \mathrm{V}$ ratios. Calculated NL(i) values for selected cation components are presented in Table 18, while NL(i) values are plotted as a 
Table 18. Normalized Release $\left(\mathrm{g} / \mathrm{m}^{2}\right)$ of Elements for $340 \mathrm{~m}^{-1}$ Batch Leach Tests

\begin{tabular}{|c|c|c|c|c|c|c|c|c|}
\hline \multirow[b]{2}{*}{ Element } & \multicolumn{8}{|c|}{ IV9202A- } \\
\hline & $14-1$ & $28-1$ & $28-2$ & $56-1$ & $56-2$ & $56-3$ & $56-4$ & $180-3$ \\
\hline NL(Si) & 0.2271 & 0.3813 & 0.2929 & 0.4081 & 0.4805 & -0.0610 & 0.2180 & 0.5920 \\
\hline NL(Mg) & -0.0314 & 0.0000 & -0.0167 & 0.0222 & 0.0877 & 0.3287 & -0.0219 & 0.0937 \\
\hline $\mathrm{NL}(\mathrm{Ca})$ & -1.4798 & -1.4649 & -1.3361 & -1.5477 & -1.5709 & -0.9034 & -1.2941 & -1.4863 \\
\hline $\mathrm{NL}(\mathrm{Na})$ & 0.6735 & 0.8280 & 0.8779 & 1.1872 & 1.2633 & -1.0188 & 0.8196 & 1.8687 \\
\hline NL(K) & 0.5896 & 0.6171 & 0.7750 & 0.8298 & 0.9779 & -0.1061 & 0.5636 & 1.2307 \\
\hline NL(Mn) & 0.0604 & 0.0603 & 0.0728 & 0.0907 & 0.1419 & 0.8192 & 0.0384 & 0.1804 \\
\hline $\mathrm{NL}(\mathrm{Li})$ & 1.0319 & 1.1728 & 1.2254 & 1.4320 & 1.6113 & 0.5556 & 1.1386 & 2.1738 \\
\hline NL(B) & 0.7001 & 0.8116 & 0.7987 & 1.0281 & 1.4244 & 0.4073 & 0.7152 & 1.9645 \\
\hline \multirow[t]{3}{*}{$\mathrm{NL}(\mathrm{U})$} & 0.5571 & 0.3980 & 1.2656 & 0.2802 & 0.3045 & 0.2343 & 0.3639 & 0.4605 \\
\hline & \multicolumn{8}{|c|}{ IV9202A- } \\
\hline & $180-4$ & $360-1$ & $360-2$ & $360-3$ & $540-1$ & $540-2$ & $720-1$ & $720-2$ \\
\hline NL(Si) & 0.6555 & 0.6992 & 0.3055 & 0.5457 & 0.4174 & 0.3753 & 0.9624 & 0.4357 \\
\hline NL(Mg) & 0.4237 & 0.7116 & 0.0592 & 0.1057 & -0.0989 & -0.0461 & 0.6262 & 0.0000 \\
\hline $\mathrm{NL}(\mathrm{Ca})$ & -1.2235 & -1.0574 & -1.3947 & -0.7794 & -1.6164 & 1.7844 & -1.0668 & -1.3628 \\
\hline $\mathrm{NL}(\mathrm{Na})$ & 1.6448 & -2.3819 & 1.7269 & 3.1182 & 2.4384 & 2.5232 & 3.1501 & 2.9849 \\
\hline $\mathrm{NL}(\mathrm{K})$ & 1.2960 & 1.4642 & 1.6010 & 2.1378 & 1.6836 & 1.7917 & 2.3830 & 2.0422 \\
\hline NL(Mn) & 0.3561 & 0.5223 & 0.0970 & 0.1656 & 0.0454 & 0.0475 & 0.4965 & 0.0694 \\
\hline NL(Li) & 1.9167 & 2.0595 & 2.2137 & 2.8600 & 2.7577 & 2.7027 & 3.1874 & 2.9443 \\
\hline NL(B) & 1.6367 & 1.7386 & 2.2189 & 5.1222 & 2.4607 & 2.1812 & 2.8573 & 2.5519 \\
\hline \multirow[t]{3}{*}{ NL(U) } & 0.3510 & 0.3994 & 0.3771 & 0.5718 & 0.5784 & 0.4396 & 0.4894 & 0.5200 \\
\hline & \multicolumn{8}{|c|}{ IV9165A- } \\
\hline & $91-3$ & $180-3$ & $180-4$ & $360-1$ & $360-2$ & $720-1$ & $720-2$ & \\
\hline $\mathrm{NL}(\mathrm{Si})$ & 0.0984 & 0.2446 & 0.2105 & 0.2164 & 0.4540 & 0.7044 & 0.7457 & \\
\hline NL(Mg) & -0.1144 & -0.1956 & -0.1970 & -0.1899 & -0.0426 & 0.0245 & 0.0209 & \\
\hline $\mathrm{NL}(\mathrm{Ca})$ & -1.0573 & -0.9940 & -0.8063 & -1.0506 & -0.9222 & -0.6449 & -0.6873 & \\
\hline NL(Na) & 0.5818 & 1.0970 & 1.1600 & 1.0864 & 1.4493 & 3.1682 & 2.7063 & \\
\hline $\mathrm{NL}(\mathrm{K})$ & 0.3950 & -4.6556 & -2.4107 & -0.4665 & -0.5497 & -9.4552 & -8.8378 & \\
\hline NL(Mn) & 0.0409 & 0.0408 & 0.0201 & 0.0218 & 0.1543 & 0.3045 & 0.3576 & \\
\hline $\mathrm{NL}(\mathrm{Li})$ & 1.1607 & 1.8601 & 1.6908 & 1.8732 & 2.1918 & 4.4783 & 3.5826 & \\
\hline $\mathrm{NL}(\mathrm{B})$ & 0.6762 & 1.3244 & 0.8556 & 0.9961 & 1.3115 & 3.7557 & 4.2873 & \\
\hline NL(U) & 0.2644 & 0.3876 & 0.2863 & 0.3148 & 0.4408 & 0.0761 & 0.0788 & \\
\hline
\end{tabular}


Table 18 - contd.

\begin{tabular}{|c|c|c|c|c|c|c|}
\hline \multirow[b]{2}{*}{ Element } & \multicolumn{6}{|c|}{ IV9131A- } \\
\hline & 14-1 & $\overline{14-2}$ & $28-1$ & $28-2$ & $56-1$ & $56-2$ \\
\hline NL(Si) & 0.4955 & 0.5210 & 0.4527 & 0.7224 & 0.8116 & $\overline{0.8221}$ \\
\hline NL(Mg) & 0.0847 & 0.1271 & 0.0019 & 0.2037 & 0.2061 & 0.2821 \\
\hline $\mathrm{NL}(\mathrm{Ca})$ & -2.3763 & -2.3624 & -1.9200 & -2.2666 & -2.2917 & -2.3142 \\
\hline $\mathrm{NL}(\mathrm{Na})$ & 1.5629 & 1.6347 & 0.6515 & 2.2150 & 1.5064 & 2.2751 \\
\hline NL(K) & 1.3101 & 1.3058 & 0.6189 & 1.7927 & 1.8586 & 1.6994 \\
\hline NL(Mn) & 0.0735 & 0.1217 & 0.0562 & 0.1688 & 0.1748 & 0.2165 \\
\hline $\mathrm{NL}(\mathrm{Li})$ & 1.9037 & 1.9439 & 1.7033 & 2.5191 & 2.7151 & 2.5333 \\
\hline$\overline{N L}(\bar{B})$ & 1.7225 & $1.774 \overline{8}$ & $0.6 \overline{8} \overline{8} 0$ & $2 . \overline{506 \overline{2}}$ & 2.6669 & 2.6477 \\
\hline \multirow[t]{2}{*}{ NL(U) } & 0.5490 & 0.5587 & 0.3311 & 0.4673 & 0.3885 & 0.3394 \\
\hline & \multicolumn{6}{|c|}{ IV9131A- } \\
\hline Element & $56-3$ & $56-4$ & $\overline{180-3}$ & $720-1$ & $\overline{720-2}$ & \\
\hline$\overline{\mathrm{NL}(\mathrm{Si})}$ & 1.0604 & $\overline{0.6114}$ & 0.8827 & 1.0795 & 2.1189 & \\
\hline NL(Mg) & 0.0751 & 0.3252 & 0.4060 & 0.2094 & 1.6738 & \\
\hline $\mathrm{NL}(\mathrm{Ca})$ & -2.2260 & -2.1757 & -2.1766 & -1.0207 & -0.3454 & \\
\hline $\mathrm{NL}(\mathrm{Na})$ & 3.5808 & 2.1499 & 3.4343 & 5.9416 & 6.1525 & \\
\hline$N L(K)$ & 2.3559 & 1.6747 & 2.3832 & 3.3720 & 3.8869 & \\
\hline NL(Mn) & 0.0710 & 0.1976 & 0.2972 & 0.2386 & 1.0518 & \\
\hline NL(Li) & 3.2883 & 2.4400 & 3.5194 & 5.2431 & 5.5275 & \\
\hline NL(B) & 3.2480 & 2.4598 & 3.7767 & 5.2988 & 5.7692 & \\
\hline NL(U) & 0.3693 & 0.4460 & 0.3964 & 0.5075 & 0.4889 & \\
\hline
\end{tabular}

Normalized Release $=((($ Dilution corrected leachate concentration $(\mu \mathrm{g} / \mathrm{ml}) * \mathrm{ml}$ leachant $)-\mu \mathrm{g}$ present in EJ-13 leachant $\left.) / 10^{6}\right) /\left(\right.$ sample surface area $\left.\left(\mathrm{mm}^{2}\right) / 10^{6}\right) *$ element weight fraction in glass.

Negative values indicate leachate concentration levels that have been reduced below the level of the initial EJ-13 leachant. 

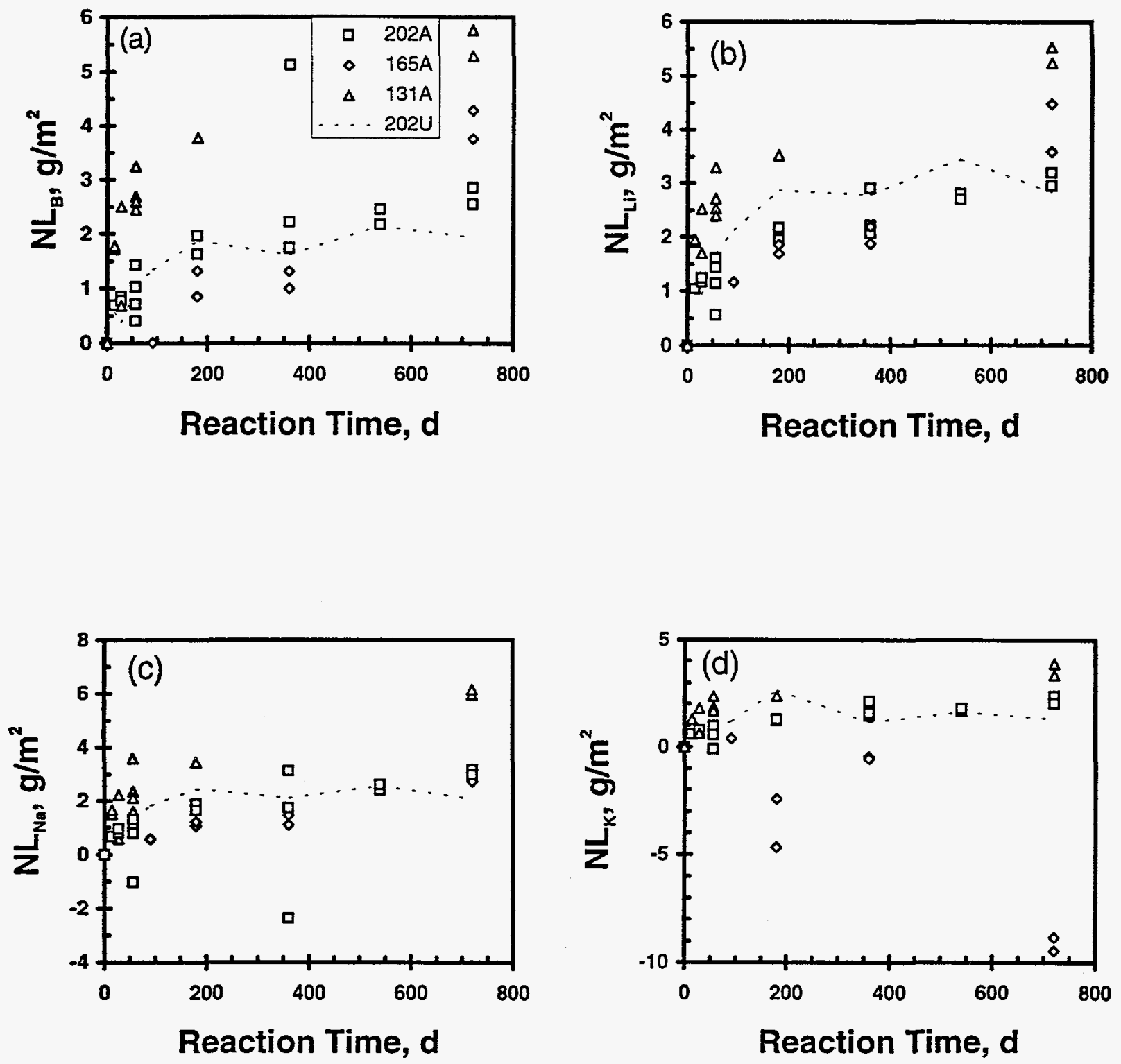

Fig. 17. Normalized Element Release Results from $340 \mathrm{~m}^{-1}$ Batch Tests Exposed to an External ${ }^{60} \mathrm{Co}$ Gamma Radiation Source of $\sim 35 \mathrm{~Gy} / \mathrm{h}$. Data are presented for individual solution analyses of tests conducted with SRL 202A, SRL 165A, and SRL 131A glasses. Averaged results from nonirradiated tests with SRL 202U glass [34] are included for reference (dotted lines). Data are expressed in grams of element " $\mathrm{i}$ " released per surface area $\left(\mathrm{m}^{2}\right)$ of glass. (a) boron, (b) lithium, (c) sodium, (d) potassium, (e) magnesium, (f) calcium, (g) silicon, and (h) uranium. 

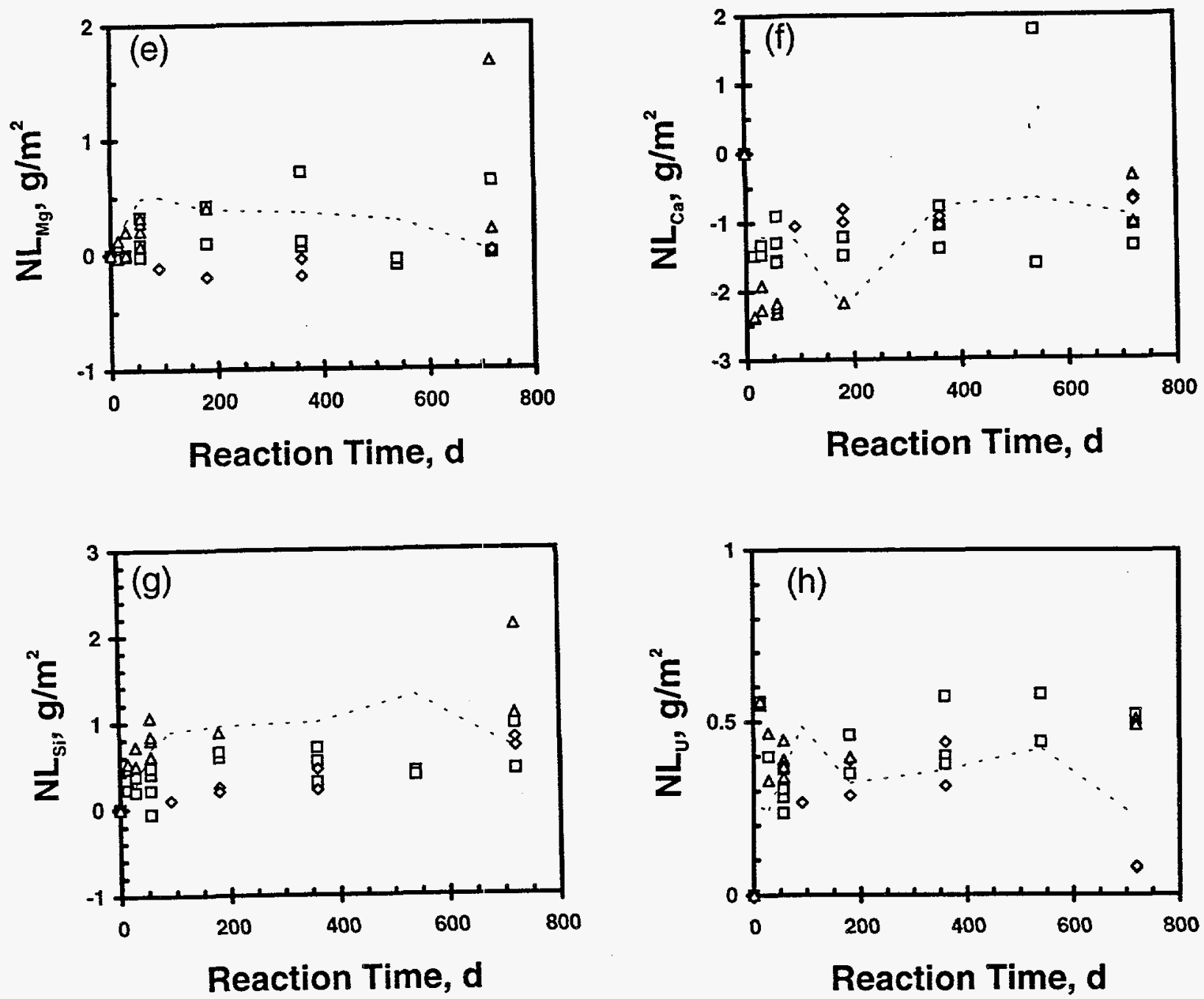
function of reaction time in Fig. 17. As with the solution concentration data previously reported, these results are also compared with those of the SRL $202 \mathrm{U}$ glass samples from the study of Ebert [34]. These latter tests were conducted under identical conditions as the present samples, except that no external gamma radiation exposure was used. Normalized elemental release trends from the irradiated and nonirradiated tests will be used to evaluate the effect that radiation has on glass performance.

The NL(B) trends indicate a similar release pattern as described previously for the element concentration results. Values for NL(B) increase progressively for the SRL 202A glass throughout the entire 720 days of testing (Fig. 17a and Table 18). Values from the nonirradiated SRL 202U glass display the same overall trend as the irradiated SRL 202A samples. With the 360- to 720-day samples, however, NL(B) values from the irradiated tests tended to increase slightly over those of the nonirradiated tests. Tests with SRL $131 \mathrm{U}$ glasses display a rapid increase in NL(B) values throughout the tests. Total NL(B) from the SRL 131A glass tests greatly exceeded the mass losses from tests with the other glasses. Results from the tests with SRL 165A glass display the lowest NL(B) values during 360 days of testing, but then display a large increase in boron release between 360 and 720 days. The final boron mass release from the 720 -day tests with the SRL 165A glass is greater than that of the SRL 202A glass, but less than that of the SRL 131A glass.

In tests with SRL 202A glass, the NL(i) values for the alkali metals ( $\mathrm{Li}, \mathrm{Na}$, and $\mathrm{K}$ ) increased during the first 180 days, then plateaued during 180 to 720 days (Figs. $17 \mathrm{~b}-\mathrm{d}$ and Table 18). Mass release trends are also very similar for the SRL 202A and SRL 202U glasses. The SRL 131A samples also display rapid mass losses of all three alkali metal components during the first 56 days and more moderated mass losses thereafter. With the SRL 165A samples, NL(Li) and NL(Na) mass losses increase continuously throughout the test interval. Negative values were obtained for all $\mathrm{NL}(\mathrm{K})$ calculations with the SRL 165A glass, except for the 90-day sample (Table 18, 90-3). This negative value indicates a net removal of potassium from the EJ-13 solution, with sorption of potassium probably occurring on the glass samples.

The NL(Mg) trends for tests with SRL 202A and SRL 202U glasses show initial increases during the first 56 days of testing followed by constant (but somewhat scattered values thereafter), while NL(Ca) values are constant throughout the duration of the tests (Fig. 17e-f and Table 18). There is no apparent mass loss difference for either element when SRL 202U samples are compared to their irradiated analogues. With the SRL 131A glasses, normalized mass losses increase throughout the tests for both magnesium and calcium; however, the $\mathrm{NL}(\mathrm{Mg})$ values for the 720-day tests display considerable data scatter. Tests with SRL 165A glass show relatively constant normalized mass loss values for these two elements throughout the tests. Slightly negative NL(Mg) values for the SRL 165 glass, as well as negative NL(Ca) values exhibited by all three glass compositions, indicate a net removal of these elements from solution, with probable sorption on the altered glass surfaces.

Silicon mass loss trends increased during the first 180 days of testing with the SRL 202A glasses and then remained relatively constant thereafter (Fig. 17g and Table 18). The NL(Si) values from the unirradiated tests with SRL 202U glasses are generally higher than those of the irradiated SRL 202A tests for the time periods of 56 to 540 days, while the mass loss from the initial and final time periods is similar for both tests. The NL(Si) values increase rapidly during the first 56 days with SRL 131A glasses and then gradually increase thereafter. The SRL 165A glasses display a gradual increase in NL(Si) values throughout the entire 720 days of testing. Normalized silicon mass loss values are generally highest for the SRL 131A glasses for all time periods tested, while the values are generally similar for the SRL 165A and SRL 202A glasses. 
The NL(U) results from the SRL 202A and SRL 131A glasses indicate a rapid increase and peak at 14 days, a decrease up to 56 days, and then a gradual increase thereafter (Fig. 17h and Table 18). Uranium mass losses in irradiated tests with SRL 202A glass are generally slightly higher than those in nonirradiated tests with SRL 202U glass. The relative differences in NL(U) noted between the irradiated and nonirradiated tests parallel both the concentration trends displayed for silicon and uranium (Fig. $16 \mathrm{~g}-\mathrm{h}$ ) and the NL(Si) trends (Fig. 17g). Results from tests with the SRL 131A and SRL 202A glasses also display the highest NL(U) values relative to the SRL 165A glasses. The SRL 165A samples display NL(U) values that increase up to 360 days and then decrease sharply with the 720 day samples.

\section{Transuranic Element and Technetium Concentrations}

Transuranic $\left({ }^{241} \mathrm{Am},{ }^{239} \mathrm{Pu}\right.$, and ${ }^{237} \mathrm{~Np}$ ) concentrations were obtained by alpha spectroscopy performed on separate aliquots of the bulk unfiltered solution, 5.0-nm filtered solution, and acid strip solution taken from the $340 \mathrm{~m}^{-1}$ batch tests. Calculated transuranic concentrations are presented in Fig. 18, while results of the counting analyses are given in Table C.1. The unfiltered solution represents the total transuranic content in solution, including soluble, colloidal, and particulate material. The $5.0-\mathrm{nm}$ filtered portion represents the fraction of the above component that is truly soluble and/or colloids that pass through the filter. The acid strip aliquot represents materials rernoved from the glass and precipitated on the surface of the stainless steel test vessel. The acid strip component was mainly derived from soluble or colloidal materials that have been released from the glass and subsequently sorbed to the stainless steel vessel surface. In a potential repository scenario, this fraction would represent radionuclides that were released from the glass and sorbed onto the stainless steel waste container.

Release patterns of transuranics were similar for all three glass types. Both ${ }^{239} \mathrm{Pu}$ and ${ }^{241} \mathrm{Am}$ show relatively low amounts of release in the 5.0-nm filtered fraction for all glass types (Figs. 18a-f). This feature probably reflects the low solubilities of these elements in the test solutions. The amounts of ${ }^{239} \mathrm{Pu}$ and ${ }^{241} \mathrm{Am}$ released in the bulk (unfiltered) solution fraction were also relatively low. Combined with the filtered tests, these findings suggest that migration of ${ }^{239} \mathrm{Pu}$ and ${ }^{241} \mathrm{Am}$ in the solution fraction is relatively minor, with the bulk of the solution fraction being dominated by the $>5.0-\mathrm{nm}$ fraction. By far, the largest release of ${ }^{239} \mathrm{Pu}$ and ${ }^{241} \mathrm{Am}$ was recovered in the acid strip solution, with release amounts from this fraction typically being five to ten times greater than that of the bulk solution.

Relative to the different glass types, the total amount of ${ }^{239} \mathrm{Pu}$ and ${ }^{241} \mathrm{Am}$ released in the bulk solution was lowest from the SRL 165A, intermediate from the SRL 202A, and greatest from the SRL 131A. This ranking was similar to that exhibited by the release of most other cation components from the glass.

An accurate determination of the aliquot concentrations of neptunium is complicated by the relatively small ${ }^{237} \mathrm{~Np}$ peak heights and an overlap of the ${ }^{237} \mathrm{~Np}$ with the ${ }^{239} \mathrm{Pu}$ spectra. Despite this caveat, the ${ }^{237} \mathrm{~Np}$ release patterns are distinctly different from those of ${ }^{241} \mathrm{Am}$ and ${ }^{239} \mathrm{Pu}$. The release is dominated by the $<5.0-\mathrm{nm}$ size fraction (Figs. $18 \mathrm{~g}-\mathrm{i}$ ). This pattern indicates that ${ }^{237} \mathrm{~Np}$ release from the glass is being controlled by a soluble and/or $<5.0-\mathrm{nm}$ colloidal component. The calculated ${ }^{237} \mathrm{~Np}$ released fractions from filtered solution sometimes exceeded those of the calculated unfiltered solution. This calculation error probably results from the previously mentioned analytical uncertainties in the ${ }^{237} \mathrm{~Np}$ analyses. The released component from the acid strip was generally negligible; however, some of the longer term (one to two year) tests with SRL 165A and SRL 202A did begin to display sporadic release increases in the acidstrip ${ }^{237} \mathrm{~Np}$ component. The overall release trends for ${ }^{237} \mathrm{~Np}$ indicate that release is being dominated by a soluble or fine colloidal $(<5.0 \mathrm{~nm})$ suspension in these tests, with some precipitation of ${ }^{237} \mathrm{~Np}$ on the stainless steel test vessel walls occurring in the long-term tests, in response to increasing ${ }^{237} \mathrm{~Np}$ concentrations in solution. 

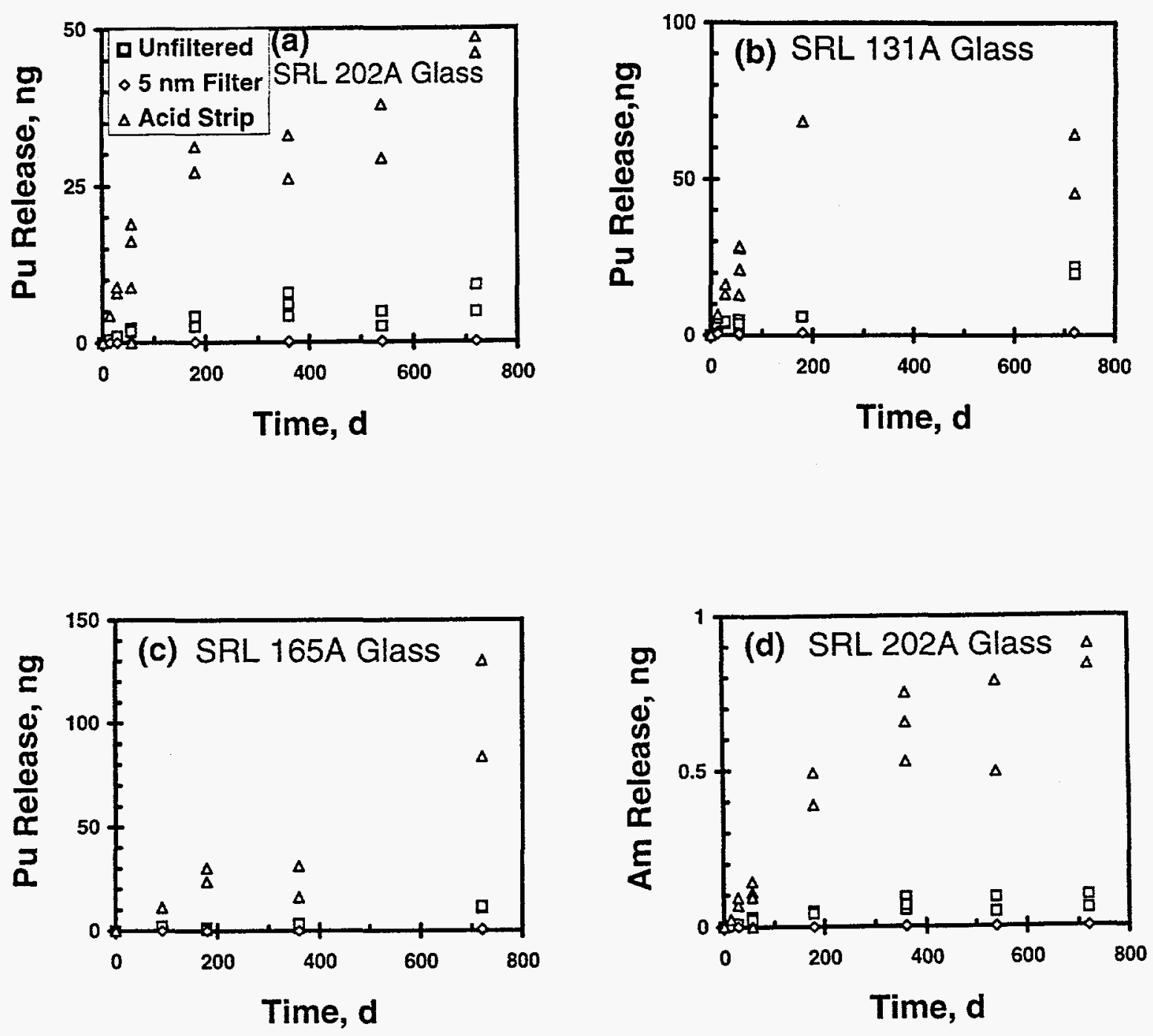

Fig. 18. Transuranic Element Release from $340 \mathrm{~m}^{-1}$ Batch Tests Exposed to External ${ }^{60} \mathrm{Co}$ Gamma Radiation Source of $\sim 35 \mathrm{~Gy} / \mathrm{h}$. Data are presented for individual solution analyses of tests conducted with SRL 202A, SRL 165A, and SRL 131A glasses, and recovered fractions from unfiltered test leachates, 5-nm filtrates, and an acid strip component obtained by soaking the reaction vessel in a dilute nitric acid solution overnight at $90^{\circ} \mathrm{C}$. (a) Plutonium release from SRL 202A glass, (b) plutonium release from SRL 131A glass, (c) plutonium release from SRL 165A glass, (d) americium release from SRL 202A glass, (e) americium release from SRL 131A glass, (f) americium release from SRL 165A glass, (g) neptunium release from SRL 202A glass, (h) neptunium release from SRL 131A glass, and (i) neptunium release from SRL 165A glass. 

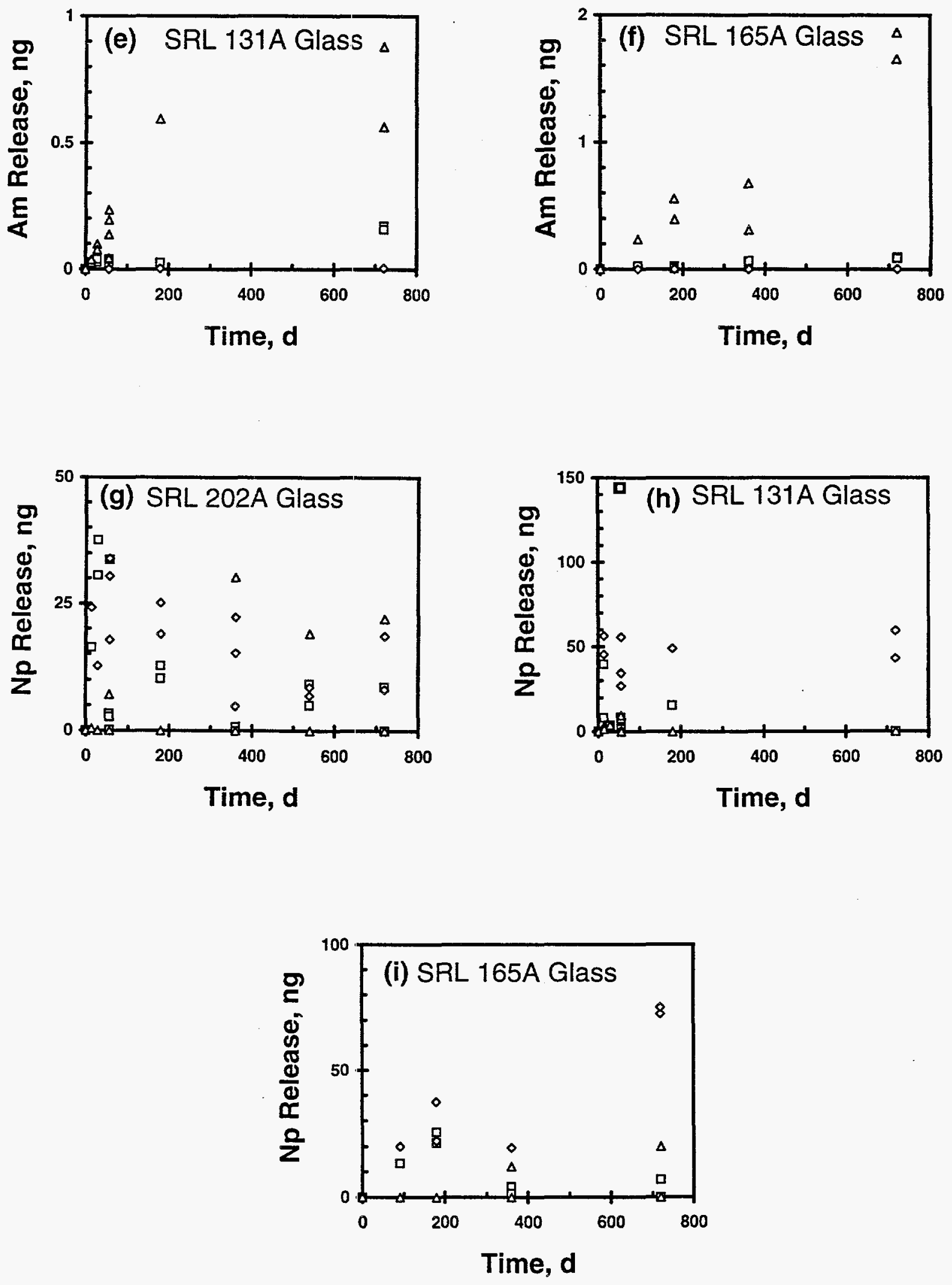


\section{SURFACE ANALYTICAL RESULTS}

Glass surface analyses were completed for glass samples reacted in the $340 \mathrm{~m}^{-1}$ batch leach tests and the vapor hydration tests.

A.

Batch Leach Tests at $340 \mathrm{~m}^{-1}$

\section{1. $\quad$ SRL 202A Glass}

The reacted surfaces of SRL 202A samples from the $340 \mathrm{~m}^{-1}$ batch tests were characterized by relatively thin alteration layers. When samples reacted for periods of 14 to 56 days were examined under the optical microscope, the reacted surfaces were found to have developed fine-grained, white scaly patches of precipitates and a peacock green-blue discoloration. The latter feature suggests the development of surface layers enriched in iron oxide and manganese. After 56 days, the altered surfaces are characterized by an enveloping white to light-gray, clay-like layer (which thickens with time) and reddish to reddish-brown ironoxide patches. After 91 days, the samples also developed surfaces with increasing reflectivity to optical light (i.e., high albedo). This type of reflectivity is often associated with the formation of illite clay minerals $\left[\left(\mathrm{K}_{1.0-1.5} \mathrm{Al}_{4}\right)\left(\mathrm{Si}_{6.5-7} \mathrm{Al}_{1.0-1.5} \mathrm{O}_{20}\right)(\mathrm{OH})_{4}\right]$ with the c-axes of the clays oriented normal to the sample surface. This orientation results in the flat plate-like surfaces of the clays lying parallel to the sample surface and, combined with the reflective surfaces of the mica-illite minerals, results in a highly reflective surface.

After 360 to 540 days, the clay layer forming on these samples had developed to a point where it was thick enough to mask the presence of most of the 600 -grit finish polishing scratches formed during the sample preparation steps. By 720 days, all visible traces of the scratches had been covered by the developing reacted layer. The alteration surfaces on the SRL 202A glasses also show some evidence for layer delamination. For example, the 360-day samples displayed a relatively thick alteration layer, which curled into cuspid-shaped polygonal plates that partially detached from the sample during drying. This curling is a feature commonly observed with smectite clays $\left[(1 / 2 \mathrm{Ca}, \mathrm{Na})_{0.7}(\mathrm{Al}, \mathrm{Mg}, \mathrm{Fe})_{4}(\mathrm{Si}, \mathrm{Al})_{8} \mathrm{O}_{20}(\mathrm{OH})_{4} \cdot \mathrm{nH}_{2} \mathrm{O}\right]$, being associated with shrinkage of the clay layers as a result of the loss of interlayer water. The intensity of this curling and flaking progressively increased with sample reaction time. This increase in flaking is also believed to result from the decreasing adherence of the layer to the glass as the alteration layer progressively increased in thickness.

Pre-test examination of the glass monoliths had identified smooth-faced, chattered edges, that formed along the sample corners, and smooth-surfaced vesicles (solidified gas bubbles in the melt). The vesicle surfaces remained unpolished during the sample preparation and thus had a smoother surface finish than the majority of the sample. These portions of the samples displayed an absence of significant clay development after being reacted in the test chamber. This finding emphasizes the importance of the surface finish in influencing reaction progress on glass. All detailed investigations of surface reactions were conducted only on the 600 -grit polished surfaces of these samples so that regions influenced by differential surface finishes would not be compared. The surface finish may also influence the release rates of elements to solution during the $340 \mathrm{~m}^{-1}$ tests [41]. This effect, however, should be minor, considering the relatively large surface area of the 600-grit finish portion of the samples compared to the fracture and vesicle surfaces.

Examinations of the 28-day samples by AEM (Fig. 19a) indicate that a reaction layer up to $0.5-\mu \mathrm{m}$ thick has formed on the sample surfaces. This layer is divided into two reaction zones, a $0.3-\mu \mathrm{m}$-thick inner layer separated from a $0.2-\mu \mathrm{m}$-thick relatively coarsegrained outer layer. The segregation into two zones does not, however, occur in all regions of 

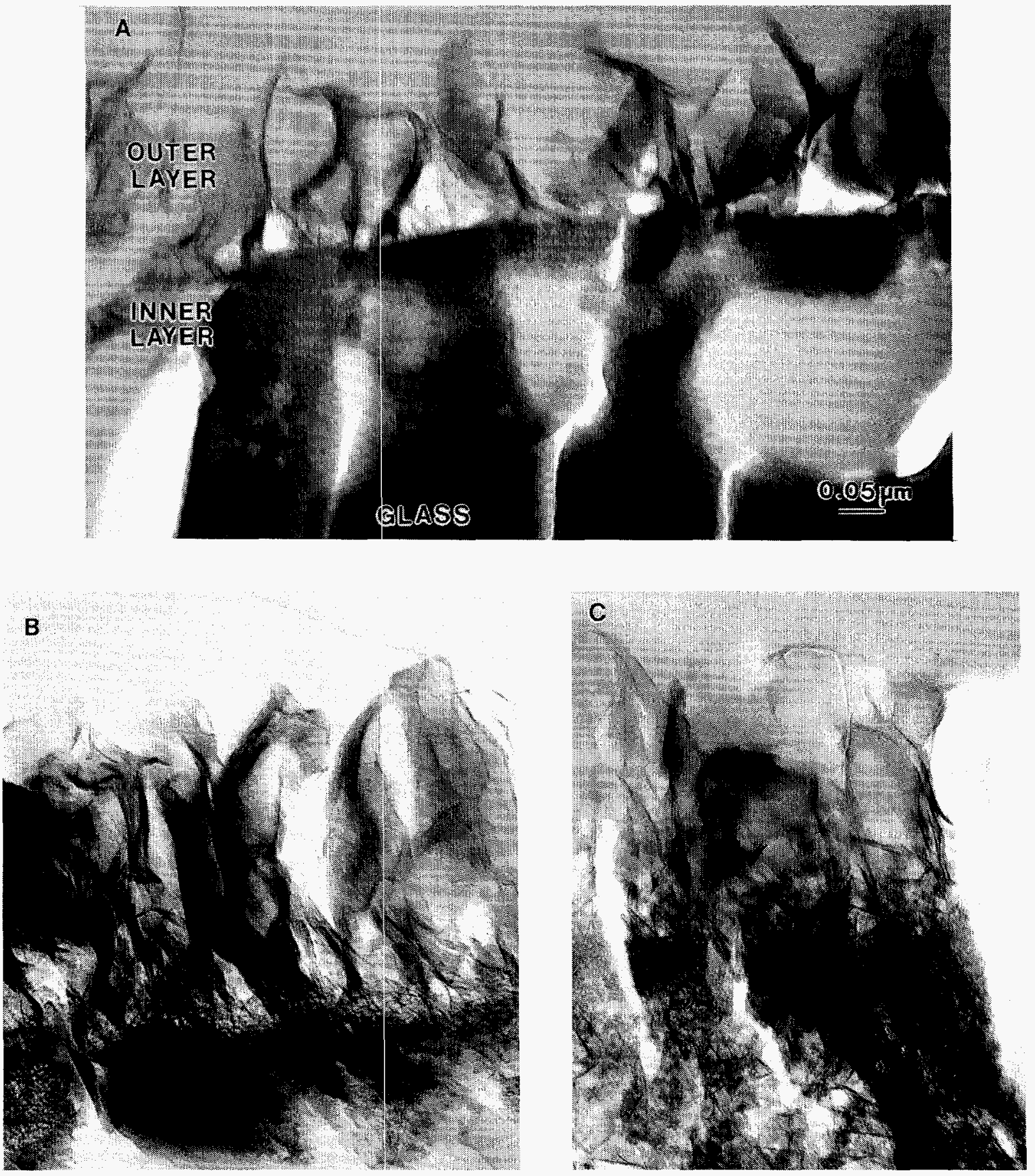

Fig. 19. AEM Photomicrograph of Alteration Layer Cross Sections from $340 \mathrm{~m}^{-1}$ Batch Test with SRL 202A Glass: (a) 28-day sample displaying 200-nm thick outer coarsegrained clay layer, a 300-nm thick inner fine-grained clay layer, and glass; (b) outer coarse-grained and inner fine-grained clay layers from 360-day sample, scale same as in Fig. 19a; and (c) outer coarse-grained and inner fine-grained clay layers from 720day sample, scale same as in Fig. 19a. 
the sample. The inner layer appeared to be mostly amorphous with some developed regions of porosity. The outer layer appeared crystalline and compact. An unidentified fine-grained precipitate phase enriched in $\mathrm{Si}, \mathrm{U}$, and $\mathrm{Ti}$ was also detected in the inner layer on this sample This phase was similar to the 180-day U-Ti colloids discussed below (Fig. 20).

Sections of the 360-day samples also display two reaction zones (Fig. 19b). Because portions of the inner layer had been plucked from the sample during sectioning, complete layer thicknesses could not be determined, but minimum thicknesses of $0.5 \mu \mathrm{m}$ were visible in these samples. The inner layer has developed some porosity, especially along the contact with the unaltered glass.

Sections prepared from the 540 - and 720-day samples were not intact due to the fragmentation of the layer during the ultramicrotoming process used to prepare the sample sections. Layer thicknesses up to 0.5 to $0.8 \mu \mathrm{m}$ were measured during the AEM examination of these specimens; however, because of the poor preservation of the layer, these sections measurements should be considered minimums. The coarse-grained outer layer and fine-grained inner layer were also replicated on these samples, with outer layer thicknesses ranging from 0.1 to $0.4 \mu \mathrm{m}$ and inner layer thicknesses approximating $0.4 \mu \mathrm{m}$ (Fig. 19c). Analytical electron microscopy/energy dispersive X-ray spectroscopy (AEM/EDS) analysis indicates an enrichment of $U$ and $T h$ in the clays relative to the unaltered glass. Fine-grained U-Ti crystallites (possibly brannerite $\left.\left[\mathrm{U}_{2} \mathrm{Ti}_{4} \mathrm{O}_{12}(\mathrm{OH})_{2}\right]\right)$, calcium-rich particles, and uranium-rich particles were also noted to occur within the inner clay layer.

Colloid samples were also collected from the tests with SRL 202A glass. These samples were obtained by wicking a small amount of leachate through a "holey" (perforated) carbon grid filter, which was then dried and prepared for AEM analysis. Colloids collected from the 56-day tests include aluminum- and calcium-rich clay particles. A number of trace constituents were also detected on these colloids, including $\mathrm{Pb}, \mathrm{Cr}, \mathrm{U}, \mathrm{Hg}, \mathrm{Fe}, \mathrm{Zn}, \mathrm{Mn}, \mathrm{Ti}$, and $\mathrm{Sn}$. This observation suggests that colloids may act as a transport mechanism for some radionuclide and hazardous elements contained in the glass. The 180-day colloid residues revealed the presence of unidentified U-Ti phases (Fig. 20). These phases were elongated and may be consanguineous to the $\mathrm{Si}-\mathrm{U}-\mathrm{Ti}$ phases previously noted to occur within the reacted layer attached to the glass. Attempts to obtain diffraction patterns from this phase were unsuccessful. Smectite clays were also noted to occur as colloidal particulates on these grids. Colloidal material was not detected in the holey carbon filters collected from the 360- and 720-day leachates.

\section{2. $\quad$ SRL $165 \mathrm{~A}$ Glass}

The SRL 165A samples reacted in a similar manner as the SRL 202A glasses, with the overall thickness of the altered layers appearing to be slightly thinner than that of the SRL 202A glasses. The shortest tests with these glasses were for 91 days. Surfaces of these samples were characterized by the presence of a light-gray clay layer, which progressively thickened with time. The increasing layer thicknesses also progressively masked the appearance of polishing scratches on the samples; however, the scratches were still readily visible, even after 720 days of reaction. Flaking of the clay layer along the sample edges and some minor curling of the layers were also noted on the 720-day samples.

Sections of the 360- and 720-day samples for SRL 165A glass had been prepared for AEM analysis; however, none of the alteration layer remained intact during the ultramicrotome sectioning. The 360-day sample displayed partially preserved layer thicknesses of 0.2 to $0.5 \mu \mathrm{m}$, while only fragmented sections of the clay layer and altered glass interface region were preserved in the 720 -day samples. The 720 -day sections were noted to display some 
etch pitting at the glass-layer interface. The fragmentation of these samples is believed to result from the poor adherence of the alteration layer to the glass.

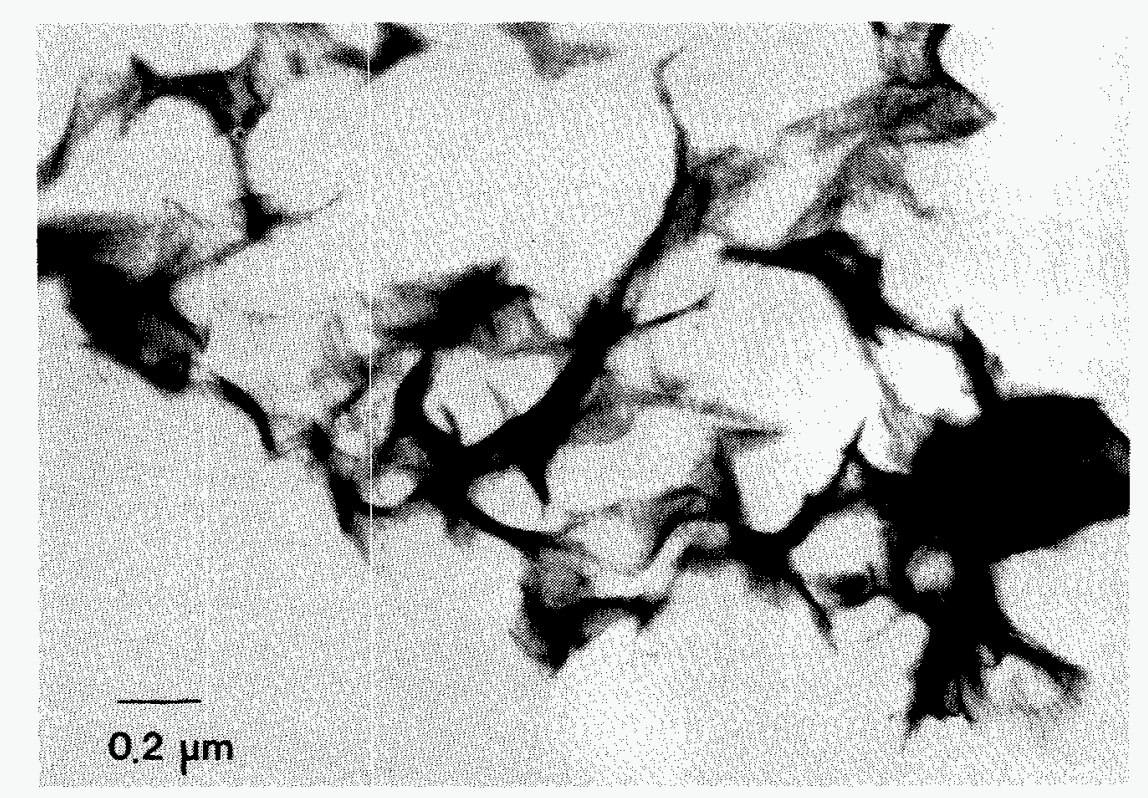

Fig. 20. AEM Photomicrograph of Colloidal Material from $340 \mathrm{~m}^{-1}$ Batch Test with SRL 202A Glass at 180 Days. Shown are a complex mixture of filtered particles of clay and an unidentified U-Ti needle shaped phase.

Agglomerates of iron-rich colloids attached to clay minerals were noted in holey carbon filtered residues from the 91-day tests with SRL 165A glass. The agglomerates were up to $1 \mu \mathrm{m}$ in diameter and sometimes displayed a clay center rimmed by the iron-rich material (Fig. 21). This pattern suggests an electrostatic attraction of the positively charged iron colloids to the negatively charged clay surfaces. Trace amounts of uranium were also detected in these colloids.

\section{3. $\quad$ SRL 131A Glass}

The SRL 131A monolith samples reacted in a similar manner as the SRL 165A and SRL 202A glasses, with the thickness of the altered layers appearing to be slightly greater than that of the SRL 202A glasses. Scaly white precipitates were first observed on the 14-day SRL 131A monoliths. These tests also displayed the formation of the patchy blue-green, "peacock coloration" suggestive of the presence of iron- and manganese-oxide phase(s). By 180 days, the reaction layer had progressively thickened to the point where most of the surface polishing scratches were no longer visible. By 720 days, all traces of polishing scratches had been obliterated by the reaction layer, which was now flaking along the sample edges. 


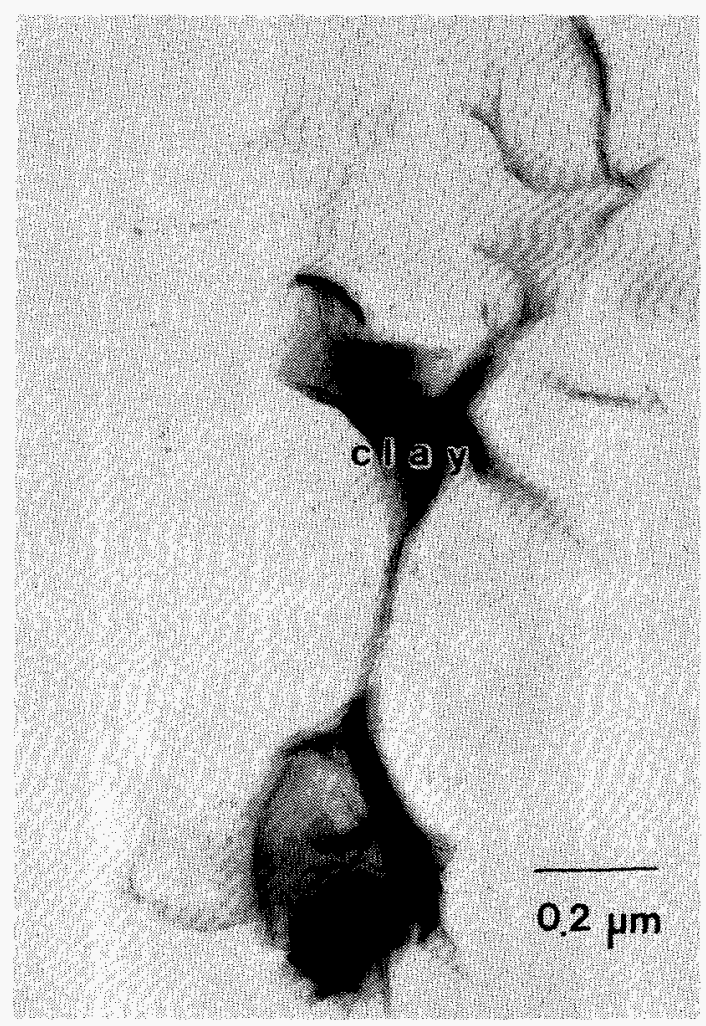

Fig. 21. AEM Photomicrograph of Colloidal Clay Particles Filtered from $340 \mathrm{~m}^{-1}$ Test with SRL 165A Glass at 91 Days

Colloid samples were also taken from the tests with SRL 131A glasses. The 180-day colloid residues revealed the presence of the same unidentified U-Ti phases that characterized colloids of the SRL 202A tests (Fig. 22). These phases displayed a maximum length of $0.1 \mu \mathrm{m}$ and appeared to be crystalline due to their regular elongated morphology, but attempts to obtain diffraction patterns from this phase were not successful. Identifiable clay lattice fringe spacings were also noted from colloidal particulates that had been collected on these grids. These clays displayed a high magnesium content and a $1.0-\mathrm{nm}$ spacing. These features suggest the presence of a collapsed (dehydrated) saponite clay [ideal composition; $(1 / 2 \mathrm{Ca}, \mathrm{Na})_{0.7}(\mathrm{Mg})_{6}\left(\mathrm{Si}_{7.3} \mathrm{Al}_{0.7}\right) \mathrm{O}_{20}(\mathrm{OH})_{4} \bullet \mathrm{nH}_{2} \mathrm{O}$ ]. Holey carbon filtered leachates from $360-$ and 720-day samples did not reveal the presence of any additional colloidal material.

The AEM/EDS analysis of the 720-day sample indicates the presence of an oriented outer clay layer that is $0.2-\mu \mathrm{m}$ thick and a randomly oriented inner clay layer that is 1.0-1.3 $\mu \mathrm{m}$ thick and contains fine-grained U-Ti crystallites. 


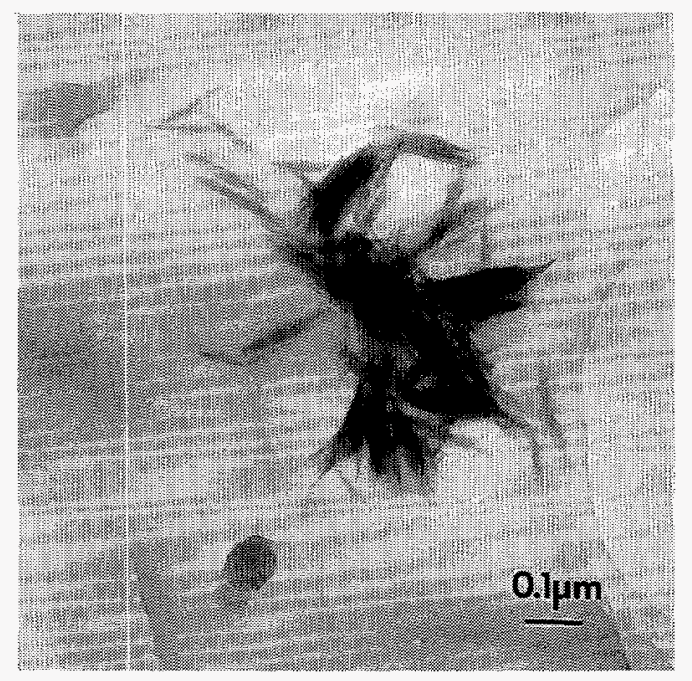

Fig. 22. AEM Photomicrograph of Colloidal Material from $340 \mathrm{~m}^{-1}$ Test with SRL 131A Glass at 180 Days. Shown are the filtered particles of an unidentified U-Ti needleshaped phase similar to that which occurs in the SRL 202A tests (see Fig. 20).

\section{B. Vapor Hydration Tests}

Vapor hydration tests were performed on SRL 202U, SRL 202A, SRL 165A, SRL 131U, and SRL 131A glass compositions. These tests are being used to examine the effects of radiation on glass behavior and secondary phase formation in a simulated unsaturated repository environment. The combination of high temperatures $\left(150-200^{\circ} \mathrm{C}\right)$ and high S/V ratios is used in the vapor hydration tests to accelerate the glass reactions [42]. The actual reaction of the glass sample takes place in a thin-film of water that condenses on the glass sample surface under the influence of the confining pressure of the test vessel. The accelerated reactions thus allows the evaluation of long-term reaction processes based on the results of relatively short-term tests. Water contact with waste materials at the proposed Yucca Mountain nuclear waste repository will not occur until the temperature of the environment surrounding the waste package falls below the local boiling point of water $\left(\sim 96^{\circ} \mathrm{C}\right)$. Previous studies have shown that temperatures up to $200-240^{\circ} \mathrm{C}$ can be used without changing the alteration mechanism affecting the glass reaction $[39,43]$.

The samples were exposed to a ${ }^{60} \mathrm{Co}$ source at a dose rate of $36 \mathrm{~Gy} / \mathrm{h}(\sim 3600 \mathrm{rad} / \mathrm{h})$, a level that would be expected for nuclear waste glasses several hundred years after waste emplacement into a repository. Testing was concentrated on the SRL 202U and SRL 202A glasses since their composition more closely approaches that expected for actual waste glass to be produced at the Savannah River Plant. Most tests were conducted for periods of 7 to 56 days; however, some SRL $131 \mathrm{U}$ samples were allowed to continue up to 720 days so that the phases developed in these tests could be compared with phases developed on samples from shorter term tests with irradiated SRL 131A glass samples.

Mineral phases were identified by using a combination of analytical techniques. Compositional information gained from SEM/EDS and AEM/EDS analysis was used to determine quantitative, semiquantitative, or qualitative information pertaining to the chemical composition of various phases. Mineral structure data were obtained from XRD and/or SAED analysis. Crystal morphologies and textures were also used to aid in the identification of these mineral phases. Other techniques, such as acid dissolution and light-reflectance, were also used to identify some phases. Mineral identification studies are often complicated by the overlapping chemical compositions and solid-solution substitution common in many mineral phases, overlapping structural features, interference of structural data from sample impurities, small 
crystal sizes, and polymorphous crystalline morphologies. Because of these inherent problems, the reliance on a single identification technique is often not conclusive enough to positively identify many mineral phases. The characterization of alteration phases, therefore, is most reliable when two or more identification techniques are used.

1. $\quad$ SRL 202 Glass

\section{a. $\quad$ SRL 202U Samples after 7 Days}

Optical microscopy examinations of the SRL 202U samples after 7 days reveal the presence of white patches of discoloration and scattered, fine-grained white precipitates (Fig. 23a). Detailed SEM investigations have revealed the presence of a nearly continuous honeycomb-like growth of clay minerals across the surface of the sample. The EDS spectrum from this clay layer displays a K-Fe-Mn-Al-Si composition with minor $\mathrm{Ti}, \mathrm{Mg}, \mathrm{Ca}$, and $\mathrm{Ni}$ peaks (Fig. 24a). This layer was depleted in $\mathrm{Na}$ and $\mathrm{Ca}$, but enriched in $\mathrm{Mg}$ and $\mathrm{Al}$ relative to the unaltered glass (Fig. 24b). Major d-spacings measured from SAED patterns of clay minerals from this layer on the 14-day sample indicate the presence of smectite clays, while compositional analyses for SRL 202U glasses reacted for all time periods are consistent with the presence of nontronite $\left[(1 / 2 \mathrm{Ca}, \mathrm{Na})_{0.7}\left(\mathrm{Fe}^{3+}\right)_{4}\left(\mathrm{Si}_{73} \mathrm{Al}_{07}\right) \mathrm{O}_{20}(\mathrm{OH})_{4} \bullet \mathrm{HH}_{2} \mathrm{O}\right]$ and/or ferroan saponite.

Average measured thicknesses of the reacted layer for all vapor hydration test samples are given in Table 19. Reaction layer thicknesses measured during SEM examinations averaged $3.7 \pm 0.8 \mu \mathrm{m}$ for the 7-day samples. These measurements are slightly greater than those determined by AEM examination, where the altered layer was determined to be a maximum thickness of $2.35 \mu \mathrm{m}$. The AEM examinations also indicate a composite structure for the altered layer, with a $0.35-\mu \mathrm{m}$ outer clay layer, with individual clay laths oriented perpendicular to the original glass surface being underlain by a randomly oriented inner layer with thicknesses ranging from 0.3 to $2.0 \mu \mathrm{m}$. The random orientation of the inner layer and its composition as determined during SEM/EDS analysis suggest that it formed in situ, that is, with the components for the clay minerals derived as a residue after the selective dissolution of more soluble glass components. The preferred orientation of the outer layer suggests that these minerals precipitated out of the solution that was in contact with the altered sample surface. The oriented texture arose as the developing crystals preferentially formed with their crystal edges attached to the sample surface.

Examination of phases forming on the SRL $202 \mathrm{U}$ glasses after 7 days has identified relatively few types of surface precipitates. The absence of significant amounts of surface precipitates gives the samples the appearance of being relatively unaltered, except for the chalky white film across the surfaces and a few very fine grain white rosettes and clear diskshaped precipitates (Figs. 25a and 25b). The SEM/EDS analyses indicated that the white rosettes are composed mostly of $\mathrm{Ca}$ and $\mathrm{Si}$, with lesser $\mathrm{K}$ and $\mathrm{Al}$ (Fig. 24c). A phase with a similar morphology and composition had been previously identified as gyrolite $\left[\mathrm{Ca}_{4} \mathrm{Si}_{6} \mathrm{O}_{15}\left(\mathrm{OH}_{2}\right)_{2} \cdot 3 \mathrm{H}_{2} \mathrm{O}\right]$ $[44,45]$. However, XRD patterns taken from a similarly shaped $\mathrm{Ca}-\mathrm{Si}$ phase present on the 35-day sample from this study (sample IVE202U-35-1a, see Table 20) and SAED patterns taken during AEM analysis of the 21-day samples did not match with existing data file standards for gyrolite or any other $\mathrm{Ca}-\mathrm{Si}$ phases with a similar composition [46]. This $\mathrm{Ca}-\mathrm{Si}$ phase thus remains unidentified. 

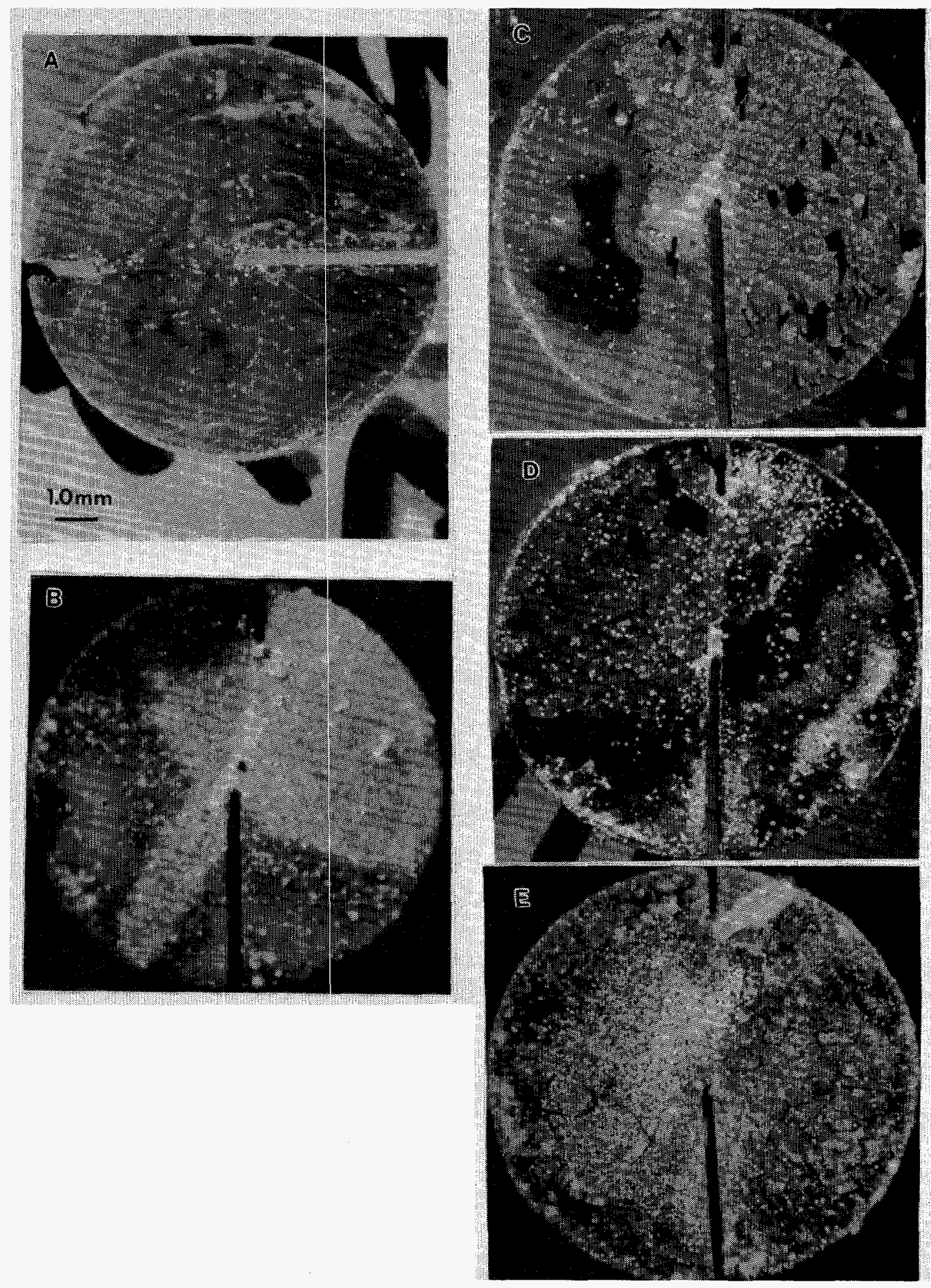

Fig. 23. Optical Photographs of the Reacted Surfaces of SRL 202U Glass Samples from Vapor Hydration Tests at $200^{\circ} \mathrm{C}$ : (a) 7-Day Sample, (b) 14-Day Sample, (c) 21-Day Sample, (d) 35-Day Sample, and (e) 56-Day Sample. All photographs at 9X, with disk diameter $\sim 10 \mathrm{~mm}$. 

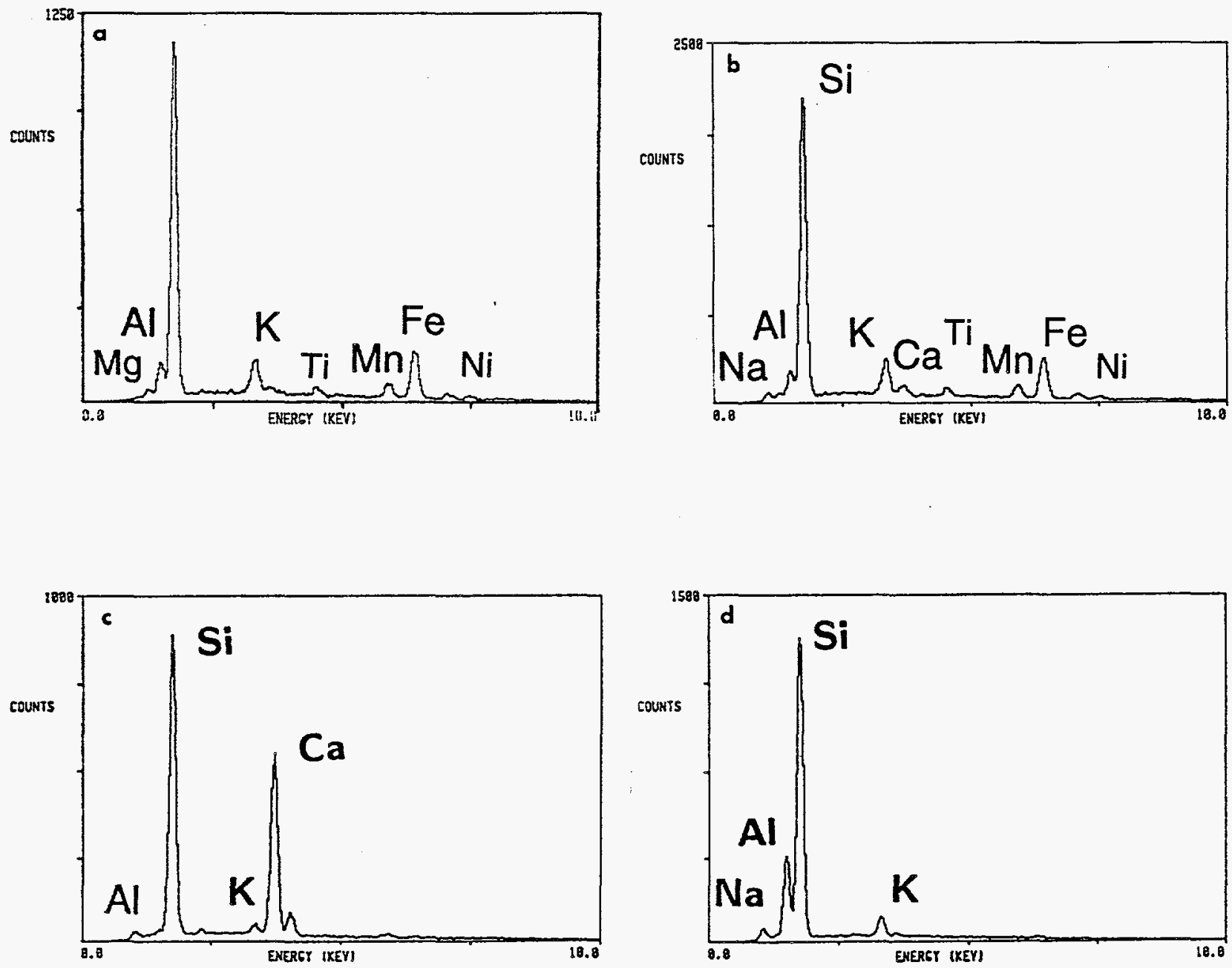

Fig. 24. SEM/EDS Spectra of SRL 202U Sample from $200^{\circ} \mathrm{C}$ Vapor Hydration Test at 7 Days: (a) Clay Alteration Surface, (b) Unaltered Glass, (c) Unidentified Ca-Si Rosette Phase at 21 Days, and (d) Na-Chabazite var. Herschelite. All spectra taken at $20 \mathrm{keV}$ accelerating potential. 
Table 19. Averaged Alteration Layer Thickness from Vapor Hydration Tests

\begin{tabular}{|c|c|c|c|c|c|c|}
\hline $\begin{array}{l}\text { Glass }^{\mathrm{a}} \\
\text { Composition }\end{array}$ & $\begin{array}{c}\begin{array}{c}\text { Test } \\
\text { Length } \\
\text { (days) }\end{array} \\
\end{array}$ & $\begin{array}{c}\text { Layer } \\
\text { Thickness } \\
(\mu \mathrm{m})\end{array}$ & $\begin{array}{l}\text { Thickness } \\
\text { Stand. } \\
\text { Dev. }(\mu \mathrm{m})\end{array}$ & $\begin{array}{c}\text { Periodic } \\
\text { Reaction } \\
\text { Rate } \\
(\mu \mathrm{m} / \text { day })\end{array}$ & $\begin{array}{c}\text { Cumulative } \\
\text { Reaction } \\
\text { Rate } \\
(\mu \mathrm{m} / \text { day }) \\
\end{array}$ & $\begin{array}{l}\text { Number of } \\
\text { Measurements }\end{array}$ \\
\hline SRL 202U & $\begin{array}{r}7 \\
14 \\
21 \\
35 \\
56 \\
\end{array}$ & $\begin{array}{c}3.7 \\
18.7 \\
19.4 \\
51.0(54.8)^{\mathrm{b}} \\
94.9(132.1)^{\mathrm{b}}\end{array}$ & $\begin{array}{c}0.8 \\
3.8 \\
8.6 \\
8.6(10.9)^{\mathrm{b}} \\
30.2(14.8)^{\mathrm{b}} \\
\end{array}$ & $\begin{array}{c}0.5 \\
2.1 \\
0.1 \\
2.3(2.5)^{\mathrm{b}} \\
2.1(3.7)^{\mathrm{b}} \\
\end{array}$ & $\begin{array}{c}0.5 \\
1.3 \\
0.9 \\
1.5(1.6)^{b} \\
1.7(2.4)^{b}\end{array}$ & $\begin{array}{l}21 \\
20 \\
20 \\
15 \\
15 \\
\end{array}$ \\
\hline SRL 202A & $\begin{array}{r}7 \\
14 \\
21 \\
35 \\
56 \\
\end{array}$ & $\begin{array}{r}39.3 \\
177.3 \\
289.2 \\
438.8 \\
490^{\circ} \\
\end{array}$ & $\begin{array}{r}3.8 \\
5.0 \\
13.4 \\
15.8 \\
-\quad \\
\end{array}$ & $\begin{array}{c}5.6 \\
19.7 \\
16.0 \\
10.7 \\
>2.4^{\mathrm{c}} \\
\end{array}$ & $\begin{array}{r}5.6 \\
12.7 \\
13.8 \\
12.5 \\
>8.8^{\mathrm{c}}\end{array}$ & $\begin{array}{l}20 \\
20 \\
20 \\
13 \\
-\end{array}$ \\
\hline SRL 165A & $\begin{array}{r}7 \\
14 \\
21 \\
35 \\
56 \\
\end{array}$ & $\begin{array}{r}15.5 \\
23.6 \\
28.9 \\
108.6 \\
170.5 \\
\end{array}$ & $\begin{array}{r}3.1 \\
2.9 \\
2.8 \\
14.3 \\
8.5 \\
\end{array}$ & $\begin{array}{l}2.2 \\
1.2 \\
0.8 \\
5.7 \\
2.9 \\
\end{array}$ & $\begin{array}{l}2.2 \\
1.7 \\
1.4 \\
3.1 \\
3.0 \\
\end{array}$ & $\begin{array}{l}20 \\
20 \\
20 \\
20 \\
20 \\
\end{array}$ \\
\hline SRL $131 \mathrm{U}$ & $\begin{array}{r}3 \\
5 \\
7 \\
14 \\
28 \\
28 \\
56 \\
91 \\
180 \\
365 \\
540 \\
720 \\
\end{array}$ & $\begin{array}{r}2.7 \\
3.0 \\
3.7 \\
5.1 \\
4.9 \\
4.9 \\
9.2 \\
14.5 \\
18.4 \\
31.8 \\
53.1 \\
76.6\end{array}$ & $\begin{array}{l}0.7 \\
0.5 \\
0.8 \\
0.9 \\
0.9 \\
0.9 \\
1.1 \\
1.1 \\
1.2 \\
2.1 \\
4.8 \\
5.6\end{array}$ & $\begin{array}{l}0.9 \\
0.2 \\
0.4 \\
0.2 \\
0.0 \\
0.0 \\
0.2 \\
0.2 \\
0.0 \\
0.1 \\
0.1 \\
0.1\end{array}$ & $\begin{array}{l}0.9 \\
0.6 \\
0.5 \\
0.4 \\
0.2 \\
0.2 \\
0.2 \\
0.2 \\
0.1 \\
0.1 \\
0.1 \\
0.1\end{array}$ & $\begin{array}{l}20 \\
20 \\
20 \\
20 \\
20 \\
20 \\
20 \\
20 \\
20 \\
20 \\
20 \\
12\end{array}$ \\
\hline SRL 131A & $\begin{array}{r}7 \\
14 \\
21 \\
35 \\
56 \\
\end{array}$ & $\begin{array}{r}3.0 \\
4.2 \\
11.0 \\
14.1 \\
35.5 \\
\end{array}$ & $\begin{array}{l}0.5 \\
1.3 \\
2.8 \\
1.7 \\
3.6 \\
\end{array}$ & $\begin{array}{l}0.4 \\
0.2 \\
1.0 \\
0.2 \\
1.0 \\
\end{array}$ & $\begin{array}{l}0.4 \\
0.3 \\
0.5 \\
0.4 \\
0.6 \\
\end{array}$ & $\begin{array}{l}14 \\
20 \\
11 \\
20 \\
20\end{array}$ \\
\hline
\end{tabular}

${ }^{\mathrm{a} S R L} 202 \mathrm{~A}$, SRL 202U, and SRL $16.5 \mathrm{~A}$ samples reacted at $200^{\circ} \mathrm{C}$; SRL 131A and SRL 131U samples at $150^{\circ} \mathrm{C}$; " $U$ " suffix samples reacted without external radiation exposure; "A" suffix samples reacted during exposure to external gamma source at dose rate of $\sim 36 \mathrm{~Gy} / \mathrm{h}$.

bValues in parenthesis include thickness of overlying precipitated illite layer.

'Sample reacted through entire monolith during testing period. Minimum thickness was determined from half-width of starting glass monolith.

${ }^{\mathrm{d}}$ Measurements taken from 600-grit polished sections along both sides of a single sample. 
The disk-shaped precipitates (Fig. 25b) display a K-Na-Al-Si composition and have XRD patterns similar to the zeolite minerals chabazite $\left(\mathrm{Ca}_{2} \mathrm{Al}_{4} \mathrm{Si}_{8} \mathrm{O}_{24} \bullet 12 \mathrm{H}_{2} \mathrm{O}\right)$ and stilbite $\left(\mathrm{NaCa}_{2} \mathrm{Al}_{5} \mathrm{Si}_{13} \mathrm{O}_{36} \cdot 14 \mathrm{H}_{2} \mathrm{O}\right)$ (sample IVE202U-7-26, see Table 20). Sodium- and potassium-bearing chabazites have previously been observed in volcanic-sedimentary environments, with compositional range for chabazites given by Calligaris [47] $\left[\mathrm{K}_{3.2} \mathrm{Na}_{0.75} \mathrm{Al}_{3.8} \mathrm{Si}_{8.2} \mathrm{O}_{24}{ }^{\bullet}\left(\mathrm{H}_{2} \mathrm{O}\right)_{8.4}\right]$ matching closely with the SEM/EDS compositional analyses from these samples (Fig. 24d). Since a wide range of $\mathrm{Ca}, \mathrm{Na}$, and $\mathrm{K}$ compositions are possible in the chabazite-herschelite group, the alkali-alkaline earth compositions will not be particularly diagnostic in zeolite phase identification. The disk-shaped morphology noted on the samples from this study also similar to that noted by Gottardi and Galli [48]. The XRD patterns generated from the present samples (sample IVE202U-7-26, see Table 20) also match XRD patterns of JCPDS-34-137 (chabazite) and JCPDS-19-1178 (herschelite). The combined SEM/EDS, XRD, and morphological characteristics suggest that this phase is Na-rich chabazite (var. herschelite, $\mathrm{Na}_{4} \mathrm{Al}_{4} \mathrm{Si}_{8} \mathrm{O}_{24} \cdot 12 \mathrm{H}_{2} \mathrm{O}$ ).

Trace amounts of an additional Na-K-Ca-U-Si phase was also detected on the samples. This phase developed as needle-like precipitates with crusted surfaces but was generally too small to obtain effective SEM images or accurate EDS spectra. The appearance and composition of this phases suggest the presence of a uranyl silicate and/or uranyl alkali silicate with a covering of evaporative salts.

\section{b. $\quad$ SRL 202U Samples after 14 Days}

The optical microscopy examinations of the 14-day samples have revealed a substantial increase in the thickness of the alteration layer covering the glass disks and also an increase in the covering of surface precipitates (Fig. 23b). Development of the alteration layer and surface precipitates occurred in a nonuniform manner, with highly altered and relatively unaltered regions occurring on the same samples. Precipitate development was most enhanced along regions in contact with the Teflon hanging thread in the test vessel and the lower-most hanging portion of the sample, i.e., those features demarking the accumulation of liquid water on 

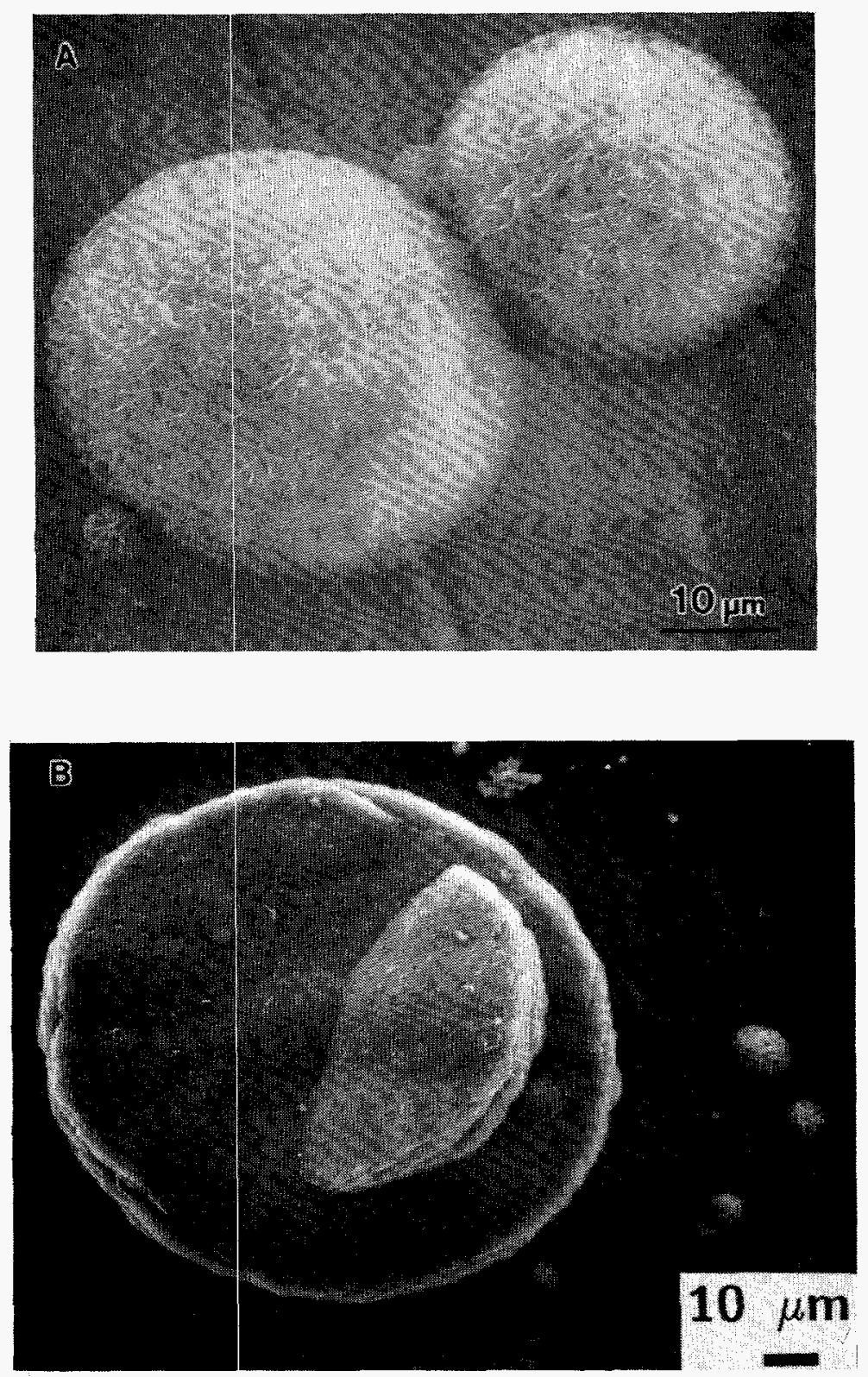

Fig. 25. SEM Images of Secondary Alteration Phases Formed on SRL 202U Glass Reacted 7 Days in Vapor Hydration Test. Images in secondary electron mode. (a) Ca-Si rosettes (1250X and (b) twinned crystal of Na-rich chabazite (var. herschelite) $(600 X)$. 
Table 20. X-Ray Diffraction Indexing Data for Selected Mineral Phases Obtained from Sample Surfaces in Vapor Hydration Tests with 202U Glass

\begin{tabular}{|c|c|c|c|c|c|}
\hline \multicolumn{2}{|c|}{ Sample IVE202U-35-2a } & \multicolumn{2}{|c|}{ Sample IVE202U-21-1b } & \multicolumn{2}{|c|}{ IVE202U-7-2b } \\
\hline $\mathrm{dA}^{\mathrm{a}}$ & I & $\mathrm{dA}$ & I & $\mathrm{dA}$ & I \\
\hline 11.1 & 100 & 8.15 & 30 & 9.3 & 100 \\
\hline 5.45 & 20 & 7.1 & 100 & 6.88 & 40 \\
\hline 3.5 & 10 & 5.35 & 20 & 5.55 & 30 \\
\hline 3.09 & 30 & 5.00 & 30 & 4.98 & 70 \\
\hline 2.97 & 30 & 4.98 & 25 & 4.32 & 70 \\
\hline 2.82 & 20 & 4.29 & 15 & 4.15 & 35 \\
\hline 2.77 & 25 & 4.09 & 20 & 3.87 & 30 \\
\hline 2.505 & 5 & 3.67 & 10 & 3.73 & 15 \\
\hline 2.225 & 5 & 3.23 & 40 & 3.57 & 70 \\
\hline 2.14 & 5 & 3.22 & 40 & 3.43 & 30 \\
\hline 1.995 & 15 & 3.19 & 100 & 3.22 & 20 \\
\hline \multirow[t]{2}{*}{1.835} & 20 & 2.94 & 25 & 3.17 & 20 \\
\hline & & 2.73 & 30 & 2.92 & 100 \\
\hline \multirow{14}{*}{\multicolumn{2}{|c|}{ Phase ID - Tobermorite }} & 2.675 & 25 & 2.88 & 65 \\
\hline & & 2.55 & 15 & 2.68 & 15 \\
\hline & & 2.505 & 15 & 2.60 & 20 \\
\hline & & 2.245 & 15 & 2.495 & 30 \\
\hline & & 2.045 & 20 & 2.295 & 10 \\
\hline & & 1.985 & 10 & 2.11 & 10 \\
\hline & & 1.96 & 15 & 2.08 & 15 \\
\hline & & 1.778 & 10 & 1.86 & 20 \\
\hline & & 1.772 & 20 & 1.842 & 20 \\
\hline & & 1.714 & 20 & 1.80 & 30 \\
\hline & & 1.675 & 15 & 1.72 & 30 \\
\hline & & 1.662 & 15 & & \\
\hline & & & & \multirow{2}{*}{\multicolumn{2}{|c|}{ Phase ID - Herschelite }} \\
\hline & & \multicolumn{2}{|c|}{ Phase ID - Phillipsite } & & \\
\hline \multicolumn{2}{|c|}{ Sample IVE202U-56-2b } & \multicolumn{2}{|c|}{ Sample IVE202U-56-2b } & & \\
\hline$\overline{\mathrm{dA}}$ & $\overline{\mathrm{I}}$ & $\mathrm{dA}$ & $\mathrm{I}$ & & \\
\hline 10.2 & 80 & 11.2 & 100 & & \\
\hline 4.45 & 40 & 5.45 & 30 & & \\
\hline 4.14 & 100 & 3.495 & 20 & & \\
\hline 3.77 & 40 & 3.09 & 25 & & \\
\hline 3.42 & 40 & 2.96 & 25 & & \\
\hline 3.04 & 10 & 2.815 & 15 & & \\
\hline 2.6 & 30 & 2.77 & 35 & & \\
\hline 2.42 & 10 & 2.26 & 5 & & \\
\hline 2.23 & 10 & 2.33 & 20 & & \\
\hline 2.2 & 10 & 2.14 & 5 & & \\
\hline 2.08 & 15 & 2.00 & 10 & & \\
\hline 1.719 & 15 & 1.83 & 10 & & \\
\hline 1.519 & 30 & 1.618 & 10 & & \\
\hline 1.313 & 30 & & & & \\
\hline 1.198 & 15 & Phase ID & orite & & \\
\hline Phase ID & & & & & \\
\hline
\end{tabular}


84

Table 20 - contd.

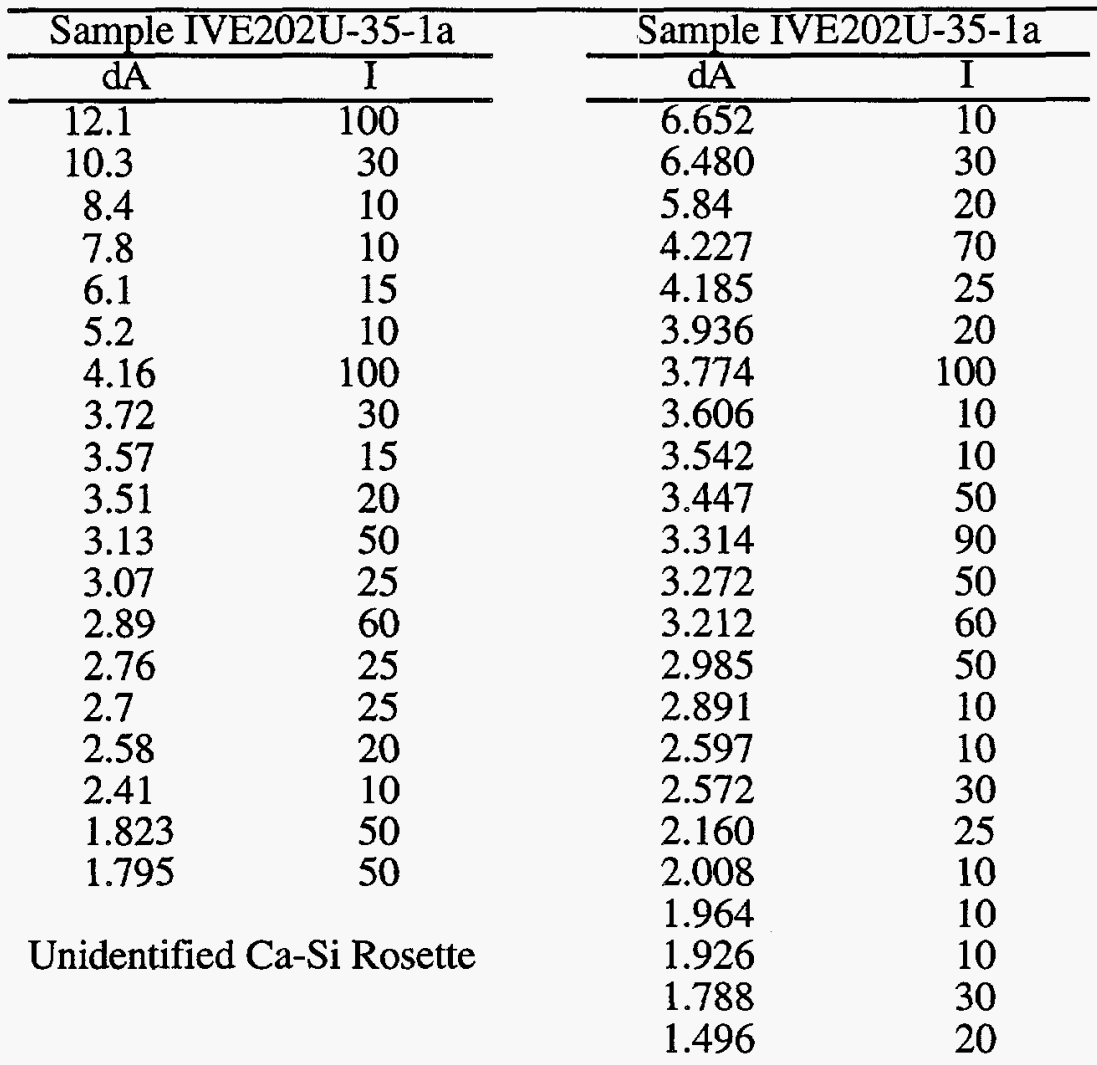

Phase ID - Adularia

${ }^{\mathrm{a}} \mathrm{dA}$, basal spacing in angstrom units; $\mathrm{I}$, relative peak height intensity. Phase ID based on combined XRD and SEM/EDS data. 
the sample surface during testing. Reaction layer thicknesses measured during SEM examinations averaged $18.7 \pm 3.8 \mu \mathrm{m}$ (Table 19). These clay layers retained the same honeycomb-textured patterns that characterized the 7-day samples (Fig. 26a).

The main altered surface layer was enriched in $\mathrm{Ca}$ and $\mathrm{Mg}$, while being depleted in $\mathrm{Na}$ and $\mathrm{K}$ relative to the unaltered glass. Figures $27 \mathrm{a}$ and $27 \mathrm{~b}$ show the EDS spectra while the corresponding quantitative analysis results for the unaltered glass and altered layer, are presented in Table 21. The derived composition in Table 21 reflects a weight normalized calculation for the eight listed elements. A thin and discontinuous surface layer enriched in uranium was also identified during SEM/EDS and AEM investigations of this sample. This layer was similar in composition to the $\mathrm{Na}-\mathrm{K}-\mathrm{Ca}-\mathrm{U}-\mathrm{Si}$ phase observed in the 7-day test.

A low-magnification bright-field AEM image with SAED pattern and two high-resolution images of the reacted clay layer are presented in Fig. 28. The major d-spacings measured from the SAED pattern (Fig. 28a, inset) are $0.16,0.27$, and $0.47 \mathrm{~nm}$. The highresolution images (Figs. 28b and 28c) display both the characteristic curved lattice images typical of smectite clays, and the $0.47-$ and 1.4-nm lattice spacings. These spacings are characteristic of expanded smectite clays. The combined compositional and lattice imaging characteristics of this clay layer are consistent with the presence of saponite, ferroan saponite, and/or nontronite from the smectite group of clay minerals.

Major alteration phases identified on the 14-day samples included Na-rich chabazite (herschelite), which now displays a pseudo-hexagonal, rhombohedral crystalline morphology with an irregular blocky surface texture (Fig. 26b). The EDS spectrum for this phase is similar to that of Fig. 24d, confirming that this was the same mineral phase present on the 7-day samples. Figure 29 shows the high-resolution AEM image and SAED patterns for the herschelite phase. A $0.96-\mathrm{nm}$ spacing was measured in both the SAED and high-resolution AEM images. Other phases identified on the sample include a potassium-rich phase (adularia?, $\left.\mathrm{KAlSi}_{3} \mathrm{O}_{8}\right)$, analcime $\left(\mathrm{NaAlSi}_{2} \mathrm{O}_{6} \cdot \mathrm{H}_{2} \mathrm{O}\right)$, weeksite $\left[\mathrm{K}_{2}\left(\mathrm{UO}_{2}\right)_{2}\left(\mathrm{Si}_{2} \mathrm{O}_{5}\right)_{3} \bullet 4 \mathrm{H}_{2} \mathrm{O}\right]$, and tobermorite $\left[\mathrm{Ca}_{5}(\mathrm{OH})_{2} \mathrm{Si}_{6} \mathrm{O}_{16} \cdot 4 \mathrm{H}_{2} \mathrm{O}\right]$. Very limited amounts of sodium-weeksite $\left[\mathrm{Na}_{2}\left(\mathrm{UO}_{2}\right)_{2}\left(\mathrm{Si}_{2} \mathrm{O}_{5}\right)_{3} \cdot 4 \mathrm{H}_{2} \mathrm{O}\right]$ and phillipsite $\left[(\mathrm{K}, \mathrm{Na})_{10}\left(\mathrm{Al}_{10} \mathrm{Si}_{22} \cdot 20 \mathrm{H}_{2} \mathrm{O}\right)\right]$ were also noted.

Additional precipitates were noted on the sample surfaces, but these phases could not be positively identified. These unidentified phases include P-Si phases rich in alkali and alkali earth, and the same $\mathrm{Ca}$-Si rosette that was present in the 7-day tests. Figure 30 shows a SEM photomicrograph, high-resolution AEM photomicrograph, and SAED pattern from the $\mathrm{Ca}-\mathrm{Si}$ rosette phase. The major d-spacings measured from the SAED pattern (Fig. 30b) are $0.19,0.28,0.32,0.37$, and $0.44 \mathrm{~nm}$. Despite an extensive search, we could find no phase matching these $\mathrm{d}$-spacings and $\mathrm{Ca}-\mathrm{Si}$ composition. A number of the secondary phases were also covered with a fine dusting of amorphous sodium salts, possibly including $\mathrm{NaCl}$ (halite), $\mathrm{NaNO}_{3}$ (soda niter), and/or $\mathrm{Na}_{2} \mathrm{SO}_{4}$.

\section{c. $\quad$ SRL 202U Samples after 21 Days}

Optical microscopy examinations of the 21-day samples indicate a continued increase in the development of the alteration layer (Fig. 23c). The surface precipitate cover developed in a nonuniform manner, with highly altered and relatively unaltered regions occurring on the samples. As with the previous tests, precipitate development was enhanced along regions in contact with the Teflon hanging thread and the lower-most hanging portion of the sample. The alteration layer was also sufficiently thick enough where portions of the layer cracked and flaked off after the sample was air-dried following termination of the test. Clay reaction layer thicknesses measured during SEM examinations averaged $19.4 \pm 8.6 \mu \mathrm{m}$, only a slight increase over what was observed from the 14-day test sample. (Table 19 and Fig. 31 a). 
The honeycomb-textured smectite clay surfaces of the 21-day samples are both compositionally and morphologically indistinguishable from the 7-day sample surfaces.
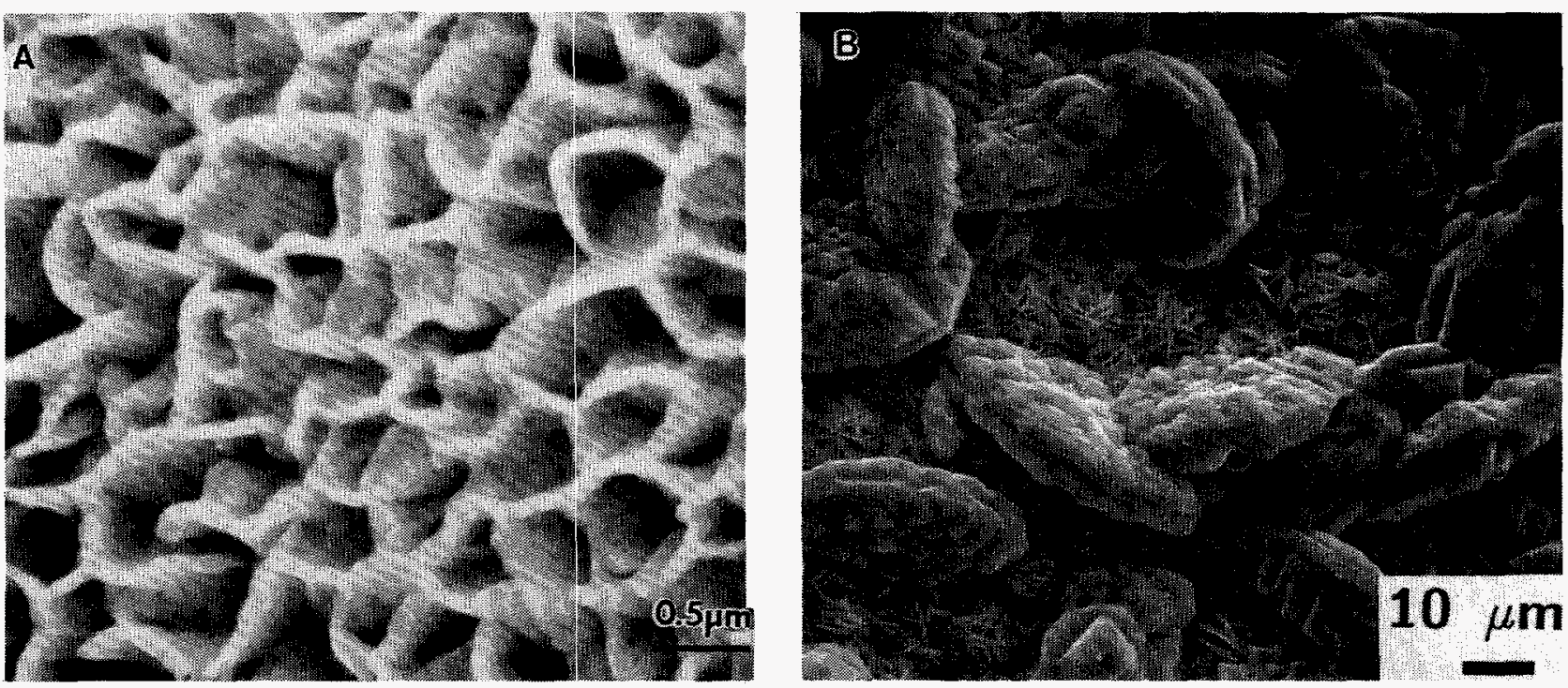

Fig. 26. Secondary Electron Image of Secondary Alteration Phase Formed on SRL 202U Glass Reacted 14 Days in Vapor Hydration Test. (a) Honeycomb-textured surface of clay layer $(20,000 \mathrm{X})$ and (b) Na-rich chabazite (herschelite) disks overlying smectite clay surface $(750 \mathrm{X})$. 
(a)

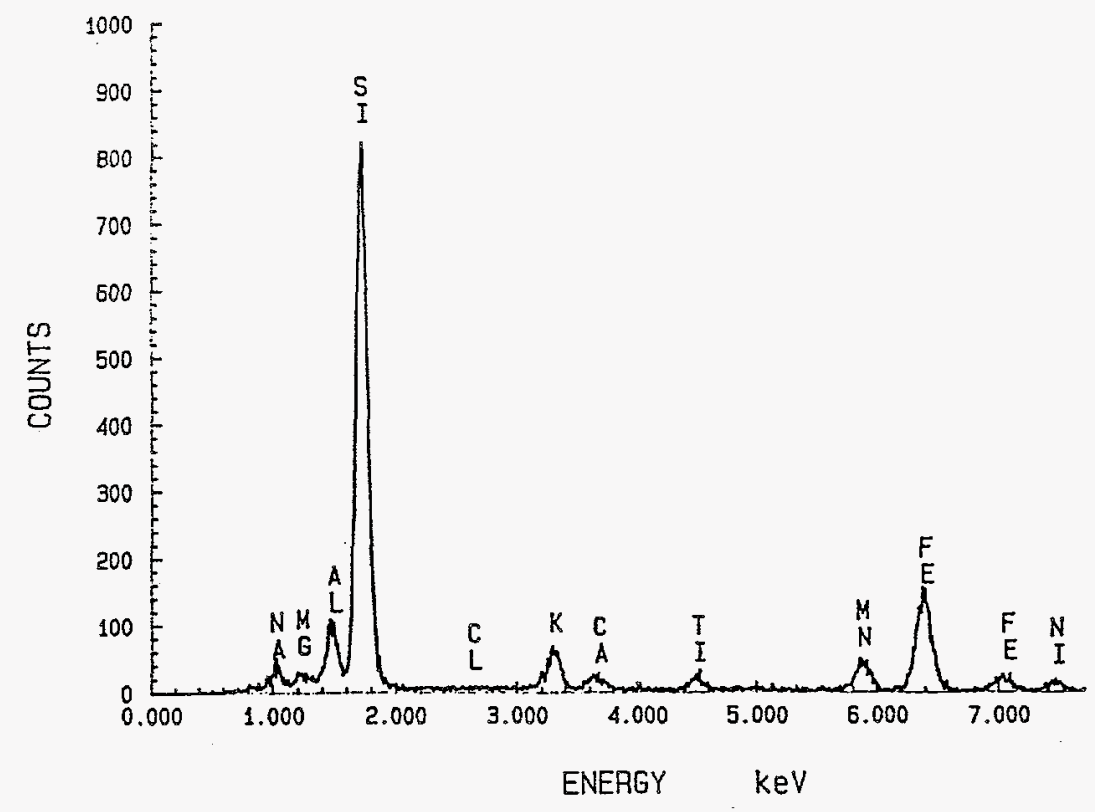

(b)

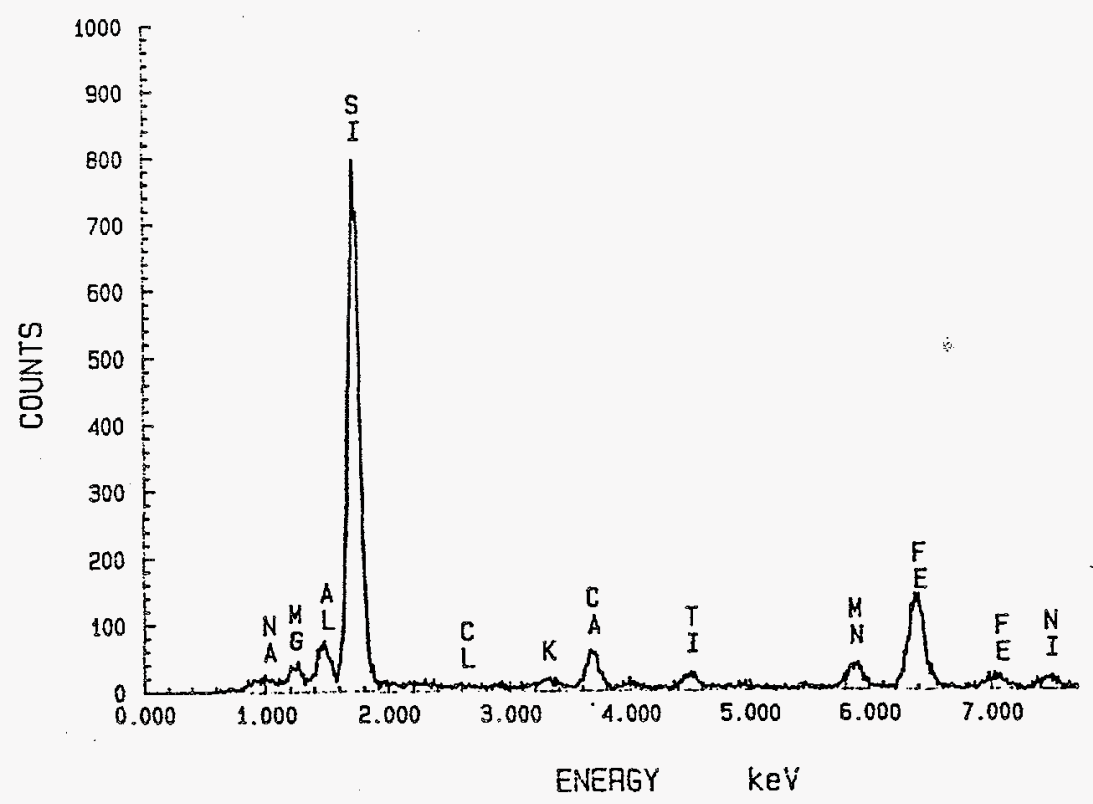

Fig. 27. AEM/EDS Spectra from SRL 202U Glass Samples Used in Vapor Hydration Testing: (a) Unaltered Glass and (b) Altered Clay Layer 
Table 21. SEM/EDS Compositional Analysis of Clay Layer and Unaltered Glass from SRL 202U Sample Reacted in Vapor Hydration Test for 14 Days at $200^{\circ} \mathrm{C}$

\begin{tabular}{lcccc}
\hline & & & Element & Oxide \\
Sample & Element & Atomic \% & Wt \% & Wt $^{\mathrm{a}}$ \\
\hline \multirow{3}{*}{ Clay Layer } & & & & \\
& $\mathrm{Si}$ & 25.14 & 32.41 & 69.46 \\
& $\mathrm{Al}$ & 2.29 & 2.84 & 5.37 \\
& $\mathrm{~K}$ & 0.36 & 0.65 & 0.79 \\
& $\mathrm{Ca}$ & 1.66 & 3.05 & 4.27 \\
& $\mathrm{Mn}$ & 0.99 & 2.50 & 3.96 \\
$\mathrm{Mg}$ & 0.91 & 1.00 & 1.67 \\
& $\mathrm{Fe}$ & 3.65 & 9.42 & 13.45 \\
& $\mathrm{Na}$ & 0.72 & 0.76 & 1.02 \\
& & & & \\
& $\mathrm{Si}$ & 24.53 & 31.55 & 67.62 \\
& $\mathrm{Al}$ & 2.62 & 3.24 & 6.13 \\
$\mathrm{~K}$ & 1.63 & 2.92 & 3.52 \\
& $\mathrm{Ca}$ & 0.64 & 1.18 & 1.66 \\
& $\mathrm{Mn}$ & 1.02 & 2.57 & 4.07 \\
& $\mathrm{Mg}$ & 0.59 & 0.65 & 1.08 \\
& $\mathrm{Fe}$ & 3.57 & 9.18 & 13.11 \\
& $\mathrm{Na}$ & 1.98 & 2.09 & 2.82 \\
\hline
\end{tabular}

${ }^{\mathrm{a}} \mathrm{Fe}$ as $\mathrm{Fe}_{2} \mathrm{O}_{3}$. 


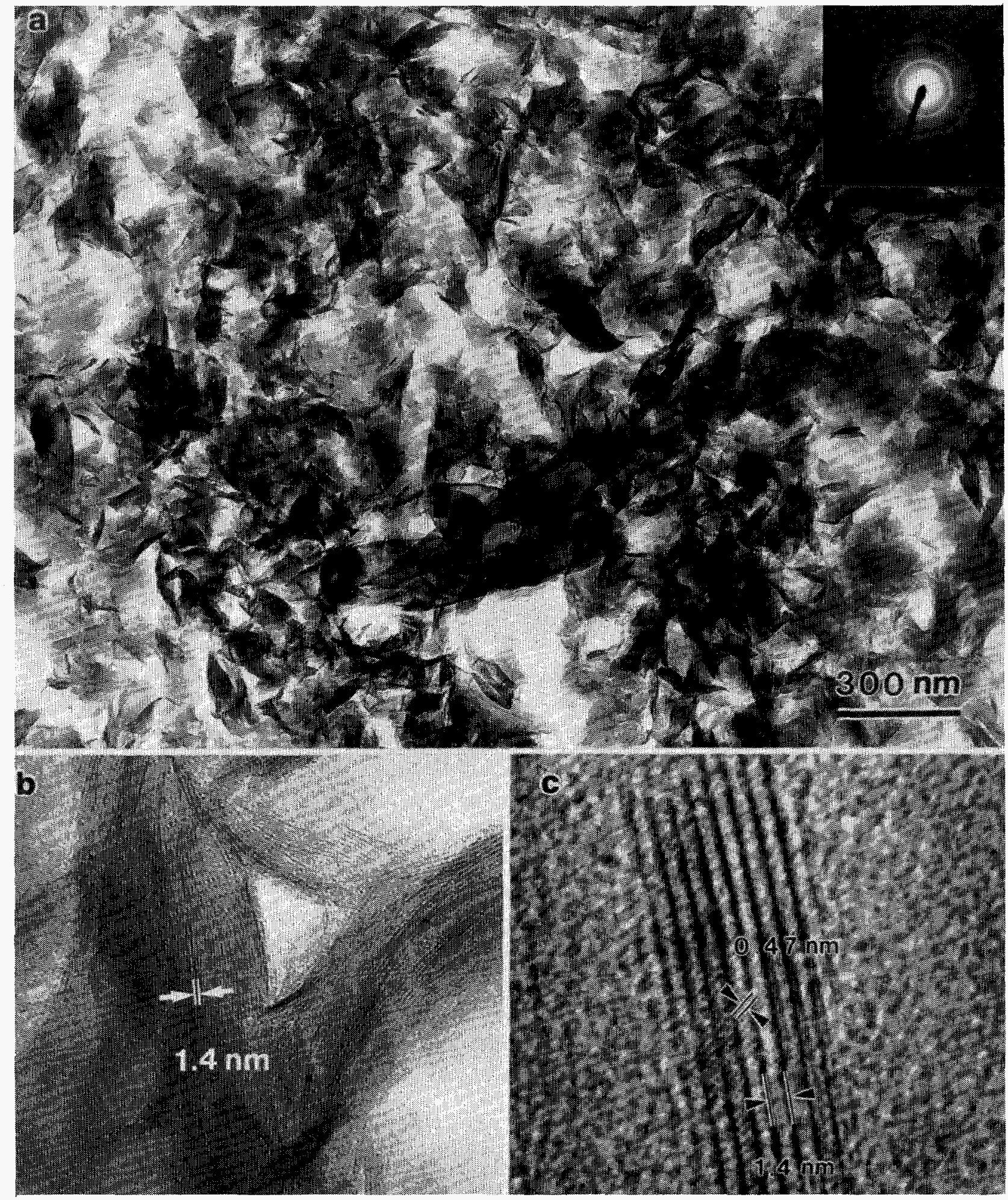

Fig. 28. AEM Images of Slightly Porous Smectite Layer from SRL 202U Glass Reacted for 14 Days in Saturated Vapor Environment at $200^{\circ} \mathrm{C}$. (a) Low-magnification image with SAED pattern, inset to upper right, and (b) and (c) high-resolution electron micrographs showing lattice fringe spacings in smectite layer. 
(a)

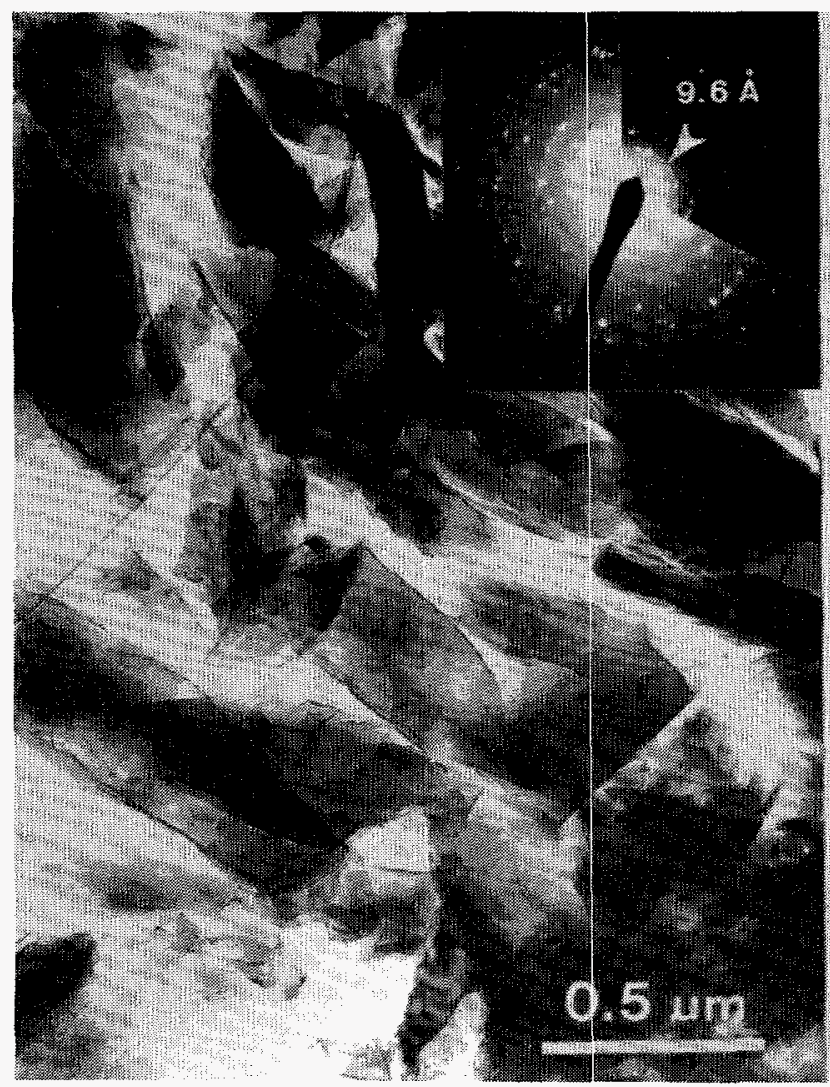

(b)

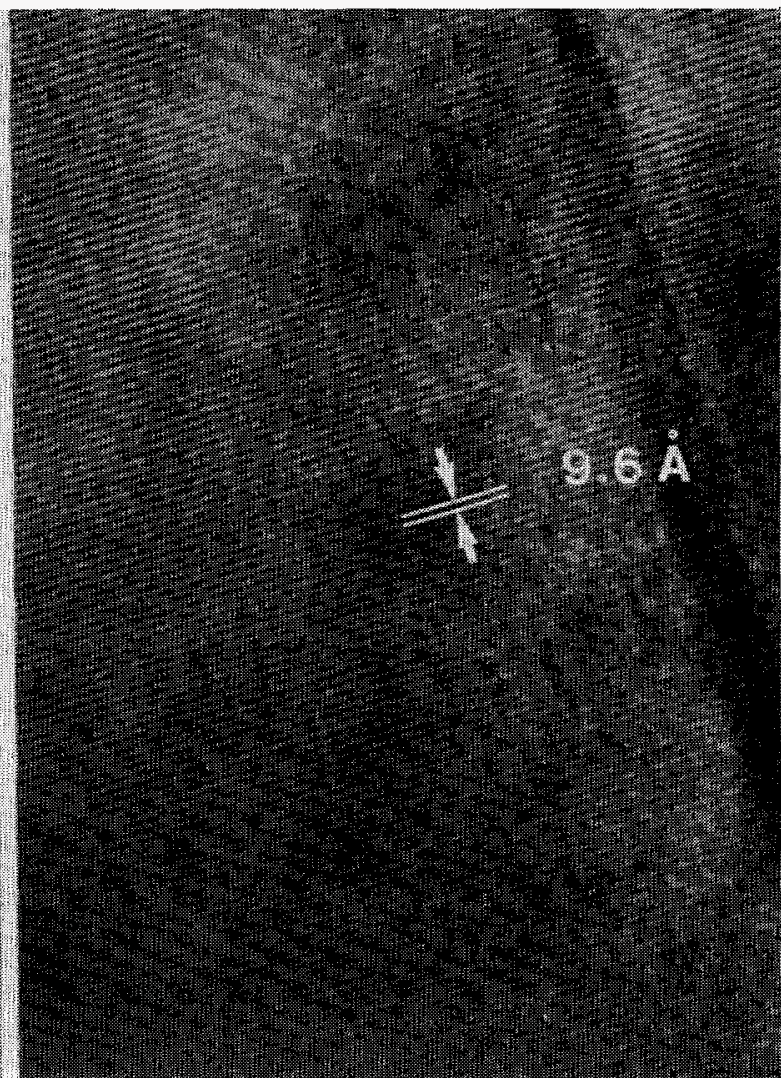

Fig. 29. AEM Images of Na-Rich Chabazite (var. Herschelite) Phase from SRL 202U Glass Reacted for 14 Days in Saturated Vapor Environment at $200^{\circ} \mathrm{C}$. (a) Lowmagnification image with SAED pattern, inset to upper right, and (b) high-resolution electron micrograph image showing lattice fringe spacings of Na-rich chabazite. 
(a)
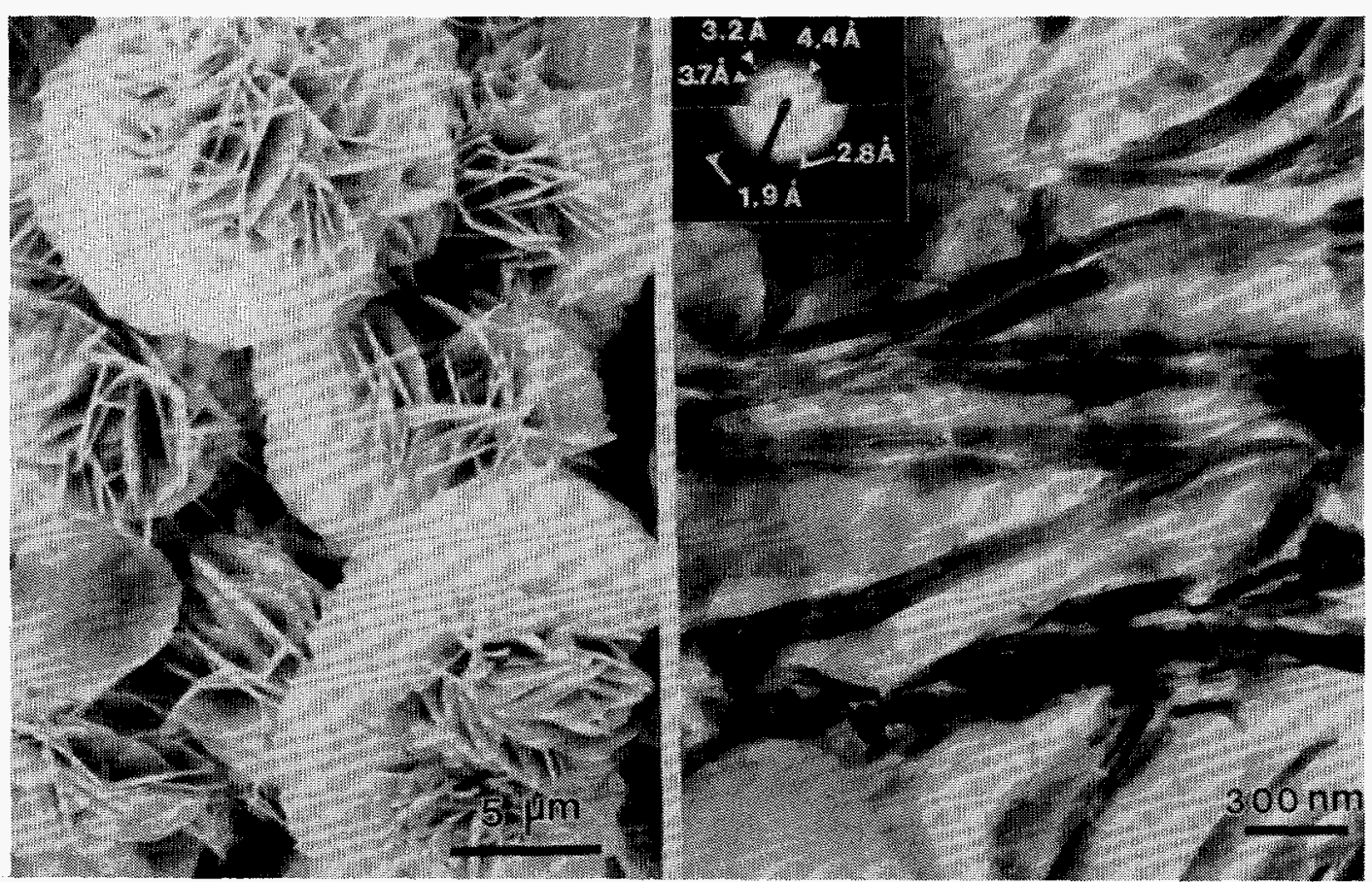

Fig. 30. Photomicrographs of Unidentified Ca-Si Phase from SRL 202U Glass Reacted for 14 Days in Saturated Vapor Environment at $200^{\circ} \mathrm{C}$. (a) SEM image showing platy habit and (b) low-magnification transmission electron image of individual platy grains with SAED pattern, inset to upper left.

(b) 
(a)

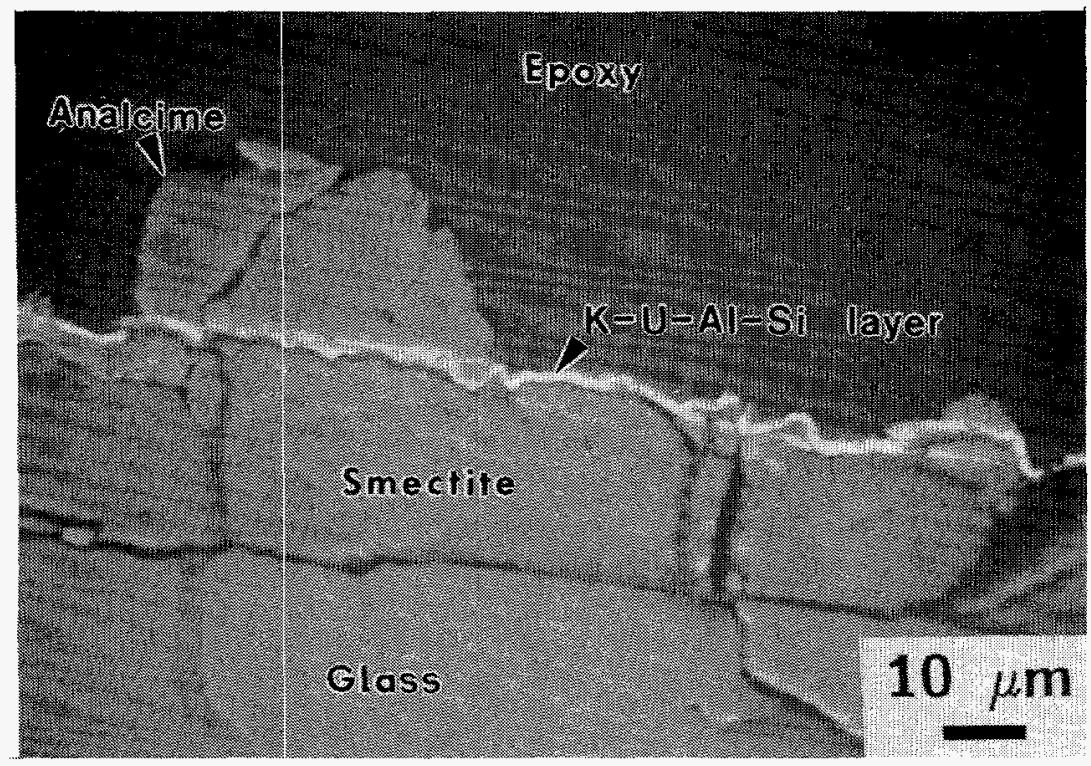

(b)

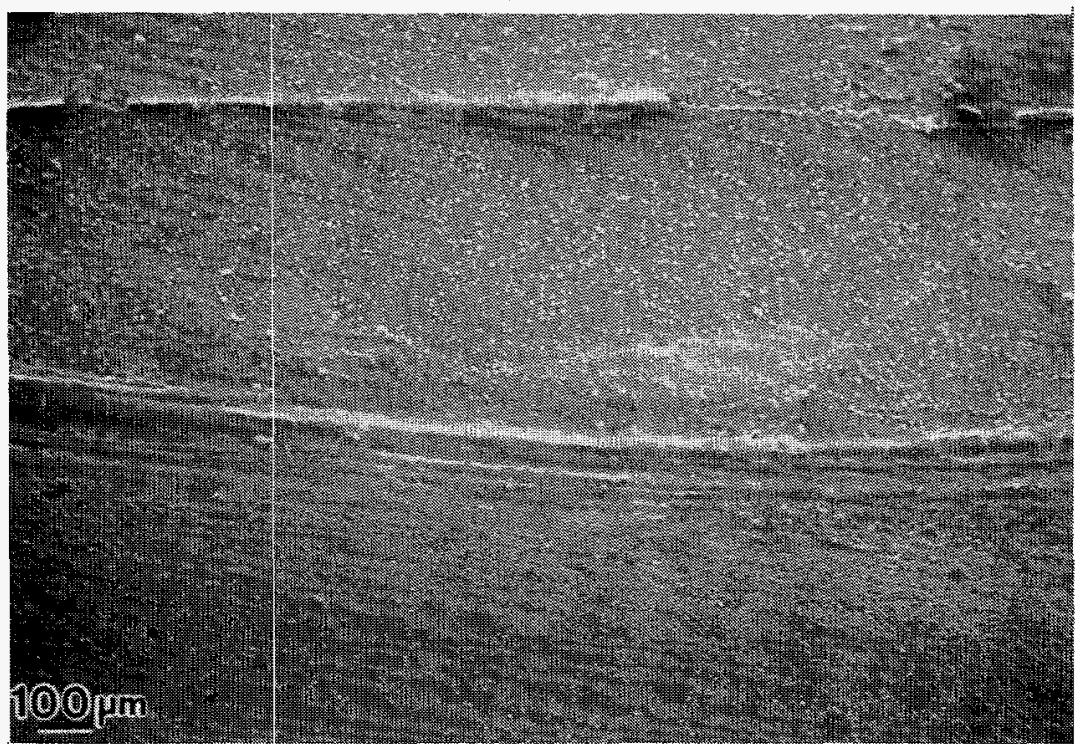

Fig. 31. SEM Images of Secondary Alteration Phases Formed on SRL 202U Glass Reacted for 21 Days in Saturated Vapor Environment at $200^{\circ} \mathrm{C}$. (a) Backscattered electron image of polished cross section showing (from bottom to top) unaltered glass, smectite clay layer, thin K-U-Al-Si outer layer, large isolated analcime crystal, and epoxy (750X). Note fractures progressing through smectite layer and into glass. (b) Surface of successive ridges of silicon-rich crust (secondary electron image, 50X). 
After 21 days, the glass samples begin to display irregular-banded zones of precipitates. These bands occur as crusty white layers, which apparently demarcate the edge of water films that have migrated across the glass surfaces at various times (Fig. 31b). The SEM/EDS analysis of the white bands indicate the presence of an amorphous silicon-rich material with minor amounts of $\mathrm{K}, \mathrm{Na}, \mathrm{Ca}, \mathrm{Al}, \mathrm{Fe}$, and $\mathrm{Mn}$ (Fig. 32a; Fe and Mn not shown). Desiccation cracks that formed on these ridges indicate that this band may be composed of a hydrated silicon-rich phase.

Several distinct crystalline phases were also observed on these surfaces. The most abundant phase on the samples is the unidentified $\mathrm{Ca}-\mathrm{Si}$ rosette that was so prominent on the 7-and 14-day samples. The Ca-Si rosette phase on the 21-day samples also contained minor amounts of $\mathrm{K}, \mathrm{Na}$, and $\mathrm{Fe}$ and displayed a well-developed platy habit. Also present, but less abundant, are phillipsite, analcime (Fig. 32b), weeksite, K-Al-Si rosettes, a K-Fe-Si "mosslike" overgrowth, unidentified $\mathrm{Na}-\mathrm{K}-\mathrm{Ca}-\mathrm{Al}-\mathrm{Si}$ needles (Fig. 32c), and unidentified $\mathrm{Na}-\mathrm{K}-\mathrm{Ca}-\mathrm{U}-\mathrm{Si}-\mathrm{Fe}$ fibers (Fig. 32d). The identification of phillipsite in this sample had been confirmed by XRD diffraction patterns (Table 20; sample IVE202U-21-1b). Portions of the sample surface, including the precipitate phases, appeared to be covered with a salt film. This film may have given rise to some of the alkali and alkaline earth enrichments noted in these samples. The AEM investigations also revealed the presence of an amorphous $\mathrm{Ca}-\mathrm{Si}-\mathrm{O}$ material.

The discontinuous uranium-enriched layer first observed on the 14-day samples (Fig. 31a) had evolved into a layer that enveloped most of the sample in the present tests. This layer was enriched in $\mathrm{Si}, \mathrm{K}, \mathrm{U}$, and $\mathrm{Fe}$ and was localized on the outer smectite clay surface, but sometimes was itself overlain by other precipitate phases.

\section{d. $\quad$ SRL 202U Samples after 35 Days}

The surfaces of the 35-day samples displayed continued growth of the alteration layer and precipitate material. Layer development appeared to be more uniform on these samples relative to those of previous tests (see Fig. 23d). Composite layer thicknesses measured during SEM examinations averaged $54.8 \pm 10.9 \mu \mathrm{m}$ (Table 19). The composite layer was now clearly segregated into two distinct layers, an outer illite and inner smectite layer of clays (Fig. 33).

The outermost illite layer was relatively thin, with layer measurements indicating considerable variation in thickness. The illite layer averaged about $4 \mu \mathrm{m}$ in thickness but varied from being completely absent to being about $25-\mu \mathrm{m}$ thick (Fig. 33a). The SEM/EDS compositional analyses revealed an enrichment of potassium in the outer layer (Fig. 34a) relative to the underlying smectite layer (Fig. 34b). The XRD analysis from similar precipitate material taken from a 56-day sample confirms that this layer is composed of the clay mineral illite (Table 20; sample IVE202U-56-2b), a finding consistent with the potassium enrichment observed in SEM/EDS. The illite layer displayed poor adherent properties with respect to the underlying clay layer, and in many locations could be seen to be delaminating from the sample surface (Fig. 33b). Most of this delamination occurred after the tests were terminated and the samples had been allowed to air dry. The illite displayed a high reflectance of light (high albedo) during optical examinations and was relatively porous when compared to the underlying layer (Fig. 33a). In some locations the illite layer appeared to coat some of the large precipitate minerals, while in other locations, mineral precipitates were clearly overlying the illite layer. Small amounts of illite were also detected on the support wires for these sample, indicating that this phase precipitated out of the solution that was in contact with the glass. This mode of formation differs from that of the underlying smectite layer, which appeared to have formed in situ. 

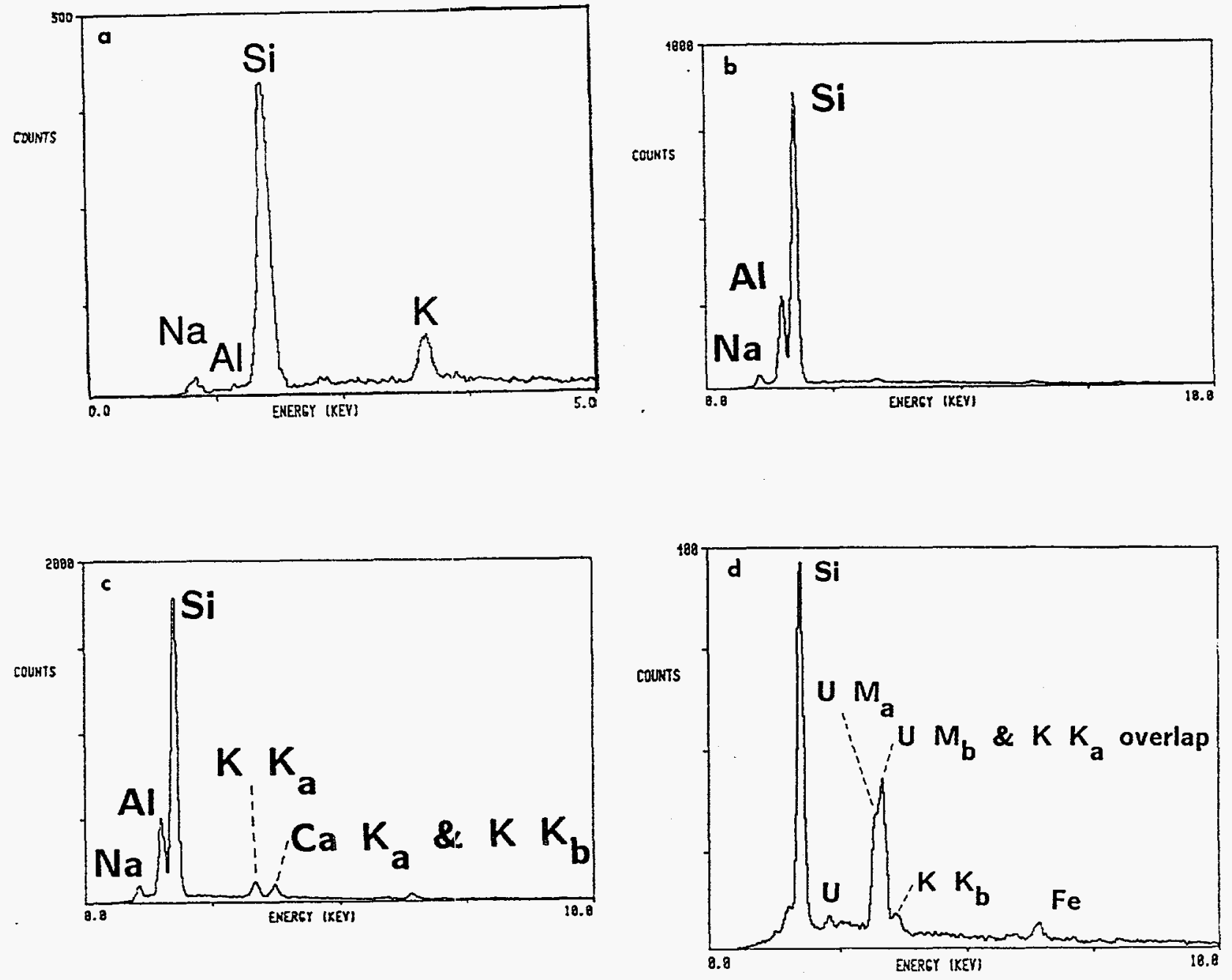

Fig. 32. SEM/EDS Spectra of Phases from SRL 202U Samples Reacted For 21 Days in Saturated Vapor Environment at $200^{\circ} \mathrm{C}$ : (a) Si-Rich Crust, (b) Analcime, (c) Unidentified Na-K-Ca-A.l-Si Needles, and (d) Unidentified Na-K-Ca-U-Si-Fe Fibers. All spectra taken at $20 \mathrm{keV}$ accelerating potential. 
(a)

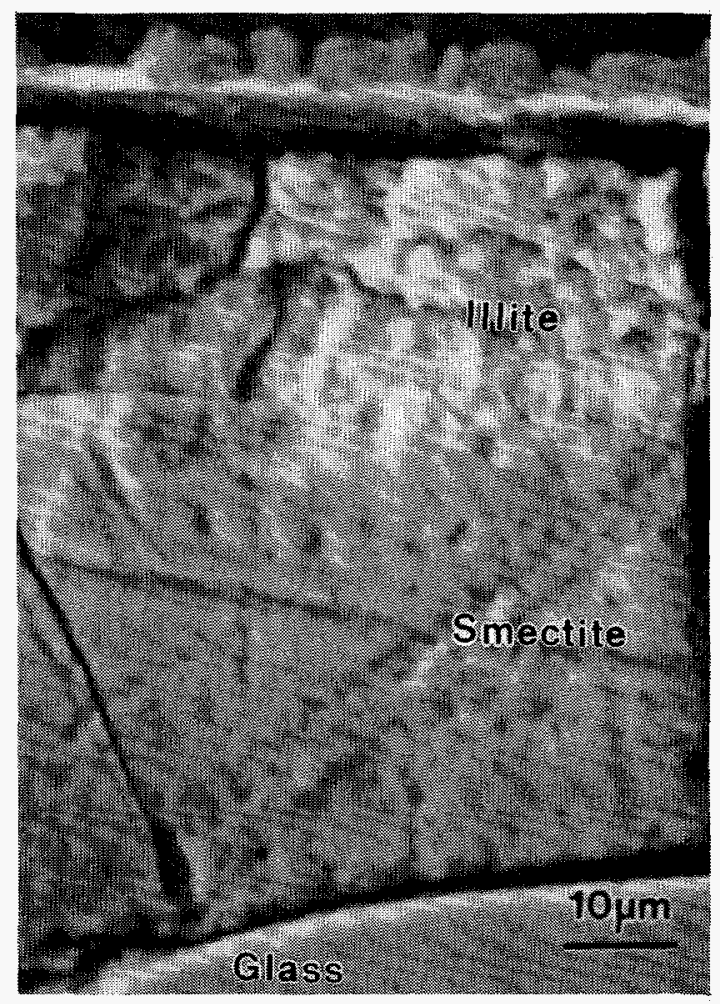

(b)

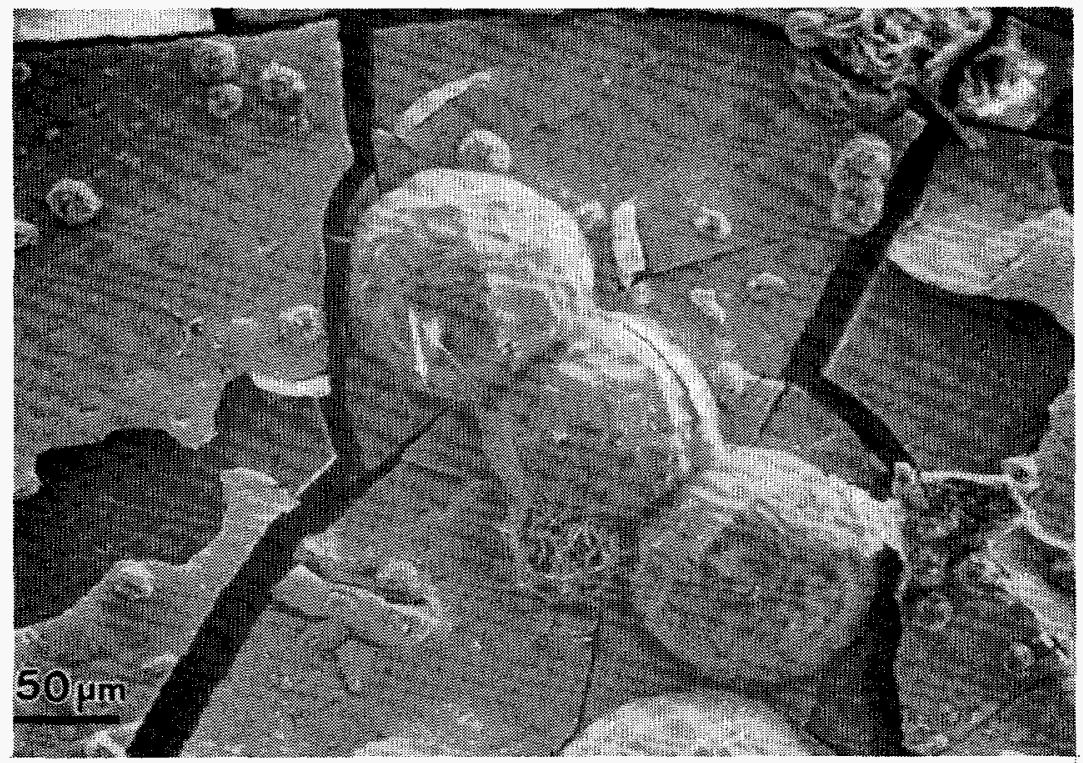

Fig. 33. SEM Images of Secondary Alteration Phases Formed on SRL 202U Glass Reacted for 35 Days in Saturated Vapor Environment at $200^{\circ} \mathrm{C}$. (a) Backscattered electron image of polished cross section showing (from bottom to top) unaltered glass, inner smectite layer, outer illite layer, precipitate cover, and epoxy (1000X). (b) Reacted sample surface displaying illite layer detaching from surface, underlying smectite layer, and large rounded adularia grains (secondary electron image, 200X). 

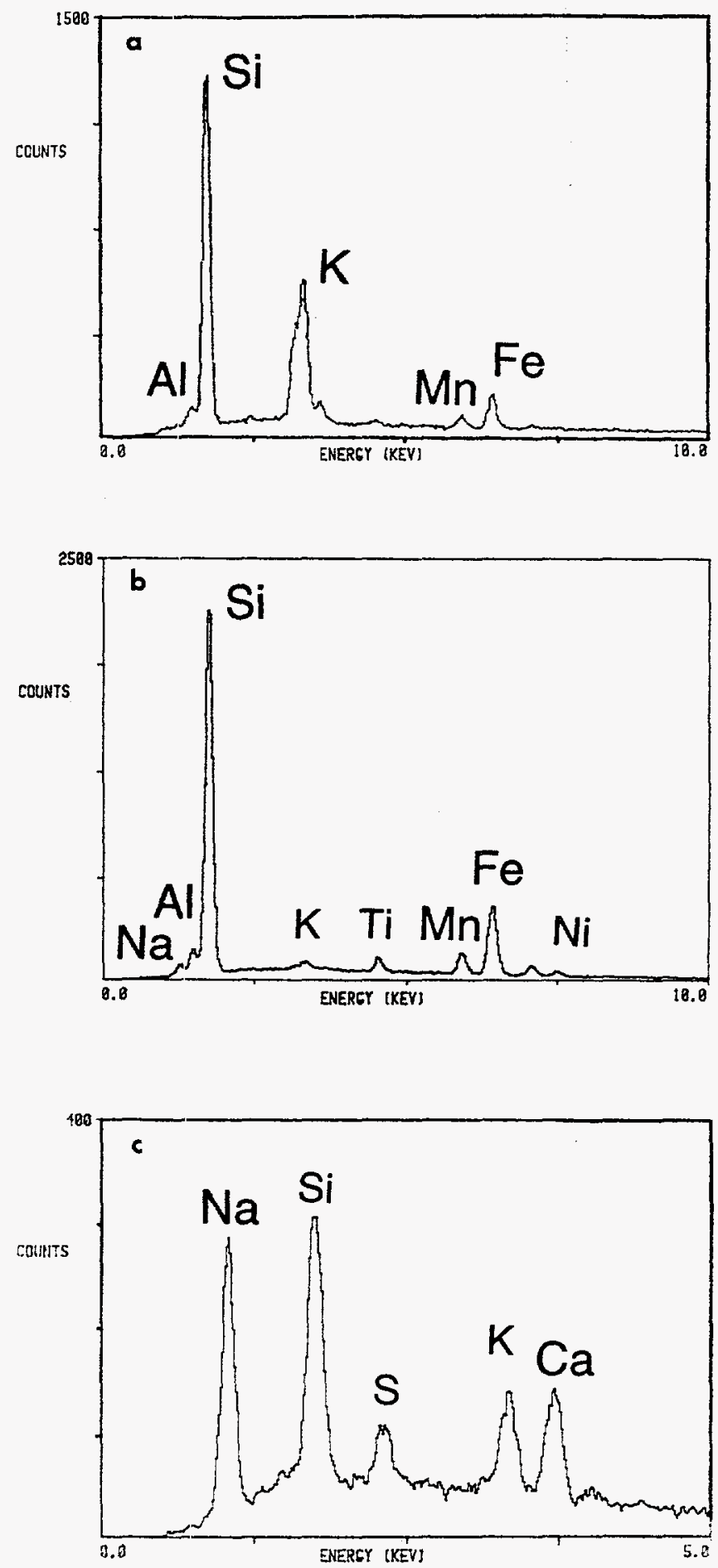

Fig. 34. SEM/EDS Spectra of SRL 202U Glass from 35-Day Vapor Hydration Test: (a) Illite Clay Layer, (b) Smectite Clay Layer, and (c) Unidentified Na-Si-K-Ca-S Needles. All spectra taken at $20 \mathrm{keV}$ accelerating potential. 
The underlying layer displayed the same SEM/EDS composition as previous tests, indicating the presence of smectite clays of probable nontronite and/or ferroan saponite composition (Fig. 34b). The smectite layer was of a relatively uniform thickness, with measured layer thicknesses averaging $51 \pm 8.6 \mu \mathrm{m}$ (Table 19). This layer also appeared to be more compact (and thus presumably less permeable) than the overlying illite clay layer (Fig. 33a). In some locations, especially along the corners of the samples, the smectite material had penetrated into the sample along fractures that penetrated into the glass.

Both SEM/EDS and AEM examinations also revealed the continued presence of the U-K-enriched outer layer on these samples. The SEM examination in backscattered electron mode indicates that this layer is less conspicuous than previously observed in the 21-day samples. The cumulative reaction layer was $\sim 50 \mu \mathrm{m}$ thick, as measured by AEM, and the layer had become rather discontinuous, being present on only a portion of the sample surface. It appears that the uranium added to this layer on the 21-day samples was now being incorporated into distinct uranyl silicate phases, as described below. The potassium from this layer was probably being incorporated into either the uranyl alkali silicate phases or the illite layer.

The most conspicuous precipitate phase present on the 35-day samples is large crystals of euhedral analcime that are several hundreds of microns in diameter. These crystals appear as clear, vitreous grains that often take on a gray or cloudy coloration when they form overgrowths on previously deposited phases. Clear, multi-faceted crystals often display a compound "bugs-eye image" of underlying grains. The SEM/EDS examination of this phase reveals a pure $\mathrm{Na}-\mathrm{Al}-\mathrm{Si}$ composition similar to that of analcime grains observed on the 21-day samples (see Fig. 32b). Several different analcime crystal morphologies were noted on the samples, including equidimensional, convex, multi-faceted crystals and multi-faceted crystals with concave central depressions ("dimpled analcime,; see Fig. 35a). The equidimensional convex crystals were concentrated adjacent to the hanging thread and/or the down-hanging portion of the sample, while the dimpled analcime grains were associated with the top-hanging portions of the samples, in regions away from the Teflon thread. This spatial segregation of crystal morphologies suggests that the dimpled analcime formed where the water film covering the sample was of insufficient depth to completely envelop the growing crystals. Where the surface film was of a sufficient depth, the analcime grains formed the convex-shaped equidimensional grains. These patterns of crystal growth indicate that the film of water contacting the samples varied in thickness, with the thickest portions of the film occurring at the bottoms of the samples due to the influence of gravity, and adjacent to the hanging thread/wire due to the electrostatic forces of water molecules.

Several different varieties of crystalline $\mathrm{Ca}-\mathrm{Si}$ phases were identified on the SRL 202U samples. The SEM/EDS examinations indicate that these phases may contain variable, but relatively minor concentrations of $\mathrm{Na}, \mathrm{K}$, and/or $\mathrm{S}$. The most common crystalline variety occurs as euhedral, stellar masses and is identical in composition and morphology to $\mathrm{Ca}-\mathrm{Si}$ rosettes previously found on the 7-, 14-, and 21-day samples. The XRD diffraction obtained from these samples could not be matched with existing standards in the JCPDS data file. The remaining varieties of $\mathrm{Ca}-\mathrm{Si}$ crystals are relatively minor in abundance. They differ from the rosette-shaped grains in having a sinuous crystalline texture to more fibrous, needle-like varieties. The XRD spectra obtained from the needle-like grains indicate the presence of tobermorite $\left[\mathrm{Ca}_{5}(\mathrm{OH})_{2} \mathrm{Si}_{6} \mathrm{O}_{16} \cdot 4 \mathrm{H}_{2} \mathrm{O}\right]$. It is not known if any of the remaining unidentified crystal types are polymorphous varieties of the $\mathrm{Ca}-\mathrm{Si}$ rosettes or tobermorite, or if they were completely different phases altogether. Some variations in SEM/EDS compositions were noted, but some of these differences could be attributed to spectral interferences from adjacent mineral grains and/or surface-covering salt deposits. 


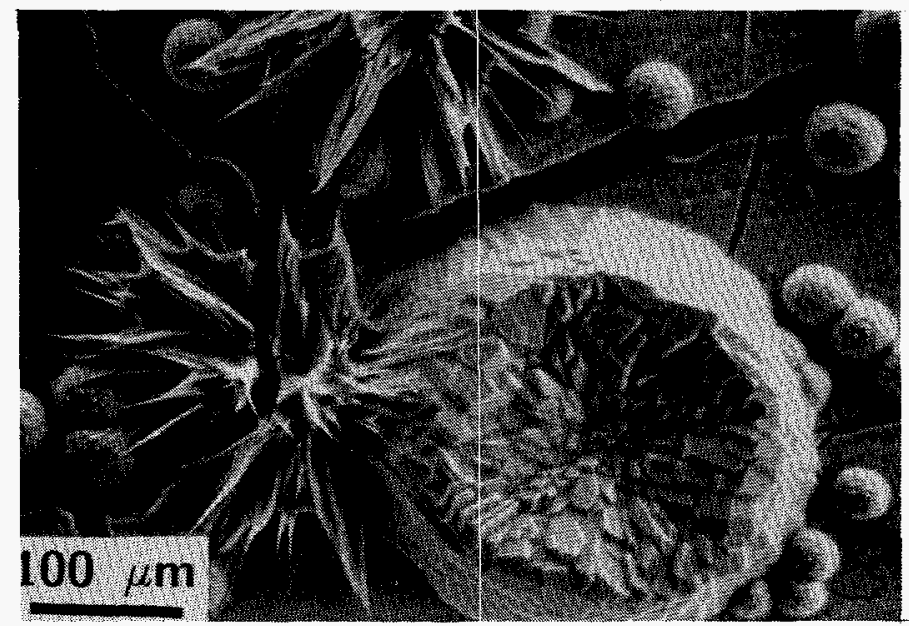

(a)

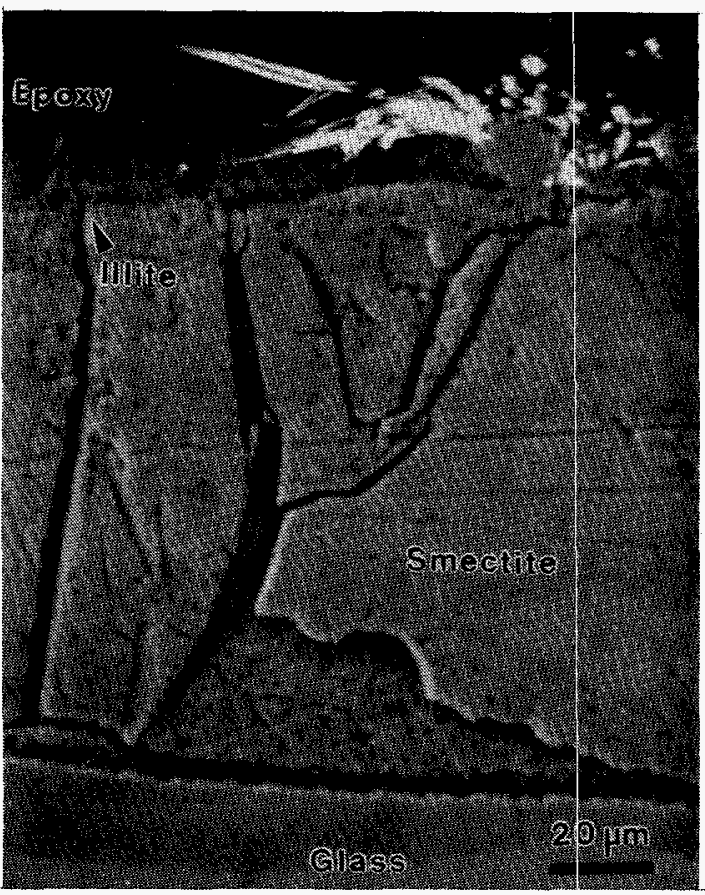

(b)

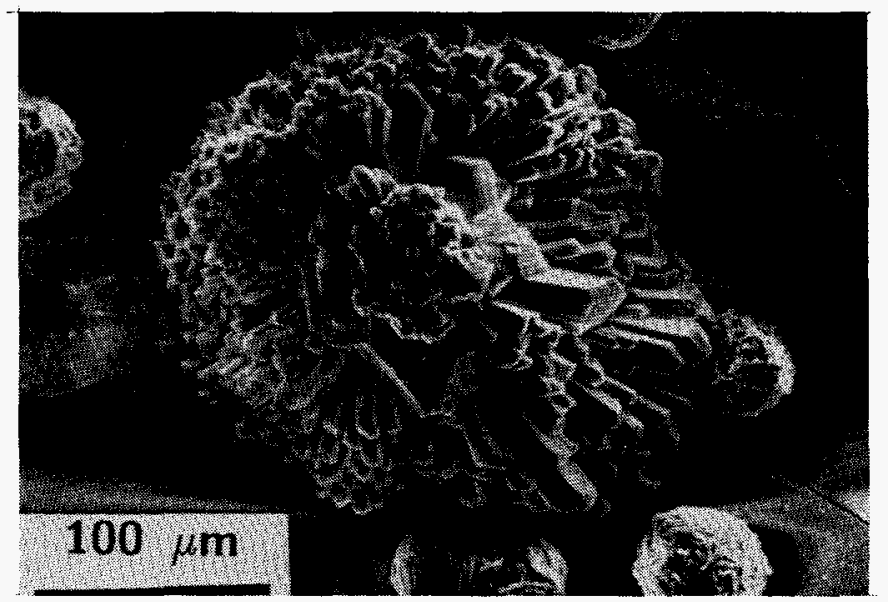

(c)

Fig. 35. SEM Images of Secondary Alteration Phases Formed on SRL 202U Glass from Vapor Hydration Tests. (a) Large dimpled analcime, unidentified Na-Si-K-Ca-S needles, and small rounded adularia grains lying on fractured clay surface ( 35 day, secondary electron image, 250X). (b) Large rounded agglomerate of phillipsite needles, rounded adularia grains, and unidentified $\mathrm{Ca}-\mathrm{Si}$ rosettes ( $35 \mathrm{day}$, secondary electron image, 250X). (c) Backscattered electron image of polished cross section showing (from bottom to top) unaltered glass, heterogeneous smectite layer, outer illite layer, precipitate cover, and epoxy (56 day, backscattered electron image, $500 \mathrm{X})$. 
Webbed-like, vitreous, radiating crystals are also noted to occur in numerous patches across the sample surfaces (Fig. 35a). Individual grains are typically 100 to $200 \mu \mathrm{m}$ in diameter, and where the grains grow in large accumulations they often coalesce to form white, "hair-like" mats. The SEM/EDS examinations of this phase indicate a $\mathrm{Na}$-Si-dominated composition with lesser $\mathrm{K}, \mathrm{Ca}$, and $\mathrm{S}$ (Fig. 34c). The absence of aluminum in these grains is also particularly diagnostic. The fibrous radiating habits combined with the $\mathrm{Na}$ and Si compositions suggest that this phase may be a Na-rich amphibole mineral.

Numerous K-Al-Si buttons were noted on the 35-day sample surfaces. These grains occur as spherical masses with small blocky crystals projecting from the sample surface (Figs. 33b and 35a). Morphologically, this phase is similar to the K-Al-Si blocks found on the 14-day samples, and K-Al-Si rosettes first observed in the 21-day samples. The XRD patterns have confirmed that this precipitate is a $\mathrm{K}$-feldspar phase (Table 20; sample IVE202U-35-1a). Although the SEM/EDS and XRD data cannot determine specifically which variety of potassium-feldspar mineral has formed on these samples, the experimental temperature of $200^{\circ} \mathrm{C}$ suggests that the most likely potassium-feldspar phase to be stable under these conditions is adularia [49].

Phillipsite grains previously noted on the 14- and 21-day samples had evolved into relatively large masses of radiating rod-shaped crystals (Fig. $35 \mathrm{~b}$ ). Combined morphological, compositional, and XRD characteristics have been used to identify this phase. Phillipsite $\left[(\mathrm{K}, \mathrm{Na})_{10}\left(\mathrm{Al}_{10} \mathrm{Si}_{22}\right) \cdot 20 \mathrm{H}_{2} \mathrm{O}\right]$ and merlinoite $\left[\mathrm{K}_{5} \mathrm{Ca}_{2}\left(\mathrm{Al}_{9} \mathrm{Si}_{23} \mathrm{O}_{64}\right) \cdot 24 \mathrm{H}_{2} \mathrm{O}\right]$ both display compositional, structural, and morphological similarities to the phase present on the test samples. Differentiation of these two phases is difficult in potassium-rich systems, and merlinite has often been mistakenly identified as phillipsite [50]. The basis used here to identify the phase as phillipsite was based on the XRD patterns (Table 20; sample IVE202U-21-1b). The XRD patterns for the two phases are similar, except that merlinoite has two additional lines at $d=10.0$ and $4.48 \AA$. The intensities of these two lines obtained from samples in our study are much weaker than those noted in the JCPDS merlinoite data files. Because of these data, the phase present on this sample was judged to be potassium-rich phillipsite. However, the presence of merlinoite could not be ruled out on these samples, since diffraction data were obtained from only a limited number of specimens, and the phillipsite observed often displayed changing morphologies throughout the testing periods.

Several trace phases have also been identified during examination of these altered glass surfaces. The uranyl silicate phase, weeksite $\left[\mathrm{K}_{2}\left(\mathrm{UO}_{2}\right)_{2}\left(\mathrm{Si}_{2} \mathrm{O}_{5}\right)_{3} \bullet 4 \mathrm{H}_{2} \mathrm{O}\right]$, has been identified in SEM/EDS examinations. This phase appears as long acicular yellow crystals, although short prismatic grains thought to be weeksite have also been noted during SEM/EDS examination. Also present are a Na-K-Ca-U-Si phase, similar in morphology and composition to the phase previously described for the 21 -day tests, except that in the present specimens, iron is absent.

\section{e. SRL 202U Samples after 56 Days}

The surface of the 56-day sample was relatively similar in appearance to that of the 35-day sample. The proportion of surface precipitates and the covering of the illite clay layer appear to have continued to increase with time, as evidenced in the relative increases of these materials on the present sample (Fig. 23e). The samples continued to display increasingly thicker and more uniform layer thicknesses, with layer measurements during SEM examinations averaging $132.1 \pm 14.8 \mu \mathrm{m}$ (Table 19). The composite layer continues to be segregated into an outer illite clay and inner smectite layers (Fig. 35c). These two layers individually were less consistent in thickness than the composite layer as a whole, with average 
thicknesses of $37.2 \pm 28.3$ and $94.9 \pm 30.2 \mu \mathrm{m}$, respectively. The inner smectite layer also began to display variations in texture, with a highly irregular and localized porous zone developing adjacent to the glass (Fig. 35c). The SEM/EDS examinations did not reveal any compositional variations between the two different textured regions of the smectite layer.

Surface examinations of the clays revealed several distinct compositions. In addition to the enveloping illite and smectite layers, other isolated "clay-like" patches included (1) a K-Fe-Mn-Al-Si composition similar to that observed on the 7-and 21-day samples, (2) small star-like raised projections with a Ca-K-Fe-P-Si composition, and (3) a K-U-Al-Si composition clay or mixture of clay and other crystals (Fig. 36). This later phase(s) appeared as a folded blanket along the outer edges of the glass wafer, suggesting that this layer may have become unstable and was beginning to slough off the unaltered glass surface during the reaction (Fig. 37a). This blanket material appears to be the same composition as that of the U-K enriched layer previously observed on the 14-, 21-, and 35-day tests.

As with the 35-day samples, analcime and the Ca-Si rosette are the most common precipitate phases to occur on the 56-day samples, with both phases displaying more coarse crystalline textures in these latter tests.

The adularia buttons first identified in the 35-day samples continue to exhibit changing crystalline habits. The XRD patterns from the 35-day samples have confirmed that this precipitate is a K-feldspar phase. The present grains occur as agglomerates of blocky crystals that are intergrown into button-shaped mounds (Fig. 37b). Despite the different morphologies, these grains are compositionally similar to the adularia present on the 35-day sample surfaces. In some instances, the individual blocks also appear to be delaminating along a preferred crystalline axis (Fig. 37c), possibly indicating an episode of corrosive activity.

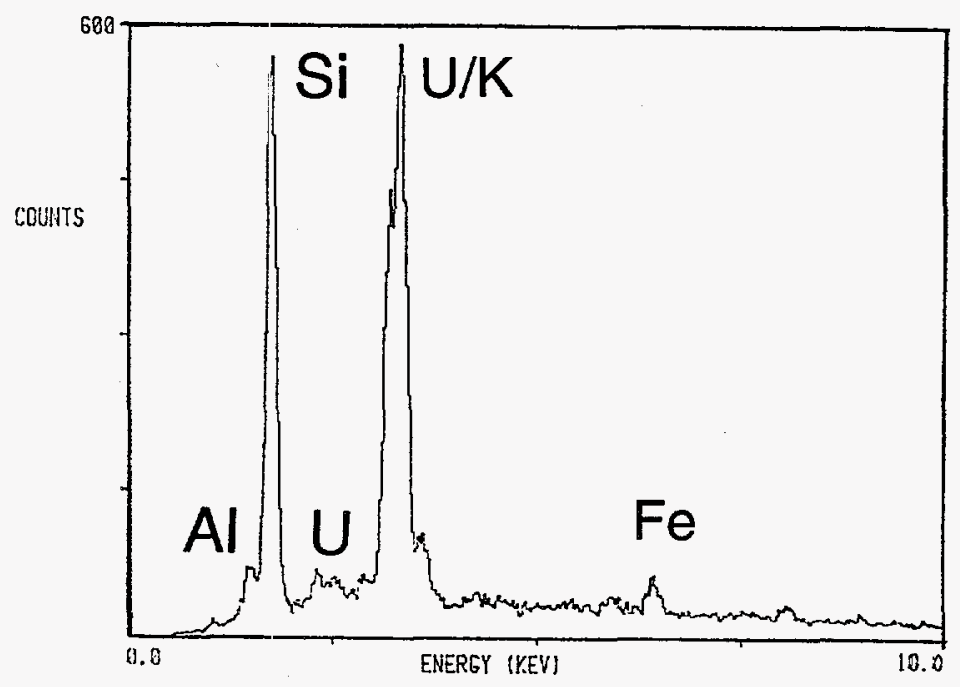

Fig. 36. SEM/EDS Spectrum of SRL 202U Sample from Vapor Hydration Test at 56 Days: K-U-Al-Si Layer Material 


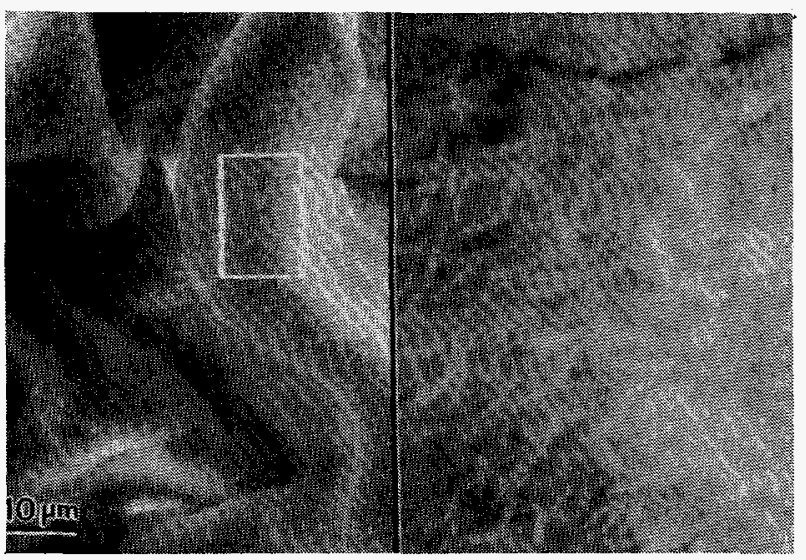

(a)

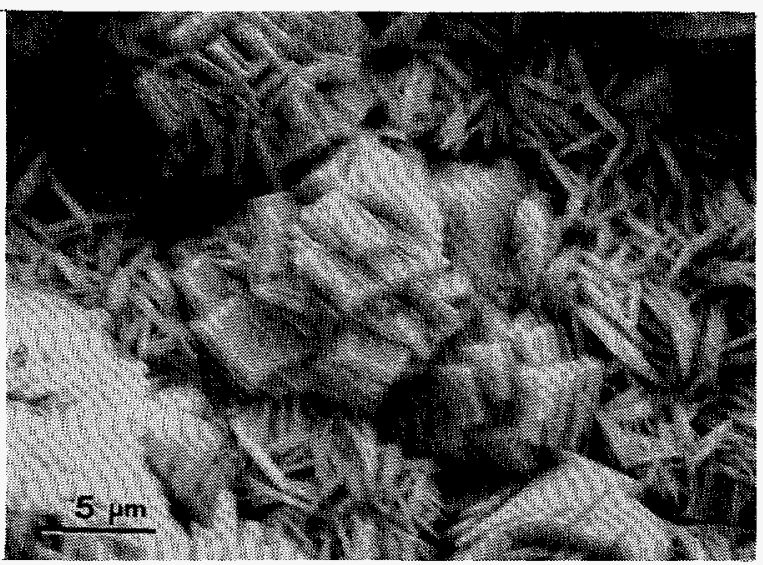

(c)

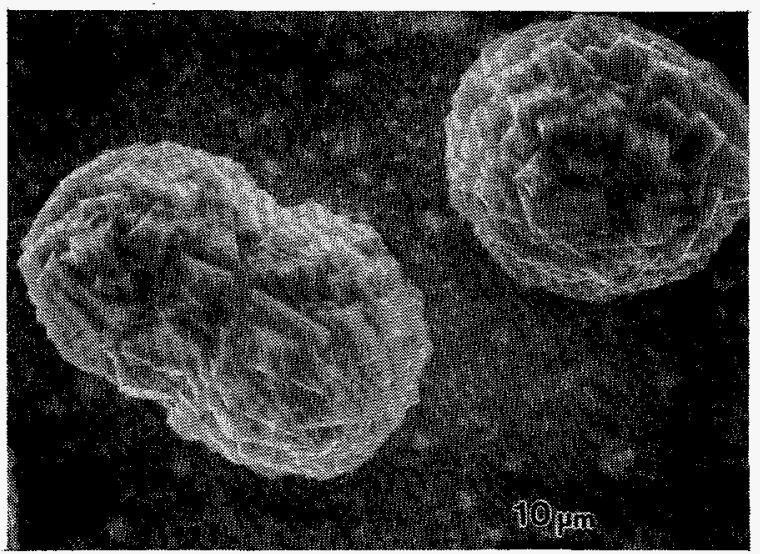

(b)

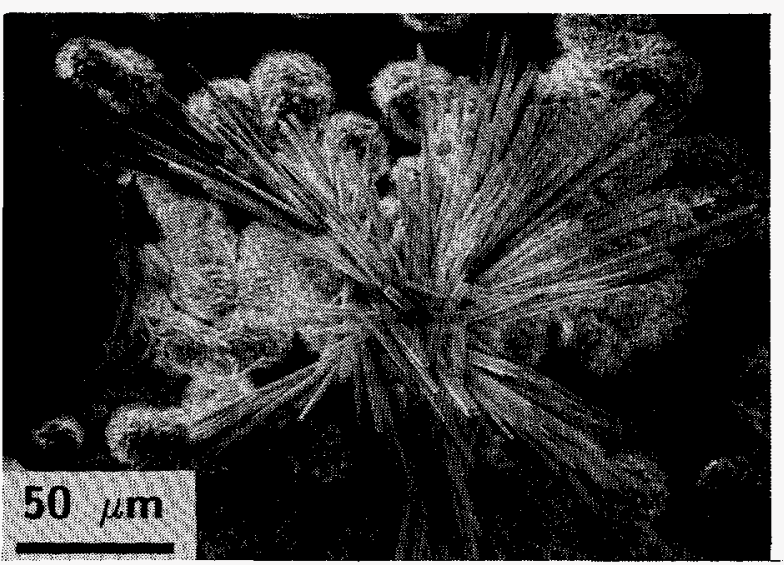

(d)

Fig. 37. SEM Images of Secondary Alteration Phases Formed on SRL 202U Glass from Vapor Hydration Test at 56 Days. All images in secondary electron mode. (a) Folded blanket-like layer of clay surface along sample edge $(1000 \mathrm{X})$, inset to right displays detailed image of fine-grained clays in area outlined in left photo $(5000 \mathrm{X})$. (b) Adularia grains on clay surface $(1250 \mathrm{X})$. (c) Delaminating adularia grains (center) and weeksite needles (2500X). (d) Tobermorite needles, unidentified Ca-Si phase, and large analcime grain (left center) (350X). 
The occurrence of weeksite has become much more prevalent on these longer term samples. This phase was identified as long, yellow-to-green crystals readily observed during optical examination. A specimen of weeksite exposed during the crosssectioning of the 56-day sample is apparent in Fig. 35c, while a very-fine grained, unusual morphology of weeksite needles forms a mat like deposit visible in Fig. 37c.

The 56-day samples also developed localized as semblages of fairly largesized accumulations of white acicular crystalline masses (Fig. 37d). These grains displayed a $\mathrm{K}-\mathrm{Ca}-\mathrm{Al}-\mathrm{Si}$ composition during SEM/EDS analysis (Fig. 38a). The XRD analysis of similar material from this and the previously described 35-day samples indicates the presence of tobermorite (Table 20; sample IVE202U-35-2a). The presence of potassium in this phase, combined with the crystalline morphology, also suggests the possibility of mordenite $\left[\mathrm{Na}_{3} \mathrm{KCa}_{2}\left(\mathrm{Al}_{8} \mathrm{Si}_{4} \mathrm{O}_{96}\right) \cdot 28 \mathrm{H}_{2} \mathrm{O}\right]$; however, no confirmatory mordenite XRD patterns had been obtained from this sample.

Several other minor assemblages were noted to occur on these samples, including (1) Ca-Sr feathers and buttons (Figs. 38b and 39a), (2) honeycomb-textured $\mathrm{Ca}-\mathrm{Sr}-\mathrm{Na}-\mathrm{S}$ (anhydrite?) needles (Figs. 38c and 39b), and (3) Ca-Si anemone-like projections with a composition similar to that of the $\mathrm{Ca}$-Si rosettes (Figs. 38d and 39c). None of these latter phases could be isolated for XRD analysis because of their rare occurrence and relatively small crystal sizes.

\section{f. SRL 202A Samples after 7 Days}

The SRL 202A glass samples at 7 days rapidly developed a thick white coat of alteration minerals after the samples were exposed to the irradiated test atmosphere (Fig. 40a). The layers were of a sufficient thickness where flakes of surface layer material readily formed after the tests were terminated, and the samples were air dried. This flaking was especially prevalent along the sample edges.

During cross-section examination, the secondary clay minerals appeared as a single continuous layer and displayed a SEM/EDS composition consistent with the presence of smectite plus illite clays. These samples displayed a dual-layer assemblage similar to that developed on the longer term SRL 202U samples. The alteration layers, however, were more uniform in thickness and were also much thicker than the layers formed in the nonirradiated analogue tests. Layer measurements during SEM examinations of these 7-day samples averaged $39.3 \pm 3.8 \mu \mathrm{m}$, an increase of $\sim 10$ fold relative to the nonirradiated SRL 202U samples (Table 19). Discrete illite grains were also detected on the Pt-Rh support wires on these tests, indicating that this phase had precipitated out of the solution in contact with the glass rather than forming in situ (Figs. 41a and 42a).

Precipitate phases identified on these glasses include analcime (Fig. 41a), the unidentified $\mathrm{Ca}-\mathrm{Si}$ rosettes that were prevalent on the SRL 202U glasses, mixed weeksite and haiweeite ( $\left[\mathrm{Ca}\left(\mathrm{UO}_{2}\right)_{2} \mathrm{Si}_{6} \mathrm{O}_{15} \cdot 5 \mathrm{H}_{2} \mathrm{O}\right]$, Figs. $41 \mathrm{~b}$ and $42 \mathrm{~b}$ ), and a $\mathrm{K}-\mathrm{Al}$-Si phase believed to be adularia (Figs. $41 \mathrm{~b}$ and $42 \mathrm{c}$ ). The dipyramidal-shaped morphology of the adularia phase is unusual with respect to previous samples observed on the SRL 202U glasses. Minor amounts of unidentified $\mathrm{Na}-\mathrm{P}$ (Fig. 41a) and $\mathrm{Na}-\mathrm{K}-\mathrm{Ca}-\mathrm{Al}-\mathrm{Si}$ phases were also noted as fine-grained deposits on the $\mathrm{Pt}-\mathrm{Rh}$ wire. 

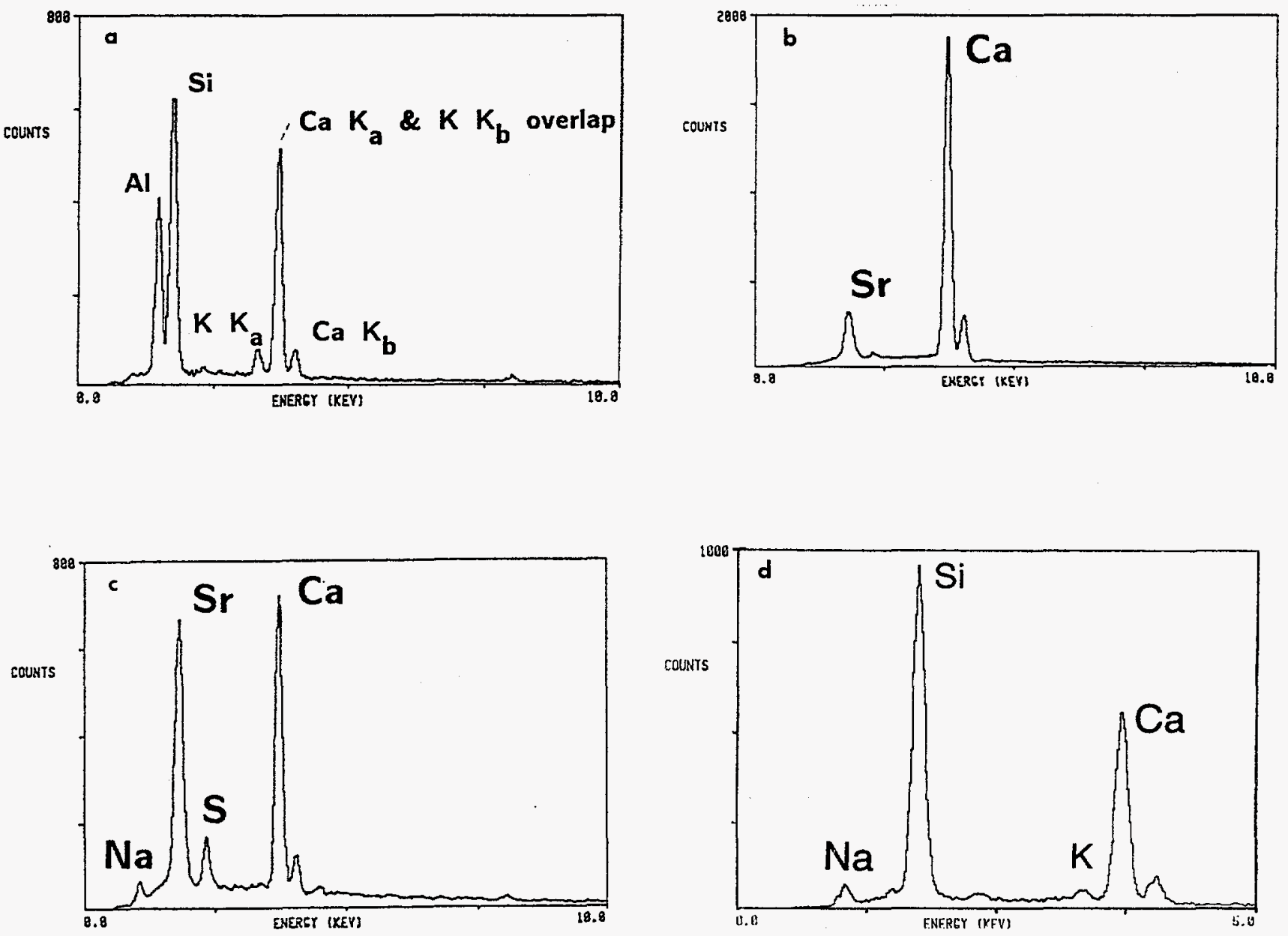

Fig. 38. SEM/EDS Spectra of SRL 202U Samples from Vapor Hydration Testing at 56 Days: (a) Adularia (potassium feldspar), (b) Unidentified Ca-Sr Phase, (c) Unidentified $\mathrm{Ca}-\mathrm{Sr}-\mathrm{Na}-\mathrm{S}$ Phase, and (d) Unidentified Ca-Si Anemone-Shaped Phase. All spectra taken at $20 \mathrm{keV}$ accelerating potential. 


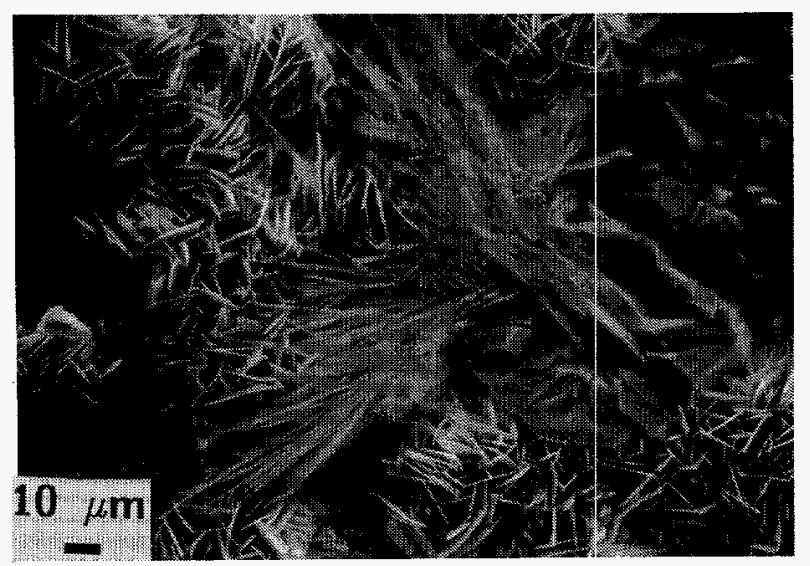

(a)

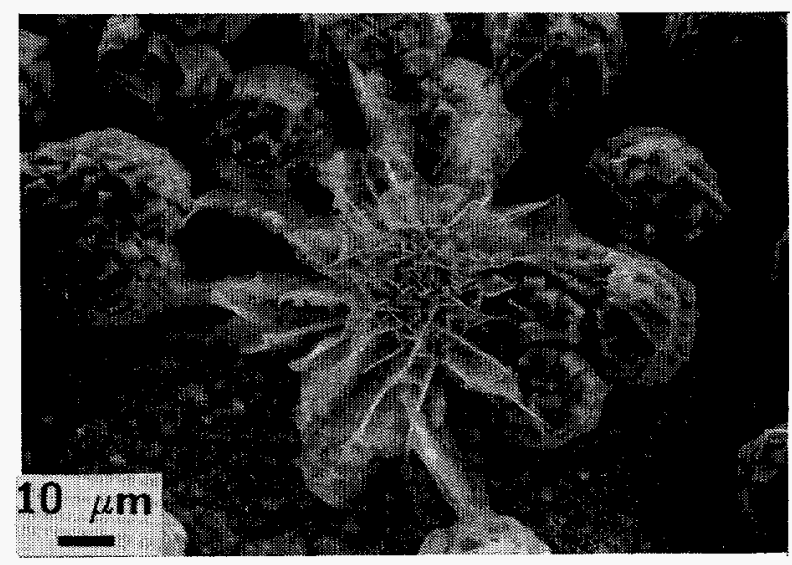

(b)

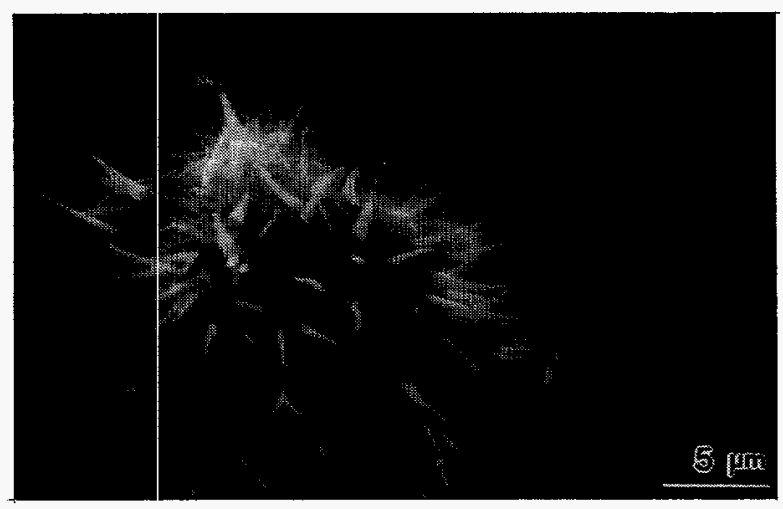

(c)

Fig. 39. SEM Images of Secondary Alteration Phases Formed on SRL 202U Glass from Vapor Hydration Tests at 56 Days. All images in secondary electron mode. (a) Unidentified $\mathrm{Ca}-\mathrm{Sr}$ phase (center), blocky analcime (right-center), and plate-like surfaces of $\mathrm{Ca}-\mathrm{Si}$ rosettes $(450 \mathrm{X})$, (b) unidentified $\mathrm{Ca}-\mathrm{Sr}-\mathrm{Na}-\mathrm{S}$ phase (center) lying on top of adularia grains (700X), and (c) unidentified Ca-Si anemone-shaped phase (2500X). 

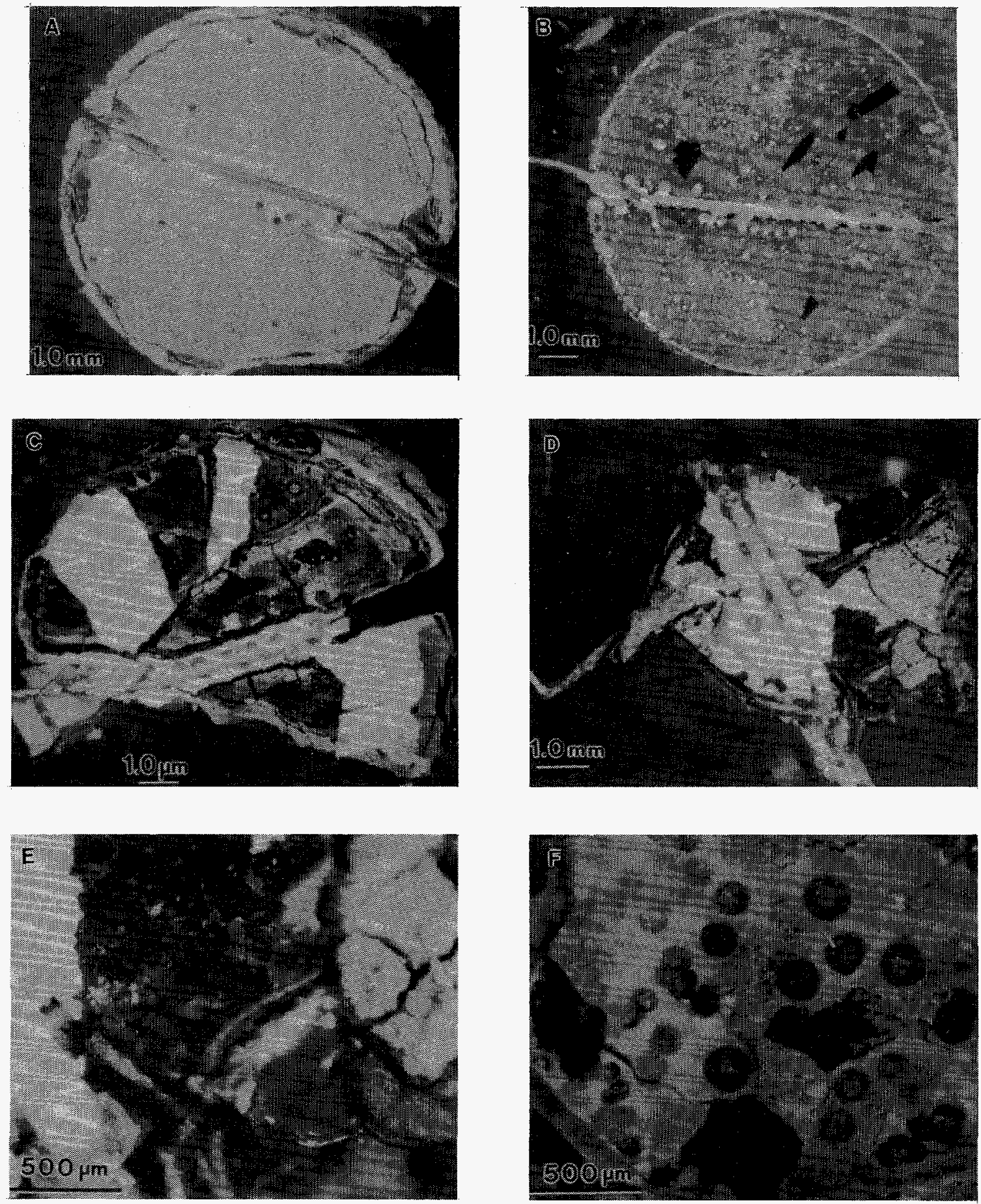

Fig. 40. Optical Photographs of the Reacted Surfaces of SRL 202A Glass Samples from the $200^{\circ} \mathrm{C}$ Vapor Hydration Tests Exposed to a Dose Rate of $\sim 35 \mathrm{~Gy} / \mathrm{h}$. Some samples are shown with Pt-Rh support wire attached: (a) 7-Day Sample at 9X, (b) 14-Day Sample at 9X, (c) 21-Day Sample at 9X showing fragmentation, note alteration material along sinuous fracture surface exposed at bottom of sample, (d) 35-Day Sample at $12 \mathrm{X}$ showing severe fragmentation, (e) 35-Day Sample at 50X showing $\mathrm{Fe}$ - and Si-rich core material, (f) 56-Day Sample at 50X showing analcime buttons, yellow weeksite, and crusted alteration surface. 


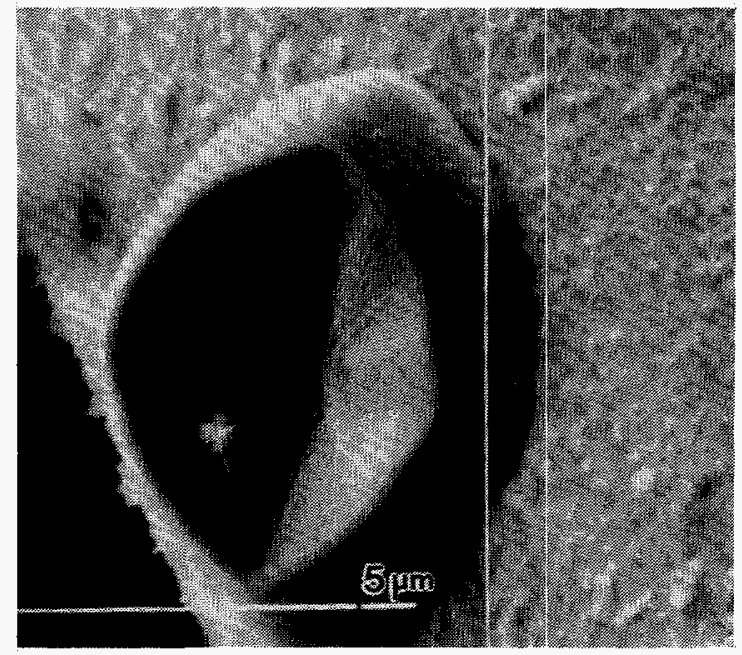

(a)

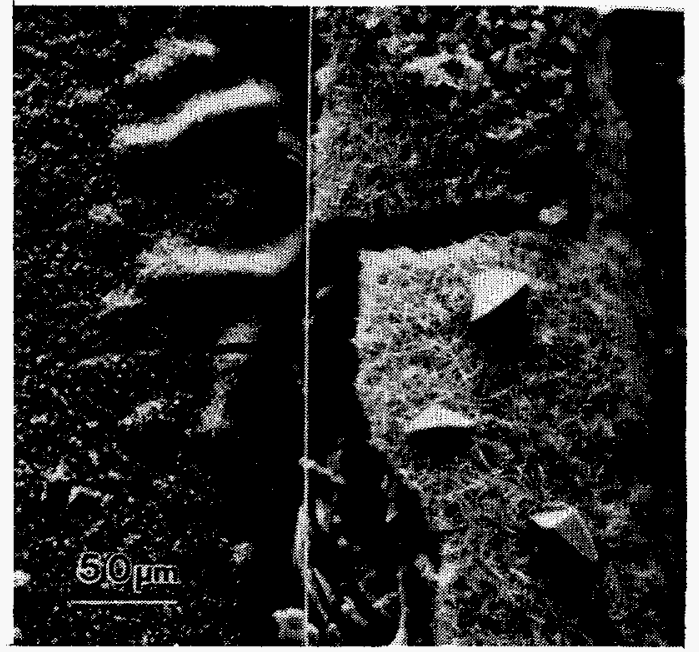

(b)

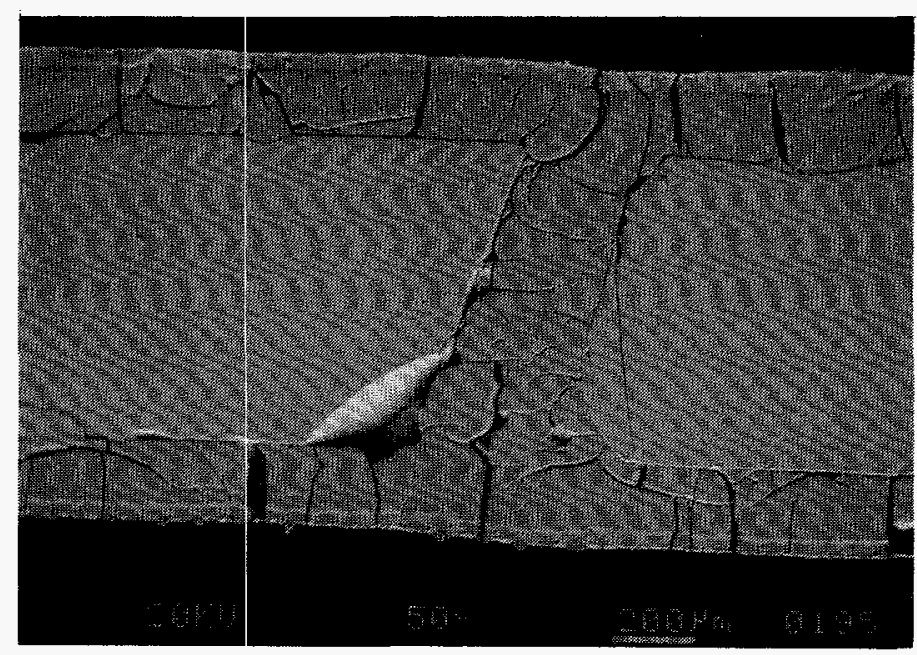

(c)

Fig. 41. SEM Images of Secondary Alteration Phases Forming on SRL 202A Glass Samples from the $200^{\circ} \mathrm{C}$ Vapor Hydration Tests Exposed to a Dose Rate of $\sim 35 \mathrm{~Gy} / \mathrm{h}$. Vertical shite bar in some photographs is from electronic distortion. (a) Dimpled analcime grain, with small stellar sodium-phosphate phase deposited on the analcime surface. Also present are fine flakes of illite that have precipitated directly on the platinum-rhodium support wire used in these tests ( 7 day, secondary electron image, 1500X). (b) Dipyramidal grains of adularia overlying needle-like substrate of uranophane to right of sample. Left half of sample shows underlying clay substrate where the overlying adularia, weeksite, and haiweeite layer has flaked off ( 7 day, secondary electron image, 300X). (c) Backscattered electron image of polished cross section showing unaltered glass cores surrounded and cross cut by clay layer (14 day, 50X). The inner smectite and outer illite layers are visible in the surficial clay layers at the top and bottom of the photos. 

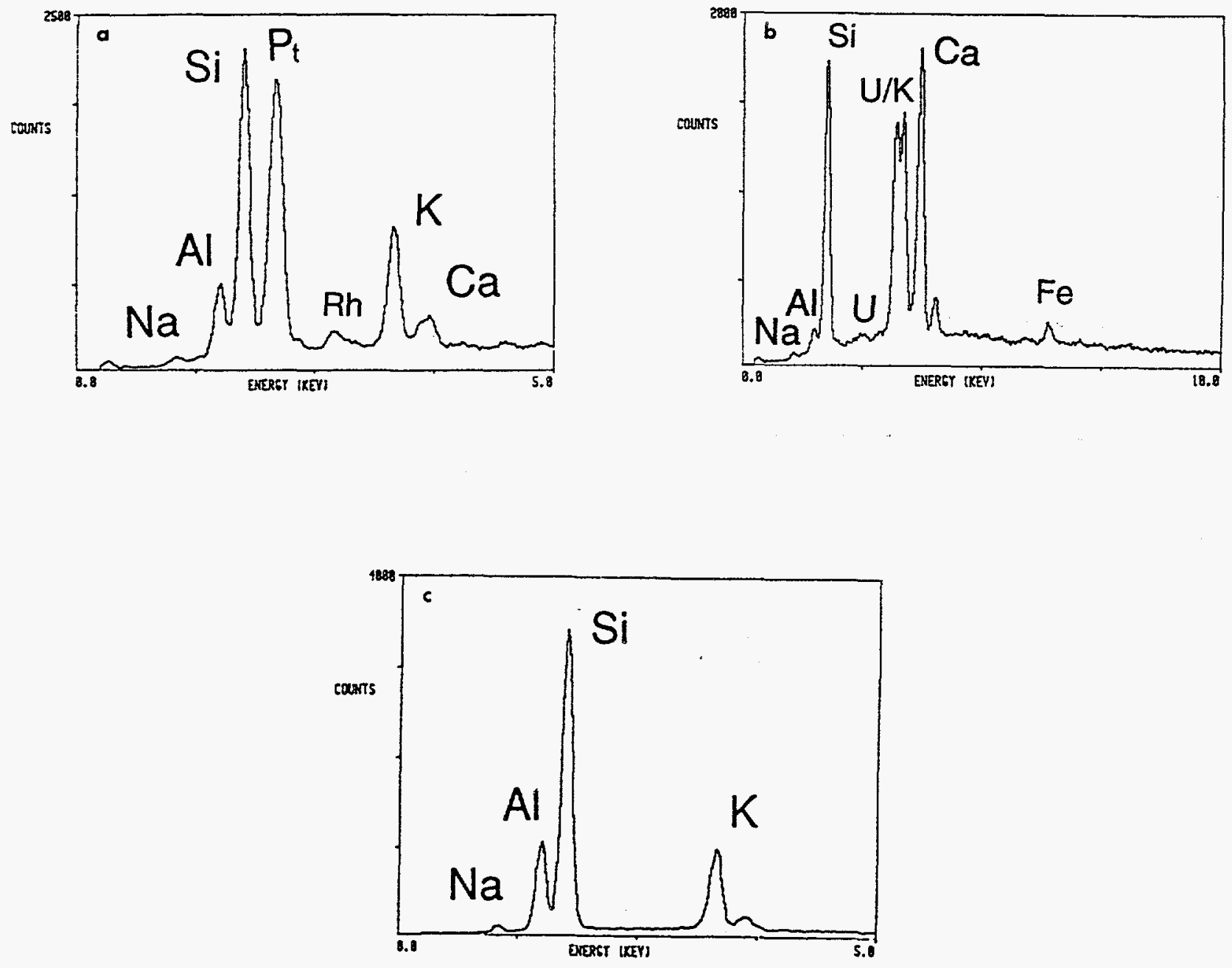

Fig. 42. SEM/EDS Spectra from Various Alteration Phases Formed on the 7-Day SRL 202A Samples from the $200^{\circ} \mathrm{C}$ Vapor Hydration Tests Exposed at Dose Rate of $\sim 35 \mathrm{~Gy} / \mathrm{h}$. (a) Illite deposited on Pt-Rh support wire, (b) mixed weeksite and haiweeite, and (c) adularia. All spectra taken at $20 \mathrm{keV}$ accelerating potential. 


\section{g. SRL 202A Samples at 14 Days}

The SRL 202A glass samples at 14 days were characterized by the development of a thick alteration layer and the formation of a number of secondary phases on the sample surfaces (Fig. 40b). These sample surfaces also displayed a minor amount of fracturing when the samples were air dried following the termination of the tests.

The composite altered surface includes both outer and inner clay layers. The outer layer was enriched in $\mathrm{Si}-\mathrm{Fe}-\mathrm{K}$ with lesser $\mathrm{Al}-\mathrm{Mn}-\mathrm{Ca}-\mathrm{Ti}-\mathrm{Ni}$, suggesting an illite plus smectite composition. This enrichment of illite in the outer layer followed the same pattern exhibited by the nonirradiated SRL 202 U glasses. By contrast, the inner layer was strongly depleted in potassium relative to the outer layer, suggesting a composition-dominated smectite. This inner layer progressed nonuniformly into the glass samples, with rapidly developed alteration fronts occurring along preferential zones that completely enveloped semi-rounded and apparently unaltered glass cores (Fig. 41c). These zones may represent the loci of fractures in the glass that, in turn, may have localized the rapidly developed alteration fronts. Alteration layer development also was enhanced along the sample corners relative to the interior portions of the sample. Layer thickness measurements made during SEM examinations of these samples excluded the altered fracture zones. Composite alteration layer thicknesses were remarkably uniform, averaging $177 \pm 5 \mu \mathrm{m}$ (Table 19). This thickness represents a 10-fold increase over that observed in the nonirradiated SRL 202U samples. The outer illite layer of the SRL 202A sample averaged $38 \pm 7 \mu \mathrm{m}$, while the inner smectite layer averaged $139 \pm 7 \mu \mathrm{m}$.

The surface precipitates include phillipsite, analcime (Fig. 43a), the unidentified $\mathrm{Ca}-\mathrm{Si}$ rosettes (Figs. 43a and 43b), tobermorite (Fig. 43b), weeksite (Fig. 43a), sodium-weeksite, haiweeite, adularia (Fig. 43a), unidentified $\mathrm{Na}-\mathrm{Ca}-\mathrm{Fe}-\mathrm{Mn}-\mathrm{P}-\mathrm{Si}$ nodules and flakes, and low-Z salts.

Also of great interest are micron-sized Tc-K-Ca solids that were observed to occur only as deposits located on the $\mathrm{Pt}-\mathrm{Rh}$ support wire or on other phases that themselves were located on the wire (Fig. 43c). Precipitates of this type were not located directly on any portion of the altered glass surface. The Pt-Rh wire, thus, may have acted as a catalyst toward the precipitation of these technetium deposits.

The Tc-K-Ca phase occurred as small submicron-sized amorphous grains that were first located by their strong backscatter electron signal. It was impossible to isolate this phase for more detailed AEM analysis due to the small size of the particles. Compositional spectra obtained during SEM/EDS investigations always display the presence of interference spectra from adjacent minerals. This interference occurs due to size limitations in focusing the electron beam to diameters smaller than the particles of interest, and also possibly due to signal response generated from electrons passing through the target phase. A qualitative comparison of the SEM analyses of technetium particles and their adjacent mineral substrate indicates a large increase in the spectrum response for $\mathrm{Tc}$ and $\mathrm{K}$, with a minor increase also recorded for calcium.

Literature reviews indicate several potential mineral phases that are consistent with this SEM/EDS signal. These phases include $\mathrm{KTcO}_{4}, \mathrm{~K}_{2} \mathrm{Tc}(\mathrm{OH})_{9}$, and $\mathrm{K}_{2} \mathrm{TcF}_{6}$. The short wavelength $\mathrm{X}$-ray signal generated by $\mathrm{H}, \mathrm{O}$, and $\mathrm{F}$ would not be detected during standard SEM/EDS analysis due to detector limitations. The first two phases represent solids of the oxidized $\mathrm{Tc}^{7+}$ state, while the latter represents the reduced $\mathrm{Tc}^{4+}$ state. The presence of phases with the higher oxidation state is favored for the following reasons: the observed spatial association of uranyl silicate and ferric iron phases, and the expected production of $\mathrm{H}_{2} \mathrm{O}_{2}, \mathrm{O}_{2}^{-}$, and $\cdot \mathrm{OH}$ in the radiolytic environment that solutions in these tests were exposed to [2-4]. 


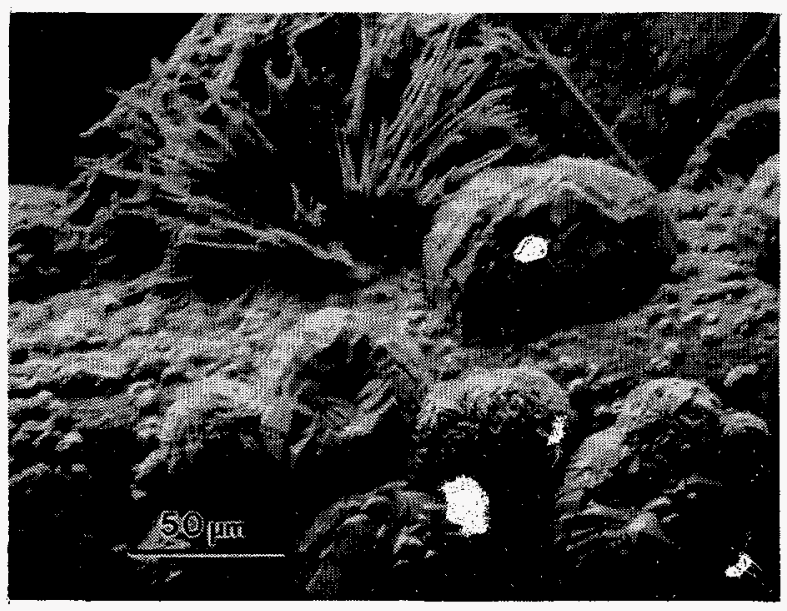

(a)

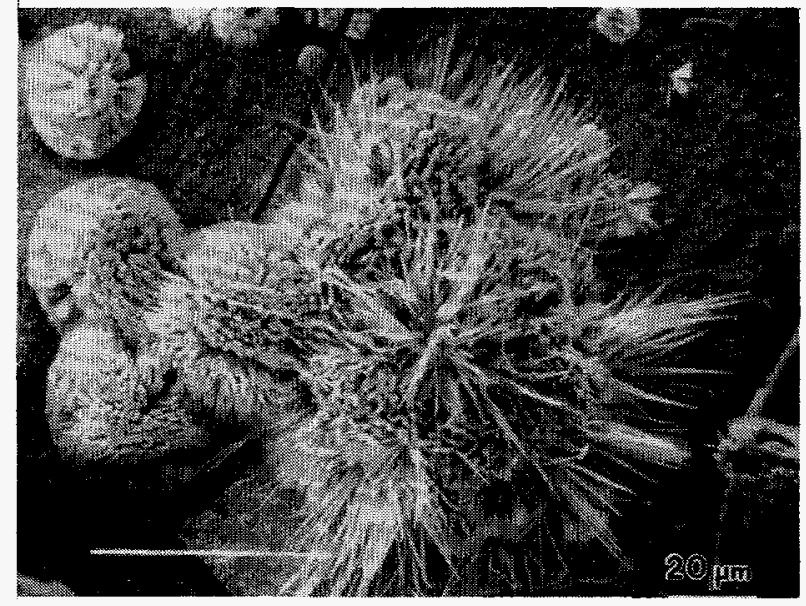

(b)

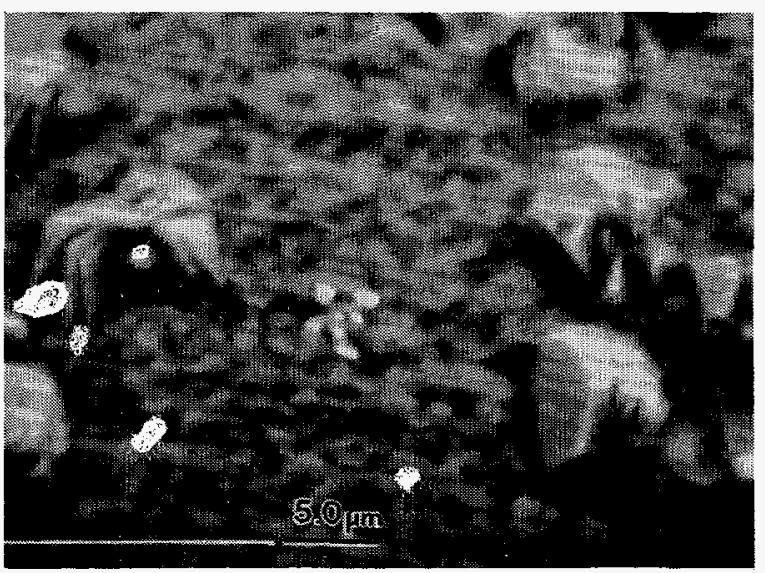

(c)

Fig. 43. SEM Images of Secondary Alteration Phases Forming on SRL 202A Glass Samples from the $200^{\circ} \mathrm{C}$ Vapor Hydration Tests Exposed for 14 Days at Dose Rate of $\sim 35 \mathrm{~Gy} / \mathrm{h}$. (a) Large dimpled analcime, fine-grained adularia buttons, large $\mathrm{Ca}-\mathrm{Si}$ rosettes, weeksite needles showing salt encrustation, all phases deposited on Pt-Rh wire (secondary electron image, 500X). (b) Tobermorite needles intergrown with $\mathrm{Ca}-\mathrm{Si}$ rosettes (secondary electron image, 750X). (c) Backscattered electron image of very fine-grained $\mathrm{K}-\mathrm{Tc}-\mathrm{Ca}$ phase, larger blocky adularia crystals, and $\mathrm{Na}-\mathrm{Ca}-\mathrm{P}-\mathrm{Si}$ substrate $(3000 \mathrm{X})$. 
h. $\quad$ SRL 202A Sample at 21 Days

Optical examinations of the 21-day samples indicate the presence of a highly altered specimen (Fig. 40c). The glass disks from the 21-day samples were almost completely transformed into secondary reaction products, and the alteration was severe enough to cause fragmentation of some of the glass monoliths during the testing interval. With these tests, altered fragments of the samples were found in the bottom of the test vessel when the tests were terminated. Due to potential interactions with the test vessel, these fallen fragments were not examined further.

Clay layer measurements during SEM examinations of these 21-day samples averaged $289 \pm 13 \mu \mathrm{m}$ (Table 19). This thickness represents a 15 -fold increase over the reaction layer thickness observed on the corresponding nonirradiated SRL 202U glasses. As with the previous tests, altered fracture zones have completely penetrated through the $\sim 1.0-\mathrm{mm}$ glass monoliths in some regions of the samples. These fractured regions were not included in the layer measurements. The composite layer was also divided into the same outer illite and inner smectite layers observed on earlier samples. The thicknesses of these sublayers are $60 \pm 20$ and $229 \pm 17 \mu \mathrm{m}$, respectively. The distinction between these layers was not as readily apparent as in the past samples. In some regions the contact between the outer illite and inner smectite layers was difficult to distinguish during SEM/EDS examination, while in other regions several bands of illite minerals could be detected in the outer layer. The smectite layer is similar in composition to that in the 14-day sample, with the exception that the present sample shows a slight enrichment of $\mathrm{Ca}, \mathrm{Ti}$, and $\mathrm{Mg}$.

The 21-day samples were characterized by the formation of adularia (Fig. 44), analcime, weeksite, sodium-weeksite, tobermorite, haiweeite, the ubiquitous $\mathrm{Ca}-\mathrm{Si}$ rosettes, unidentified $\mathrm{Na}-\mathrm{Ca}-\mathrm{Fe}-\mathrm{Mn}-\mathrm{P}-\mathrm{Si}$ nodules and flakes, limited amounts of $\mathrm{Cu}$ nodules [Fig. 44; probably copper oxide, cuprite $\left(\mathrm{Cu}_{2} \mathrm{O}\right)$ or tenorite $(\mathrm{CuO})$ ], and alkali-alkaline earth-P-Si phases.

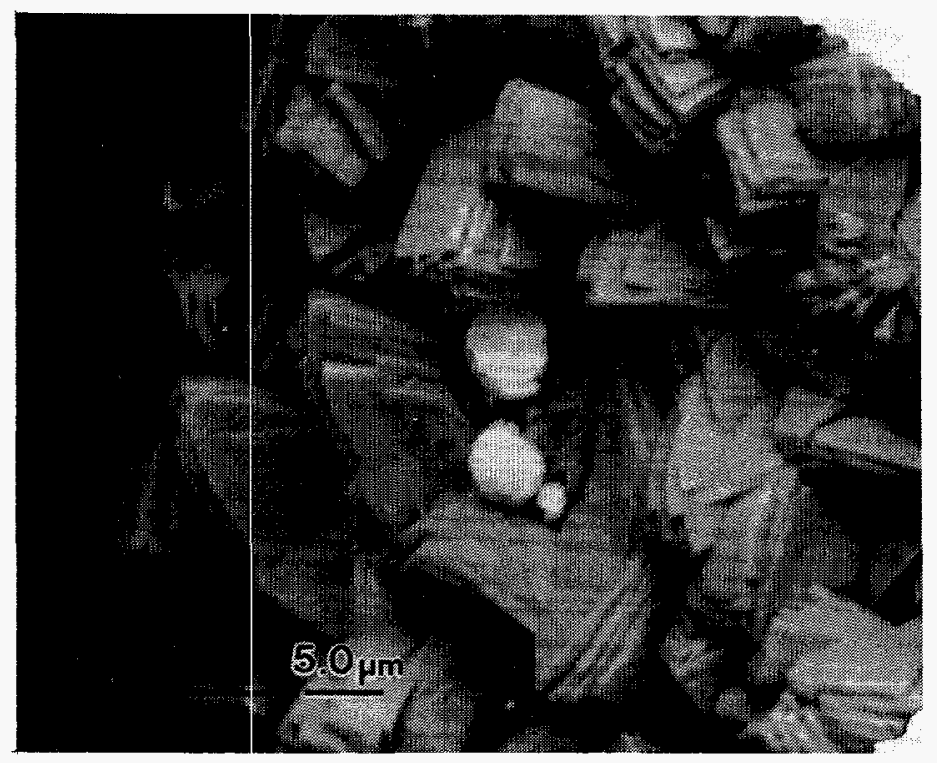

Fig. 44. SEM Image of Blocky Adularia Crystals and Bright Copper Oxide Crystals Forming on 21-Day SRL 202A Sample from the $200^{\circ} \mathrm{C}$ Vapor Hydration Test Exposed to Dose Rate of $\sim 35 \mathrm{~Gy} / \mathrm{h}$ (backscattered electron image, 2000X) 


\section{i. SRL 202A Samples at 35 Days}

The 35-day samples also displayed evidence of severe corrosion and fragmentation of the sample monoliths (Figs. 40d and 40e). As with the 21 -day samples, altered fracture zones have completely penetrated the $\sim 1.0-\mathrm{mm}$ glass monoliths in some regions of the samples. The sample surfaces were characterized by the formation of several blanketing layers and the near complete alteration of glass material.

An unusual material occurring as "altered cores" within the samples exhibited both mottled and banded textures in different regions of the sample (Figs. 40e and 45a). The banded sections exhibited more than 50 individual layers, ranging in thickness from 1 to $10 \mu \mathrm{m}$. An EDS spectral analysis of the banded material (Fig. 46a) revealed a composition dominated by $\mathrm{Fe}$ and $\mathrm{Si}$, with lesser $\mathrm{Al}, \mathrm{Na}, \mathrm{Mn}, \mathrm{Ca}, \mathrm{K}, \mathrm{Ti}$, and $\mathrm{Ni}$. The predominance of relatively insoluble components such as $\mathrm{Fe}, \mathrm{Si}, \mathrm{Al}$, and other transition metals suggests that the material in this layer accumulated by a residual enrichment process after the more soluble components of the glass had been leached away.

Up to three additional layers enveloped this Si-Fe core, with some of these overlying layers also displaying banded textures (Fig. 45b). The layer immediately overlying the Fe-Si core appeared as a brownish clay layer that was lighter in color at its base and displayed numerous pits on its surface when examined by SEM/EDS (Figs. 45b and 45c). This layer also displayed several distinct bands when examined in cross section. The SEM/EDS composition is consistent with a smectite clay composition, although the presence of potassium also suggests 

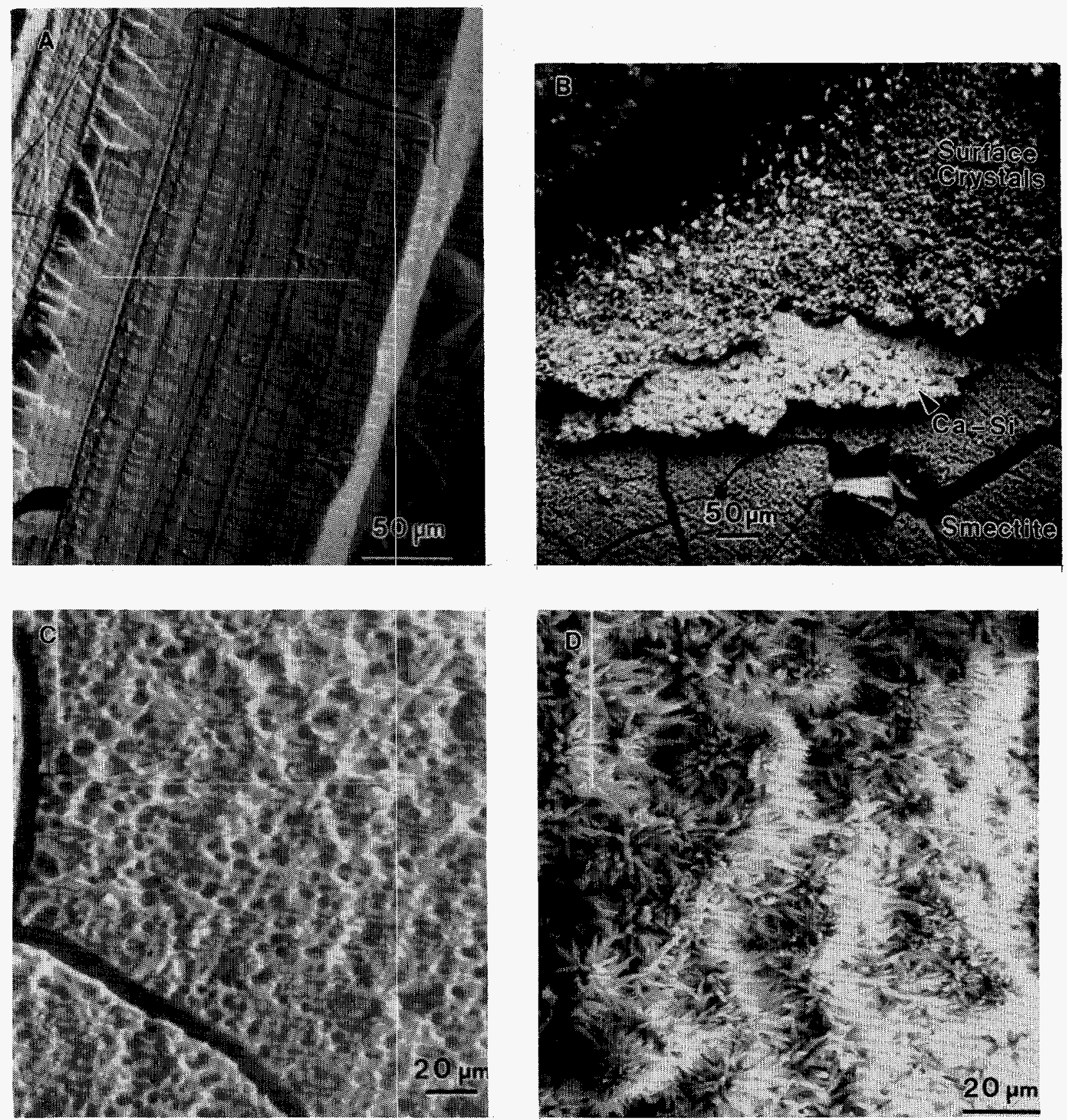

Fig. 45. SEM Images of Secondary Alteration Phases Forming on 35-Day SRL 202A Samples from the $200^{\circ} \mathrm{C}$ Vapor Hydration Tests Exposed to Dose Rate of $\sim 35 \mathrm{~Gy} / \mathrm{h}$. White bar down center of some photographs is from electronic distortion. (a) Autofractured section of glass showing banded textured Si-Fe core material (secondary electron image, 150X). (b) Autofractured section of reacted layer surface showing underlying smectite layer, $\mathrm{Ca}-\mathrm{Si}$ crystal layer, and heterogeneous mixture of surface crystals (backscattered electron image, 150X). (c) High-magnification image of underlying smectite clay from Fig. $45 \mathrm{~b}$ showing pitted surface material (secondary electron image, 1000X). (d) High-magnification image of Ca-Si crystal layer from Fig. 45b (secondary electron image, 1500X). 

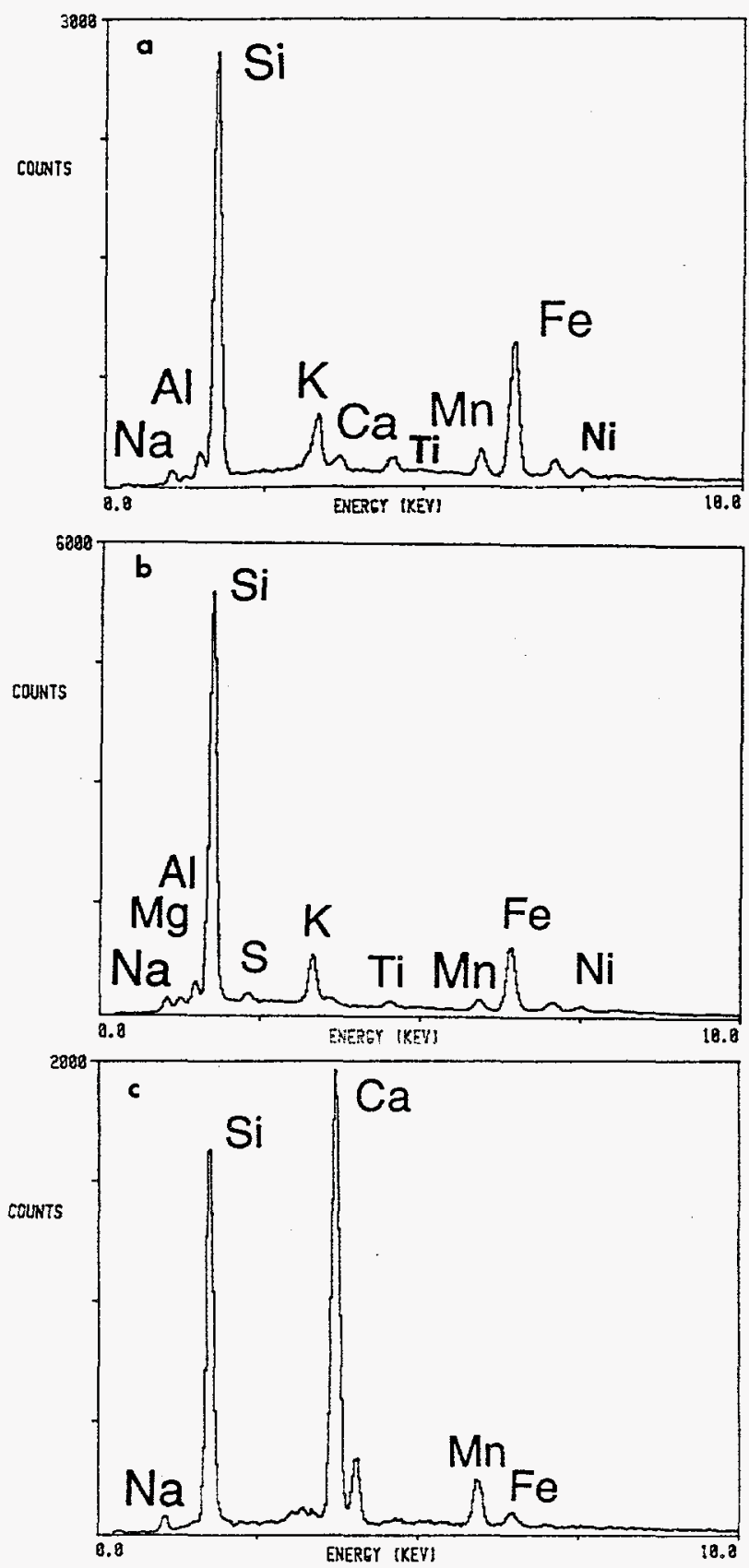

Fig. 46. SEM/EDS Spectra from Various Alteration Phases Formed on 35-Day SRL 202A Samples from the $200^{\circ} \mathrm{C}$ Vapor Hydration Tests Exposed to a Dose Rate of $\sim 35 \mathrm{~Gy} / \mathrm{h}$. (a) Banded Fe-Si zone, (b) smectite/illite clay mixture, and (c) $\mathrm{Ca}-\mathrm{Si}$ crystals. All spectra taken at $20 \mathrm{keV}$ accelerating potential. 
that some illite clays are present (Fig. 46b). Thickness measurements made on this clay layer during SEM examinations of cross-sectioned samples averaged $439 \pm 16 \mu \mathrm{m}$ (Table 19). These measurements were taken from nonfractured regions of the sample and represent an eight-fold increase in layer thickness relative to the layer from the unirradiated SRL 202U samples.

Overlying this brown layer is a thin sheet of white, anemone-shaped, CaSi crystals of unknown identity (Figs. 45b, 45d, and 46c). This Ca-Si layer was, in turn, overlain by a surface covering mixture of heterogeneous crystalline precipitate material that was intergrown to the extent where it behaved as a cohesive layer (Figs. 45b, 47a, and 47b). It appeared that both illite and smectite were intergrown with the surface precipitates in this upper layer material.

Distinct mineral precipitates were identified both in the outermost altered layer and as isolated alteration phases occurring on the sample surface and Pt-Rh wire supporting the specimen. Detailed optical and SEM/EDS examination of the samples indicate the presence of analcime (Fig. 47b), adularia, and tobermorite. A number of unidentified phases were also noted during SEM/EDS examinations, including a Si-Al-Na-Ca blocky phases, the Ca-Si rosettes, $\mathrm{Ca}-\mathrm{Si}$ nodules, $\mathrm{Si}-\mathrm{S}-\mathrm{P}-\mathrm{Ca}-\mathrm{K}-\mathrm{Na}-\mathrm{Al}$ needles, $\mathrm{Na}-\mathrm{P}$ needles, copper oxides, amorphous $\mathrm{Si}-\mathrm{S}-\mathrm{Ca}$ phases, and $\mathrm{Na}-\mathrm{Ca}-\mathrm{Fe}-\mathrm{Mn}-\mathrm{P}-\mathrm{Si}$ nodules.

Uranium was distributed among a wide variety of mineral phases on these samples. An examination of SEM/EDS peak heights from the 35-day sample indicates that both weeksite and boltwoodite $\left[\mathrm{K}\left(\mathrm{H}_{3} \mathrm{O}\right) \mathrm{UO}_{2}\left(\mathrm{SiO}_{4}\right) \bullet \mathrm{nH}_{2} \mathrm{O}\right]$ were present on the sample surface. The boltwoodite occurred as a single large stellar mass of green needles growing in close proximity to the more prevalent yellow weeksite crystals (Fig. 47b). The boltwoodite was distinguished from weeksite based on larger $\mathrm{U}_{\mathrm{Ma}} / \mathrm{Si}_{\mathrm{Ka}}$ and $\mathrm{K}_{\mathrm{Kb}} / \mathrm{Si}_{\mathrm{Ka}}$ peak ratios (compare Figs. 48a and 48b). The relative peak height ratios of Si:U:K for boltwoodite also match well with stoichiometric ratios of 1:1:1 for boltwoodite when compared with the $3: 1: 1$ ratio exhibited by the weeksite phase. These ratios also match with a boltwoodite phase previously identified as an alteration product on uranium oxide pellets [51]. In addition to the potassium uranyl silicates, both haiweeite (Fig. 48c) and a Na- or Na-Ca-weeksite type phase (Fig. 48d) were noted to occur on the sample surface.

\section{j. $\quad$ SRL 202A Sarnples at 56 Days}

The extent of alteration and sample fragmentation in the 56-day SRL 202A sample (Fig. 40f) is similar to that described previously for the 35-day sample. The samples were noted to be fragmented when the tests were terminated; however, despite the extensive fragmentation, relatively little delamination of the individual layers has occurred on these samples. Cross-section examinations of these samples revealed that the glass was completely transformed to secondary alteration products. Minimum reaction layer thicknesses were calculated using half of the thickness of the starting glass monoliths. The thickest monoliths used in these tests had a measured thickness of $980 \mu \mathrm{m}$, thus indicating a minimum reaction layer thickness of $490 \mu \mathrm{m}$ (Table 19). A comparison of this value with alteration trends from samples terminated from tests of shorter duration suggests that the potential reaction layer thickness is considerably greater than the $490 \mu \mathrm{m}$ measured for these samples.

The central cores of the 56-day samples were replaced by a microcrystalline iron- and silicon-rich material with a massive-to-banded texture appearance. Up to four additional layers could be distinguished overlying the core material, with some of these layers also displaying evidence for microlayering. By contrast, only two alteration layers were detected in the 56-day glasses reacted without radiation exposure. 
(a)

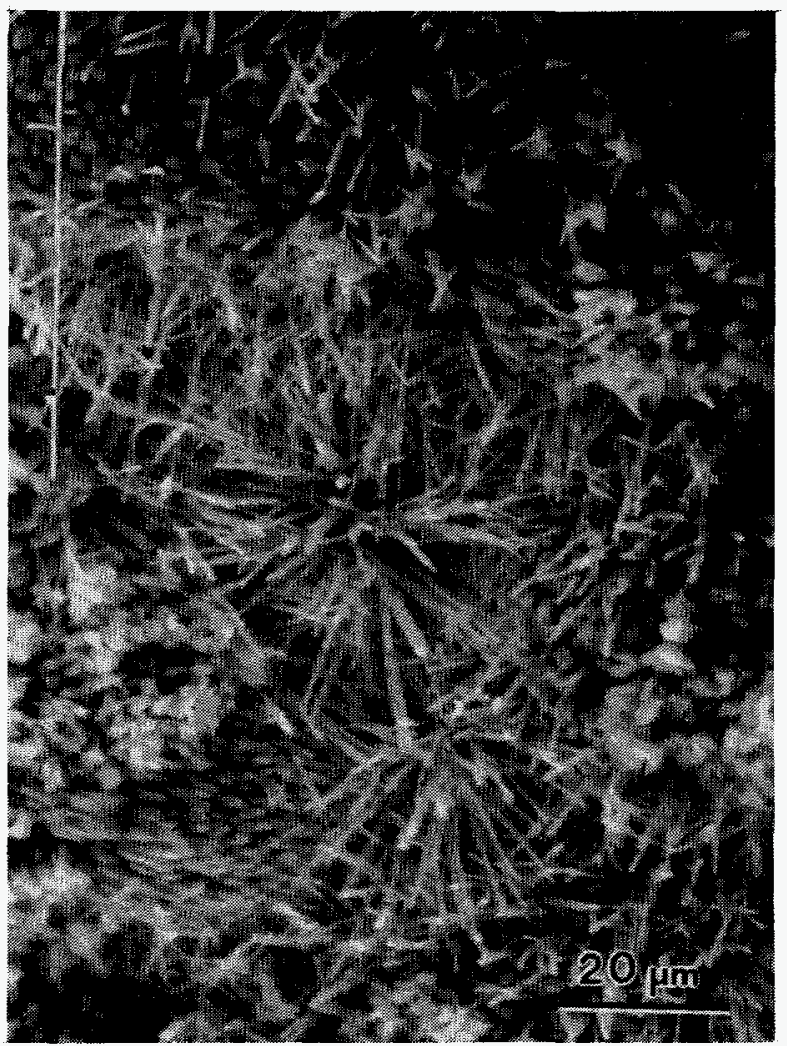

(b)

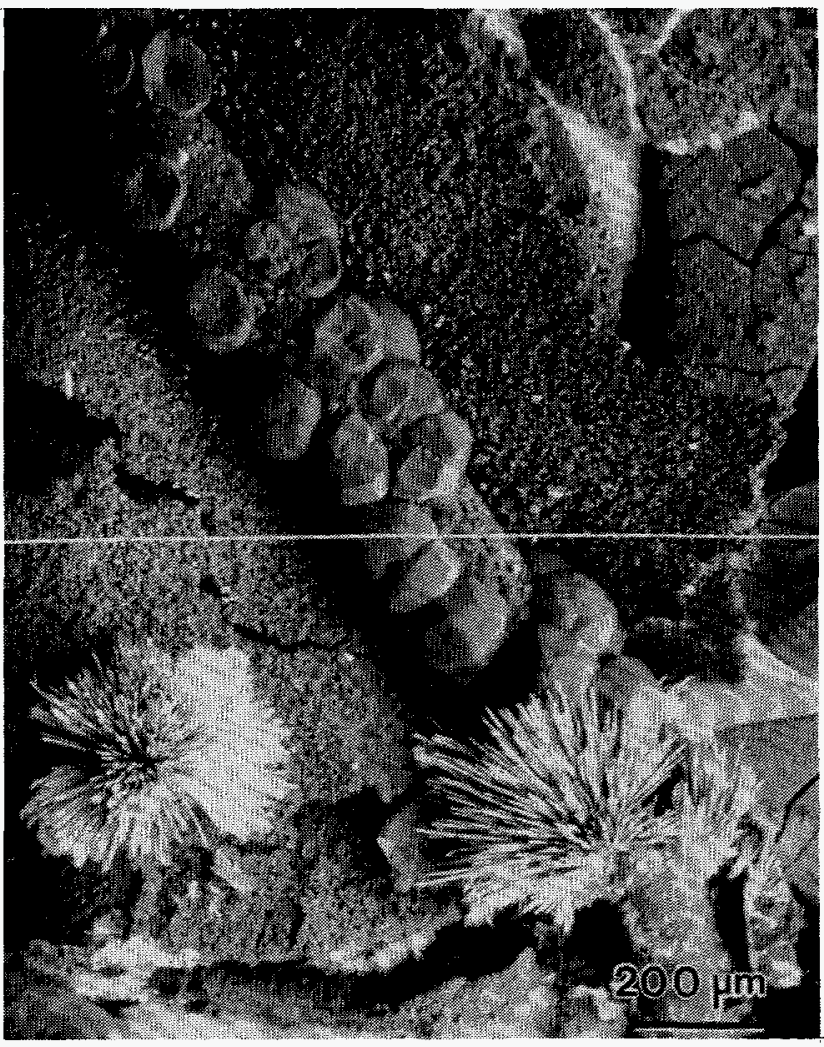

Fig. 47. SEM Images of Secondary Alteration Phases Forming on 35-Day SRL 202A Samples from the $200^{\circ} \mathrm{C}$ Vapor Hydration Tests Exposed to Dose Rate of $\sim 35 \mathrm{~Gy} / \mathrm{h}$. White bar down center of some photographs is from electronic distortion. (a) Highmagnification image of heterogeneous mixture of crystals from Fig. 45b. Included in this outer layer were $\mathrm{Ca}-\mathrm{Si}$ needles, assemblages of $\mathrm{Si}-\mathrm{Na}$ rods, and both illite and smectite clays (secondary electron image, 200X). (b) Large stellar weeksite and boltwoodwite, large trapezohedron and dimpled analcime (deposits on Pt-Rh wire running from lower left to upper right), and bright BSE images of fine-grained Naweeksite phase (bright linear features in upper right corner result from silver mounting medium that has impregnated fractures) (backscattered electron image, 50X). 

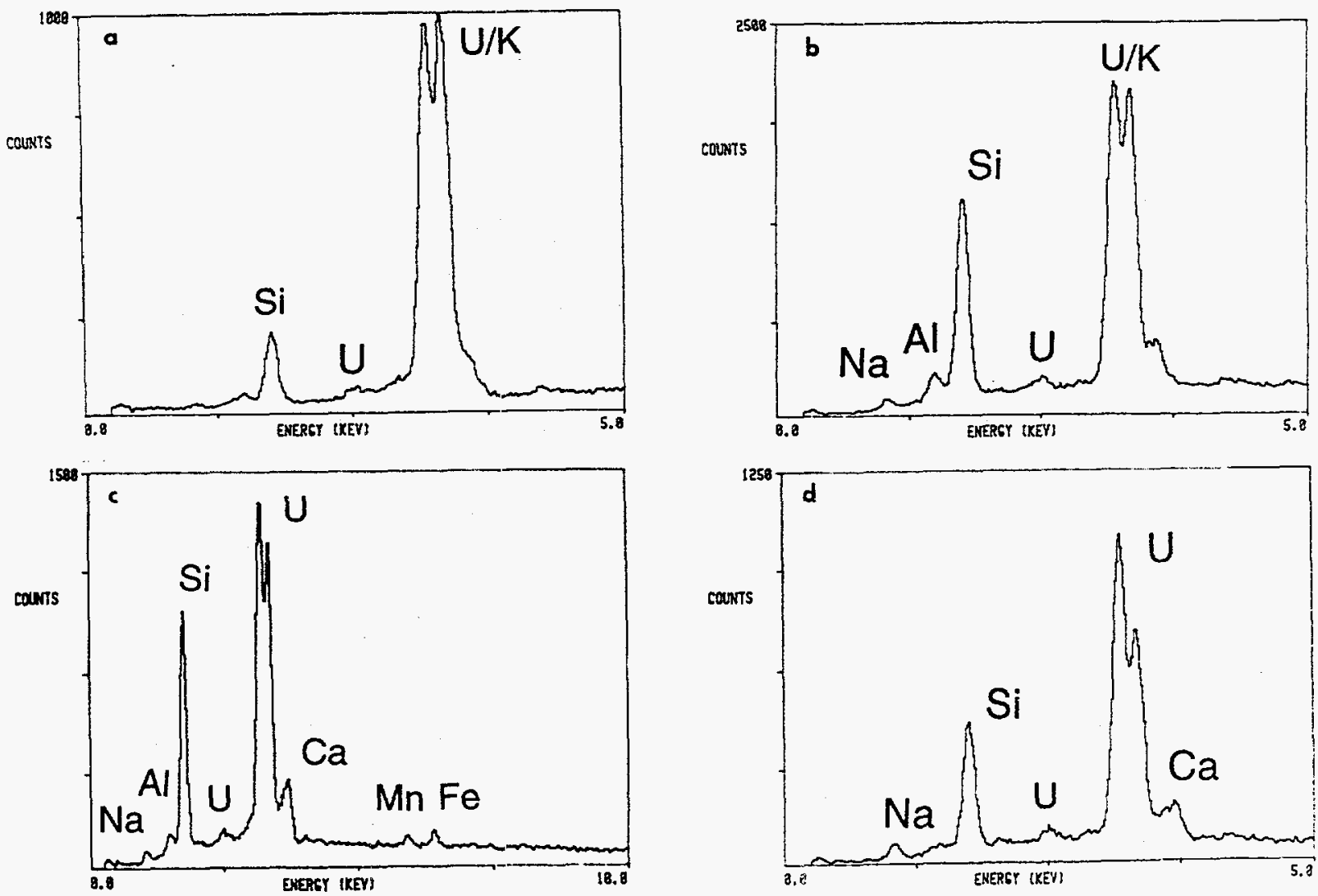

Fig. 48. SEM/EDS Spectra from. Various Alteration Phases Formed on 35-Day SRL 202A Samples from the $200^{\circ} \mathrm{C}$ Vapor Hydration Tests Exposed to Dose Rate of $\sim 35 \mathrm{~Gy} / \mathrm{h}$ : (a) Boltwoodite, (b) Weeksite, (c) Haiweeite, and (d) Sodium-Weeksite. All spectra taken at $20 \mathrm{keV}$ accelerating potential.

Surface precipitates were also detected on the samples, including weeksite, haiweeite (Fig. 49a), tobermorite, Ca-Si rosettes, copper oxides, and unidentified $\mathrm{Cu}-\mathrm{S}$ nodules. Adularia (Figs. 49a and 49b) and analcime (Fig. 49c) were also detected on these samples, with both phases displaying the formation of surface pits and crevasses indicative of a period of corrosive activity. Small crystals of what appeared to be albite $\left(\mathrm{NaAlSi}_{3} \mathrm{O}_{8}\right)$ formed on the pitted surface of the adularia grains (Fig. 49b). These combined features and the composition of the various phases suggest that both adularia and analcime may be replaced in the long-term mineral formation sequence by albite and another potassium-bearing phase.

\section{SRL 165 Glass}

\section{a. SRL 165A Sanaples at 7 Days}

Optical microscopy examinations of the 7-day SRL 165A samples indicate that only a limited extent of surface reaction has occurred (Fig. 50a). A thin dusting of finegrained crystals was noted along with a light discoloration of the surface due to the formation of clay minerals. The surface was extensively fractured, especially along the sample edges; however, despite this fracturing, very little flaking of surface material was noted. As with previous SRL 202 glass samples, precipitate development was enhanced along regions in contact with the hanging wire and the lower-most hanging portion of the sample. Clay reaction layer thicknesses measured during SEM cross-section examinations averaged $15.5 \pm 3.1 \mu \mathrm{m}$ (Table 19). The altered clay surface is enriched in Si-Fe-Al, suggesting a nontronite composition. 
(a)

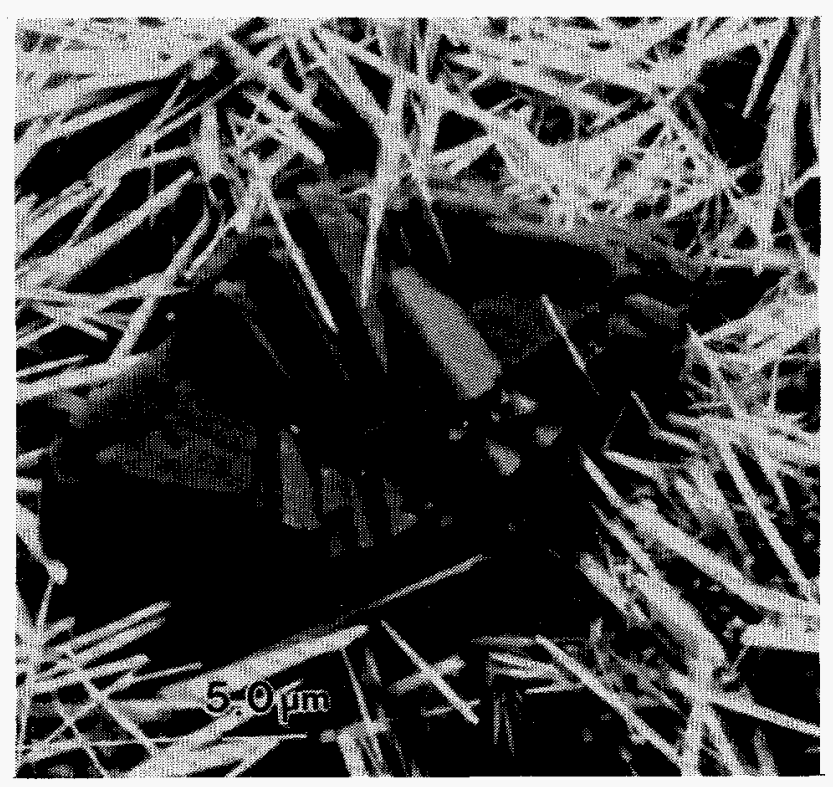

(b)

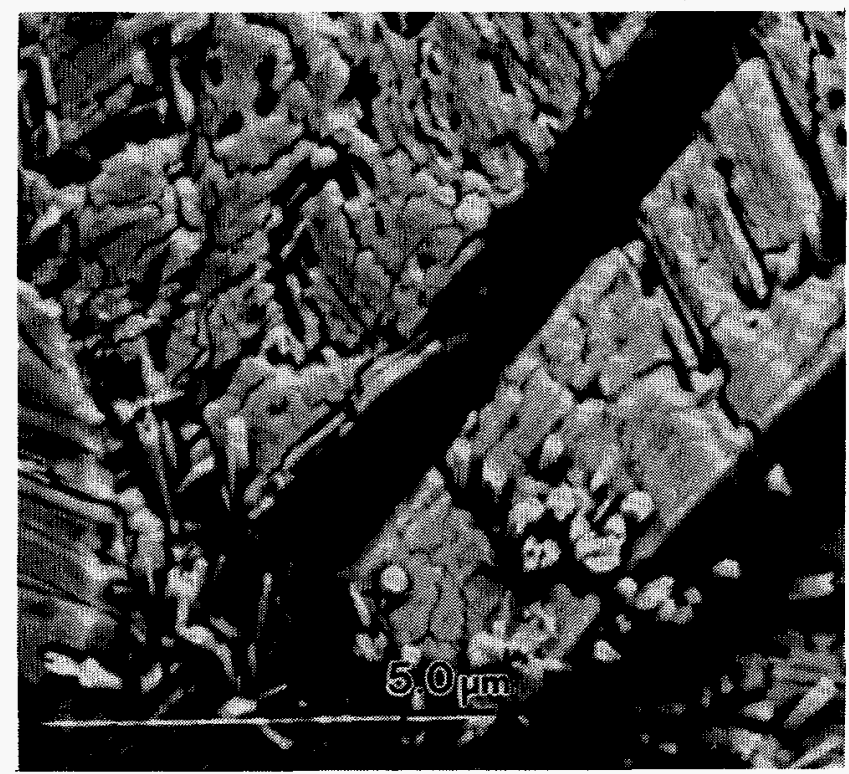

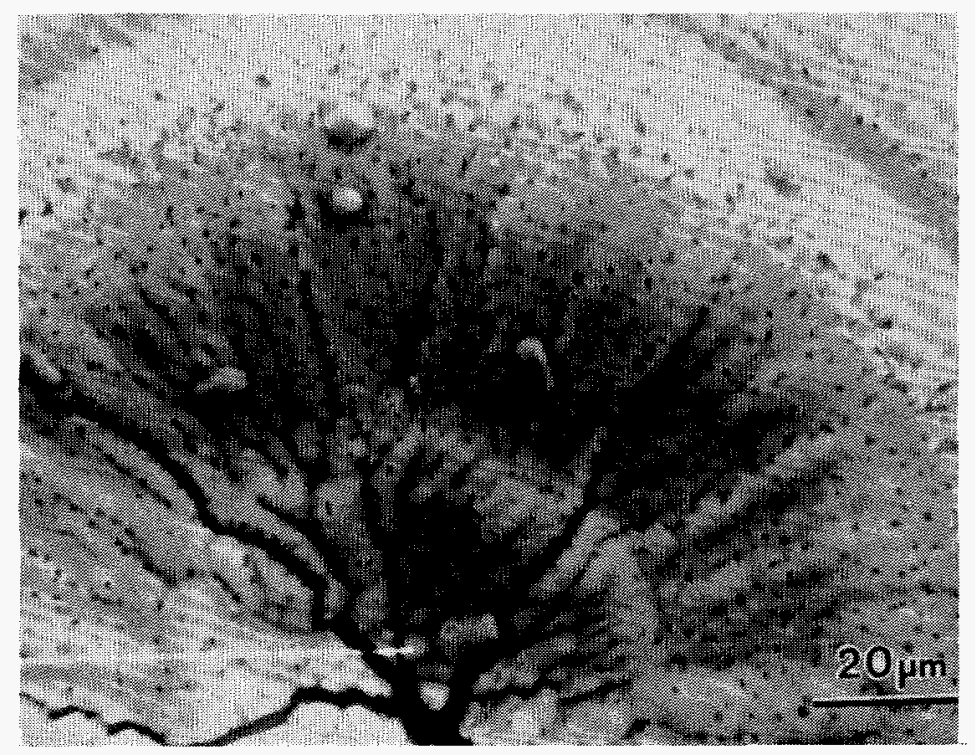

(c)

Fig. 49. SEM Images of Secondary Alteration Phases Forming on 56-Day SRL 202A Samples from the $200^{\circ} \mathrm{C}$ Vapor Hydration Tests Exposed to Dose Rate of $\sim 35 \mathrm{~Gy} / \mathrm{h}$. All images in secondary electron mode. White bar in photograph is from electronic distortion. (a) Large blocky adularia grain and haiweeite needles (2000X). (b) High-magnification image of adularia grain showing dissolution pits and crevasses $(2000 \mathrm{X})$. (c) Surface of large dimpled analcime grain displaying dissolution pits and crevasses, as well as ingrowths of small albite crystals (1000X). 

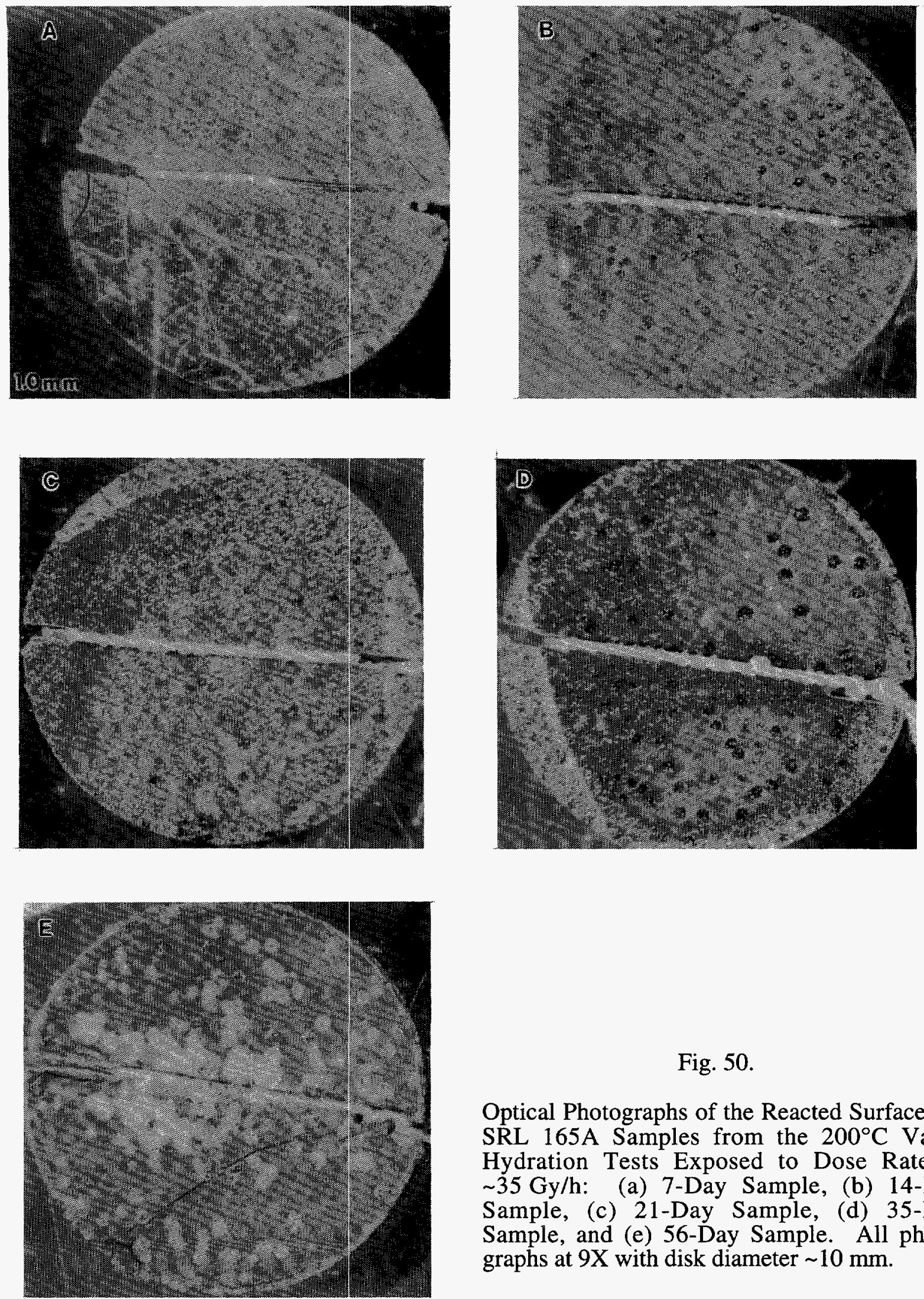

Fig. 50 .

Optical Photographs of the Reacted Surfaces of SRL 165A Samples from the $200^{\circ} \mathrm{C}$ Vapor Hydration Tests Exposed to Dose Rate of 35 Gy/h: (a) 7-Day Sample, (b) 14-Day Sample, (c) 21-Day Sample, (d) 35-Day Sample, and (e) 56-Day Sample. All photographs at $9 \mathrm{X}$ with disk diameter $\sim 10 \mathrm{~mm}$. 
The surface precipitate mineralogy displayed by the SRL 165A glass samples is relatively simple compared to the patterns exhibited by SRL 131A and SRL 202A glasses. The 7-day tests displayed an abundance of analcime, and lesser weeksite, sodiumweeksite, iron oxides, $\mathrm{Na}-\mathrm{Si}$ threads, amorphous $\mathrm{Ca}-\mathrm{Si}$ material, unidentified $\mathrm{Ca}-\mathrm{Na}-\mathrm{Al}-\mathrm{U}-\mathrm{S}$ needles, and salt crusts containing low-atomic weight elements.

\section{b. $\quad$ SRL 165A Samples at 14 Days}

The altered sample surfaces of the 14-day SRL 165A samples were similar in appearance as the 7-day samples. The samples had developed a brownish alteration layer that was partially covered with a dusting of whitish surface precipitates (Fig. 50b). Some samples also displayed extensive fracturing of the surface layer material. Clay reaction layer thicknesses measured during SEM cross-section examinations averaged $23.6 \pm 2.9 \mu \mathrm{m}$ (Table 19). The altered clay surface is enriched in Si-Al-Fe, suggesting a nontronite composition (Fig. 51a).

Surface precipitates include analcime, and lesser sodium-weeksite (Figs. 52a and 52b), haiweeite (Fig. 52a), Na-Si needles, Na-Si-P needles (Figs. 52b and 51b), $\mathrm{Ca}-\mathrm{Si}$ rosettes (Fig. 52b), amorphous silicon, and low-Z salts.

\section{c. $\quad$ SRL 165A Samples at 21 Days}

The altered sample surfaces of the 21-day SRL 165A samples (Fig. 50c) display the same brownish alteration layer and white precipitate covering that characterized the 7- and 14-day samples. This surface layer is also similar in composition to the 7-day sample. Clay reaction layer thicknesses measured during SEM cross-section examinations averaged $28.9 \pm 2.8 \mu \mathrm{m}$ (Table 19). A detailed examination of the sample surface also indicates the presence of a fine-grained Ca-P material (possibly apatite) intergrown with the honeycombtextured smectite clays.

Surface precipitate minerals formed on these samples include analcime (Fig. 52c), Ca-Si rosettes (Figs. 52c and 51c), Ca-Si spheres (Figs. 52c and 51d), sodiumweeksite, unidentified $\mathrm{Na}-\mathrm{Si}$ needles (Fig. 51e), amorphous silicon, low-Z salts, and the previously mentioned apatite phase.

\section{d. SRL 165A Samples at 35 Days}

The altered clay surface of the 35-day SRL 165A samples (Fig. 50d) is identical in appearance to the samples reacted for 7,14 , and 21 days. Clay reaction layer thicknesses measured during SEM cross-section examinations averaged $109 \pm 14 \mu \mathrm{m}$ (Table 19).

The surface precipitates that formed on these samples include analcime (Figs. 53a and 53b), U-Si needles [possibly soddyite $\left(\mathrm{U}_{6} \mathrm{Si}_{2} \mathrm{O}_{16} \cdot \mathrm{H}_{2} \mathrm{O}\right)$ ] (Fig. 54a), Ca-Si spheres (Fig. 53c), Ca-Si blades (Figs. 53c and 54b), radiating Ca-Si prisms (Figs. 53c and 54c), Si-P moss-like growths (Figs. 53b and 54d), apatite (Figs. 53d and 54e), and Si-rich needles. The soddyite also contained minor amounts of $\mathrm{Na}$ and $\mathrm{K}$, suggesting some spectral interference from clay minerals and/or the presence of weeksite and sodium-weeksite. The diversity of Ca-Si bearing phases was unusual with respect to previously examined samples. It is not known if these phases are all separate phases or polymorphs of the same phase. 

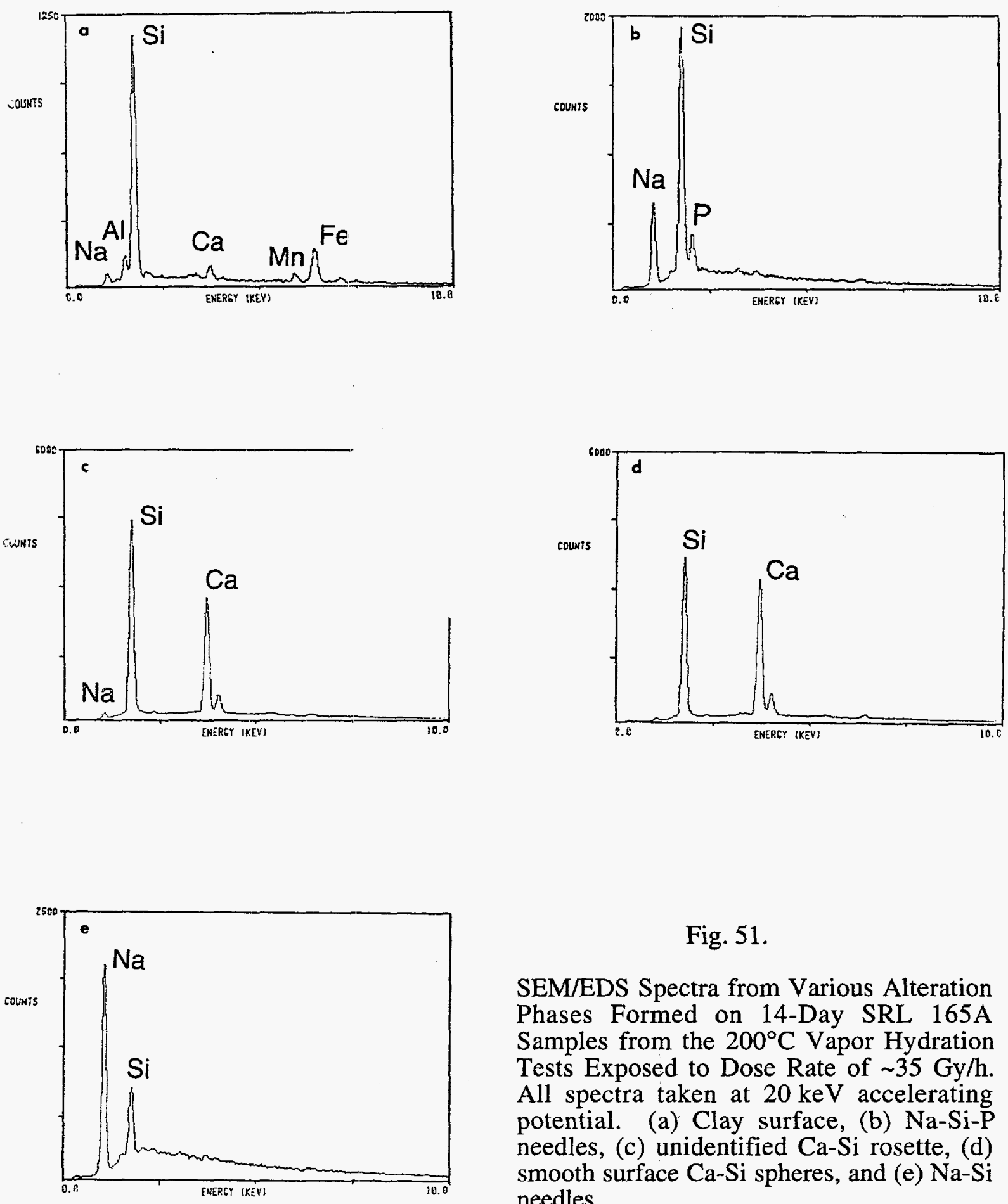

Fig. 51.

SEM/EDS Spectra from Various Alteration Phases Formed on 14-Day SRL 165A Samples from the $200^{\circ} \mathrm{C}$ Vapor Hydration Tests Exposed to Dose Rate of $\sim 35 \mathrm{~Gy} / \mathrm{h}$. All spectra taken at $20 \mathrm{keV}$ accelerating potential. (a) Clay surface, (b) Na-Si-P needles, (c) unidentified $\mathrm{Ca}-\mathrm{Si}$ rosette, (d) smooth surface $\mathrm{Ca}-\mathrm{Si}$ spheres, and (e) $\mathrm{Na}-\mathrm{Si}$ needles. 


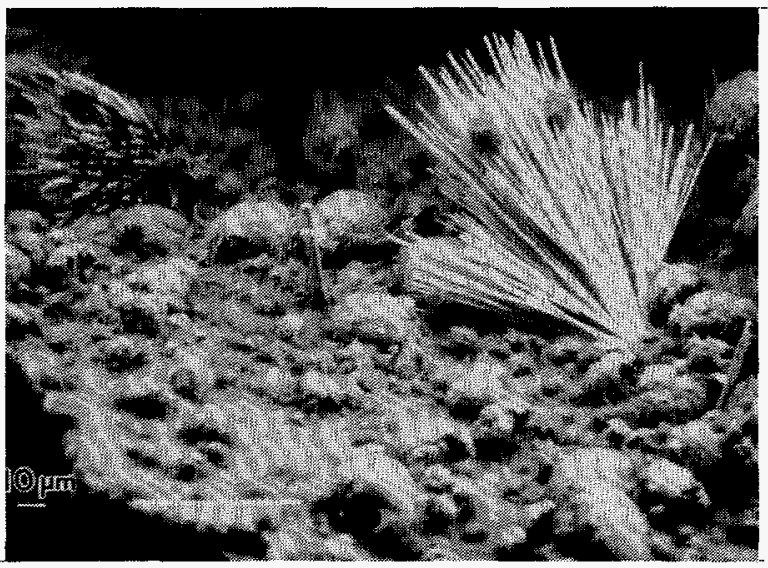

(a)

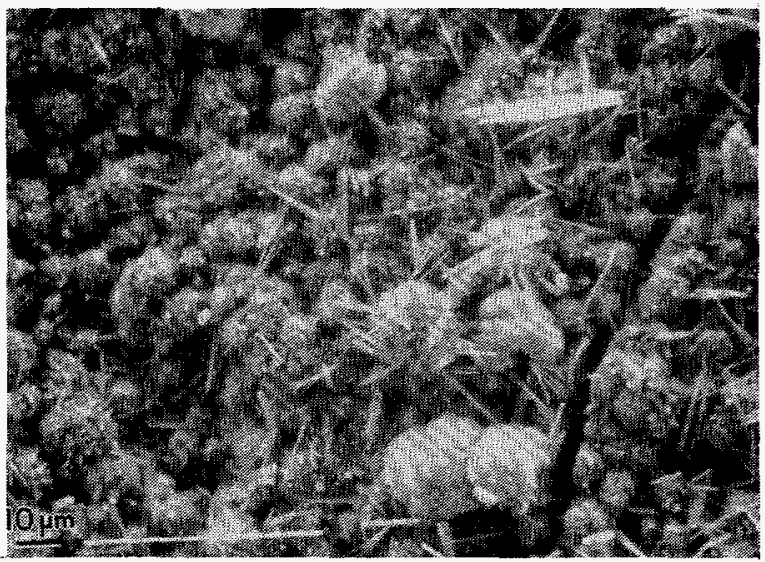

(b)

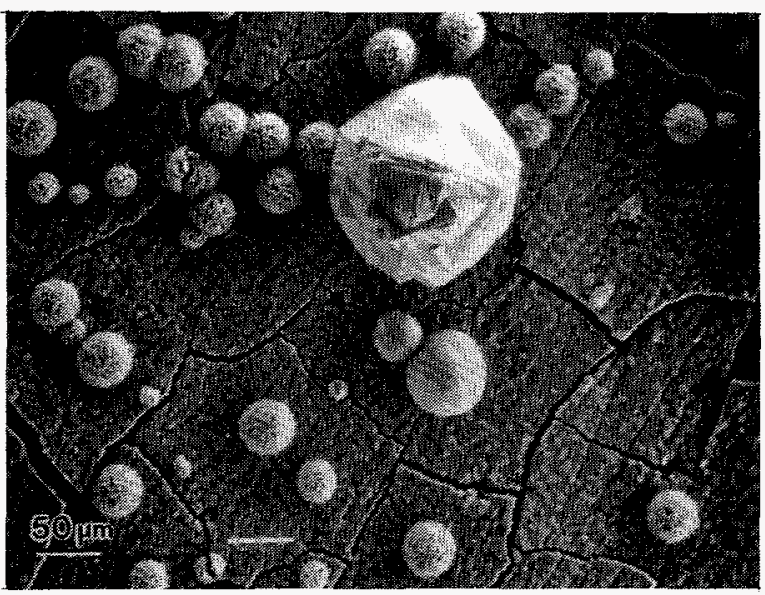

(c)

Fig. 52. SEM Images of Secondary Alteration Phases Forming on SRL 165A Samples from the $200^{\circ} \mathrm{C}$ Vapor Hydration Tests Exposed to Dose Rate of $\sim 35 \mathrm{~Gy} / \mathrm{h}$. (a) Two haiweeite needles (center of photo); large fan-shaped sodium-weeksite needle assemblages (to right and upper left); scattered fine-grained, dark-colored $\mathrm{Na}-\mathrm{Si}$ needles; and unidentified Ca-Si spheres (14 days, backscattered electron image, $500 \mathrm{X}$ ). (b) Bright sodium-weeksite needles, dark Na-Si-P needles, Ca-Si rosettes, and clay surface (14 day, backscattered electron image, 700X). (c) Large multifaceted analcime, smooth surface $\mathrm{Ca}-\mathrm{Si}$ spheres, and $\mathrm{Ca}-\mathrm{Si}$ rosettes (21 day, secondary electron image, $200 \mathrm{X}$ ). 
(a)

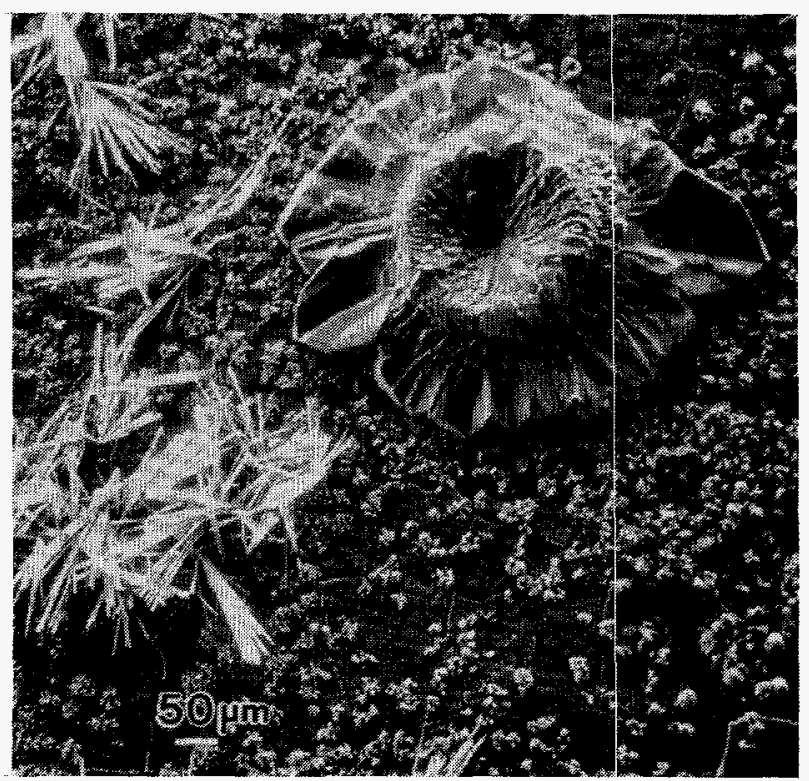

(c)

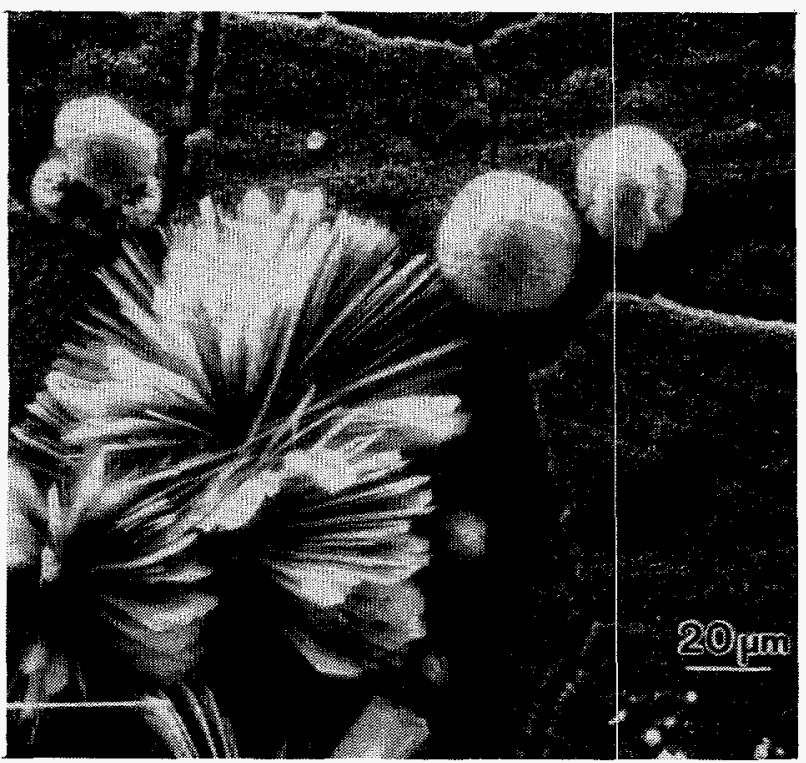

(b)

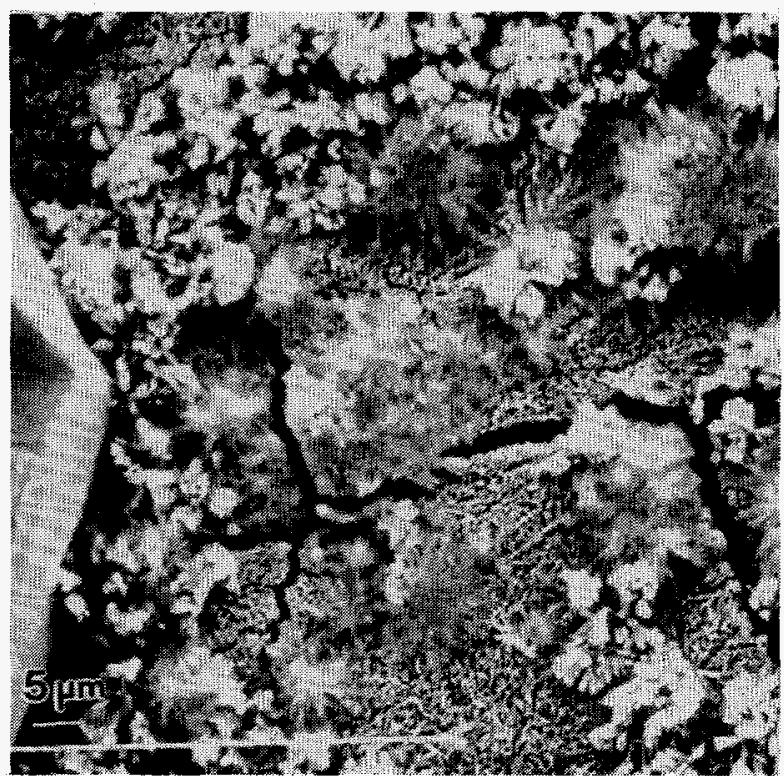

(d)

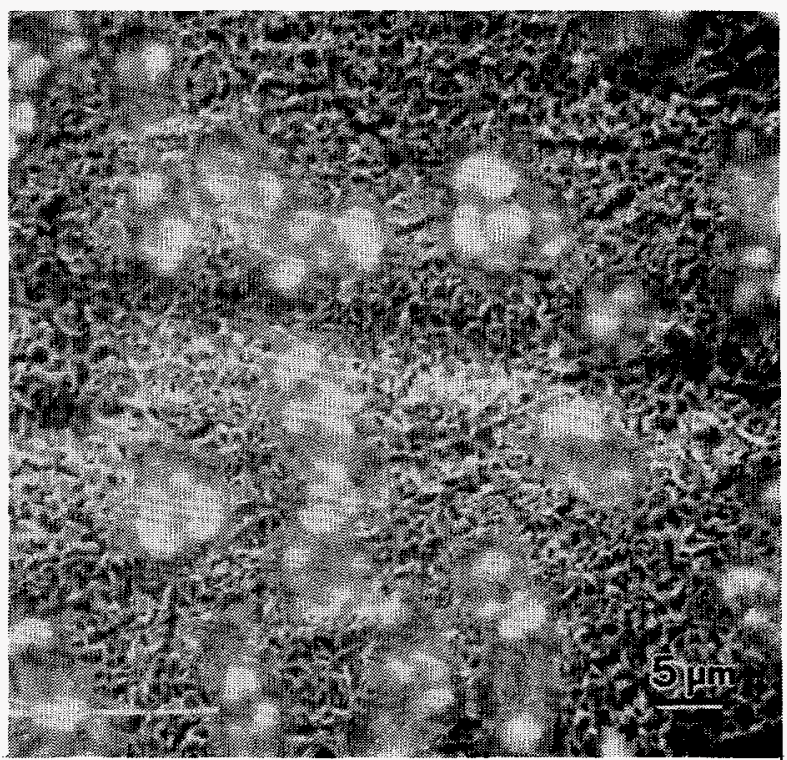

Fig. 53. SEM Images of Secondary Alteration Phases Forming on 35-Day SRL 165A Samples from the $200^{\circ} \mathrm{C}$ Vapor Hydration Tests Exposed to Dose Rate of $\sim 35 \mathrm{~Gy} / \mathrm{h}$. All images in backscattered electron mode. (a) Dimpled analcime, mixed sodiumand potassium-weeksite needles, and small radiating Ca-Si prisms (35 day, 1000X). (b) Analcime (to left), small radiating Ca-Si prisms, Si-P moss-like phase (35 day, 1000X). (c) Ca-Si spheres and Ca-Si blades (35 day, 500X). (d) Small buttons of apatite on clay surface ( 35 day, 1500X). 

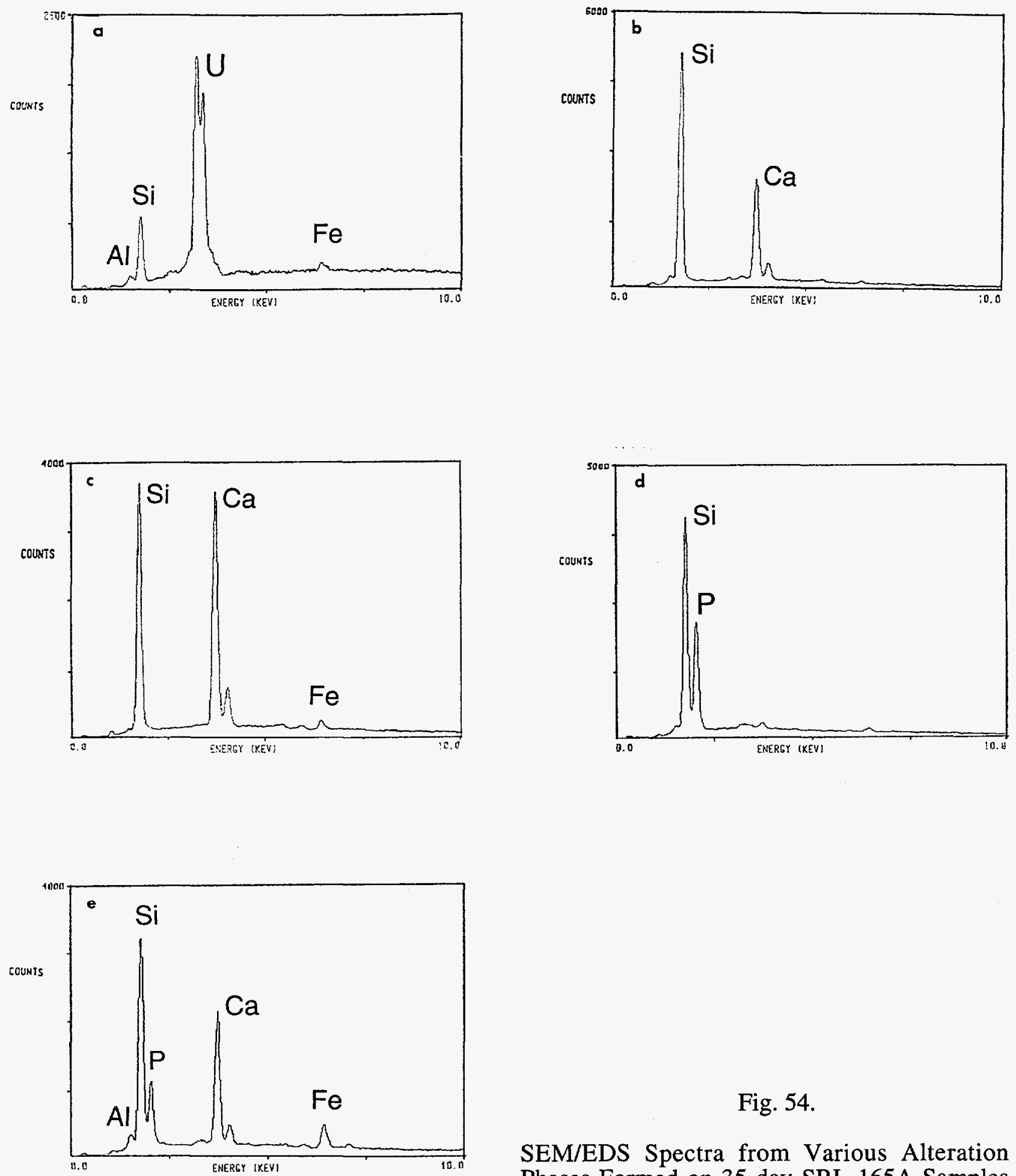

Fig. 54.

SEM/EDS Spectra from Various Alteration Phases Formed on 35-day SRL 165A Samples from the $200^{\circ} \mathrm{C}$ Vapor Hydration Tests Exposed to Dose Rate of $\sim 35 \mathrm{~Gy} / \mathrm{h}$. All spectra taken at $20 \mathrm{keV}$ accelerating potential. (a) Soddyite crystals, (b) Ca-Si blades, (c) radiating $\mathrm{Ca}-\mathrm{Si}$ prisms, (d) Si-P moss-like phase, and (e) apatite. 


\section{e. $\quad$ SRL $165 A$ Samples at 56 Days}

The altered surfaces of the 56-day SRL 165A samples (Fig. 50e) are typically more grayish than earlier samples. Upon termination of the tests, a thin film of solution remained in contact with the samples for several days, eventually drying into a salt residue. Some samples also displayed swelled or puffy surface regions, suggesting that areas with internal voids had developed within the samples. Despite some fracturing of the sample surface, the altered layer remained intact and attached to the glass. The SEM/EDS spectra of the clay layer suggest the presence of smectite clays. Clay reaction layer thicknesses measured during SEM cross-section examinations averaged $171 \pm 9 \mu \mathrm{m}$ (Table 19). A Na-Si-P rich substrate was also noted where the overlying layer had flaked off.

The surface precipitate minerals displayed by these samples include very fine-grained heterogeneous particles of a $\mathrm{Zn}-\mathrm{S}-\mathrm{Fe}-\mathrm{Pb}-\mathrm{Cl}$ (Fig. 55a), mixed sodium- and potassium-weeksite (Fig. 55b), copper oxides, iron oxides, Na-Si-S needles, and low-Z salts (Fig. 55b). Both blocky and dimpled morphologies of analcime were visible on these sample surfaces (Fig. 55b). Elongated needles of a Na-Si-S composition were commonly noted in association with the analcime grains. The sodium EDS spectra of this phase is enriched relative to the analcime substrate; thus, the presence of sodium in this phase appears to be real. The presence of silicon in these precipitates cannot be determined with any certainty due to their small size and the presence of silicon in the underlying analcime substrate.

3. $\quad$ SRL 131 Glass

\section{a. SRL 131U Sanples at 3-7 Days}

Previous tests with borosilicate glasses indicate that the SRL 131 composition glass is less resistant to aqueous alteration than the SRL 202 and SRL 165 glasses $[45,60]$. For this reason, experimental temperatures of $150^{\circ} \mathrm{C}$ were used for the reactions of the SRL 131 glass. Alteration surfaces on the 3-, 5-, and 7-day samples on SRL 131U glass developed in a similar manner and thus will be described together here. Upon exposure to the steam environment, the surfaces of the nonirradiated samples rapidly developed a brown alteration layer and a ubiquitous cover of white precipitates, that formed as the solution contacting the glass became saturated with respect to certain alteration products.

Detailed AEM examinations of the alteration layers from the nonirradiated SRL 131U glass tests have revealed the presence of a thin $(0.2 \mu \mathrm{m})$ outer clay layer. This layer is relatively coarse-grained with individual platy particles that are oriented perpendicular to the original glass surface and an inner layer of relatively fine-grained clays (Fig. 56a). The outer layer displays a curved crystal habit that is characteristic for the smectite clays and a $1.2-\mathrm{nm}$ basal spacing that is indicative of sodium-smectites with a single interlayer of water (Fig. 56b). In some surface regions of samples treacted for five or more days, this oriented outer layer has doubled into two parallel layers with an aggregate thickness of $0.5 \mu \mathrm{m}$ (Fig. 57), while in other regions only a single layer exists.

Lying between the coarse outer layer and the unaltered glass is an inner layer of fine-grained, randomly oriented clay (Figs. 56c and 57). This inner layer progressively thickens during the course of the tests by advancing along an alteration front into the glass.

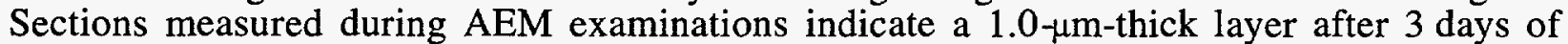
reaction (Fig. 56c), while both the 5- and 7-day samples displayed the formation of $1.5-\mu \mathrm{m}$-thick inner layers. The layer in the 3-day sample became progressively more crystalline as traverses were made from the unaltered glass and inner layer interface, to the inner layer and outer layer interface (Fig. 56c). This feature suggests that the alteration layers progressively crystallized from the outside surface inward. 
(a)

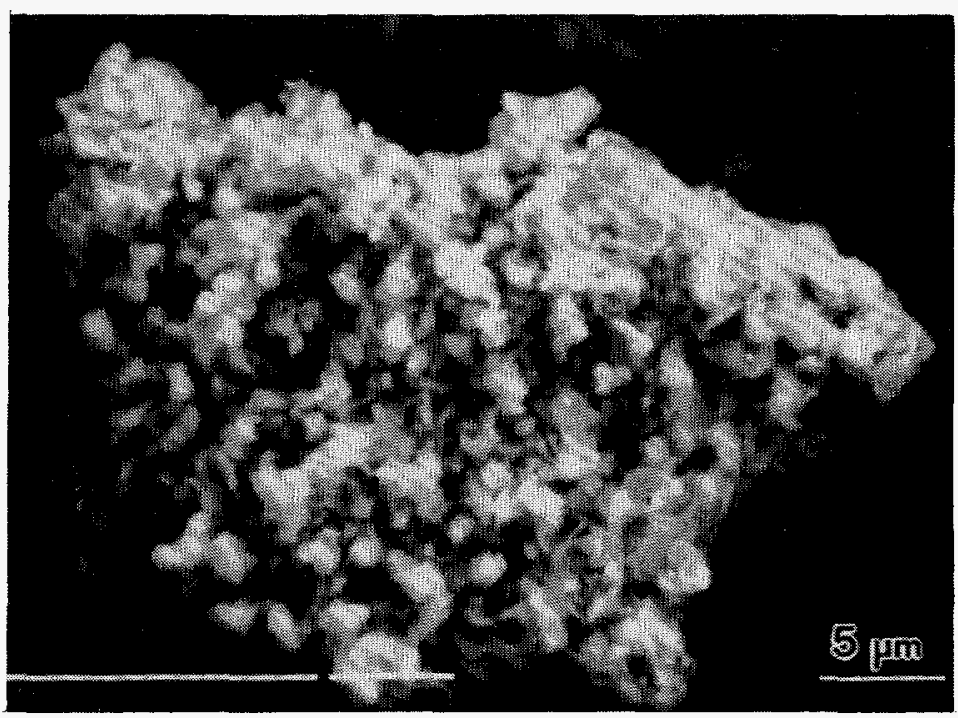

(b)

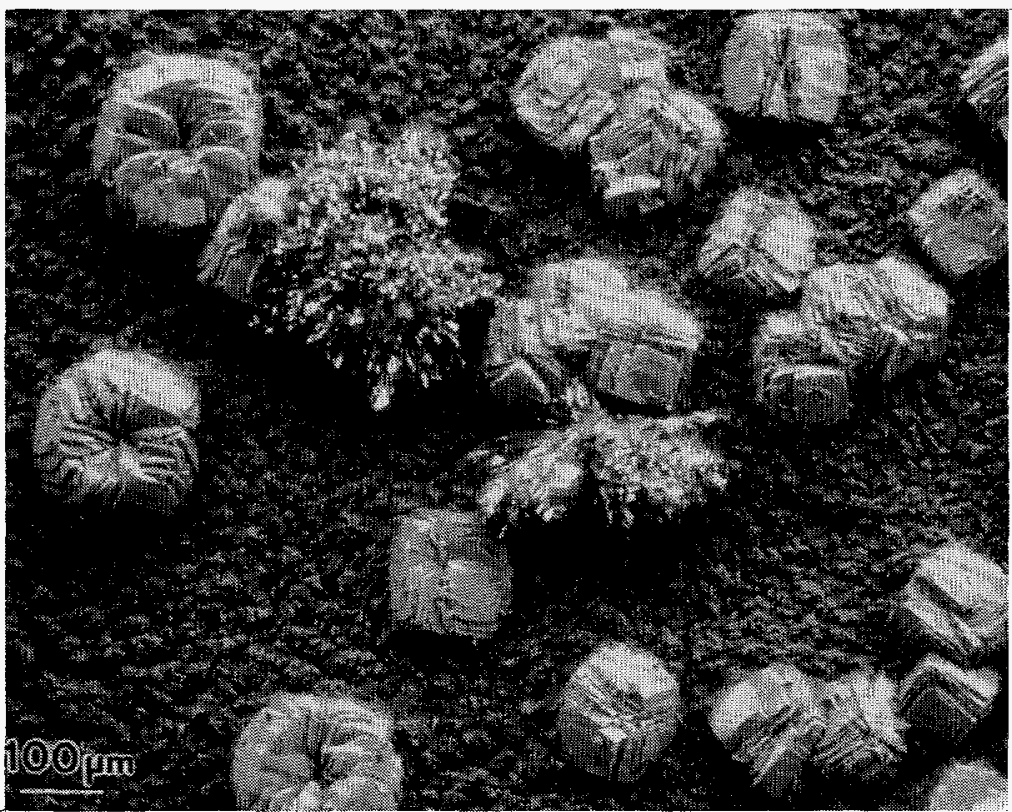

Fig. 55. SEM Images of Secondary Alteration Phases Forming on 56-Day SRL 165A Samples from the $200^{\circ} \mathrm{C}$ Vapor Hydration Tests Exposed to Dose of $\sim 35 \mathrm{~Gy} / \mathrm{h}$. Both images in backscattered electron mode. (a) Heterogeneous $\mathrm{Zn}-\mathrm{S}-\mathrm{Fe}-\mathrm{Pb}-\mathrm{Cl}$ precipitate $(3000 \mathrm{X})$. (b) Blocky and dimpled analcime, mixed sodium- and potassium-weeksite showing salt encrustation (100X). 

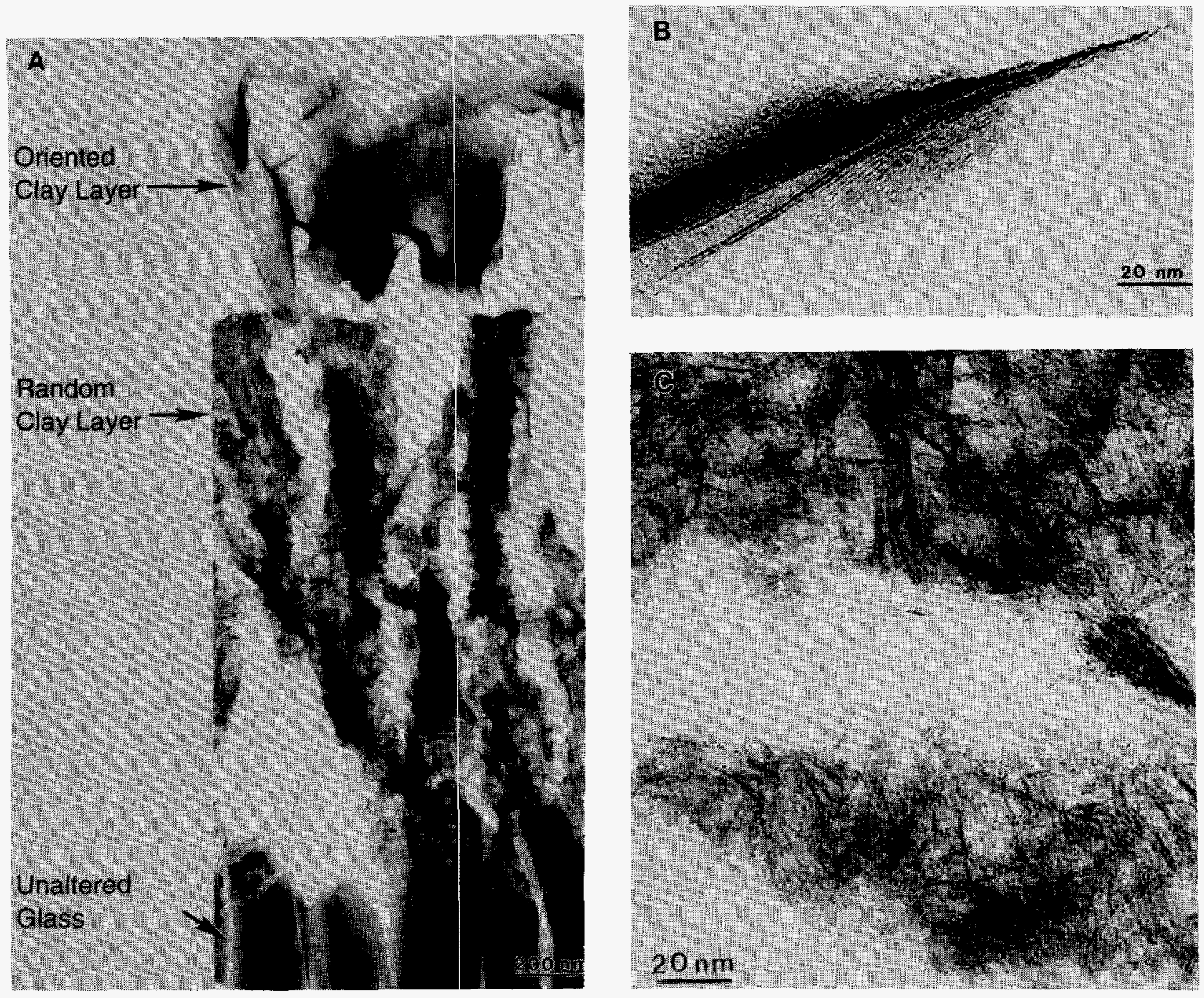

Fig. 56. AEM Photomicrographs of Alteration Layer Cross Section from SRL 131U Glass Samples Reacted in Vapor Hydration Test at $150^{\circ} \mathrm{C}$ for Three Days. (a) Lowmagnification image of entire cross-section showing an oriented outer clay layer, random-oriented inner clay layer, and unaltered glass. (b) High-magnification image of oriented outer clay material displaying wavy morphology characteristic of smectite clays. Lattice fringes from these clays have a measured spacing of about $1.2 \mathrm{~nm}$. (c) High-magnification image of random-oriented inner clay material. Lattice fringes from these clays also have a measured spacing of about $1.2 \mathrm{~nm}$. 


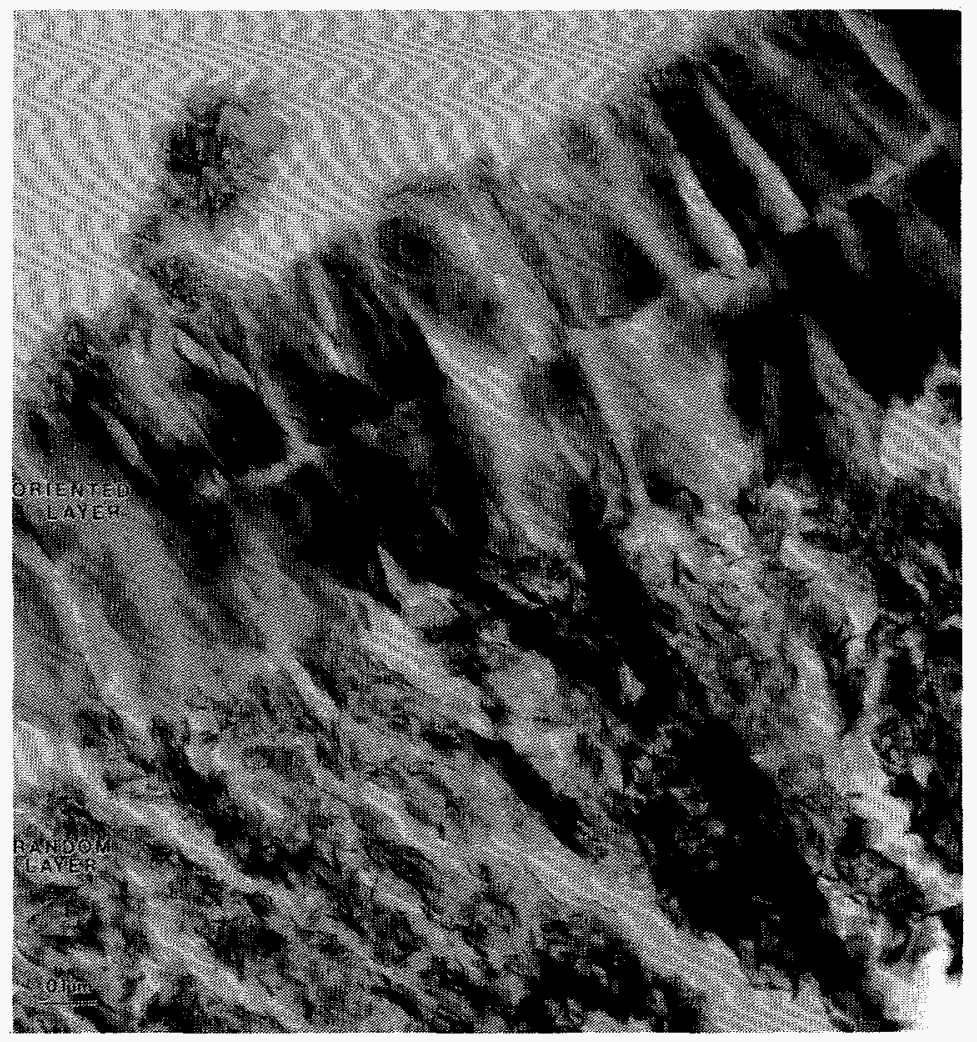

Fig. 57. Low-Magnification AEM Photomicrograph of Alteration Layer Cross Section from SRL $131 \mathrm{U}$ Glass Samples Reacted in Vapor Hydration Test at $150^{\circ} \mathrm{C}$ for Five Days Showing Oriented Outer Clay Layer and Random-Oriented Inner Clay Layer. Note the precipitation of fine-grained clay material on the outermost sample surface.

The smectite clays appeared as a honeycomb-textured layer that was readily apparent on the altered glass surface in as little as three days of reaction (Fig. 58). The layer measurements taken during SEM examinations of cross-sectioned samples were consistently thicker than those measured during the AEM examinations. Averaged SEM measurements from cross-sectioned samples of the 3-, 5-, and 7-day tests were $2.7 \pm 0.7$, $3.0 \pm 0.5$, and $3.7 \pm 0.8 \mu \mathrm{m}$, respectively (Table 19). These measurements were for the composite layer thickness, with the resolution of the SEM not being sufficiently good enough to image the outer and inner layers previously identified during AEM examinations. The cause for the discrepancy between the layer thickness measurements using the SEM and AEM techniques probably results from the AEM sections being cut nonperpendicular to the sample surfaces.

Selected area diffraction (SAD) analyses obtained during AEM study indicate that the clay layers are composed of smectite-group clay minerals (Table 22). This finding agrees with the qualitative compositional data obtained during SEM/EDS analysis (Table 23), which are consistent with the presence of a clay layer that has a nontronite-type smectite composition.

Semiquantitative AEM/EDS analyses have also been used to calculate a stoichiometric mass balance of clays in the reacted layer, with results for these calculations presented in Table 24. Caution must be exercised here in interpreting the phase calculations in this table because the derivative calculations include a number of simplistic assumptions about clay compositions and also use semiquantitative AEM/EDS analyses for the minor elements present in the clays. Despite these caveats, these phase calculations can provide useful 
information on the clay layer composition, especially when combined with the detailed AEM and SEM layer investigations performed in this study.

The mass balance calculations for the 3-day sample were derived by using the normalized AEM/EDS composition determined for the outer layer and were found to be consistent with a mixture of $60 \%$ nontronite, $30 \%$ saponite (as $\left.(1 / 2 \mathrm{Ca}, \mathrm{Na})_{0.66}(\mathrm{Mn}, \mathrm{Ni}, \mathrm{Mg})_{6}\left[\left(\mathrm{Si}_{7.34} \mathrm{Al}_{0.66}\right) \mathrm{O}_{20}\right]\left(\mathrm{OH}_{4}\right) \cdot \mathrm{nH}_{2} \mathrm{O}\right)$, and 10\% montmorillonite (Table 24). The results from these calculations also indicate excess titanium in the layer, which may be present as anatase or rutile $\left(\mathrm{TiO}_{2}\right)$, excess silicon, and a deficiency in the alkali-alkaline earth interlayer component. This latter deficiency probably results from the volatility of sodium during electron beam irradiation and a lack of sensitivity in detecting the light atomic weight alkali elements. The 5- and 7-day samples display a progressive decrease in the proportion of nontronite with time, as evidenced by the decreasing iron concentrations in the clay layer. The 7-day sample also displays evidence for decreasing proportions of saponite relative to the previous tests. A corresponding increase in the proportion of montmorillonite is indicated because of the increasing Al and Si concentrations in these latter samples.

The 3-day inner smectite layer displays a composition consistent with the presence of $55 \%$ nontronite, $40 \%$ saponite, and $<5 \%$ montmorillonite (Table 24). The inner layer also displays an increasing montmorillinite component at the expense of decreasing nontronite and saponite components over the 3- to 7-day interval. The 7-day sample has a calculated composition of approximately $40 \%$ nontronite, $30 \%$ saponite, and $30 \%$ montmorillonite. Excess aluminum occurred in the 3-day sample, while silicon was in excess for the 5- and 7-day samples. As with the previous outer smectite layer, titanium was presumed to occur in a separate phase, and alkali-alkaline earth concentrations were deficient for the calculated clay concentrations.

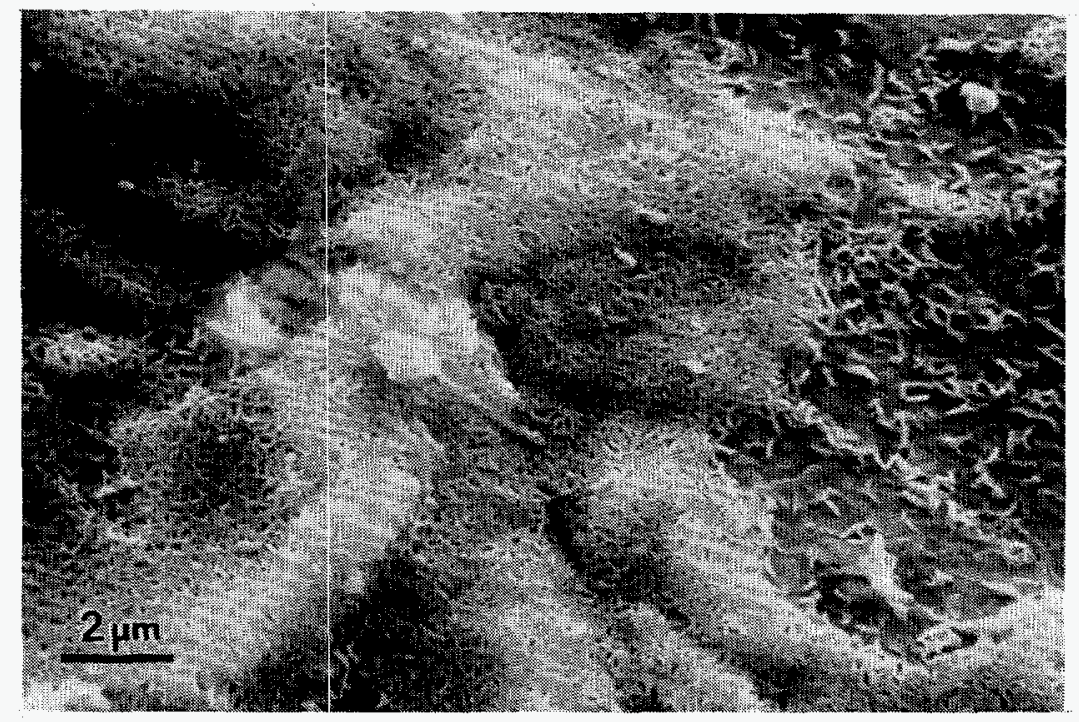

Fig. 58. SEM Image of Fine-Grained Smectite Growing over Glass Surface on 3-Day Sample of SRL $131 \mathrm{U}$ Glass (secondary electron image, 5000X). 
Table 22. Selected Area Diffraction Data Obtained from Inner Clay Layer of Three-Day $131 \mathrm{U}$

Samples and JCPDS Data for Montmorillonite

\begin{tabular}{|c|c|c|}
\hline \multicolumn{2}{|c|}{$\begin{array}{c}\text { 3-Day 131U } \\
\text { d-Spacings }(\mathrm{nm}) \\
\end{array}$} & $\begin{array}{c}\text { JCPDS 29-11499 } \\
(\mathrm{nm})\end{array}$ \\
\hline 0.453 & 0.012 & $\begin{array}{l}0.045 \\
0.315\end{array}$ \\
\hline 0.258 & 0.005 & 0.256 \\
\hline 0.17 & 0.003 & 0.169 \\
\hline 0.152 & 0.002 & 0.1495 \\
\hline 0.131 & 0.002 & 0.1325 \\
\hline
\end{tabular}


Table 23. AEM/EDS Compositional Analysis of Clay Layers Formed on SRL 131U Glass Samples Reacted in Vapor Hydration Tests

\begin{tabular}{|c|c|c|c|c|c|c|c|c|c|c|c|c|c|c|}
\hline \multicolumn{15}{|c|}{ Outer-Oriented Clay Layer } \\
\hline & \multicolumn{2}{|c|}{$131 \mathrm{U}-3-2 \mathrm{~b}$} & \multicolumn{2}{|c|}{$131 \mathrm{U}-5-2 b$} & \multicolumn{2}{|c|}{$131 \mathrm{U}-7-1 \mathrm{~b}$} & \multicolumn{2}{|c|}{$131 \mathrm{U}-14-1 \mathrm{~b}$} & \multicolumn{2}{|c|}{$131 \mathrm{U}-56-1 \mathrm{a}$} & \multicolumn{2}{|c|}{$131 U-91-1 a$} & \multicolumn{2}{|c|}{$131 \mathrm{U}-180-1 \mathrm{a}$} \\
\hline & Ave. & s.d. & Ave. & s.d. & Ave. & s.d. & Ave. & s.d. & Ave. & s.d. & Ave. & s.d. & Ave. & s.d. \\
\hline$\overline{\mathrm{Si}}$ & 49.50 & 1.44 & 52.74 & 0.84 & 56.50 & 0.00 & 53.42 & 0.22 & 56.51 & 2.24 & 55.15 & 0.30 & N.A. & $\overline{-}$ \\
\hline $\mathrm{Na}$ & 0.19 & 0.19 & 0.11 & 0.22 & 0.42 & 0.42 & 0.01 & 0.01 & N.A. & - & N.A. & - & N.A. & - \\
\hline $\mathrm{Mg}$ & 1.92 & 0.22 & 2.28 & 0.43 & 0.97 & 0.01 & 2.42 & 0.17 & 3.66 & 0.38 & 2.04 & 0.68 & N.A. & - \\
\hline $\mathrm{Al}$ & 5.79 & 0.64 & 7.88 & 0.91 & 10.75 & 0.02 & 9.51 & 0.13 & 6.47 & 0.75 & 0.02 & 0.00 & N.A. & - \\
\hline $\mathrm{K}$ & 0.09 & 0.07 & 0.01 & 0.01 & 0.01 & 0.01 & 0.00 & 0.00 & N.A. & - & N.A. & - & N.A. & - \\
\hline $\mathrm{Ca}$ & 0.96 & 0.26 & 0.32 & 0.10 & 0.35 & 0.11 & 0.25 & 0.03 & 1.52 & 0.35 & 3.10 & 1.03 & N.A. & - \\
\hline Ti & 0.61 & 0.26 & 0.65 & 0.54 & 0.63 & 0.20 & 0.40 & 0.07 & N.A. & - & N.A. & - & N.A. & - \\
\hline $\mathrm{Mn}$ & 8.51 & 0.58 & 7.65 & 0.18 & 6.45 & 0.13 & 7.36 & 0.35 & 6.92 & 1.16 & 6.22 & 0.25 & N.A. & - \\
\hline $\mathrm{Fe}$ & 31.81 & 0.59 & 26.99 & 0.94 & 23.18 & 1.00 & 26.18 & 0.47 & 22.54 & 2.08 & 30.40 & 0.59 & N.A. & - \\
\hline \multirow[t]{2}{*}{$\mathrm{Ni}$} & 0.63 & 0.21 & 1.37 & 0.81 & 0.75 & 0.10 & 0.48 & 0.02 & 2.39 & 1.24 & 3.19 & 0.09 & N.A. & - \\
\hline & $n=9$ & & $n=5$ & & $n=2$ & & $n=2$ & & $n=5$ & & $n=2$ & & & \\
\hline \multicolumn{15}{|c|}{ Inner Random-Oriented Fine-Grained Clay Layer } \\
\hline & \multicolumn{2}{|c|}{$131 \mathrm{U}-3-2 b$} & \multicolumn{2}{|c|}{$131 \mathrm{U}-5-2 \mathrm{~b}$} & \multicolumn{2}{|c|}{$131 \mathrm{U}-7-1 \mathrm{~b}$} & \multicolumn{2}{|c|}{$131 \mathrm{U}-14-1 \mathrm{~b}$} & \multicolumn{2}{|c|}{$131 \mathrm{U}-56-1 \mathrm{a}$} & \multicolumn{2}{|c|}{$131 \bigcup-91-1 \mathrm{a}$} & \multicolumn{2}{|c|}{$131 \mathrm{U}-180-1 \mathrm{a}$} \\
\hline & Ave. & s.d. & Ave. & s.d. & Ave. & s.d. & Ave. & s.d. & Ave. & s.d. & Ave. & s.d. & Ave. & s.d. \\
\hline$\overline{\mathrm{Si}}$ & 48.06 & 1.77 & 52.49 & 1.52 & 55.18 & 0.59 & 49.81 & 0.48 & 56.28 & 6.32 & 55.56 & 1.87 & 55.70 & 5.39 \\
\hline $\mathrm{Na}$ & 0.68 & 0.43 & 0.61 & 0.67 & 0.15 & 0.12 & 0.29 & 0.20 & N.A. & - & N.A. & - & N.A. & - \\
\hline $\mathrm{Mg}$ & 0.71 & 0.39 & 0.61 & 0.18 & 1.05 & 0.07 & 0.99 & 0.17 & 1.62 & 1.03 & 2.05 & 0.46 & 4.31 & 1.70 \\
\hline $\mathrm{Al}$ & 5.38 & 0.50 & 7.17 & 0.62 & 9.15 & 0.15 & 8.57 & 0.11 & 5.58 & 2.16 & 1.23 & 1.33 & 4.89 & 1.95 \\
\hline $\mathrm{K}$ & 0.18 & 0.14 & 0.03 & 0.05 & 0.16 & 0.16 & 0.30 & 0.08 & N.A. & - & N.A. & - & N.A. & - \\
\hline $\mathrm{Ca}$ & 1.66 & 0.45 & 0.52 & 0.24 & 0.30 & 0.09 & 0.70 & 0.05 & 2.45 & 1.58 & 2.85 & 1.02 & 0.03 & 0.01 \\
\hline $\mathrm{Ti}$ & 1.68 & 0.39 & 1.36 & 0.08 & 1.03 & 0.02 & 1.45 & 0.06 & N.A. & - & N.A. & - & N.A. & - \\
\hline Mn & 8.71 & 0.53 & 7.76 & 0.40 & 7.11 & 0.13 & 7.76 & 0.18 & 5.57 & 1.91 & 6.16 & 1.43 & 4.79 & 3.20 \\
\hline $\mathrm{Fe}$ & 27.91 & 1.28 & 25.12 & 0.17 & 22.09 & 0.06 & 25.70 & 0.31 & 25.41 & 6.12 & 28.50 & 1.75 & 27.70 & 3.17 \\
\hline \multirow[t]{2}{*}{$\mathrm{Ni}$} & 5.04 & 0.75 & 4.34 & 0.17 & 3.81 & 0.22 & 4.47 & 0.31 & 3.09 & 1.24 & 3.67 & 0.45 & 2.59 & 1.60 \\
\hline & $n=13$ & & $\mathrm{n}=4$ & & $n=2$ & & $n=2$ & & $n=12$ & & $n=4$ & & $n=4$ & \\
\hline
\end{tabular}

All values in normalized element wt \%; s.d. is standard deviation; $n$ is number of analyses; N.A. = not analyzed. 
Table 24. Calculated Clay Mineral Fractions (in percent) in Reacted Clay Layers Based on Analyzed Layer Composition of 131 U Samples

\begin{tabular}{|c|c|c|c|c|}
\hline $\begin{array}{l}\text { Test } \\
\text { Length (days) }\end{array}$ & Saponite & Nontronite & Montmorillonite & Illite \\
\hline \multicolumn{5}{|c|}{ Outer Layer } \\
\hline 3 & $\overline{31}$ & 60 & 9 & 1 \\
\hline 5 & 29 & 49 & 22 & 0 \\
\hline 7 & 19 & 42 & 39 & 0 \\
\hline 14 & 24 & 46 & 30 & 0 \\
\hline 56 & 38 & 45 & 17 & 0 \\
\hline 91 & 37 & 63 & 0 & 0 \\
\hline 180 & N.A. & N.A. & N.A. & N.A. \\
\hline \multicolumn{5}{|c|}{ Inner Layer } \\
\hline 3 & $\overline{42}$ & 53 & 3 & 1 \\
\hline 5 & 35 & 47 & 18 & 0 \\
\hline 7 & 31 & 40 & 28 & 1 \\
\hline 14 & 36 & 48 & 15 & 2 \\
\hline 56 & 32 & 54 & 13 & 0 \\
\hline 91 & 39 & 61 & 0 & 0 \\
\hline 180 & 29 & 46 & 6 & 0 \\
\hline
\end{tabular}

N.A. = not analyzed.

Compositions of clays used in analysis:

saponite $(1 / 2 \mathrm{Ca}, \mathrm{Na})_{0.7} \mathrm{Mg}_{6}\left(\mathrm{Si}_{7.34} \mathrm{Al}_{0.66} \mathrm{O}_{20}\right)(\mathrm{OH})_{4} \bullet^{n} \mathrm{H}_{2} \mathrm{O}$, nontronite $(1 / 2 \mathrm{Ca}, \mathrm{Na})_{0.7} \mathrm{Fe}_{4}\left(\mathrm{Si}_{7.34} \mathrm{Al}_{0.66} \mathrm{O}_{20}\right)(\mathrm{OH})_{4} \cdot n^{2} \mathrm{H}_{2} \mathrm{O}$, montmorillonite $(1 / 2 \mathrm{Ca}, \mathrm{Na})_{0.7}\left(\mathrm{Al}_{3.34} \mathrm{Mg}_{0.66}\right)\left(\mathrm{Si}_{8} \mathrm{O}_{20}\right)(\mathrm{OH})_{4} \bullet \mathrm{nH}_{2} \mathrm{O}$, illite $\mathrm{K}_{1-1.5} \mathrm{Al}_{4}\left(\mathrm{Si}_{6.5-7} \mathrm{Al}_{1-1.5} \mathrm{O}_{20}\right)(\mathrm{OH})_{4}$.

Surface precipitates were relatively rare on these early samples. Very fine-grained spherical masses with a Na-Al-Si-Fe composition were noted on the 3-day samples. These may be early-formed analcime, but isolation of these phases for further analysis was impossible due to their extremely small size. Flat, hexagonal-shaped Na-rich chabazite (herschelite) grains first appeared on the sample surfaces after seven days.

\section{b. $\quad$ SRL 131U Samples at 14 Days}

Altered glass monoliths were covered with the same brownish alteration layer and thin outer cover of white precipitates that characterized the earlier samples (Fig. 59a). The SEM/EDS analysis indicates that this layer was of a similar nontronite-dominated mineralogy as that present in the earlier SRL 131U samples. Layer measurements during SEM examinations of these samples averaged $5.1 \pm 0.9 \mu \mathrm{m}$ (Table 19).

Quantitative AEM/EDS analyses (Table 23) indicate a mineralogic composition for the 14-day outer smectite layer that is consistent with the presence of approximately $45 \%$ nontronite, $30 \%$ montmorillonite, and $25 \%$ saponite (Table 24 ). The results from these calculations also indicate excess $\mathrm{Si}$ and $\mathrm{Ti}$ and a deficiency in the alkali-alkaline earth interlayer component for the calculated clay mineral compositions. The 14-day inner smectite layer has a calculated composition of approximately $50 \%$ nontronite, $35 \%$ saponite, and $15 \%$ 

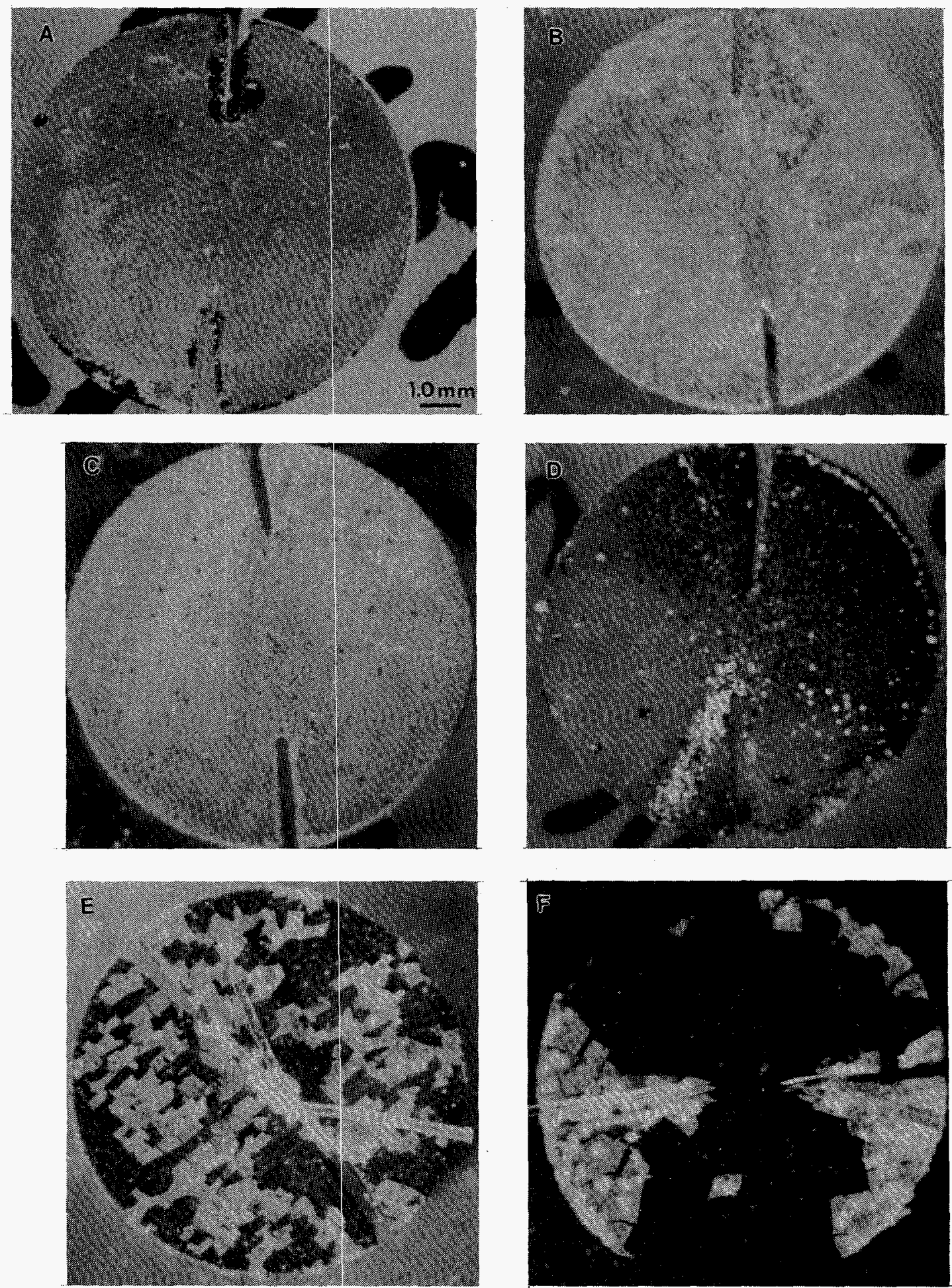

Fig. 59. Optical Photographs of Reacted Surfaces on SRL $131 \mathrm{U}$ Samples from the $150^{\circ} \mathrm{C}$ Vapor Hydration Tests. All photographs at $9 \mathrm{X}$ with disk diameter $\sim 10 \mathrm{~nm}$ : (a) 14-day sample, (b) 56-day sample, (c) 91-day sample, (d) 180-day sample, (e) 365-day sample displaying flaked off portion of surface layer. 
montmorillonite. These calculations also indicate excess $\mathrm{Al}$ and $\mathrm{Ti}$ and a deficiency in the alkalialkaline earth interlayer component for the calculated clay mineral compositions.

Surface precipitate phases were still relatively rare on these samples. Hexagonal plates of Na-rich chabazite (herschelite), identical to those present on the surfaces of the 7 -day samples, were detected on these samples (Fig. 60). The SEM/EDS analysis indicates a $\mathrm{Na}-\mathrm{Al}-\mathrm{Si}$ composition for this phase that was similar to that forming on the SRL 202U glasses (see Fig. 24d). Analcime grains were also noted on the surfaces of these samples, with this phase exhibiting a pure $\mathrm{Na}-\mathrm{Al}-\mathrm{Si}$ composition.

\section{c. $\quad$ SRL 131U Samples at 28 Days}

The SRL 131U surfaces on 28-day samples are characterized by an increased presence of the whitish surface layer. Some samples also display increased growth of surface precipitates along portions of the sample in contact with the Teflon hanging thread and portions of the sample hanging lowest in the test chamber. As with previous samples, this feature is attributed to the preferential accumulation of water on these regions of the sample as a result of gravitation effects and electrostatic attractive forces of water. The thicker films of water in these regions, in turn, were more conducive to crystal formation because they induced less stress on crystal growth than areas of the sample covered with only a thin film of water.

Analytical electron microscopy analysis of the clay layers from SRL 131U glasses indicate the presence of a thin outer smectite layer with relatively coarse, platy, clay grains oriented perpendicular to the glass surface and an inner layer of randomly oriented finegrained smectite. Quantitative AEM/EDS analyses were not performed on these altered glass samples. The SEM/EDS analyses indicate that both layers are depleted in $\mathrm{Na}$ and $\mathrm{K}$, while being enriched in $\mathrm{Si}, \mathrm{Al}, \mathrm{Mg}, \mathrm{Mn}, \mathrm{Fe}$, and Ti relative to the unaltered glass. Layer measurements made during SEM examinations of these samples averaged $4.9 \pm 0.9 \mu \mathrm{m}$ and thus were virtually indistinguishable in layer thickness from the 14-day samples (Table 19).

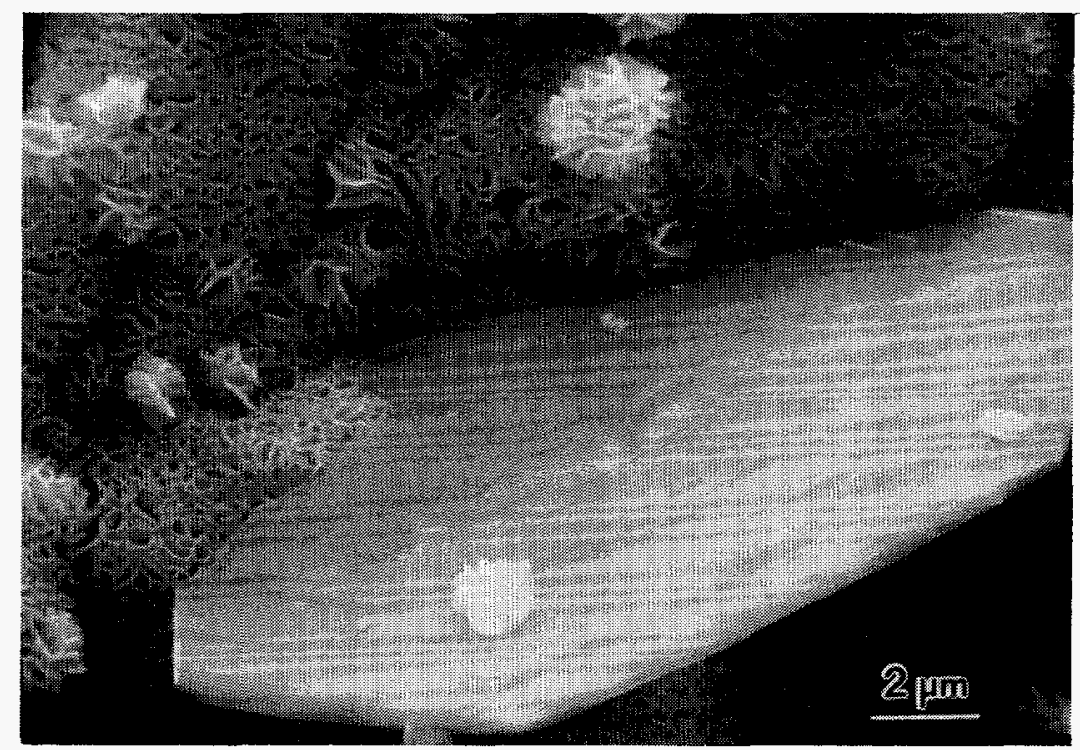

Fig. 60. Secondary Electron Image of Na-Rich Chabazite (Herschelite) Disk Growing on Smectite Surface for 14-Day Sample of SRL 131U Glass (5000X). 
Surface precipitate phases were still relatively sparse on these samples. Phases that were located on these samples include analcime, rhombohedral calcite, and unidentified $\mathrm{Ca}-\mathrm{Si}$ nodules. Patches of low-Z salts were also noted on these samples. These salts probably give rise to the whitish discoloration of the sample surfaces, as previously noted.

\section{d. $\quad$ SRL 131U Samples at 56 Days}

The 56-day samples with the SRL $131 \mathrm{U}$ glasses continued to display an increase in the development of the outer white precipitate zone. Sample surfaces at this time appeared as a light gray to white covering over a brownish clay layer (Fig. 59b). Layer thickness measurements taken during SEM examinations of cross-sectioned samples averaged $9.2 \pm 1.1 \mu \mathrm{m}$ (Table 19).

Analytical electron microscopy examination indicates that the smectite surface is still segregated into a distinct coarse-grained outer and fine-grained inner layers (Fig. 61). The outer layer is approximately $1-\mu \mathrm{m}$ thick, and has developed with the individual clay particles oriented with their long axes oriented normal to the glass surface. The inner layer displays a general random orientation of finer-grained clay particles and has an aggregate thickness of $4 \mu \mathrm{m}$. However, this sample also displayed an unusual development of fine grained banded zones in the inner layer, indicating that the growth orientation of clays in this layer was not completely random. There are at least six bands of fine grained clay, parallel to the sample surface, that are visible within the inner clay layer depicted in Fig. 61a. This banded feature was not observed on any of the sectional samples from the SRL 131 glass, nor any of the other glass compositions examined in this study. The rhythmic bands record a history of episodic growth for this inner layer, possibly indicating a diffusion influenced composition for the interpore fluids from which those clays were formed.

The nucleation of the inner smectite clay layer appears to have occurred in a heterogeneous manner, with multinucleation sites occurring spontaneously within the altered glass layer, rather than forming along a definite reaction front that progressed into the glass. The reaction layer appears to have progressed through a crystallization sequence where amorphous material first crystallized into fine-grained clays, and then recrystallized again into intermediatesized clay particles. Evidence used to support this interpretive sequence includes the presence of numerous crystal nucleation sites lying between regions of amorphous material. These features are evident where relatively coarse-grained smectites (the nucleation centers) laterally grade first into crystallized finer-grained smectite, and finally into zones of amorphous material (Fig. 61b). Figures $61 \mathrm{c}$ and $61 \mathrm{~d}$ display the SAED patterns from the regions with large and small smectite crystals, respectively. The major d-spacings measured from both regions are virtually the same $(0.47,0.27$, and $0.16 \mathrm{~nm})$. However, the diffraction rings from the relatively coarser-grained region (Fig. 61c) are less continuous than those of the finer-grained region (Fig. 61d), indicating that a small number of crystals with a nonrandom orientation give rise to the electron diffraction patterns in the former. 

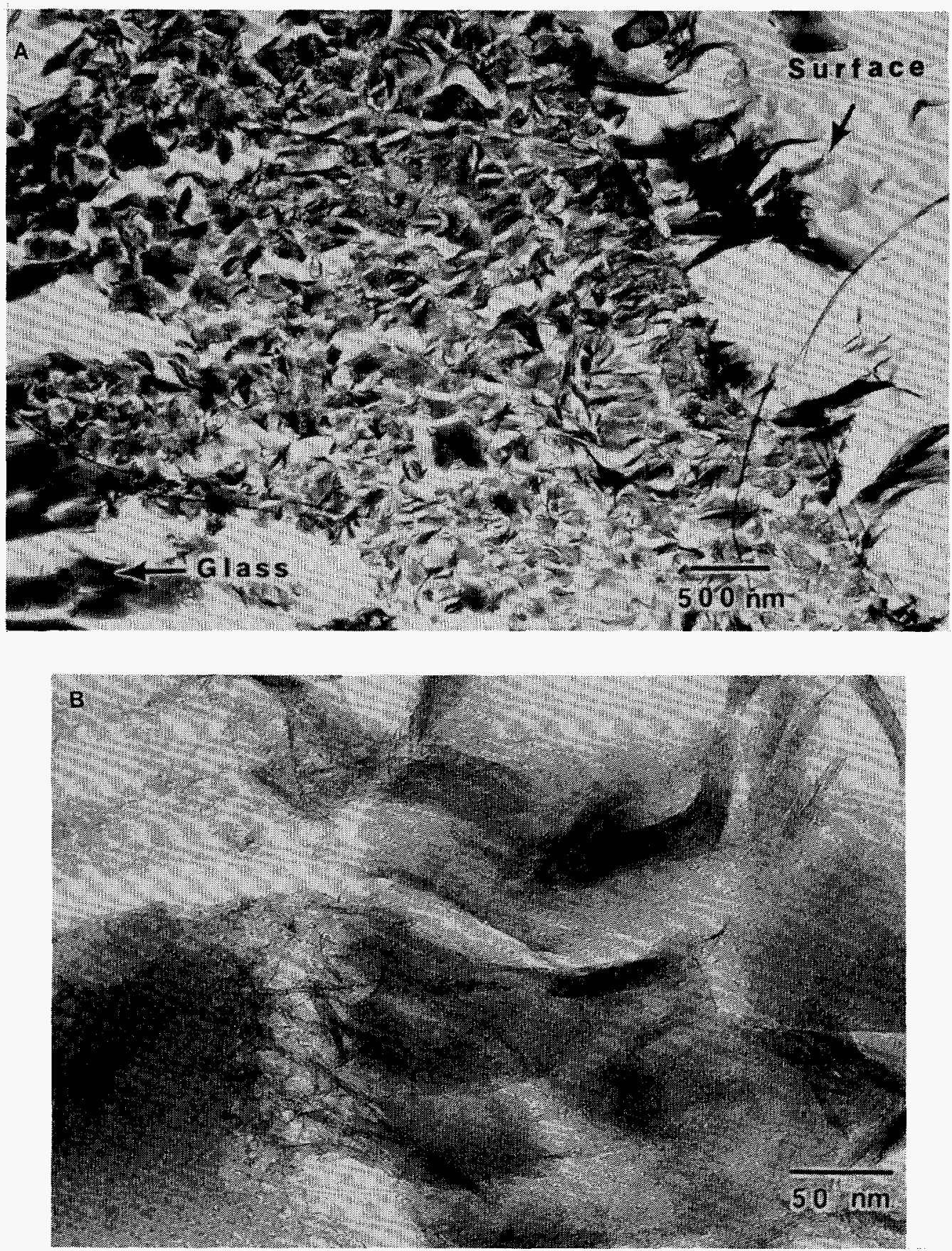

Fig. 61. AEM Photomicrograph of Alteration Layer Cross Section from SRL 131U Glass Samples Reacted in Vapor Hydration Test at $150^{\circ} \mathrm{C}$ for 56 Days. (a) Lowmagnification image of entire cross section showing an oriented outer surface clay layer, random-oriented inner clay layer, and unaltered glass. (b) High-magnification image of random-oriented inner clay layer displaying lateral gradation from amorphous to fine-grained banded zone clays to coarse-grained clays. (c) Selected area electron diffraction image of coarse-grained smectite displaying discontinuous diffraction ring pattern. (d) Selected area electron diffraction image of fine-grained smectite displaying continuous diffraction ring pattern. (e) Microvugs developed within randomly oriented clay layer. 
Fig. 61 (contd.)
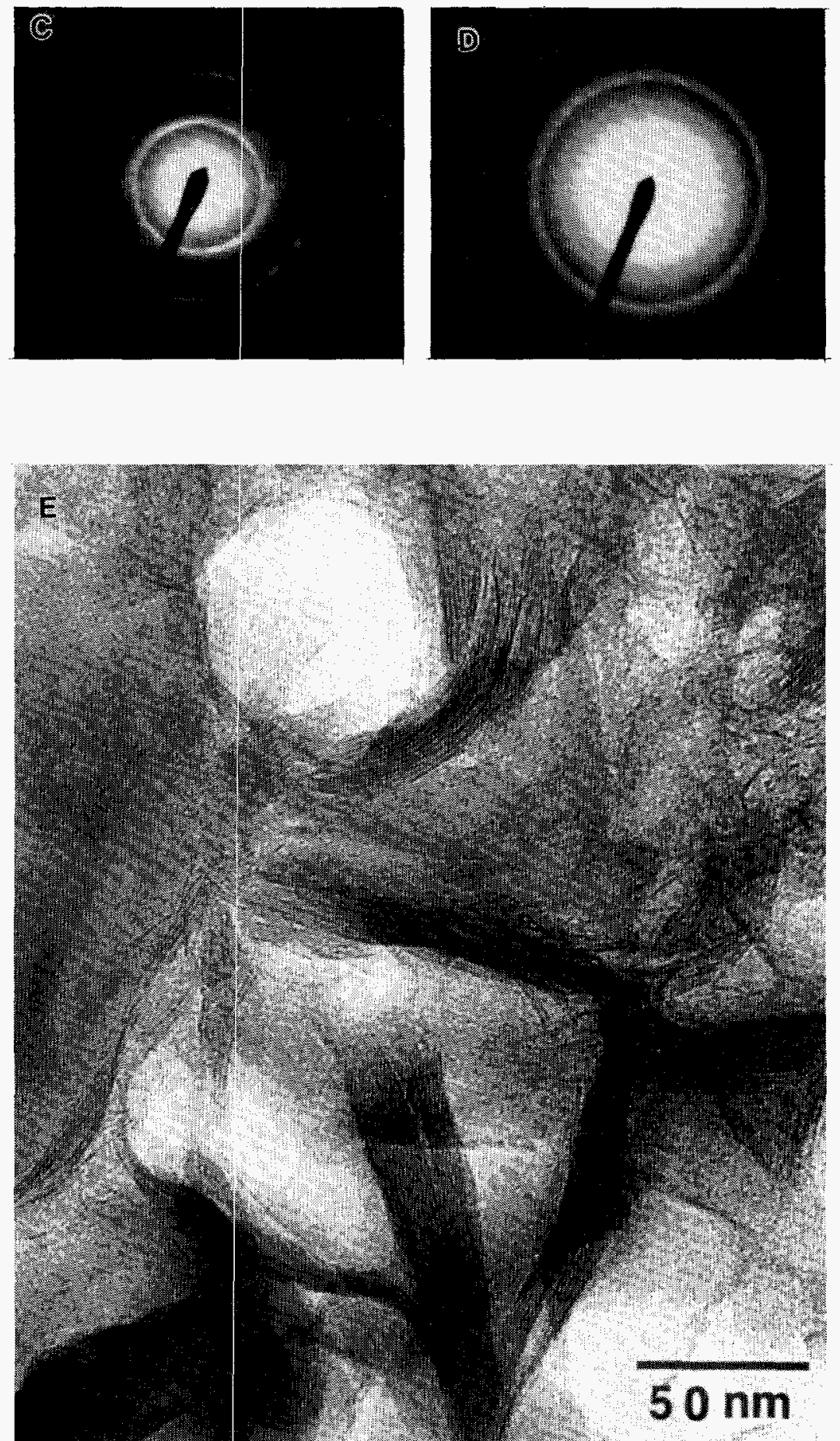
Microvugs or cavities that are rimmed by intermediate-sized clay grains also provide evidence for recrystallization of the reacted layer (Fig. 61e). These latter features arise due to the higher density of the clay relative to its precursor glass and/or amorphous source material. The result of this recrystallization of amorphous material into a higher density phase would necessitate the formation of microvugs in the layer or, alternatively, a decrease in the total layer thickness. It is considered to be unlikely that the microvugs existed in this layer prior to the recrystallization. If the microvugs were primary features present in the amorphous glass, then one would expect the clay particles precipitating in this open-space feature to orient themselves in a ring around the inside of the microvug, with their long axes perpendicular to the microvug surface. This process would be analogous to the preferred orientation of the smectites on the outer layer, as previously described. This type of orientation was not observed. Instead, the clays occurred with either nonoriented directions relative to the microvugs (Fig. 6le) or with clay crystals that were deformed during microvug growth, with their long axes parallel to the evolving microvug surface. These features are suggestive of microvug growth during recrystallization of the layer.

Quantitative AEM/EDS analyses (Table 23) indicate a mineralogic composition for the 56-day outer smectite layer that is consistent with the presence of approximately $45 \%$ nontronite, $40 \%$ saponite, and $15 \%$ montmorillonite (Table 24). The composition of the inner layer, as determined by AEM/EDS analysis, is consistent with the presence of approximately $55 \%$ nontronite, $30 \%$ saponite, and $15 \%$ montmorillonite. This inner layer is also heterogeneous on a fine scale, with regions of both amorphous material and small clay crystals that were enriched in $\mathrm{Ca}$ and depleted in Fe relative to the amorphous zones. The calculations of mineral composition for both the inner and outer smectite layers indicate a sufficiently large excess silicon component and a depletion in the alkali-alkaline earth component. These AEM/EDS analyses did not include $\mathrm{Na}, \mathrm{K}$, and Ti. The exclusion of the two former elements possibly gave rise to the alkaline and alkaline earth shortages in the calculations.

Surface precipitates were relatively rare on these samples, with analcime and copper oxides being the only phases detected. The copper oxides may have been derived as a contaminant from the copper gasket used to send the reaction vessel.

\section{e. $\quad$ SRL 131U Samples at 91 Days}

The SRL 131U samples after 91 days displayed a decrease in the amount of white surface material relative to that of the previous tests at 56 days (Fig. 59c). Relatively coarser-grained precipitates also began to be more prevalent on these samples relative to the shorter term tests. The whitish film that covers these samples has also become more strongly developed along the portion of the sample that hangs lowest in the sample vessel.

Analytical electron microscopy examinations of the altered layer material indicated that the layer is still segregated into an outer layer of relatively coarse-grained, oriented smectite and an inner layer of random-oriented smectite. The outer layer displays smectite lengths of up to $200 \mathrm{~nm}$ or larger, with basal spacings of 1.2 to $1.5 \mathrm{~nm}$ (Fig. 62a). The inner layer is composed almost entirely of fine- to intermediate-sized clay aggregates with dimensions of 20 to $40 \mathrm{~nm}$ long and less than $10 \mathrm{~nm}$ wide (Fig. $62 \mathrm{~b}$ ). Unlike the previous samples, there were no remaining amorphous regions or large crystals found in this inner layer. This homogenization signifies the near complete crystallization of this altered layer. The absence of the large crystals that were present in the previous 56-day tests indicates that either the samples have altered in a different manner, or the large crystals were originally present in this sample but have recrystallized into finer-grained material between 56 and 91 days. The SAED patterns from the relatively coarser-grained region (Fig. 62a inset) are less continuous than those of the finergrained region (Fig. 62b inset), indicating a smaller number of crystals with a less random 
orientation in the former. Layer measurements during SEM examinations of these samples averaged $14.5 \pm 1.1 \mu \mathrm{m}$ (Table 19).
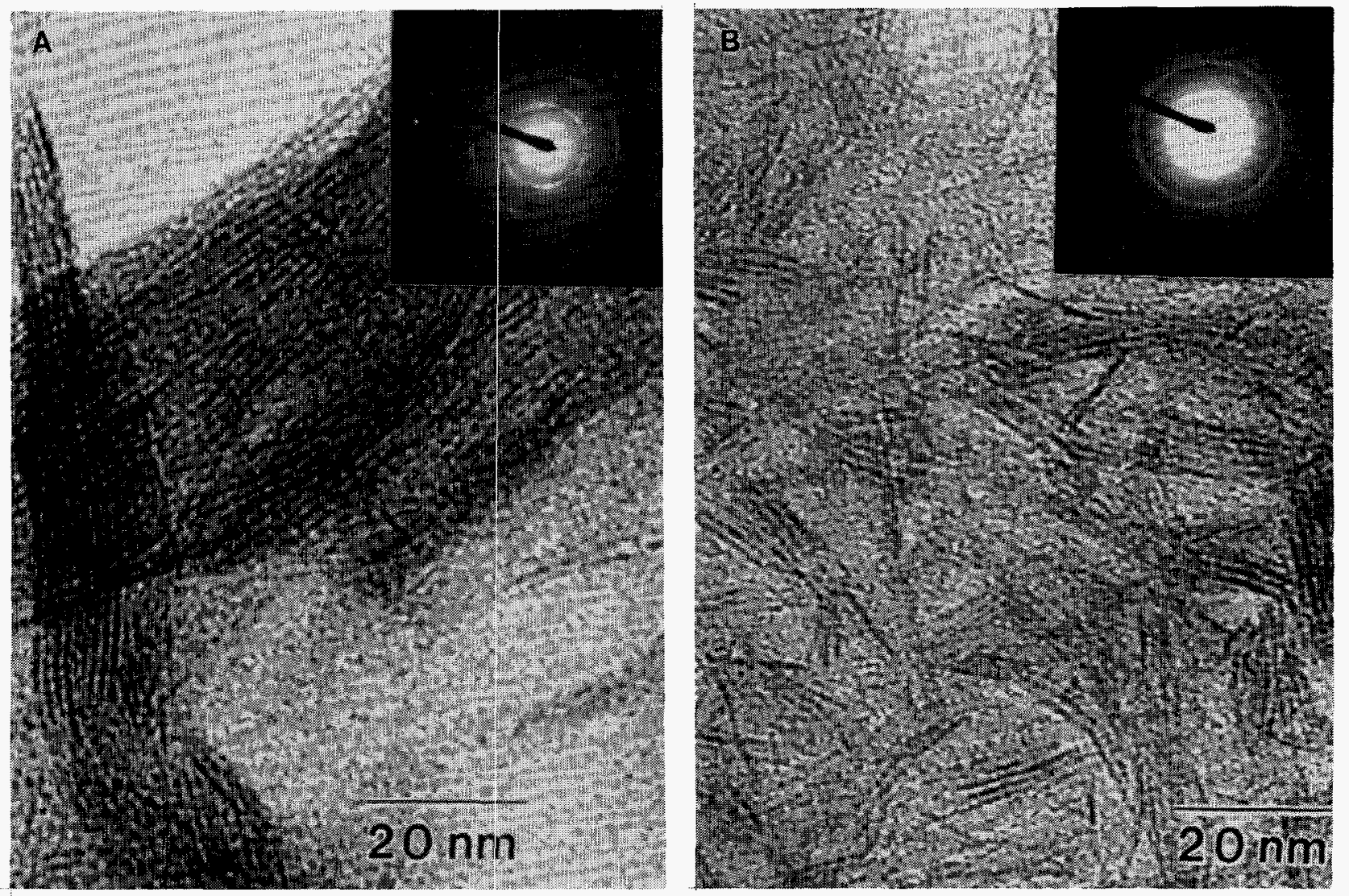

Fig. 62. AEM Photomicrograph of Alteration Layer Cross Section from SRL 131U Glass Samples Reacted in Vapor Hydration Test at $150^{\circ} \mathrm{C}$ for 91 Days. (a) Highmagnification image of outer-oriented clay layer displaying wavy morphology characteristic of smectite clays. Lattice fringes from these clays have measured spacings of 1.2 to $1.5 \mathrm{~nm}$. Inset diagram shows selected area electron diffraction image of coarse-grained smectite with discontinuous diffraction ring pattern. (b) High-magnification image of inner random-oriented clay layer displaying wavy morphology characteristic of smectite clays. Inset diagram shows selected area electron diffraction image of fine-grained smectite with continuous diffraction ring patterns. 
Quantitative AEM/EDS analyses (Table 23) indicate a mineralogic composition for the 91-day outer smectite layer that is consistent with the presence of approximately $65 \%$ nontronite and $35 \%$ saponite (Table 24 ). Aluminum concentrations were anomalously low and probably indicate some analytical error. The inner smectite layer has a calculated mineralogic composition consistent with the presence of approximately $60 \%$ nontronite and $40 \%$ saponite. Although aluminum concentrations were low in both of these samples, the amount of calcium was sufficient to satisfy the alkali-alkaline earth component for these clay mineral calculations.

Surface precipitates were still relatively sparse on the sample surfaces. The most predominant phase is analcime. Unidentified Ca-Si nodules (Fig. 63) and very finegrained Si-Al-P-La phases (Fig. 64) were also detected on the sample surfaces.

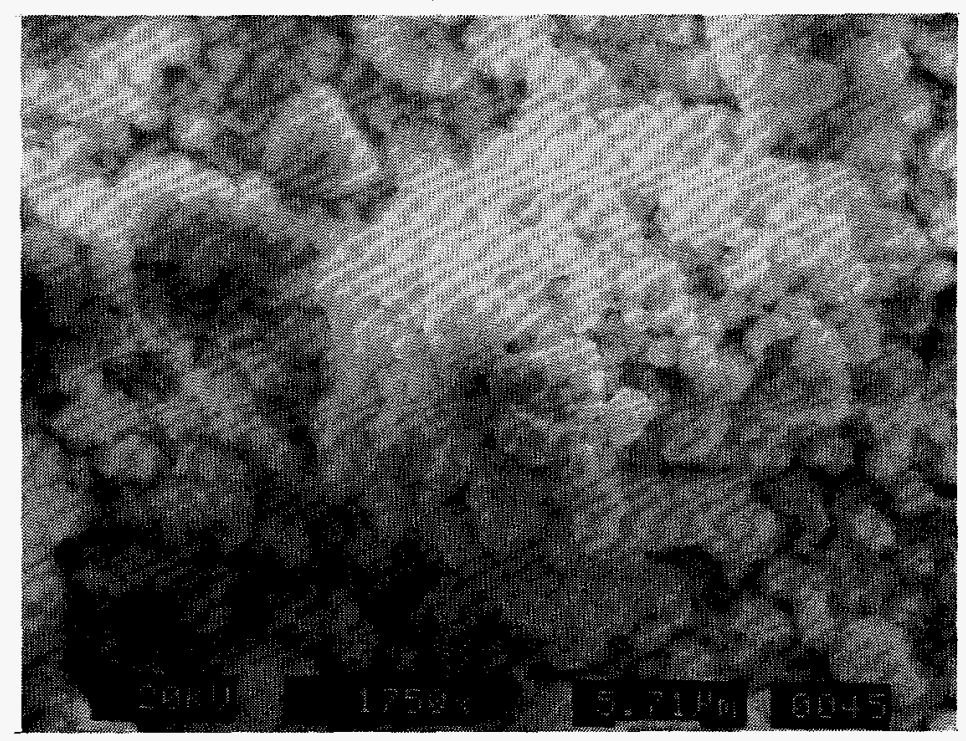

Fig. 63. SEM Image of Ca-Si Nodules Forming on 91-Day Sample of SRL 131U Glass (Secondary Electron Image, 1750X)

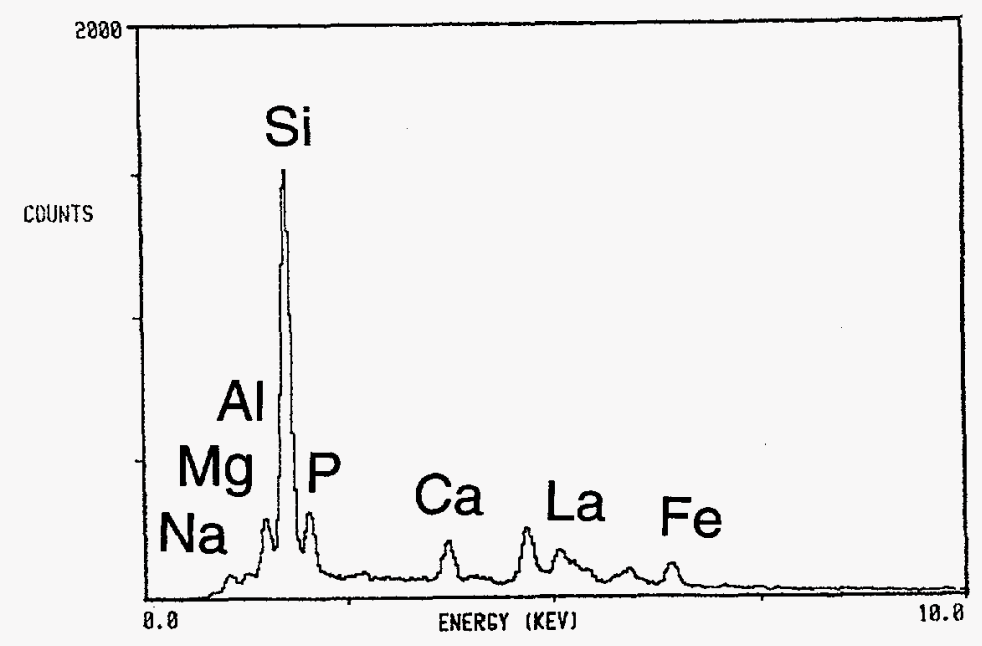

Fig. 64. SEM/EDS Spectrum of Si-Al-P-La Phase Formed on the SRL 131U Samples from Vapor Hydration Tests at 91 Days. Spectrum taken at $20 \mathrm{keV}$ accelerating potential. 


\section{f. $\quad$ SRL 131U Samples at 180 Days}

The 180-day samples of SRL $131 \mathrm{U}$ glass were characterized by the development of a brown alteration layer and the formation of scattered white and clear precipitates (Fig. 59d). Analytical electron microscopy examinations indicated the presence of a fine- to intermediate-sized, randomly oriented clay layer (Fig. 65). The oriented outer clay layer that was present on the earlier samples appears to have been inadvertently removed during the sectioning process for this sample. The AEM/EDS and d-spacings taken from the remaining "inner layer" material are consistent with the presence of smectite clays. The clay crystals in this layer were also agglomerated into 20 to $40 \mathrm{~nm}$ long by $10 \mathrm{~nm}$ wide clumps that were similar in appearance to those of the previous 56 -day tests.

During SEM examinations of the sample surface, a separate layer of coarse-grained smectite can be observed overgrowing a relatively finer-grained smectite layer (Fig. 66a). Both layers appear to have grown with their long axes oriented perpendicular to the surface of the glass and thus are probably representative of the outer smectite layer Other surface regions display the growth of small mounds of clay material. The SEM/EDS spectra indicate a similar composition for the layer and mound materials, with compositions being consistent with the presence of nontronite-dominated clay material. Layer measurements made during SEM examinations of cross-sectioned samples averaged 18.4 $\pm 1.2 \mu \mathrm{m}$ (Table 19).

Quantitative AEM/EDS analyses were only performed on the inner layer, with the outer layer apparently being stripped off during AEM sample preparation. Calculations for the inner layer indicate a mineralogic composition that is consistent with the presence of approximately $45 \%$ nontronite, $30 \%$ saponite, and $5 \%$ montmorillonite (Table 24). Once again, $\mathrm{Na}, \mathrm{K}$, and Ti were not determined in these tests.

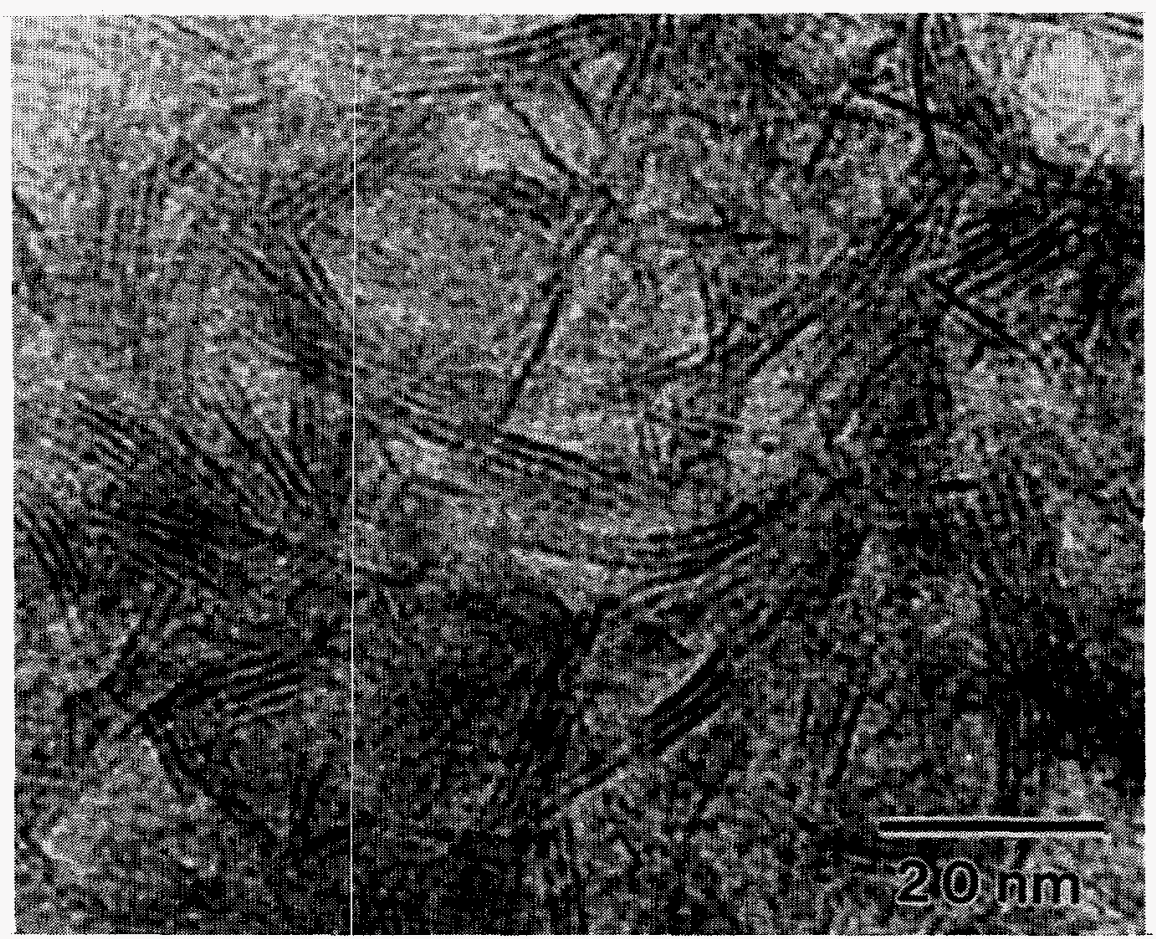

Fig. 65. AEM Photomicrograph of Random-Oriented Inner Clay Layer from SRL 131U Glass Samples Reacted in Vapor Hydration Tests at $150^{\circ} \mathrm{C}$ for 180 Days 
(a)

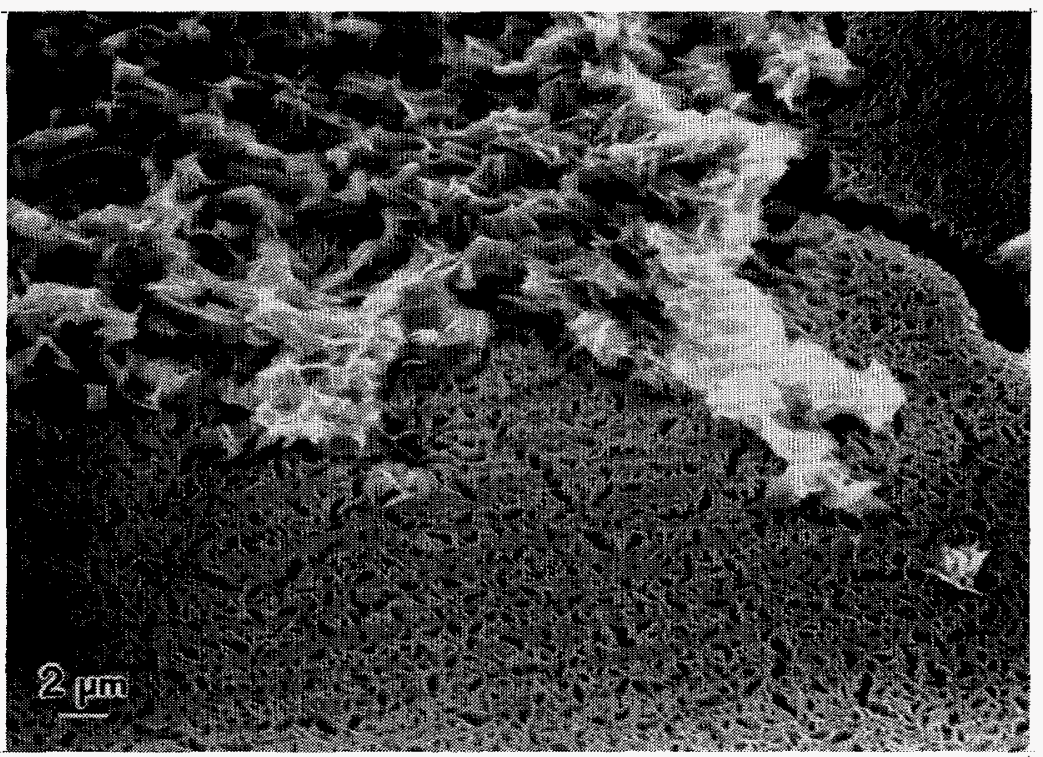

(b)

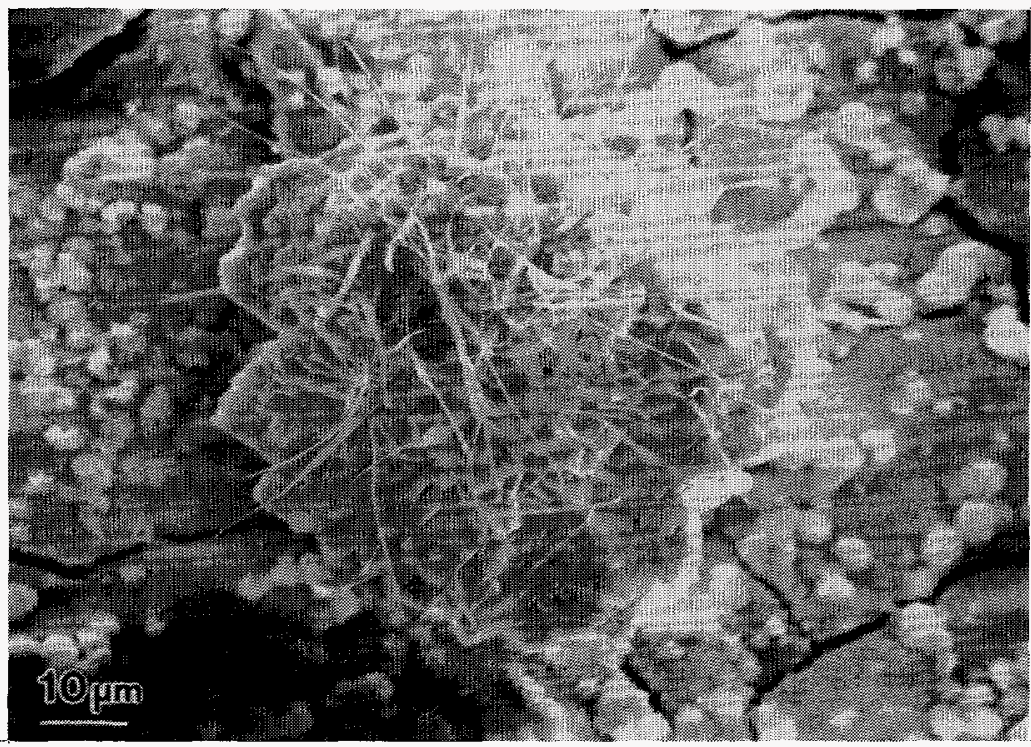

Fig. 66. SEM Images of Secondary Alteration Phases Forming on 182-Day Sample of SRL 131U Glass: Both images in secondary electron mode. (a) Coarse-Grained Smectite Layer Growing over Relatively Finer-Grained Smectite (2000X) and (b) Haiweeite Needles Intergrown with Rhombohedral-Shaped Calcite (800X). 
The diversity and amount of surface precipitate material appears to have increased on these samples relative to the shorter-term tests. Precipitates consisting of analcime, rhombohedral-shaped calcite (Fig. 66b), very fine-grained Si-Al-P-La phases, sodium-weeksite, and haiweeite (Figs. 66b and 67) were identified on the sample surfaces. Identification of calcite was aided by immersing an isolated grain in dilute hydrochloric acid while observing the sample under the microscope. The calcite rapidly dissolved in this solution, effervescing gas bubbles (presumably $\mathrm{CO}_{2}$ ) and leaving behind a residue of unidentified clear solid material.

\section{g. SRL 131U Samples at 365 Days}

After 365 days, the SRL $131 \mathrm{U}$ samples were covered with gray-brown surface clays, as well as abundant surface precipitates (Fig. 59e). After removal from the test vessels and air drying, the surfaces displayed the formation of a highly fragmented surface layer. The surface material became progressively more delaminated from the underlying glass material as the surface layer curled during dehydration. Curled and partially detached sections of the surface layer are visible in Fig. 59e. The gray-brown clays were the volumetrically most important phase, with SEM/EDS compositional analyses indicating an enrichment in Fe-Mn-Al-Si (Fig. 68a). Layer measurements taken during SEM examinations of crosssectioned samples averaged $31.8 \pm 2.1 \mu \mathrm{m}$ (Table 19). Areas where the surface layers had delaminated were avoided in these measurements.

Exposure of the underlying glass substrate has also revealed some interesting alteration features. The exposed glass surface is optically less reflective than pristine glass, indicating that some hydration and/or clay formation has occurred on this surface. In some regions of the sample, however, a second thin "Coke-bottle" green layer was observed. Portions of this surface have themselves delaminated, revealing the vitreous surface of the underlying glass. The SEM/EDS spectra from the green layer indicate the presence of abundant iron, with lesser Mn-Si-Ca-Ti-Ni (Fig. 68b). The green color also suggests that the iron is in a reduced (ferrous) state, as opposed to the ferric iron present in the surficial nontronite clays. Polygonal fracture traces also progress from the surface clay layer, through the green layer, and into the underlying glass. These features suggest that the alteration layer progression into the glass sample occurs in a nonuniform manner, with more rapid advances into the glass occurring along fracture surfaces.

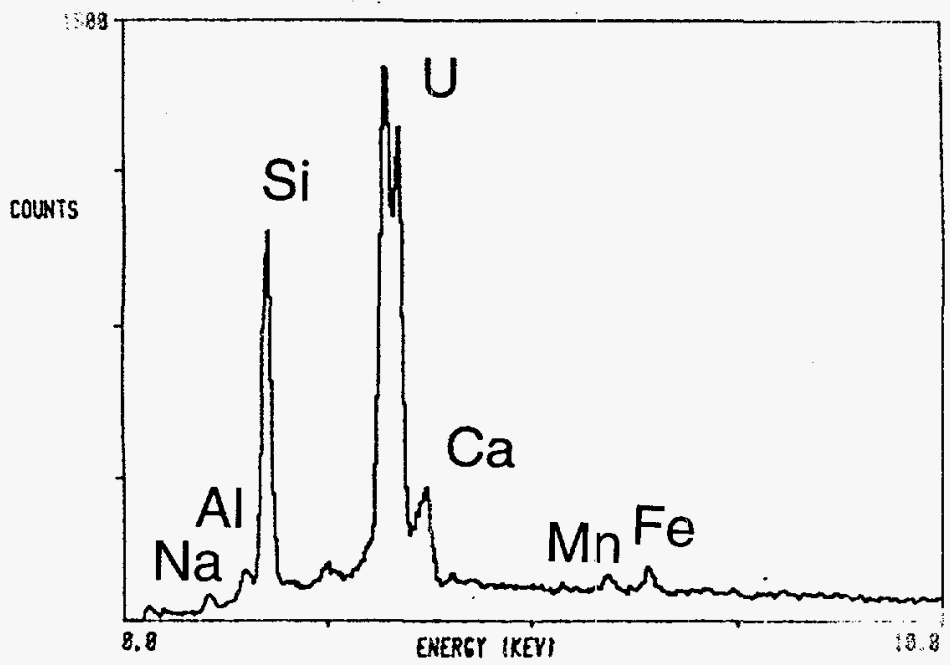

Fig. 67.

SEM/EDS Spectrum of Haiweeite Formed on 182-Day SRL $131 \mathrm{U}$ Sample from Vapor Hydration Tests. Spectrum taken at $20 \mathrm{keV}$ accelerating potential. 

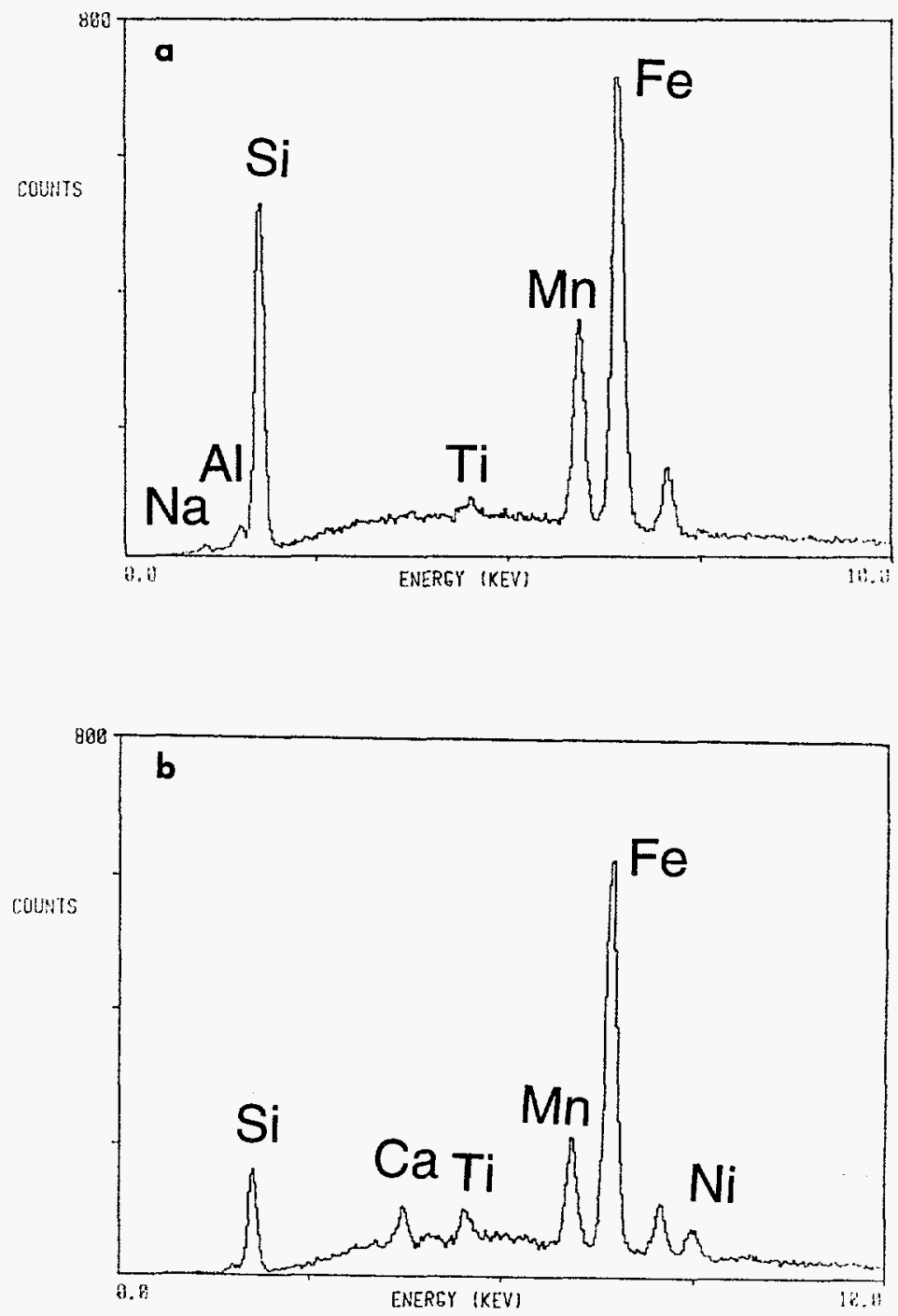

Fig. 68. SEM/EDS Spectra of Alteration Phases Formed on 365-Day SRL 131U Samples from Vapor Hydration Test: (a) Surface Clays and (b) Inner Fe-Rich Green Layer. Spectra taken at $20 \mathrm{keV}$ accelerating potential. 
Surface mineral precipitates included analcime (Figs. 69a and 69b), calcite (Figs. 69b, 69c, and 70a), Na-Si needles (Fig. 70b), Si-P-Ca-Na buttons (Fig. 70c), very finegrained Si-Al-P-La phases, and low atomic weight salts (Fig. 70d). The broad spectrum background in Fig. 70d results from the bremsstrahlung effect, where secondary X-rays are generated underneath the sample surface after interacting with primary X-rays. The calcite grains also display evidence for localized pitting corrosion (Fig. 69c). Dissolution pitting on these calcite grains appeared to occur along preferential zones within the crystals, suggesting that fine-scale compositional differences within layers in the calcite grains may have influenced the corrosion process.

\section{h. SRL 131U Samples at 540 Days}

The SRL $131 \mathrm{U}$ samples after 540 days were covered with a gray surface layer of altered material and an abundance of surface precipitates. The surface layer delamination was much less extensive in this sample relative to both the 360- and 720-day samples. The SEM/EDS compositional analyses indicated a Si-Mn-Fe-Al dominated surface layer consistent with the presence of smectite clays. These clays occurred in the same moundlike fashion as the previous 180- and 365-day samples. Layer measurements made during SEM examinations of cross-sectioned samples averaged 53.1 $\pm 4.8 \mu \mathrm{m}$ (Table 19).

Several mineral precipitates, including analcime, calcite, tobermorite (Fig. 71), unidentified Si-P buttons (Fig. 72), and low atomic weight salts were noted to form on the samples.

\section{i. $\quad$ SRL 131U Samples at 720 Days}

The final SRL $131 \mathrm{U}$ sample from the vapor hydration test had a relatively thick brown alteration layer that was partially covered by white precipitate (Fig. 59f). As with the 360-day sample, dehydration of the clay material after test termination has resulted in extensive delamination of the surface layer. The SEM/EDS compositional analyses indicated a $\mathrm{Si}-\mathrm{Mn}-\mathrm{Fe}-\mathrm{Al}$ dominated composition consistent with the presence of smectite clays. Layer measurements made during SEM examinations of the cross-sectioned samples averaged $76.6 \pm 5.6 \mu \mathrm{m}$ (Table 19).

Surface precipitate phases were relatively abundant on the sample surface. These phases included analcime, tobermorite (Fig. 73a), Si-P buttons, soddyite $\left(\mathrm{U}_{5} \mathrm{Si}_{2} \mathrm{O}_{19} \cdot 6 \mathrm{H}_{2} \mathrm{O}\right)$ (Figs. 74 and 73b), weeksite, and amorphous silica.

\section{j. $\quad$ SRL 131A Samples at 7 Days}

Optical examinations of the SRL 131A samples reacted in an irradiated atmosphere for 7 days revealed the presence of a relatively pristine sample surface (Fig. 75a). The absence of significant surficial clay layers and surface precipitates suggests that these samples have reacted to a minimal extent during the first seven days of testing. The sample surfaces were also covered with relatively scattered, white, needle-like precipitates and clear, disk-shaped precipitates.

The altered clay surface exhibited a Si-Fe-K-Mn-Al composition, suggesting a smectite or mixed smectite/illite mineralogy. Layer measurements during SEM examinations of these samples averaged $3.0 \pm 0.5 \mu \mathrm{m}$ (Table 19). These layers are actually thinner on average than those measured for the 7-day SRL 131U glasses reacted without radiation exposure, although these measurements are not significantly different (within one standard deviation of the mean value). 

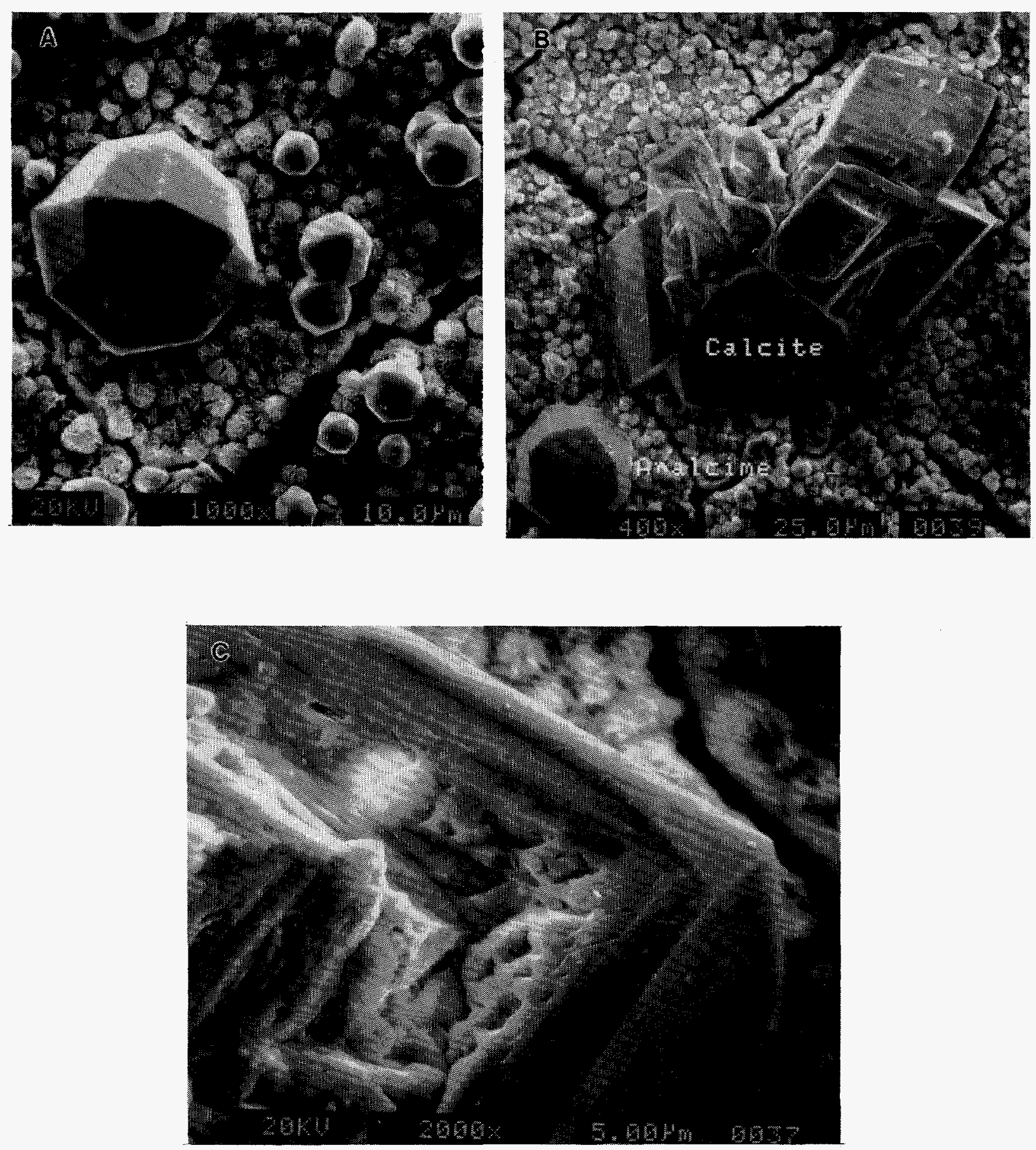

Fig. 69. SEM Images of Secondary Alteration Phases Forming on 365-Day Sample of SRL 131U Glass. All images in secondary electron mode. (a) Analcime crystals displaying characteristic trapezohedron morphology growing on clay surface $(1000 \mathrm{X})$, (b) rhombohedral-shaped calcite and trapezohedron-shaped analcime $(400 \mathrm{X})$, and (c) calcite crystal displaying crystal zonation and preferential dissolution pits in inner layer (2000X). 

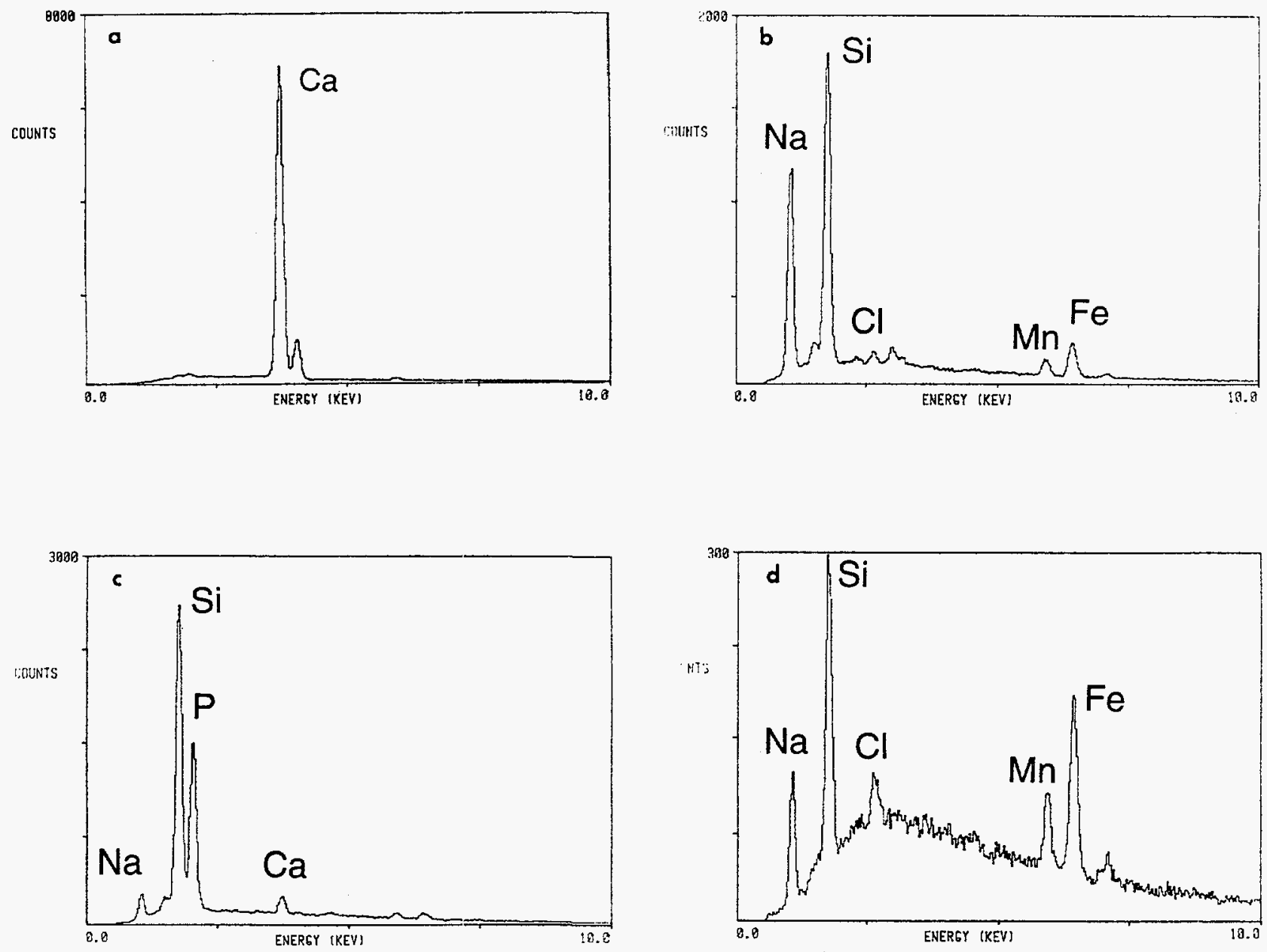

Fig. 70. SEM/EDS Spectra of Alteration Phases Formed on the SRL 131U Samples in Vapor Hydration Tests at 365 Days: (a) Calcite, (b) Na-Si Needles, (c) Si-P-Ca-Na Buttons, and (d) Salt Overgrowth on Clay Surface Displaying Typical Broad Background Response Due to Bremsstrahlung Effect. Spectra taken at $20 \mathrm{keV}$ accelerating potential. 


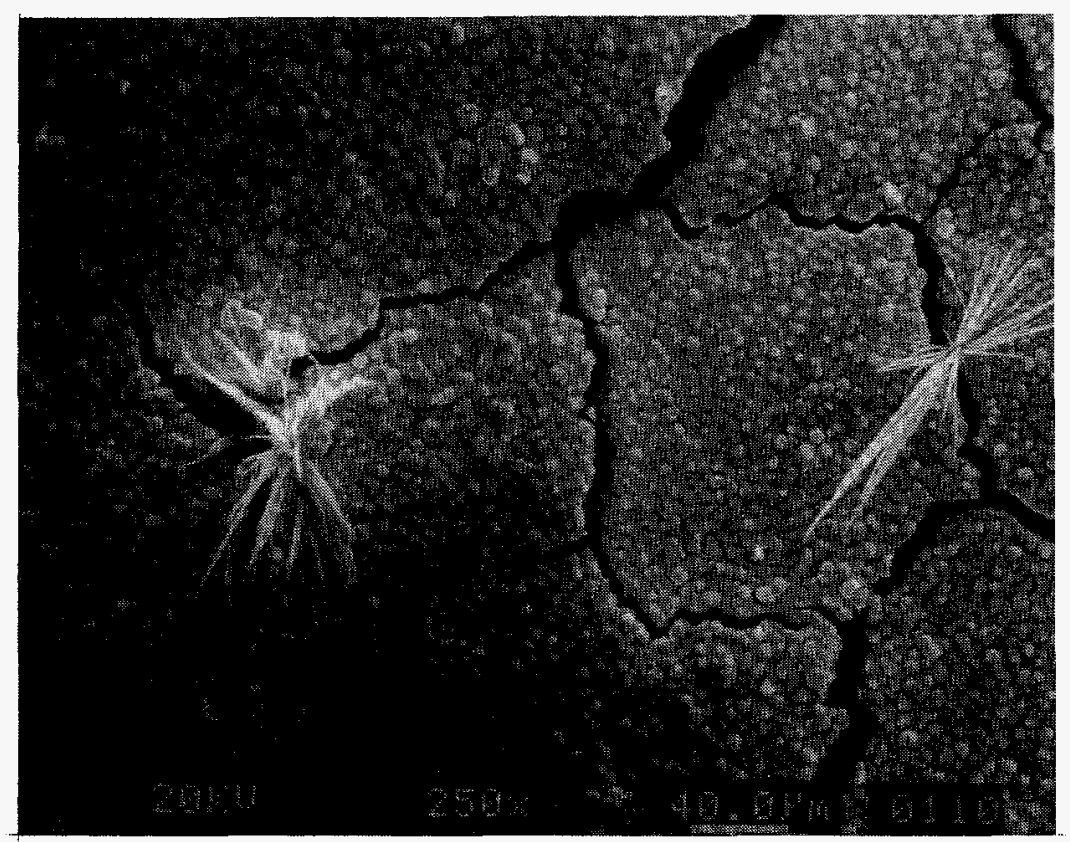

Fig. 71. SEM Secondary Electron Image of Tobermorite Needles on Clay Surface of 540-Day Sample of SRL 131U Glass (250X)

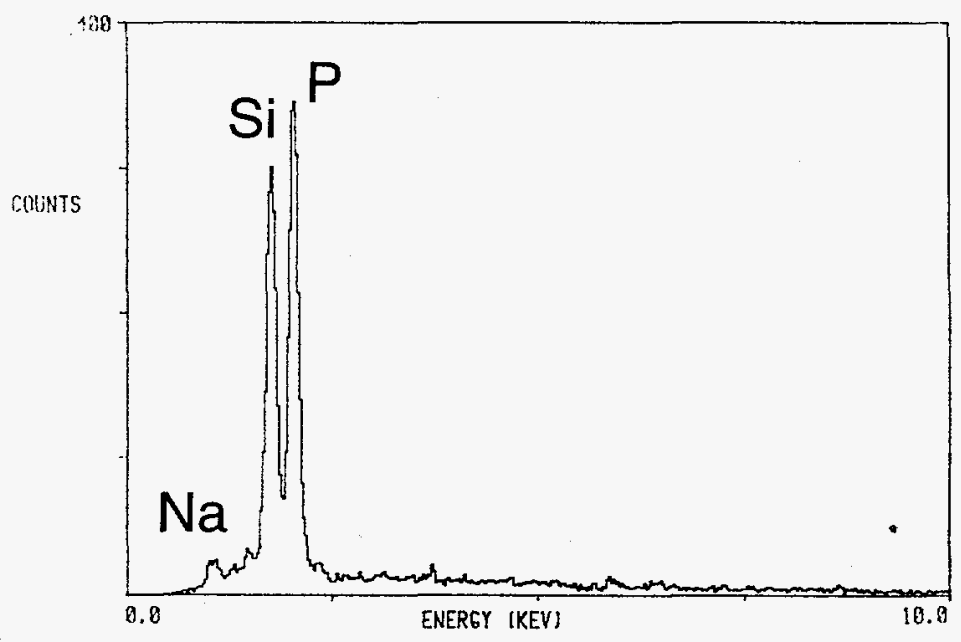

Fig. 72. SEM/EDS Spectrum of Si-P Buttons Formed on the SRL 131U Samples from Vapor Hydration Test after 540 Days. Spectrum taken at $20 \mathrm{keV}$ accelerating potential. 

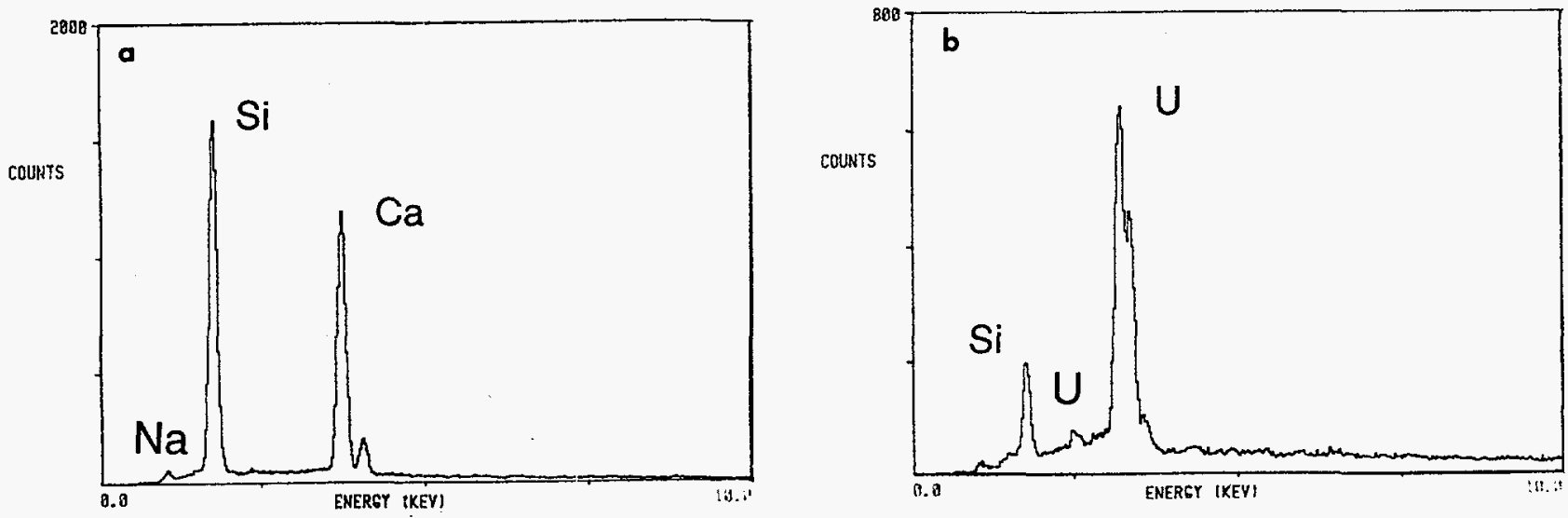

Fig. 73. SEM/EDS Spectra of (a) Tobermorite and (b) Soddyite Formed on the SRL 131U Sample in Vapor Hydration Tests after 720 Days. Spectra taken at $20 \mathrm{keV}$ accelerating potential.

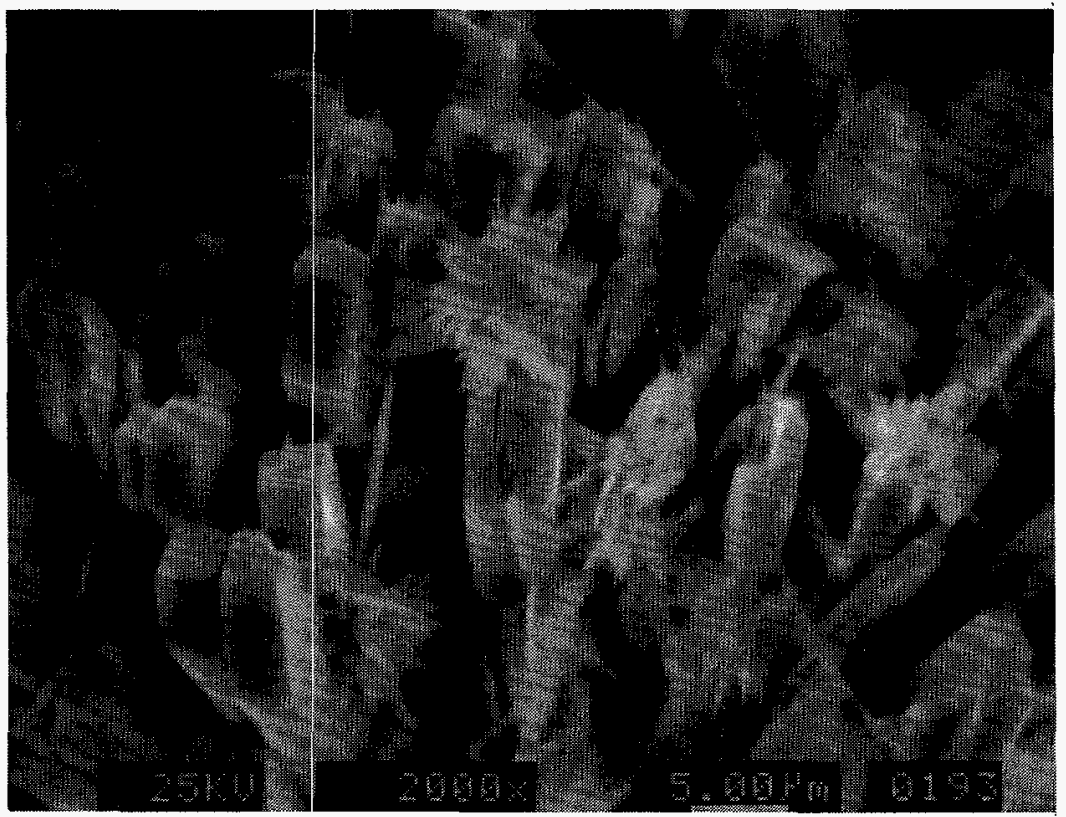

Fig. 74. SEM Secondary Electron Image of Bladed Soddyite Crystals Displaying Delamina-tion Features and Fine-Grained Radiating Ca-Si Phase on 720-Day Sample of SRL 131U Glass (2000X) 

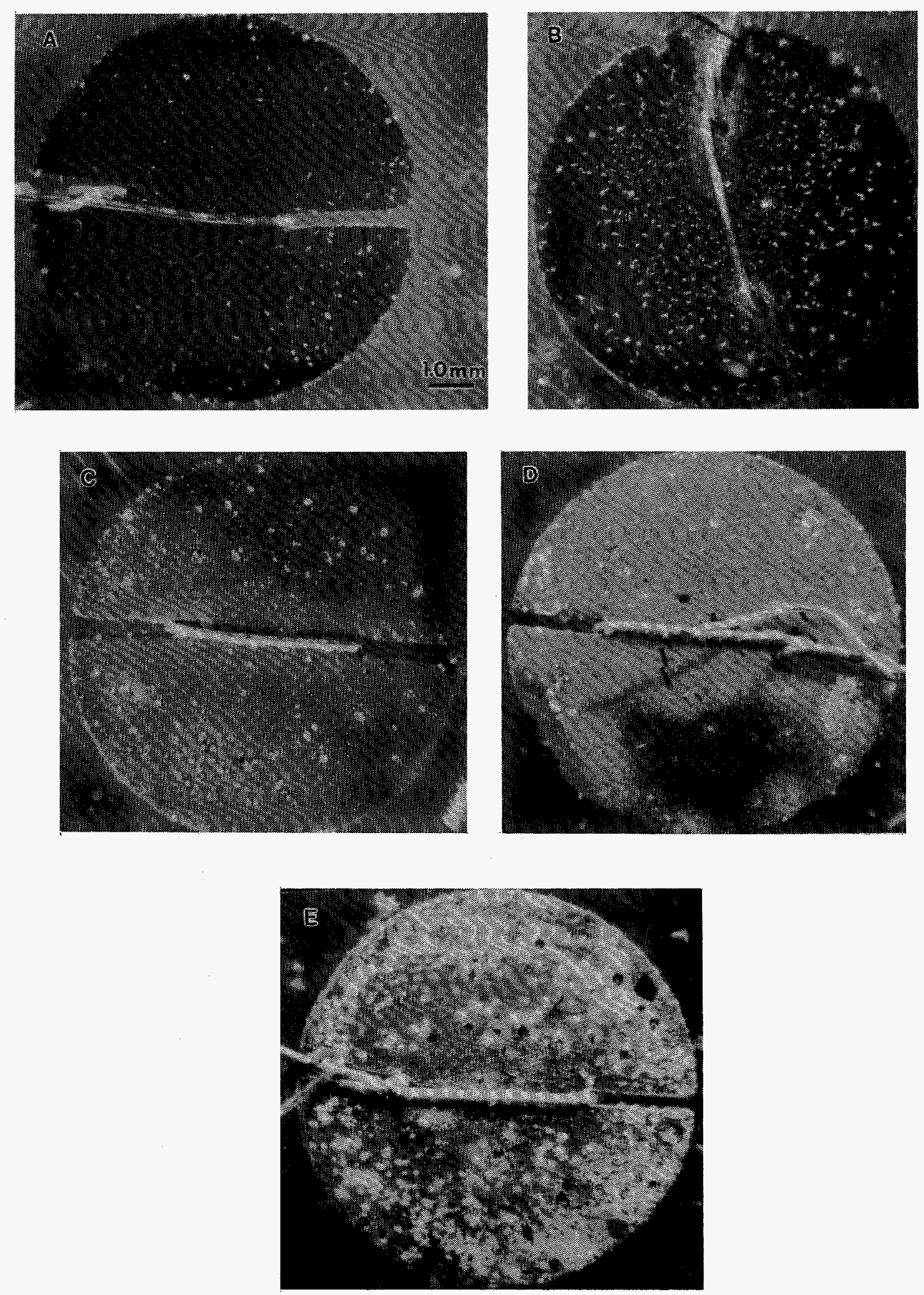

Fig. 75. Optical Photographs of Reacted Surfaces of SRL 131A Glass Samples from the $150^{\circ} \mathrm{C}$ Vapor Hydration Tests Exposed to a Dose Rate of $\sim 35 \mathrm{~Gy} / \mathrm{h}$. All photographs at $9 \mathrm{X}$ with disk diameter $\sim 10 \mathrm{~mm}$ : (a) 7-day sample, (b) 14-day sample, (c) 21-day sample, (d) 35-day sample, (e) 56-day sample. 
Precipitate phases identified in these tests include phillipsite, weeksite (Fig. 76), analcime, Ca-Si fibers (possibly tobermorite; Figs. 76 and 77), Ca-Si flowers (Fig. 76), a fine-grained $\mathrm{Ca}-\mathrm{P}-\mathrm{Si}$ precipitate that appears to be $\mathrm{Si}$-enriched apatite, amorphous iron oxides, and several low atomic weight (low-EDS response) salt deposits.

\section{k. $\quad$ SRL 131A Samples at 14 Days}

The optical examinations of SRL 131A sample surfaces after 14 days indicate the development of a brownish discoloration layer and enhanced layer growth occurring in regions in contact with the Pt-Rh support wire. The surfaces were also covered with scattered spots of a golden-yellow crusty material, which appeared to be a dried salt phase (Fig. 75b), and relatively abundant, but scattered white, needle-like precipitates identical to those that occurred on the 7-day samples.

The altered clay surface exhibited a Si-Fe-K-Na-Mn-Al composition, suggesting a smectite or mixed smectite/illite mineralogy. Layer measurements during SEM examinations of these samples averaged $4.2 \pm 1.3 \mu \mathrm{m}$ (Table 19). These layer thicknesses are again less than those determined for the 14-day SRL 131U glasses reacted without radiation exposure, although these differences are not significantly different (within one standard deviation of the mean value).

The types of alteration phases present on the 14-day samples were similar to those observed on the 7-day sarnples. These phases include phillipsite, weeksite (Figs. 78a and 79a), calcite (Fig. 78a), analcime, Ca-Si fibers, Ca-Si flowers, radiating Na-Si crystals, apatite disks (Figs. $78 \mathrm{~b}$ and $79 \mathrm{~b}$ ), an amorphous $\mathrm{Si}-\mathrm{Fe}-\mathrm{K}$ phase, an amorphous Ca-Si-K-U (possibly haiweeite) phase, and low atomic weight salt deposits.

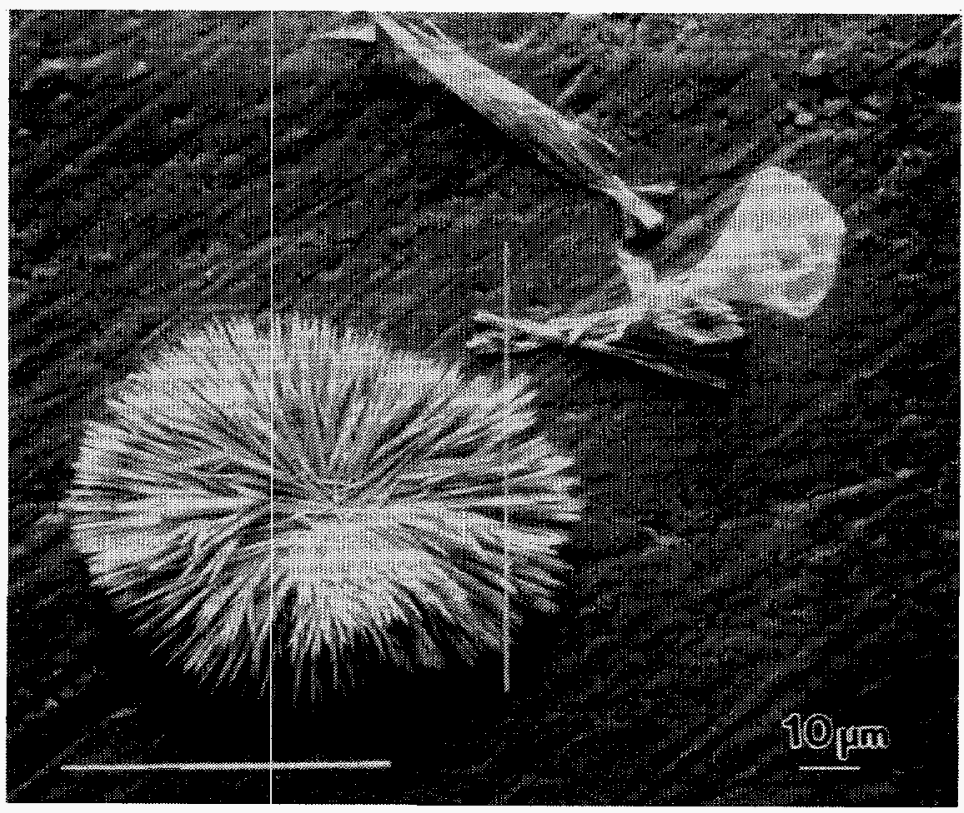

Fig. 76. SEM Image of Large Agglomeration of Radiating Weeksite Needles, Ca-Si Fibers (upper-center), and Ca-Si Flower-Shaped Phase on 7-Day Sample of SRL 131A Glass from the $150^{\circ} \mathrm{C}$ Vapor Hydration Tests Exposed to Dose Rate of $\sim 35 \mathrm{~Gy} / \mathrm{h}$ (Secondary Electron Image, 700X) 


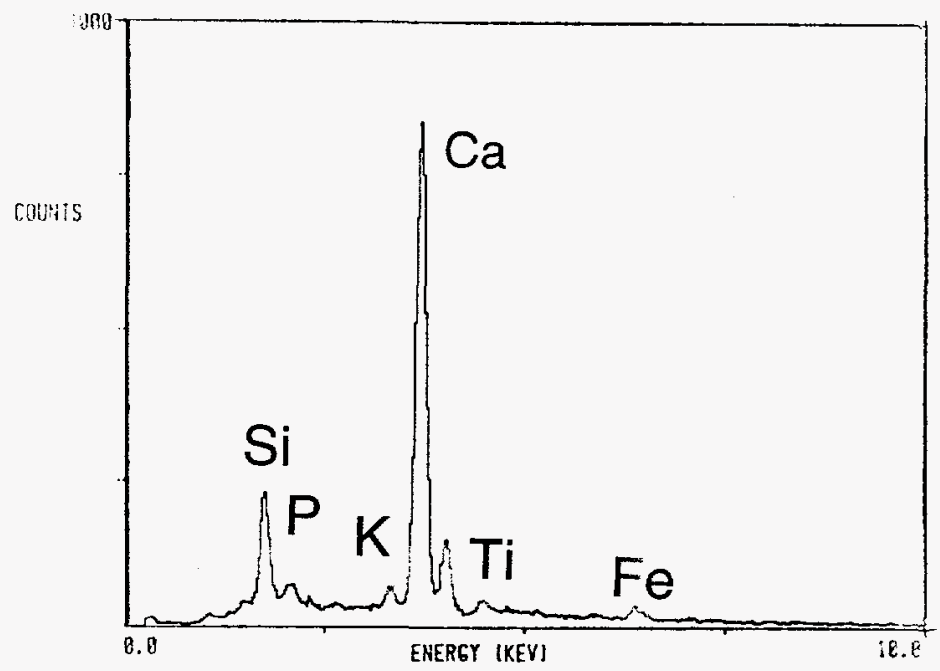

Fig. 77. SEM/EDS Spectrum of Ca-Si Needles Formed on SRL $131 \mathrm{~A}$ Samples from $150^{\circ} \mathrm{C}$ Vapor Hydration Tests Exposed to Dose Rate of $\sim 35$ Gy/h at 7 Days. Spectrum taken at $20 \mathrm{keV}$ accelerating potential.

(a)

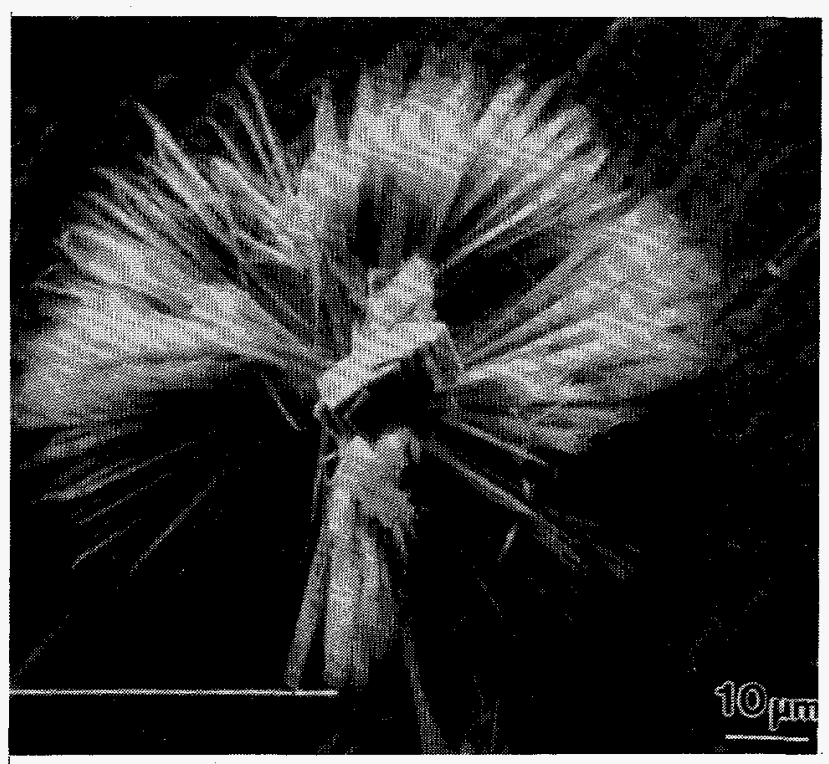

(b)

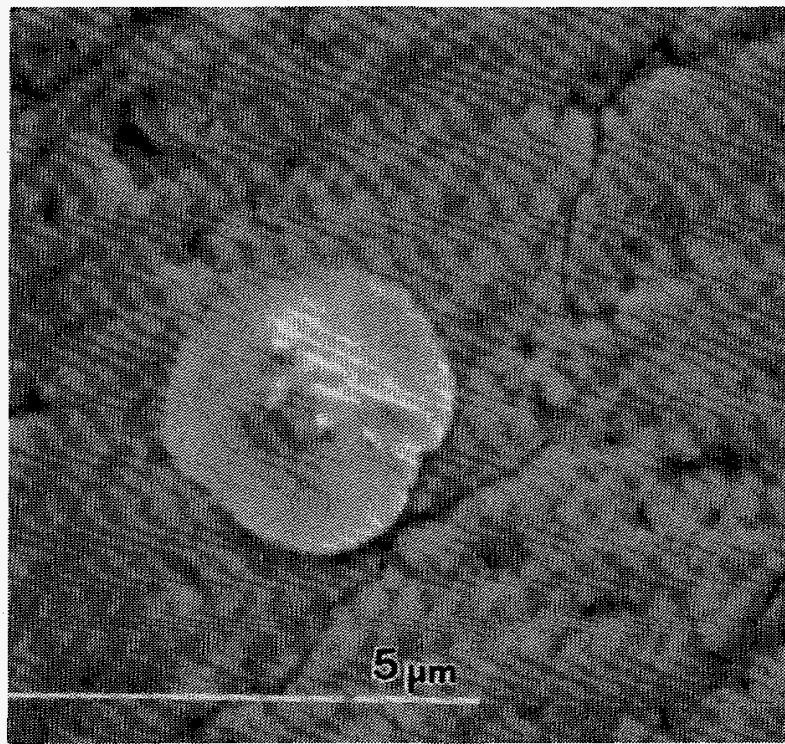

Fig. 78. SEM Images of Secondary Alteration Phases Forming on 14-Day Sample of SRL 131A Glass from the $150^{\circ} \mathrm{C}$ Vapor Hydration Tests Exposed to Dose Rate of $\sim 35 \mathrm{~Gy} / \mathrm{h}$. Both images in secondary electron mode. (a) Radiating weeksite needles intergrown with calcite (1000X) and (b) apatite disk on clay surface (3000X). 

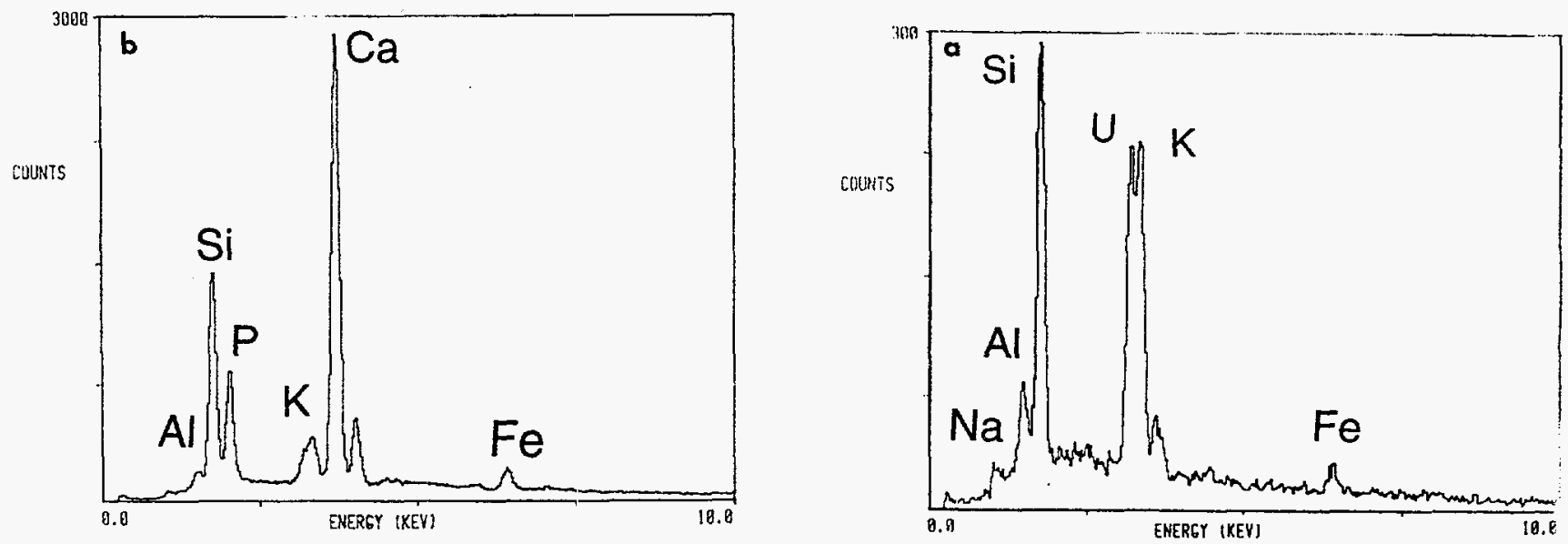

Fig. 79. SEM/EDS Spectrum of (a) Weeksite and (b) Ca-Si-P Disks Formed on the SRL 131A Samples from the $150^{\circ} \mathrm{C}$ Vapor Hydration Tests Exposed to Dose Rate of $\sim 35 \mathrm{~Gy} / \mathrm{h}$. Spectra taken at $20 \mathrm{keV}$ accelerating potential.

The AEM/EDS analyses also indicate the presence of an outer clay layer that is oriented perpendicular to the sample surface and $0.5-\mu \mathrm{m}$ thick (Figs. $80 \mathrm{a}$ and $80 \mathrm{~b}$ ) and an inner clay layer that is randomly oriented and $4-\mu \mathrm{m}$ thick (Figs. $80 \mathrm{a}, 80 \mathrm{~b}$, and $80 \mathrm{~d}$ ). The outer layer material was enriched in iron and potassium relative to both the inner clay layer and the unaltered glass material (Fig. 81). The presence of potassium-enriched and relatively straight clay crystals in the outer layer (Fig. 80c) suggests the presence of illite clays, with the illite being intermixed with the iron-rich smectites. The inner layer was composed entirely of fine-grained, randomly oriented smectite crystals, with sodium being depleted from this layer relative to the underlying glass (Figs. 81b and 81c).

\section{1. $\quad$ SRL 131A Samples at 21 Days}

Optical examinations of SRL 131A samples have revealed the development of a relatively thick alteration: layer on the sample surface after 21 days (Fig. $75 \mathrm{c}$ ). The layer developed in a nonuniform manner, with the thickest layer occurring toward the bottom hanging portions of the samples and areas in contact with the $\mathrm{Pt}-\mathrm{Rh}$ hanging wire. Surface precipitate phases include scattered white needles and clear crystals.

The altered clay surface exhibited a Si-Fe-K-Mn-Al-Ca-Ti-Ni composition (Fig. 82a), suggesting a smectite or mixed smectite/illite mineralogy. Layer measurements during SEM examinations of these samples averaged $11.0 \pm 2.8 \mu \mathrm{m}$ (Table 19). These thicknesses are significantly greater than those measured for 28-day tests with unirradiated SRL 131U glasses. A detailed examination of the glass surface beneath the layer reveals a network of ridges that underlie the polygonal fractures evident in the surface alteration layer (Figs. 83a and 83b). Also present are randomly distributed mounds that underlie the polygonal clay fragments. These ridges and mounds are depleted in $\mathrm{K}, \mathrm{Mn}$, and $\mathrm{Fe}$, while being enriched in $\mathrm{Na}$ relative to the adjacent, flat-lying substrate material (Figs. $82 \mathrm{~b}$ and $82 \mathrm{c}$ ). 

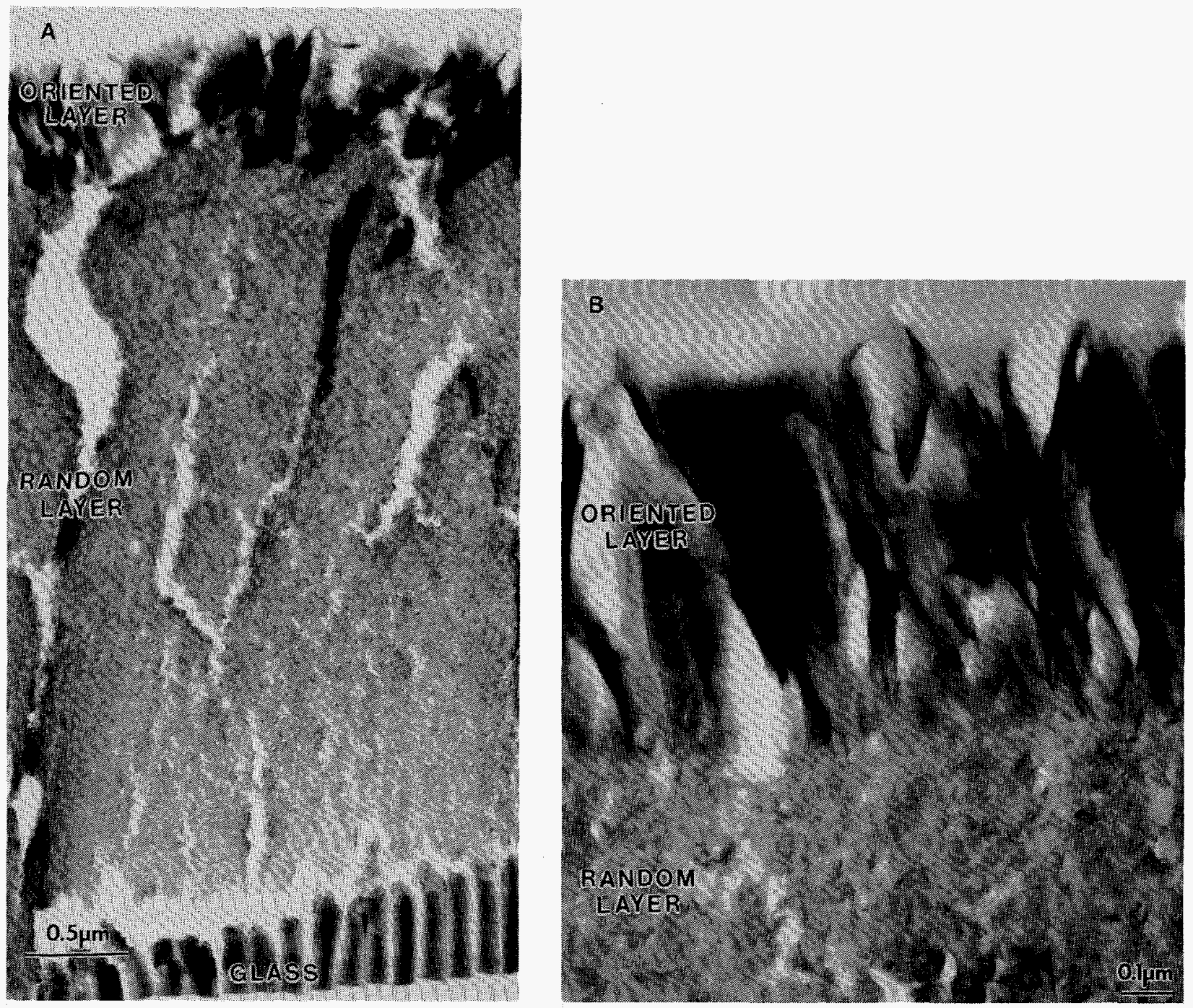

Fig. 80. AEM Photomicrograph of Alteration Layer Cross Section from SRL 131A Glass Samples Reacted in Vapor Hydration Test at $150^{\circ} \mathrm{C}$ for 14 Days. This sample was exposed to a ${ }^{60} \mathrm{Co}$ gamma radiation source at a dose rate of $\sim 35 \mathrm{~Gy} / \mathrm{h}$. (a) Lowmagnification image of entire cross section showing an outer-oriented clay layer, inner random-oriented clay layer, and unaltered glass. (b) High-magnification image of outer oriented and upper section on inner random-oriented clay material displaying wavy morphology characteristic of smectite clays. (c) Lattice image of relatively straight illite clays from outer-oriented layer. (d) High magnification image of glass layer interface region displaying precipitation of clays directly on the corroded glass surface interface across most of sample, and localized regions (upper left and lower right) where the glass surface appears to display evidence for pitting. 
Fig. 80. (contd.)
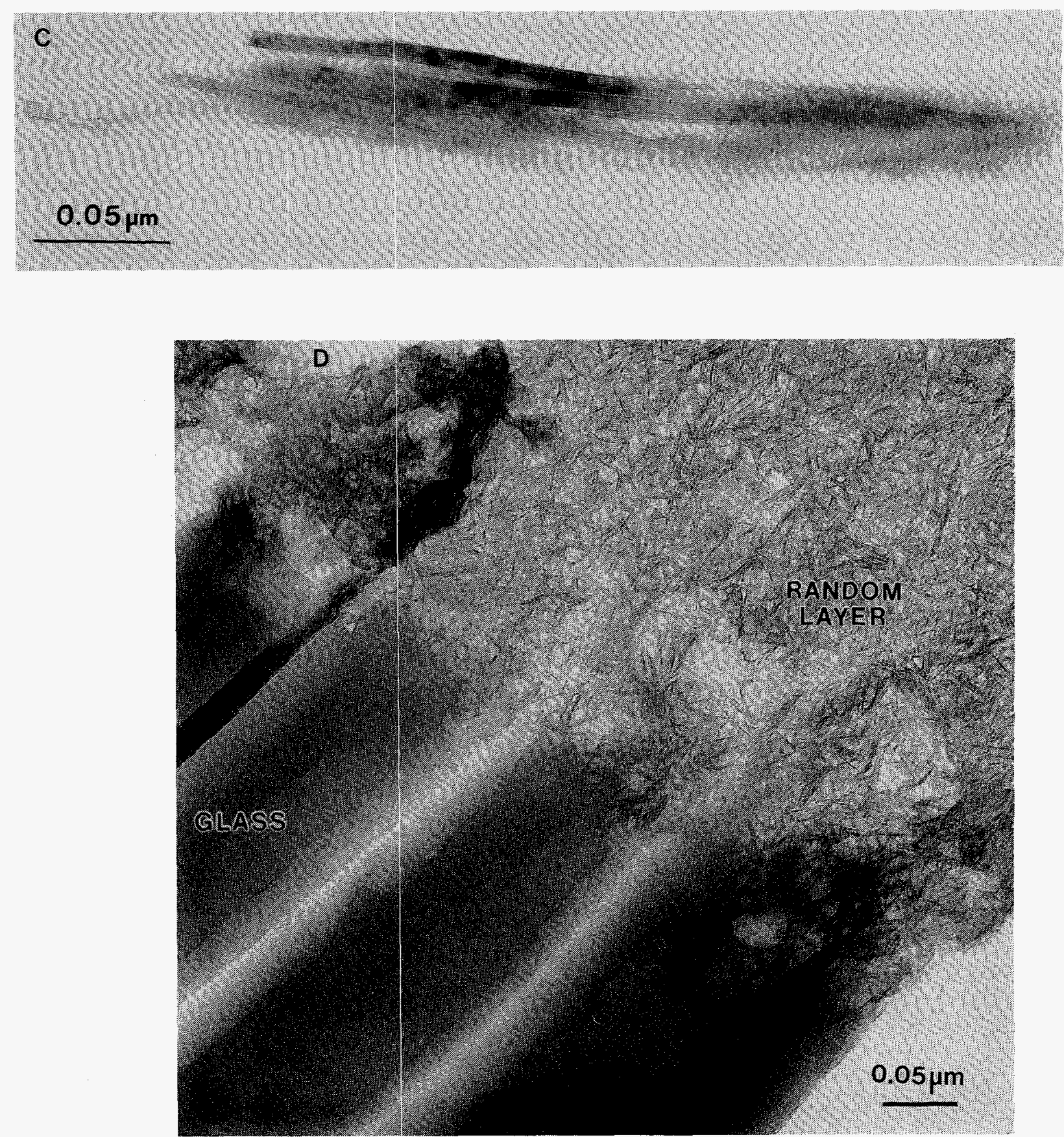


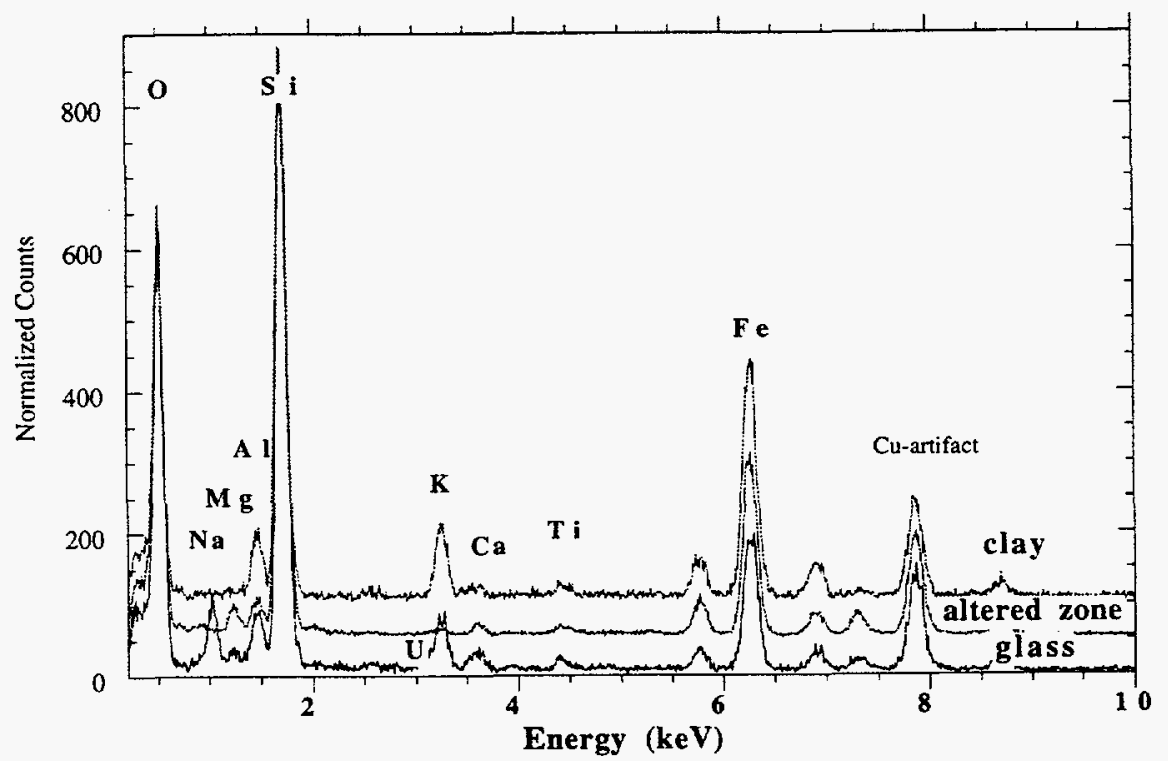

Fig. 81. AEM/EDS Spectra from 14-Day SRL 131A Glass Sample from Fig. 80a. This sample was also exposed to a ${ }^{50} \mathrm{Co}$ gamma radiation source at a dose rate of $\sim 35 \mathrm{~Gy} / \mathrm{h}$. Figure shows outer oriented clay layer displaying enrichment of $\mathrm{Fe}$ and $\mathrm{K}$, inner smectite (altered zone) sample, and unaltered glass. Cu-artifact is from grid holder.

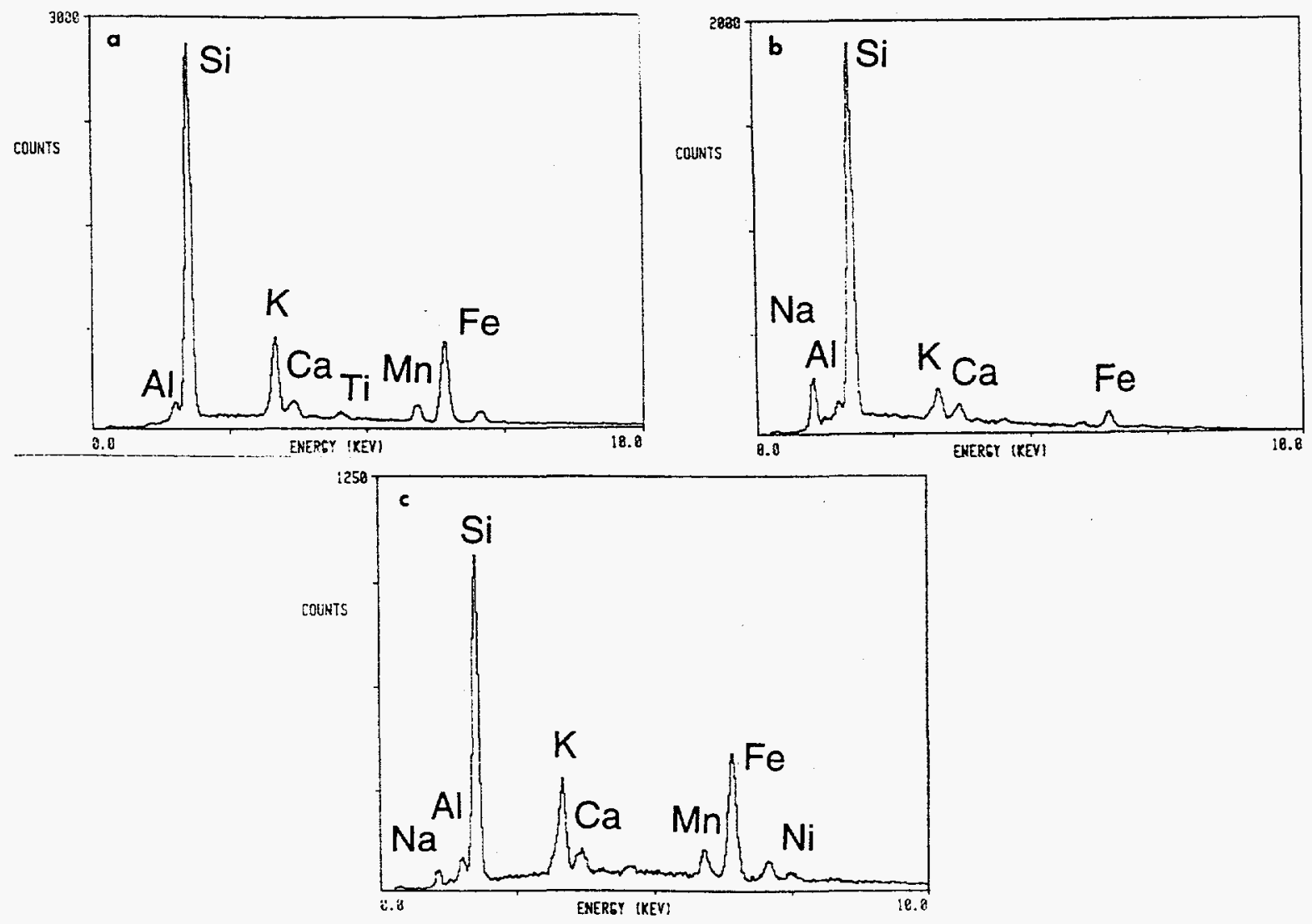

Fig. 82. SEM/EDS Spectrum of (a) Surface Clays, (b) Raised Mounds, and (c) Flat-Lying Altered Glass Surface Formed on SRL $131 \mathrm{~A}$ Samples from the $150^{\circ} \mathrm{C}$ Vapor Hydration Tests Exposed to Dose Rate of $\sim 35 \mathrm{~Gy} / \mathrm{h}$ for 21 Days. Spectra taken at $20 \mathrm{keV}$ accelerating potential. 


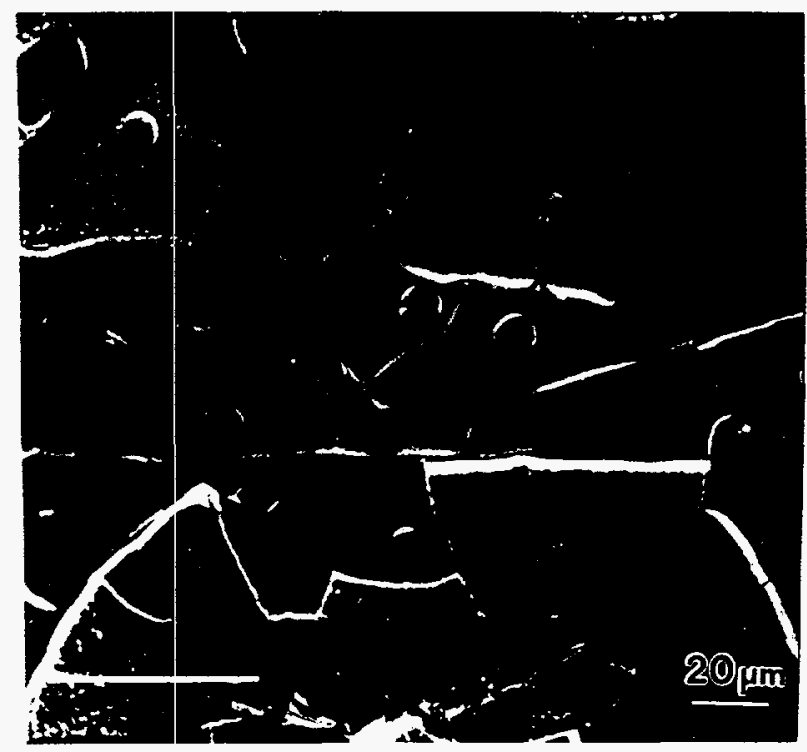

(a)

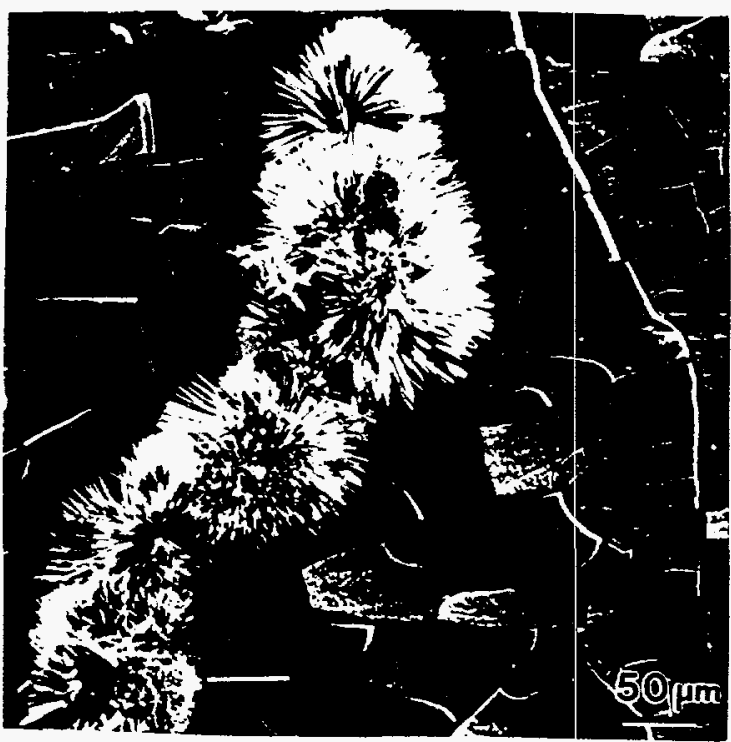

(b)

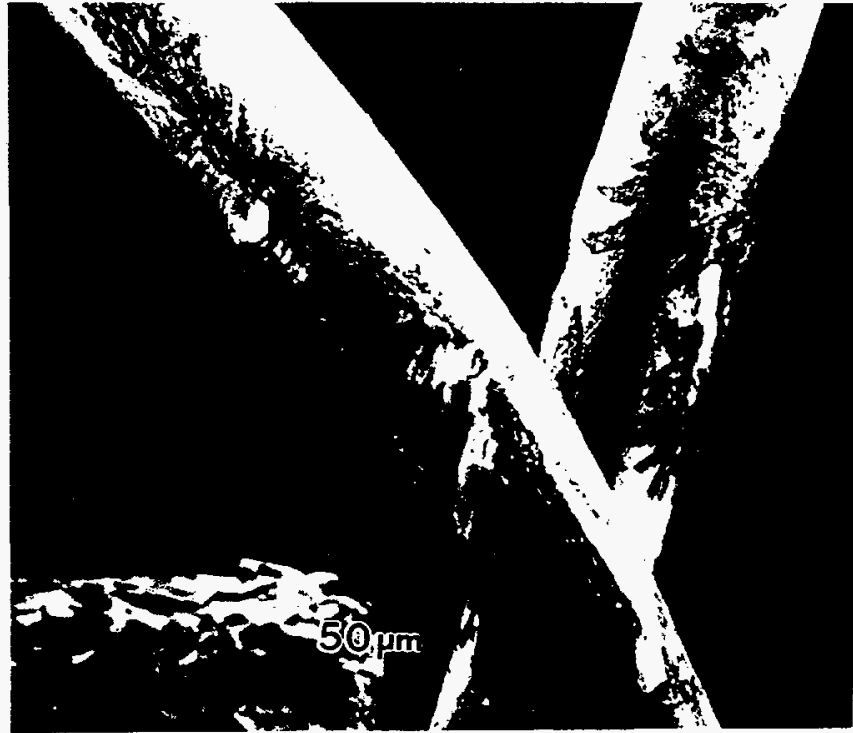

(c)

Fig. 83. SEM Images of Secondary Alteration Phases Forming on 21-Day Sample of SRL 131A Glass from the $150^{\circ} \mathrm{C}$ Vapor Hydration Tests Exposed to Dose Rate of $\sim 35 \mathrm{~Gy} / \mathrm{h}$. All images in backscattered electron mode. (a) Exposed ridges on glass surface demarking locations of polygonal fractures in the alteration layer and random circular mounds and craters (500X), note intact clay layer to bottom of photo, (b) sinuous arrangement of weeksite needles lying on highly fractured clay surface (200X), and (c) dendritic patches of low atomic weight salts on Pt-Rh wire (100X). 
Surface precipitate phases include phillipsite, weeksite (Fig. 83b), calcite, analcime, $\mathrm{Ca}-\mathrm{Si}$ fibers (possibly tobermorite), and low atomic weight salt deposits (Fig. 83c). This mineral assemblage was similar to that observed on the 7-and 14-day tests. The absence of high background noise in the salts in Fig. 84, relative to the previous EDS salt spectra in Fig. 70d, results from the high-density Pt-Rh wire substrate in the present tests, which prevents significant penetration of the SEM electrons below the salt material.

\section{m. SRL 131A Samples at 35 Days}

The 35-day SRL 131A samples are characterized by a nonuniformly thick alteration layer and the precipitation of scattered white needle-like aggregates and clear crystals (Fig. 75d). Some regions of the surface on the upward hanging side of the sample appear relatively unaltered, indicating minimal water contact in these regions. Polygonal fracture patterns have developed where the alteration layer has increased to a moderate thickness. During the air drying after the tests were terminated, small portions of the dehydrated clay layer began to curl and delaminate from the sample surface.

The altered clay surface exhibited a Si-Fe-K-Mn-Al-Ca composition suggesting a smectite or mixed smectite/illite mineralogy. The surface composition has remained relatively constant throughout of these tests. Layer thickness measurements during SEM examinations of these samples averaged $14.1 \pm 1.7 \mu \mathrm{m}$ (Table 19).

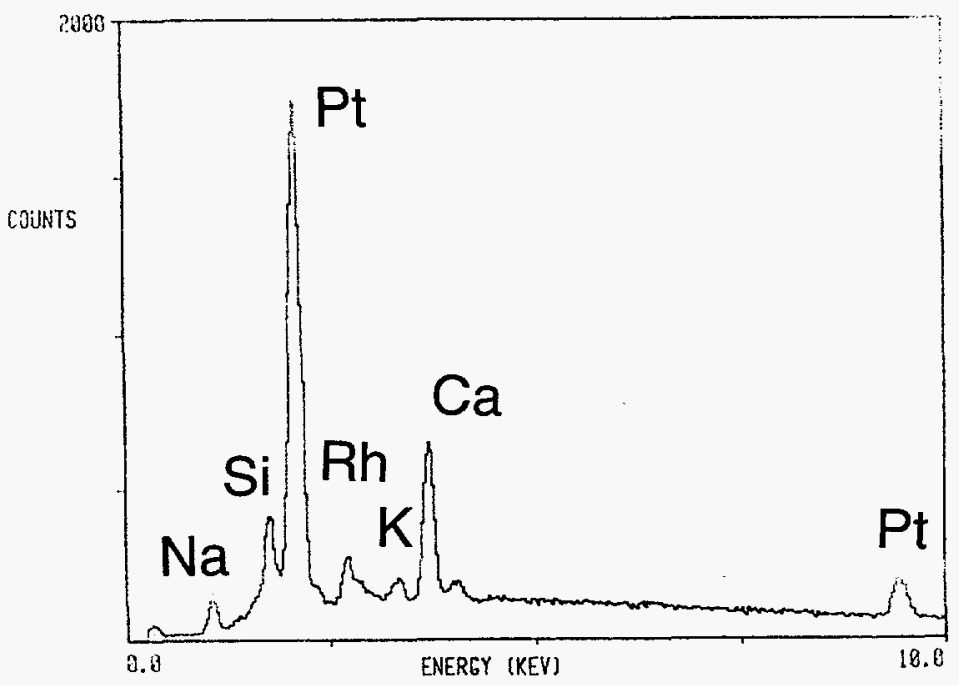

Fig. 84. SEM/EDS Spectrum of Low Atomic Weight Salt Deposited on Pt-Rh Wire from Vapor Hydration Test Sample with SRL 131A Glass at $150^{\circ} \mathrm{C}$. Sample was also exposed to dose rate of $\sim 35 \mathrm{~Gy} / \mathrm{h}$ for 35 days. Spectrum taken at $20 \mathrm{keV}$ accelerating potential.

Surface precipitate phases include phillipsite (Fig. 85a), weeksite, analcime, Ca-Si fibers (possibly tobermorite), and apatite (Figs. 85b and 86). This latter phase also displays some evidence for pitting corrosion. In addition, low atomic weight salt deposits were detected on the samples, with salt compositions varying widely from region to region. All salts contained variable concentrations of $\mathrm{Na}, \mathrm{K}$, and $\mathrm{Si}$. Calcium and sulfur were also detected in some of these salt deposits. It is probable that some of the more soluble, low atomic weight elements ( $\mathrm{B}$ and $\mathrm{Li}$ ) were also present, but these elements could not be detected in the samples because of detector limitations. 
n. SRL 131A Samples at 56 Days

Optical examinations of the surface on 56-day SRL 131A samples revealed a uniformly thick alteration layer and an extensive cover of white needles and clear crystals (Fig. 75e). Most of the surface layer displays the polygonal fracture surfaces that are characteristic of the thicker alteration layers; however, very little delamination of layer material has occurred.

The altered clay surface exhibited the same Si-Fe-K-Mn-Al-Na-Ti composition as previous samples, suggesting a smectite or mixed smectite/illite mineralogy (Fig. 87). The AEM/EDS analyses of reacted surfaces of these samples confirmed the presence of two separate layers of clay minerals that were similar to the layers developed in shorter-term samples. The oriented outer layer was composed of mixed potassium-rich, relatively straight crystals of illite and more wavy textured iron-rich smectite clays. These analyses also revealed the presence of $\mathrm{Ca}-\mathrm{Si}$-oxide crystals, which contained relatively minor amounts of barium and several rare earth elements (Fig. 88). Composite layer measurements taken during SEM examinations of cross-sectioned samples averaged $35.5 \pm 3.6 \mu \mathrm{m}$ (Fig. 89; Table 19). These thicknesses are approximately four-fold greater than those measured for SRL 131U glasses reacted for the same time period under nonirradiated conditions

(a)

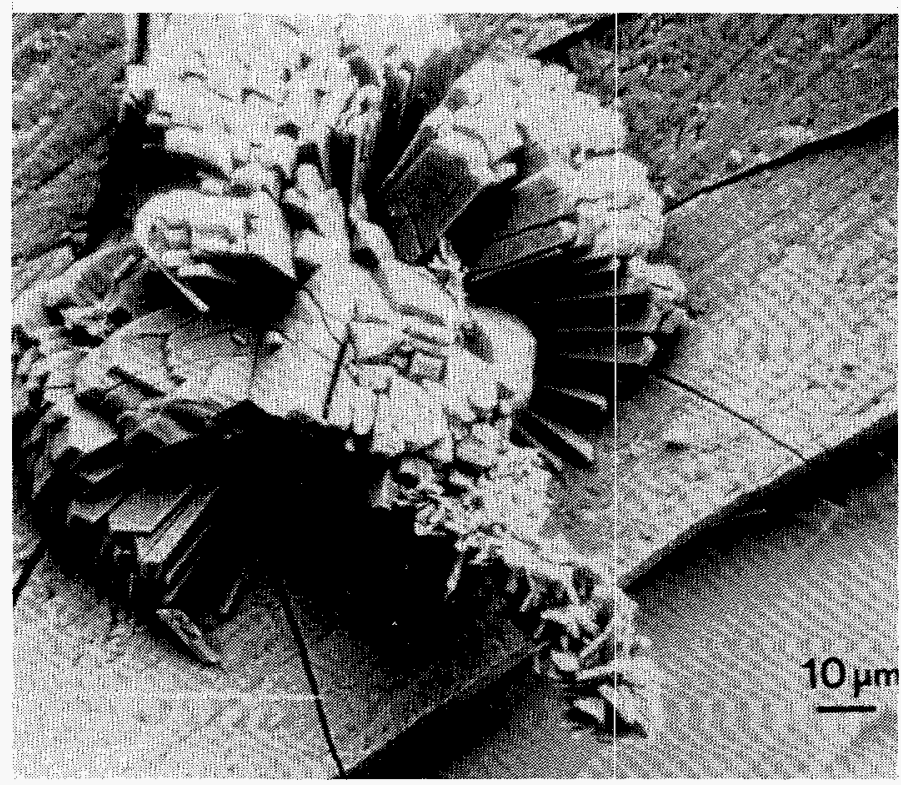

(b)

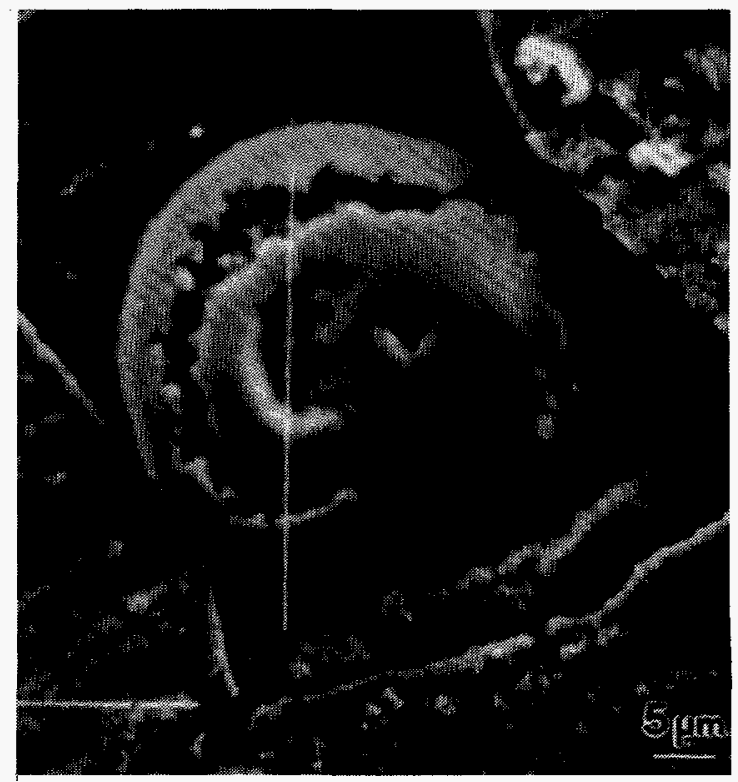

Fig. 85. SEM Images of Secondary Alteration Phases Forming on 35-Day Sample of SRL 131A Glass from the $150^{\circ} \mathrm{C}$ Vapor Hydration Tests Exposed to Dose Rate of $\sim 35 \mathrm{~Gy} / \mathrm{h}$. (a) Phillipsite (700X) and (b) apatite showing dissolution pits (1500X). 


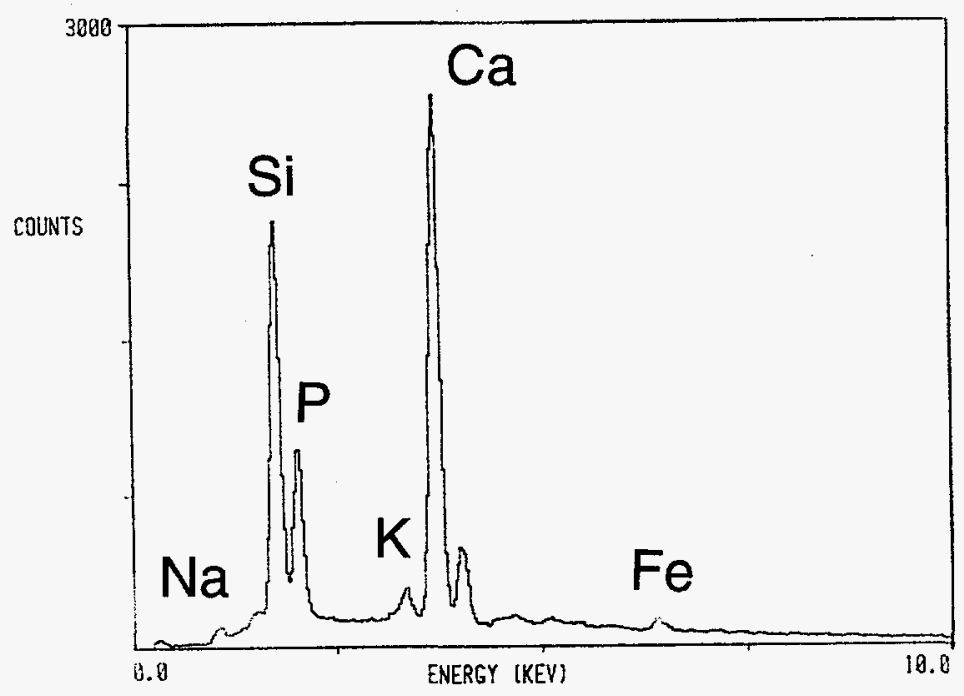

Fig. 86. SEM/EDS Spectrum of Apatite Formed on 35-Day SRL 131A Samples from $150^{\circ} \mathrm{C}$ Vapor Hydration Tests Exposed to Dose Rate of $\sim 35 \mathrm{~Gy} / \mathrm{h}$. Si, Fe, and $\mathrm{K}$ spectra are derived from underlying layer. Spectrum taken at $20 \mathrm{keV}$ accelerating potential.

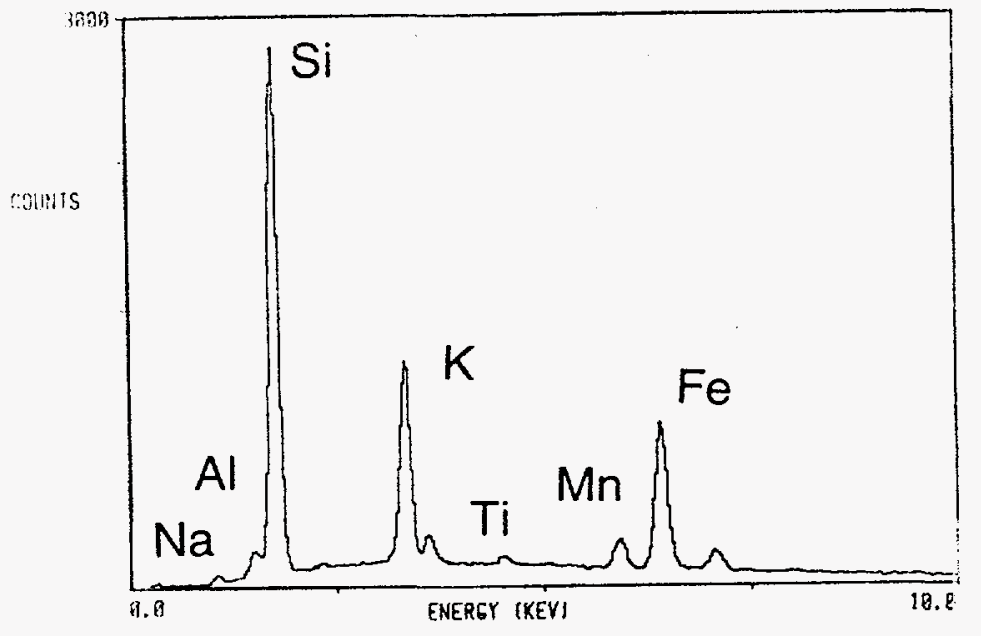

Fig. 87. SEM/EDS Spectrum of Smectite Clay Layer Formed on 56-Day SRL 131A Samples from the $150^{\circ} \mathrm{C}$ Vapor Hydration Tests Exposed to Dose Rate of $\sim 35 \mathrm{~Gy} / \mathrm{h}$. Spectrum taken at $20 \mathrm{keV}$ accelerating potential. 


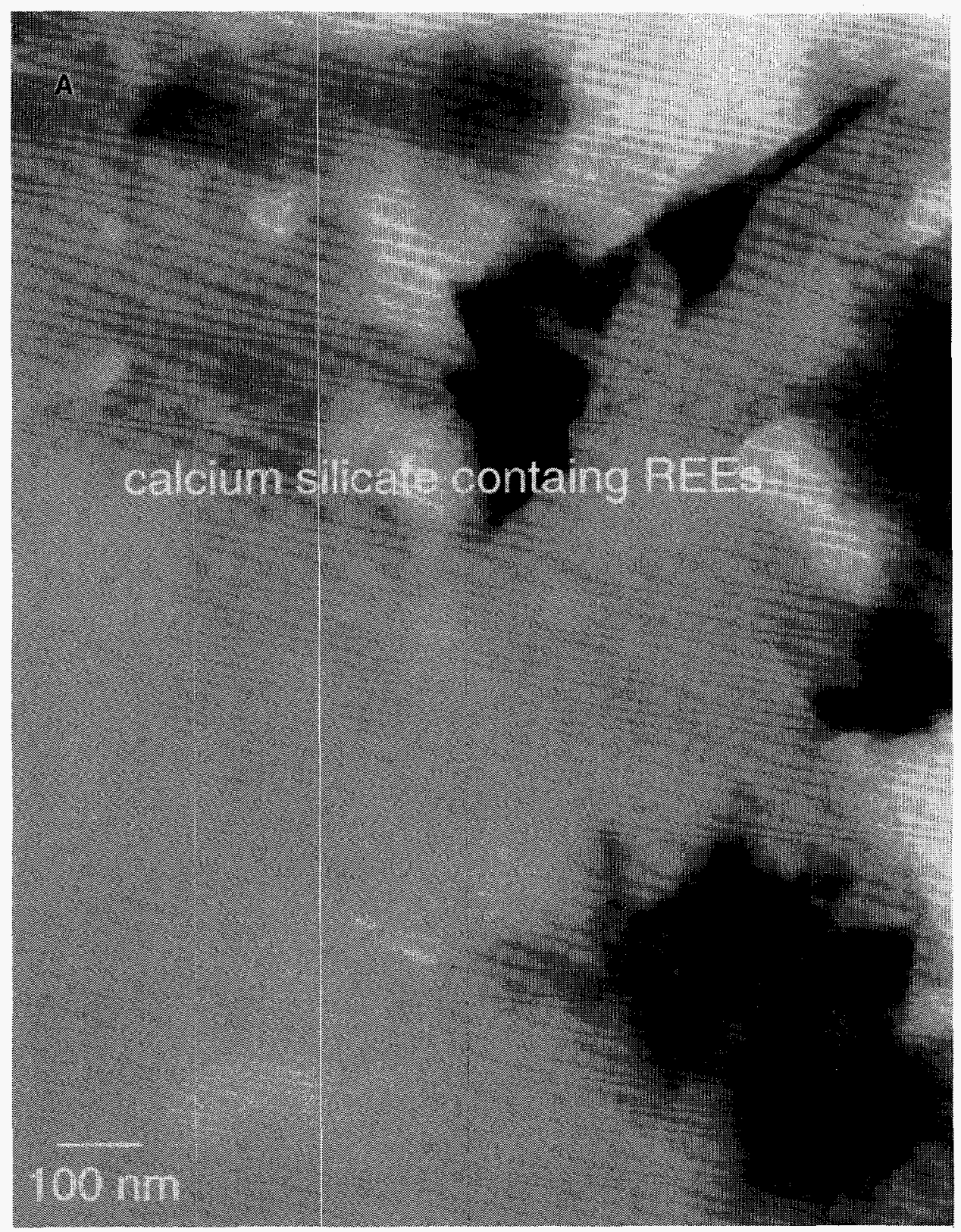

Fig. 88. Calcium-Silicon Phase (possibly tobermorite) Found within Altered Clay Layer in SRL 131A Glass Sample Reacted in Vapor Hydration Test at $150^{\circ} \mathrm{C}$ for 56 Days. This sample was exposed to dose rate of $\sim 35 \mathrm{~Gy} / \mathrm{h}$. (a) Low-magnification AEM photomicrograph of $\mathrm{Ca}$-Si-oxide phase, (b) EDS spectrum $\mathrm{Ca}$-Si-oxide phase $(\mathrm{Cu}$ peak from AEM support grid), (c) electron energy loss, second difference spectra displaying the presence of trace amounts of $\mathrm{Ba}$ and several light rare earth elements (La, Ce, Pr, and $\mathrm{Nd}$ ). 
Fig. 88 (contd.)
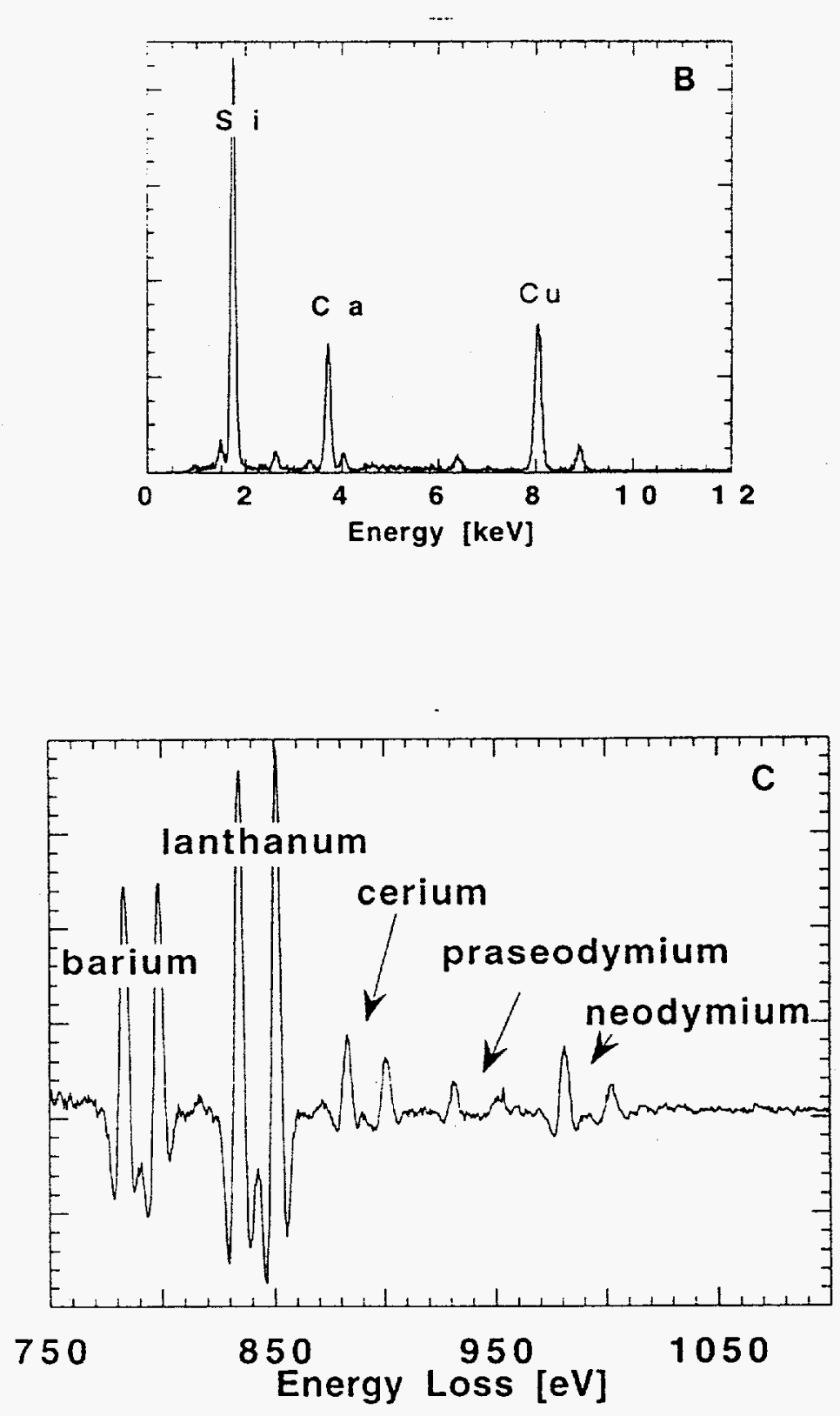


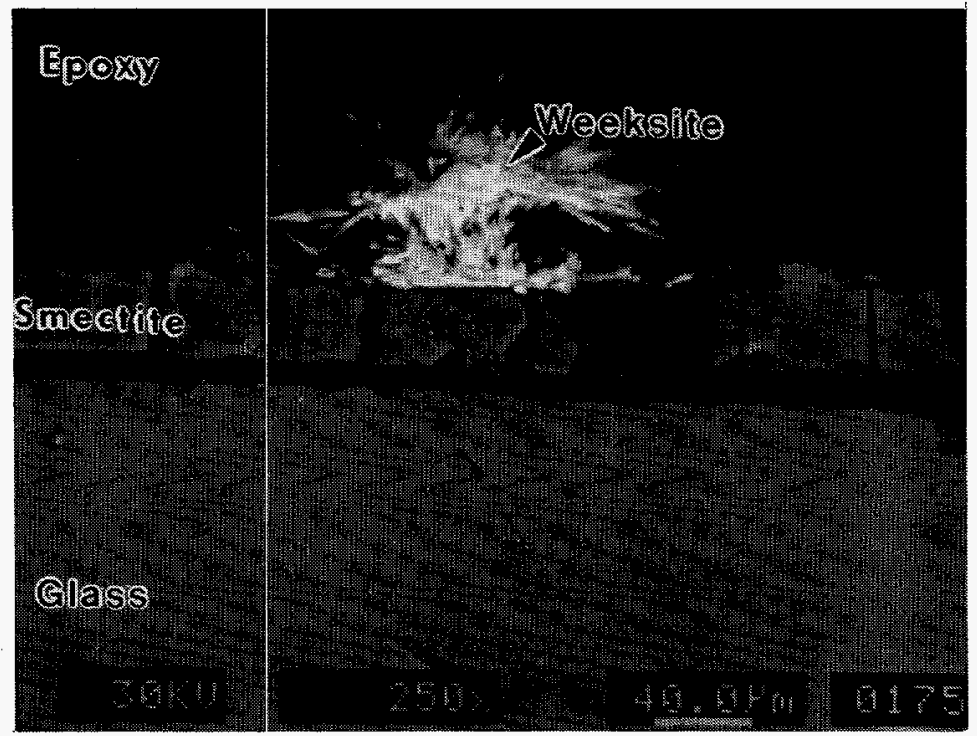

Fig. 89. SEM Backscattered Electron Image of Phase Forming on 56-Day Sample of SRL 131A Glass from $150^{\circ} \mathrm{C}$ Vapor Hydration Tests Exposed to Dose Rate of $\sim 35 \mathrm{~Gy} / \mathrm{h}$. The cross-section profile displays, from bottom to top, unaltered glass, smectite clay layer, surface precipitates including prominent weeksite phase, and epoxy (250X).

Surface precipitate phases identified in these samples include phillipsite, weeksite (Fig. 89), analcime, illite, Ca-Si fibers (possibly tobermorite) iron oxides, K-Al-Si-Na mounds, and several low atomic weight salt deposits. A comparison of reaction patterns for all time periods indicates that the sequence of mineral phases has changed relatively little throughout these tests. 


\section{DISCUSSION}

\section{A. Blank Irradiation Tests}

Under the geologically unsaturated conditions expected at the proposed Yucca Mountain nuclear waste repository, only limited amounts of water, if any, are expected to come in contact with the nuclear waste materials. Limited water ingress into the waste package environment is most likely to occur by dripping (or wicking) of water from the geologic medium surrounding the emplacement drift or by condensation of small amounts of liquid water from the moist repository atmosphere onto the waste form. These processes will affect both the surfaces of waste containers and their contained nuclear waste solids in the event of canister breaching. The accumulation of liquid water may vary from container to container depending on the mechanism of water contact, the number and location of canister breaches, and the hydrological conditions of the local repository geologic strata. It is, therefore, likely that limited quantities of water will come in contact with the waste containers and exposed waste forms in breached waste containers as thin films or pools of standing water on the solid surfaces. This type of contact mode is likely to result in a high-G/L environment in most places where water contacts the waste.

Any bicarbonate present in the thin films of water may be quickly overwhelmed by nitric acid produced during radiolysis reactions [23]. Any nitric acid that subsequently condenses on the glass will acidify the solution and may accelerate the reaction rates of the waste containers or nuclear waste forms. With the present set of tests, the confining pressures of the test vessel may have kept the bicarbonate solution concentration at relatively high levels; however, in an actual waste repository, the effectiveness of the bicarbonate buffer will be further reduced as radiolytic heating reduces the solubility of $\mathrm{CO}_{2}$ in aqueous solutions.

The limited quantities of solution used in the present gamma tests and the use of glass test vessels in the alpha radiation tests do not allow for unambiguous interpretation of radiation effects on solution $\mathrm{pH}$. However, the relatively high $\mathrm{NO}_{3}{ }^{-}$concentrations (Table 12) and calculated $\mathrm{pH}$ values as low as 3.6 (Fig. 11) from the alpha radiation tests confirm that acids are being produced in these tests, and that these acids are being concentrated in the limited quantities of solution present. These results are consistent with previously published observations noting the acidification of deionized water in irradiated air-water systems at high $\mathrm{G} / \mathrm{L}$ ratios $[3,4,8,11,14,15,17,52]$.

Test results indicate that nitrate + nitrite concentrations vary inversely with temperature, with the lowest quantities being detected for the higher temperature experiments (Fig. 6). This trend is important from the standpoint of waste form and container stability in that the highest radiation dose rates will occur early in the life of the repository, when waste package heating due to radioactive decay will also be the greatest. Thus, the elevated temperatures expected early in the life of the repository may mitigate against large decreases in $\mathrm{pH}$ resulting from nitrogen acid production. It is not known if the elevated temperatures affect the $\mathrm{NO}_{3}{ }^{-}$concentrations by lowering the production rate of $\mathrm{NO}_{3}^{-}$or by destabilizing the $\mathrm{NO}_{3}^{-}$that has already formed. Elevated repository temperatures may also hinder the formation of carboxylic acids as a consequence of the reduced solubility of $\mathrm{CO}_{2}$ in water at higher temperatures.

Results from the present experiments allow a comparison between the $\mathrm{NO}_{3}^{-}$yields from the $90^{\circ} \mathrm{C}$ gamma tests in this study and the $80^{\circ} \mathrm{C}$ neutron-dominated irradiation tests of Linacre and Marsh [11] (Fig. 6). The average yield from our study, $\mathrm{G}\left(\mathrm{NO}_{3}{ }^{-}+\mathrm{NO}_{2}{ }^{-}\right)=1.3 \pm 1.0$, is reduced slightly relative to the Linacre and Marsh data, $\mathrm{G}\left(\mathrm{NO}_{3}{ }^{-}\right)=1.9$, although the data from the present study display a considerable amount of scatter. A slight decrease in G yields at $90^{\circ} \mathrm{C}$ is expected based on previously discussed temperature effects on nitrogen acid yields. The $25^{\circ} \mathrm{C}$ yields are 
considerably larger than the ambient temperature $1.0-\mathrm{MeV}$ electron irradiation results of Jones, $\mathrm{G}\left(\mathrm{NO}_{3}{ }^{-}\right)=1.2$ [8]. Alpha yields from the present experiments, $\mathrm{G}\left(\mathrm{NO}_{3}{ }^{-}+\mathrm{NO}_{2}{ }^{-}\right)=2.4 \pm 1.1$, are also similar to the determined $\mathrm{G}\left(\mathrm{NO}_{3}{ }^{-}\right)$yield of $\sim 2.0$ for alpha energy deposited in air above a ${ }^{244} \mathrm{Cm}$-doped solution at $23^{\circ} \mathrm{C}[14]$.

The apparent alpha yields are slightly lower than the $3.2 \pm 0.7$ values determined for gamma radiation exposure at $25^{\circ} \mathrm{C}$ in this study. The decrease may be attributed to partial attenuation of the alpha particles in the thin film of water condensed on the ${ }^{241} \mathrm{Am}$ foils. If the energy transfer process (i.e., G-value yields) were identical for both gamma and alpha radiation absorbed by the gas phase, then the observed decrease in alpha yields would represent an attenuation of approximately $25 \%$ of the alpha particle energy by the thin film of water. Additional tests need to be performed with a single phase in $100 \%$ relative humidity systems to better constrain the nitrogen-species $\mathrm{G}$ values for alpha radiation.

Burns et al. [3,4] indicate that complete attenuation of alpha particles should occur in a $40 \mu \mathrm{m}$ film, while Garisto [53] indicates that $>90 \%$ of the alpha particles will be attenuated within a $20 \mu \mathrm{m}$ film of water. These values suggest an exponential change in the rate of alpha attenuation as a function of the thickness of the water film on the sample. Extrapolating these values to the observed decrease in alpha relative to gamma yields suggests that an average water film thickness of $\sim 0.2 \mu \mathrm{m}$ should result in the $25 \%$ attenuation of alpha energy deposited in the gas phase. Under actual test conditions, however, the thickness of the water film was nonuniform. The thickness of the water film notably varied from the top to the bottom of the foil under the influence of gravity and as a result of water periodically dripping from the bottom of the foil. The data scatter for the alpha tests may therefore partially reflect variable attenuation rates of alpha particles through films of water that are of an uneven thickness.

Alternatively, the observed variation in nitrate + nitrite yields between the alpha and gamma tests may represent a fundamental difference in the mechanism or efficiency of radiolytic product formation during ionization of moist air. Burns et al. [3,4] note that $\mathrm{G}$ values for liquid-water radiolysis species differ between alpha- and gamma-irradiated systems. It is conceivable, therefore, that $G$ values for primary radiolytic species may also differ for the two forms of radiation in moist-air environments. These differences may arise due to variations in the efficiencies of different forms of radiation in breaking bonds in atmospheric gases.

Gamma irradiation tests run at $\sim 500 \mathrm{~Gy} / \mathrm{h}(50,000 \mathrm{rad} / \mathrm{h})$ and $36 \mathrm{~Gy} / \mathrm{h}(\sim 3600 \mathrm{rad} / \mathrm{h})$ show little variation in nitrogen species production as a function of cumulative dose (Fig. 5). This lack of variation in nitrogen acid yields indicates that dose rate does not directly affect nitric acid yields, and that the radiolysis product yields can be accurately modeled by considering variations in the dose rate over time. Linacre and Marsh [11] had previously found that the formation of nitric acid was proportional to the absorbed dose up to a cumulative dose exposure of $2.3 \times 10^{7} \mathrm{~Gy}$ $(2300 \mathrm{Mrad})$. The present set of experiments examined the production of nitric acid at dose exposures that are more relevant to actual repository conditions (up to $\sim 1.4 \times 10^{6} \mathrm{~Gy}$ or $\sim 140$ Mrad).

A decrease in the concentration of nitrogen acid species was noted in only the highest cumulative dose experiments (Fig. 5). This decrease after $\sim 1.4 \times 10^{6}$ Gy exposure appears to be an anomaly in light of the consistency observed between the results from the present tests with lower dose rates and those of Linacre and Marsh. The decrease observed in the $\sim 1.4 \mathrm{x} 10^{6} \mathrm{~Gy}$ results may be attributable to several processes, including reactions with the test vessel during radiolytic acidification of the solution, analytical error, errors in dosimetry measurements, consumption of air components necessary for nitrogen species production, or radiolytic decomposition of nitric acid (and/or precursor components) during irradiation. Potential reaction with the test vessel is not supported by data obtained during testing, since there is not any observed 
systematic increase of released vessel components to solution as a function of cumulative dose absorbed by the system. Although analytical or dosimetry errors are possible, it is unlikely that they would influence only the two long-term tests and none of the others. Of the nitrogen acid forming components present in air, oxygen will be of the shortest supply. However, only $\leq 6$ mole $\%$ of the oxygen present in the tests is calculated to be consumed during the production of the 5 to $8 \mu$ moles of nitrogen acid species produced in the $\sim 1.4 \times 10^{6}$ Gy exposure tests. Radiolytic decomposition of nitrogen acids, or alternatively, a precursor species to the acid formation, may potentially influence the balance of nitrogen acid species formed in the longer term tests. A similar phenomenon has been observed by Reed [18], however, the decrease observed in that study was more gradual than that observed in the present test results.

The presence of large volumes of liquid water in these air-water systems may influence the radiolysis product concentrations by displacing equivalent volumes of the air phase from the test vessels. Since the nitrogen acids are predominantly produced in air, displacement of air from the test vessel is likely to reduce the amount of nitrogen acid produced. A comparison of the tests conducted at different $\mathrm{G} / \mathrm{L}$ ratios $\left(90^{\circ} \mathrm{C} ; \mathrm{G} / \mathrm{L}=10\right.$ and 100$)$, however, indicates little variation in mean $G$ yields, although there is a large degree of scatter for both sample sets (Table 10). The presence of the larger volumes of water in the low $\mathrm{G} / \mathrm{L}$ ratio tests also may dilute the radiolysis products that form and offer a bicarbonate $\mathrm{pH}$ buffering reservoir to the radiolysis reaction products [23].

Radiolysis of water may influence the redox potential (Eh) of the irradiated solution. During water irradiation, equal amounts of reducing and oxidizing species will be formed. The $\mathrm{H}_{2}$ produced during radiolysis is chemically inert toward low-temperature $\left(<100^{\circ} \mathrm{C}\right)$ aqueous reactions and has a high diffusional mobility relative to other radiolytic products $[2,6,54]$. The $\mathrm{H}_{2}$ produced by radiolysis reactions may thus separate from the aqueous system, increasing the overall oxidation state of the irradiated fluid.

For radiolysis reactions occurring with spent fuel, $\mathrm{O}_{2}^{-}$and $\bullet \mathrm{OH}$ are the predominant oxidizing agents, followed by $\mathrm{H}_{2} \mathrm{O}_{2}$, which in turn, is followed by $\mathrm{O}_{2}$ [55]. Measured oxidation potentials had been increased by $0.70 \mathrm{eV}$ within $30 \mathrm{~mm}$ of an alpha radiation source, but these potentials rapidly decrease with distance from the source, falling to the local ambient potential at distances of $70 \mathrm{~mm}[55,56]$. Since the alpha-particle travel distance is limited to a maximum of $\sim 40 \mu \mathrm{m}$ in aqueous systems [57], the region affected by the alpha-associated redox change will be $\sim 1750$-fold greater than the region directly interacting with the alpha particles themselves. These trends suggest that the size of the region affected by the redox changes is likely to be influenced by the diffusion capacity of the short-lived radiolytically produced oxidants. Because alpha-particle travel distances in air are $\sim 40 \mathrm{~mm}$, the region affected by radiolytic oxidants in air-dominated systems may be somewhat larger than that of aqueous systems. The actual region affected by radiolytic oxidants in air dominated systems should, therefore, be influenced by the availability of liquid water, the convection of water aerosols, and the attenuation of alpha particles in the thin film of water contacting the alpha source. In either water or moist-air dominated systems, however, the effects of alpha radiation on the redox potential of aqueous systems will be localized to the general area immediately surrounding the radioactive material.

An examination of the rates of $\mathrm{NO}_{3}^{-}$versus $\mathrm{NO}_{2}^{-}$production in the irradiated test solutions may offer some insight into the redox environment of the present tests. Nitrous acid $\left(\mathrm{HNO}_{3}\right)$ is thermally unstable and will decompose to nitrate according to the following reaction $[58,59]$ :

$$
3 \mathrm{HNO}_{2} \rightarrow \mathrm{H}^{+}+\mathrm{NO}_{3}{ }^{-}+2 \mathrm{NO}+\mathrm{H}_{2} \mathrm{O}
$$

The NO produced in this reaction would be rapidly oxidized in irradiated solutions to $\mathrm{NO}_{2}$, which would then react with water to form equimolar amounts of $\mathrm{NO}_{3}^{-}$and $\mathrm{NO}_{2}^{-}$[59]. The $\mathrm{NO}_{2}^{-}$formed 
in this later step would then reprotonate to renew the cycle. Nitrous acid may also dissociate in the presence of radiolytically produced hydrogen peroxide to form nitrate according to the following reaction [58]:

$$
\mathrm{H}_{2} \mathrm{O}_{2}+\mathrm{H} \mathrm{NO}_{2} \rightarrow \mathrm{H}^{+}+\mathrm{NO}_{3}^{-}+\mathrm{H}_{2} \mathrm{O}
$$

The result of the reactions in Eqs. 3 and 4 is that all $\mathrm{NO}_{2}^{-}$initially formed during radiolysis reactions will eventually be converted to $\mathrm{NO}_{3}{ }^{-}[59]$.

A comparison of the nitrate and nitrite levels in the present tests indicates that $\mathrm{NO}_{3}{ }^{-}$is the predominant species formed under most test conditions, while $\mathrm{NO}_{2}^{-}$concentrations are typically below levels of determination (Tables 8 and 12). Total recovered $\mathrm{NO}_{2}^{-}$values are generally $<0.05 \mu \mathrm{mol}$ for the $0.22 \mathrm{~mL}$ of solution in the gamma tests and $<1.0 \mu \mathrm{mol}$ for the $\sim 18 \mathrm{~mL}$ of solution in the alpha tests. An interesting feature of the gamma tests is that the $\mathrm{NO}_{3}^{-} / \mathrm{NO}_{2}^{-}$ratios are lower in the rinse aliquots due to a slight increase in $\mathrm{NO}_{2}^{-}$levels (Tables 8 and 16). Reactions between the thin films of condensed water and the stainless steel vessel walls may have caused this increase due to consumption of $\mathrm{H}_{2} \mathrm{O}_{2}$ and a consequent inhibition of the reaction of Eq. $4[3,4]$. Alternatively, $\mathrm{Fe}^{2+}$ released during reactions with the vessels may reduce $\mathrm{NO}_{3}^{-}$to $\mathrm{NO}_{2}^{-}$[59]. Minor surface pits were observed on some vessels after numerous rounds of high-temperature testing.

Only slight increases in $\mathrm{NO}_{3}-\mathrm{NO}_{2}^{-}$ratios occurred as a function of decreasing reaction temperatures for the gamma tests at the same dose rate (Fig. 90). By contrast, large increases in $\mathrm{NO}_{3}^{-} / \mathrm{NO}_{2}^{-}$ratios are noted in the $\sim 500 \mathrm{~Gy} / \mathrm{h}$ tests relative to the $\sim 30 \mathrm{~Gy} / \mathrm{h}$ tests up to $\sim 4 \mathrm{x} 10^{5} \mathrm{~Gy}$ cumulative exposure. A large increase in the amount of $\mathrm{NO}_{2}^{-}$produced is noted for gamma tests exposed to more than $\sim 6.8 \times 10^{5}$ Gy of cumulative radiation energy (Table 8 and Fig. 90). This change probably occurs when the increased acidification of the solutions due to nitric acid generation destabilizes nitrous acid [59]. Oxygen depletion in the gas phase would probably not be responsible for the observed $\mathrm{NO}_{2}^{-}$increase because the consumption of only $3 \%$ of the oxygen present in the vessels is required to produce the amounts of $\mathrm{NO}_{3}^{-}$formed in these tests. Furthermore, oxygen consumption rnay actually be lower if oxygen contributions derived from water radiolysis products contribute to nitric acid generation. Reaction between test solutions and $\mathrm{Fe}^{2+}$ in the stainless steel vessels may also have contributed to the $\mathrm{NO}_{2}^{-}$increase in the high-gamma dose tests, but the magnitude of the $\mathrm{NO}_{3}{ }^{-} \mathrm{NO}_{2}{ }^{-}$change is probably too large to be accounted for by such reactions with the stainless steel vessels alone.

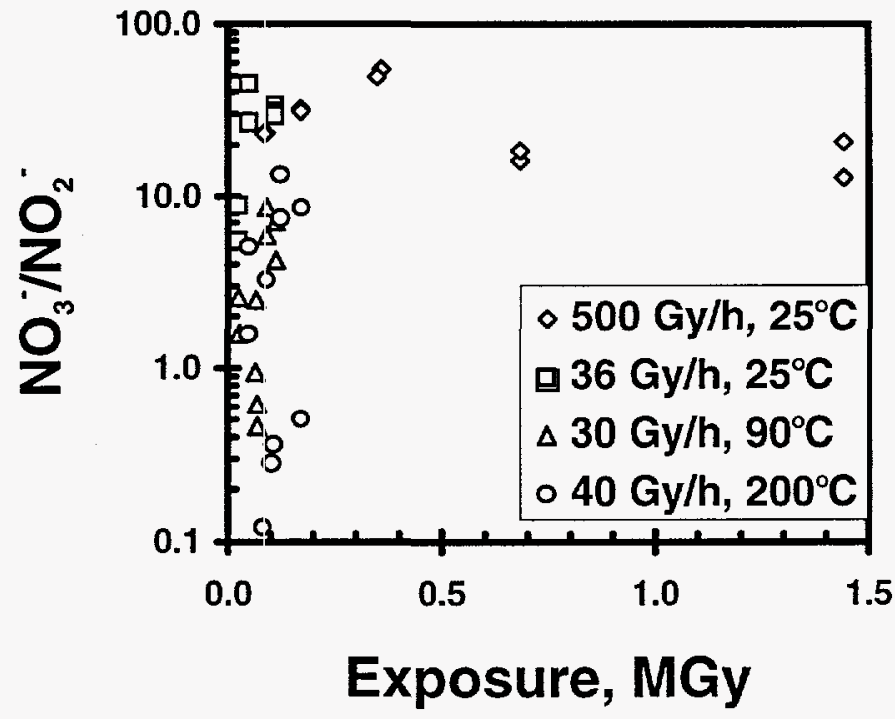

Fig. 90. Nitrate/Nitrite Ratio for Blank Gamma Tests 
Overall patterns developed with respect to carbon contents of the test solutions are difficult to evaluate due to the large amount of data scatter. Averaged values for rinse solution fractions of organic carbon (OC) and inorganic carbon (IC) increased during the low-dose gamma tests at 25 and $90^{\circ} \mathrm{C}$ relative to the levels that characterized background tests without irradiation (Table 9 and Figs. 8 and 9). The most likely source for the OC generation in these solutions is the conversion of dissolved carbonic acid species into OC [5]. The absence of a corresponding decrease in the IC carbon fraction during $\mathrm{OC}$ formation suggests that dissolution of $\mathrm{CO}_{2}$ from the gas phase or reactions with the test vessel walls have released additional IC to the solution. It is not understood, however, why the IC fraction in the irradiated tests should increase over that of the corresponding background tests conducted without irradiation exposure. Analyses were not performed on the test vessel atmosphere to examine for $\mathrm{CO}_{2}$ depletion in the gas phase.

Increases were also observed in the OC and IC contents of the alpha-test rinse solutions relative to the prerinse fraction. It appears, however, that the test vessel apparatus may have contributed to this increase because similar concentrations were also observed in the rinse solutions of the background tests (Table 9).

Formate and oxalate were noted in the rinse solution fractions during both alpha and gamma radiation exposure. These organic species account for $\sim 50 \%$ of the OC observed in the alpha tests and $90^{\circ} \mathrm{C}$ gamma tests, but only $5 \%$ of the $\mathrm{OC}$ observed in the $25^{\circ} \mathrm{C}$ gamma tests at low dose. Increases were not observed in the $\mathrm{OC}$ fraction of the prerinse solutions from either the gamma or alpha tests. The formation of oxalate and formate is favored under low-pH and/or oxygen-free conditions that prevent carboxyl radicals $\left(\mathrm{CO}_{2}{ }^{-}\right)$from transferring charge to oxygen, thus allowing sufficient time for their dimerization into oxalate or reaction with hydrogen ions to produce formate [5,59]. Favorable conditions may exist for the formation of formate and oxalate in the thin films of water contacting the vessel components in these tests. Although it was previously noted that $\mathrm{NO}_{3}{ }^{-}$has preferentially collected in the prerinse solution (Sec. III.A.1), the volume of water that condensed on the vessel walls, top, and support rods (in the alpha tests only) may be considerably less than that at the bottom. If true, then the nitric acid concentration in this limited volume of fluid present on the vessel walls may be greater than that of the prerinse solution collected at the bottom of the test vessel. Higher nitric acid concentrations, combined with less oxidizing conditions (as evidenced by the reduced $\mathrm{NO}_{3}{ }^{-} / \mathrm{NO}_{2}^{-}$ratios shown in Table 16) of the condensed fluid on the walls would favor the formation of formate and oxalate.

Organic carbon production and the amounts of oxalate and formate are diminished in the blank gamma tests at $25^{\circ} \mathrm{C}$ and high dose and those at $200^{\circ} \mathrm{C}$ and low dose, relative to levels produced in tests at $25^{\circ} \mathrm{C}$ and low dose (Figs. 8 and 9). The lower amount of these species in the high-dose rate tests does not appear to reflect the cumulative dose because there is a notable difference in the yields of organic species where the cumulative doses for the high- and low-dose tests overlap. Thus, it appears that the variable dose rates have influenced the carboxylic acid concentrations in these tests. The reduced levels in the $200^{\circ} \mathrm{C}$ tests are probably associated with the decreased solubility of carboxyl radicals and/or carbonic acids in aqueous fluids.

\section{B. Batch Tests at $340 \mathrm{~m}^{-1}$}

Elemental release patterns from the batch tests at $\mathrm{S} / \mathrm{V}=340 \mathrm{~m}^{-1}$ can provide an important indicator of the extent of glass corrosion. Release trends of more soluble components in the glass (for example, B) may directly reflect the extent of glass corrosion. Less soluble glass components may be released in a nonstoichiometric manner due to the selective removal of soluble components and the residual enrichment of remaining elements in the alteration layers [6]. This leaching process would result in a residual enrichment of the less soluble components, along with a corresponding decrease in the "apparent release" of these components to solution. Elements may also be selectively removed from solution by the precipitation of mineral phases on the sample 
surface and/or test vessel walls. Thus, an examination of altered glass surfaces is important, in addition to monitoring solution composition, for determining the extent of glass corrosion.

A compositional and mineralogic analysis of the altered surface material can be extremely useful in determining the extent of residual enrichment of elements on the altered glass surface. For example, the accumulation of residual elements due to selective ion exchange may be represented on the glass surface by the formation of a gel layer enriched in iron and silica, or a layer of clay minerals that have formed as recrystallized gel material. In addition, the precipitation of elements from solution may be recorded by examining the formation of isolated crystalline phases on the sample surface. The combined use of solution analytical results with solid phase microanalysis is thus the best method for examining the reaction progress affecting the solids.

Nitrogen acid formation occurs in irradiated air-water systems due to the ionization-induced splitting of $\mathrm{N}_{2}$ in the atmosphere and the recombination of elemental nitrogen with other air and water radiolysis products to form nitric and nitrous acids. Results from the batch tests at $340 \mathrm{~m}^{-1}$ record an increase in concentration of $\mathrm{NO}_{3}^{-}+\mathrm{NO}_{2}^{-}$with increasing test length (and a corresponding increase in dose exposure) (Table 15). The $\mathrm{NO}_{3}^{-}+\mathrm{NO}_{2}^{-}$concentrations in these tests may have several potential sources. Nitrogen acids have previously been shown to be produced radiogenically by exposure of moist air systems to radiation sources (see Sec. V.A). The blank tests were run under similar conditions as the $340 \mathrm{~m}^{-1}$ tests, except that a deionized water leachant was used and no glass was present in the test vessels. Concentrations of $\mathrm{NO}_{3}{ }^{-}+\mathrm{NO}_{2}^{-}$in the blank tests were increased to 2-4 ppm after 56 to 120 days of exposure (Table 8). Adding this radiolytic contribution to the $8.2 \mathrm{ppm} \mathrm{NO}_{3}^{-}+\mathrm{NO}_{2}^{-}$initially present in the EJ-13 leachant (Table 15) would result in a total concentration of 10 to $12 \mathrm{ppm}$. This total is generally below the level exhibited by the leachants taken from the $340 \mathrm{~m}^{-1}$ tests at 56 days $(17 \pm 3 \mathrm{ppm}$; Table 15$)$. The source of the excess $\mathrm{NO}_{3}^{-}$and $\mathrm{NO}_{2}^{-}$detected in the $340 \mathrm{~m}^{-1}$ tests is unknown, but the components for nitrogen acids may have been derived from corroding glass samples.

The concentrations of other anions from the irradiated $340 \mathrm{~m}^{-1}$ tests (Table 15) indicate only minor variations with no demonstrable changes that can be correlated as a function of radiation exposure. Fluorine, chlorine, and sulfate are typically enriched 30 to $50 \%$ over the concentration of the starting EJ-13 leachant. It is likely that these increases were derived from anion components released from the glass during the alteration process.

Carbon analytical results for the $340 \mathrm{~m}^{-1}$ tests display an overall increase in TC and IC as a function of time, while OC levels clecrease relative to the starting EJ-13 solution. The results shown in Table 15 also display an overall increase in TC and IC that can be correlated to increasing radiation exposure; however, there is considerable scatter in the obtained data. With the blank gamma and alpha tests, small increases in both the IC and OC fractions with time were observed (Tables 9 and 13). These increases were previously attributed to the absorption of $\mathrm{CO}_{2}$ from the vessel atmosphere and the partial conversion of IC to OC, respectively. The batch tests with glass samples display a much larger increase in IC over the starting EJ-13 solution, relative to the increase for the alpha and gamma tests over their starting deionized water solutions. The magnitude of the increase for the tests with glass requires that a significant contribution of IC come from the dissolving glass. The OC fraction actually decreases in the $340 \mathrm{~m}^{-1}$ tests relative to the starting EJ-13 solution. This trend is opposite to that of the blank gamma and alpha tests, where OC increased due to radiation exposure as IC was converted into carboxylic acids. With the 340 $\mathrm{m}^{-1}$ tests, it is apparent that the $\mathrm{OC}$ (including the radiogenically produced carboxylic acids) is being consumed through reactions with the glass samples. The decrease in OC is also paralleled by decreasing concentrations of carboxylic acids, especially formate, in the glass tests relative to the blank gamma tests (compare results from Tables 9 and 15).

For the $340 \mathrm{~m}^{-1}$ tests, the $\mathrm{pH}$ of the irradiated test solution in contact with SRL 202A glass initially increases during the first 180 days, then decreases up to 540 days (Fig. 15). The pH 
values for the irradiated tests are slightly lower than those of the nonirradiated throughout the duration of the experiments. This trend is interpreted to reflect a temporal change in the rate of glass reaction relative to a steady rate of radiolytically produced nitric acid. During the initial alteration process, alkali and alkaline earths present in the glass are exchanged for $\mathrm{H}^{+}$ions from the test solution, thereby neutralizing the radiolytic acids produced. The result of excess alkali release is an increase in the $\mathrm{pH}$ of the test solution. In the longer-term tests, the increasing thickness of the alteration layer may act as a partial diffusion barrier to migrating ions. A lessening in the release rates of alkalis B and Mg from the SRL 202A glasses after 180 days is consistent with such an interpretation (Figs. 16a-e). The resultant decrease in the ion-exchange process would allow the radiolytic acids to concentrate in the leachate and subsequently decrease the $\mathrm{pH}$ of the solution. A maximum decrease of $\sim 1 \mathrm{pH}$ unit would be expected, based on the increase in $\mathrm{NO}_{2}^{-}+\mathrm{NO}_{3}^{-}$ observed in the leachate solution over that of the EJ-13 leachant (Table 15). This change would assume that the aqueous system does not have a significant buffering effect on protons released by the radiogenic acids. This calculated $\mathrm{pH}$ shift is consistent with the changes observed between the irradiated versus nonirradiated experimental systems (Fig. 15). The cause of the increase in $\mathrm{pH}$ observed in the final 720-day tests has not been determined.

Results from irradiated SRL 131A glasses similarly show an initial increase in pH followed by a decrease (Fig. 15). The $\mathrm{pH}$ values for the SRL 131A glasses reach a peak at 56 days and then decline thereafter. This peak in $\mathrm{pH}$ values occurred earlier than previously exhibited by the SRL 202A glasses, indicating that the SRL 131A glasses have reacted more rapidly under the present test conditions. The rapid development of a thick alteration layer would quickly induce a diffusion barrier for ion exchange and thus would lead to early and enhanced preservation of radiogenically produced acids, as evidenced in the $\mathrm{pH}$ decreases of these tests. Such an interpretation is consistent with the solution data from these tests, which indicate rapid increases in the release of alkalis, $\mathrm{B}$, and $\mathrm{Mg}$ up to 56 days, and then a relatively gradual release thereafter (Figs. 16a-e).

Although the SRL 165A glasses show some data scatter at 180 days, the overall $\mathrm{pH}$ trend appears to show values that increase throughout the tests (Fig. 15). This pattern may reflect relatively limited development of alteration layers on these samples and thus continued alkali release from the glass at sufficient levels to neutralize the radiogenic acids produced during gamma irradiation. The relatively thinner alteration layers present on the SRL 165 glasses can be explained by a more stoichiometric release of glass components relative to other glasses. It is also interesting to note that the SRL $165 \mathrm{~A}$ samples at 720 days display the highest $\mathrm{pH}$ values of the four sample compositions shown in Fig. 15.

Comparisons of $\mathrm{pH}$ values for the unirradiated SRL 202U versus irradiated SRL 202A $340 \mathrm{~m}^{-1}$ tests indicate that the irradiated test solutions have slightly decreased $\mathrm{pH}$ values (Fig. 15). This $\mathrm{pH}$ decrease is $\sim 0.5 \mathrm{pH}$ units lower for most time periods tested. The irradiated SRL 202A tests also display lower NL(Si) release values for most time periods (Fig. 17g). The lower total silicon concentrations apparently developed in response to the lower $\mathrm{pH}$ values of the irradiated tests. This pattern has been reported from previous studies with the decrease in silicon concentrations arising through the lowering of the activity of silicic acid with decreasing $\mathrm{pH}$ [30-33].

The production of radiolytic acids in these tests has also resulted in lower rates for the release of other glass components into solution, such as $\mathrm{Li}$ and $\mathrm{Mg}$ (Figs. 17b and 17e). The release patterns for these elements apparently follow the same trend as that of silicon into solution. The NL(i) release trends for $\mathrm{B}, \mathrm{Na}, \mathrm{K}$ and $\mathrm{Ca}$ do not display the same dependence on the $\mathrm{pH}$ values, with release trends for these elements being similar for the SRL 202A and SRL 202U glasses (Figs. 17a, 17c, 17d, and 17f).

The 720-day samples provide an interesting example for the $\mathrm{pH}$ effect on the NL(i) release values discussed in the previous paragraph. The SRL 202A samples display a progressive 
decrease in $\mathrm{pH}$ between 180 and 540 days, and then plateau or possibly even display a slight increase in $\mathrm{pH}$ for the final 720-day sample (Fig. 15). The SRL 202U samples display a relatively constant $\mathrm{pH}$ between 56 and 540 days, and then a decrease up to the final 720 -day sample. A comparison of NL(i) release values indicates that the $\mathrm{pH}$ increase displayed by the 720-day irradiated SRL 202A glasses corresponds to an increase in release rates for $\mathrm{Li}, \mathrm{Mg}$, and Si (Figs. 17b, 17e, and 17g). By contrast, the $\mathrm{pH}$ decrease in the unirradiated SRL 202U tests corresponds to lower NL(i) release values for the same three elements plus uranium. While the decreased release rates in themselves are not a priori evidence for decreased glass reaction rates (i.e., sequestering of elements by secondary alteration phases may also decrease element release rates), their correlation with the timing of the $\mathrm{pH}$ change provides supporting evidence for a change in the rate of the ionic exchange process as a function of the radiation-influenced $\mathrm{pH}$ values. Uranium release may have also been enhanced by the increased stability of bicarbonate uranyl complexes at the higher $\mathrm{pH}$ values.

Relative to both the SRL 202A and SRL 165A glasses, the SRL 131A samples consistently display higher $\mathrm{NL}(\mathrm{i})$ release values for $\mathrm{B}, \mathrm{Li}, \mathrm{Na}, \mathrm{K}, \mathrm{Mg}$, and $\mathrm{Si}$ (Fig. 17). This trend indicates that, under the present test conditions, the SRL 131A glass is the least durable of the three glass compositions. These high NL(i) values are displayed by both network-forming and networkmodifying elements in the SRL 131A glass. Both the SRL 131A and SRL 202A glasses also display higher NL(U) values than the SRL 165A glasses.

The NL(Ca) values were reduced in the SRL 131A glasses relative to the other two glass types tested, especially in the short term tests (Fig. 17f). This trend was unusual in that the calcium did not follow a similar path as magnesium, the other alkaline earth element analyzed with these samples. Both elements would be expected to behave in a similar manner during the alteration process affecting the glass samples. This finding suggests that calcium may be preferentially incorporated into alteration phases that are present on the glass surface. Other than the smectite clays, no distinct Ca-bearing phases were observed to have formed on the $340 \mathrm{~m}^{-1}$ batch test samples. Since the NL(Ca) values of almost all SRL 131A tests displayed negative release numbers, it appears that the release of calcium from the glass samples was insufficient to balance the proportion of calcium being incorporated in alteration phases forming on the glass surface. The negative NL(Ca) values, therefore, reflect calcium concentrations that were depleted below levels of the starting EJ-13 leachant. The lower NL(Ca) values in these tests also may reflect the lower calcium concentrations in the SRL 131A glass relative to the other two glass compositions (Table 4).

A comparison of the NL(i) trends between the SRL 165A and SRL 202A glasses indicates that the releases of $\mathrm{B}, \mathrm{Na}$, and $\mathrm{Mg}$ are lower for the SRL 165A glasses for all test periods prior to 720 days, while for the 720-day tests, the NL(i) values for these elements are similar to, or higher than, those of tests with SRL 202A glass (Fig. 17). Silicon also displays a similar trend, with the $\mathrm{NL}(\mathrm{Si})$ values for SRL 165A approaching those of the SRL $202 \mathrm{~A}$ glasses between 365 and 720 days (Fig. 17g). The NL(i) values for $\mathrm{Li}$ and Ca release from SRL 165A are similar to, or higher than, those of the SRL 202A glasses for all time periods (Figs. 17b and 17f). All of the NL(K) values for SRL 165A are negative, reflecting a strong depletion of potassium in solution relative to the starting EJ-13 leachant solution (Fig. 17d). The removal of potassium from the solution in contact with these samples reflects the very low initial potassium concentrations of the SRL 165A glass (Table 4) and the formation of potassium-bearing alteration phases on the sample surfaces. The NL(U) values for the SRL 131A and SRL 202A glasses are also generally similar, with the SRL 165A glasses displaying lower values, especially for the final 720-day samples (Fig. 17h). The conclusion from these comparisons is that the normalized release rates of most components from the SRL 165A glass are generally slightly lower than those from the SRL 202A glass under the present experimental conditions, which includes the exposure of the samples to an external gamma radiation source during testing. 
Alpha counting results for unfiltered, 5-nm filtered, and acid-strip aliquots from the $340 \mathrm{~m}^{-1}$ batch tests up to 720 days indicate that $\mathrm{Am}$ and Pu plutonium release is dominated by the acid strip component, with the $<5 \mathrm{~nm}$ fraction is negligible for most time periods (Figs. 18a through 18f). This trend indicates that most of the $\mathrm{Am}$ and $\mathrm{Pu}$ released from the glass will subsequently plate out on the stainless steel vessel walls, while the remainder is dominated by filterable grains $>5 \mathrm{~nm}$ in size. A similar fractionation trend has previously been documented by Bates and others [65]. These results are of interest because they indicate that (1) stainless steel waste canisters will act as a sorption barrier to Am and Pu migration in a repository scenario, and (2) iron oxide particulates and colloids liberated from degrading canisters may serve as a transport medium for actinide migration. The former scenario will be a benefit to repository performance in retarding the migration of these actinide species, while the latter scenario is clearly one that should be taken into consideration from actinide migration standpoint.

Neptunium displays a fractional release distribution different from that exhibited by Am and $\mathrm{Pu}$ (see Figs. 18h and 18i). With neptunium, the greatest release is observed in the 5-nm filtered or unfiltered fractions, with only a minor fraction of the total release being attributable to the acidstrip component. These trends indicate that neptunium migration will be dominated by the fraction present in a soluble form and/or colloids $<5 \mathrm{~nm}$ in size. In contrast to the behavior of $\mathrm{Am}$ and $\mathrm{Pu}$, very little neptunium is expected to be adsorbed onto stainless steel components present in the repository or be transported by iron-oxide colloids.

In a number of gamma radiolysis tests on air-water systems without the presence of glass, $\mathrm{NO}_{3}{ }^{-}$was found to be the predominant nitrogen species present $[3,4,8,11]$. Blank gamma tests run without the presence of glass, but otherwise under similar test conditions as the $340 \mathrm{~m}^{-1}$ batch tests [24], display higher $\mathrm{NO}_{3}^{-} / \mathrm{NO}_{2}^{-}$ratios and elevated $\mathrm{NO}_{3}^{-}$concentrations relative to the glass tests. By contrast, the redox sensitive ratio of $\mathrm{NO}_{3}{ }^{-} / \mathrm{NO}_{2}^{-}$generally varies from 0.1 to 0.4 for the irradiated glass tests. This ratio is significantly decreased from that of both the starting EJ-13 solution ( $>16)$ and the blank tests (Table 16). This decreased $\mathrm{NO}_{3}{ }^{-} \mathrm{NO}_{2}^{-}$ratio results from a one to two orders of magnitude increase in the concentration of $\mathrm{NO}_{2}^{-}$during a period where the $\mathrm{NO}_{3}{ }^{-}$ contents remained the same or even decreased slightly relative to the starting EJ-13 solution.

In previous gamma studies, the radiolytic reduction of $\mathrm{NO}_{3}^{-}$to $\mathrm{NO}_{2}^{-}$was suggested to result from a reduced Eh of the irradiated solutions [7,66]. This proposed radiolysis-induced $\mathrm{Eh}$ decrease conflicts with the results from this study (see Sec. V.A.) and several studies conducted with spent nuclear fuel oxidation $[2,54,55]$. With the blank gamma and alpha tests, the oxidized $\mathrm{NO}_{3}^{-}$species dominated the nitrogen systematics of the irradiated environment (Table 16). Since the irradiation tests with glass were conducted under similar test conditions as the blank tests, and $\mathrm{NO}_{3}^{-}$would therefore have formed in these tests if the glass had not been present, we can conclude that the presence of the glass samples has either (1) inhibited the formation of $\mathrm{NO}_{3}^{-}$, or (2) the glass surface has reduced the $\mathrm{NO}_{3}^{-}$to $\mathrm{NO}_{2}^{-}$. The former conclusion is favored since the concentration of $\mathrm{NO}_{3}^{-}$present in the irradiated $340 \mathrm{~m}^{-1}$ leachates has remained similar to that of the starting EJ-13 leachant (i.e., neither the irradiation exposure nor the presence of the altered glass surface appears to have reduced the concentration of $\mathrm{NO}_{3}^{-}$present in the EJ-13 leachant).

The role of $\mathrm{H}_{2} \mathrm{O}_{2}$ in oxidizing $\mathrm{NO}_{3}{ }^{-}$to $\mathrm{NO}_{2}^{-}$has previously been documented by Van Konynenburg [Eq. 4 and 5]. The results from the Van Konynenburg study indicate that $\mathrm{H}_{2} \mathrm{O}_{2}$ will be decomposed through reactions with materials, such as manganese oxides, which are also present on the surface of altered glass. This argument would thus suggest that the conversion of $\mathrm{NO}_{2}^{-}$to $\mathrm{NO}_{3}^{-}$may have been blocked in these tests through the consumption of $\mathrm{H}_{2} \mathrm{O}_{2}$ on the glass surface. Although $\mathrm{H}_{2} \mathrm{O}_{2}$ contests were not measured in the present tests, the slightly lower $\mathrm{NO}_{3}^{-} / \mathrm{NO}_{2}{ }^{-}$ratios obtained from the blank gamma rinse solutions relative to the prerinse solution (see Sec. V.A) may also be attributed to $\mathrm{H}_{2} \mathrm{O}_{2}$ consumption on the stainless steel vessel walls. Although both the rinse and prerinse aliquots are in contact with the stainless steel, the liquid 
volume/vessel surface area ratio is much greater in the rinse solution, thus allowing relatively greater consumption of $\mathrm{H}_{2} \mathrm{O}_{2}$ in this aliquot.

The decreased $\mathrm{NO}_{3}^{-}-\mathrm{NO}_{2}^{-}$ratio of irradiated solutions in the $340 \mathrm{~m}^{-1}$ tests, relative to the gamma blank tests (Tables $8 \mathrm{~b}$ and 15 ) may also be attributed to the stability of the $\mathrm{NO}_{2}^{-}$radical in the presence of an alkali solution buffered by the reacting glass. Linacre and Marsh [11] note that reactions converting $\mathrm{NO}_{2}^{-}$to $\mathrm{NO}_{3}{ }^{-}$may be catalyzed by acids; therefore, neutralization of the acids through reactions with the glass may inhibit such a reaction from taking place. This interpretation, however, conflicts with the reaction expressed in equation 4.

\section{Vapor Hydration Tests}

\section{Reaction Layer Development on SRL 202 Glass}

One of the objectives of these experiments is to examine the cumulative effect of radiolytic product formation on the performance of a simulated borosilicate waste glass under unsaturated test conditions. To examine this parameter, several different glass compositions were reacted under both gamma-irradiated and nonirradiated steam environments. Following this exposure, the reacted samples were examined for the presence of secondary mineral precipitants and thicknesses of reaction layers. The differences observed between the irradiated and nonirradiated samples indicate the influence that irradiation has on the stability of both the glass waste form and secondary alteration minerals.

Published studies of glass alteration in water-vapor-dominated environments with high $\mathrm{S} / \mathrm{V}$ ratios $\left(>20,000 \mathrm{~m}^{-1}\right)$ are relatively rare compared to the vast number of studies where glass samples are immersed in a bath of liquid water at relatively low S/V ratios. Bates and coworkers have examined the reactions of a number of simulated waste glass compositions, including SRL 131, SRL 165, SRL 202, and SRL 211 glasses under unirradiated vapor hydration conditions in a series of papers $[37,42,45,60,67]$. These studies were conducted under a variety of test temperatures and relative humidity conditions and also form a basis for which the mineral phases identified in the present study will be compared. Additional results of SRL 202 and SRL 131 glass reactions under saturated test conditions will supplement this study $[68,69]$.

Several of the previously mentioned studies also have compared the formation of mineral phases under water-saturated and unsaturated conditions. Differences in the timing of the formation of alteration minerals in the two tests have been attributed to different rates of saturation levels of the fluid with respect to the alteration phases that develop $[45,60,67]$. In the high-S/V environment characteristic of the vapor hydration tests, the thin film of fluid contacting the waste is rapidly saturated with respect to certain secondary alteration phases. This rapid saturation results in the precipitation of secondary alteration phases on the glass samples, which, in turn, lowers the concentration of the constituent elements in solution. The development of this "corrosion product sink" of secondary alteration phases will lower the concentration of these elements in solution, thereby kinetically favoring continued dissolution of the waste glass. Conversely, under lower $\mathrm{S} / \mathrm{V}$ test conditions, the concentration of corrosion products in solution is diluted by the larger volumes of fluid in the tests. Because of these larger solution volumes, the saturation levels of the fluids with respect to the secondary alteration products are attained more slowly than the corresponding tests conducted at higher $S / N$ ratios. The continued buildup of corrosion products in these low $\mathrm{S} / \mathrm{V}$ tests will inhibit the release of additional elements from the glass. The progression of alteration phase development under water-saturated conditions (low $\mathrm{S} / \mathrm{V}$ ) may, therefore, be retarded relative to the formation of these phases under unsaturated (high $S / V$ ) conditions.

A single study of glass alteration in an irradiated vapor hydration test, by Yokahima et al., has identified the formation of deposits enriched in Mo and $\mathrm{Ba}$ [70]. Similar deposits were 
not present on glass samples from parallel tests conducted under saturated water conditions, indicating that the high-S/ $\mathrm{V}$ conditions have likely accelerated the glass reactions during radiation exposure. These authors, however, did not compare results from reactions in irradiated versus nonirradiated environments, therefore, results from the Yokahima et al. study could not be extracted in a quantifiable comparison of the effects of irradiation exposure on glass reactions.

With the present tests, both the nonirradiated and irradiated samples rapidly developed an in situ alteration layer and a cover of precipitates formed as the solution contacting the glass became saturated with leached components [23,24,71]. The SRL 202U samples (reacted without external radiation) developed a $\sim 130-\mu \mathrm{m}$-thick alteration layer after only 56 days (Table 20). Two individual layers were identified on SRL 202U glasses. An in situ layer of smectite was detected on the surfaces of all $202 \mathrm{U}$ samples terminated prior to 21 days. This layer was depleted in $\mathrm{Na}$ and $\mathrm{K}$, but enriched in $\mathrm{Mg}, \mathrm{Ti}, \mathrm{Fe}, \mathrm{Mn}$, and $\mathrm{Ca}$ relative to the unaltered glass. Major d-spacings measured from selected area diffraction patterns, combined with the compositional analyses, suggest that this layer may be composed of nontronite and saponite clays. After 35 days, the smectite was blanketed with a layer of high-albedo clay material. Both SEM/EDS and XRD data confirm that this layer is composed of the mineral illite. Small amounts of illite were also detected on the support wires for these samples, indicating that this phase precipitated from the solution in contact with the glass surface rather than forming in situ.

Measured SRL 202U glass alteration layer thicknesses from the present study samples are comparable to those of SRL 202U layer measurements published by Ebert and Bates [45] for the first 21 days. The layer measurements from the 35- and 56-day samples from the present study are, however, approximately two-fold higher than those from the 56-day samples of Ebert and Bates. When the illite layer is included (illite was not observed by Ebert and Bates), the reaction layer in the present study is thicker by approximately three-fold. These differences probably arise due to variations in the kinetics of alteration phase development in the two tests, with more rapid phase development leading to the thicker layers. The differences in the two studies thus underscore the potential for significant variations in reaction progress for vapor hydration tests run under similar conditions. These differences also reinforce the need to examine large numbers of samples when using the vapor hydration test.

In the present study, the development of alteration layers in the irradiated SRL 202A glasses is considerably more complex than that of the SRL 202U glasses. The thin films of water in contact with the glass wafers in the irradiated tests with SRL 202A glass turned out to be so corrosive that the samples had begun to react completely through their $0.7-$ to $0.9-\mathrm{mm}$ thicknesses along preferred zones in as little as 14 days. This results suggests a glass reaction rate that locally exceeded $30 \mu \mathrm{m} /$ day. This alteration layer progressed into the samples along a nonuniform front along preferential fracture zones that completely enveloped rounded and apparently unaltered glass cores (Figs. 40c and 41c). In some regions of the sample the remnant cores themselves remained intact, while in others they were highly fractured but otherwise appeared unaltered. These latter fractures appear to have formed from post-test residual stresses in the glass samples.

It is not known for certain whether the fracturing of the glass cores in the irradiated SRL 202A glass is related to (1) release of thermal stresses incorporated during sample casting, (2) stresses induced by water diffusion into the glass, or (3) radiation damage to the glass. Radiation damage appears to offer the least plausible explanation because fracture damage resulting from radiolytic processes would be expected to be uniformly distributed throughout the glass. The $\sim 10^{4} \mathrm{~Gy}$ of cumulative gamma dose that these samples received is also two to three orders of magnitude less than that required to cause structural damage to the glass $[72,73]$. If the fracturing resulted from thermal stresses, then glasses reacted without radiation would be expected to fracture in a similar manner as those exposed to radiation. However, only minor fracturing was observed 
from tests with the nonradioactive glass, with these cracks being isolated along the sample monolith edges. It thus appears that water diffusion-related stresses may be responsible for these fractures. Additional studies are warranted to verify this conclusion.

After 35 to 56 days, the remnant glass cores in the irradiated $202 \mathrm{~A}$ tests were completely replaced by a microcrystalline, silicon- and iron-rich material that is mottled- or bandedtextured (Figs. 40e and 45a). Fracturing normal to the banded layers often revealed differential parting zones, exhibited as alternating ridge and valley structures, between the individual layers. In some locations, the differential parting layers displayed preferred cleavage fractures that propagated into the sample between the individual banded layers. By analogy with geologic systems, these parting layers are most likely caused by deposition of thin layers of oriented clay between the banded iron- and silicon-rich material. SEM/EDS analyses, however, have not revealed any compositional differences between the layers. Thus, if the clays are present, they may be in layers that are too thin to be detected by SEM/EDS analysis.

The envelopment of these cores by several other alteration layers, the banded texture, and the smooth-to-botryoidal outer surfaces of the banded zones suggest that the iron- and silicon-rich bands developed as precipitated material inside a cavity or fracture within the glass. Alternatively, these layers may have formed as a partially recrystallized gel layer, which restructured after more soluble components had been leached away. . If the layer did originate through restructuring, it still appears that the smooth-to-botryoidal innermost band was deposited from solution within an open space. Both the banded- and mottled-textured zones rich in $\mathrm{Fe}$ and $\mathrm{Si}$ probably developed as the last remnants of the glass cores had been altered. As such, these samples can provide important insights into the behavior of the alteration layers as the influence of the glass dissolution products on solution compositions is eliminated.

The thicknesses measured for clay alteration layers formed in gamma-irradiated $(\sim 35 \mathrm{~Gy} / \mathrm{h})$ versus nonirradiated tests have been plotted for SRL 202 glasses reacted in the vapor hydration tests (Fig. 91, Table 19). These layer thicknesses were measured during SEM/EDS analysis of cross-sectioned samples and exclude the measurements of isolated surface precipitates and fractured regions of the glass. A comparison of the plotted trends indicates that the layer development rapidly diverges between the two sets of data after 7 days. An averaged increase in cumulative reaction layer thickness of $\sim 10$-fold is noted for the 7- to 35-day irradiated SRL 202A samples relative to the nonirradiated tests. The differences in cumulative layer thickness are greatest for the 21 -day tests $(\sim 15$ fold) and smallest for the 35 day tests ( $\sim$ fold). The final irradiated SRL 202A layer thickness for the 56-day tests represents a minimum value, since the glass disks had reacted completely through their $\sim 1-\mathrm{mm}$ thicknesses somewhere between 35 and 56 days of testing. The final "long-term" reaction rates determined from the cumulative 35-day tests suggest an alteration rate of $\sim 12.5 \mu \mathrm{m} /$ day for the irradiated tests and $1.6 \mu \mathrm{m} /$ day for the nonirradiated tests (Table 19).

The increase in alteration layer thickness is attributed to the effects of radiation exposure, with accelerated alteration rates resulting from the formation and/or condensation of radiolytic products such as nitrogen acids, carboxylic acids, and water dissociation radicals in the thin films of water contacting the samples at the high $\mathrm{S} / \mathrm{V}$ conditions. These products result in a fluid that is corrosive toward the glass, leading to the enhanced rates of ion exchange between the glass and the thin film of water, and a resultant increase in the rate of alteration layer formation.

The efficiency of $\mathrm{HNO}_{3}+\mathrm{HNO}_{2}$ acid production in independently evoking these changes has yet to be experimentally determined. Nitrogen acid species production rates from the $200^{\circ} \mathrm{C}$ gamma irradiation tests (Section III.A.1) indicate that $\sim 0.1 \mu$ moles of acid species will be 


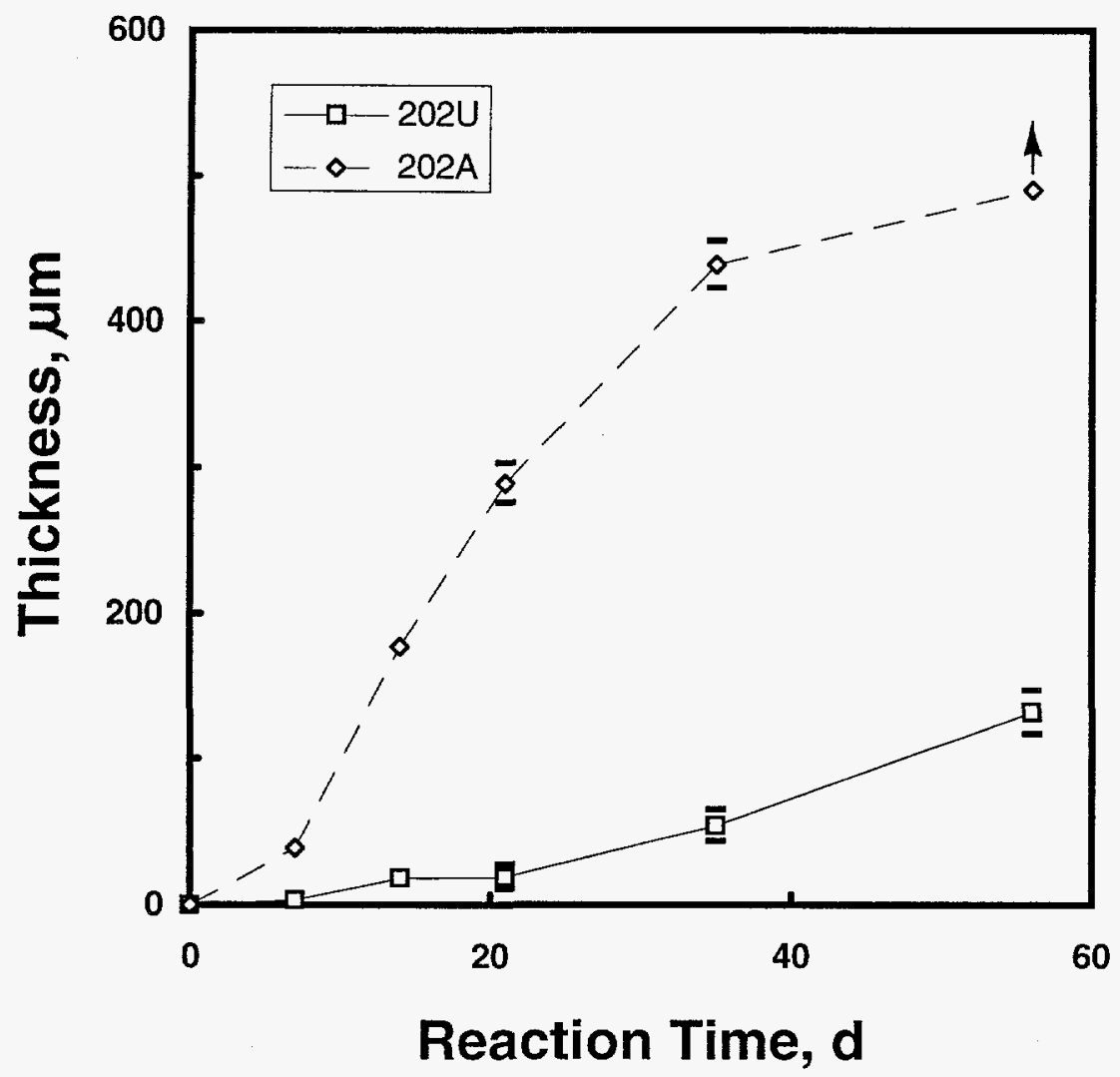

Fig. 91. Average Alteration Layer Thickness Measured from Cross-Sectioned SRL 202A and SRL 202U Glass Samples Reacted in Vapor Hydration Tests at $200^{\circ} \mathrm{C}$. The SRL $202 \mathrm{~A}$ glass was exposed to dose rate of $\sim 35 \mathrm{~Gy} / \mathrm{h}$, while the SRL $202 \mathrm{U}$ glass was reacted without any external radiation exposure. Brackets indicate layer measurements \pm 1 standard deviation for 20 cross-section measurements on each sample. Brackets have been omitted where the standard deviation is less that the height of the plotted symbol. The final 56-day SRL 202A reaction layer thickness is plotted as a minimum value because the sample monolith had reacted completely through its $\sim 1000-\mu \mathrm{m}$ thickness somewhere between 35 and 56 days.

generated following 56 days of gamma radiation exposure under the present test conditions. Such a quantity of acid species would result in a pH of $\sim 3$ for the $\sim 0.1 \mathrm{~mL}$ of liquid water expected to be present in the vapor hydration test vessels (ignoring the effects of the bicarbonate and glass buffering systems). The bicarbonate system is expected to be overwhelmed by the acids produced in the vessels due to the limited quantity of $\mathrm{CO}_{2}$ initially present in the vessel atmosphere $(0.033 \%$ $\mathrm{CO}_{2}$ ), solubility limits of $\mathrm{H}_{2} \mathrm{CO}_{3}$ in water, and the decreased solubility of $\mathrm{CO}_{2}$ in water as a function of increasing temperature and decreasing $\mathrm{pH}$. It is important to note that while the buffering capacity of the bicarbonate in the sealed test vessels may be constrained by the limited volume of air initially present, such a constraint will not exist in the open-air system of an unsaturated nuclear waste repository, thus the effect of bicarbonate buffering on radiolytic acid formation may be more important under actual repository conditions than the presents tests would indicate. 


\section{Surface Precipitate Formation on SRL 202 Glass}

Surface precipitates that developed on both the irradiated and nonirradiated SRL 202 glasses were characterized by the formation of a variety of zeolite, clay, Ca-Si, and alkali metal or alkaline earth uranyl silicate phases. A paragenetic sequence has been devised for alteration phases forming on both the SRL 202U and SRL 202A glass surfaces exposed to $200^{\circ} \mathrm{C}$ vapor hydration environments (Fig. 92). A comparison of these sequences indicates what effect the irradiation of the test vessel (including solution composition changes) has on the formation of alteration phases. This sequence has been developed through extensive optical microscopy, SEM/EDS, AEM, and XRD examinations of glass surfaces that were altered between 7 and 56 days. The trends displayed in Fig. 92 suggest the following simplified temporal paths for the major cations leached from the nonirradiated SRL 202U glasses (major phases in bold letters):

$\mathrm{Na}$ - Incorporated early into Na-rich chabazite (var. herschelite), and later into analcime. Of lesser importance is phillipsite.

$\mathrm{K}$ - Incorporated early into Na-rich chabazite, followed by phillipsite, and in the later stages is distributed between adularia, illite, and weeksite.

$\mathrm{Ca}$ - Path is dominated by unidentified Ca-Si rosette. Of lesser importance in later tests is tobermorite.

$\mathrm{U}$ - Incorporated early into unidentified Na-K-Ca-U-Si webbed-shaped phases, followed by the formation of a discontinuous U-K-Si surface layer, and finally into weeksite.

These results are similar to those of the SRL 202A glasses that were exposed to an external gamma source at a dose rate of $\sim 35 \mathrm{~Gy} / \mathrm{h}$, where the following cation pathways have been identified:

$\mathrm{Na}$ - Path is dominated by unidentified analcime. Of lesser importance is early phillipsite and latter sodium-weeksite and albite.

$\mathrm{K}$ - Incorporated early into illite and phillipsite and later into adularia, weeksite, and boltwoodite.

$\mathrm{Ca}$ - Incorporated into $\mathrm{Ca}$-Si rosette, tobermorite, and haiweeite.

$\mathrm{U}$ - Incorporated into a variety of phases, including weeksite, haiweeite, sodium-weeksite, and boltwoodite.

Ebert and Bates [45] had identified the formation of analcime, gyrolite $\left[\mathrm{Ca}_{4}\left(\mathrm{Si}_{6} \mathrm{O}_{16}\right)(\mathrm{OH})_{2} \cdot 3 \mathrm{H}_{2} \mathrm{O}\right]$, orthoclase $\left(\mathrm{KAlSi}_{3} \mathrm{O}_{8}\right)$, hydroxy apatite, and $\mathrm{LiPO}_{4}$ and possibly tobermorite, and weeksite on SRL $202 \mathrm{U}$ glasses reacted in a $200^{\circ} \mathrm{C}$ saturated vapor environment for periods of up $\sim 70$ days; and iron-rich clays, weeksite, and clinoptilolite $\left[(\mathrm{Na}, \mathrm{K})_{6}\left(\mathrm{Al}_{6} \mathrm{Si}_{30} \mathrm{O}_{72}\right) \cdot 20 \mathrm{H}_{2} \mathrm{O}\right]$ on SRL $202 \mathrm{U}$ glasses reacted in $90^{\circ} \mathrm{C}$ Product Consistency Tests (PCTs) in EJ-13 solution reacted for periods of up to one year. Some of these phases were the same as the ones from the present investigation, while the following phases differed from those of the present study:

(1) The gyrolite phase identified by Ebert and Bates may be similar to the unidentified $\mathrm{Ca}-\mathrm{Si}$ rosettes noted in the present investigation. Attempts to match the XRD and SAED patterns of the Ca-Si rosettes from the present study with gyrolite and other Ca-Si phases were not successful; and hence, 


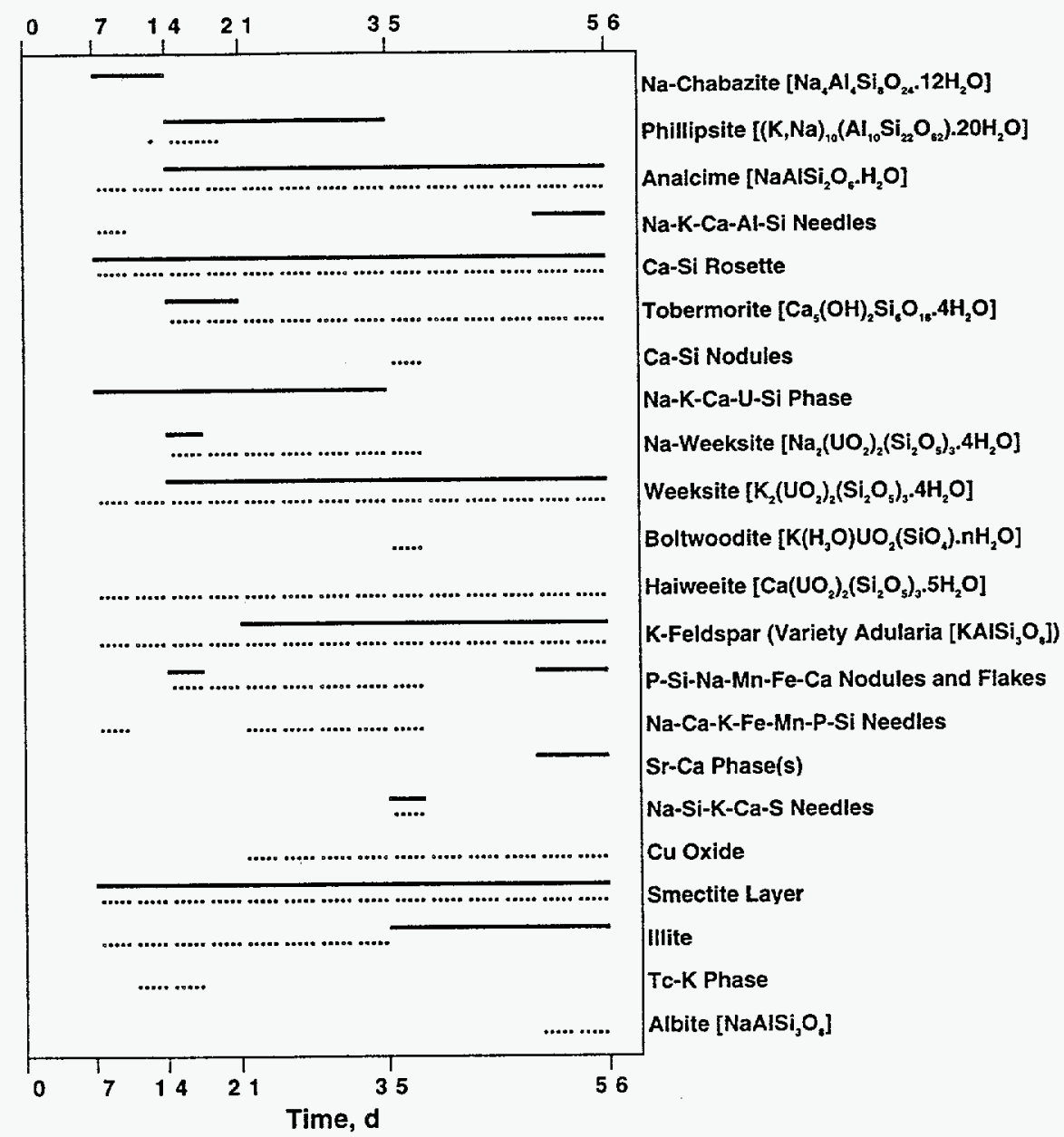

Fig. 92. Alteration Mineral Paragenetic Sequence Identified for SRL 202 Glasses Reacted in Vapor Hydration Tests at $200^{\circ} \mathrm{C}$. Dotted lines represent SRL 202A glass samples that were exposed to dose rate of $\sim 35 \mathrm{~Gy} / \mathrm{h}$, while solid lines represent SRL 202U samples reacted without any external radiation.

this phase remains unidentified. Ebert and Bates reported that the gyrolite present on their samples was identified by SEM/EDS and XRD analysis, but neither set of data was included in their paper. The reaction time for this sample was also not reported. In a later paper, Ebert et al. [74] report that XRD patterns obtained from Ca-Si phases reacted on West Valley Project glass did not match the JCPDS data for gyrolite. The XRD patterns of the $\mathrm{Ca}-\mathrm{Si}$ phase from the present study (Table 20) match well with the patterns exhibited by Ebert et al.'s [74] West Valley Ca-Si (C1) phase.

Byers et al. [44] also have reported the presence of gyrolite in their study of synthetic basalt glass reacted in a vapor saturated environment at $240^{\circ} \mathrm{C}$ for an unspecified period of time. This phase exhibits morphological and SEM/EDS characteristics that are similar to the $\mathrm{Ca}-\mathrm{Si}$ rosettes in the present study; however, the XRD pattern of the Byers et al. gyrolite phase is distinctly different from the Ca-Si rosette identified in the present study (Table 20). Caurel et al. [75] also report the presence of gyrolite and/or okenite 
$\left[\mathrm{CaO}\left(\mathrm{SiO}_{2}\right)_{2} \cdot 2 \mathrm{H}_{2} \mathrm{O}\right]$ forming on French $\mathrm{R} 7 \mathrm{~T} 7$ glass altered in a saturated environment at $250^{\circ} \mathrm{C}$. This phase was similar in morphology to both gyrolite identified by Byers et al. and the unidentified $\mathrm{Ca}-\mathrm{Si}$ rosette noted in the present study.

(2) Ebert and Bates [45] identified the potassium-feldspar phase occurring on their samples as orthoclase. With the present study, this phase has been identified as the potassium-feldspar adularia. Both phases are similar in composition and mineral structure, thus distinguishing them based on SEM/EDS and XRD data is difficult. The classification of adularia in the present study is based on the typical environment in which this mineral is formed. Adularia commonly is deposited in relatively low-temperature $\left(<350^{\circ} \mathrm{C}\right)$ geologic environments, such as hydrothermal systems and diagenetic-sedimentary environments [49]. Orthoclase, alternatively, is commonly associated with much higher temperature $\left(\geq 800^{\circ} \mathrm{C}\right)$ igneous systems. Based on these data, the potassium-feldspar phase identified in both studies is probably adularia.

(3) Ebert and Bates reported the presence of hydroxy-apatite $\left[\mathrm{Ca}_{5}\left(\mathrm{PO}_{4}\right)_{3} \mathrm{OH}\right]$ and $\mathrm{Li}_{3} \mathrm{PO}_{4}$ but did not note the presence of either phase in their mineral summary (Table II) [45]. Since the concentration of phosphorus is negligible in SRL $202 \mathrm{U}$ glasses (T'able 4), it is unlikely that either phosphorus-bearing phase would be developed to any great extent on the altered surfaces of SRL 202U glasses. No phosphorus-bearing phases were identified in the present study. The detection of lithium was not possible in the present study due to limitations of the EDS detector.

(4) Ebert and Bates [45] reported the presence clinoptilolite on altered glass samples from $90^{\circ} \mathrm{C}$ PCTs. Clinoptilolite is a common alteration product for volcanic glass deposited in saline-alkaline lakes and basalt extruded in ocean settings [76]. Clinoptilolite was not observed on any altered samples from the present study. This absence may be due to the $200^{\circ} \mathrm{C}$ test temperatures used in the vapor hydration tests. In a summarization of the temperature conditions under which clinoptilolite has been observed, Gottardi and Galli [48] indicate a maximum temperature stability range for noted occurrences at $150^{\circ} \mathrm{C}$.

(5) Additional surface precipitate phases listed in Fig. 92 were identified as reaction products in the present tests, but were not noted in the Ebert and Bates study [45].

Discrete boron-bearing phases were not observed on any of the altered samples of SRL 202 glasses examined in this study. The boron released from the glass is probably deposited within the salt crusts that form as water present on the sample surfaces is evaporated in air at the conclusion of the testing period.

Faster glass alteration rates are observed in the irradiated SRL 202A vapor-rich environment relative to the nonirradiated SRL $202 \mathrm{U}$ glass tests, as evidenced by the greater abundance of secondary precipitates on the sample surfaces of the former (Fig. 93). Despite the advanced degree of alteration of the glass monoliths in the irradiated SRL 202A tests, most of the phases are similar to those noted during the examination of the nonirradiated SRL 202U samples. The paragenetic sequences for both test conditions (Fig. 92) indicate that secondary mineral formation has been accelerated in the irradiated tests relative to the nonirradiated tests. For example, analcime, weeksite, and adularia were detected on earlier tests of the SRL 202A relative 
(a)

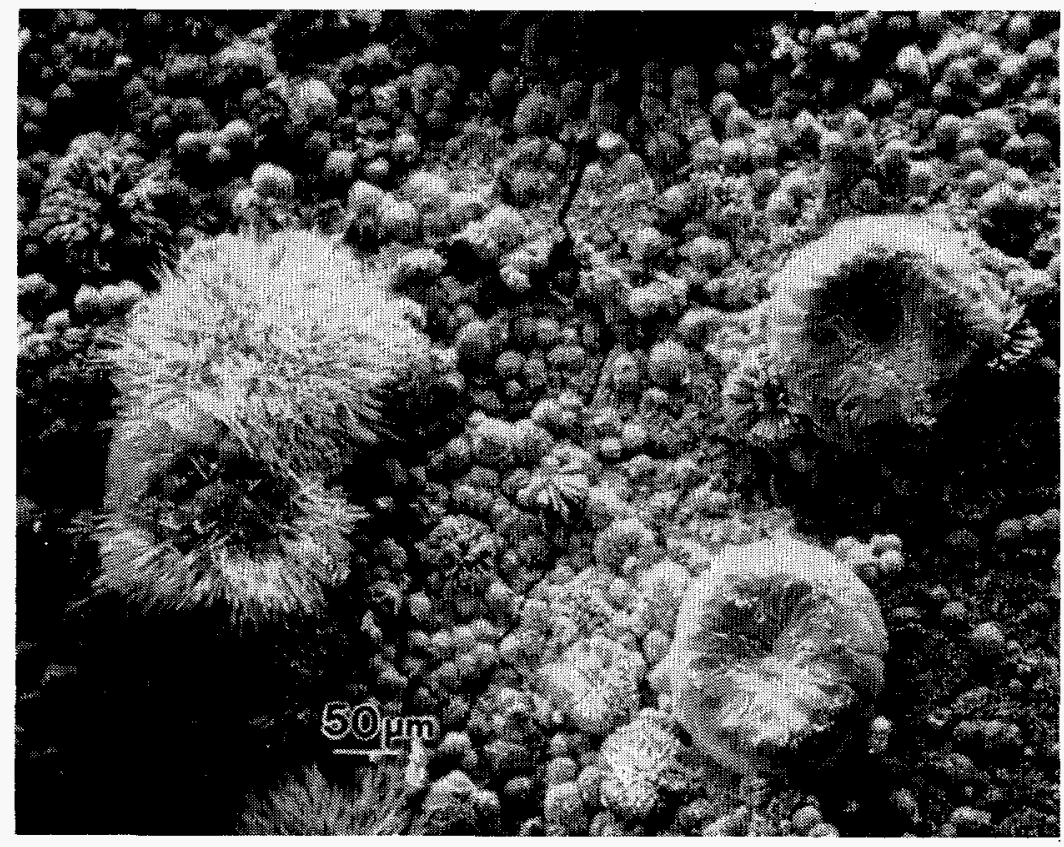

(b)

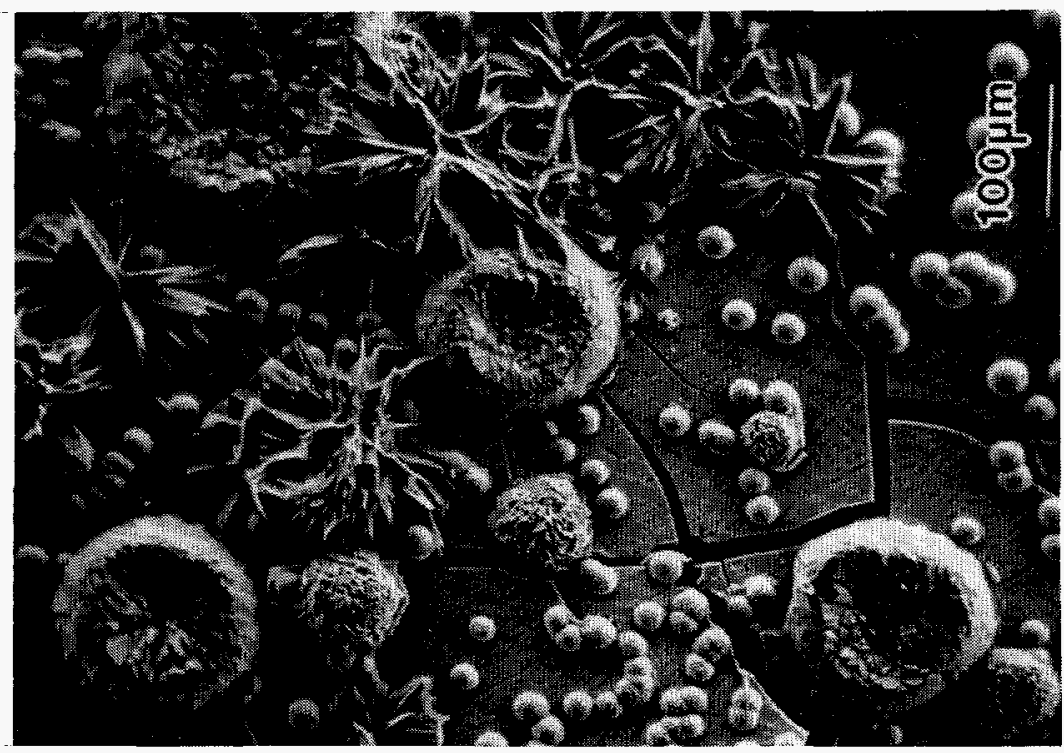

Fig. 93. SEM Photomicrographs of SRL 202 Glasses Reacted in the Vapor Hydration Tests for 35 Days at $200^{\circ} \mathrm{C}$. (a) The surface of the SRL 202A glass that was reacted while exposed to dose rate of $\sim 35 \mathrm{~Gy} / \mathrm{h}$. (b) The surface of the SRL 202U glass reacted without any external radiation exposure. Note that the SRL 202A glass has developed a thick alteration cover with a relatively dense overgrowth of secondary precipitates, while the SRL 202U glass has developed a thin alteration cover with a sparse overgrowth of precipitates. Both photomicrographs at $100 \mathrm{X}$ with scale bars equivalent to $100 \mu \mathrm{m}$. 
to the SRL 202U glasses. Transient phases such as Na-rich chabazite (herschelite) and phillipsite, which formed early in the SRL 202U tests, were not detected on the SRL 202A samples, although it is possible that these phases first precipitated and then dissolved prior to the collection of the first samples at 7 days. A number of secondary precipitates were also detected on the longer-term SRL 202A samples, but not on the SRL 202U samples. The most notable of these phases are sodiumweeksite, haiweeite, and albite. It is thought that these phases would eventually have formed on the SRL 202U glasses if the tests had been allowed to continue for longer time periods.

The similarity of alteration phases noted between the nonirradiated and irradiated tests suggests that the solution environment (especially $\mathrm{pH}$ ) in contact with the surfaces of the samples has remained within the stability field of the various alteration phases, despite the radiolytic production of $\mathrm{HNO}_{3}$ in the former tests. These trends also indicate that the glass monoliths will react at a faster rate in the irradiated tests, with the increase in associated glass reaction products apparently neutralizing whatever radiolytic products that have formed. Although accelerated glass reaction is not desirable in terms of glass performance, the apparent neutralization of radiolytic acids by dissolving glass components may stabilize the radionuclide-bearing alteration phases that have formed on the altered glass surface.

Electrons accelerated to a $30 \mathrm{kV}$ voltage were also used during SEM/EDS analysis to excite the $\mathrm{L}$ shell electrons of the transuranic isotopes ${ }^{241} \mathrm{Am},{ }^{239} \mathrm{Pu}$, and ${ }^{237} \mathrm{~Np}$ and thus allowing for the detection of alteration phases that may contain these elements. Transuranic elements were not identified in any of the surface precipitate phases present on the altered glass surfaces. The inability to detect these elements in the surface precipitates suggests that the transuranics were largely retained in the altered layer at concentrations that were below the detection limit of the SEM/EDS system rather than being concentrated in discrete precipitate phases on the altered sample surfaces.

Release of technetium from a nuclear waste repository is of great concern due to the relatively long half-life of the ${ }^{99} \mathrm{Tc}$ isotope, its high-energy beta decay, and its common complexation of technetium as the very soluble pertechnetate anion $\left(\mathrm{TcO}_{4}{ }^{-}\right)$. Because of its anionic state, the pertechnetate ion will not sorb onto the negatively charged surfaces of clay and zeolite minerals present in the potential Yucca Mountain repository horizon, and may therefore be readily mobilized from the waste package environment. Very fine-grained Tc-K-Ca solids were identified on the 14-day SRL 202A sample, occurring as submicron particles deposited on the Pt-Rh support wire. No deposits of this type were identified directly on the altered glass surface. The Pt-Rh wire may have acted as a catalyst toward the formation of these deposits containing technetium. The high ionic strength of the leachate solution on the sample surface may also possibly contribute to the precipitation of the technetium by balancing the net negative charge of the pertechnetate with excess cations in the solution.

Literature reviews indicate several potential mineral phases that are consistent with the SEM/EDS signal received for the Tc-K-Ca solids identified on the 14-day SRL 202A sample from the vapor hydration tests. These phases include $\mathrm{KTcO}_{4}, \mathrm{~K}_{2} \mathrm{Tc}(\mathrm{OH})_{9}$, and $\mathrm{K}_{2} \mathrm{TcF}_{6}$. The first two phases represent solids of the oxidized $\mathrm{Tc}^{7+}$, while the later represents the reduced $\mathrm{Tc}^{4+}$ state. The presence of phases with the higher oxidation state is favored, due to the observed close spatial association of uranyl silicate and ferric iron phases and the expected production of $\mathrm{H}_{2} \mathrm{O}_{2}, \mathrm{O}_{2}{ }^{-}$, and - $\mathrm{OH}$ in the radiolytic environment that solutions in these tests were exposed to [2,54].

Based on its electron configuration and valency, technetium may be expected to behave in a similar manner as rhenium. Because rhenium exists naturally in two isotopic forms (stable ${ }^{185} \mathrm{Re}$ and the long-lived radioactive ${ }^{187} \mathrm{Re}$ ), its behavior in natural systems may be useful in predicting the behavior of technetium. Literature surveys indicate that rhenium is commonly associated with naturally occurring deposits of platinum group elements (PGE: Pt, Pd, Rh, Ir, Os, 
$\mathrm{Ru})$, columbite-tantalite $\left[(\mathrm{Fe}, \mathrm{Mn}) \mathrm{NbO}_{6}-(\mathrm{Fe}, \mathrm{Mn}) \mathrm{Ta}_{2} \mathrm{O}_{6}\right]$, molybdenite $\left[\mathrm{MoS}_{2}\right]$, gadolinite $\left[(\mathrm{Ce}, \mathrm{La}, \mathrm{Y})_{2} \mathrm{FeBe}_{2} \mathrm{Si}_{2} \mathrm{O}_{10}\right]$, and a variety of copper sulfides. These relationships suggest that a variety of $\mathrm{PGE}, \mathrm{Nb}$, Ta, Mo, and $\mathrm{Cu}$ phases may act as sorbents for technetium. Supporting evidence for this correlation is also found in the association of technetium with the Pt-Rh support wires in this study, and in the occurrence of metallic alloy inclusions of $\mathrm{Tc}, \mathrm{Mo}, \mathrm{Ru}, \mathrm{Pd}$, and $\mathrm{Rh}$ that are found in spent nuclear fuel [77].

\section{Reaction Layer Development on SRL 165 Glass}

The vapor hydration test with SRL $165 \mathrm{U}$ samples reacted without external radiation [45] exposure developed an alteration layer that was $\sim 170-\mu \mathrm{m}$ thick after 56 days (Table 19). The pattern of the reaction layer growth for the SRL 165A glasses was initially rather subdued for the first 21 days but increased greatly thereafter (Fig. 94). Reaction layer development increased from an initial $\sim 1.4 \mu \mathrm{m} /$ day for the first 21 days to $\sim 3.0 \mu \mathrm{m} /$ day for the $35-$ and 56 -day tests. The initial low rate of layer development differed from patterns observed with the SRL 202A (Fig. 91) and SRL 131A (Fig. 96) glasses, where increases in accelerated reaction rates occurred much earlier.

Of great interest with regard to the performance of nuclear waste is the cause of the reaction rate increase observed between the 21 - and 35-day samples. Reaction rate increases have been previously attributed to the precipitation of certain mineral phases on the sample surfaces that lowered the effective concentration of elements in the solution contacting both the glass and the crystal phases [60]. These phases thus act as a "sink" for elements, thus allowing the elements to be released from the glass at a higher rate than they would if solution concentrations had continued to increase. Since the solubility of a particular element in solution will depend on the equilibrium constant of particular mineral phases, these phases may indirectly control the rate of glass reaction. A systematic approach to identify which particular mineral phase(s) is responsible for these accelerated reactions has yet to be conducted. Bates et al. [60] had noted an increase in the rate of glass alteration in 120 to $240^{\circ} \mathrm{C}$ vapor hydration tests that correlated with first occurrence of tobermorite. With the present tests, the rate change in reaction layer development observed between 21 and 35 days (Fig. 94) did not correlate with any major change in alteration layer paragenesis (Fig. 95). This lack of correlation suggests that the formation of a single specific mineral phase may not be the major determining factor in the rate of layer development, although this hypothesis is certainly worthy of additional study. Possibly, $\mathrm{pH}$ changes associated with the build up of radiolysis products have exerted a stronger influence on reaction layer development under the present test conditions.

A comparison of reaction layer development between the SRL 202A and SRL 165A glasses will reveal the relative durabilities of the two glasses, with both glass compositions being reacted in vapor hydration tests at $200^{\circ} \mathrm{C}$. The cumulative alteration layer development for the SRL 202A glasses was $\sim 13 \mu \mathrm{m} /$ day after both 21 and 35 days of reaction (Table 19). The superior durability of the SRL $165 \mathrm{~A}$ glass is revealed in reaction layer rates of development that were on the order of $\sim 3 \mu \mathrm{m} /$ day after both the 35- and 56-day tests. The rate of layer formation of the SRL 165A glass is thus reduced four-fold relative to the rate for the SRL 202A glasses.

Gamma-irradiated ( $-35 \mathrm{~Gy} / \mathrm{h})$ versus nonirradiated clay alteration layer thicknesses have been plotted for SRL 165A (this study) and SRL 165U [45], both glass types being reacted in a saturated steam-air environment at $200^{\circ} \mathrm{C}$ (Fig. 94). Alteration layer thicknesses for the irradiated SRL 165A glasses were initially similar to, or only slightly greater than, those of the unirradiated SRL $165 \mathrm{U}$ samples during the first 21 days. Thereafter, the rate of reaction layer development was increased significantly for the irradiated samples. After 35 days, the SRL 165A reaction layers were four-fold thicker than those reported for unirradiated glasses, while 56-day layers displayed a five-fold increase in layer thickness. This increase in layer development due to radiation exposure is significantly less than that previously reported for SRL 202 glass reacted 


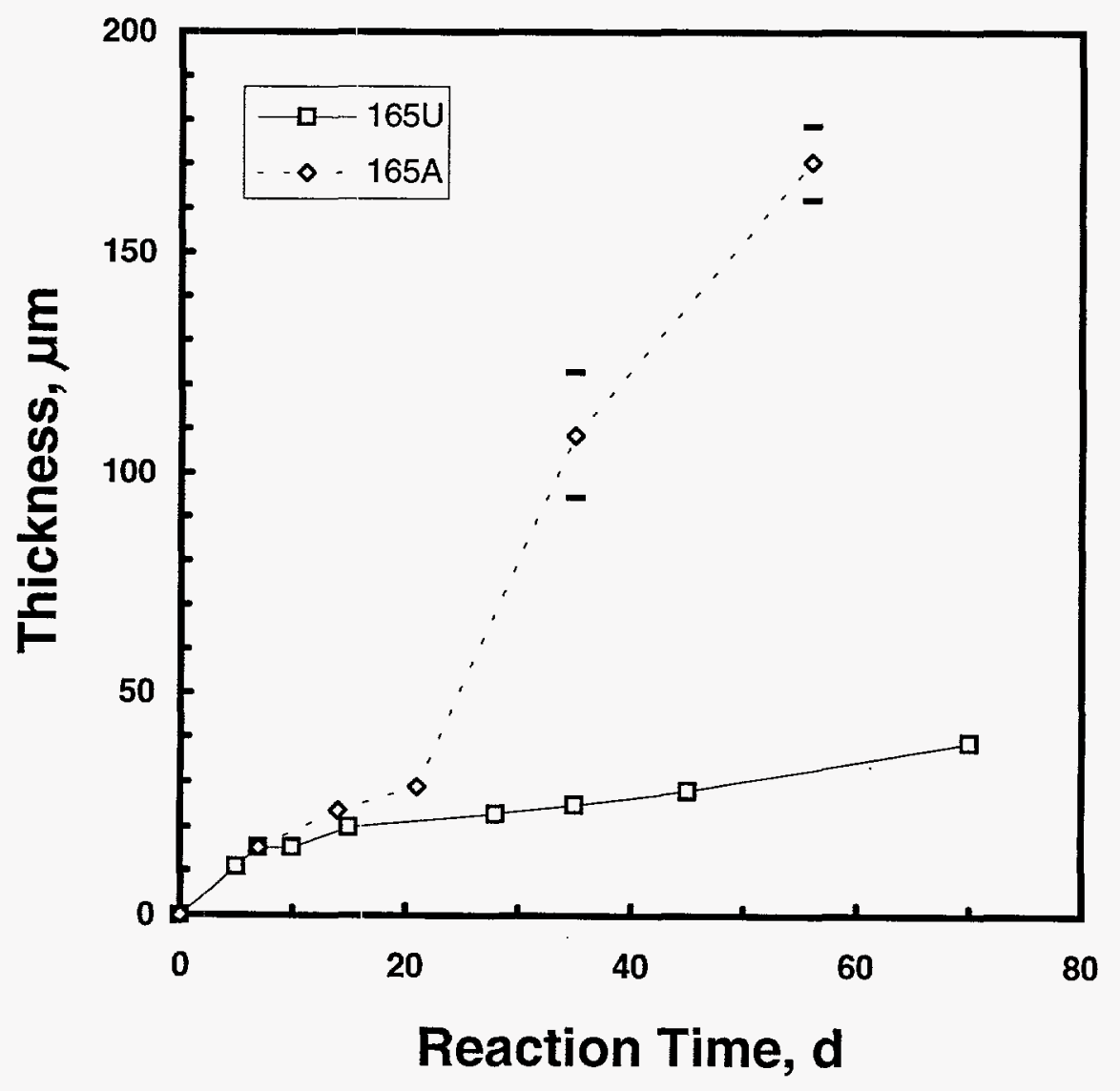

Fig. 94. Average Alteration Layer Thickness Measured from Cross-Sectioned SRL 165A and SRL 165U Glass Samples Reacted in Vapor Hydration Tests at $200^{\circ} \mathrm{C}$. The SRL 165A glass was exposed to dose rate of $\sim 35 \mathrm{~Gy} / \mathrm{h}$ while the SRL $165 \mathrm{U}$ glass was reacted without any external radiation exposure. The SRL $165 \mathrm{U}$ data are derived from the Ebert et al. [45]. Brackets indicate layer measurements \pm 1 standard deviation for 20 cross-section measurements on each sample. Brackets have been omitted where the standard deviation is less that the height of the plotted symbol. 


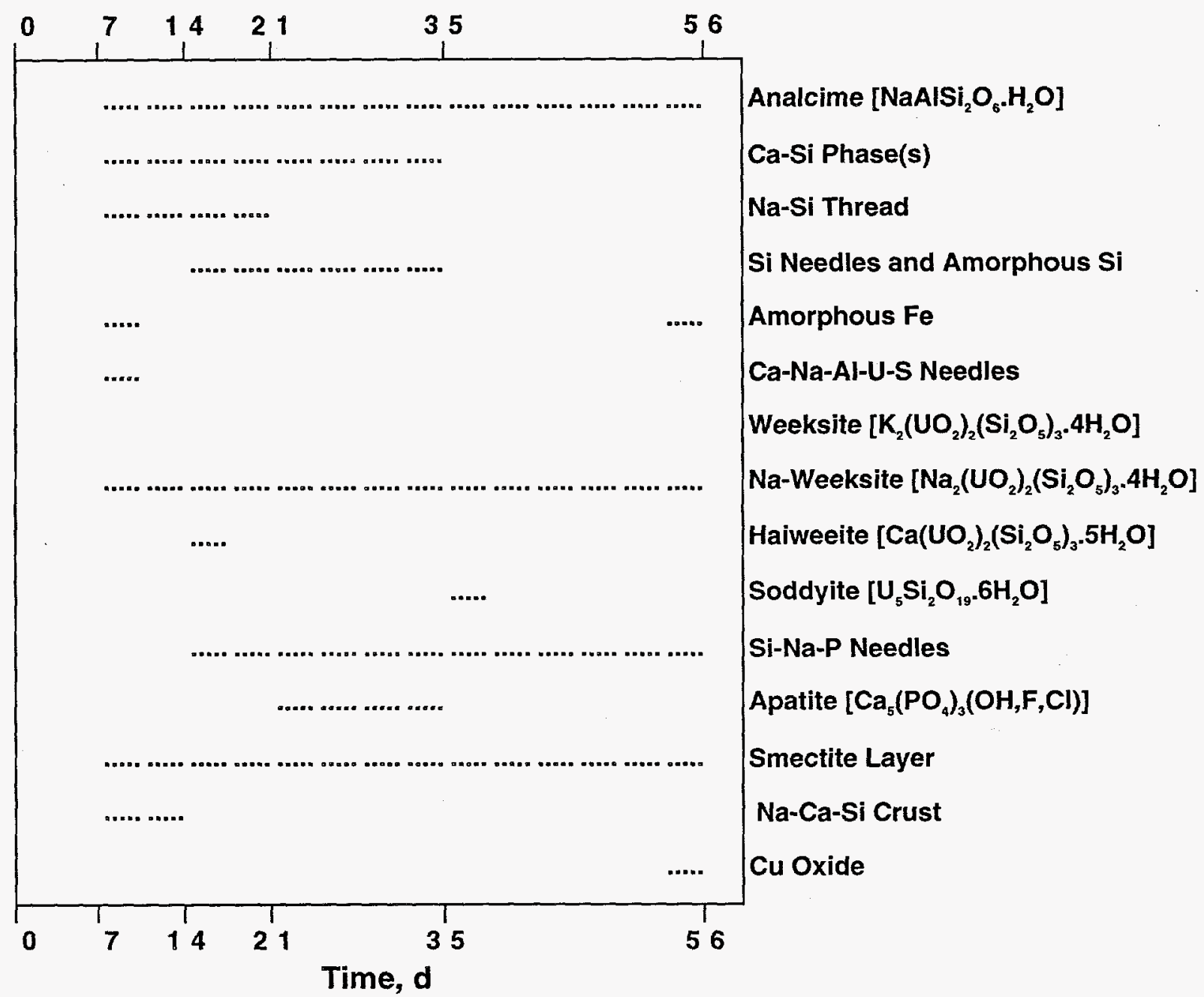

Fig. 95. Alteration Mineral Paragenetic Sequence Identified for SRL 165 Glasses Reacted in Vapor Hydration Tests at $200^{\circ} \mathrm{C}$ While Exposed to a Gamma Dose Rate of $\sim 35 \mathrm{~Gy} / \mathrm{h}$ 
under similar test conditions ( 10-fold increase). As with the SRL 202 glass tests, these accelerated alteration rates appear to have resulted from the formation and/or condensation of corrosive radiolytic products such as nitrogen acids, carboxylic acids, and water dissociation radicals in the thin films of water contacting the samples. The increased reaction layer thickness results from increased ion exchange rates between radiolytic acids and glass components.

Some hummocky surface textures noted on the SRL 165A glasses during vapor alteration may be a result of cavity formation within the glass. An examination of the crosssectioned reaction layers indicates some separation of the clay layer from the unaltered glass core, but it is also possible that this separation may have occurred when the samples were allowed to dry after the tests were terminated, or when the samples were immersed in the epoxy mounting medium used in forming the cross-sectioned samples.

The composition and mineral texture of the altered clay layer are similar to those previously described for the SRL 202 glasses, with results being consistent with the presence of smectite clays.

\section{Surface Precipitate Formation on SRL 165 Glass}

The mineral sequence developed on the SRL 165A samples was compared to results from tests of SRL $165 \mathrm{U}$ glasses that were also reacted at $200^{\circ} \mathrm{C}$ in vapor hydration tests [45]. With the SRL 165A glasses from the present study, surface precipitate minerals were characterized by the formation of analcime, several uraniferous phases (sodium-weeksite, haiweeite, and soddyite), apatite, and a number of unidentified phases, including the $\mathrm{Ca}-\mathrm{Si}$ rosettes that were common on the SRL 202 glasses (Fig. 95). Of interest are the occurrence of the amorphous-to-fibrous silicon phases and the formation of soddyite. The presence of these relatively Si-rich phases indicates that the ratio between the activity of silicon species and the activity of alkali and alkaline earths must have increased in the solution contacting the SRL 165A glass relative to that of the SRL 131A and SRL 202A glasses. Such a pattern is expected given the higher silica concentration of the SRL, 165A glass relative to the other glass compositions (Table 4).

The mineralogic sequence developed on the SRL 165A glasses was similar to that of published results from previous studies with SRL $165 \mathrm{U}$ glasses. Ebert and Bates [45] had identified analcime, gyrolite, sodium-weeksite, and possibly tobermorite in their examination of SRL $165 \mathrm{U}$ glasses reacted in vapor hydration tests at $200^{\circ} \mathrm{C}$ for periods of up to $\sim 70$ days. Analcime, the $\mathrm{Ca}-\mathrm{Si}$ phase resembling the gyrolite phase of Ebert and Bates, and sodium-weeksite were also identified in the present study. No phase resembling tobermorite was identified from the present tests. A large number of additional phases listed in Fig. 95 were not noted in the Ebert and Bates study.

\section{Reaction Layer Development on SRL 131 Glass}

The SRL 131U samples reacted without external radiation in vapor hydration tests $\left(150^{\circ} \mathrm{C}\right.$ ) developed an alteration layer that was $\sim 75-\mu \mathrm{m}$ thick after 720 days (Table 19). Reaction layer development steadily declined $0.9 \mu \mathrm{m}$ /day for the first three days to a cumulative value of $0.1 \mu \mathrm{m} /$ day at 180 days. The layer thickness development (averaged periodic and cumulative) remained at $\sim 0.1 \mu \mathrm{m} /$ day for the remainder of the time periods tested.

Alteration layer thicknesses for gamma-irradiated $(\sim 35 \mathrm{~Gy} / \mathrm{h})$ versus nonirradiated tests have been plotted for SRL 131 glasses reacted in a saturated steam-air environment at $150^{\circ} \mathrm{C}$. Alteration layer thicknesses for the irradiated SRL 131A glasses developed approximately four-fold faster that the corresponding nonirradiated SRL $131 \mathrm{U}$ glasses for time periods of up to 56 days 
(Fig. 96). This increase was less than that for SRL 202 glass ( 10 -fold increase) and similar to that of the SRL 165 glasses, although both the SRL 202 and SRL 165 glasses were reacted at higher temperatures $\left(200^{\circ} \mathrm{C}\right)$. As with the SRL 202 glass tests, these accelerated alteration rates in the irradiated tests resulted from the formation and/or condensation of corrosive radiolytic products such as nitrogen acids, carboxylic acids, and water dissociation radicals in the thin films of water contacting the samples.

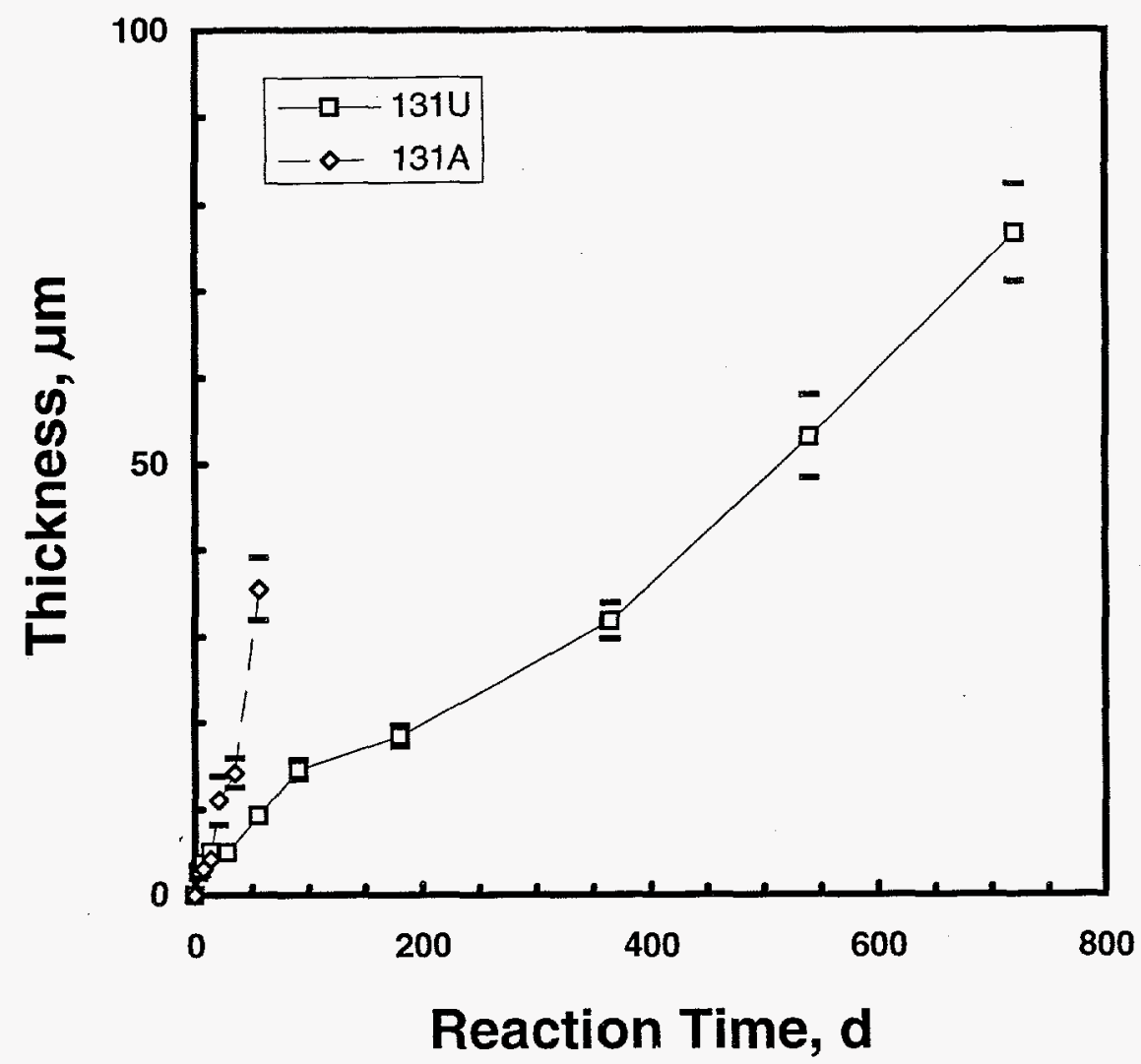

Fig. 96. Average Alteration Layer Thickness Measured from Cross-Sectioned SRL 131A and SRL 131U Glass Samples Reacted in Vapor Hydration Tests at $150^{\circ} \mathrm{C}$. The SRL 131A glass was exposed to gamma dose rate of $\sim 35 \mathrm{~Gy} / \mathrm{h}$, while the SRL $131 \mathrm{U}$ glass was reacted without any external radiation exposure. Brackets indicate layer measurements \pm 1 standard deviation for 20 cross-section measurements on each sample. Brackets have been omitted where the standard deviation is less that the height of the plotted symbol.

The results from alteration layer thicknesses measurements from 3- to 91-day samples in the present study have been compared to published layer measurements from Bates et al. [60] for SRL $131 \mathrm{U}$ glasses under a variety of temperatures (Fig. 97). Layer thickness measurements taken from the present $150^{\circ} \mathrm{C}$ samples are intermediate between the 120 and $202^{\circ} \mathrm{C}$ results obtained by Bates et al., although the data indicate a proportionally larger increase between 150 and $200^{\circ} \mathrm{C}$ as opposed to increase between 120 and $150^{\circ} \mathrm{C}$. These data may suggest that the Arrhenius relationship for the initial hydration process determined by Bates et al. may not be valid for long-term layer development. It is uncertain whether this patterns reflects a fundamental change in the reaction mechanism controlling the glass reactions or differences in the rate of alteration layer development in association with the first appearance of certain mineral phases. 


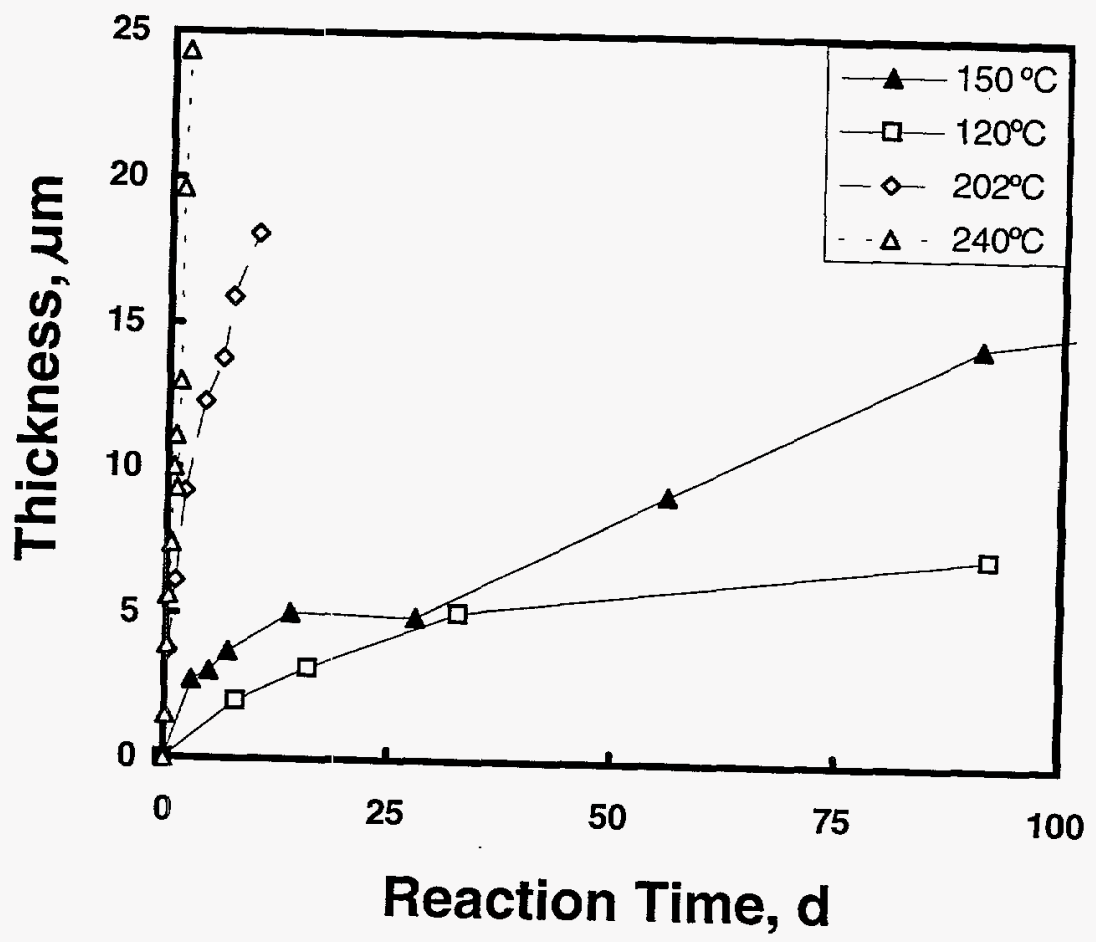

Fig. 97. Average Alteration Layer Thickness Measured from Cross-Sectioned SRL 131U Glass Samples Reacted in Vapor Hydration Tests as a Function of Reaction Time and Temperature. Tests at 120,202 , and $240^{\circ} \mathrm{C}$ are from the results of Bates et al. [60], while the $150^{\circ} \mathrm{C}$ results are from the present study.

Major d-spacings measured from selected area diffraction patterns, combined with the SEM/EDS and AEM/EDS compositional analyses, suggest that the material in the SRL 131 glasses is composed of nontronite and saponite clays. The AEM/EDS compositional analyses of the SRL 131U glass indicate that both the inner random and outer oriented smectite clay layers are depleted in $\mathrm{Na}$ and $\mathrm{K}$, while being enriched in $\mathrm{Al}, \mathrm{Mn}$, $\mathrm{Ti}$, and $\mathrm{Fe}$ relative to the unaltered glass (Fig. 98). Although it is difficult to fully interpret the trends in layer concentrations due to the semiquantitative nature of these analyses, consistent element variations developed over the course of several time periods may be useful in evaluating the glass reaction trends.

Nickel was depleted in the outer oriented layer for the first 14 days of testing relative to the inner layer, while nickel values were comparable between the two layers after 56 days, with both layers displaying as slight enrichment relative to the unaltered glass (Fig. 98a). The enrichment of nickel in the inner layer during the first 14 days is thought to reflect a residual process, where more soluble components are removed from the reacted glass layer by ion exchange processes, thereby leaving more insoluble components, such as $\mathrm{Ni}, \mathrm{Al}$, and $\mathrm{Fe}$ to be enriched in the residual layer material. The depletion of nickel in the outer layer during this period provides confirmatory evidence for this theory since the solution in which this layer precipitated would have been depleted in nickel, relative to the glass, due to the residual enrichment of nickel in the inner layer clays. After 56 days, both layers have near equal nickel concentrations; however, the residual nature of nickel is still reflected in its enrichment in both layers relative to the unaltered glass. The pattern for titanium is similar to that of nickel for the first 14 days, also suggesting a 
residual enrichment process in the altered clay layers as well (Fig. 98b). Longer-term analyses of titanium were not performed for the altered glass layers.
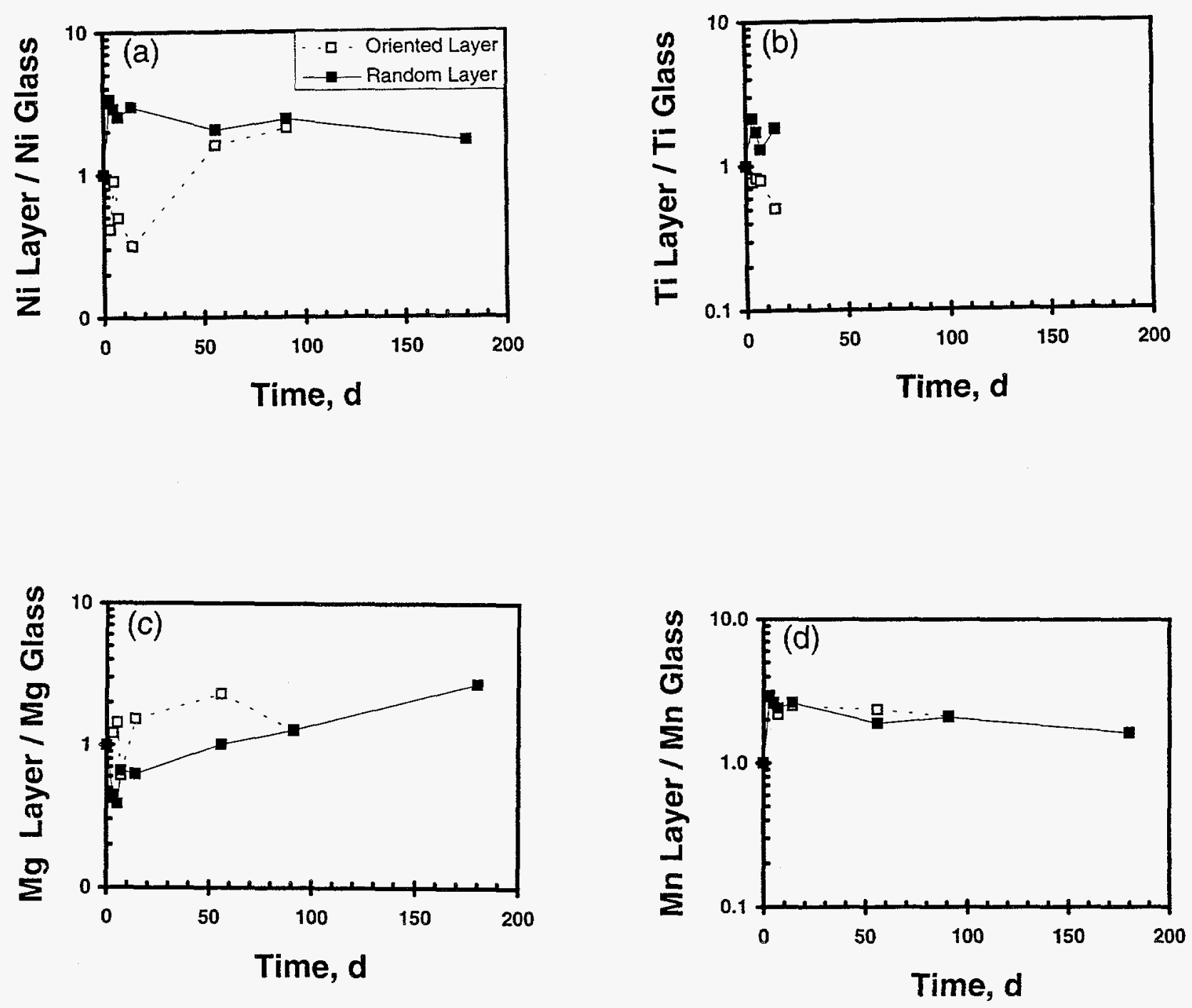

Fig. 98. Element Concentrations in the Outer Oriented and Inner Random Clay Alteration Layers in SRL 131U Glass Normalized to the Concentration of the Respective Elements in the Unaltered Glass: (a) Nickel, (b) Titanium, (c) Magnesium, (d) Manganese, (e) Iron, (f) Aluminum, (g) Sodium, (h) Potassium, (i) Silicon, and (j) Calcium. Enrichments in the altered layer are plotted as values $>1$, while depletions relative to the glass are plotted as values $<1$. Values were not obtained for the outer oriented layer section with the 180-day samples due to lack of preservation of this layer material during the sectioning process. 
Fig. 98. contd.
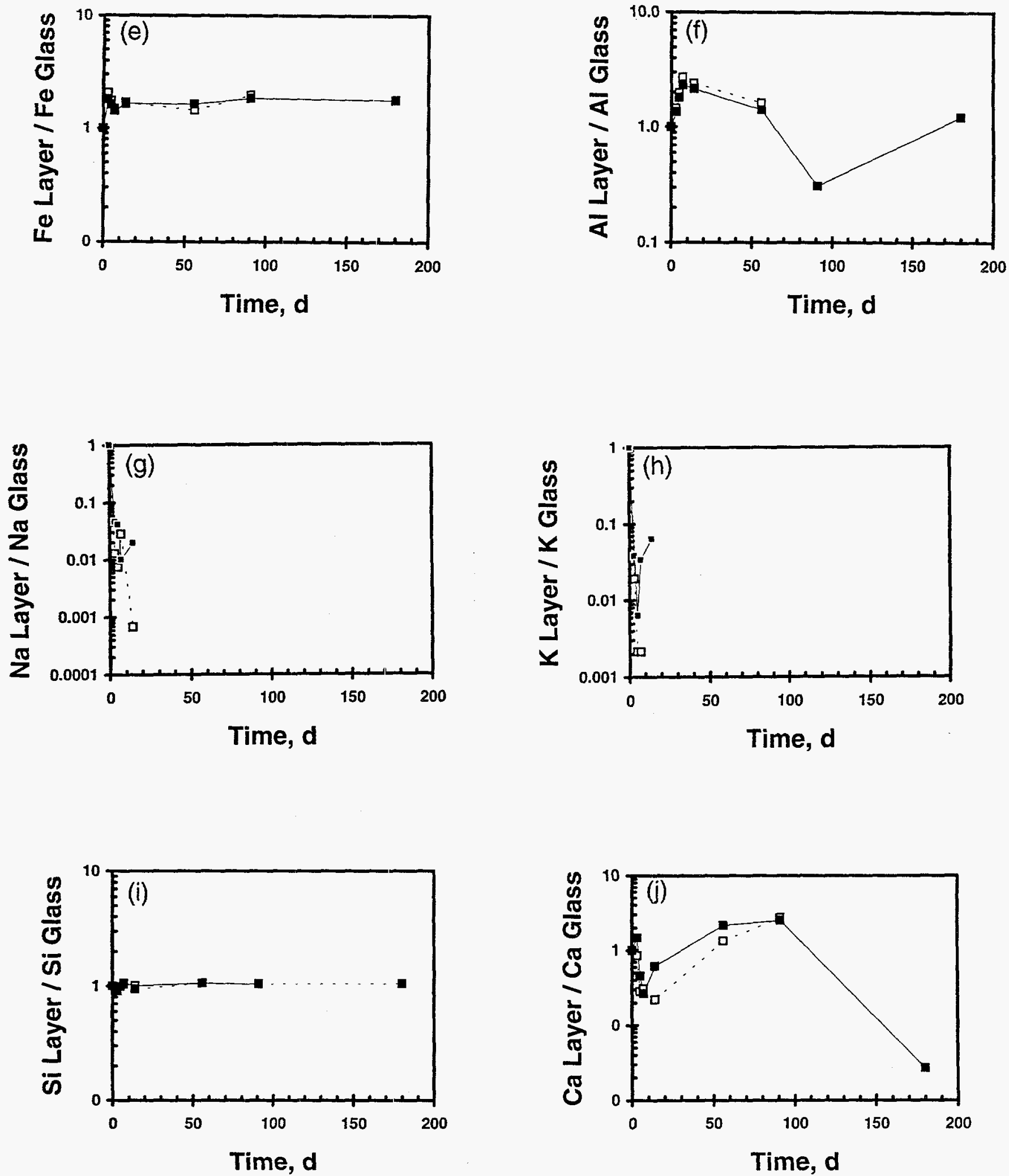
Magnesium distributions reflect an inverse pattern of concentration relative to $\mathrm{Ni}$ and Ti. Magnesium is depleted in the inner layer relative to the unaltered glass for the early testing periods, with concentrations progressively increasing for all reaction times after five days (Fig. 98c). The outer smectite layer is enriched in magnesium relative to the unaltered glass for most time periods tested. This enrichment probably reflects the incorporation of magnesium in saponite clays in the outer clay layer.

Other relatively insoluble components, such as $\mathrm{Mn}, \mathrm{Fe}$, and $\mathrm{Al}$, are also enriched in both the inner and outer clay layers relative to the unaltered glass for most time periods tested (Figs. 98d, 98e, and 98f, respectively). This finding suggests that a residual enrichment process has concentrated these elements in the clays as other more soluble glass components were leached away.

Sodium and potassium are depleted in the clay layer relative to the unaltered glass (Figs. 98g and 98h). The inclusion of these constituents in common surface precipitate phases such as analcime, illite, and adularia attests to their solubility within the alteration layer. Both $\mathrm{Na}$ and $\mathrm{K}$ show an increase in concentration within the inner layer at 14 days. This feature may reflect the residual enrichment of these elements in clays as the concentration of leached components in the solution contacting the layer increases, thus retarding the leaching process that removes these elements from the alteration layer.

Silicon concentrations in the clay layers are relatively constant throughout the duration of the tests (Fig. 98i). Both the inner and outer clay layers display silicon concentrations that are approximately the same as those of the unaltered glass. These patterns suggest that silicon is slowly being released from the alteration layer in a proportional manner to the release of other glass components. This release is of a sufficient magnitude to keep silicon values increasing in the layer via a residual enrichment process.

Calcium values show considerable data scatter during the tests (Fig. 98j). Although no consistent trends developed, calcium is generally depleted in both layers relative to the unaltered glass, but does show some enrichment in the outer layer after 56 days and a large depletion in the inner layer after 180 days.

The enrichment of residual components such as $\mathrm{Ni}, \mathrm{Ti}, \mathrm{Mn}, \mathrm{Fe}$, and $\mathrm{Al}$ in the inner layer, combined with its random crystal orientation, suggests that the inner layer formed in situ as a residual material that remained after the more soluble components had been leached from the glass. The random crystal orientation of these clays arises from the formation of multiple crystal nucleation sites for the clay minerals within the restructuring layer. The preferred orientation of the outer layer, with the crystallographic c-axis oriented parallel to the original glass surface, suggests that these clays precipitated directly from the film of water covering the sample surface. The depletion of less-soluble components from this layer relative to the inner layer material also supports a precipitation origin for this outer layer material.

The presence of the "coke-bottle green," iron-rich inner layer in contact with the glass surface on the SRL $131 \mathrm{U}$ sample at 360 days may offer some insight into the redox environment within the reacting glass layer. The color of this layer suggests that the iron is present in the reduced ferrous state $\left(\mathrm{Fe}^{2+}\right)$. This is in contrast to the oxidized ferric state $\left(\mathrm{Fe}^{3+}\right)$ of the iron present in the main smectite layer. Although the redox states were not measured in the present set of glasses, previous studies indicate that the $\mathrm{Fe}^{2+} / \mathrm{Fe}$ total ratio of SRL 165 glasses produced under the same conditions as the present glasses had a $\mathrm{Fe}^{2+} / \mathrm{Fe}$ total ratio of $0.31 \pm 0.09$ [78]. An oxidizing environment for the outer layer is also supported by the presence of a variety of uranyl $\left(\mathrm{U}^{6+}\right)$ alteration phases, copper oxides, and iron oxides on the altered sample surfaces. These mineral patterns suggest that the altered layers become progressively more oxidizing when moving 
from the glass surface to the outer surface of the alteration layer. These changes in redox conditions across the sample surface may have important implications for the mobility of many redox sensitive radionuclides, such as the actinide elements and technetium.

\section{Surface Precipitate Formation on SRL 131 Glass}

A variety of zeolite, smectite, calcium and sodium silicates, phosphate, evaporitic salts, and uranyl silicate phases were deposited on the sample surfaces of both the irradiated and nonirradiated SRL 131 glasses. With few exceptions, the types of phases present on the reacted glass surface appear to be similar for both the SRL 131U and SRL 131A glasses (Fig. 99). The main differences are the greater abundance of secondary precipitates (Fig. 100) and the accelerated paragenetic sequence observed on the SRL 131A glasses (Fig. 99). An example of this accelerated sequence is evident in the development of analcime and tobermorite. These phases appear in the SRL 131A reaction sequence as early as 7 days, while they appear in the SRL 131U sequence only after 56 and 540 days, respectively. Accelerated glass corrosion rates and the resultant rapid saturation of the leachate with respect to secondary phases appear to be responsible for the more rapid advancement of the paragenetic sequence observed in the irradiated tests. The accelerated glass corrosion rates, in turn, resulted as glass components and were released to solution in response to the production of radiolytic acids. This pattern is the same as that exhibited by the SRL 202 glasses. There are also some differences observed in element-mineral pathways between the irradiated and nonirradiated tests. For example, uranium is contained in sodium-weeksite, haiweeite, and soddyite in the SRL $131 \mathrm{U}$ tests, while it occurs chiefly in weeksite and to a lesser extent, haiweeite, in the SRL 131A tests.

The alteration minerals noted in the present study were compared with several published investigations of SRL $131 \mathrm{U}$ glass. Bates and coworkers $[37,42,60]$ examined glass samples reacted in vapor hydration tests at temperatures of 75 to $240^{\circ} \mathrm{C}$ and noted the formation of analcime, tobermorite, smectite clays, and gyrolite. The phases identified in these studies match those presented in Fig. 99. Biwer et al. [67] examined the reaction of SRL 131 glass in vapor hydration tests at $75^{\circ} \mathrm{C}$. Altered surfaces were characterized by the formation of smectite clays, calcite, analcime, $\mathrm{Na}-\mathrm{Cl}$, and $\mathrm{Na}-\mathrm{S}$ phases in tests conducted at relative humidities of 60 to $100 \%$. These phase identifications agree with those in the present study with the $\mathrm{Na}-\mathrm{Cl}$ and $\mathrm{Na}-\mathrm{S}$ phases appearing as multielement, low atomic weight salt deposits.

Previous studies have also examined the reaction of SRL 131 glass under water saturated test conditions. Using a variety of leachant compositions, Strachan et al. [79] examined the behavior of SRL 131 glass in MCC-1 static leach tests at 40 and $90^{\circ} \mathrm{C}$. While the $40^{\circ} \mathrm{C}$ tests developed amorphous gel layers on their altered surfaces, the $90^{\circ} \mathrm{C}$ tests were characterized by the formation of several different crystalline materials, including $\mathrm{Mn}-\mathrm{Fe}-\mathrm{Si}$ phase, uranium-crystallites, and magnesium-silicates.

With deionized water in MCC- 1 static leach tests at $90^{\circ} \mathrm{C}$, Biwer et al [67] identified the formation of smectites, birnessite $\left[\left(\mathrm{Na}_{0.7} \mathrm{Ca}_{0.3}\right) \mathrm{Mn}_{7} \mathrm{O}_{14} \cdot 2.8 \mathrm{H}_{2} \mathrm{O}\right]$, and a U-Ti-oxide phase [possibly brannerite $\left(\mathrm{U}_{3} \mathrm{Ti}_{5} \mathrm{O}_{16}\right)$ ]. Abrajano et al. [80] also examined the reaction of SRL 131 glass under similar conditions and identified smectite, birnessite, "serpentine-kaolinite types" $\left[\mathrm{Mg}_{3}\left(\mathrm{Si}_{2} \mathrm{O}_{5}\right)(\mathrm{OH})_{4}\right.$ and $\mathrm{Al}_{4}\left(\mathrm{Si}_{4} \mathrm{O}_{10}\right)(\mathrm{OH})_{8}$, respectively], magnetite $\left(\mathrm{Fe}_{3} \mathrm{O}_{4}\right)$, iron hydroxides, barite $\left(\mathrm{BaSO}_{4}\right)$, and rutile $\left(\mathrm{TiO}_{2}\right)$; and with a second glass of a composition similar to the SRL 131, Abrajano et al., [81] noted the presence of smectite, birnessite, serpentine, and U-Ti oxides. In a similar study, Mazer et al. [68] noted the presence of smectite, birnessite, and serpentine on altered SRL 131 glasses. Bates et al. [82] also examined SRL 131 glass under similar test conditions and identified birnessite, smectites (saponite and nontronite), serpentine, a U-Ti oxide phase, and a Ca-Ti-P phase (also containing $\mathrm{Am}$ and $\mathrm{Pu}$ ) in the altered layer. Other than the smectite clays and rare occurrences of iron oxides, none of the alteration phases reported in the previously mentioned MCC-1 studies were noted to form on the vapor hydration test samples. 


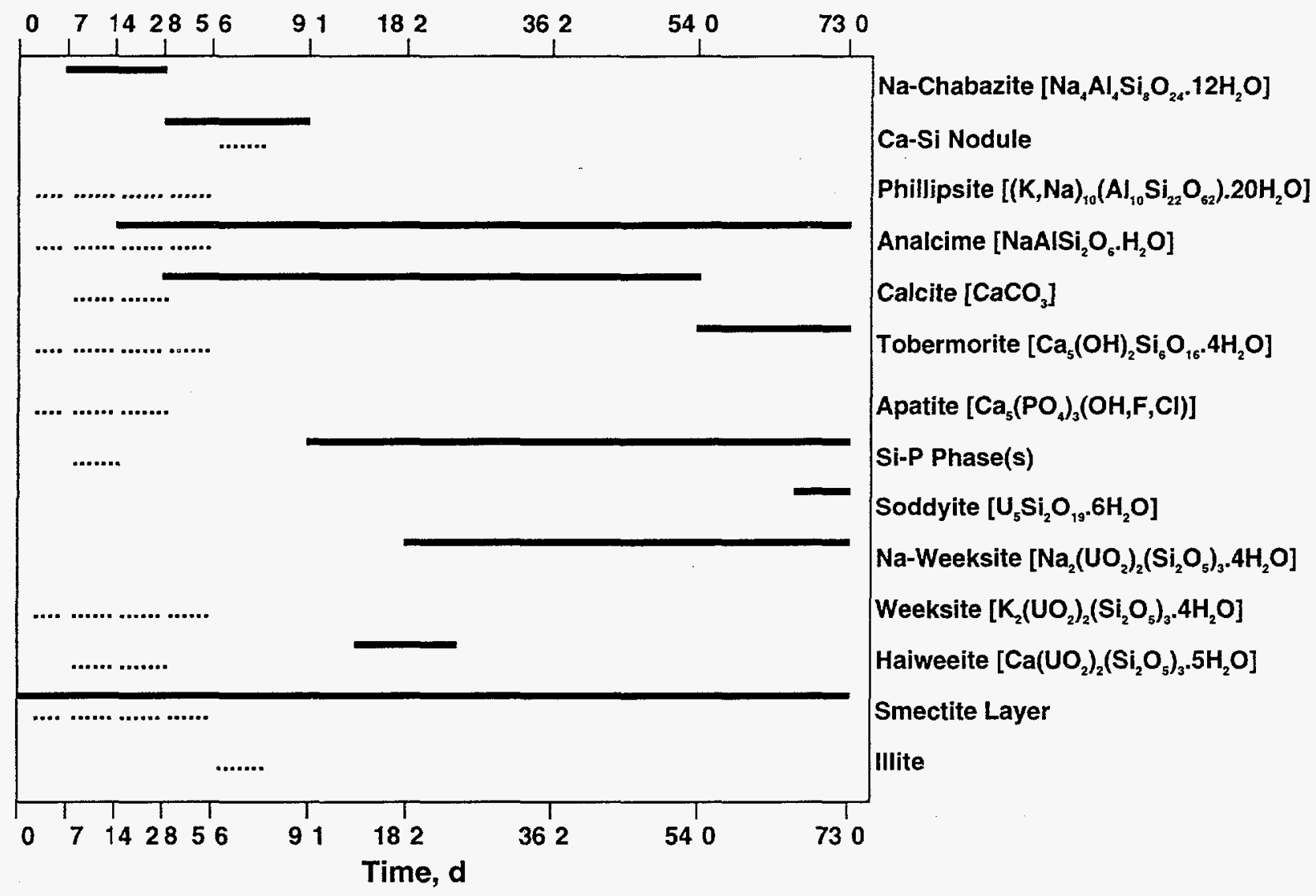

Fig. 99. Alteration Mineral Paragenetic Sequence Identified for SRL 131 Glasses Reacted in Vapor Hydration Tests at $150^{\circ} \mathrm{C}$. Dotted lines represent SRL 131A glass samples that were exposed to gamma dose rate of $\sim 35 \mathrm{~Gy} / \mathrm{h}$, while solid lines represent SRL $131 \mathrm{U}$ samples reacted without any external radiation exposure. SRL 131A samples were reacted for a maximum time period of 56 days. 
(a)

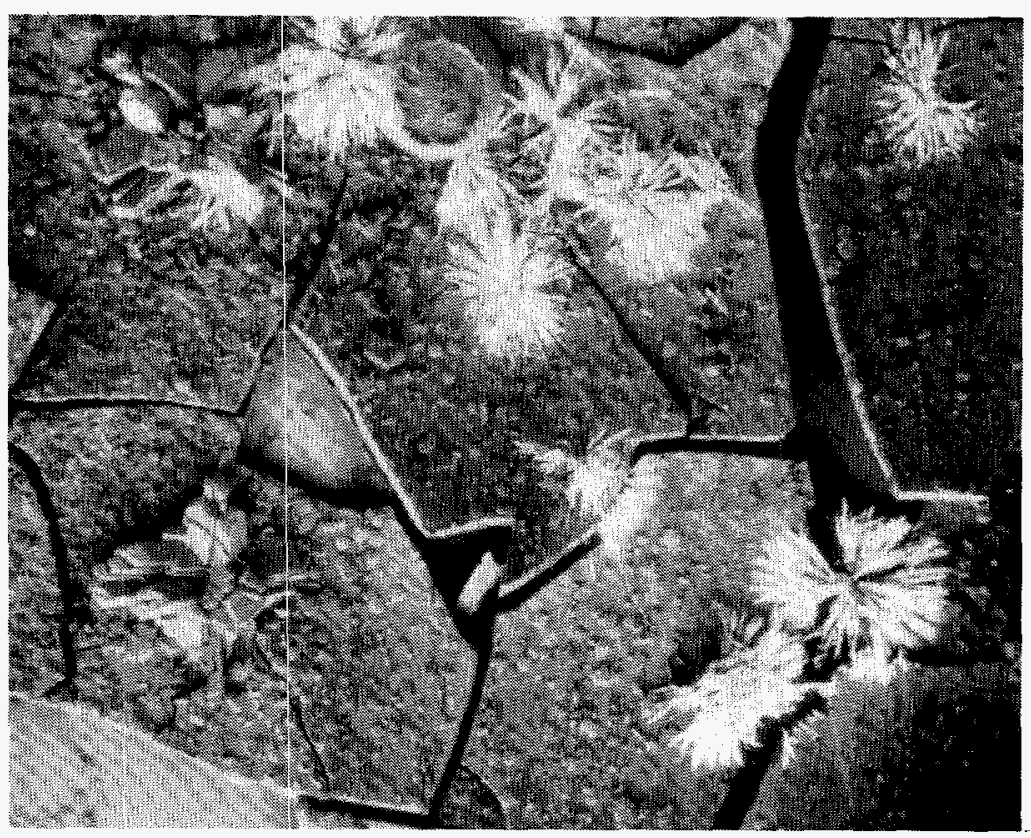

(b)

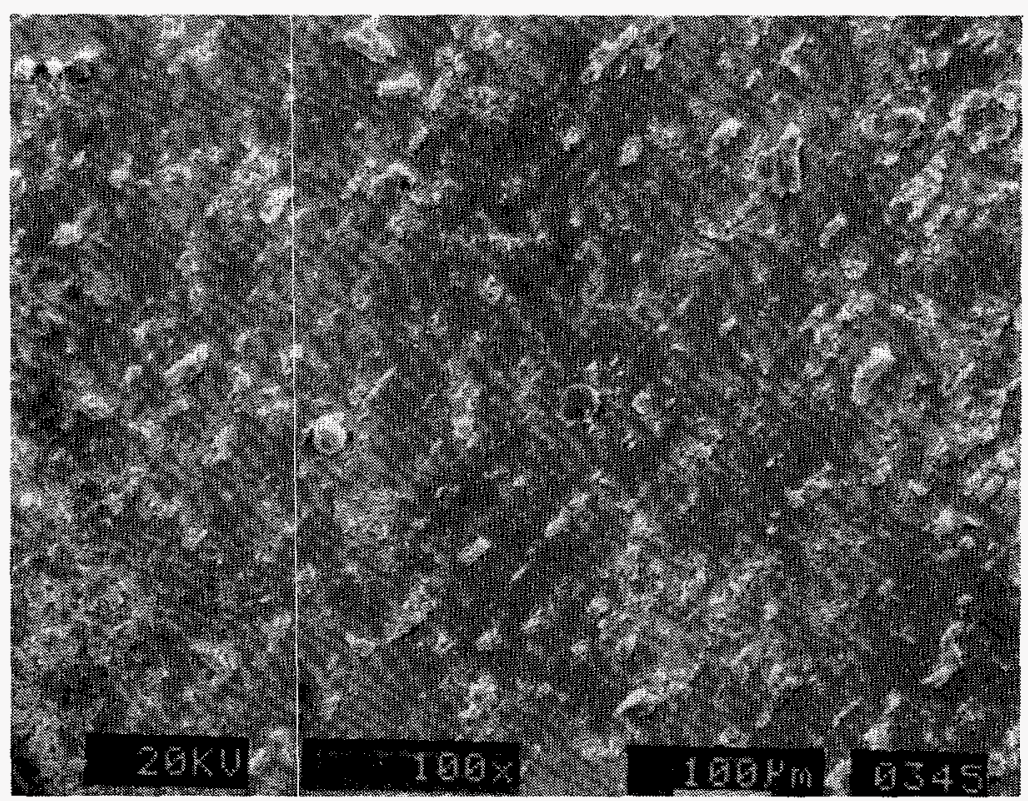

Fig. 100. SEM Photomicrographs of SRL 131 Glasses Reacted in Vapor Hydration Tests for 35 Days at $150^{\circ} \mathrm{C}$. (a) The surface of the SRL 131A glass that was reacted while exposed to gamma dose rate of $\sim 35 \mathrm{~Gy} / \mathrm{h}$. (b) The surface of the SRL $131 \mathrm{U}$ glass reacted without any external radiation exposure. Note that the SRL $131 \mathrm{~A}$ glass has developed a thick alteration cover with a relatively dense overgrowth of secondary precipitates, while the SRL $131 \mathrm{U}$ glass has developed a thin alteration cover with a sparse overgrowth of precipitates. Both photomicrographs at $100 \mathrm{X}$ with scale bar equivalent to $100 \mu \mathrm{m}$. 
Nuñez et al., [83] also studied the effect of alteration processes on the behavior of SRL 131/llR composition glass in $90^{\circ} \mathrm{C} \mathrm{PCTs}$ at $2000 \mathrm{~m}^{-1}$. This glass was produced by melting a blend of SRL 131 glass frit with actual radioactive sludge obtained from waste storage tanks at the Savannah River Site. The SRL 131/llR glass internally generates a radioactive field that is similar in magnitude to the external gamma-radiation field used in the present vapor hydration tests with SRL 131A, SRL 202A, and SRL 165A glasses. Alteration sequences developed on the SRL 131/11R glass over a period of five years are characterized by the formation of smectite, analcime, calcite, and $\mathrm{Ca}-\mathrm{Si}$ fibers that appear to be the phase tobernorite. The sequence of phases developed in the PCTs are also noted to form in the vapor hydration tests of the present study (Fig. 99), indicating that the short-term vapor hydration tests will repeat the alteration behavior of long-term PCTs with the SRL 131 glass.

As with the SRL 202A and SRL 165A glass samples, the SRL 131A monoliths were also scanned at electron accelerating voltages of $30 \mathrm{kV}$ during SEM/EDS analysis to excite the more diagnostic inner electron shells of $\mathrm{Tc}, \mathrm{Am}, \mathrm{Pu}$, and $\mathrm{Np}$. None of the transuranic elements were detected in surface precipitates or the clay alteration layer forming on the SRL 131A glass despite the examination of an extensive suite of sample regions. This absence of discrete transuranic-bearing phases suggests that these elements were being retained as insoluble residue within the inner alteration layer, rather than dissolving and being reprecipitated on the sample surface. Other studies, however, have noted the enrichment of $\mathrm{Np}$, up to several hundred $\mathrm{ppm}$, in sodium weeksite that had precipitated on the surface of altered SRL 131A glass [84]. Technetium was not observed to form any discrete alteration phases with the SRL 131A glasses, although Tc$\mathrm{K}$ phases were observed to form during vapor hydration tests with the SRL 202A glass. (Fig. 43c).

Discrete B- and Li-bearing phases were also not observed on any of the altered samples of SRL 131 glasses examined in this study. The detection of lithium is beyond the capabilities of the SEM/EDS system used in the present study, and boron detection requires wavelength dispersive detector capabilities. As with the SRL 202 glasses, the boron released from the glass is probably deposited within the salt crusts that form as water present on the sample surfaces is evaporated in air at the conclusion of the tests. Because of the relatively high release rate of lithium observed in the $340 \mathrm{~m}^{-1}$ batch tests (Fig. 17b), this element is also expected to be a major component in the salt crusts deposited on the sample surfaces. Lithium, however, can also be incorporated into smectite clays that form during glass alteration.

\section{Comparison with Natural Analogues}

The alteration mineral paragenetic trends developed during the examination of the present test samples have been compared to those developed during the alteration of volcanic glasses deposited in naturally occurring geologic deposits. A comparison of test results with natural analogue occurrences is important in verifying that the (1) minerals cited in the present study have been accurately identified, (2) the elevated temperatures and S/V ratios used in the present tests have not influenced the stability of mineral phases that formed relative to the ones expected to form in an actual repository setting, and (3) these elevated temperatures and S/V ratios have not changed the underlying reaction mechanism controlling glass reactions.

Paragenetic trends developed in the vapor hydration tests with SRL 202A glass are nearly identical to those that are found when volcanic glass alteration occurs in saline-alkaline lacustrine (lake) environments [43]. For example, several studies have noted that volcanic glass altered in a saline-alkaline lacustrine setting displays a well-defined lateral zonation of mineral formation, where the following sequence is displayed from the basin margin to the basin center [61-63] (Fig. 101a): 
unaltered glass + smectite ---> alkali-silica-zeolites --->

analcime ---> K-feldspar + illite $--->$ albite

This lateral zonation in mineralogy has been interpreted to reflect changes in volcanic glass alteration trends as basinal brine fluids become progressively more saline and alkaline toward the basin center.

[a]

Basin Margin

Basin Center

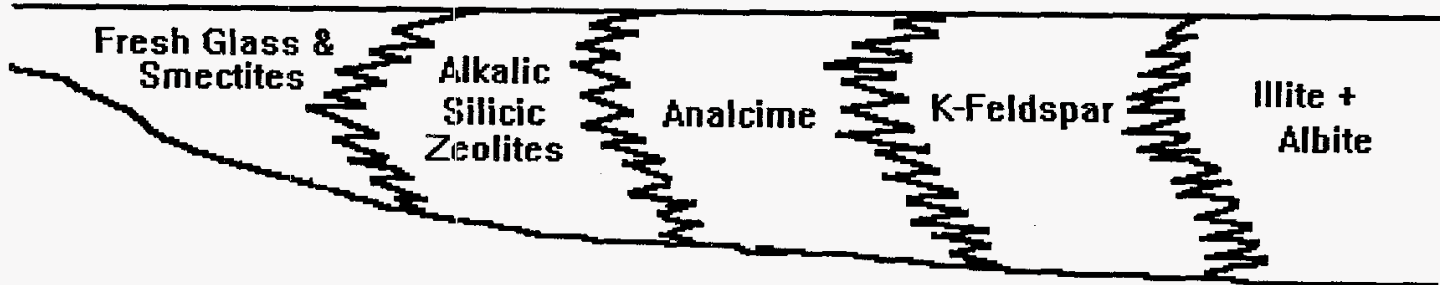

[b]

Unaltered Glass<smiles>C[As][As](C)(C)C</smiles>
$\rightarrow$ Na-Chabazite Analcime Phillipsite $\rightarrow$ K-Feldspar $\longrightarrow$ Illite $\longrightarrow$ Albite

Fig. 101. (a) Mineral Zonation Patterns and Paragenesis Developed during Volcanic Glass Alteration in Saline-Alkaline Lakes as Function of Increasing Water Salinity and Alkalinity from Basin Margin to Basin Center [61-63]. Both illite and albite have also been detected in advanced alteration stages of saline-alkaline lake sediments from the southwestern U.S. (C.E. Chapin, New Mexico Bureau of Mines and Mineral Resources, personal communication, 1993). (b) Mineral Zonation Patterns and Paragenesis Developed during Simulated SRL 202U Borosilicate Waste Glass Alteration in Vapor Saturated-Air Environment at $200^{\circ} \mathrm{C}$. Displayed sequence develops as a function of increasing reaction time, from zero to 56 days, and is interpreted to reflect a progressive increase in both the salinity and alkalinity of the thin film of water contacting the samples as alkaline components are released from the glass matrix [43]. 
Glass alteration trends as a function of increasing depth of burial have also been described from vertical profiles in volcanic rock sequences [64]. Vertical zonations in these sequences have been attributed to increasing temperatures with depth of burial. Volcanic glass material in these settings has been altered according to the following trend:

unaltered glass + smectite ---> clinoptilolite + K-feldspar + smectite --->

clinoptilolite + analcime + K-feldspar + smectite + illite ---> K-feldspar + albite + illite

Once again, these paragenetic trends match remarkably well with those developed in the vapor hydration tests of the present study.

Hay and Guildman [62] have also correlated the vertically zoned mineralogic trends with both the changes in the $\mathrm{pH}$ of associated pore fluids, as well as with stratigraphic age by correlation with dated stratigraphic horizons. Volcanic glasses in contact with moderately saline and slightly alkaline $(\mathrm{pH} \sim 7.5)$ pore fluids were transformed into smectites, clinoptilolite, analcime, and opal, while glass in contact with highly saline and alkaline ( $\mathrm{pH} \sim 9.5)$ pore fluids was transformed first into phillipsite and/or merlinoite and then K-feldspar and searlesite $\left(\mathrm{NaBSi}_{2} \mathrm{O}_{6} \cdot \mathrm{H}_{2} \mathrm{O}\right)$. Data from this study also indicate that the transformation of phillipsite (and/or merlinite) to $\mathrm{K}$-feldspar required between 45,000 to 140,000 years to complete. The temperature of this transformation was not specified in the paper, but since the depths of transformation were $<300 \mathrm{~m}$, the ambient pore fluid temperatures should not be significantly above that of near-surface temperatures. These temperatures suggest that the paragenetic sequence occurring in the present study should be in progress during the 10,000 - to 100,000 -year period of regulatory concern following emplacement of nuclear waste glass into a geologic repository. The nuclear waste paragenetic reactions will also be accelerated relative to the natural analogue deposits because the repository temperatures will be raised as a result of the decay of radioactive material.

A similar alteration sequence has also been described by Bish and Aronson [85] from three drill cores taken from the Yucca Mountain repository site. This study examined vertical mineral profiles as a function of burial temperature. Clinoptilolite became unstable above $100^{\circ} \mathrm{C}$, mordinite was not a major phase above $130^{\circ} \mathrm{C}$, and analcime transformed to albite above $175-200^{\circ} \mathrm{C}$. The transformation of clinoptilolite to analcime also correlated with the appearance of calcite, chlorite, and smectite, while increasing temperatures also corresponded to increasing illite/smectite ratios. The correlation between the naturally occurring alteration minerals at Yucca Mountain with those formed during the vapor hydration tests provides strong evidence that the mineral sequence produced during SRL $202 \mathrm{U}$ glass alteration is likely to be the same one that will occur in an actual repository setting at Yucca Mountain.

In another interesting natural analogue study to the present vapor hydration tests, Hernandez et al. [86] found phillipsite, chabazite, analcime, K-feldspar, and calcite forming as a result of the action of water trapped within the pores of recently deposited volcanic rocks. This alteration sequence was attributed to the rapid reaction of water vapor with the volcanic glass at the high-depositional temperatures of the volcanic rocks. The rapid hydration, dissolution, and subsequent formation of alteration minerals in these volcanic rock pores thus occurred in a hightemperature, closed-system environment that was analogous to the reaction of SRL 202 glass within the confined space of the stainless steel test vessels.

With the SRL 202U samples, the glass material is progressively transformed through the reaction sequence illustrated in Fig. 101b. This sequence is remarkably similar to that observed in both the saline-alkaline lacustrine and burial diagenesis scenarios (Figs. 101a and 102, respectively). This similarity arises because the release of corrosion products from the simulated SRL 202 waste glass in the unsaturated vapor hydration environment quickly transforms the thin film of water contacting the samples into a saline-alkaline fluid. 


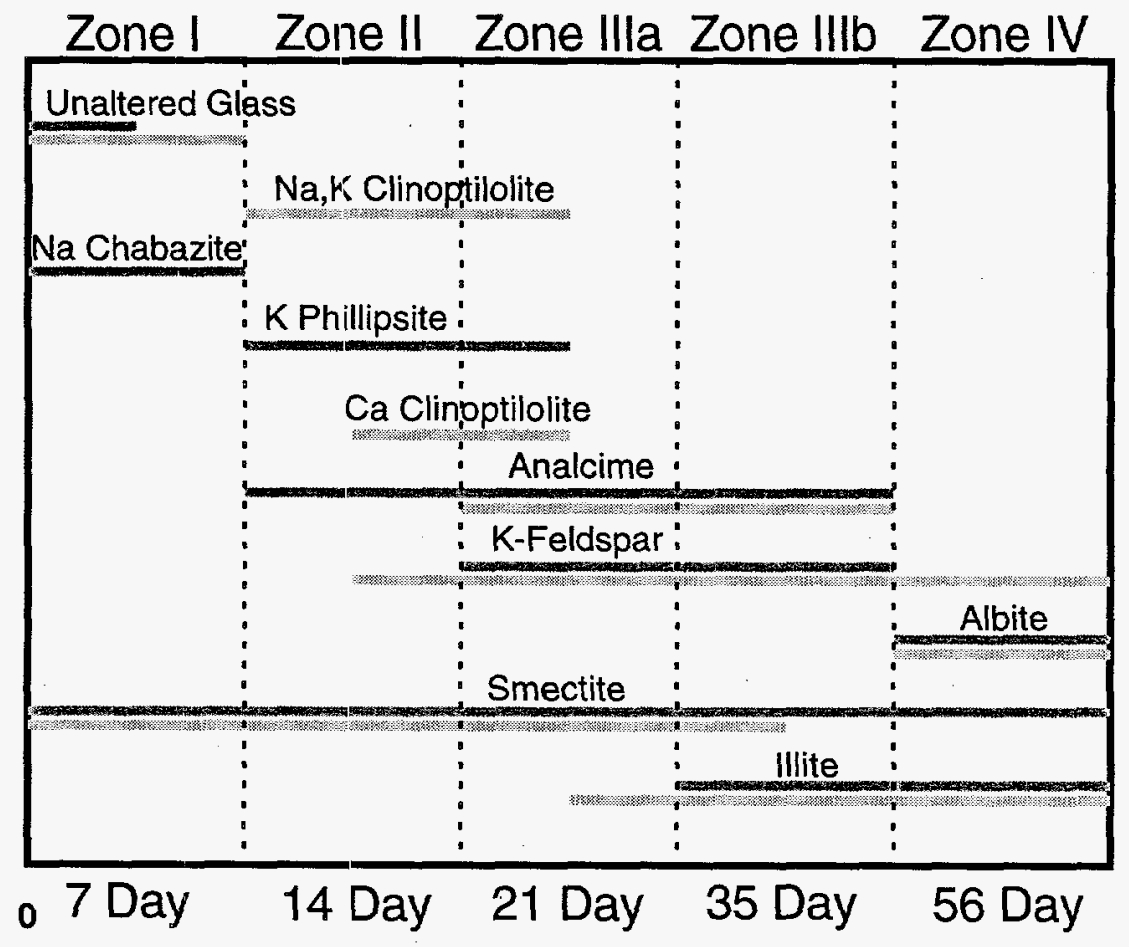

Fig. 102. Mineral Paragenesis Developed During Silicic Volcanogenic Sediment Alteration (Light-Shaded Bars Bracketed by Zones I through IV, Top Axis), and Mineral Zonation Patterns Developed During Simulated SRL 202U Borosilicate Waste Glass Alteration in a Vapor Saturated-Air Environment at $200^{\circ} \mathrm{C}$ (Dark-Shaded Bars Bracketed by 7 Day to 56 Day Periods, Bottom Axis). Silicic sediment alteration is interpreted to reflect decreasing hydration rates, increasing temperature, and increasing pore fluid salinity when moving from near-surface (Zone I) to deeply buried conditions (Zone IV) [64]. Sequence for the SRL 202U glass develops in response to increasing salinity and alkalinity of the thin-film of water contacting the samples as alkaline components are released from the glass matrix.

Paragenetic trends similar to those described here have also been noted in experimental studies with geologic materials. Naturally occurring volcanic glasses were reacted in distilled water at temperatures of 60 to $200^{\circ} \mathrm{C}$, with developed paragenetic trends that were similar to those of the present tests [87]. Chermak [88] reacted a shale sample composed predominantly of quartz $\left(\mathrm{SiO}_{2}\right)$-kaolinite-illite-calcite in 150 to $200^{\circ} \mathrm{C} \mathrm{KOH}$ solutions prepared to a $\mathrm{pH}$ of 12.83 . Results from these tests also correlate well with the observed paragenetic trends from the reacted SRL 202U glasses. With this latter study, the use of clays as a starting material simply bypassed the first step in the glass paragenesis trends, the transformation of glass to smectite clays. 
The similarities in mineral paragenesis observed between the saline-alkaline lacustrine and burial diagenesis settings versus those of the present tests with simulated nuclear waste glass can be correlated to changes occurring in the composition of fluids contacting the glass materials. The mineral patterns displayed in the lacustrine setting are a function of spatial variations in solution composition, which is a function of the distance of the fluid from the basin margin [63]. In the burial environment, the release of components from the volcanic glass raised the $\mathrm{pH}$, activity of dissolved silicon in solution, and the activity ratio of $\left(\mathrm{Na}^{+}+\mathrm{K}^{+}\right) / \mathrm{H}^{+}$; in addition, the increasing temperatures with burial depth have resulted in the observed mineral paragenetic trends $[62,64,85]$. By analogy, the paragenetic patterns displayed by the altered SRL 202U glasses reflect temporal changes in solution composition, with both the salinity and alkalinity of the fluid in contact with the glass surface increasing with time because of the release of glass components to solution. These correlations indicate that the accelerated mineral paragenetic trend observed in the vapor hydration tests is likely to be replicated at lower temperatures that are more relevant to repository disposal conditions, and thus, the observed paragenetic trend appears to be realistic for predicting nuclear waste glass alteration trends in an actual disposal scenario. The saline-alkaline lake settings and thermally heated volcanic rock sequences also offer excellent natural analogue research targets for investigating element migration and alteration mineral stability patterns associated with the geologic disposal of nuclear waste glasses. 


\section{CONCLUSIONS}

Radiolysis tests were conducted with air-water systems at high $\mathrm{G} / \mathrm{L}$ ratios to examine the formation rates of radiolytic products, locations of radiolytic product condensates, effects of radiolytic products on glass corrosion, and effects of radiolytic products on alteration phase development. In tests conducted without glass, the following conclusions were reached:

(1) Nitrate (nitric acid) is the predominant radiolytic product formed during radiolysis tests at high $\mathrm{S} / \mathrm{V}$ conditions. The rate of nitrogen acid $\left(\mathrm{NO}_{3}^{-}+\mathrm{NO}_{2}^{-}\right)$production in the air-filled portion of the vessel was proportional to the cumulative dose, with little variation occurring as a function of dose rate or gas/liquid ratio. Most of the nitrogen acids that formed in the tests had collected in the leachate present at the bottom of the vessel, either by aerosol condensation and/or dripping from the test vessel component surfaces.

(2) Nitrogen acid production varied inversely with temperature. Respective $\mathrm{G}\left(\mathrm{NO}_{3}^{-}+\mathrm{NO}_{2}^{-}\right)$values for the 25,90 , and $200^{\circ} \mathrm{C}$ gamma irradiated experiments are $3.2 \pm 0.7,1.3 \pm 1.0$, and $0.4 \pm 0.3$. This trend is important from the standpoint of waste form and container stability in that the elevated temperatures expected early in the life of the repository may mitigate against large decreases in $\mathrm{pH}$ resulting from nitrogen acid production.

(3) High $\mathrm{NO}_{3}^{-}-\mathrm{NO}_{2}^{-}$ratios indicate that oxidizing conditions persisted throughout the irradiation tests. An increase in nitrite levels is noted in the high cumulative dose and low $\mathrm{G} / \mathrm{L}$ ratio tests. These later increases are believed to result from reactions between the test solutions and stainless steel test vessel walls.

(4) Apparent alpha radiation $\mathrm{G}\left(\mathrm{NO}_{3}^{-}+\mathrm{NO}_{2}^{-}\right)$values of $2.4 \pm 1.1$ for the air-filled portion of the vessel are slightly lower than the $3.2 \pm 0.7$ values determined for gamma radiation exposure at $25^{\circ} \mathrm{C}$. The lower yields may result from partial attenuation of alpha particles in the thin film of water condensed on the ${ }^{241} \mathrm{Am}$ foils.

(5) The formation of nitrogen acids in alpha-irradiated environments with high $\mathrm{G} / \mathrm{L}$ ratio indicates that nitrogen acids may still play a role in influencing nuclear waste form reactions under long-term (>300 yr) unsaturated storage conditions, when alpha radiation from long-lived transuranic elements dominates emissions from the nuclear waste forms. Thus, the alpha radiation being emitted from the waste after 300 years may still be sufficient to acidify thin films of water that may contact the waste. The acidification of these solutions will ultimately depend on the rate of acid formation versus the ability of the bicarbonate buffering reservoir to regenerate itself and neutralize the radiolytic acids that form. Fluids in contact with glass waste forms will also be buffered by the release of glass components into solution. This latter process will lead to enhanced leach rates for glass components.

(6) Formate, oxalate, and total organic carbon were noted to increase as a consequence of radiation exposure. These organic fractions were preferentially concentrated in thin films of water that had condensed on the walls of the test vessels. The formation of oxalate and formate is favored under relatively low $\mathrm{pH}$ conditions that may be present in these thin films. These organics may influence the retentive properties of nuclear waste forms by complexing with radionuclides and transporting them away from the waste form. 
(7) No enrichment of organic components was noted when test vessels were irradiated at $200^{\circ} \mathrm{C}$ or at dose rates of $\sim 500 \mathrm{~Gy} / \mathrm{h}$.

In tests conducted with glass present, but otherwise under identical conditions as the previous tests, the following conclusions were reached:

(8) Radiolytic products, including nitrogen acids, have led to enhanced rates of glass alteration under vapor hydration test conditions. Layer development may be accelerated by a factor of between 4 - and 15-fold due to radiation exposure at high $\mathrm{S} / \mathrm{V}$ conditions, relative to identical tests conducted under nonirradiated conditions. The magnitude of the difference in accelerated layer development varied as a function of glass composition and, possibly, also reaction temperature. Enhanced rates of glass reaction occur due to the formation of nitrogen acids and their condensation in the film of water located on the glass surface. This process led to increased rates of ion exchange between the glass and the acids contained in the water film. The increased rates of glass reaction apparently buffer against a major decrease in the $\mathrm{pH}$ of the film of water, as evidenced by the formation of a suite of alteration phases that are representative of those forming naturally in alkaline systems.

(9) With tests at $340 \mathrm{~m}^{-1}$, the release rates of most glass components were accelerated a maximum of four-fold under gamma irradiation conditions relative to identical tests conducted under nonirradiated conditions. The release rates of some elements, including $\mathrm{Si}$, were actually reduced in the irradiated tests, possibly as a result of the decreased stability of certain complexants at lower solution $\mathrm{pH}$ values.

(10) Release patterns from irradiation tests at $340 \mathrm{~m}^{-1}$ indicate that relatively minor amounts of ${ }^{239} \mathrm{Pu}$ and ${ }^{241} \mathrm{Am}$ are released in a soluble form $(<5.0-\mathrm{nm}$ filtered fraction), while the largest release component for these elements is obtained after an acid strip of the stainless steel vessel walls. This trend indicates that ${ }^{239} \mathrm{Pu}$ and ${ }^{241} \mathrm{Am}$ are deposited as relatively insoluble phases on the test vessel walls during the experiments. By contrast, release patterns of ${ }^{237} \mathrm{~Np}$ are dominated by the $<5.0-\mathrm{nm}$ size fraction, indicating that ${ }^{237} \mathrm{~Np}$ release from the glass is being controlled by a soluble and/or $<5.0-\mathrm{nm}$ colloidal component.

(11) Alteration layer development also appeared to progress along preferential fractures and pits with the irradiated tests, while in the nonirradiated tests alteration fronts were more planer. The fractures appeared to be related to more rapid alteration rates rather than the radiation exposure, since the cumulative doses received by the glass were well below those which would be sufficient to cause solid-phase radiation damage. The distribution of fractures was also nonuniform in the reacted glass samples, suggesting that solid-phase radiation damage was not the underlying cause of the fractures.

(12) The irradiation environment accelerated the formation of many alteration minerals relative to identical tests conducted under a nonirradiated environment. In some instances, the irradiation exposure led to the precipitation of different phases. These changes may be related to changes in solution composition caused by radiolytic product formation or, alternatively, by enhanced reaction kinetics that are indirectly related to radiation exposure through the enhanced release rates of glass components.

(13) A very fine-grained Tc-K-Ca solid phase was identified on the SRL 202A sample at 14 days, occurring as submicron particles deposited on the Pt-Rh support wire. No deposits of this type were identified directly on the altered glass surface. The Pt-Rh wire may have acted as a catalyst toward the formation of these technetium deposits. 
The presence of an oxidized $\mathrm{Tc}^{7+}$ phase is suspected as evidenced by the close spatial association of uranyl silicate and ferric iron phases, along with the expected production of $\mathrm{H}_{2} \mathrm{O}_{2}, \mathrm{O}_{2}$, and $\bullet \mathrm{OH}$ water radiolysis products.

(14) The presence of the "coke-bottle green," iron-rich inner layer in contact with the glass surface on some SRL 131U samples from vapor hydration tests suggests that the ferrous iron is present below the ferric iron-iron main smectite layer. Changes in redox conditions across the sample surface may have important implications for the mobility of many redox-sensitive radionuclides, such as the actinide elements and technetium.

(15) The similarities in mineral paragenesis observed between volcanic glass in both saline-alkaline lacustrine and thermal-burial settings versus those of the present tests with simulated nuclear waste glass can be correlated to changes occurring in the composition of fluids contacting the glass materials. Thus, accelerated mineral paragenesis observed in the 150 to $200^{\circ} \mathrm{C}$ vapor hydration tests are likely to be replicated at lower temperatures that are more relevant to repository disposal conditions, and thus, the observed paragenetic trend appears to be realistic for nuclear waste glasses. Furthermore, saline-alkaline lake settings and thermally heated volcanic rock sequences offer excellent natural analogue research tools for investigating element migration and alteration mineral stability patterns associated with the geologic disposal of nuclear waste glasses. 


\section{ACKNOWLEDGMENTS}

This research has been supported by the U.S. Department of Energy, Office of Environmental Management, under contract W-31-109-ENG-38. Appreciation is acknowledged for analytical support provided by E. Huff and M. Gong (ICP cations analyses); D. Bowers and F. Jeffries (anions analyses); C. Bradley, N. Brown, N. Dietz, and J. Fortner (AEM analyses); B. Tani (XRD analysis); and M. Gong (alpha counting). Technical support from T. Gerding is gratefully acknowledged. R. Riel's assistance in manuscript preparation is also appreciated. D. Reed provided assistance with dosimetry measurements and guidance into the discipline of radiation chemistry. Access to unpublished data of W. Ebert and early efforts of $J$. Young in initiating some of the tests in this study are also appreciated. This manuscript has been improved through reviews by W. Ebert, C. Bradley, S. Vogler, J. Harmon, and several unidentified reviewers. 


\section{REFERENCES}

1. W. J. Weber, "The Effect of Radiation on Nuclear Waste Forms," JOM, pp. 35-39 (July 1991).

2. S. Sunder and D. W. Shoesmith, Chemistry of Uranium Dioxide Fuel Dissolution in Relation to the Disposal of Used Nuclear Fuel, Atomic Energy of Canada Ltd. Report AECL-10395 (1992).

3. W. G. Burns, A. E. Hughes, J. A. C. Marples, R. S. Nelson, and A. M. Stoneham, "Effects of Radiation on the Leach Rates of Vitrified Radioactive Waste," J. Nucl. Mater. $\underline{107}, 245$ (1982).

4. W. G. Burns, A. E. Hughes, J. A. C. Marples, R. S. Nelson, and A. M. Stoneham, "Radiation Effects and the Leach Rates of Vitrified Radioactive Waste," Nature 295, 130-132 (1982).

5. Aa. Barkatt, Al. Barkatt, and W. Sousanpour, "Gama Radiolysis of Aqueous Media and Its Effects on the Leaching Processes of Nuclear Waste Disposal Materials," Nucl. Technol. $\underline{60}, 218-227$ (1983).

6. J. E. Mendel (compiler), "Radiation Effects," in Final Report of the Defense High-Level Waste Leaching Mechanisms Program, Pacific Northwest Laboratory Report PNL-5157, pp. 5.0-5.18 (1984).

7. N. E. Bibler, "Leaching Fully Radioactive SRP Nuclear Waste Glass in Tuff Groundwater in Stainless Steel," Adv. Ceram. 20, 619-626 (1986).

8. A. R. Jones, "Radiation-Induced Reactions in the $\mathrm{N}_{2}-\mathrm{O}_{2}-\mathrm{H}_{2} \mathrm{O}$ System," Radiat. Res. 10 , 655-663 (1959).

9. L. Dolle and J. Rozenberg, "Radiolytic Yields in Water Reactor System and Influence of Dissolved Hydrogen and Nitrogen," Water Chemistry of Nuclear Reactor Systems, British Nuclear Society, London (1978).

10. D. Rai, R. G. Strickert, and J. L. Ryan, "Alpha Radiation Induced Production of $\mathrm{HNO}_{3}$ During Dissolution of Pu Compounds," Inorg. Nucl. Lett. 16, 551-555 (1980).

11. J. K. Linacre and W. R. Marsh, The Radiation Chemistry of Heterogenous and Homogenous Nitrogen and Water Systems, UKAEA Harwell Laboratory, Oxfordshire, AERE Report R-10027 (1981.).

12. W. J. Gray and G. L. McVay, Nitric Acid Formation During Gamma Irradiation of Air/Water Mixtures, Pacific Northwest Laboratories Report PNL-SA-12309 (1984).

13. H. K. Rae, G. M. Allison, A. R. Bancroft, W. Makintosh, J. F. Palmer, E. E. Winter, J. E. Lesurf, and S. R. Hatcher, "Experince with the Chemistry of Water in Moderator and Coolant Systems," Third Proc. of Int. Conf. on Peaceful Uses for Atomic Energy, Geneva, Vol. 9, p. 318 (1985).

14. D. T. Reed and D. L. Bowers, "Alpha Particle-Induced Formation of Nitrate in the Cm-Sulfate Aqueous System,” Radiochim. Acta 51, 119-125 (1990). 
15. W. Primak and L. H. Fuchs, "Nitrogen Fixation in a Nuclear Reactor," Nucl Technol. 13, 38 (1955).

16. D. T. Reed, "Effect of Ionizing Radiation on Moist Air Systems," Mater. Res. Soc. Symp. Proc. 112, 393-404 (1987).

17. D. T. Reed and R. A. Van Konynenburg, "Effect of Ionizing Radiation on the Waste Package Environment," Am. Nucl. Soc. Proc. High Level Radioactive Waste Management Conference, Las Vegas, Nevada, April 28-May 3, 1991, pp. 1396-1403 (1991).

18. D. T. Reed, "Progress in Assessing the Effect of Ionizing Radiation on the Anticipated Waste Package Environment at the Yucca Mountain Potential Repository Site," Proc. of the Topical Meeting on Nucl. Waste Pkg. Focus '91, Las Vegas, Nevada, Sept. 29-Oct. 2, 1991, pp. 58-67 (1992).

19. G. L. McVay and C. Q. Buckwater, "The Nature of Glass Leaching," Nucl. Technol. 1ㅣ, 123-129 (1980).

20. G. L. McVay, W. J. Weber, and L. R. Pederson, "Effects of Radiation on the Leaching Behavior of Nuclear Waste Forms," Nucl. and Chem. Waste Manage. 2, 103-108 (1981).

21. G. L. McVay, "Effects of Gamma Irradiation on Leaching," Unpublished Proc. Workshop on Testing of High Level Waste Forms Under Repository Conditions, October 17-28, 1981, pp. 6-15 (1981).

22. W. G. Burns, W. G. Marsh, and W. S. Walters, "The Gamma Irradiation-Enhanced Corrosion of Stainless and Mild Steels by Water in the Presence of Air, Argon and Hydrogen," Radiat. Phys. Chem. 21, 259-279 (1983).

23. D. J. Wronkiewicz, J. E. Young, and J. K. Bates, "Effects of Alpha and Gamma Radiation on Glass Reaction in an Unsaturated Environment," Mater. Res. Soc. Symp. Proc. 212, 99-106 (1991).

24. D. J. Wronkiewicz, L. M. Wang, J. K. Bates, and B. S. Tani, "Effects of Radiation Exposure on Glass Alteration in a Steam Environment," Mater. Res. Soc. Symp. Proc. 294, 183-190 (1993).

25. D. J. Wronkiewicz, "Radinuclide Decay Effects on Waste Glass Corrosion," Mater. Res. Soc. Symp. Proc. 333, 83-97 (1994).

26. D. J. Wronkiewicz, Effects of Radionuclide Decay on Waste Glass Behavior -- A Critical Review, Argonne National Laboratory Report ANL-93/45 (1994).

27. D. J. Wronkiewicz, "Effects of Radionuclide Decay," Chapter 2.5, in High-Level Nuclear Waste Borosilicate Glass: A Compendium of Corrosion Characteristics, J. C. Cunnane, Compiler (in press).

28. G. L. McVay and L. R. Pederson, "Effect of Gama Radiation on Glass Leaching," J. Am. Ceram. Soc. 64, 154-158 (1984).

29. L. R. Pederson and G. L. McVay, "Influence of Gamma Irradiation on Leaching of Simulated Nuclear Waste Glass: Temperature and Dose Rate Dependence in Deaerated Water," J. Am. Ceram. Soc. 66, 863-867 (1983). 
30. J. K. Bates, D. F. Fischer, and T. J. Gerding, The Reaction of Glass During Gamma Irradiation in a Saturated Tuff Environment, Part I: SRL 165 Glass, Argonne National Laboratory Report ANL-86-62 (1986).

31. P. Van Iseghem, Performance of Vitrious Waste Forms and Barriers Under Clay Repository Conditions, International Atomic Energy Agency, IAEA Report, (1988).

32. P. Van Iseghem, K. Berghman, and W. Timmermans, "The Interaction between Nuclear Waste Glasses and Clay-II," Mater. Res. Soc. Symp. Proc. 176, 291-298 (1990).

33. P. Van Iseghem, C. Cantale, M. Coquerelle, J. L. Dussossoy, G. Malow, and H. Roggendorf, "Corrosion Mechanisms of Vitrified High-Level Waste," in Radioactive Waste Management and Disposal, L. Cecille, Ed., Elsevier, New York, pp. 275-286 (1991).

34. W. L. Ebert, The Effects of the Glass Surface Area/Solution Volume Ratio on Glass Corrosion: A Critical Review, Argonne National Laboratory Report ANL-94/34 (1995).

35. J. W. T. Spinks and R. J. Woods, An Introduction to Radiation Chemistry, 2nd Edition, John Wiley and Sons, New York, pp. 93-103 (1976).

36. G. Faure, Principles of Isotope Geology, John Wiley and Sons, New York (1986).

37. T. A. Abrajano, Jr., J. K. Bates, and J. J. Mazer, "Aqueous Corrosion of Natural and Nuclear Waste Glasses II. Mechanisms of Vapor Hydration of Nuclear Waste Glasses," J. Non-Cryst. Solids 108, 269-288 (1989).

38. G. G. Wicks, W. C. Mosley, P. G. Whitkop, and K. A. Saturday, "Durability of Simulated Waste Glass -- Effects of Pressure and Formation of Surface Layers," J. NonCryst. Sol. $\underline{49}, 413-428$ (1982).

39. T. A. Abrajano, Jr., J. K. Bates, T. J. Gerding and W. L. Ebert, The Reaction of Glass During Gamma Irradiation in a Saturated Tuff Environment, Part III: Long Term Experiments at $1 \times 10^{4} \mathrm{Rad} / \mathrm{Hour}$, Argonne National Laboratory Report, ANL-88-14 (1988).

40. J. C. Walton, "Effects of Evaporation and Solute Concentration on Presence and Composition of Water in and Arround the Waste Package at Yucca Mountain," Waste Manage. 13, 293-301 (1993).

41. J. L. Dussossoy, C. Dubois, E. Vernaz, and A. Chambaudet, "Effect of Surface Finish on Nuclear Glass Dissolution Rate,” Mater. Res. Soc. Symp. Proc. 176, 339-346 (1990).

42. J. K. Bates, L. J. Jardine, and M. J. Steindler, "Hydration Aging of Nuclear Waste Glasses," Science 218, 51-54 (1982).

43. D. J. Wronkiewicz, C. R. Bradley, and J. K. Bates, "A Comparison of Alteration Paragenesis between High-Level Nuclear Waste Materials and Geologic Analogues," Geological Society of America, Abstract with Program, p. A-185 (1993). 
44. C. D. Byers, M. J. Jercinovic, and R. C. Ewing, A Study of Natural Glass Analogues as Applied to Alteration of Nuclear Waste Glass, Argonne National Laboratory Report NUREG/CR-4842, ANL-86-46 (1987).

45. W. L. Ebert and J. K. Bates, "The Reaction of Synthetic Nuclear Waste Glass in Steam and Hydrothermal Solution," Mater. Res. Soc. Symp. Proc. 176, 339-346 (1990).

46. Joint Committee on Powder Diffraction Standards, International Centre for Diffraction Data, Mineral Powder Diffraction File, Group Data Book, International Center for Diffraction Data, Swarthmore, PA (1983).

47. M. Calligaris, G. Nardin, and L. Randaccio, "Cation Size Location in Hydrated Chabazite. Crystal Structure of Potassium- and Silver-Exchanged Chabazite," Zeolites $\underline{3}$, 205-208 (1983).

48. G. Gottardi and E. Galli, Natural Zeolites, Springer-Verlag, Berlin (1985).

49. W. A. Deer, R. A.Howie, and J. Zussman, An Introduction to the Rock-Forming Minerals, 2nd Edition, Longman Scientific and Technical Publishers, Essex, England, pp. 396-430 (1992).

50. R. Donahoe, J. G. Liou, and S. Guildman, "Synthesis and Characterization of Zeolites in the System $\mathrm{Na}_{2} \mathrm{O}-\mathrm{K}_{2} \mathrm{O}-\mathrm{Al}_{2} \mathrm{O}_{3}-\mathrm{H}_{2} \mathrm{O}$," Clays and Clay Miner. $\underline{32}$, 433-443 (1984).

51. D. J. Wronkiewicz, J. K. Bates, and E. Veleckis, "Uranium Release and Secondary Phase Formation during Unsaturated Drip Testing of $\mathrm{UO}_{2}$ at $90^{\circ} \mathrm{C}$," J. Nucl. Mater. $\underline{190}$, 107-127 (1992).

52. S. L. Nicolosi, "A General Model for the Analysis of Groundwater Radiolysis," Mater. Res. Soc. Symp. Proc. 44, 631-640 (1985).

53. F. Garisto, "The Energy-Spectrum of Alpha-Particles Emitted from Used CANDU Fuel," Annal. Nucl. Energy 16, 33-38 (1989).

54. S. Sunder and H. Christensen, "Gamma Radiolysis of Water Solutions Relevant to the Nuclear Fuel Waste Management Program, “ Nuclear Technology (in press).

55. D. W. Shoesmith, S. Sunder, L. H. Johnson, and M. G. Bailey, "Oxidation of CANDU $\mathrm{UO}_{2}$ Fuel by the Alpha-Radiolysis Products of Water," Mater. Res. Soc. Symp. Proc. $\underline{50}$, 309-316 (1985).

56. M. G. Bailey, L. H. Johnson, and D. W. Shoesmith, "The Effects of the Alpha-Radiolysis of Water on the Corrosion of $\mathrm{UO}_{2}$," Corros. Sci. 25, 233-238 (1985).

57. W. J. Weber, J. W. Wald, and G. L. McVay, "Effects of Alpha-Radiolysis on Leaching of a Nuclear Waste Glass,” J. Am. Ceram. Soc. 68, C253-C255 (1988).

58. W. L. Jolly, The Inorganic Chemistry of Nitrogen, W.A. Benjamin Publishers, New York (1964).

59. R. A. Van Konynenburg, Radiation Chemical Effects in Experiments to Study the Reaction of Glass in an Environment of Gamma-Irradiated Air, Groundwater, and Tuff, Lawrence Livermore National Laboratory Report UCRL-53719 (1986). 
60. J. K. Bates, L. J. Jardine, and M. J. Steindler, The Hydration Process of Nuclear Waste Glass: An Interim Report, Argonne National Laboratory Report ANL-82-11 (1982).

61. M. Utada, "Zonal Distribution of Authigenig Zeolites in the Tertiary Pyroclastic Rocks in Mogami District, Yamagata. Prefecture," Sci. Papers Coll. Gen. Educ. Univ. Tokyo 15, 173-216 (1965).

62. R. L. Hay, "Geologic Occurrences of Zeolites," in Natural Zeolites Occurrence, Properties, Use, L.B. Sand and F.A. Mumpton, Eds., Pergamon Press, Oxford, pp. 135-143 (1978).

63. R. C. Surdam and R. A. Sheppard, "Zeolites in Saline, Alkaline-Lake Deposits," in Natural Zeolites Occurrence, Properties, Use, L.B. Sand and F.A. Mumpton, Eds., Pergamon Press, Oxford, pp. 145-174 (1978).

64. A. IIjima, "Geologic Occurrences of Zeolites in Marine Environments," in Natural Zeolites Occurrence, Properties, Use, L.B. Sand and F.A. Mumpton, Eds., Pergamon Press, Oxford, pp. 175-198 (1978).

65. J. K. Bates, W. L. Ebert, X. Feng, and W. L. Bourcier, "Issues Affecting the Prediction of Glass Reactivity in an Unsaturated Environment," J. Nucl. Mater. 190, 198-227 (1982).

66. W. L. Ebert, J. K. Bates, and T. J. Gerding, The Reaction of Glass During Gamma Irradiation in a Saturated Tuff Environment, Argonne National Laboratory ANL-90-13 (1990).

67. B. M. Biwer, J. K. Bates, T. A. Abrajano, Jr., and J. P. Bradley, "Comparison of the Layer Structure of Vapor Phase and Leached SRL Glass by Use of AEM," Mater. Res. Soc. Symp. Proc. 176, 255-263 (1990).

68. J. J. Mazer, J. K. Bates, B. M. Biwer, and C. R. Bradley, "AEM Analysis of SRL 131 Glass as a Function of SA/V," Mater. Res. Soc. Symp. Proc. 257, 73-81 (1992).

69. W. L. Ebert, J. K. Bates, E. C. Buck and C. R. Bradley, "Accelerated Glass Reaction Under PCT Conditions,” Mater. Res. Soc. Symp. Proc. 294, 569-576 (1993).

70. H. Yokayama, H. P. Hermansson, H. Christenson, I. K. Bjorner, and L. Werme, "Corrosion of Simulated Nuclear Waste Glass in a Gamma Radiation Field," Mater. Res. Soc. Symp. Proc. 44, 601-608 (1985).

71. D. J. Wronkiewicz, C. R. Bradley, L. M. Wang, and J. K. Bates, "Effects of Radiation Exposure on SRL 131 Glass in a Steam Environment," Mater. Res. Soc. Symp. Proc. $\underline{333}, 259-267$ (1994).

72. S. Sato, H. Furuya, K. Asakura, K. Ohita, and T. Tamai, "Radiation Effect on Simulated Waste Glass Irradiated with Ion, Electron, and Gamma Ray," Nucl. Instru. Meth. Phys. Res. B1, 534-537 (1984).

73. D. G. Howitt, H. W. Chan, J. F. DeNatale, and J. P. Heuer, "Mechanism for the Radiolytically Induced Decomposition of Soda-Silicate Glasses," J. Am. Ceram. Soc. 74, 1145-1147 (1991).

74. W. L. Ebert, J. K. Bates, and W. L. Bourcier, "The Hydration of Borosilicate Waste Glass in Liquid Water and Steam at $200^{\circ} \mathrm{C}$," Waste Manage. 11, 205-221 (1991). 
75. J. Caurel, E. Vernaz, and D. Beaufort, "Hydrothermal Leaching of R7T7 Borosilicate Glass,” Mater. Res. Soc. Symp. Proc. 176, 309-318 (1990).

76. M. J. Jercinovic, K. Keil, M. R. Smith and R. A. Schmitt, "Alteration of Basaltic Glasses from North-Central British Columbia, Canada," Geochim. Cosmochim. Acta $\underline{54}$, 2679-2696 (1990).

77. R. S. Forsyth and L. O. Werme, "Spent Fuel Corrosion and Dissolution," J. Nucl. Mater. 190, 3-19 (1992).

78. J. K. Bates and T. J. Gerding, Application of the NNWSI Unsaturated Test Method to Actinide Doped SRL 165 Type Glass, Argonne National Laboratory Report ANL-89/24 (1990) 83p.

79. D. M. Strachan, L. R. Pederson, and R. O. Lokken, "Results from the Long-Term Interaction and Modeling of SRL-131 Glass With Aqueous Solutions," Mater. Res. Soc. Symp. Proc. 50, 195-202 (1985).

80. T. A. Abrajano, Jr. and J. K. Bates, "Analytical Electron Microscopy of Leached Nuclear Waste Glasses," Ceram. Trans. 9, 211-228 (1990).

81. T. A. Abrajano, Jr., J. K. Bates, A. B. Woodland, J. P. Bradley, and W. L. Bourcier, "Secondary Phase Formation During Nuclear Waste-Glass Dissolution," Clays and Clay Miner. 38, 537-548 (1990).

82. J. K. Bates, W. L. Ebert, J. J. Mazer, J. P. Bradley, C. R. Bradley, and N. L. Dietz, "The Role of Surface Layers in Glass Leaching Performance," Mater. Res. Soc. Symp. Proc. $\underline{212}, 77-87$ (1991).

83. Personal Communication, L. Nuñez, Argonne National Laboratory (1995).

84. W. L. Ebert, J. K. Bates, E. C. Buck, M. Gong, and S. F. Wolf, "Disposition of Actinides Released from High Level Waste Glass," Ceram. Trans. (1994) 231-241.

85. D. L. Bish and J. L. Aronson, "Paleogeothermal and Paleohydrologic Conditions in Silicic Tuff From Yucca Mountain, Nevada,” Clay and Clay Miner. 41, 148-161 (1993).

86. J. E. Garcia Hernandez, J. S. Notario Del Pino, M. M. Gonzalez Martin, F. Hernan Reguera, and J. A. Rodriguez Losada, "Zeolites in Pyroclastic Deposits in Southeastern Tenerife (Canary Islands)," Clay and Clay Miner. 41, 521-526 (1993).

87. M. De'Gennaro, C. Colella, F. Franco, and D. Stanzione, "Hydrothermal Conversion of Trachytic Glass into Zeolite 1. Reactions with Deionized Water," Neues Jahrbuch Fur Mineralogie-Abhandlungen $\underline{4}, 149-158$ (1988).

88. J. A. Chermak, "Low Temperature Experimental Investigations of the Effect of High $\mathrm{pH}$ $\mathrm{KOH}$ Solutions on the Opalinus Shale, Switzerland," Clay and Clay Miner. 41, 365-372 (1993). 
APPENDIX A

Raw Analytical Data 
Table A.1. Dosimetry Analysis for Gamma Blank and Batch Tests.

Exposure Tube Scale O.D. O.D. Delta Dose Rate Bldg, Test

\begin{tabular}{|c|c|c|c|c|c|c|c|c|c|c|}
\hline Date & Time (h) & No. & Factor & Fricke Soln. & Blank Soln. & O.D. & Gy/h & Location & Oven & Comments \\
\hline $8 / 8 / 90$ & 1 & 5 & 1 & 0.216 & 0.058 & 0.158 & 43.54 & 223 & 3 & \\
\hline $8 / 8 / 90$ & 1 & 6 & 1 & 0.213 & 0.058 & 0.155 & 42.71 & 223 & 3 & \\
\hline $8 / 8 / 90$ & 1 & 3 & 1 & 0.207 & 0.058 & 0.149 & 41.06 & 223 & 3 & \\
\hline $8 / 8 / 90$ & 1 & 4 & 1 & 0.183 & 0.055 & 0.128 & 35.27 & 223 & 3 & \\
\hline $8 / 8 / 90$ & 1 & 8 & 1 & 0.145 & 0.055 & 0.090 & 24.80 & 223 & 3 & \\
\hline $8 / 13 / 90$ & 2 & 1 & 1 & 0.313 & 0.074 & 0.239 & 32.93 & 223 & 3 & \\
\hline $8 / 13 / 90$ & 2 & 2 & 1 & 0.348 & 0.074 & 0.274 & 37.75 & 223 & 3 & \\
\hline $8 / 13 / 90$ & 2 & 3 & 1 & 0.349 & 0.074 & 0.275 & 37.89 & 223 & 3 & \\
\hline $8 / 13 / 90$ & 2 & 4 & 1 & 0.329 & 0.075 & 0.254 & 35.00 & 223 & 3 & \\
\hline $8 / 13 / 90$ & 2 & 5 & 1 & 0.399 & 0.075 & 0.324 & 44.64 & 223 & 3 & \\
\hline $8 / 13 / 90$ & 2 & 6 & 1 & 0.434 & 0.075 & 0.359 & 49.46 & 223 & 3 & \\
\hline $8 / 13 / 90$ & 2 & 7 & 1 & 0.389 & 0.076 & 0.313 & 43.13 & 223 & 3 & \\
\hline $8 / 13 / 90$ & 2 & 8 & 1 & 0.388 & 0.076 & 0.312 & 42.99 & 223 & 3 & \\
\hline $8 / 13 / 90$ & 2 & 9 & 1 & 0.217 & 0.076 & 0.141 & 19.43 & 223 & 3 & \\
\hline $8 / 13 / 90$ & 2 & 10 & 1 & 0.230 & 0.075 & 0.155 & 21.36 & 223 & 3 & \\
\hline $8 / 13 / 90$ & 2 & 11 & 1 & 0.239 & 0.075 & 0.164 & 22.60 & 223 & 3 & \\
\hline $8 / 13 / 90$ & 2 & 12 & 1 & 0.250 & 0.075 & 0.175 & 24.11 & 223 & 3 & \\
\hline $8 / 13 / 90$ & 2 & 13 & 1 & 0.297 & 0.075 & 0.222 & 30.59 & 223 & 3 & \\
\hline $8 / 14 / 90$ & 3 & 1 & 1 & 0.528 & 0.080 & 0.448 & 41.15 & 223 & 4 & \\
\hline $8 / 14 / 90$ & 3 & 2 & 1 & 0.538 & 0.080 & 0.458 & 42.07 & 223 & 4 & \\
\hline $8 / 14 / 90$ & 3 & 3 & 1 & 0.543 & 0.080 & 0.463 & 42.53 & 223 & 4 & \\
\hline $8 / 14 / 90$ & 3 & 4 & 1 & 0.530 & 0.078 & 0.452 & 41.52 & 223 & 4 & \\
\hline $8 / 14 / 90$ & 3 & 5 & 1 & 0.659 & 0.078 & 0.581 & 53.37 & 223 & 4 & \\
\hline $8 / 14 / 90$ & 3 & 6 & 1 & 0.710 & 0.078 & 0.632 & 58.05 & 223 & 4 & \\
\hline $8 / 14 / 90$ & 3 & 7 & 1 & 0.729 & 0.082 & 0.647 & 59.43 & 223 & 4 & \\
\hline $8 / 14 / 90$ & 3 & 8 & 1 & 0.701 & 0.082 & 0.619 & 56.86 & 223 & 4 & \\
\hline $8 / 14 / 90$ & 3 & 9 & 1 & 0.324 & 0.082 & 0.242 & 22.23 & 223 & 4 & \\
\hline $8 / 14 / 90$ & 3 & 10 & 1 & 0.347 & 0.077 & 0.270 & 24.80 & 223 & 4 & \\
\hline $8 / 14 / 90$ & 3 & 11 & 1 & 0.338 & 0.077 & 0.261 & 23.97 & 223 & 4 & \\
\hline $8 / 14 / 90$ & 3 & 12 & 1 & 0.341 & 0.077 & 0.264 & 24.25 & 223 & 4 & \\
\hline $8 / 14 / 90$ & 3 & 13 & 1 & 0.454 & 0.077 & 0.377 & 34.63 & 223 & 4 & \\
\hline $2 / 19 / 91$ & 0.2028 & 1 & 1 & 0.423 & 0.020 & 0.403 & 547.59 & 200 & None & \\
\hline $2 / 19 / 91$ & 0.2028 & 2 & 1 & 0.399 & 0.020 & 0.379 & 514.98 & 200 & None & \\
\hline $2 / 19 / 91$ & 0.2028 & 3 & 1 & 0.398 & 0.020 & 0.378 & 513.62 & 200 & None & \\
\hline $2 / 19 / 91$ & 0.2028 & 4 & 1 & 0.433 & 0.020 & 0.413 & 561.17 & 200 & None & \\
\hline $2 / 19 / 91$ & 0.2028 & 5 & 1 & 0.356 & 0.020 & 0.336 & 456.55 & 200 & None & \\
\hline $2 / 19 / 91$ & 0.2028 & 6 & 1 & 0.380 & 0.020 & 0.360 & 489.16 & 200 & None & \\
\hline
\end{tabular}


Table A.1. Dosimetry Analysis for Gamma Blank and Batch Tests.

\begin{tabular}{|c|c|c|c|c|c|c|c|c|c|c|}
\hline Date & $\begin{array}{l}\text { Exposure } \\
\text { Time (h) }\end{array}$ & $\begin{array}{l}\text { Tube } \\
\text { No. }\end{array}$ & $\begin{array}{l}\text { Scale } \\
\text { Factor }\end{array}$ & $\begin{array}{c}\text { O.D. } \\
\text { Fricke Soln. }\end{array}$ & $\begin{array}{c}\text { O.D. } \\
\text { Blank Soln. }\end{array}$ & $\begin{array}{l}\text { Delta } \\
\text { O.D. }\end{array}$ & $\begin{array}{c}\text { Dose Rate } \\
\text { Gy/h }\end{array}$ & $\begin{array}{c}\text { Bldg. } \\
\text { Location }\end{array}$ & $\begin{array}{l}\text { Test } \\
\text { Oven }\end{array}$ & Comments \\
\hline $2 / 19 / 91$ & 4.0 & 7 & 1 & 0.550 & 0.020 & 0.530 & 36.51 & 200 & South & \\
\hline $2 / 19 / 91$ & 4.0 & 8 & 1 & 0.527 & 0.020 & 0.507 & 34.93 & 200 & South & \\
\hline $2 / 19 / 91$ & 4.0 & $9 a$ & 1 & 0.461 & 0.020 & 0.441 & 30.38 & 200 & South & \\
\hline $2 / 19 / 91$ & 4.0 & $9 b$ & 1 & 0.466 & 0.020 & 0.446 & 30.72 & 200 & South & \\
\hline 2/19/91 & 4.0 & $9 c$ & 1 & 0.469 & 0.020 & 0.449 & 30.93 & 200 & South & \\
\hline $2 / 19 / 91$ & 4.0 & 10 & 1 & 0.509 & 0.020 & 0.489 & 33.69 & 200 & South & \\
\hline $2 / 19 / 91$ & 4.0 & $11 a$ & 1 & 0.476 & 0.020 & 0.456 & 31.41 & 200 & South & \\
\hline $2 / 19 / 91$ & 4.0 & $11 b$ & 1 & 0.467 & 0.020 & 0.447 & 30.79 & 200 & South & \\
\hline $2 / 19 / 91$ & 4.0 & $11 c$ & 1 & 0.468 & 0.020 & 0.448 & 30.86 & 200 & South & \\
\hline $2 / 14 / 91$ & 0.5 & 1 & 0.1 & 0.790 & 0.550 & 0.240 & 13.23 & 200 & South & I" Pb shield \\
\hline $2 / 14 / 91$ & 0.5 & 2 & 0.1 & 0.768 & 0.550 & 0.218 & 12.01. & 200 & South & I"Pb shield \\
\hline $2 / 14 / 91$ & 0.5 & 3 & 0.1 & 0.745 & 0.550 & 0.195 & 10.75 & 200 & South & I" Pb shield \\
\hline $2 / 14 / 91$ & 0.5 & 4 & 0.1 & 0.743 & 0.550 & 0.193 & 10.64 & 200 & South & 1" Pb shield \\
\hline $2 / 14 / 91$ & 0.5 & 5 & 0.1 & 0.703 & 0.550 & 0.153 & 8.43 & 200 & South & 1" Pb shield \\
\hline
\end{tabular}

O.D., optical density of solution.; Blank, nonirradiated Fricke solution; $G y /$ h determined by eqn. $D D=$ delta O.D. $x$ 2.7556 E2/liradiation Time (h). Tube No. 9a, 9b, 9c and 11a, 11b, $11 \mathrm{c}$ represent triplicate ultraviolet analyses performed with multiple aliquots. 
APPENDIX B

Alpha Counting Results 
Table B.1.1. Anion Solution Volumes For Gamma Blank Tests.

\begin{tabular}{cccc} 
Test Number & $\begin{array}{c}\text { Test } \\
\text { Solution } \\
\text { Volume }(\mathrm{ml})\end{array}$ & $\begin{array}{c}\text { Aliquots } \\
\text { Volume } \\
\text { Removed }(\mathrm{ml})\end{array}$ & $\begin{array}{c}\text { Rinse } \\
\text { Volume } \\
\text { Added (ml) }\end{array}$ \\
\hline IV9000-7-G1B-100 & 0.22 & 0.10 & 0.97 \\
IV9000-7-G2B-100 & 0.22 & 0.10 & 1.43 \\
IV9000-14-G1B-100 & 0.22 & 0.10 & 2.14 \\
IV9000-14-G2B-100 & 0.22 & 0.10 & 1.79 \\
IV9000-28-G1B-100 & 0.21 & 0.10 & 1.73 \\
IV9000-28-G2B-100 & 0.21 & 0.10 & 1.60 \\
IV9000-56-G1B-100 & 0.22 & 0.10 & 0.81 \\
IV9000-56-G2B-100 & 0.22 & 0.10 & 1.21 \\
IV9000-120-G1B-100 & 0.21 & 0.10 & 1.22 \\
IV9000-120-G2B-100 & 0.22 & 0.10 & 1.01 \\
& & & \\
IV9000-28-G1-100 & 0.22 & 0.10 & 1.26 \\
IV9000-28-G2-100 & 0.21 & 0.10 & 1.40 \\
IV9000-56-G1-100 & 0.21 & 0.10 & 1.47 \\
IV9000-56-G2-100 & 0.22 & 0.10 & 1.14 \\
IV9000-120-G1-100 & 0.19 & 0.10 & 2.03 \\
IV9000-120-G2-100 & 0.20 & 0.10 & 2.07 \\
IV9000-14-G1-100 & 0.17 & 0.10 & 1.60 \\
IV9000-14-G2-100 & 0.20 & 0.10 & 1.50 \\
& & & \\
IV1000-56-G3-100 & 0.22 & 0.10 & 3.22 \\
IV1000-56-G4-100 & 0.22 & 0.10 & 1.93 \\
IV1000-85-G1-100 & 0.20 & 0.10 & 1.33 \\
IV1000-85-G2-100 & 0.21 & 0.10 & 1.14 \\
IV1000-120-G1-100 & 0.22 & 0.10 & 3.13 \\
IV1000-120-G2-100 & 0.22 & 0.10 & 3.95 \\
IV1000-150-G1-100 & 0.22 & 0.10 & 1.84 \\
IV1000-150-G2-100 & 0.21 & 0.10 & 1.37 \\
IV1000-56-G1-100 & 0.22 & 0.10 & 2.24 \\
IV1000-56-G2-100 & 0.22 & 0.10 & 2.08 \\
\hline
\end{tabular}


Table B.1.1. (cont.). Anion Solution Volumes For Gamma Blank Tests.

\begin{tabular}{cccc} 
Test Number & $\begin{array}{c}\text { Test } \\
\text { Solution } \\
\text { Volume (ml) }\end{array}$ & $\begin{array}{c}\text { Aliquots } \\
\text { Volume } \\
\text { Removed (ml) }\end{array}$ & $\begin{array}{c}\text { Rinse } \\
\text { Volume } \\
\text { Added (ml) }\end{array}$ \\
\hline IV2000-56-G3-100 & 0.22 & 0.10 & 2.04 \\
IV2000-56-G4-100 & 0.22 & 0.10 & 2.52 \\
IV2000-85-G1-100 & 0.21 & 0.10 & 1.09 \\
IV2000-85-G2-100 & 0.19 & 0.10 & 0.79 \\
IV2000-120-G1-100 & 0.17 & 0.10 & 3.66 \\
IV2000-120-G2-100 & 0.16 & 0.10 & 2.61 \\
IV2000-150-G1-100 & 0.22 & 0.10 & 0.72 \\
IV2000-150-G2-100 & 0.16 & 0.10 & 0.75 \\
IV2000-56-G1-100 & 0.22 & 0.10 & 2.73 \\
IV2000-56-G2-100 & 0.14 & 0.10 & 2.4 \\
& & & \\
IV1000-56-G1-10 & 1.99 & 0.85 & 1.26 \\
IV1000-56-G2-10 & 1.98 & 0.85 & 2.05 \\
IV1000-120-G1-10 & 2.00 & 0.8 & 0.93 \\
IV1000-120-G2-10 & 1.99 & 0.8 & 1.04 \\
& & & \\
IV9000-28-BLANK-100 & 0.22 & 0.10 & 2.36 \\
IV9000-120-BLANK-100 & 0.22 & 0.10 & 1.57 \\
IV1000-56-BLANK-100 & 0.22 & 0.10 & 0.7 \\
IV1000-120-BLANK-100 & 0.22 & 0.10 & 1.72 \\
IV2000-56-BLANK-100 & 0.21 & 0.10 & 2.32 \\
IV2000-120-BLANK-100 & 0.21 & 0.10 & 1.44 \\
\hline
\end{tabular}


Table B.1.2. Anion Solution Volumes For Alpha Blank Tests.

\begin{tabular}{cccc} 
Test Number & $\begin{array}{c}\text { Test } \\
\text { Solution } \\
\text { Volume }(\mathrm{ml})\end{array}$ & $\begin{array}{c}\text { Aliquots } \\
\text { Volume } \\
\text { Removed }(\mathrm{ml})\end{array}$ & $\begin{array}{c}\text { Rinse } \\
\text { Volume } \\
\text { Added (ml) }\end{array}$ \\
\hline IV9000-14-A1-100 & 17.8 & 10.85 & 13.2 \\
IV9000-14-A2-100 & 18.03 & 10.85 & 15 \\
IV9000-28-A1-100 & 17.66 & 11 & 18 \\
IV9000-28-A2-100 & 17.68 & 11 & 18.5 \\
IV9000-56-A1-100 & 17.45 & 11.35 & 18.9 \\
IV9000-56-A2-100 & 17.52 & 10.85 & 16.8 \\
& & & \\
IV9000-14-A1S-100 & 17.1 & 10.85 & 13.6 \\
IV9000-14-A2S-100 & 16.56 & 10.85 & 14.7 \\
IV9000-28-A1S-100 & 16.18 & 10.85 & 14.5 \\
IV9000-28-A2S-100 & 14.26 & 10.85 & 13.4 \\
IV9000-45-A1S-100 & 15.25 & 11 & 26.07 \\
IV9000-45-A2S-100 & 14.69 & 11 & 25.81 \\
IV9000-56-A1S-100 & 12.74 & 10.85 & 14 \\
IV9000-56-A2S-100 & 14.41 & 10.85 & 12.9 \\
IV9000-85-A1S-100 & 15.14 & 11 & 16.24 \\
IV9000-85-A2S-100 & 14.42 & 11 & 15.5 \\
IV9000-110-A1S-100 & 7.31 & 7.06 & 15.68 \\
IV9000-110-A2S-100 & 5.5 & 5.42 & 22.83 \\
IV9000-120-A1S-100 & 12.2 & 11.3 & 35.4 \\
IV9000-120-A2S-100 & 10.3 & 9.1 & 65.5 \\
& & & \\
IV9000-45-BLANK-100 & 13.83 & 11 & 23.43 \\
IV9000-110-BLANK-100 & 9.47 & 9.26 & 25.72 \\
\hline
\end{tabular}


Table B.2.1. Row Cation Results, Batch Leach Tests at $340 \mathrm{~m}^{-1}$ (Values in ppm).

\begin{tabular}{|c|c|c|c|c|c|c|c|c|c|c|c|c|c|c|c|c|c|c|c|c|}
\hline Test Number & Al & B & $\mathrm{Ba}$ & $\mathrm{Ca}$ & $\mathrm{Cr}$ & $\mathrm{Cu}$ & $\mathrm{Fe}$ & $K$ & $\mathrm{Li}$ & $\mathrm{Mg}$ & $\mathrm{Mn}$ & $\mathrm{Na}$ & $\mathrm{Ni}$ & $\mathrm{Si}$ & $\mathrm{Sr}$ & Th & U & Ti & $Z n$ & $\mathrm{Zr}$ \\
\hline V9202A-14-1 & 0.11 & 0.50 & 0.02 & $0.09<$ & 0.02 & 0.03 & 0.19 & 1.11 & 0.57 & 0.03 & 0.03 & 5.69 & 0.03 & $5.28<$ & $0.01<$ & 0.1 & $0.151<$ & 0.01 & 0.01 & 0.02 \\
\hline V9202A-28-1 & 12 & 0.56 & 0.02 & 09 & 0.02 & .04 & 0.24 & 1.1 & 0.63 & & & & (2007 & & 01 & & & & & 0.0 \\
\hline $8-2$ & 0.10 & 54 & 0.02 & 2 & 0.02 & & 0.23 & 1.21 & 0.64 & & & & & 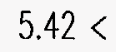 & $01<$ & 0.1 & 26 & 01 & & 0.02 \\
\hline V920 & 0.10 & 69 & 0.02 & 07 & 0.02 & 0.05 & 0.28 & 1.25 & 0.75 & 04 & & 6.3 & 0.03 & $6.13<$ & $0.01<$ & 0.1 & .072 & 0.01 & 0.02 & 0.02 \\
\hline$-2<$ & 0.10 & .96 & 0.02 & $.06<$ & 0.02 & 0.08 & 0.42 & 1.38 & 0.85 & 0.05 & & 6.46 & 0.04 & 6.6 & $0.01<$ & 0.1 & 079 & 0.01 & 0.02 & 0.02 \\
\hline $\mathrm{V} 9202$ & 0.33 & 0.50 & 0.03 & .39 & 0.64 & 9.22 & 4.22 & 0.87 & 0.52 & 0.18 & 66 & 4.37 & 0.35 & 5.84 & 0.01 & 0.05 & & 0.04 & & 0.05 \\
\hline 920 & 0.10 & 0.55 & 0.02 & 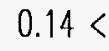 & 0.02 & 0.02 & 0.16 & 1.17 & 0.68 & 0.03 & & 6.4 & 0.03 & & & 35 & $r$ & . & & 0.05 \\
\hline-3 & 10 & 80 & 0.02 & & 0.02 & 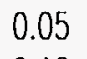 & 0.47 & 1.54 & 1.11 & 5 & & & 0.03 & 7.0 & or & .05 & & 0.02 & & 0.05 \\
\hline-4 & 0.22 & .08 & 0.02 & & 0.02 & & 1.08 & 61 & & & & 02 & 15 & 7.5 & & 15 & 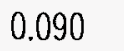 & 0.03 & & 0.05 \\
\hline-3 & 0.16 & .15 & 0.02 & 0 & $0.03<$ & 0.02 & 0.29 & 2.16 & 1.59 & 5 & & 06 & 0.03 & 6.5 & . & 05 & 0.137 & 0.02 & & 0.05 \\
\hline-1 & 0.10 & 1.6 & 0.02 & 0.05 & 0.26 & 0.04 & 0.46 & 1.92 & $1.42<$ & 0.01 & 0. & 32 & 0.05 & 6.01 & $0.01<$ & .05 & 0.14 & 0.02 & & 0.05 \\
\hline 2 & 0.10 & 1.34 & 0.02 & 0.77 & $0.05<$ & 0.02 & 0.17 & 1.91 & 1.30 & 0.02 & 0.02 & $03<$ & 0.03 & 5.49 & ( & 0.05 & & 0.02 & & 0.05 \\
\hline-1 & 0.37 & 1.85 & 0.02 & 0.17 & 0.08 & 0.18 & 1.49 & 2.45 & 1.63 & 016 & & & 0.09 & 9.22 & . & & & . & & 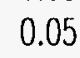 \\
\hline & 0.1 & & & & & & & & & & & & & & & & & & & \\
\hline & & & & & & & & & & & & & & & & & & & & \\
\hline & 10 & $1 \mathrm{~J}$ & 0.02 & 12 & 0.02 & 0.02 & 0.08 & 35 & 1.01 & 0.01 & & 68 & 03 & 5.4 & & 05 & & 1.0 & & 0.05 \\
\hline & 0.10 & & 0.02 & 0.19 & 0.02 & 0.02 & 0.06 & 0.49 & 95 & 0.01 & 0. & 27 & 0.03 & 0.4 & 0.0 & 05 & & 0.0 & & 0.05 \\
\hline-1 & 0.10 & 57 & 0.02 & 0.1 & 0.02 & 0.02 & 0.02 & 0.55 & 0.9 & 0.0 & 0.0 & & 0.03 & 5.0 & 0.0 & 0.05 & & & & 0.05 \\
\hline-2 & 0.10 & .83 & 0.02 & 0.16 & 0.05 & 0.07 & 0.43 & 0.61 & 1.26 & 3 & & 21 & 0.03 & 7.40 & 0.01 & 0.05 & 0.2 & 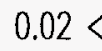 & & . \\
\hline-1 & 0.30 & 11 & 0.01 & & 0.27 & 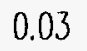 & 0.5 & 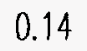 & 3.41 & 0.0 & & & 0 & 12.2 & no & & . & 0.0 & & 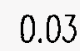 \\
\hline $0-2$ & 0.40 & 360 & 0.01 & 31 & 0.04 & 0.02 & 0.55 & .18 & 2.77 & 005 & 0.25 & 14.9 & 0.07 & 12.7 & no & ח & 0.055 & .01 & 年 & 0.03 \\
\hline & & & 002 & & $0.02<$ & 002 & 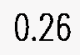 & 174 & & & & & & & & & & & & $00 ?$ \\
\hline & 0 & & 0.02 & 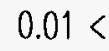 & 0.02 & 0.05 & 0.33 & 1.77 & 0 & & & & 0.03 & 6.8 & 0.6 & & & & & 0. \\
\hline-1 & 0.12 & 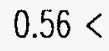 & 0.02 & 0.09 & 0. & 0.1 & 0.24 & 1.10 & 0.63 & & & & 0.03 & & 0.0 & 0.10 & & & & 0.1 \\
\hline & 0. & 2.00 & 0.02 & & 0.02 & 0.0 & 0.52 & 2.09 & 0 & & & & 0.03 & 7.5 & & 0. & & & & 0.02 \\
\hline$-1<$ & 0.10 & 180 & 0.02 & 0.02 & 0.02 & 0.0 & 0.48 & 1.91 & 0.89 & & & $93<$ & 0.03 & & 001 & & & 1 & & 0.02 \\
\hline $6-2$ & 0.13 & $2.06<$ & $0.02<$ & $0.02<$ & 0.02 & 0.04 & 0.63 & 1.96 & 0.91 & 0.09 & 0. & & 0.04 & & 0.01 & 0.10 & 0.231 & 0.01 & 0.01 & 0.02 \\
\hline$-56-3$ & 0.10 & 220 & 0.02 & $0.03<$ & 0.02 & 0.02 & 0.19 & 2.18 & 1.03 & 0.04 & 0. & 0.8 & 0.03 & 7.9 & 0.01 & 0.05 & 0.219 & 0.02 & 0.0 & 0.05 \\
\hline $6-4$ & 0.15 & 1. & 0.02 & 0.04 & 0.03 & 0.02 & 0.55 & 2.00 & 0.91 & & & & 0.04 & 6.97 & 0.01 & 0.05 & 0.31 & 0.02 & 0.0 & 0.05 \\
\hline & 0.16 & 2.8 & 0.02 & 0. & 0.04 & 0. & 0.89 & 2.45 & 1.23 & & & & 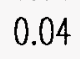 & 7.96 & 0.01 & 政 & & 0.0 & & 0.05 \\
\hline & 0.2 & 3.9 & 0.01 & 0. & 0. & 0.1 & 0.58 & 3.1 & 1.78 & & & & 0.01 & .0 & na & & 0.329 & 0.02 & 0.6 & 0.01 \\
\hline $19131 \mathrm{~A}-720-2$ & 0.55 & 4.21 & 0.01 & 0.34 & 0.25 & 0.03 & 2.29 & 3.56 & 1.87 & 0.35 & 0.48 & 17.3 & 0.30 & 13.9 & na & 0.01 & 0.313 & 0.05 & 0.04 & 0.02 \\
\hline
\end{tabular}


Table B.2.2. Cation Results, Batch Leach Tests at $340 \mathrm{~m}^{-1}$ (Total $\mu \mathrm{g}$ Released).

Soln. Vol

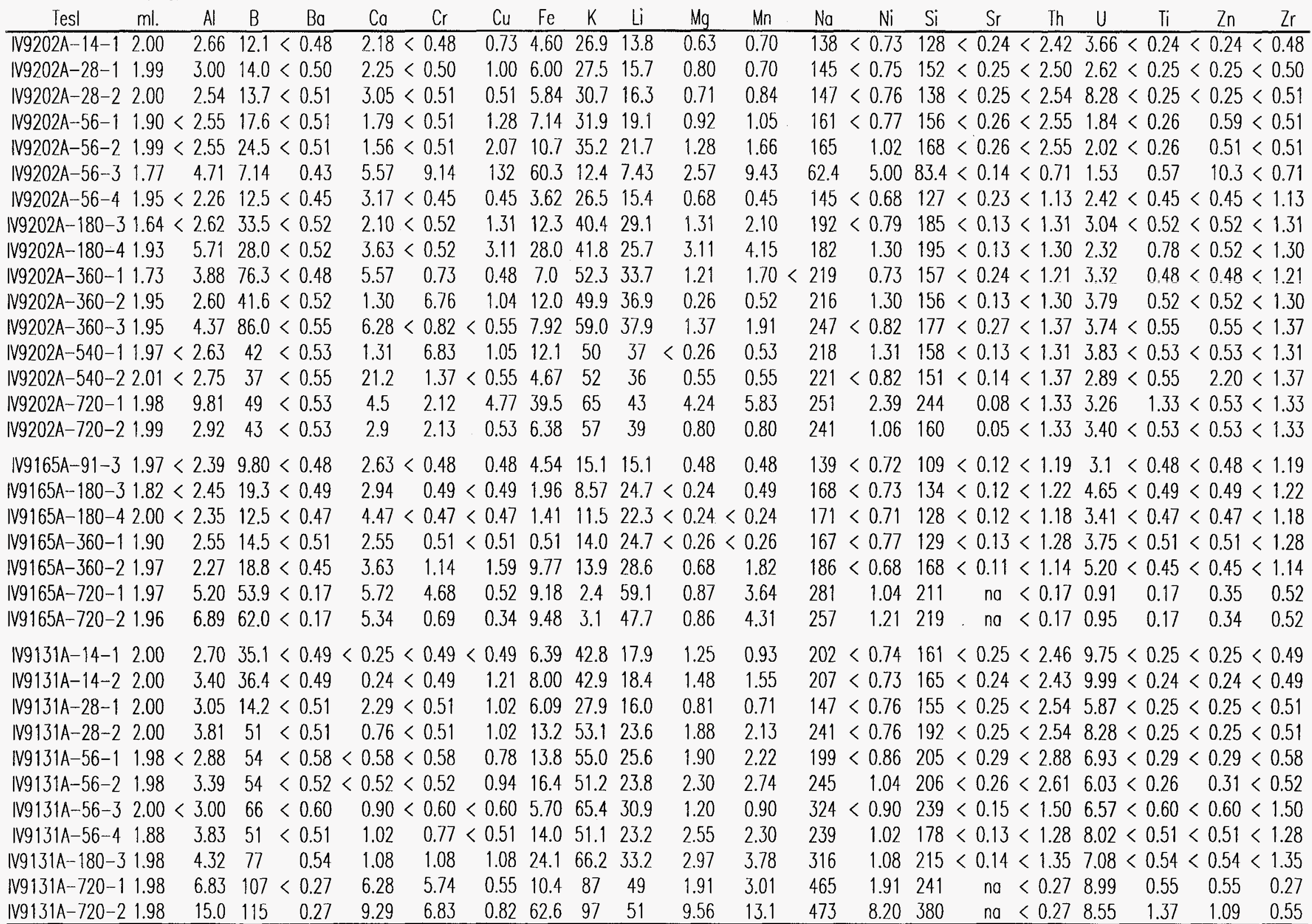
Total ug released $=$ Soln. Conc. $($ ppm) $*$ Initial Soln. Vol. $(\mathrm{mll})$ 
Table B.2.3. Sample Weights and Aliquot Volumes, Batch Leach Tests at $340 \mathrm{~m}^{-1}$.

Tare Wgt. w/ Dilluted Sample Dillution ACL-DIW Carbon Anion . Planch pH ... Planch Weight Sample Weight Volume Volume Added Aliquot Aliquot Aliquot Aliquot Aliquot

\begin{tabular}{|c|c|c|c|c|c|c|c|c|c|c|c|}
\hline Test Number & (g) & (g) & (g) & $(\mathrm{mL})$ & $(\mathrm{mL})$ & $(\mathrm{mL})$ & $(\mathrm{mL})$ & $(\mathrm{mL})$ & $(\mathrm{mL})$ & $(\mathrm{mL})$ & $(\mathrm{mL})$ \\
\hline IV9202A-14-1 & 24.28 & 25.63 & 35.62 & 1.35 & 9.99 & 5 & none & none & none & none & 0.05 \\
\hline IV9202A-28-1 & 24.24 & 25.54 & 35.56 & 1.30 & 10.02 & 5 & 0.25 & 0.1 & 0.05 & none & 0.05 \\
\hline IV9202A-28-2 & 24.09 & 25.37 & 35.35 & 1.28 & 9.98 & 5 & 0.25 & 0.1 & 0.05 & none & none \\
\hline IV9202A-56-1 & 24.31 & 25.52 & 35.55 & 1.21 & 10.03 & 5 & 0.25 & 0.1 & 0.05 & none & 0.05 \\
\hline IV9202A-56-2 & 24.28 & 25.55 & 35.57 & 1.27 & 10.02 & 5 & 0.25 & 0.1 & 0.05 & none & 0.05 \\
\hline IV9202A-56-3 & 11.08 & 12.51 & 22.62 & 1.43 & 10.11 & 0 & 0.05 & 0.05 & 0.05 & none & none \\
\hline IV9202A-56-4 & 11.07 & 12.5 & 22.67 & 1.43 & 10.17 & 5 & 0.05 & 0.1 & 0.05 & none & 0.05 \\
\hline IV9202A-180-3 & 11.07 & 12.07 & 22.05 & 1.00 & 9.98 & 5 & 0.1 & 0.1 & 0.05 & none & 0.10 \\
\hline IV9202A-180-4 & 11.09 & 12.29 & 22.22 & 1.20 & 9.93 & 5 & 0.1 & 0.1 & 0.05 & none & 0.10 \\
\hline IV9202A-360-3 & 19.16 & 20.36 & 30.96 & 1.20 & 10.60 & 5 & 0.1 & 0.1 & 0.05 & none & 0.10 \\
\hline IV9202A-540-1 & 11.08 & 12.28 & 22.07 & 1.20 & 9.79 & 5 & 0.1 & 0.1 & 0.05 & none & 0.05 \\
\hline IV9202A-540-2 & 11.01 & 12.21 & 22.42 & 1.20 & 10.21 & 5 & 0.1 & 0.1 & 0.05 & none & 0.10 \\
\hline IV9202A-720-1 & 19.19 & 20.39 & 30.26 & 1.20 & 9.87 & 5 & 0.1 & 0.1 & 0.05 & none & 0.05 \\
\hline IV9202A-720-2 & 19.30 & 20.5 & 30.33 & 1.20 & 9.83 & 5 & 0.1 & 0.1 & 0.05 & none & 0.05 \\
\hline IV9165A-91-3 & 11.13 & 12.33 & 22.11 & 1.20 & 9.78 & 5 & 0.1 & 0.1 & 0.05 & none & 0.05 \\
\hline IV9165A-180-3 & 11.04 & 12.24 & 22.18 & 1.20 & 9.94 & 5 & 0.1 & 0.1 & 0.05 & none & 0.10 \\
\hline IV9165A-180-4 & 11.43 & 12.83 & 22.88 & 1.40 & 10.05 & 5 & 0.1 & 0.1 & 0.05 & none & 0.10 \\
\hline IV9165A-360-1 & 10.96 & 12.18 & 22.34 & 1.22 & 10.16 & 5 & 0.1 & 0.1 & 0.05 & none & 0.10 \\
\hline IV9165A-360-2 & 11.03 & 12.47 & 22.63 & 1.44 & 10.16 & 5 & 0.05 & 0.1 & 0.05 & none & none \\
\hline IV9165A-720-1 & 11.07 & 12.37 & 22.50 & 1.30 & 10.13 & 0 & 0.1 & 0.1 & 0.05 & none & 0.10 \\
\hline IV9165A & 11.09 & 12.39 & 22.52 & 1.30 & 10.13 & 0 & 0.1 & 0.1 & 0.05 & none & 0.10 \\
\hline IV9131 & 24.44 & 25.77 & 35.78 & 1.33 & 10.01 & 5 & none & none & none & none & none \\
\hline IV9131A-14-2 & 24.3 & 25.65 & 35.67 & 1.35 & 10.02 & 5 & none & none & none & none & none \\
\hline IV9131A-28-1 & 24.35 & 25.63 & 35.6 & 1.28 & 9.97 & 5 & 0.25 & 0.1 & 0.05 & none & 0.05 \\
\hline IV9131A-28-2 & 24.2 & 25.48 & 35.45 & 1.28 & 9.97 & 5 & 0.25 & none & 0.05 & none & 0.05 \\
\hline IV9131A-56-1 & 24.4 & 25.51 & 35.53 & 1.11 & 10.02 & 5 & 0.25 & 0.1 & 0.05 & none & 0.05 \\
\hline IV9131A-56-2 & 24.48 & 25.71 & 35.70 & 1.23 & 9.99 & 5 & 0.25 & 0.1 & 0.05 & none & 0.05 \\
\hline IV9131A-56-3 & 11.09 & 12.17 & 22.30 & 1.08 & 10.13 & 5 & 0.05 & 0.1 & 0.05 & none & 0.10 \\
\hline IV9131A-56-4 & 11.09 & 12.29 & 22.39 & 1.20 & 10.10 & 5 & 0.1 & 0.1 & 0.05 & none & 0.10 \\
\hline IV9131A-180-3 & 11.01 & 12.21 & 22.39 & 1.20 & 10.18 & 5 & 0.1 & 0.1 & 0.05 & none & 0.10 \\
\hline IV9131A-720-1 & 11.03 & 11.15 & 22.24 & 1.20 & 11.09 & 0 & 0.1 & 0.1 & 0.05 & none & 0.05 \\
\hline IV9131A-720-2 & 11.11 & 11.21 & 22.30 & 1.20 & 11.09 & 0 & 0.1 & 0.1 & 0.05 & none & 0.10 \\
\hline
\end{tabular}


Appendix B.3.1. Glass Monolith Geometric Surface Areas.

\begin{tabular}{|c|c|c|c|c|c|c|c|}
\hline Test Number & $\begin{array}{c}\text { Disk } \\
\text { ID }\end{array}$ & $\begin{array}{l}\text { Mass } \\
\text { (g) }\end{array}$ & $\begin{array}{c}\text { Rodius } \\
\mathrm{mm}\end{array}$ & $\begin{array}{c}\text { Thickness } \\
\text { mm }\end{array}$ & $\begin{array}{l}\text { Calc. } \\
\text { Vol. } \\
\mathrm{mm}^{3}\end{array}$ & $\begin{array}{c}\text { Surface } \\
\text { Area } \\
\mathrm{mm}^{2}\end{array}$ & $\begin{array}{l}\text { Total } \\
\text { Area } \\
\mathrm{mm}^{2}\end{array}$ \\
\hline \multirow[t]{4}{*}{ IV9202A-14-1 } & $A$ & 0.15067 & $4.800 !$ & 0.7548 & 54.64 & 167.54 & \\
\hline & B & 0.16012 & 4.8001 & 0.8021 & 58.06 & 168.96 & \\
\hline & $\mathrm{C}$ & 0.17156 & 4.8001 & 0.8594 & 62.21 & 170.69 & \\
\hline & $D$ & 0.16803 & 4.8001 & 0.8418 & 60.93 & 170.16 & 677.35 \\
\hline \multirow[t]{4}{*}{ IV9202A-28-1 } & $A$ & 0.18732 & 4.8001 & 0.9384 & 67.93 & 173.07 & \\
\hline & $B$ & 0.16041 & 4.8001 & 0.8036 & 58.17 & 169.01 & \\
\hline & $\mathrm{C}$ & 0.15345 & 4.8001 & 0.7687 & 55.64 & 167.96 & \\
\hline & $D$ & 0.15790 & 4.8001 & 0.7910 & 57.26 & 168.63 & 678.66 \\
\hline \multirow[t]{4}{*}{ IV9202A-28-2 } & $A$ & 0.14489 & 4.8001 & 0.7258 & 52.54 & 166.66 & \\
\hline & B & 0.15747 & 4.8001 & 0.78899 & 57.10 & 168.56 & \\
\hline & $\mathrm{C}$ & 0.15519 & 4.8001 & 0.7774 & 56.28 & 168.22 & \\
\hline & D & 0.17392 & 4.8001 & 0.8713 & 63.07 & 171.05 & 674.49 \\
\hline \multirow{4}{*}{ IV $9202 A-56-1$} & $A$ & 0.15978 & 4.8001 & 0.80014 & 57.94 & 168.91 & \\
\hline & B & 0.16204 & 4.8001 & 0.8118 & 58.76 & 169.25 & \\
\hline & C & 0.16348 & 4.8001 & 0.8190 & 59.28 & 169.47 & \\
\hline & $D$ & 0.16230 & 4.8001 & 0.8131 & 58.85 & 169.29 & 676.93 \\
\hline \multirow[t]{4}{*}{ IV9202A-56-2 } & $A$ & 0.17217 & 4.8001 & 0.8625 & 62.43 & 170.78 & \\
\hline & $B$ & 0.16350 & 4.8001 & 0.8191 & 59.29 & 169.47 & \\
\hline & $C$ & 0.16866 & 4.8001 & 0.8449 & 61.16 & 170.25 & \\
\hline & $D$ & 0.18975 & 4.8001 & 0.9506 & 68.81 & 173.44 & 683.95 \\
\hline \multirow[t]{4}{*}{ IV9202A-56-3 } & $A$ & 0.15853 & 4.8001 & 0.7942 & 57.49 & 168.72 & \\
\hline & $B$ & 0.17386 & 4.8001 & 0.8710 & 63.05 & 171.04 & \\
\hline & $\mathrm{C}$ & 0.14625 & 4.8001 & 0.7327 & 53.03 & 166.87 & \\
\hline & $D$ & 0.14385 & 4.8001 & 0.7206 & 52.16 & 166.51 & 673.14 \\
\hline \multirow[t]{4}{*}{ IV9202A-56-4 } & $A$ & 0.17621 & 4.8001 & 0.8827 & 63.90 & 171.39 & \\
\hline & $B$ & 0.17295 & 4.8001 & 0.8664 & 62.72 & 170.90 & \\
\hline & C & 0.18199 & 4.8001 & 0.9117 & 65.99 & 172.27 & \\
\hline & $D$ & 0.17375 & 4.8001 & 0.8704 & 63.01 & 171.02 & 685.59 \\
\hline \multirow[t]{4}{*}{ IV9202A-180-3 } & $A$ & 0.14996 & 4.8001 & 0.7512 & 54.38 & 167.43 & \\
\hline & $B$ & 0.17846 & 4.8001 & 0.8940 & 64.71 & 171.73 & \\
\hline & C & 0.17522 & 4.8001 & 0.8778 & 63.54 & 171.24 & \\
\hline & $D$ & 0.16850 & 4.8001 & 0.8441 & 61.10 & 170.23 & 680.64 \\
\hline
\end{tabular}


Appendix B.3.1. (cont.). Glass Monolith Geometric Surface Areas.

\begin{tabular}{|c|c|c|c|c|c|c|c|}
\hline Test Number & $\begin{array}{c}\text { Disk } \\
\text { ID }\end{array}$ & $\begin{array}{c}\text { Mass } \\
(\mathrm{g})\end{array}$ & $\begin{array}{c}\text { Rodius } \\
\mathrm{mm}\end{array}$ & $\begin{array}{c}\text { Thickness } \\
\mathrm{mm}\end{array}$ & $\begin{array}{l}\text { Calc. } \\
\text { Vol. } \\
\mathrm{mm}^{3}\end{array}$ & $\begin{array}{c}\text { Surface } \\
\text { Areo } \\
\mathrm{mm}^{2} \\
\end{array}$ & $\begin{array}{l}\text { Total } \\
\text { Area } \\
\mathrm{mm}^{2} \\
\end{array}$ \\
\hline \multirow[t]{4}{*}{ IV9202A-180-4 } & A & 0.16488 & 4.8001 & 0.8260 & 59.79 & 169.68 & \\
\hline & $B$ & 0.17121 & 4.8001 & 0.8577 & 62.08 & 170.64 & \\
\hline & C & 0.17008 & 4.8001 & 0.8520 & 61.67 & 170.47 & \\
\hline & 0 & 0.17119 & 4.8001 & 0.8576 & 62.08 & 170.64 & $\theta$ \\
\hline \multirow[t]{4}{*}{ IV9202A-360-1 } & A & 0.15951 & 4.8001 & 0.7991 & 57.84 & 168.87 & \\
\hline & $B$ & 0.15837 & 4.8001 & 0.7934 & 57.43 & 168.70 & \\
\hline & $C$ & 0.16033 & 4.8001 & 0.8032 & 58.14 & 169.00 & \\
\hline & $D$ & 0.16520 & 4.8001 & 0.8276 & 59.90 & 169.73 & 676. \\
\hline \multirow[t]{4}{*}{ IV9202A-360-2 } & A & 0.17126 & 4.8001 & 0.8579 & 62.10 & 170.65 & \\
\hline & $B$ & 0.15793 & 4.8001 & 0.7912 & 57.27 & 168.63 & \\
\hline & C & 0.16372 & 4.8001 & 0.8202 & 59.37 & 169.51 & \\
\hline & $D$ & 0.14386 & 4.8001 & 0.7207 & 52.17 & 166.51 & 675.2 \\
\hline \multirow[t]{4}{*}{ IV9202A-360-3 } & A & 0.15071 & 4.8001 & 0.7550 & 54.65 & 167.54 & \\
\hline & B & 0.15777 & 4.8001 & 0.7904 & 57.21 & 168.61 & \\
\hline & $C$ & 0.14955 & 4.8001 & 0.7492 & 54.23 & 167.37 & \\
\hline & D & 0.17240 & 4.8001 & 0.8637 & 62.52 & 170.82 & 674.3 \\
\hline \multirow[t]{4}{*}{ N9202A-540-1 } & A & 0.16715 & 4.8001 & 0.8374 & 60.61 & 170.03 & \\
\hline & B & 0.18478 & 4.8001 & 0.9257 & 67.01 & 172.69 & \\
\hline & C & 0.16522 & 4.8001 & 0.8277 & 59.91 & 169.73 & \\
\hline & D & 0.16853 & 4.8001 & 0.8443 & 61.11 & 170.23 & 682 \\
\hline \multirow[t]{4}{*}{ IV $9202 A-540-2$} & A & 0.16266 & 4.8001 & 0.8149 & 58.98 & 169.35 & \\
\hline & B & 0.15442 & 4.8001 & 0.7736 & 56.00 & 168.10 & \\
\hline & C & 0.17350 & 4.8001 & 0.8692 & 62.91 & 170.98 & \\
\hline & D & 0.16218 & 4.8001 & 0.8125 & 58.81 & 169.27 & 677 \\
\hline \multirow[t]{4}{*}{ IV9202A-720-1 } & A & 0.23105 & 4.8001 & 1.1575 & 83.78 & 179.68 & \\
\hline & B & 0.16600 & 4.8001 & 0.8316 & 60.20 & 169.85 & \\
\hline & C & 0.14822 & 4.8001 & 0.7425 & 53.75 & 167.17 & \\
\hline & D & 0.16695 & 4.8001 & 0.8363 & 60.54 & 170.00 & 686 \\
\hline \multirow[t]{4}{*}{ N9202A-720-2 } & A & 0.15927 & 4.8001 & 0.7979 & 57.75 & 168.83 & \\
\hline & B & 0.15398 & 4.8001 & 0.7714 & 55.84 & 168.04 & \\
\hline & C & 0.16233 & 4.8001 & 0.8132 & 58.86 & 169.30 & \\
\hline & D & 0.15319 & 4.8001 & 0.7674 & 55.55 & 167.92 & \\
\hline
\end{tabular}

SRL 202A Glass - Calculated Average Density $=2.76 \mathrm{~g} / \mathrm{cm}^{3}$ 
Appendix B.3.1. (cont.). Glass Monolith Geometric Surface Areas.

\begin{tabular}{|c|c|c|c|c|c|c|c|}
\hline Test Number & $\begin{array}{c}\text { Disk } \\
\text { ID } \\
\end{array}$ & $\begin{array}{c}\text { Mass } \\
(\mathrm{g})\end{array}$ & $\begin{array}{c}\text { Rodius } \\
\mathrm{mm}\end{array}$ & $\begin{array}{c}\text { Thickness } \\
\text { mm }\end{array}$ & $\begin{array}{l}\text { Calc. } \\
\text { Vol. } \\
\mathrm{mm}^{3}\end{array}$ & $\begin{array}{c}\text { Surface } \\
\text { Area } \\
\mathrm{mm}^{2}\end{array}$ & $\begin{array}{l}\text { Total } \\
\text { Area } \\
\mathrm{mm}^{2} \\
\end{array}$ \\
\hline \multirow[t]{4}{*}{ IV9165A-91-3 } & $A$ & 0.15285 & 4.7865 & 0.7442 & 55.85 & 166.33 & \\
\hline & B & 0.15917 & 4.7915 & 0.8331 & 58.16 & 169.33 & \\
\hline & $C$ & 0.15781 & 4.7715 & 0.8077 & 57.66 & 167.27 & \\
\hline & $D$ & 0.14779 & 4.7230 & 0.7772 & 54.00 & 163.22 & 666.15 \\
\hline \multirow[t]{4}{*}{ IV9165A-180-3 } & $A$ & 0.17216 & 4.8001 & 0.8690 & 62.90 & 170.98 & \\
\hline & B & 0.17069 & 4.8001 & 0.8616 & 62.37 & 170.76 & \\
\hline & C & 0.15782 & 4.8001 & 0.7966 & 57.66 & 168.80 & \\
\hline & $D$ & 0.17332 & 4.8001 & 0.8749 & 63.33 & 171.16 & 681.69 \\
\hline \multirow[t]{4}{*}{ IV9165A-180-4 } & $A$ & 0.15400 & 4.8001 & 0.7773 & 56.27 & 168.22 & \\
\hline & B & 0.17792 & 4.8001 & 0.8981 & 65.01 & 171.86 & \\
\hline & C & 0.16600 & 4.8001 & 0.8379 & 60.65 & 170.04 & \\
\hline & $D$ & 0.14400 & 4.8001 & 0.7269 & 52.61 & 166.69 & 676.81 \\
\hline \multirow[t]{4}{*}{ IV9165A-360-1 } & $A$ & 0.17269 & 4.8001 & 0.8717 & 63.10 & 171.06 & \\
\hline & B & 0.15541 & 4.8001 & 0.7845 & 56.78 & 168.43 & \\
\hline & C & 0.17197 & 4.8001 & 0.8680 & 62.83 & 170.95 & \\
\hline & $D$ & 0.14265 & 4.8001 & 0.7200 & 52.12 & 166.49 & 676.93 \\
\hline \multirow[t]{4}{*}{ IV9165A-360-2 } & $A$ & 0.14836 & 4.8001 & 0.7489 & 54.21 & 167.36 & \\
\hline & B & 0.14558 & 4.8001 & 0.7343 & 53.19 & 166.93 & \\
\hline & $c$ & 0.14749 & 4.8001 & 0.7445 & 53.89 & 167.22 & \\
\hline & $D$ & 0.15752 & 4.8001 & 0.7951 & 57.55 & 168.75 & 670.27 \\
\hline \multirow[t]{4}{*}{ IV9165A-720-1 } & $A$ & 0.17490 & 4.8001 & 0.8828 & 63.90 & 171.40 & \\
\hline & B & 0.16171 & 4.8001 & 0.8163 & 59.09 & 169.39 & \\
\hline & $c$ & 0.16173 & 4.8001 & 0.8164 & 59.09 & 169.39 & \\
\hline & D & 0.15864 & 4.8001 & 0.8008 & 57.96 & 168.92 & 679.10 \\
\hline \multirow[t]{4}{*}{ IV9165A-720-2 } & $A$ & 0.14694 & 4.8001 & 0.7417 & 53.69 & 167.14 & \\
\hline & $B$ & 0.17903 & 4.8001 & 0.9037 & 65.41 & 172.03 & \\
\hline & C & 0.21006 & 4.8001 & 1.0603 & 76.75 & 176.75 & \\
\hline & 0 & 0.15875 & 4.8001 & 0.8013 & 58.00 & 168.94 & 684 \\
\hline
\end{tabular}

SRL 165 A Glass - Calculated Average Density $=2.74 \mathrm{~g} / \mathrm{cm}^{3}$ 
Appendix B.3.1. (cont.). Glass Monolith Geometric Surface Areas.

\begin{tabular}{|c|c|c|c|c|c|c|c|}
\hline Test Number & $\begin{array}{c}\text { Disk } \\
\text { ID } \\
\end{array}$ & $\begin{array}{c}\text { Mass } \\
\text { (g) }\end{array}$ & $\begin{array}{c}\text { Radius } \\
\mathrm{mm}\end{array}$ & $\begin{array}{c}\text { Thicknes } \\
\mathrm{mm}\end{array}$ & $\begin{array}{l}\text { Calc. } \\
\text { Vol. } \\
\mathrm{mm}^{3}\end{array}$ & $\begin{array}{c}\text { Surface } \\
\text { Area } \\
\mathrm{mm}^{2} \\
\end{array}$ & $\begin{array}{l}\text { Total } \\
\text { Area } \\
\mathrm{mm}^{2} \\
\end{array}$ \\
\hline \multirow[t]{4}{*}{ IV9131A-14-1 } & A & 0.17089 & 4.8001 & 0.8155 & 59.03 & 169.37 & \\
\hline & B & 0.16380 & 4.8001 & 0.7817 & 56.58 & 168.35 & \\
\hline & C & 0.14954 & 4.8001 & 0.7136 & 51.65 & 166.29 & \\
\hline & $D$ & 0.16607 & 4.8001 & 0.7925 & 57.36 & 168.67 & 672.68 \\
\hline \multirow[t]{4}{*}{ IV9131A-14-2 } & A & 0.17546 & 4.8001 & 0.8373 & 60.61 & 170.02 & \\
\hline & $B$ & 0.16873 & 4.8001 & 0.8052 & 58.28 & 169.06 & \\
\hline & C & 0.16914 & 4.8001 & 0.8071 & 58.42 & 169.11 & \\
\hline & D & 0.16885 & 4.8001 & 0.8058 & 58.32 & 169.07 & 677.27 \\
\hline \multirow[t]{4}{*}{ IV9131A-28-1 } & $A$ & 0.19200 & 4.8001 & 0.9162 & 66.32 & 172.40 & \\
\hline & $B$ & 0.14952 & 4.8001 & 0.7135 & 51.65 & 166.29 & \\
\hline & C & 0.15786 & 4.8001 & 0.7533 & 54.53 & 167.49 & \\
\hline & $D$ & 0.14316 & 4.8001 & 0.6832 & 49.45 & 165.38 & 671.56 \\
\hline \multirow[t]{4}{*}{ IV $9131 \mathrm{~A}-28-2$} & $A$ & 0.15920 & 4.8001 & 0.7597 & 54.99 & 167.68 & \\
\hline & B & 0.15631 & 4.8001 & 0.7459 & 53.99 & 167.27 & \\
\hline & $C$ & 0.16236 & 4.8001 & 0.7748 & 56.08 & 168.14 & \\
\hline & $D$ & 0.16177 & 4.8001 & 0.7720 & 55.88 & 168.05 & 671.14 \\
\hline \multirow[t]{4}{*}{ IV9131A-56-1 } & A & 0.16138 & 4.8001 & 0.7701 & 55.74 & 168.00 & \\
\hline & B & 0.17336 & 4.8001 & 0.8273 & 59.88 & 169.72 & \\
\hline & C & 0.16614 & 4.8001 & 0.7928 & 57.39 & 168.68 & \\
\hline & $D$ & 0.17037 & 4.8001 & 0.8130 & 58.85 & 169.29 & 675.69 \\
\hline \multirow[t]{4}{*}{ IV9131A-56-2 } & A & 6745 & 4.8001 & 0.7991 & 57.84 & 168.87 & \\
\hline & B & 0.17466 & 4.8001 & 0.8335 & 60.33 & 169.91 & \\
\hline & C & 0.14683 & 4.8001 & 0.7007 & 50.72 & 165.90 & \\
\hline & $D$ & 0.16385 & 4.8001 & 0.7819 & 56.60 & 168.35 & 673.04 \\
\hline \multirow[t]{4}{*}{ IV9131A-56-3 } & A & 0.16458 & 4.8001 & 0.7854 & 56.85 & 168.46 & \\
\hline & B & 0.15549 & 4.8001 & 0.7420 & 53.71 & 167.15 & \\
\hline & C & 0.14623 & 4.8001 & 0.6978 & 50.51 & 165.82 & \\
\hline & D & 0.19216 & 4.8001 & 0.9170 & 66.38 & 172.43 & 673.85 \\
\hline \multirow[t]{4}{*}{ IV $131 A-56-4$} & A & 0.16901 & 4.8001 & 0.8065 & 58.38 & 169.10 & \\
\hline & B & 0.16395 & 4.8001 & 0.7824 & 56.63 & 168.37 & \\
\hline & C & 0.19683 & 4.8001 & 0.9393 & 67.99 & 173.10 & \\
\hline & D & 0.17895 & 4.8001 & 0.8539 & 61.81 & 170.53 & 681.09 \\
\hline
\end{tabular}


222

Appendix B.3.1. (cont.). Glass Monolith Geometric Surface Areas.

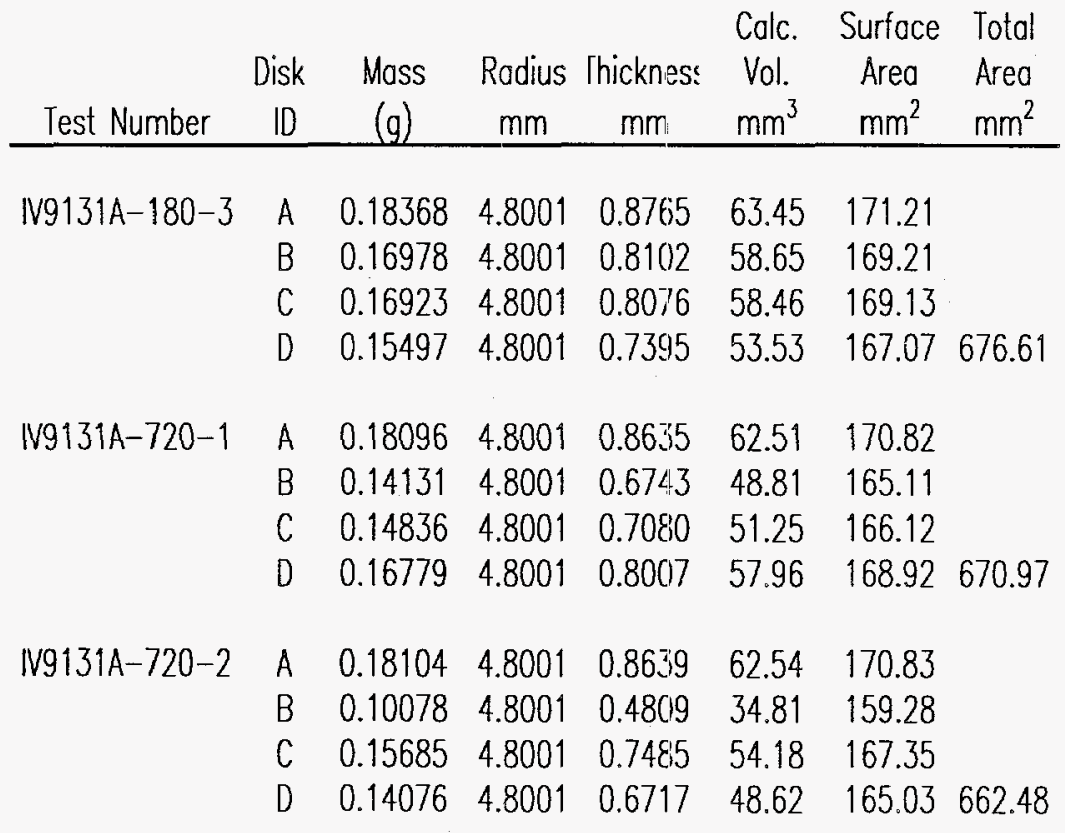

$131 \mathrm{~A}$ Glass - Average Density $=2.8950 \mathrm{~g} / \mathrm{cm}^{3}$ 
Appendix B.3.2. Sampling Disk Weights for Batch Leach Tests $\left(340 \mathrm{~m}^{-1}\right)$.

\begin{tabular}{|c|c|c|c|c|c|c|c|c|}
\hline Test Number & $\begin{array}{l}\text { Weight } \\
\text { in/out }\end{array}$ & Disk A & $\begin{array}{l}\text { Sample } \\
\text { Disk B }\end{array}$ & $\begin{array}{l}\text { Weight }(g) \\
\text { Disk C }\end{array}$ & Disk D & Total & $\begin{array}{c}\text { Weight } \\
\text { Differential }(\mathrm{g})\end{array}$ & $\begin{array}{c}\% \\
\text { Change }\end{array}$ \\
\hline \multirow[t]{2}{*}{ IV9202A-14-1 } & in & 0.15067 & 0.16012 & 0.17156 & 0.16803 & 0.65038 & 0.01026 & 1.578 \\
\hline & out & 0.16072 & 0.16017 & 0.17164 & 0.16811 & 0.66064 & & \\
\hline \multirow[t]{2}{*}{ IV9202A-28-1 } & in & 0.18732 & 0.16041 & 0.15345 & 0.15790 & 0.65908 & 0.00007 & 0.011 \\
\hline & out & 0.18733 & 0.16042 & 0.15346 & 0.15794 & 0.65915 & & \\
\hline \multirow[t]{2}{*}{ IV9202A-28-2 } & in & 0.14489 & 0.15747 & 0.15519 & 0.17392 & 0.63147 & 0.00007 & 0.011 \\
\hline & out & 0.14489 & 0.15750 & 0.15523 & 0.17392 & 0.63154 & & \\
\hline \multirow[t]{2}{*}{ IV9202A-56-1 } & in & 0.15978 & 0.16204 & 0.16348 & 0.16230 & 0.64760 & 0.00020 & 0.031 \\
\hline & out & 0.15983 & 0.16209 & 0.16351 & 0.16237 & 0.64780 & & \\
\hline \multirow[t]{2}{*}{ IV9202A-56-2 } & in & 0.17217 & 0.16350 & 0.16866 & 0.18975 & 0.69408 & 0.00006 & 0.009 \\
\hline & out & 0.17221 & 0.16349 & 0.16869 & 0.18975 & 0.69414 & & \\
\hline \multirow[t]{2}{*}{ IV9202A-56-3 } & in & 0.15853 & 0.17386 & 0.14625 & 0.14385 & 0.62249 & 0.00045 & 0.072 \\
\hline & out & 0.15871 & 0.17403 & 0.14629 & 0.14391 & 0.62294 & & \\
\hline \multirow[t]{2}{*}{ IV9202A-56-4 } & in & 0.17621 & 0.17295 & 0.18199 & 0.17375 & 0.70490 & 0.00067 & 0.095 \\
\hline & out & 0.17637 & 0.17311 & 0.18219 & 0.17390 & 0.70557 & & \\
\hline \multirow[t]{2}{*}{ IV9202A-180-3 } & in & 0.14996 & 0.17846 & 0.17522 & 0.16850 & 0.67214 & -0.00167 & -0.248 \\
\hline & out & 0.14950 & 0.17836 & 0.17517 & 0.16744 & 0.67047 & & \\
\hline \multirow[t]{2}{*}{ IV9202A-180-4 } & in & 0.16488 & 0.17121 & 0.17008 & 0.17119 & 0.67736 & 0.00004 & 0.006 \\
\hline & out & 0.16490 & 0.17118 & 0.17015 & 0.17117 & 0.67740 & & \\
\hline \multirow[t]{2}{*}{ IV9202A-360-1 } & in & 0.15951 & 0.15837 & 0.16033 & 0.16520 & 0.64341 & -0.00045 & -0.070 \\
\hline & out & 0.15939 & 0.15824 & 0.16022 & 0.16511 & 0.64296 & & \\
\hline \multirow[t]{2}{*}{ IV9202A-360-2 } & in & 0.17126 & 0.15793 & 0.16372 & 0.14386 & 0.63677 & -0.00045 & -0.071 \\
\hline & out & 0.17105 & 0.15786 & 0.16363 & 0.14378 & 0.63632 & & \\
\hline \multirow[t]{2}{*}{ IV9202A-360-3 } & in & 0.15071 & 0.15777 & 0.14955 & 0.17240 & 0.63043 & & \\
\hline & out & & urface Lay & ers Floking & Off Sampl & & & \\
\hline \multirow[t]{2}{*}{ IV9202A-540-1 } & in & 0.16715 & 0.18478 & 0.16522 & 0.16853 & 0.68568 & -0.00030 & -0.044 \\
\hline & out & 0.16710 & 0.18466 & 0.16518 & 0.16844 & 0.68538 & & \\
\hline \multirow[t]{2}{*}{ IN9202A-540-2 } & in & 0.16266 & 0.15442 & 0.17350 & 0.16218 & 0.65276 & -0.00047 & -0.072 \\
\hline & out & 0.16261 & 0.15429 & 0.17337 & 0.16202 & 0.65229 & & \\
\hline \multirow[t]{2}{*}{ IV9202A-720-1 } & in & 0.23105 & 0.16600 & 0.14822 & 0.16695 & 0.71222 & & \\
\hline & out & & urface Lay & ers Flaking & Off Sampl & & & \\
\hline \multirow[t]{2}{*}{ IV9202A-720-2 } & in & 0.15927 & 0.15398 & 0.16233 & 0.15319 & 0.62877 & & \\
\hline & out & & urface Lay & ers Flaking & Off Sampl & & & \\
\hline
\end{tabular}


Appendix B.3.2 (cont.). Sompling Disk Weights for Batch Leach Tests $\left(340 \mathrm{~m}^{-1}\right)$.

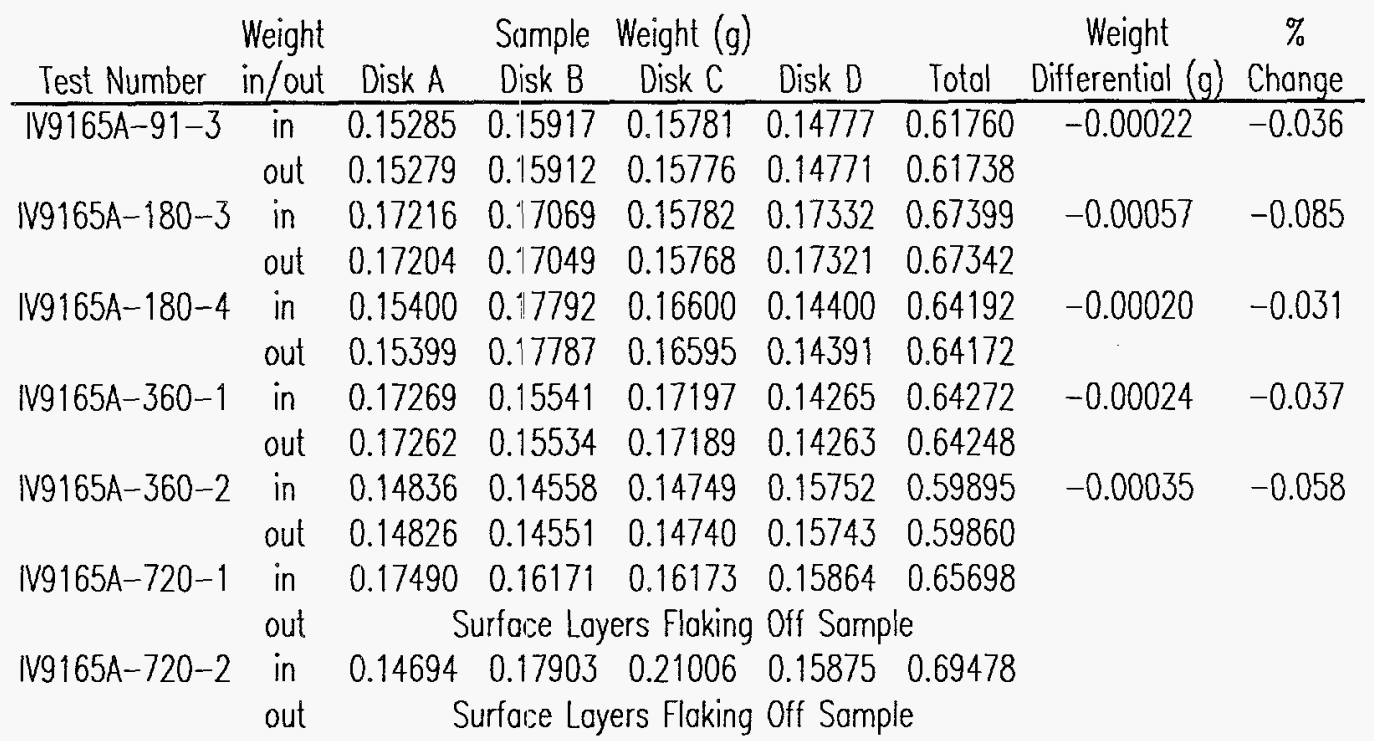

\begin{tabular}{|c|c|c|c|c|c|c|c|c|}
\hline \multirow[t]{2}{*}{ IN9131A-14-1 } & in & 0.17089 & 0.16380 & 0.14954 & 0.16607 & 0.65030 & -0.00019 & -0.029 \\
\hline & out & 0.17083 & 0.16374 & 0.14953 & 0.16601 & 0.65011 & & \\
\hline \multirow[t]{2}{*}{ IV9131A-14-2 } & in & 0.17546 & 0.16873 & 0.16914 & 0.16885 & 0.68218 & -0.00031 & -0.045 \\
\hline & out & 0.17540 & 0.16866 & 0.16907 & 0.16874 & 0.68187 & & \\
\hline \multirow[t]{2}{*}{ IV9131A-28-1 } & in & 0.19200 & 0.14952 & 0.15786 & 0.14316 & 0.64254 & -0.00071 & -0.110 \\
\hline & out & 0.19177 & 0.14931 & 0.15774 & 0.14301 & 0.64183 & & \\
\hline \multirow[t]{2}{*}{ IV9131A-28-2 } & in & 0.15920 & 0.15631 & 0.16236 & 0.16177 & 0.63964 & -0.00060 & -0.094 \\
\hline & out & 0.15905 & 0.15615 & 0.16223 & 0.16161 & 0.63904 & & \\
\hline \multirow[t]{2}{*}{ IV9131A-56-1 } & in & 0.16138 & 0.17336 & 0.16614 & 0.17037 & 0.67125 & -0.00099 & -0.147 \\
\hline & out & 0.16121 & 0.17310 & 0.16585 & 0.17010 & 0.67026 & & \\
\hline \multirow[t]{2}{*}{ IV9131A-56-2 } & in & 0.16745 & 0.17466 & 0.14683 & 0.16385 & 0.65279 & -0.00107 & -0.164 \\
\hline & out & 0.16717 & 0.17439 & 0.14659 & 0.16357 & 0.65172 & & \\
\hline \multirow[t]{2}{*}{ IN9131A-56-3 } & in & 0.16458 & 0.15549 & 0.14623 & 0.19216 & 0.65846 & -0.00069 & -0.105 \\
\hline & out & 0.16439 & 0.15530 & 0.14609 & 0.19199 & 0.65777 & & \\
\hline \multirow[t]{2}{*}{ IV $9131 \mathrm{~A}-56-4$} & in & 0.16901 & 0.16395 & 0.19683 & 0.17895 & 0.70874 & -0.00061 & -0.086 \\
\hline & out & 0.16882 & 0.16378 & 0.19674 & 0.17879 & 0.70813 & & \\
\hline \multirow[t]{2}{*}{ N9131A-180-3 } & in & 0.18368 & 0.16978 & 0.16923 & 0.15497 & 0.67766 & -0.00106 & -0.156 \\
\hline & out & 0.18340 & 0.16946 & 0.16897 & 0.15477 & 0.67660 & & \\
\hline \multirow[t]{2}{*}{ N9131A-720-1 } & in & 0.18096 & 0.14131 & 0.14836 & 0.16779 & 0.63842 & & \\
\hline & out & & urface Laye & ers Flaking & Off Sampl & & & \\
\hline \multirow[t]{2}{*}{ IV9131A-720-2 } & in & 0.18104 & 0.10078 & 0.15685 & 0.14076 & 0.57943 & & \\
\hline & out & & c & $\Gamma 1$. & Off Camol & & & \\
\hline
\end{tabular}


APPENDIX C

Gamma Counting Results 
Table C.1. Alpha Spectroscopy Counting Results.

\begin{tabular}{|c|c|c|c|c|c|c|c|c|c|c|}
\hline Sample & File \# & $\begin{array}{l}\text { Count } \\
\text { Date }\end{array}$ & $\begin{array}{l}\text { Soln. } \\
\text { Vol. } \\
\text { (ml) }\end{array}$ & $\begin{array}{l}\text { Aliquot } \\
\text { Vol. } \\
\text { (ml) }\end{array}$ & $\begin{array}{l}\text { Live } \\
\text { Time } \\
\text { (sec) }\end{array}$ & $\begin{array}{l}\text { Detector } \\
\text { Efficiency } \\
\text { Cnts./dsnt. }\end{array}$ & $\begin{array}{c}\text { Total } \\
\text { Counts }\end{array}$ & $\begin{array}{l}\text { Bkg. Np } \\
\text { Counts }\end{array}$ & $\begin{array}{l}\text { Bkg. Pu } \\
\text { Counts }\end{array}$ & $\begin{array}{l}\text { Bkg. Am } \\
\text { Counts }\end{array}$ \\
\hline IV9202A-14-1* & $\mathrm{AO} 42$ & $10 / 28 / 90$ & 2.00 & 0.05 & 84,840 & 0.2700 & 1,230 & 0.000035 & 0.000014 & 0.000021 \\
\hline IV9202A-14-1** & $\mathrm{A} 046$ & $10 / 30 / 90$ & 2.00 & 0.05 & 171,789 & 0.2700 & 822 & 0.000035 & 0.000014 & 0.000021 \\
\hline IV9202A-14-1*** & A039 & $10 / 28 / 90$ & 16.65 & 0.10 & 86,201 & 0.2270 & 1,531 & 0.000043 & 0.000035 & 0.000021 \\
\hline IV9202A-28-1 * & $\mathrm{A} 029$ & $10 / 25 / 90$ & 2.00 & 0.05 & 85,723 & 0.2230 & 1,964 & 0.000007 & 0.000000 & 0.000000 \\
\hline IV9202A-28-1** & $\mathrm{A} 048$ & $10 / 30 / 90$ & 2.00 & 0.05 & 171,789 & 0.2640 & 698 & 0.000064 & 0.000021 & 0.000057 \\
\hline IV9202A-28-1 $1^{\star \star \star}$ & $\mathrm{AO} 44$ & $10 / 28 / 90$ & 16.10 & 0.10 & 84,840 & 0.2640 & 3,741 & 0.000064 & 0.000021 & 0.000057 \\
\hline IV9202A-28-2 & $A 030$ & $10 / 25 / 90$ & 2.00 & 0.05 & 85,723 & 0.2700 & 2,639 & 0.000035 & 0.000014 & 0.000021 \\
\hline IV9202A-28-2 & NA & & & & & & & & NA & \\
\hline IV9202A-28-2 ${ }^{\star \star \star}$ & $A 050$ & $11 / 15 / 90$ & 17.52 & 0.10 & 86,261 & 0.2700 & 4,267 & 0.000035 & 0.000014 & 0.000021 \\
\hline IV9202A-56-1 * & $\mathrm{A} 090$ & $11 / 15 / 90$ & 1.90 & 0.05 & 81,393 & 0.2700 & 3,603 & 0.000035 & 0.000014 & 0.000021 \\
\hline IV9202A-56-1** & A093 & $11 / 16 / 90$ & 1.90 & 0.05 & 172,915 & 0.2230 & 1,028 & 0.000007 & 0.000000 & 0.000000 \\
\hline IV9202A-56-1 $1^{* * *}$ & A099 & $11 / 20 / 90$ & 18.49 & 0.10 & 87,237 & 0.2270 & 6,621 & 0.000043 & 0.000035 & 0.000021 \\
\hline IV9202A-56-2* & A089 & $11 / 15 / 90$ & 2.00 & 0.05 & 81,394 & 0.2230 & 7,211 & 0.000007 & 0.00 & 000 \\
\hline IV9202A-56-2 $2^{\star \star}$ & A095 & $11 / 16 / 90$ & 2.00 & 0.05 & 172,914 & 0.2270 & 1,075 & 0.000043 & 0.000035 & 0.000021 \\
\hline IV9202A-56-2 $2^{\star \star \star}$ & $A 098$ & $11 / 20 / 90$ & 17.42 & 0.10 & 87,237 & 0.2700 & 7,606 & 0.000035 & 0.000014 & 0.000021 \\
\hline IV9202A-56-3* & A717 & $10 / 7 / 91$ & 1.77 & 0.05 & 143,508 & 0.2960 & 10,780 & 0.000100 & 0.000080 & 0.000040 \\
\hline IV9202A-56-3 & NA & & & & & & & & NA & \\
\hline IV9202A-56-3*** & A712 & $10 / 7 / 91$ & 16.87 & 0.10 & 23,035 & 0.3030 & 1,347 & 100 & 0.000040 & 030 \\
\hline IV9202A-56-4* & A736 & $10 / 12 / 91$ & 1.95 & 0.05 & 86,232 & 0.3030 & 4,720 & 0.000100 & 0.000040 & 030 \\
\hline IV9202A-56-4 * & A729 & $10 / 41 / 91$ & 1.95 & 0.05 & 226,964 & 0.2960 & 897 & 0.000100 & 0.000080 & 0.000040 \\
\hline IV9202A-56-4 & A731 & $10 / 12 / 91$ & 16.73 & 0.10 & 30,679 & 0.2560 & 1,912 & 0.000100 & 0.000200 & 0.000500 \\
\hline IV9202A- & A878 & $1 / 1 / 92$ & 1.64 & 0.05 & 108,156 & 0.2988 & 11,991 & 130 & 0.0 & 0.0 \\
\hline IV9202A-1 & A897 & $1 / 16 / 92$ & 1.64 & 0.10 & 103,544 & 0.2718 & 1,183 & 0.000190 & 0.000180 & 0.000880 \\
\hline IV9202A-180-3*** & A884 & $1 / 3 / 92$ & 16.05 & 0.10 & 162,642 & 0.2988 & 40,684 & 0.000130 & 0.000011 & 0.000040 \\
\hline IV9202A-180-4* & A875 & $12 / 30 / 91$ & 1.93 & 0.05 & 167,356 & 0.2770 & 18,288 & 0.000056 & 0.000110 & 0.000045 \\
\hline IV9202A-180-4 ${ }^{\star *}$ & $A 871$ & $12 / 27 / 91$ & 1.93 & 0.10 & 253,677 & 0.2770 & 3,501 & 0.000056 & 0.000110 & 0.000045 \\
\hline IV9202A-180-4 $4^{\star * *}$ & $A 881$ & $1 / 2 / 92$ & 15.41 & 0.10 & 86,148 & 0.2988 & 18,708 & 0.000130 & 0.000011 & 0.000040 \\
\hline IV9202A-360-1" & A681 & $9 / 25 / 91$ & 1.73 & 0.05 & 91,472 & 0.2960 & 23,293 & 0.000100 & 0.000080 & 0.000040 \\
\hline IV9202A-360-1** & A692 & $9 / 30 / 91$ & 1.73 & 0.10 & 172,516 & 0.3030 & 680 & 0.000100 & 0.000040 & 0.000030 \\
\hline IV9202A-360-1*** & A752 & $10 / 20 / 91$ & 16.15 & 0.10 & 234,266 & 0.3030 & 56,377 & 0.000100 & 0.000040 & 0.000030 \\
\hline IV9202A-360-2* & A663 & $9 / 19 / 91$ & 1.95 & 0.05 & 85,538 & 0.2560 & 9,219 & 0.000100 & 0.000200 & 0.000500 \\
\hline IV9202A-360-2** & A662 & $9 / 19 / 91$ & 1.95 & 0.10 & 85,538 & 0.2750 & 950 & 0.000030 & 0.000020 & 0.000050 \\
\hline IV9202A-360-2 $2^{\star * *}$ & A753 & $10 / 20 / 91$ & 17.36 & 0.10 & 87,236 & 0.2960 & 23,682 & 0.000100 & 0.000080 & 0.000040 \\
\hline IV9202A-360-3* & C78 & $8 / 15 / 92$ & 1.95 & 0.05 & 85,992 & 0.2831 & 14,110 & 0.000080 & 0.000070 & 0.000060 \\
\hline IV9202A-360-3** & $\mathrm{C} 77$ & $8 / 15 / 92$ & 1.95 & 0.10 & 85,992 & 0.2921 & 1,133 & 0.000050 & 0.000040 & 0.000070 \\
\hline IV9202A-360-3*** & $\mathrm{C} 75$ & $8 / 15 / 92$ & 16.19 & 0.10 & 85,993 & 0.2521 & 36,738 & 0.000040 & 0.000007 & 0.000077 \\
\hline IV9202A-360-3* & C199 & $1 / 18 / 93$ & 1.95 & 0.05 & 86,663 & 0.2476 & 11,921 & 0.000020 & 0.000050 & 0.000100 \\
\hline IV9202A-360-3 ${ }^{\star \star}$ & $\mathrm{C} 201$ & $1 / 18 / 93$ & 1.95 & 0.10 & 114,486 & 0.2639 & 1,395 & 0.000066 & 0.000020 & 0.000046 \\
\hline IV9202A-360- $3^{* * *}$ & $\mathrm{C} 200$ & $1 / 18 / 93$ & 16.19 & 0.10 & 86,663 & 0.2403 & 35,541 & 0.000400 & 0.000400 & 0.001500 \\
\hline
\end{tabular}


Table C.1 (cont.). Alpha Spectroscopy Counting Results.

\begin{tabular}{|c|c|c|c|c|c|c|c|c|c|c|}
\hline Sample & File \# & $\begin{array}{l}\text { Count } \\
\text { Date }\end{array}$ & $\begin{array}{l}\text { Soln. } \\
\text { Vol. } \\
(\mathrm{ml}) \\
\end{array}$ & $\begin{array}{l}\text { Aliquot } \\
\text { Vol. } \\
\text { (ml) } \\
\end{array}$ & $\begin{array}{l}\text { Live } \\
\text { Time } \\
\text { (sec) } \\
\end{array}$ & $\begin{array}{l}\text { Detector } \\
\text { Efficiency } \\
\text { Cnts./dsnt. }\end{array}$ & $\begin{array}{c}\text { Total } \\
\text { Counts } \\
\end{array}$ & $\begin{array}{l}\text { Bkg. Np } \\
\text { Counts }\end{array}$ & $\begin{array}{l}\text { Bkg. Pu } \\
\text { Counts }\end{array}$ & $\begin{array}{c}\text { Bkg. Am } \\
\text { Counts }\end{array}$ \\
\hline IV9202A-540-1* & 1964 & $2 / 28 / 92$ & 1.97 & & 245,750 & 0.2918 & 42,052 & 557 & 030 & 030 \\
\hline IV9202A-540-1** & A970 & /3/92 & 1.97 & 05 & 3,598 & 482 & & 340 & 0.000720 & 1800 \\
\hline IV9202A-540- & A967 & $3 / 2 / 92$ & 16.77 & 0.10 & 83,592 & .2482 & 16,412 & 0.000340 & 0720 & 0.001800 \\
\hline IV9202A- & A962 & $2 / 28 / 92$ & 2.01 & 05 & 245,751 & 2772 & 19,990 & 0.000060 & 020 & 0.000085 \\
\hline IV9202A & A965 & 2/28/92 & 2.01 & 10 & 245,750 & 818 & & 085 & 0.000085 & 0.000074 \\
\hline IV9202A-540-2 & A968 & $3 / 2 / 92$ & 15.45 & 0.10 & 83,592 & 918 & 29,553 & 0.000057 & 0.000030 & 0.000030 \\
\hline IV9: & & & 1.50 & & & & & & & \\
\hline IV92 & $\mathrm{C} 202$ & 193 & 1.98 & 05 & 114,485 & 300 & & 90 & 100 & 086 \\
\hline IV92 & $\mathrm{C} 204$ & $/ 93$ & 17.87 & 10 & 27,823 & & & 00 & 100 & 500 \\
\hline IV9202 & C191 & $1 / 13 / 93$ & 1.99 & 0.05 & 189,521 & 476 & 21,296 & 020 & 050 & 100 \\
\hline IV9202 & C193 & $1 / 13 / 93$ & 1.99 & 0.05 & 189,521 & 2639 & 753 & 066 & 020 & 046 \\
\hline IV92C & C192 & 93 & 16.44 & 10 & & 03 & & 100 & 100 & 500 \\
\hline & & 2/28/92 & & & & & & & 20 & 300 \\
\hline IV91 & A966 & $3 / 2 / 92$ & 1.97 & 05 & 187,890 & 772 & 8 & 60 & 20 & 085 \\
\hline IV91 & A969 & $3 / 2 / 92$ & 16.95 & 10 & 91 & 318 & 48 & 185 & 85 & 074 \\
\hline IV91 & ה & 1/1/92 & 1. & & & & & & & \\
\hline IV91 & $A 870$ & 2/27/91 & 1.82 & 0 & 77 & 88 & & 30 & & \\
\hline 1916 & A882 & $1 / 3 / 92$ & 15.95 & 0.10 & 162,643 & 718 & 33 & 90 & & 380 \\
\hline IV91 & A879 & $1 / 1 / 92$ & 2.00 & 0.05 & 194,304 & .2770 & 3,559 & 56 & 10 & 045 \\
\hline IV916 & A872 & $30 / 91$ & 2.00 & & & & & & 80 & 380 \\
\hline IV9165A- & A899 & $1 / 16 / 92$ & 15.57 & 0 & 3 & 38 & 49 & 30 & & 40 \\
\hline & & & 0 & & & & & & & 30 \\
\hline IV91 & 665 & 91 & 1.90 & 0 & & & & & 80 & 40 \\
\hline 916 & A755 & $26 / 91$ & 17.11 & & & & & & & 500 \\
\hline IV9165 & $A 678$ & $9 / 25 / 91$ & 2.00 & 0.05 & 91,472 & 0.2750 & 9,629 & 030 & 0.000020 & 050 \\
\hline IV9165 & NA & & & & & & & & & \\
\hline IV916 & A754 & 26/91 & 15 & 10 & 0 & 50 & 4 & 30 & 0.0 & 50 \\
\hline & & & & & & & & & & \\
\hline IV916 & C177 & $1 / 6 / 93$ & 1.97 & 0.10 & 565 & 330 & & 88 & 46 & 023 \\
\hline IV916 & C156 & $12 / 10 / 92$ & 15.96 & 0.10 & 268 & 83 & 16 & 70 & 30 & 300 \\
\hline IV91 & & & 1.96 & 0.05 & & & & 10 & 20 & 057 \\
\hline IV916 & C159 & $2 / 11 / 92$ & 1.96 & 0.10 & 236,841 & 628 & 12,358 & 50 & 770 & 250 \\
\hline IV9165 & $\mathrm{C} 160$ & $12 / 11 / 92$ & 16.93 & 0.10 & 236,840 & 483 & 187,077 & 370 & 330 & 300 \\
\hline & & & 2.00 & & & & & & & \\
\hline IV913 & A052 & $1 / 1 / 90$ & 2.00 & 0.05 & ,261 & 540 & 3,292 & 64 & 21 & 057 \\
\hline IV9131 & $\mathrm{A038}$ & /28/90 & 16.68 & 0. & 087 & & & & 14 & 021 \\
\hline & & $29 / 90$ & 2.00 & 0. & 34,838 & 30 & & & 00 & 000 \\
\hline & $\mathrm{A} 047$ & $/ 30 / 90$ & 2.00 & 0.05 & 171,789 & 0.2270 & & & 35 & 0.0 \\
\hline IV913 & $\mathrm{A} 037$ & /28/90 & 17.25 & 0.10 & 85,909 & 0.2230 & 2,234 & 0.000007 & 0.000000 & 0.000000 \\
\hline
\end{tabular}


Table C.1 (cont.). Alpha Spectroscopy Counting Results.

\begin{tabular}{|c|c|c|c|c|c|c|c|c|c|c|}
\hline Sample & File \# & $\begin{array}{l}\text { Count } \\
\text { Date }\end{array}$ & $\begin{array}{l}\text { Soln. } \\
\text { Vol. } \\
\text { (ml) }\end{array}$ & $\begin{array}{l}\text { Aliquot } \\
\text { Vol. } \\
\text { (ml) } \\
\end{array}$ & $\begin{array}{l}\text { Live } \\
\text { Time } \\
\text { (sec) } \\
\end{array}$ & $\begin{array}{l}\text { Detector } \\
\text { Efficiency } \\
\text { Cnts./dsnt. }\end{array}$ & $\begin{array}{c}\text { Total } \\
\text { Counts } \\
\end{array}$ & $\begin{array}{l}\text { Bkg. Np } \\
\text { Counts }\end{array}$ & $\begin{array}{l}\text { Bkg. Pu } \\
\text { Counts }\end{array}$ & $\begin{array}{c}\text { Bkg. Am } \\
\text { Counts }\end{array}$ \\
\hline IV9131A-28-1* & $\mathrm{A032}$ & $10 / 25 / 90$ & 2.00 & 0.05 & 85,723 & 0.2640 & 6,650 & 0.000064 & 0.000021 & 0.000057 \\
\hline IV9131A-28-1** & NA & & & & & & & & NA & \\
\hline IV9131A-28-1*** & $\mathrm{AO} 49$ & $11 / 1 / 90$ & 15.35 & 0.10 & 86,261 & 0.2230 & 6,284 & 0.000007 & 0.000000 & 0.000000 \\
\hline IV9131A-28-2* & A031 & $10 / 25 / 90$ & 2.00 & 0.05 & 85,723 & 0.2270 & 7,173 & 0.000043 & 0.000035 & 0.000021 \\
\hline IV9131A-28-2 & NA & & & & & & & & NA & \\
\hline IV9131A-28-2 & $\mathrm{A} 040$ & $10 / 28 / 90$ & 15.56 & 0.10 & 86,316 & 0.2640 & 5,868 & 0.000064 & 0.000021 & 0.000057 \\
\hline IV9131A & A091 & $11 / 15 / 90$ & 2.00 & 0.05 & 81,393 & 0.2270 & 7,568 & 0.000043 & 0.00 & 0.000021 \\
\hline IV9131A-56-1** & A094 & $11 / 16 / 90$ & 2.00 & 0.05 & 172,914 & 0.2700 & 1,436 & 0.000035 & 0.000014 & 0.000021 \\
\hline IV9131A-56-1*** & A097 & $11 / 20 / 90$ & 18.20 & 0.10 & 87,238 & 0.2230 & 10,258 & 0.000007 & 0.000000 & 0.000000 \\
\hline IV9131A-56-2* & $\mathrm{A} 092$ & $11 / 15 / 90$ & 2.00 & 0.05 & 81,393 & 0.2640 & 8,382 & 0.000064 & 0.000021 & 0.000057 \\
\hline IV9131A-56-2 & A096 & $11 / 16 / 90$ & 2.00 & 0.05 & 172,914 & 0.2640 & 2,874 & 0.000064 & 0.000021 & 0.000057 \\
\hline IV9131A-56-2 & A100 & $11 / 20 / 90$ & 17.33 & 0.10 & 87,237 & 0.2640 & 13,330 & 0.000064 & 0.000021 & 0.000057 \\
\hline IV9131A-56-3* & A737 & $10 / 12 / 91$ & 2.00 & 0.05 & 86,231 & 0.2960 & 3,110 & 0.000100 & 0.000080 & 0.000040 \\
\hline IV9131A-56-3** & A728 & $10 / 11 / 91$ & 2.00 & 0.10 & 226,965 & 0.3030 & 3,200 & 0.000100 & 0.000040 & 0.000030 \\
\hline IV9131A-56-3 $3^{\star \star \star}$ & A732 & $10 / 12 / 91$ & 17.25 & 0.10 & 30,679 & 0.3030 & 1,911 & 0.000100 & 0.000040 & 0.000030 \\
\hline IV9131A-56-4* & A734 & $10 / 12 / 91$ & 1.88 & 0.05 & 86,232 & 0.2750 & 8,273 & 0.000030 & 0.000020 & 0.000050 \\
\hline IV9131A-56-4** & A726 & $10 / 11 / 91$ & 1.88 & 0.10 & 226,965 & 0.2750 & 3,415 & 0.000030 & 0.000020 & 0.000050 \\
\hline IV9131A-56-4 & A723 & $10 / 10 / 91$ & 17.03 & 0.10 & 87,074 & 0.2560 & 8,741 & 0.000100 & 0.000200 & 0.000500 \\
\hline IV9131A-180-3* & A896 & $1 / 15 / 92$ & 2.00 & 0.05 & 170,902 & 0.2770 & 20,763 & 0.000056 & 0.0 & 0.0 \\
\hline IV9131A-180-3** & A874 & $12 / 30 / 91$ & 2.00 & 0.1 & 167,356 & 0.2988 & 9,436 & 0.000130 & 0.000011 & 0.000040 \\
\hline IV9131A-180-3*** & A898 & $1 / 16 / 92$ & 16.19 & 0.1 & 103,543 & 0.2494 & 37,218 & 0.000260 & 0.000200 & 0.000530 \\
\hline IV9131A-720-1* & C194 & $1 / 13 / 93$ & 1.98 & 0.05 & 189,521 & 0.2790 & 95,889 & 0.000110 & 0.00 & 0.000057 \\
\hline IV9131A-720-1** & C189 & $1 / 12 / 93$ & 1.98 & 0.05 & 81,660 & 0.2830 & 2,340 & 0.000088 & 0.000046 & 0.000023 \\
\hline IV9131A-720-1*** & C188 & $1 / 12 / 93$ & 16.48 & 0.1 & 81,661 & 0.2483 & 32,618 & 0.000370 & 0.000830 & 0.001800 \\
\hline IV9131A-720-2* & $\mathrm{C} 213$ & $1 / 22 / 93$ & 1.98 & 0.05 & 237,319 & 0.2628 & 102,261 & 0.000050 & 0.000070 & 0.000250 \\
\hline IV9131A-720-2** & $\mathrm{C} 236$ & 2/1/93 & 1.98 & 0.1 & 291,299 & 0.2790 & 16,859 & 0.000110 & 0.000120 & 0.000057 \\
\hline IV9131A-720-2 $2^{\star \star \star}$ & $\mathrm{C} 214$ & $1 / 22 / 93$ & 16.48 & 0.1 & 237,319 & 0.2483 & 93,610 & 0.000370 & 0.000830 & 0.001800 \\
\hline
\end{tabular}


Table C.1 (cont.). Alpha Spectroscopy Counting Results.

\begin{tabular}{|c|c|c|c|c|c|c|c|c|c|}
\hline Sample & $\begin{array}{l}\mathrm{Np} \\
\text { Counts }\end{array}$ & $\begin{array}{c}\mathrm{Pu} \\
\text { Counts }\end{array}$ & $\begin{array}{c}\text { Am } \\
\text { Counts }\end{array}$ & $\begin{array}{c}\text { Calculated } \\
\text { Np (ng) }\end{array}$ & $\begin{array}{l}\text { Calculated } \\
\text { Pu (ng) }\end{array}$ & $\begin{array}{c}\text { Calculated } \\
\text { Am (ng) }\end{array}$ & $\begin{array}{l}\text { Aliquot } \\
\text { Concen. } \\
\mathrm{Np}(\mathrm{ng} / \mathrm{ml})\end{array}$ & $\begin{array}{l}\text { Aliquot } \\
\text { Concen. } \\
\text { Pu (ng/ml) }\end{array}$ & $\begin{array}{l}\text { Aliquot } \\
\text { Concen. } \\
\text { Am }(\mathrm{ng} / \mathrm{ml})\end{array}$ \\
\hline IV9202A-14-1* & 246 & 633 & 351 & 0.01060956 & 0.02758193 & 0.01524522 & 8.1307 & 0.2402 & 0.0024 \\
\hline IV9202A-14-1** & 738 & 78 & 6 & 0.01578136 & 0.0016298 & 5.158E-05 & 12.0941 & 0.0142 & 8.11E-06 \\
\hline IV9202A-14-1*** & 5 & 1,166 & 360 & 6.6097E-05 & 0.05943403 & 0.01830522 & 0.0253 & 0.2587 & 0.0014 \\
\hline IV9202A-28-1• & 381 & 1,016 & 567 & 0.01989923 & 0.05314831 & 0.02966052 & 15.2498 & 0.4628 & 0.0047 \\
\hline IV9202A-28-1** & 386 & 101 & 44 & 0.00826875 & 0.00214747 & 0.00075428 & 6.3368 & 0.0187 & 0.0001 \\
\hline IV9202A-28-1*** & 0 & 2,534 & 1,207 & -0.0002424 & 0.11305703 & 0.05367354 & -0.0929 & 0.4922 & 0.0042 \\
\hline IV9202A-28-2* & 569 & 1,344 & 726 & 0.02445426 & 0.05801625 & 0.03128937 & 18.7406 & 0.5051 & 0.0049 \\
\hline IV9202A-28-2 ${ }^{* *}$ & & NA & & & NA & & & NA & \\
\hline IV9202A-28-2 & 0 & 2,715 & 1,552 & -0.0001296 & 0.11651943 & 5892 & $-0 .($ & 0.5073 & .0052 \\
\hline IV9? & 512 & 2,201 & 890 & 0.02316828 & 87 & 2055 & 17.7551 & 0.8716 & 0.0064 \\
\hline IV9202A & 805 & 215 & 8 & 0.0208452 & 0.00557574 & 0.00020747 & 15.9748 & 0.0485 & $3.26 \mathrm{E}-05$ \\
\hline IV9202A-56-1*** & 0 & 4,646 & 1,975 & -0.0001894 & 0.234 & 0.08 & -0.0726 & 1.0207 & 0.0078 \\
\hline IV9202 & 40 & 1,915 & 1,021 & 0.00217237 & 0.105 & 098 & 1.6648 & 0.9186 & 0.0088 \\
\hline IV9202 & 876 & 168 & 31 & 0.0221282 & 0.004 & 1727 & 16.9580 & .0359 & 0.0001 \\
\hline IV9202A & 0 & 5,034 & 1,894 & -0.0001296 & 0.2136 & 286 & -0.0497 & 0.9302 & 0.0063 \\
\hline IV9202A- & 20 & 6,212 & 4,548 & 0.00013299 & 0.14596918 & 0.10693135 & 0.1019 & 1.2709 & 0.0168 \\
\hline IV9202A- & & NA & & & NA & & & NA & \\
\hline IV9202A & 10 & 841 & 496 & 274 & 0.12 & 42 & 0.4 & 0.5240 & \\
\hline IV92 & 56 & 2,759 & 1,905 & 0.00 & 281 & 079 & 1.3896 & 0.9183 & 0.0115 \\
\hline IV920 & 823 & 62 & 12 & 0.01191257 & 0.000 & 4.34 & 9.1292 & 0.0057 & 6.84E-06 \\
\hline IV9202A- & & & & -0.0003906 & -0.0007813 & -0.0 & -0.1497 & -0.0034 & -0.0002 \\
\hline IV92 & 339 & & 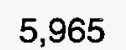 & & & & & & \\
\hline IV9202A & 868 & 187 & 128 & 0.0301433 & 0.00598236 & 0.00 & 11.5502 & 0.0260 & 0.0001 \\
\hline IV9202A & 0 & 21,680 & 19,004 & -0.0004351 & 0.44607649 & 149 & -0.1667 & 1.9420 & 0.0307 \\
\hline IV9202A- & 329 & 11,559 & 6,400 & 0.00689483 & 0.2489469 & 3462 & 5.2839 & 2.1675 & 0.0217 \\
\hline IV9202A-1 & 2,402 & 796 & 303 & 0.03398102 & 0.01093087 & 4958 & 13.0207 & 0.0476 & 0.0003 \\
\hline IV9202A-180-4 & 0 & 10,428 & 8,280 & -0.0004351 & 0.40507417 & 0.32153078 & -0.1667 & 1.7635 & 0.0253 \\
\hline IV9202A & 0 & 14,209 & 9,084 & 378 & 13 & 61 & -0 . & 69 & 28 \\
\hline IV9202A-3 & 400 & 229 & 51 & 0.00732219 & 0.00424888 & 0.00087665 & 2.8057 & 0.0185 & $6.9 E-05$ \\
\hline IV9202A-360-1*** & 370 & 26,347 & 29,660 & 0.0048825 & 0.37104283 & 0.41774917 & 1.8709 & 1.6153 & 0.0329 \\
\hline IV9202A-360-2* & 20 & 5,425 & 3,774 & 0.00052271 & 0.24696106 & 9332 & 0.4006 & 2.1503 & 0.0268 \\
\hline IV9202A & 706 & 212 & 32 & 0.02990405 & 0.00893972 & 0.00 & 11.4585 & 0.0389 & 9.27E-05 \\
\hline IV9202A-36 & 0 & 11,277 & 12,405 & -0.0003378 & 0.43645229 & 0.48 & -0.1295 & 1.9001 & 0.0378 \\
\hline IV9202A-360-3* & 0 & 8,868 & 5,242 & -0.0002826 & 0.36402597 & 0.21511511 & -0.2166 & 3.1695 & 0.0338 \\
\hline IV9202A-360-3** & 506 & 424 & 203 & 0.01997344 & 0.01674313 & 0.00784209 & 7.6534 & 0.0729 & 0.0006 \\
\hline IV9202A-360-3 $3^{\star \star \star}$ & 0 & 23,996 & 12,742 & -0.0001587 & 1.10685827 & 0.58745677 & -0.0608 & 4.8186 & 0.0462 \\
\hline IV9202 & 0 & 7,582 & 4,339 & $-8.078 E-05$ & 0.35314385 & 0.20 & -0.0619 & 3.0748 & 0.0317 \\
\hline IV9202A & 636 & 536 & 223 & 0.0208006 & 0.01766505 & 0.00720667 & 7.9703 & 0.0769 & 0.0006 \\
\hline IV9202A-360-3*** & 0 & 23,097 & 12,444 & -0.0016646 & 1.10743256 & 0.59130748 & -0.6378 & 4.8211 & 0.0465 \\
\hline
\end{tabular}


Table C.1 (cont.). Alpha Spectroscopy Counting Results.

\begin{tabular}{|c|c|c|c|c|c|c|c|c|c|}
\hline Sample & $\begin{array}{c}\mathrm{Np} \\
\text { Counts }\end{array}$ & $\begin{array}{c}\mathrm{Pu} \\
\text { Counts }\end{array}$ & $\begin{array}{c}\text { Am } \\
\text { Counts }\end{array}$ & $\begin{array}{l}\text { Calculated } \\
\mathrm{Np}(\mathrm{ng})\end{array}$ & $\begin{array}{c}\text { Calculated } \\
\mathrm{Pu}(\mathrm{ng})\end{array}$ & $\begin{array}{l}\text { Calculated } \\
\text { Am (ng) }\end{array}$ & $\begin{array}{l}\text { Aliquot } \\
\text { Concen. } \\
\mathrm{Np}(\mathrm{ng} / \mathrm{ml})\end{array}$ & $\begin{array}{c}\text { Aliquot } \\
\text { Concen. } \\
\mathrm{Pu}(\mathrm{ng} / \mathrm{ml})\end{array}$ & $\begin{array}{c}\text { Aliquot } \\
\text { Concen. } \\
\text { Am (ng/ml) }\end{array}$ \\
\hline IV9202A-540-1* & 449 & 20,347 & 21,256 & 0.00606599 & 0.28363739 & 0.29631345 & 4.6487 & 2.4696 & 0.0466 \\
\hline IV9202A-540-1** & 255 & 509 & 637 & 0.00454841 & 0.00891245 & 0.00753186 & 3.4857 & 0.0776 & 0.0012 \\
\hline IV9202A-540-1 & 90 & 8,369 & 7,953 & 0.002968 & 0.40047263 & 0.37607072 & 1.1373 & 1.7434 & 0.0296 \\
\hline IV9202A-540-2* & 234 & 9,722 & 10,014 & 0.00321856 & 0.14264208 & 0.14669401 & 2.4666 & 1.2420 & 0.0231 \\
\hline IV9202A-540-2 & 786 & 433 & 193 & 0.01104816 & 0.00595086 & 0.00252431 & 4.2334 & 0.0259 & 0.0002 \\
\hline IV9202A-540-2*** & 0 & 13,718 & $15,8: 35$ & -0.0001953 & 0.56229349 & 0.64908406 & -0.0748 & 2.4479 & 0.0511 \\
\hline IV9202A-720-1* & 0 & 3,610 & 2,143 & $-8.078 \mathrm{E}-05$ & 0.52381437 & 0.3106673 & -0.0619 & 308 & 489 \\
\hline IV9202A-720-1** & 170 & 142 & 10 & 0.00536502 & 0.0043859 & 5.1822E-06 & 4.1115 & 0.0382 & 0000 \\
\hline IV9202A-720-1 & 10 & 4,178 & 4,363 & -0.0001689 & 0.62322894 & 0.64632134 & -0.0647 & 2.7132 & .0508 \\
\hline IV9202A-720-2* & 268 & 12,923 & 8,373 & 0.00563041 & 0.27519204 & 0.1780279 & 4.3149 & 2.3961 & 0.0280 \\
\hline IV9202A-720-2** & 622 & 131 & 9 & 0.01218628 & 0.00254345 & 5.6391E-06 & 9.3390 & 0.0221 & 0.0000 \\
\hline IV9202A-720-2*** & 235 & 29,284 & 29,919 & 0.00349549 & 0.64134705 & 0.65071263 & 1.3394 & 2.7921 & 0.0512 \\
\hline IV916 & 621 & 7,870 & 5,111 & 125 & 0.126 & 0.07 & 25 & 82 & 120 \\
\hline IV9165 & 698 & 156 & 22 & 0.01318519 & 0.00292306 & 0.000 & 10.1045 & 0.0255 & .0000 \\
\hline 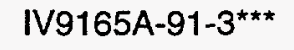 & 0 & 3,595 & 4,153 & -0.0003016 & 0.15231305 & 0.17604026 & -0.1156 & 0.6631 & 0.0138 \\
\hline IV9165 & 848 & 5,028 & 4,262 & 579 & 0.09 & 0.077 & 596 & 32 & 122 \\
\hline IV9165A & 4,109 & 915 & 786 & 0.05 & 0.012 & 0.01 & 20.6051 & 524 & 008 \\
\hline IV9165A- & 0 & 19,283 & 13,954 & -0.000699 & 0.43554241 & 0.3124186 & -0.2679 & 61 & 0246 \\
\hline IV9165A-180-4* & 905 & 1,476 & 1,178 & 0.0166125 & 0.02702658 & 0.02172448 & 12.7310 & 353 & 0.0034 \\
\hline IV9165A-180-4 ** & 1,346 & 209 & 227 & 0.02889143 & 0.00393241 & 0.0017527 & 11.0705 & 0.0171 & 0.0001 \\
\hline IV9165A-180-4*** & 0 & 10,756 & 14,093 & -0.0004351 & 0.34761897 & 0.45538054 & -0.1667 & 1.5133 & .0358 \\
\hline IV9165 & 80 & 1,628 & 1,533 & 0.00 & 0.06 & 0.05 & 26 & & 93 \\
\hline IV9165A-360-1** & 686 & 184 & 142 & 0.0267563 & 0.00699696 & 0.00547327 & 10.2524 & 305 & 004 \\
\hline IV9165A-360-1*** & 0 & 9,536 & 10,407 & -0.0003906 & 0.21242909 & 0.23073144 & -0.1497 & 248 & 0.0181 \\
\hline IV9165A-360-2* & 25 & 4,652 & 4,952 & 0.00088475 & 0.18486155 & 0.19667857 & 0.6780 & 1.6096 & 0.0309 \\
\hline IV9165A-360-2 & & NA & & & NA & & & NA & \\
\hline IV9165A-360-2 ${ }^{* \star *}$ & 105 & 22,633 & 27,256 & 0.00207634 & 0.47100294 & 0.56711542 & 0.7956 & 2.0505 & 0.0446 \\
\hline IV9165A-720-1* & 0 & 13,213 & 6,369 & -0.0001903 & 0.62610439 & 0.30097524 & -0.1458 & & 473 \\
\hline IV9165A-720-1** & 261 & 108 & 31 & 0.09610646 & 0.03973432 & 0.0113706 & 36.8257 & 0.1730 & 0.0009 \\
\hline IV9165A-720-1 & 25 & 24,168 & 26,423 & -0.0002358 & 1.20926538 & 1.31850145 & -0.0903 & 5.2645 & 0.1037 \\
\hline IV9165A-720-2* & 13 & 1,769 & 738 & 0.00447714 & 0.6624556 & 0.27634157 & 3.4311 & 5.7679 & .0435 \\
\hline IV9165A-720-2** & 6,231 & 5,151 & 976 & 0.09991937 & 0.08249158 & 0.0147295 & 38.2868 & 0.3591 & 0.0012 \\
\hline IV9165A-720-2*** & 269 & \#\#\#\#\# & 82,673 & 0.00308412 & 1.76743866 & 1.39857785 & 1.1818 & 7.6944 & 0.1100 \\
\hline IV9131A-14-1* & 502 & 1,278 & 776 & & 0.06620566 & & 19.8308 & 764 & 63 \\
\hline IV9131A-14-1** & 683 & 1,615 & 994 & 0.02974953 & 0.07083848 & 0.04343271 & 22.7987 & 0.6168 & 0.0068 \\
\hline 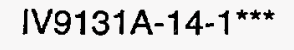 & 15 & 1,791 & 448 & 0.00051572 & 0.07700234 & 0.01919653 & 0.1976 & 0.3352 & 0.0015 \\
\hline IV9131A-14-2* & 100 & 3,066 & 1,656 & 0.00525433 & 0.16206019 & 0.08753153 & 4.0267 & 1.4110 & 0.0138 \\
\hline IV9131A-14-2** & 1,444 & 1,197 & 3,739 & 0.03683996 & 0.03054123 & 0.09578898 & 28.2324 & 0.2659 & 0.0151 \\
\hline IV9131A-14-2 & 5 & 1,715 & 514 & 0.0002296 & 0.0895198 & 0.02682984 & 0.0880 & 0.3897 & 0.0021 \\
\hline
\end{tabular}


Table C.1 (cont.). Alpha Spectroscopy Counting Results.

\begin{tabular}{|c|c|c|c|c|c|c|c|c|c|}
\hline Sample & $\begin{array}{c}\mathrm{Np} \\
\text { Counts }\end{array}$ & $\begin{array}{c}\mathrm{Pu} \\
\text { Counts }\end{array}$ & $\begin{array}{c}\text { Am } \\
\text { Counts }\end{array}$ & $\begin{array}{l}\text { Calculated } \\
\mathrm{Np}(\mathrm{ng})\end{array}$ & $\begin{array}{c}\text { Calculated } \\
\text { Pu (ng) }\end{array}$ & $\begin{array}{c}\text { Calculated } \\
\text { Am (ng) }\end{array}$ & $\begin{array}{c}\text { Aliquot } \\
\text { Concen. } \\
\mathrm{Np}(\mathrm{ng} / \mathrm{ml})\end{array}$ & $\begin{array}{c}\text { Aliquot } \\
\text { Concen. } \\
\mathrm{Pu}(\mathrm{ng} / \mathrm{ml})\end{array}$ & $\begin{array}{c}\text { Aliquot } \\
\text { Concen. } \\
\text { Am (ng/ml) }\end{array}$ \\
\hline IV9131A-28-1* & 60 & 4,665 & 1,925 & 0.00240883 & 0.20605576 & 0.08484528 & 1.8460 & 1.7941 & 0.0133 \\
\hline IV9131A-28-1** & & NA & & & NA & & & NA & \\
\hline IV9131A-28-1 *** & 10 & 4,706 & 1,568 & 0.00048846 & 0.24464202 & 0.08151268 & 0.1872 & 1.0650 & 0.0064 \\
\hline IV9131A-28-2* & 50 & 4,771 & 2,352 & 0.00238006 & 0.24502675 & 0.1207764 & 1.8240 & 2.1334 & 0.0190 \\
\hline IV9131A-28-2** & & NA & & & NA & & & NA & \\
\hline IV9131A-28-2 $2^{\star \star *}$ & 20 & 4,418 & 1,430 & 0.00063525 & 0.19379887 & 0.06253786 & 0.2434 & 0.8437 & 0.0049 \\
\hline IV9131A-56-1* & 1,741 & 4,222 & 1,605 & 0.09403973 & 0.22835559 & 0.08677584 & 72.0677 & 1.9883 & 0.0137 \\
\hline IV9131A-56-1** & 829 & 570 & 37 & 0.01762697 & 0.01215715 & 0.00071474 & 13.5085 & 0.1059 & 0.0001 \\
\hline IV9131A-56-1*** & 0 & 6,826 & 2,639 & $-3.139 E-05$ & 0.35087927 & 0.13565344 & -0.0120 & 1.5275 & 0.0107 \\
\hline IV9131A-56-2* & 120 & 5,988 & 2,274 & 0.00534217 & 0.27859152 & 0.10561208 & 4.0940 & 2.4257 & 0.0166 \\
\hline IV9131A-56-2 & 1,669 & 1,062 & 143 & 0.03631899 & 0.02318482 & 0.00291667 & 27.8332 & 0.2019 & 0.0005 \\
\hline IV9131A-56-2*** & 0 & 8,713 & 3,958 & -0.0002424 & 0.3782446 & 0.17164303 & -0.0929 & 1.6467 & 0.0135 \\
\hline IV9131A-56-3* & 129 & 1,966 & 1,015 & 0.00471613 & 0.07675378 & 0.03963059 & 3.6142 & 0.6683 & 0.0062 \\
\hline IV9131A-56-3** & 3,100 & 92 & 8 & 0.0447476 & 0.00120577 & 1.7319E-05 & 17.1462 & 0.0052 & 0.0000 \\
\hline IV9131A-56-3*** & 0 & 1,616 & 295 & -0.00033 & 0.17371055 & 0.03163586 & -0.1265 & 0.7562 & 0.0025 \\
\hline IV9131A-56-4* & 30 & 5,233 & 3,020 & 0.00115599 & 0.22060021 & 0.12717004 & 0.8859 & 1.9207 & 0.0200 \\
\hline IV9131A-56-4 & 50 & 3,214 & 151 & 0.00069199 & 0.05142102 & 0.00223746 & 0.2652 & 0.2239 & 0.0002 \\
\hline 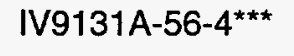 & 41 & 6,347 & 2,312 & 0.00144868 & 0.28395191 & 0.10176565 & 0.5551 & 1.2362 & 0.0080 \\
\hline IV9131A-180-3* & 485 & 16,415 & 3,862 & 0.01004293 & 0.34635171 & 0.0 & 7.6 & 3.0156 & 0.0128 \\
\hline IV9131A-180-3 & 3,222 & 5,126 & 1,088 & 0.06399708 & 0.1024707 & 0.02162348 & 24.5222 & 0.4461 & 0.0017 \\
\hline IV9131A-180-3*** & 0 & 25,115 & 12,103 & -0.0010425 & 0.97175527 & 0.46655337 & -0.3995 & 4.2305 & 0.0367 \\
\hline IV9131A-720-1* & 0 & 66,957 & 28,932 & -0.0003943 & 1.26586559 & 0.54695981 & -0.3021 & 11.0217 & 0.0860 \\
\hline IV9131A-720-1 ** & 913 & 1,072 & 355 & 0.03919598 & 0.04622457 & 0.01528013 & 30.0380 & 0.4025 & 0.0024 \\
\hline IV9131A-720-1*** & 0 & 12,954 & 19,664 & -0.0014901 & 0.63553038 & 0.9625516 & -0.5710 & 2.7667 & 0.0757 \\
\hline IV9131A-720-2* & 0 & 70,734 & 31,527 & -0.0001903 & 1.13388468 & 0.50455355 & -0.1458 & 9.8726 & 0.0794 \\
\hline IV9131A-720-2** & 4,652 & 9,635 & 2,572 & 0.0568454 & 0.11812196 & 0.03144239 & 21.7818 & 0.5142 & 0.0025 \\
\hline IV9131A-720-2 & 0 & 53,136 & 40,474 & -0.0014901 & 0.89839563 & 0.67961005 & -0.5710 & 3.9111 & 0.0535 \\
\hline
\end{tabular}


Table C.1 (cont.). Alpha Spectroscopy Counting Results.

\begin{tabular}{|c|c|c|c|}
\hline Sample & $\begin{array}{c}\text { Total Mass } \\
\mathrm{Np}(\mathrm{ng})\end{array}$ & $\begin{array}{l}\text { Total Mass } \\
\text { Pu (ng) }\end{array}$ & $\begin{array}{c}\text { Total Mass } \\
\text { Am (ng) }\end{array}$ \\
\hline IV9202A-14-1* & 16.2613 & 0.4803 & 0.0048 \\
\hline IV9202A-14-1** & 24.1882 & 0.0284 & 1.62285E-05 \\
\hline IV9202A-14-1 & 0.4217 & 4.3081 & 0.0240 \\
\hline IV9202A-28-1* & 30.4997 & 0.9255 & 0.0093 \\
\hline IV9202A-28-1 & 12.6736 & 0.0374 & 0.0002 \\
\hline IV9202A-28-1*** & -1.4956 & 7.9242 & 0.0680 \\
\hline IV9202A-28-2* & 37.4812 & 1.0103 & 0.0098 \\
\hline IV9202A-28-2** & & NA & \\
\hline IV9202A-28-2 & -0.8702 & 8.8872 & 0.0917 \\
\hline IV9202A-56-1* & 33.7347 & 1.6560 & 0.0121 \\
\hline IV9202A-56-1** & 30.3521 & 0.0922 & 6.20119E-05 \\
\hline IV9202A-56-1 $1^{\star * *}$ & -1.3421 & 18.8728 & 0.1449 \\
\hline IV9202A-56-2* & 3.3296 & 1.8372 & 0.0177 \\
\hline IV9202A-56-2 ${ }^{\star \star}$ & 33.9160 & 0.0718 & 0.0002 \\
\hline IV9202A-56-2 $2^{\star \star *}$ & -0.8653 & 16.2040 & 0.1101 \\
\hline IV9202A-56-3* & 0.1804 & 2..2496 & 0.0298 \\
\hline IV9202A-56-3 ${ }^{\star \star}$ & & NA & \\
\hline IV9202A-56-3 $3^{\star \star \star}$ & 7.1283 & 8.8398 & 0.0942 \\
\hline IV9202A-56-4* & 2.7097 & 1.7906 & 0.0223 \\
\hline IV9202A-56-4 ${ }^{\star \star}$ & 17.8020 & 0.0111 & 1.33398E-05 \\
\hline IV9202A-56-4 & -2.5041 & -0.0569 & -0.0026 \\
\hline IV9202A-180-3* & 12.6371 & 2.5123 & 0.0476 \\
\hline IV9202A-180-3** & 18.9423 & 0.0427 & 0.0002 \\
\hline 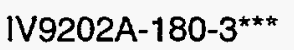 & -2.6757 & 31.1685 & 0.4935 \\
\hline IV9202A-180-4* & 10.1979 & 4.1834 & 0.0419 \\
\hline IV9202A-180-4** & 25.1300 & 0.0918 & 0.0006 \\
\hline IV9202A-180-4 $4^{\star \star \star}$ & -2.5690 & 27.1750 & 0.3897 \\
\hline IV9202A-360-1* & -0.4479 & 7.9008 & 0.0913 \\
\hline IV9202A-360-1** & 4.8538 & 0.0320 & 0.0001 \\
\hline IV9202A-360-1*** & 30.2144 & 26.0873 & 0.5307 \\
\hline IV9202A-360-2* & 0.7811 & 4.1930 & 0.0523 \\
\hline IV9202A-360-2 $2^{\star \star}$ & 22.3441 & 0.0759 & 0.0002 \\
\hline IV9202A-360-2 $2^{* \star \star}$ & -2.2473 & $32 . .9852$ & 0.6558 \\
\hline IV9202A-360-3* & -0.4223 & 6.1806 & 0.0660 \\
\hline IV9202A-360-3** & 14.9240 & 0.1421 & 0.0012 \\
\hline IV9202A-360-3*** & -0.9843 & 78.0137 & 0.7481 \\
\hline IV9202A-360-3* & -0.1207 & 5.9958 & 0.0619 \\
\hline IV9202A-360-3** & 15.5421 & 0.1500 & 0.0011 \\
\hline IV9202A-360-3 $3^{\star \star *}$ & -10.3265 & 78.0542 & 0.7530 \\
\hline
\end{tabular}


Table C.1 (cont.). Alpha Spectroscopy Counting Results.

\begin{tabular}{|c|c|c|c|}
\hline Sample & $\begin{array}{c}\text { Total Mass } \\
\mathrm{Np}(\mathrm{ng})\end{array}$ & $\begin{array}{c}\text { Total Mass } \\
\mathrm{Pu}(\mathrm{ng})\end{array}$ & $\begin{array}{c}\text { Total Mass } \\
\text { Am (ng) }\end{array}$ \\
\hline IV9202A-540-1* & 9.1579 & 4.8651 & 0.0918 \\
\hline IV9202A-540-1** & 6.8668 & 0.1529 & 0.0023 \\
\hline 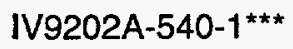 & 19.0720 & 29.2373 & 0.4961 \\
\hline IV9202A-540-2* & 4.9578 & 2.4964 & 0.0464 \\
\hline IV9202A-540-2 & 8.5091 & 0.0521 & 0.0004 \\
\hline IV9202A-540-2 $2^{\star \star *}$ & -1.1564 & 37.8202 & 0.7888 \\
\hline IV9202A-720-1* & -0.1226 & 9.0304 & 0.0968 \\
\hline IV9202A-720-1** & 8.1408 & 0.0756 & 0.0000 \\
\hline IV9202A-720-1*** & -1.1566 & 48.4846 & 0.9085 \\
\hline IV9202A-720-2* & 8.5866 & 4.7682 & 0.0557 \\
\hline IV9202A-720-2 $2^{\star \star}$ & 18.5846 & 0.0441 & 0.0000 \\
\hline IV9202A-720-2*ネ* & 22.0196 & 45.9015 & 0.8415 \\
\hline IV9165A-91-3* & 13.3025 & 2.1634 & 0.0237 \\
\hline IV9165A-91-3* & 19.9059 & 0.0501 & 0.0000 \\
\hline IV9165A-91-3 $3^{\star \star \star}$ & -1.9591 & 11.2393 & 0.2347 \\
\hline IV9165A-180-3* & 21.4206 & 1.4982 & 0.0222 \\
\hline IV9165A-180-3** & 37.5012 & 0.0954 & 0.0015 \\
\hline IV9165A-180-3*** & -4.2723 & 30.2429 & 0.3920 \\
\hline IV9165A-180-4* & 25.4621 & 0.4706 & 0.0068 \\
\hline IV9165A-180-4 & 22.1410 & 0.0342 & 0.0003 \\
\hline IV9165A-180-4 & -2.5957 & 23.5626 & 0.5577 \\
\hline IV9165A-360-1* & 4.0138 & 1.0369 & 0.0176 \\
\hline IV9165A-360-1** & 19.4795 & 0.0579 & 0.0008 \\
\hline IV9165A-360-1 & -2.5610 & 15.8233 & 0.3105 \\
\hline IV9165A-360-2* & 1.3561 & 3.2191 & 0.0619 \\
\hline IV9165A-360-2 & & NA & \\
\hline IV9165A-360-2*** & 12.0534 & 31.0648 & 0.6758 \\
\hline IV9165A-720-1* & -0.2872 & 10.7393 & 0.0933 \\
\hline IV9165A-720-1** & 72.5467 & 0.3408 & 0.0018 \\
\hline IV9165A-720-1*** & -1.4419 & 84.0207 & 1.6552 \\
\hline IV9165A-720-2* & 6.7249 & 11.3051 & 0.0852 \\
\hline IV9165A-720-2** & 75.0421 & 0.7039 & 0.0023 \\
\hline IV9165A-720-2 & 20.0073 & 130.2667 & 1.8624 \\
\hline IV9131A-14-1* & 39.6615 & 1.1529 & 0.0126 \\
\hline IV9131A-14-1** & 45.5973 & 1.2336 & 0.0137 \\
\hline IV9131A-14-1" & 3.2961 & 5.5915 & 0.0252 \\
\hline IV9131A-14-2* & 8.0533 & 2.8221 & 0.0275 \\
\hline IV9131A-14-2 & 56.4648 & 0.5318 & 0.0301 \\
\hline IV9131A-14-2 & 1.5176 & 6.7226 & 0.0364 \\
\hline
\end{tabular}


Table C.1 (cont.). Alpha Spectroscopy Counting Results.

\begin{tabular}{|c|c|c|c|}
\hline Sample & $\begin{array}{c}\text { Total Mass } \\
\text { Np (ng) }\end{array}$ & $\begin{array}{c}\text { Total Mass } \\
\text { Pu (ng) }\end{array}$ & $\begin{array}{c}\text { Total Mass } \\
\text { Am }(n g)\end{array}$ \\
\hline IV9131A-28-1" & 3.6920 & 3.5882 & 0.0267 \\
\hline IV9131A-28-1 ** & & NA & \\
\hline IV9131A-28-1*** & 2.8730 & 16.3483 & 0.0984 \\
\hline IV9131A-28-2* & 3.6479 & 4.2668 & 0.0380 \\
\hline IV9131A-28-2** & & NA & \\
\hline IV9131A-28-2*** & 3.7875 & 13.1278 & 0.0765 \\
\hline IV9131A-56-1* & 144.1353 & 3.9765 & 0.0273 \\
\hline IV9131A-56-1** & 27.0170 & 0.2117 & 0.0002 \\
\hline IV9131A-56-1*** & -0.2189 & 27.8010 & 0.1942 \\
\hline IV9131A-56-2* & 8.1880 & 4.8513 & 0.0332 \\
\hline IV9131A-56-2 & 55.6664 & 0.4037 & 0.0009 \\
\hline IV9131A-56-2 & -1.6098 & 28.5367 & 0.2340 \\
\hline IV9131A-56-3* & 7.2284 & 1.3366 & 0.0125 \\
\hline IV9131A-56-3** & 34.2925 & 0.0105 & 0.0000 \\
\hline IV9131A-56-3*** & -2.1815 & 13.0451 & 0.0429 \\
\hline IV9131A-56-4* & 1.6655 & 3.6110 & 0.0376 \\
\hline IV9131A-56-4** & 0.4985 & 0.4209 & 0.0003 \\
\hline IV9131A-56-4 & 9.4533 & 21.0519 & 0.1363 \\
\hline IV9131A-180-3* & 15.3929 & 6.0313 & 0.0256 \\
\hline IV9131A-180-3** & 49.0444 & 0.8922 & 0.0034 \\
\hline IV9131A-180-3*** & -6.4673 & 68.4914 & 0.5941 \\
\hline IV9131A-720-1* & -0.5982 & 21.8230 & 0.1704 \\
\hline IV9131A-720-1 *\# & 59.4752 & 0.7969 & 0.0048 \\
\hline IV9131A-720-1*** & -9.4098 & 45.5959 & 1.2477 \\
\hline IV9131A-720-2* & -0.2887 & 19.5477 & 0.1572 \\
\hline IV9131A-720-2** & 43.1280 & 1.0182 & 0.0049 \\
\hline IV9131A-720-2*** & -9.4098 & 64.4550 & 0.8810 \\
\hline
\end{tabular}

Disintegrations/Second $=$ [[Sample Counts/Live Time (sec)] - Bkg. Counts (disc/sec)]/Detector Efficiency Half-lives (isotope, years): Np-237, 2.14E6; Pu-239, 2.411E4; Am-24, 432.

Aliquot Concentration $(\mathrm{ng} / \mathrm{ml})=$

[[Disint./sec * $1 / 2$ life $(\mathrm{y})$ * sec/y/ln 2]/6.02205E23] * [[237 * $1 \mathrm{E} 6(\mu \mathrm{g} / \mathrm{g})] /$ Aliquot vol. $(\mathrm{ml})]$

Total Mass $(\mathrm{ng})=$ Aliquot Conc. $(\mathrm{ng} / \mathrm{ml}){ }^{*}$ Soln. Vol. $(\mathrm{ml})$

".", unfiltered solution; "..", filtered solution; "...", acid strip solution 
Distribution for ANL-97/15

Internal:

T. A. Abrajano

J. K. Bates (10)

J. E. Battles

J. C. Cunnane
J. E. Harmon

J. E. Helt

T. R. Johnson
J. J. Laidler

D. M. Strachan

TIS Files

\section{External:}

DOE-OSTI (2)

ANL-E Library

ANL-W Library

Manager, Chicago Operations Office, DOE

A. Bindokas, DOE-CH

J. C. Haugen, DOE-CH

Chemical Technology Division Review Committee Members:

H. U. Anderson, University of Missouri-Rolla, Rolla, MO

E. R. Beaver, Monsanto Company, St: Louis, MO

D. L. Douglas, Consultant, Bloomington, $\mathrm{MN}$

R. K. Genung, Oak Ridge National Laboratory, Oak Ridge, TN

J. G. Kay, Drexel University, Philadelphia, PA

R. A. Osteryoung, North Carolina State University, Raleigh, NC

G. R. St. Pierre, The Ohio State University, Columbus, OH

T. Ahn, U. S. Nuclear Regulatory Commission, Washington, DC

D. H. Alexander, USDOE, Civilian Radioactive Waste Management, Washington, DC

J. Allison, Westinghouse Savannah River Company, Aiken, SC

M. J. Apted, QuantiSci, Denver, CO

S. Bates, Idaho Falls, ID

H. Benton, B\&W Fuel Company, Las Vegas, NV

A. Berusch, USDOE, Office of Civilian Radioactive Waste Management, Washington, DC

N. E. Bibler, Westinghouse Savannah River Company, Aiken, SC

J. M. Boak, USDOE, Yucca Mountain Site, Las Vegas, NV

K. Boomer, Lockheed Martin Hanford Company, Richland, WA

W. Bourcier, Lawrence Livermore National Laboratory, Livermore, CA

E. T. Bramlitt, Defense Nuclear Agency, Kirtland Air Force Base, Kirtland, NM

A. Brandstetter, Science Applications International Corp., Las Vegas, NV

J. Bucher, Lawrence Berkeley Laboratory, Berkeley, CA

J. Canepa, Los Alamos National Laboratory, Los Alamos, NM

K. A. Chacey, USDOE, Office of Environmental Management, Germantown, MD

D. Chestnut, Lawrence Livermore National Laboratory, Livermore, CA

G. R. Choppin, Florida State University, Tallahassee, FL

S. Clark, University of Georgia, Savannah River Ecology Laboratory, Aiken, SC

P. Cloke, Science Applications International Corp., Las Vegas, NV

M. O. Cloninger, Mac Technical Services, Inc., Richland, WA

D. Codell, U. S. Nuclear Regulatory Commission, Washington, DC 
G. Colten-Bradley, U. S. Nuclear Regulatory Commission, Rockville, MD

S. Coplan, U. S. Nuclear Regulatory Commission, Washington, DC

J. Davidson, U. S. Environmental Protection Agency, Washington, DC

J. Docka, Roy F. Weston, Inc., Washington, DC

R. Dresser, Roy F. Weston, Inc., Washington, DC

R. S. Dyer, Yucca Mountain Project Office, Las Vegas, NV

R. E. Erickson, USDOE, Office of Environmental Management, Germantown, MD

E. Essington, Los Alamos National Laboratory, Los Alamos, NM

R. C. Ewing, University of Michigan, Ann Arbor, MI

D. Farr, Los Alamos National Laboratory, Los Alamos, NM

R. Fish, B\&W Fuel Company, Las Vegas, NV

J. Gauthier, Sandia National Laboratories, Albuquerque, NM

F. Gelbard, Sandia National Laboratories, Albuquerque, NM

C. P. Gertz, USDOE, Yucca Mountain Project Office, Las Vegas, NV

S. E. Gomberg, USDOE, Office of Civilian Radioactive Waste Management, Washington, DC

W. Gray, Pacific Northwest National Laboratory, Richland, WA

D. Harrison, Yucca Mountain Project Office, Las Vegas, NV

P. W. Hart, USDOE, Office of Technology Development, Germantown, MD

J. Hennessey, USDOE, Office of Waste Management, Germantown, MD

J. Herzog, Lockheed Idaho Technology Company, Idaho Falls, m

D. Hobart, LANL, TTSO Group, Germantown, MD

J. Hunt, University of California, Berkeley, CA

D. Hutchins, Martin Marietta Systems, Inc., Oak Ridge, TN

C. Interrante, U. S. Nuclear Regulatory Commission, Washington, DC

V. J. Jain, West Valley Nuclear Services, West Valley, NY

C. Jantzen, Westinghouse Savannah River Company, Aiken, SC

L. J. Jardine, Lawrence Livermore National Laboratory, Livermore, CA

P. Kearl, Oak Ridge National Laboratory, Grand Junction, CO

J. Keith, Daniel B. Stephens \& Associates, Albuquerque, NM

J. Kerrisk, Los Alamos National Laboratory, Los Alamos, NM

W. S. Ketola, USDOE, West Valley Project Office, West Valley, NY

D. A. Knecht, Idaho National Engineering and Environmental Laboratory, Idaho Falls, ID

W. L. Kuhn, Pacific Northwest National Laboratory, Richland, WA

W. Lee, Environmental Evaluation Group, Albuquerque, NM

W. W. Lee, University of California, Berkeley, CA

J. C. Lehr, USDOE, Office of Environmental Restoration, Germantown, MD

W. Lemons, Los Alamos National Laboratory, Los Alamos, NM

S. Levy, Los Alamos National Laboratory, Los Alamos, NM

D. Livingston, USDOE, Yucca Mountain Site, Las Vegas, NV

J. J. Lorenz, USDOE, Yucca Mountain Site, Las Vegas, NV

R. Luce, Nuclear Waste Technical Review Board, Arlington, VA

W. Lutze, University of New Mexico, Albuquerque, NM

H. Manaktala, Southwest Research Institute, San Antonio, TX

S. Martin, Lawrence Livermore National Laboratory, Livermore, CA

J. M. Matuszek, JMM Consulting, Del Mar, NY

J. McCarthy, Oak Ridge National Laboratory, Oak Ridge, TN

L. McDowell-Boyer, Oak Ridge National Laboratory, Grand Junction, CO 
T. W. McIntosh, USDOE, Office of Waste Management, Germantown, MD

J. Meldrum, University of Nevada, Las Vegas, NV

A. Mitchell, Los Alamos National Laboratory, Los Alamos, NM

R. Morissette, Science Applications International Corp., Las Vegas, NV

D. Morris, Los Alamos National Laboratory, Los Alamos, NM

F. A. Mumpton, SUNY---College at Brockport, Brockport, NY

W. M. Murphy, Southwest Research Institute, San Antonio, TX

P. K. Nair, Southwest Research Institute, San Antonio, TX

S. Nelson, M \& O Woodward-Clyde, Las Vegas, NV

B. Newman, Los Alamos National Laboratory, Los Alamos, NM

C. Novak, Sandia National Laboratories, Albuquerque, NM

E. Nuttall, University of New Mexico, Albuquerque, NM

W. O'Connell, Lawrence Livermore National Laboratory, Livermore, CA

G. C. S. Ordaz, USDOE, Office of Technology Development, Germantown, MD

C. Palmer, Lawrence Livermore National Laboratory, Livermore, CA

R. Palmer, West Valley Nuclear Services, West Valley, NY

H. Papenguth, Sandia National Laboratories, Albuquerque, NM

W. D. Pearson, Westinghouse Savannah River Company, Aiken, SC

T. H. Pigford, University of California, Berkeley, CA

M. J. Plodinec, Mississippi State University, Mississippi State, MS

W. Polzer, Los Alamos National Laboratory, Los Alamos, NM

P. Reimus, Los Alamos National Laboratory, Los Alamos, NM

B. Robinson, Los Alamos National Laboratory, Los Alamos, NM

P. Rogers, Los Alamos National Laboratory, Los Alamos, NM

R. Rundberg, Los Alamos National Laboratory, Los Alamos, NM

C. G. Russomanno, USDOE, Civilian Radioactive Waste Management, Washington, DC

J. Ryan, University of Colorado, Boulder, CO

R. Schulze, Los Alamos National Laboratory, Los Alamos, NM

W. C. Schutte, USDOE, Office of Technology Development, Germantown, MD

M. Siegel, Sandia National Laboratories, Albuquerque, NM

E. Siegmann, INTERA, Las Vegas, NV

M. Silva, Environmental Evaluation Group, Albuquerque, NM

R. Silva, Lawrence Livermore National Laboratory, Livermore, CA

A. Simmons, USDOE, Las Vegas, NV

E. Springer, Los Alamos National Laboratory, Los Alamos, NM

J. Sproull, Westinghouse Savannah River Company, Aiken, SC

D. Stahl, Faramatome Cogema Fuels, Las Vegas, NV

W. Steinkampf, U.S. Geological Survey, Lakewood, CO

L. Stetzenbach, University of Nevada, Las Vegas, NV

R. B. Stout, Lawrence Livermore National Laboratory, Livermore, CA

D. Stucher, USDOE, Las Vegas, NV

M. Tomozawa, Rensselaer Polytechnic Institute, Troy, NY

V. Trice, USDOE, Office of Waste Management, Germantown, MD

D. Turner, Southwest Research Institute, San Antonio, TX

B. Viani, Lawrence Livermore National Laboratory, Livermore, CA

J. Wan, New Mexico Tech, Socorro, NM

L. Wang, University of New Mexico, Albuquerque, NM 
B. Weber, Pacific Northwest National Laboratory, Richland, WA

M. Whitbeck, Desert Research Institute, University of Nevada, Reno, NV

C. N. Wilson, Lockheed Martin Hanford Company, Richland, WA

J. Wilson, New Mexico Tech, Socorro, NM

M. Wilson, Sandia National Laboratories, Albuquerque, NM

J. H. Wolfram, Montana State University, Bozeman, MT

K. Wolfsberg, Los Alamos National Laboratory, Los Alamos, NM

A. Wollerman, Science Applications International Corp., Germantown, MD

D. J. Wronkiewicz, University of Missouri, Rolla, MO

A. Yang, U.S. Geological Survey, Denver, CO

M. P. Gardiner, Harwell Laboratory, Didcot, Oxon, UNITED KINGDOM

B. Grambow, Kernforschungszentrum Karlsruhe, GmbH, GERMANY

L. Johnson, Atomic Energy of Canada, Ltd., Pinawa, Manitoba, CANADA

J. Kim, Kernforschungszentrum Karlsruhe, GERMANY

H. Nitsche, Forschungszentrum Rossendorf e.V., Institut fur Radiochemie, GERMANY

T. Payne, Australian Nuclear Science and Technology Organization, AUSTRALIA

P. Van Iseghem, Boeretang, BELGIUM

E. Vernaz, Centre d'Etudes Nucleares de la Valle du Rhone, Marcoule, FRANCE

P. Vilks, Atomic Energy of Canada, Pinawa, Manitoba, CANADA

L. Werme, Svensk Karnbranslehantering AB, Stockholm, SWEDEN

Z. P. Zagorski, Institute of Nuclear Chemistry \& Technology, Dorodna, Warszawa, POLAND 